\title{
Rh(I)-catalyzed allenic Pauson-Khand reaction to access the thapsigargin core: Influence of furan and allenyl chloroacetate groups on enantioselectivity
}

Eric D. Deihl' ${ }^{1}$, Luke T. Jesikiewicz ${ }^{1}$, Logan J. Newman¹, Peng Liu'1, 2*, and Kay M.

Brummond $^{1 *}$

${ }^{1}$ Department of Chemistry, University of Pittsburgh, Pittsburgh, Pennsylvania 15260, United

States

${ }^{2}$ Department of Chemical and Petroleum Engineering, University of Pittsburgh, Pittsburgh,

Pennsylvania 15261, United States

Corresponding authors: kbrummon@pitt.edu; pengliu@pitt.edu

\section{Table of Contents}

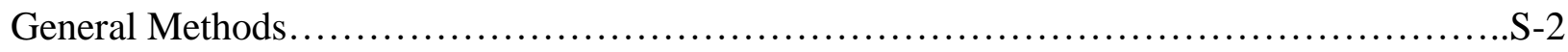

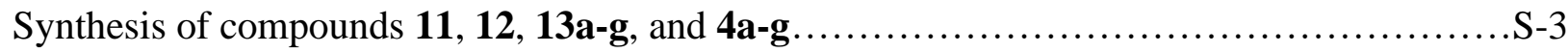

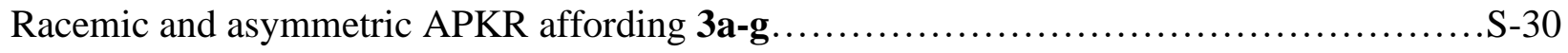

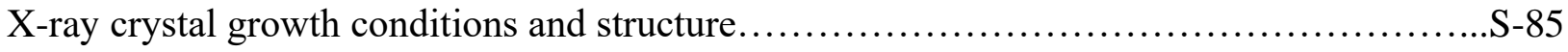

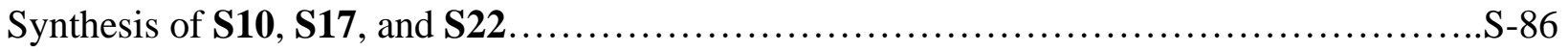

Racemic and asymmetric APKR affording S11, S18, and S23 ...................... -94

Mechanistic studies of aldehyde formation....................................... 121

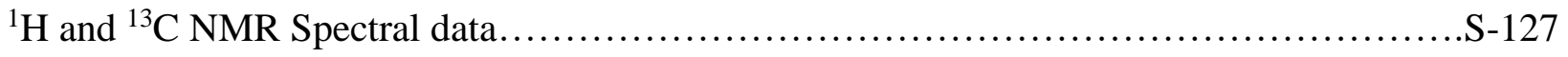

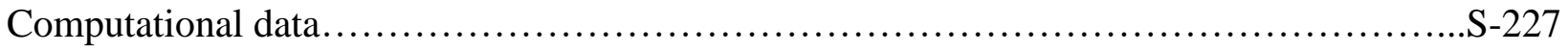

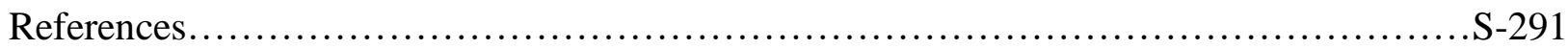




\section{General Methods}

All commercially available compounds were purchased and used as received unless otherwise noted. Tetrahydrofuran (THF), diethyl ether $\left(\mathrm{Et}_{2} \mathrm{O}\right)$, and dichloromethane $\left(\mathrm{DCM}, \mathrm{CH}_{2} \mathrm{Cl}_{2}\right)$ were purified by passing through alumina using a Sol-Tek ST-002 solvent purification system. Toluene was purified by distilling from calcium hydride. Acetone was purified by refluxing over, and distillation from, $\mathrm{KMnO}_{4}$ and stored over 4 Å molecular sieves. $o$-DCB was degassed using freezepump-thaw method (3x). Triphenylphosphine was purified by recrystallization with ethanol. Carbon monoxide (>99.99\%) and 10\% carbon monoxide in argon were purchased from Matheson. $33 \%$ (ww) $\mathrm{NaOH}$ was prepared. Purification of the compounds by flash column chromatography was performed using silica gel $(40-63 \mu \mathrm{m}$ particle size, $60 \AA$ pore size). TLC analyses were performed on silica gel F254 glass plates (250 $\mu$ m thickness). ${ }^{1} \mathrm{H}$ and ${ }^{13} \mathrm{C}$ NMR spectra were recorded on Bruker Avance 300, 400, or $500 \mathrm{MHz}$ spectrometers. Spectra were referenced to residual chloroform (7.26 ppm, ${ }^{1} \mathrm{H}$ NMR; $77.16 \mathrm{ppm},{ }^{13} \mathrm{C}$ NMR). Chemical shifts are reported in ppm, multiplicities are indicated by s (singlet), $d$ (doublet), $t$ (triplet), q (quartet), $p$ (pentet), and m (multiplet). Coupling constants, $J$, are reported in hertz (Hz). All NMR spectra were obtained at rt. IR spectra were obtained using a Nicolet Avatar E.S.P. 360 FT-IR or Perkin Elmer Spectrum Two FT-IR. ESI mass spectrometry was performed on a Waters Micromass GCT high resolution mass spectrometer, while ES mass spectrometry was performed on a Waters Q-TOF Ultima API, Micromass UK Limited high resolution mass spectrometer. Chiral HPLC was performed on a Waters 600 HPLC using a Waters 996 photodiode array detector using a Chiralcel OD chiral column or ChiralPak IA-3 chiral column. Optical rotations were measured using a Jasco P2000 polarimeter. 


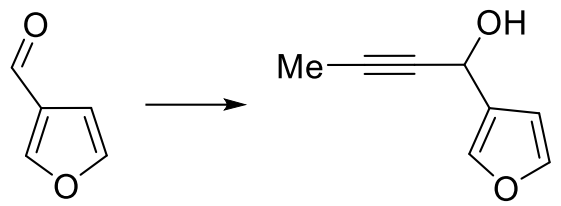

5

S1

1-(furan-3-yl)but-2-yn-1-ol (S1). This compound was prepared in a manner similar to that previously reported with some modifications. ${ }^{1}$ A flame-dried, 2-necked, 25-mL round-bottomed flask equipped with a magnetic stir bar, nitrogen inlet adapter, and septum was charged with 3furfural $(5)(0.1 \mathrm{~mL}, 1.16 \mathrm{mmol})$ and THF $(1.16 \mathrm{~mL})$. The solution was cooled by placing the flask in an ice-bath. Propynyl magnesium bromide (3.48 $\mathrm{mL}$ of a $0.5 \mathrm{M}$ solution in THF, $1.74 \mathrm{mmol})$ was added dropwise via syringe over $3 \mathrm{~min}$. After $1 \mathrm{~h}$ at $0{ }^{\circ} \mathrm{C}$, TLC showed complete consumption of starting material. Saturated aqueous ammonium chloride $(1 \mathrm{~mL})$ was added all at once. The reaction mixture was transferred to a separatory funnel. The aqueous phase was extracted with diethyl ether ( $3 \times 5 \mathrm{~mL})$, dried over magnesium sulfate, filtered, and concentrated in vacuo. The crude residue was purified by running through a short plug of silica gel (20\% ethyl acetate/hexanes) to afford $145 \mathrm{mg}(92 \%$ yield) of the title compound as a light-yellow oil.

\section{EDD5-372}

$\underline{{ }^{1} \mathrm{H} \mathrm{NMR}} \quad\left(300 \mathrm{MHz}, \mathrm{CDCl}_{3}\right)$

$7.51(\mathrm{~s}, 1 \mathrm{H}), 7.39(\mathrm{~m}, 1 \mathrm{H}), 6.50(\mathrm{~s}, 1 \mathrm{H}), 5.37(\mathrm{~s}, 1 \mathrm{H}), 2.03(\mathrm{~d}, J=5.4 \mathrm{~Hz}, 1 \mathrm{H})$, $1.9(\mathrm{~d}, J=2.4 \mathrm{~Hz}, 3 \mathrm{H}) \mathrm{ppm}$

${ }^{13} \mathrm{C} \mathrm{NMR} \quad\left(75 \mathrm{MHz}, \mathrm{CDCl}_{3}\right)$

$143.7,140.2,127.1,109.3,81.8,78.8,57.6,3.7$ ppm

$\underline{\text { IR }} \quad$ (Thin Film) 
$3371,2229 \mathrm{~cm}^{-1}$

$\underline{\text { HRMS }} \quad$ HRMS-ESI (m/z): $[\mathrm{M}+\mathrm{H}]^{+}$calcd for $\mathrm{C}_{8} \mathrm{H}_{9} \mathrm{O}_{2}, 137.0597$; found, 137.0593

$\underline{\text { TLC }} \quad R_{f}=0.41\left(35 \%\right.$ ethyl acetate/hexanes); silica gel, UV, $\mathrm{KMnO}_{4}$

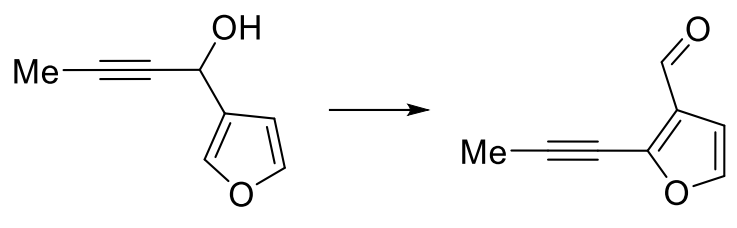

S1

11

2-(prop-1-yn-1-yl)furan-3-carbaldehyde (11). This compound was prepared in a manner similar to that previously reported with some modifications. ${ }^{1}$ A flame-dried, 2-necked, $100-\mathrm{mL}$ roundbottomed flask equipped with a magnetic stir bar, nitrogen inlet adapter, and septum was charged with 1-(furan-3-yl)but-2-yn-1-ol (S1) (1.013g, $7.44 \mathrm{mmol})$, THF (29.8 mL, 0.25 M), and water (7.44 mL). $N$-bromosuccinimide $(1.324 \mathrm{~g}, 7.44 \mathrm{mmol})$ was added in two portions about 10 minutes apart by temporary removal of the septum. After $3 \mathrm{~h}$, TLC showed complete consumption of starting material. $1 \mathrm{M} \mathrm{HCl}(7.44 \mathrm{~mL})$ was added to the solution and stirred for $10 \mathrm{~min}$. The solution was transferred to a separatory funnel and the aqueous layer was extracted with diethyl ether $(5 \mathrm{x}$ $10 \mathrm{~mL})$. The combines organic layers were washed with brine $(1 \times 10 \mathrm{~mL})$, dried over magnesium sulfate, filtered and concentrated in vacuo. The crude residue was purified by silica gel flash chromatography (7.5-15\% ethyl acetate/hexanes) to afford $693 \mathrm{mg}$ (69\% yield) of the title compound as a brown liquid that solidified to a sticky brown solid upon freezing.

\section{EDD5-375}

MP $\quad 41.6-43.3^{\circ} \mathrm{C}$

$\underline{{ }^{1} \mathrm{H} \mathrm{NMR}} \quad\left(400 \mathrm{MHz}, \mathrm{CDCl}_{3}\right)$ 
$9.98(\mathrm{~s}, 1 \mathrm{H}), 7.32(\mathrm{~d}, J=1.8 \mathrm{~Hz}, 1 \mathrm{H}), 6.73(\mathrm{~d}, J=1.8 \mathrm{~Hz}, 1 \mathrm{H}), 2.19(\mathrm{~s}, 3 \mathrm{H})$

ppm

${ }^{13} \mathrm{C} \mathrm{NMR} \quad\left(100 \mathrm{MHz}, \mathrm{CDCl}_{3}\right)$

$185.0,145.8,143.9,128.8,107.7,97.0,68.1,4.9 \mathrm{ppm}$

$\underline{\text { IR }} \quad$ (Thin Film)

$3153,3127,2833,2740,2246,2215,1786,1726,1680,1571,1504,1276 \mathrm{~cm}^{-1}$

$\underline{\text { HRMS }}$ HRMS-ESI (m/z): $[\mathrm{M}+\mathrm{H}]^{+}$calcd for $\mathrm{C}_{8} \mathrm{H}_{7} \mathrm{O}_{2}, 135.0446$; found, 135.0451

TLC $\quad R_{f}=0.56\left(35 \%\right.$ ethyl acetate/hexanes); silica gel, UV, $\mathrm{KMnO}_{4}$

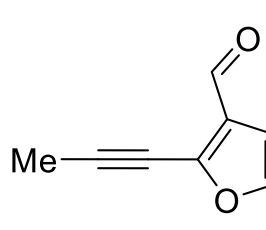

11

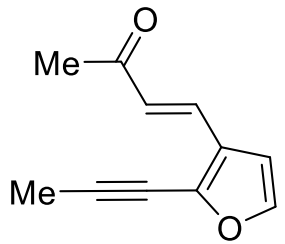

S2

(E)-4-(2-(prop-1-yn-1-yl)furan-3-yl)but-3-en-2-one (S2). This compound was prepared in a manner similar to that previously reported with some modifications. ${ }^{2}$ A flame-dried, 2-necked, 25$\mathrm{mL}$, round-bottomed flask equipped with a magnetic stir bar, nitrogen inlet adapter, and septum was charged with 2-(prop-1-yn-1-yl)furan-3-carbaldehyde (11) (822 mg, $6.12 \mathrm{mmol})$ by temporary removal of the septum. The flask was flushed with nitrogen (3x) using a double Schlenk manifold (vacuum and nitrogen). Deionized water $(5.2 \mathrm{~mL}, 1.2 \mathrm{M})$ and acetone $(1.12 \mathrm{~mL}, 15.3 \mathrm{mmol})$ were added dropwise via syringe over 2 minutes. The solution was cooled in an ice-bath, and 33\% (w/w) $\mathrm{NaOH}(0.15 \mathrm{~mL}, 1.22 \mathrm{mmol})$ was added dropwise over $1 \mathrm{~min}$. The solution was allowed to warm to $\mathrm{rt}$ and maintained for $3 \mathrm{~h}$, at which point TLC showed complete consumption of starting 
material. 10\% Sulfuric acid was added until the solution reached $\mathrm{pH} 4$ as determined using $\mathrm{pH}$ paper. The resulting solution was transferred to a separatory funnel and the organic layer separated. The aqueous layer was extracted with $\mathrm{CHCl}_{3}(5 \times 5 \mathrm{~mL})$, the combined organic layers were washed with water $(1 \times 5 \mathrm{~mL})$, and the combined aqueous layers back extracted with $\mathrm{CHCl}_{3}(1 \times 5 \mathrm{~mL})$. The combined organic layers were dried over magnesium sulfate, filtered, and concentrated in vacuo. The crude residue was purified by silica gel flash chromatography (10-15\% ethyl acetate/hexanes) to afford $713 \mathrm{mg}$ (67\% yield) of the title compound as a yellow solid.

\section{EDD4-309}

MP $\quad 63-65^{\circ} \mathrm{C}$

$\underline{{ }^{1} \mathrm{H} \mathrm{NMR}} \quad\left(400 \mathrm{MHz}, \mathrm{CDCl}_{3}\right)$

$7.45(\mathrm{~d}, J=16.2 \mathrm{~Hz}, 1 \mathrm{H}), 7.30(\mathrm{~d}, J=2.0 \mathrm{~Hz}, 1 \mathrm{H}), 6.55(\mathrm{~d}, J=2.4 \mathrm{~Hz}, 1 \mathrm{H})$,

$6.48(\mathrm{~d}, J=16.2 \mathrm{~Hz}, 1 \mathrm{H}), 2.36(\mathrm{~s}, 3 \mathrm{H}), 2.19(\mathrm{~s}, 3 \mathrm{H}) \mathrm{ppm}$

${ }^{13} \mathrm{C} \mathrm{NMR} \quad\left(100 \mathrm{MHz}, \mathrm{CDCl}_{3}\right)$

198.4, 143.8, 139.7, 133.4, 127.6, 125.1, 108.1, 96.8, 68.9, 27.6, 5.1 ppm

$\underline{\text { IR }} \quad$ (Thin Film)

$2922,2228,1666,1612,1258,1228 \mathrm{~cm}^{-1}$

$\underline{\text { HRMS }}$ HRMS-ESI (m/z): $[\mathrm{M}+\mathrm{H}]^{+}$calcd for $\mathrm{C}_{11} \mathrm{H}_{11} \mathrm{O}_{2}, 175.0759$; found, 175.0749

$\underline{\text { TLC }} \quad R_{f}=0.5$ (35\% ethyl acetate/hexanes); silica gel, UV, $p$-anisaldehyde 


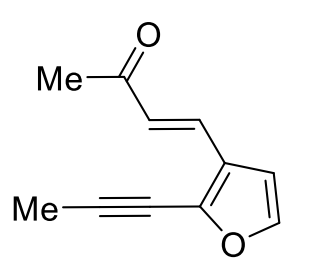

S2

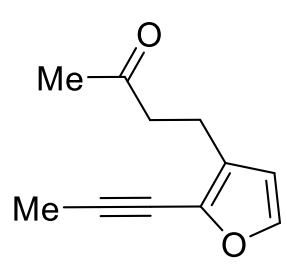

12

4-(2-(prop-1-yn-1-yl)furan-3-yl)butan-2-one (12). This compound was prepared in a manner similar to that previously reported with some modifications. ${ }^{3,4}$ A flame-dried, 2-necked, 25-mL, round-bottomed flask equipped with a magnetic stir bar, condenser with septum and nitrogen inlet needle, and septum in the second arm was charged with (E)-4-(2-(prop-1-yn-1-yl)furan-3-yl)but3-en-2-one (S2)(171 mg, $0.982 \mathrm{mmol})$ in toluene $(9.8 \mathrm{~mL}, 0.1 \mathrm{M})$ via syringe. Diethyl 2,6dimethyl-1,4-dihydropyridine-3,5-dicarboxylate (Hantzsch ester) (746 mg, $2.946 \mathrm{mmol}$ ) and silica gel $(1.473 \mathrm{~g}, 1.5 \mathrm{~g} / \mathrm{mmol})$ were added sequentially by temporary removal of the septum. The flask was lowered into a preheated $110{ }^{\circ} \mathrm{C}$ oil bath and maintained for $22 \mathrm{~h}$, at which time the TLC showed complete consumption of starting material. The solids were removed by filtration with the aid of ethyl acetate $(25 \mathrm{~mL})$. The filtrate was transferred to a separatory funnel, washed with $2 \mathrm{~N}$ $\mathrm{HCl}(5 \times 10 \mathrm{~mL})$, washed with brine, dried over magnesium sulfate, filtered, and concentrated in vасио. In cases where the residue contained Hantzsch ester as determined by ${ }^{1} \mathrm{H} \mathrm{NMR}$, it was diluted in ethyl acetate $(10 \mathrm{~mL})$ and washed with $2 \mathrm{~N} \mathrm{HCl}(3 \times 10 \mathrm{~mL})$. The combined aqueous layers were back extracted with ethyl acetate $(2 \times 5 \mathrm{~mL})$. The combined organic layers were dried over magnesium sulfate, filtered, and concentrated in vacuo. The crude residue was purified by silica gel flash chromatography (2.5-5\% ethyl acetate/hexanes) to afford $125 \mathrm{mg}$ (74\% yield) of the title compound as a yellow oil.

\section{EDD5-342}

${ }^{1} \mathrm{H} \mathrm{NMR} \quad\left(400 \mathrm{MHz}, \mathrm{CDCl}_{3}\right)$ 
$7.22(\mathrm{~d}, J=2.0 \mathrm{~Hz}, 1 \mathrm{H}), 6.23(\mathrm{~d}, J=2.0 \mathrm{~Hz}, 1 \mathrm{H}), 2.77-2.68(\mathrm{~m}, 4 \mathrm{H}), 2.16(\mathrm{~s}, 3$

H), $2.11(\mathrm{~s}, 3 \mathrm{H}) \mathrm{ppm}$; Impurities at $4.09(\mathrm{q}, J=6.8 \mathrm{~Hz}, 0.26 \mathrm{H}), 1.42(\mathrm{t}, J=7.2$

$\mathrm{Hz}, 0.37 \mathrm{H}) \mathrm{ppm}$.

${ }^{13} \mathrm{C} \mathrm{NMR} \quad\left(100 \mathrm{MHz}, \mathrm{CDCl}_{3}\right)$

208.0, 142.4, 134.8, 127.5, 111.7, 93.0, 69.5, 43.7, 30.1, 19.5, $4.8 \mathrm{ppm}$

$\underline{\mathrm{IR}} \quad$ (Thin Film)

$2919,1714,1494,1434,1362,1252,1163,1080 \mathrm{~cm}^{-1}$

$\underline{\text { HRMS }}$ HRMS-ESI (m/z): $[\mathrm{M}+\mathrm{H}]^{+}$calcd for $\mathrm{C}_{11} \mathrm{H}_{13} \mathrm{O}_{2}, 177.0916$; found, 177.0912

TLC $\quad R_{f}=0.59$ (35\% ethyl acetate/hexanes); silica gel, UV, $p$-anisaldehyde

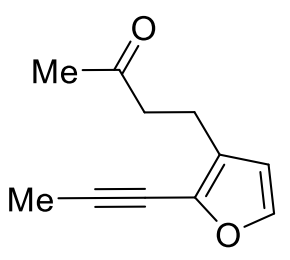

12

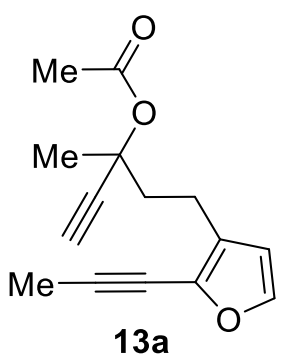

$13 a$

3-methyl-5-(2-(prop-1-yn-1-yl)furan-3-yl)pent-1-yn-3-yl acetate (13a). This compound was prepared in a manner similar to that previously reported with some modifications. ${ }^{\mathbf{5}}$ A flame-dried, 2-necked, 25-mL, round bottomed flask equipped with a magnetic stir bar, nitrogen inlet adapter, and septum was charged with 4-(2-(prop-1-yn-1-yl)furan-3-yl)butan-2-one (12) (124 mg, 0.70 mmol) and THF (2.4 mL). The solution was cooled by placing the flask in an ice-bath and ethynylmagnesium bromide (4.2 $\mathrm{mL}$ of a $0.5 \mathrm{M}$ solution in THF, $2.1 \mathrm{mmol}, 3$ equiv), was added dropwise via syringe over $5 \mathrm{~min}$. After allowing to warm to rt over $90 \mathrm{~min}$, TLC showed complete 
consumption of starting material. The flask was placed in an ice-bath and acetyl chloride $(0.25$ $\mathrm{mL}, 3.52 \mathrm{mmol}$ ) was added dropwise via syringe over $2 \mathrm{~min}$. After $2 \mathrm{~h}$, TLC showed complete consumption of the propargyl alcohol intermediate. Diethyl ether $(5 \mathrm{~mL})$ and deionized water $(5$ $\mathrm{mL}$ ) were added to the flask, the mixture was transferred to a separatory funnel, and the organic layer was separated. The aqueous layer was extracted with diethyl ether $(3 \times 15 \mathrm{~mL})$, washed with brine, the combined aqueous layers back extracted with diethyl ether $(1 \times 15 \mathrm{~mL})$, dried over magnesium sulfate, filtered, and concentrated in vacuo. The crude residue was purified by silica gel flash chromatography (5\% ethyl acetate/hexanes) to give $101 \mathrm{mg}(59 \%)$ of the title compound as a clear liquid.

\section{EDD2-106}

$\underline{{ }^{1} \mathrm{H} \mathrm{NMR}} \quad\left(400 \mathrm{MHz}, \mathrm{CDCl}_{3}\right)$

$7.23(\mathrm{~d}, J=1.6 \mathrm{~Hz}, 1 \mathrm{H}), 6.27$ (d, $J=1.6 \mathrm{~Hz}, 1 \mathrm{H}), 2.70-2.64(\mathrm{~m}, 2 \mathrm{H}), 2.60$ (s, 1

H), 2.23-2.15 (m, 1 H), 2.11 (s, 3 H), 2.08-2.05 (m, 1 H), 2.04 (s, 3 H) ppm

Trace impurities at $4.10(\mathrm{t}, J=6.3 \mathrm{~Hz}, 0.55), 3.44(\mathrm{t}, J=6.6 \mathrm{~Hz}, 0.51) \mathrm{ppm}$

$\stackrel{{ }^{13} \mathrm{C} \mathrm{NMR}}{ } \quad\left(100 \mathrm{MHz}, \mathrm{CDCl}_{3}\right)$

$169.5,142.4,134.9,127.9,111.6,92.9,83.6,74.6,73.7,69.6,41.4,26.5,22.0$,

20.3, $4.7 \mathrm{ppm}$

$\underline{\text { IR }} \quad$ (Thin Film)

$3289,2938,1743,1494,1442,1368,1239,1174,1154,1090,1064,1016 \mathrm{~cm}^{-1}$

$\underline{\text { HRMS }}$ HRMS-ESI (m/z): $[\mathrm{M}+\mathrm{H}]^{+}$calcd for $\mathrm{C}_{15} \mathrm{H}_{17} \mathrm{O}_{3}, 245.1178$; found, 245.1172

$\underline{\text { TLC }} \quad R_{f}=0.66$ (35\% ethyl acetate/hexanes); silica gel, UV, $p$-anisaldehyde 


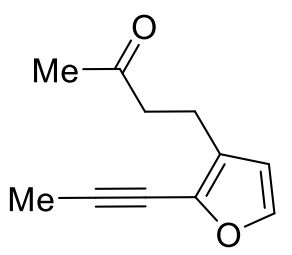

12

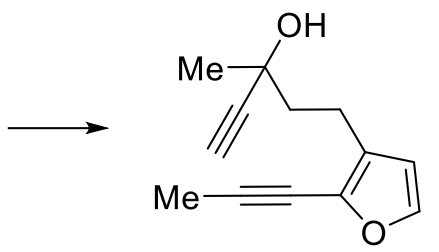

S3

3-methyl-5-(2-(prop-1-yn-1-yl)furan-3-yl)pent-1-yn-3-ol (S3). A flame-dried, single-necked, 50-mL, round-bottomed flask equipped with a magnetic stir bar and septum was charged with cerium trichloride $(389 \mathrm{mg}, 1.58 \mathrm{mmol})$ in a glovebox and sealed with a septum prior to removal. THF (11.3 mL) was added, and the solution stirred at $\mathrm{rt}$ for $17.5 \mathrm{~h}$. The flask was placed in an icebath and ethynylmagnesium bromide $(0.5 \mathrm{M}$ solution, $9.9 \mathrm{~mL}, 4.97 \mathrm{mmol})$ was added via syringe over $5 \mathrm{~min}$. After $1.5 \mathrm{~h}$, a solution of 4-(2-(prop-1-yn-1-yl)furan-3-yl)butan-2-one (12)(199 mg, $1.13 \mathrm{mmol})$ in THF (3 mL) was added via syringe over 3 min. After $1 \mathrm{~h}$, TLC showed complete consumption of starting material. Saturated aqueous ammonium chloride $(2 \mathrm{~mL})$ was added all at once, and the solution was transferred to a separatory funnel, extracted with diethyl ether ( 3 x 5 $\mathrm{mL})$, washed with brine $(1 \times 5 \mathrm{~mL})$, and the combined aqueous layers extracted with diethyl ether (1 x $5 \mathrm{~mL}$ ), dried over magnesium sulfate, filtered and concentrated in vacuo. The crude residue was purified by silica gel flash chromatography (5-20\% ethyl acetate/hexanes) to afford $210 \mathrm{mg}$ (92\% yield) of the title compound as a light-yellow oil.

\section{EDD5-321}

$\underline{{ }^{1} \mathrm{H} \mathrm{NMR}} \quad\left(400 \mathrm{MHz}, \mathrm{CDCl}_{3}\right)$

$7.23(\mathrm{~d}, J=1.6 \mathrm{~Hz}, 1 \mathrm{H}), 6.27(\mathrm{~d}, J=1.6 \mathrm{~Hz}, 1 \mathrm{H}), 2.73-2.68(\mathrm{~m}, 2 \mathrm{H}), 2.49(\mathrm{~s}, 1$

H), 2.11 (s, $3 \mathrm{H}), 1.99$ (s, $1 \mathrm{H}), 1.95-1.90$ (m, 2 H) ppm 
${ }^{13} \mathrm{C} \mathrm{NMR} \quad\left(100 \mathrm{MHz}, \mathrm{CDCl}_{3}\right)$

$142.4,134.8,128.2,111.7,92.9,87.3,71.9,69.6,68.0,43.3,30.0,20.7,4.8$ ppm

$\underline{\mathrm{IR}} \quad$ (Thin Film)

$3293,2919,1494,1451,1373,1284,1154,1099,1064,1023,952,905 \mathrm{~cm}^{-1}$

HRMS $\quad$ HRMS-ESI (m/z): $[\mathrm{M}+\mathrm{H}]^{+}$calcd for $\mathrm{C}_{13} \mathrm{H}_{15} \mathrm{O}_{2}, 203.1072$; found, 203.1063

TLC $\quad R_{f}=0.48$ (35\% ethyl acetate/hexanes); silica gel, UV, $p$-anisaldehyde

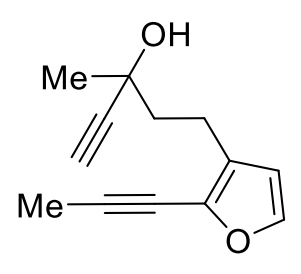

S3

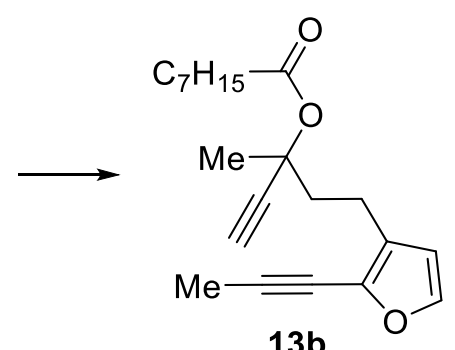

$13 b$

3-methyl-5-(2-(prop-1-yn-1-yl)furan-3-yl)pent-1-yn-3-yl octanoate (13b). A flame-dried, 2necked, $10-\mathrm{mL}$ round-bottomed flask equipped with a magnetic stir bar and septum was charged with 3-methyl-5-(2-(prop-1-yn-1-yl)furan-3-yl)pent-1-yn-3-ol (S3) (84.0 mg, $0.415 \mathrm{mmol})$ in acetonitrile $(1.7 \mathrm{~mL})$ followed by octanoic anhydride $(0.185 \mathrm{~mL}, 0.623 \mathrm{mmol})$. A second flamedried, single-necked, 5-mL, round-bottomed flask was charged with scandium triflate (4.1 mg, $0.0083 \mathrm{mmol}$ ) in a glovebox and sealed with a septum prior to removal. The scandium triflate was dissolved in acetonitrile $(0.42 \mathrm{~mL})$ and this solution was added dropwise via cannula to the first flask over $1 \mathrm{~min}$. After $15 \mathrm{~min}$, TLC showed complete consumption of starting material. Saturated aqueous sodium bicarbonate $(2 \mathrm{~mL})$ was added all at once and the mixture stirred for $30 \mathrm{~min}$. The mixture was transferred to a separatory funnel, diluted with diethyl ether $(3 \mathrm{~mL})$ and water $(2 \mathrm{~mL})$, 
extracted with diethyl ether $(2 \times 5 \mathrm{~mL})$, dried over magnesium sulfate, filtered and concentrated in vacuo. The crude residue was purified by silica gel flash chromatography $(7.5 \%$ ethyl acetate/hexanes) to give $111 \mathrm{mg}$ of the title compound containing octanoic anhydride. This mixture was dissolved in diethyl ether $(5 \mathrm{~mL})$, extracted with $1 \mathrm{~N} \mathrm{NaOH}(5 \times 2 \mathrm{~mL})$, the combined aqueous layers back extracted with diethyl ether $(1 \times 3 \mathrm{~mL})$, combined organic layers dried over magnesium sulfate, filtered and concentrated in vacuo. The title compound contained octanoic anhydride. This mixture was subjected to General Procedure A to give pure compound as a clear liquid.

\section{EDD5-324}

${ }^{1} \mathrm{HNMR} \quad\left(400 \mathrm{MHz}, \mathrm{CDCl}_{3}\right)$

$7.23(\mathrm{~d}, J=1.6 \mathrm{~Hz}, 1 \mathrm{H}), 6.27(\mathrm{~d}, J=1.6,1 \mathrm{H}), 2.69-2.64(\mathrm{~m}, 2 \mathrm{H}), 2.59(\mathrm{~s}, 1 \mathrm{H})$,

$2.27(\mathrm{t}, J=7.2,2 \mathrm{H}), 2.22-2.14(\mathrm{~m}, 1 \mathrm{H}), 2.11(\mathrm{~s}, 3 \mathrm{H}), 2.09-2.01(\mathrm{~m}, 1 \mathrm{H}), 1.73$

(s, $3 \mathrm{H}), 1.62(\mathrm{~m}, 2 \mathrm{H}), 1.30-1.25(\mathrm{~m}, 8 \mathrm{H}), 0.88(\mathrm{~m}, 3 \mathrm{H}) \mathrm{ppm}$; Impurity at 3.67

(s, 0.13), 1.30-1.25 ppm

${ }^{13} \mathrm{C} \mathrm{NMR} \quad\left(100 \mathrm{MHz}, \mathrm{CDCl}_{3}\right)$

172.3, 142.4, 134.8, 127.9, 111.6, 92.8, 83.7, 74.3, 73.7, 69.6, 41.5, 35.2, 31.8, $29.2,29.1,26.6,25.0,22.7,20.2,14.2,4.8 \mathrm{ppm}$

$\underline{\text { IR }} \quad$ (Thin Film)

$1741,1152 \mathrm{~cm}^{-1}$

HRMS HRMS-ESI (m/z): $[\mathrm{M}+\mathrm{H}]^{+}$calcd for $\mathrm{C}_{21} \mathrm{H}_{29} \mathrm{O}_{3}, 329.2117$; found, 329.2110

TLC $\quad R_{f}=0.71$ (35\% ethyl acetate/hexanes); silica gel, UV, $p$-anisaldehyde 
General Procedure A: Removal of anhydride from title compound by reaction with triethylamine, 4-(dimethylamino)pyridine, and methanol

3-methyl-5-(2-(prop-1-yn-1-yl)furan-3-yl)pent-1-yn-3-yl octanoate (13b). A flame-dried, 1necked, 5-mL round-bottomed flask equipped with a magnetic stir bar and septum with nitrogen inlet needle was charged with 3-methyl-5-(2-(prop-1-yn-1-yl)furan-3-yl)pent-1-yn-3-yl octanoate (13b) containing octanoic anhydride (107 mg, $0.326 \mathrm{mmol}, 1$ equiv) in methanol (0.54 mL, 0.6 M). Triethylamine $(0.07 \mathrm{~mL}, 0.489 \mathrm{mmol}, 1.5$ equiv) was added dropwise via syringe over $30 \mathrm{~s}$. The solution was cooled in an ice-bath and 4-(dimethylamino)pyridine (DMAP, $12 \mathrm{mg}, 0.098$ mmol, 0.3 equiv) was added by temporary removal of the septum. After $2 \mathrm{~h}$, saturated aqueous ammonium chloride $(0.5 \mathrm{~mL})$ was added and the solution transferred to a separatory funnel, extracted with diethyl ether ( $5 \times 1 \mathrm{~mL})$, dried over magnesium sulfate, filtered, and concentrated in vacuo. The crude residue was purified by silica gel flash chromatography (5\% ethyl acetate/hexanes) to give $83 \mathrm{mg}$ ( $61 \%$ from $\mathbf{S 3}$ the propargyl alcohol) of the title compound as a clear liquid.

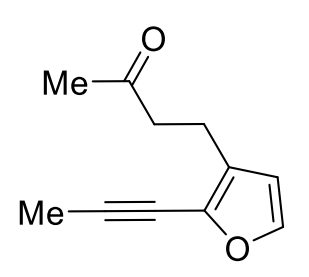

12

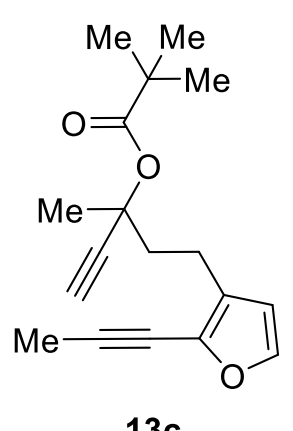

13c

3-methyl-5-(2-(prop-1-yn-1-yl)furan-3-yl)pent-1-yn-3-yl pivalate (13c). This compound was prepared in a manner similar to that previously reported with some modifications. ${ }^{5}$ A flame-dried, 
2-necked, 25-mL, round bottomed flask equipped with a magnetic stir bar, nitrogen inlet adapter, and septum was charged with 4-(2-(prop-1-yn-1-yl)furan-3-yl)butan-2-one (12) (143 mg, 0.811 mmol) and THF $(2.7 \mathrm{~mL})$. The solution was cooled by placing the flask in an ice-bath and ethynylmagnesium bromide ( $2.4 \mathrm{~mL}$ of a $0.5 \mathrm{M}$ solution in THF, $1.22 \mathrm{mmol}, 1.5$ equiv) was added dropwise via syringe over $5 \mathrm{~min}$. After $3 \mathrm{~h}$, the solution was cooled to $0{ }^{\circ} \mathrm{C}$ and ethynylmagnesium bromide (1.6 mL of a $0.5 \mathrm{M}$ solution in THF, $0.8 \mathrm{mmol}$, 1 equiv) was added and stirred for $1 \mathrm{~h}$. The flask was placed in an ice-bath and pivaloyl chloride $(0.35 \mathrm{~mL}, 1.62 \mathrm{mmol}, 3.5$ equiv) was added via syringe. After $4.5 \mathrm{~h}$, TLC showed complete consumption of the propargyl alcohol intermediate. Diethyl ether $(3 \mathrm{~mL})$ and deionized water $(3 \mathrm{~mL})$ were added to the flask, the mixture was transferred to a separatory funnel, and the organic layer was separated. The aqueous layer was extracted with diethyl ether ( $3 \times 5 \mathrm{~mL})$, combined organic layers washed with brine, dried over magnesium sulfate, filtered, and concentrated in vacuo. The crude residue was purified by silica gel flash chromatography (5-10\% ethyl acetate/hexanes) to give $111 \mathrm{mg}(48 \%)$ of the title compound as a clear oil.

\section{EDD5-379}

$\underline{{ }^{1} \mathrm{H} \mathrm{NMR}} \quad\left(500 \mathrm{MHz}, \mathrm{CDCl}_{3}\right)$

$7.24(\mathrm{~d}, J=1.5 \mathrm{~Hz}, 1 \mathrm{H}), 6.27(\mathrm{~d}, J=1.5 \mathrm{~Hz}, 1 \mathrm{H}), 2.72-2.64(\mathrm{~m}, 2 \mathrm{H}), 2.57(\mathrm{~s}, 1$ H), 2.18-2.02 (m, 2 H), 2.10 (s, 3 H), 1.72 (s, 3 H), 1.21 (s, 9 H) ppm; impurities at $1.26(\mathrm{~s}, 2.73 \mathrm{H}), 1.24(\mathrm{~s}, 1.17 \mathrm{H}) \mathrm{ppm}$

${ }^{13} \mathrm{C} \mathrm{NMR} \quad\left(125 \mathrm{MHz}, \mathrm{CDCl}_{3}\right)$

$176.7,142.4,134.9,128.0,111.6,92.8,83.7,73.9,73.4,69.5,41.9,39.4,27.2$, 26.4, 20.2, $4.7 \mathrm{ppm}$; Impurities at 26.7 and $25.7 \mathrm{ppm}$ 
$\underline{\text { IR }} \quad$ (Thin Film)

$1734,1134 \mathrm{~cm}^{-1}$

HRMS $\quad$ HRMS-ESI (m/z): $[\mathrm{M}+\mathrm{H}]^{+}$calcd for $\mathrm{C}_{18} \mathrm{H}_{23} \mathrm{O}_{3}, 287.1642$; found, 287.1642

TLC $\quad R_{f}=0.68$ (35\% ethyl acetate/hexanes); silica gel, UV, $p$-anisaldehyde

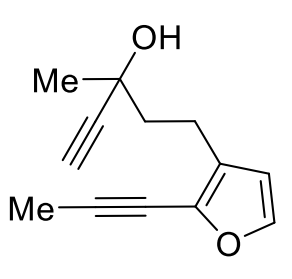

S3

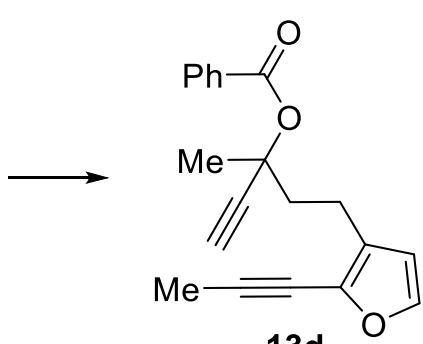

$13 d$

3-methyl-5(2-(prop-1-yn-yl)furan-3-yl)pent-1-yn-3-yl benzoate (13d). This compound was prepared in a manner similar to that previously reported with some modifications. ${ }^{5}$ A flame-dried, 2-necked, 10-mL, round-bottomed flask containing magnetic stir bar, septum and nitrogen inlet adapter was charged with 3-methyl-5-(2-(prop-1-yn-1-yl)furan-3-yl)pent-1-yn-3-ol (S3) (63 mg, $0.311 \mathrm{mmol})$ and DCM $(0.52 \mathrm{~mL}, 0.6 \mathrm{M})$. Triethylamine $(65 \mu \mathrm{L}, 0.467 \mathrm{mmol})$ was added via syringe. 4- $N, N$-(Dimethylamino)pyridine ( $11 \mathrm{mg}, 0.093 \mathrm{mmol}$ ) was added by temporary removal of the septum. The solution was cooled by placing the flask in an ice-bath. Benzoic anhydride (106 $\mathrm{mg}, 0.467 \mathrm{mmol}$ ) was added by temporary removal of the septum. After $46 \mathrm{~h}$, TLC showed small amounts of starting material, but the reaction was not progressing. Saturated aqueous ammonium chloride was added to the flask and the mixture was transferred to a separatory funnel and extracted 
with diethyl ether ( $5 \times 5 \mathrm{~mL})$, dried over magnesium sulfate, filtered, and concentrated in vacuo. The crude residue was purified by silica gel flash chromatography (5\% ethyl acetate/hexanes) to afford $47 \mathrm{mg}$ (49\% yield) of the title compound as a clear oil.

\section{EDD3-134}

${ }^{1} \mathrm{H} \mathrm{NMR} \quad\left(400 \mathrm{MHz}, \mathrm{CDCl}_{3}\right)$

8.03-8.01 (m, $2 \mathrm{H}), 7.58-7.54(\mathrm{~m}, 1 \mathrm{H}), 7.43(\mathrm{t}, J=7.8,2 \mathrm{H}), 7.24(\mathrm{~d}, J=1.8 \mathrm{~Hz}$, $1 \mathrm{H}), 6.31(\mathrm{~d}, J=1.8 \mathrm{~Hz}, 1 \mathrm{H}), 2.86-2.72(\mathrm{~m}, 2 \mathrm{H}), 2.65(\mathrm{~s}, 1 \mathrm{H}), 2.38-2.17$ (m, 2 H), $2.09(\mathrm{~s}, 3 \mathrm{H}), 1.88(\mathrm{~s}, 3 \mathrm{H}) \mathrm{ppm}$; Impurities at 4.1 (q, $J=6.3 \mathrm{~Hz}), 3.44(\mathrm{q}, J=$ $6.6 \mathrm{~Hz}), 1.21(\mathrm{t}, J=7.2 \mathrm{~Hz}$ ) (diethyl ether) ppm

${ }^{13} \mathrm{C} \mathrm{NMR} \quad\left(100 \mathrm{MHz}, \mathrm{CDCl}_{3}\right)$

$164.9,142.4,134.9,133.1,131.0,129.8,128.5,127.9,111.7,92.9,83.5,75.1$, 74.1, 69.6, 41.9, 26.7, 20.4, 4.7 ppm; 6.0 and 15.4 (diethyl ether) ppm

$\underline{\text { IR }} \quad$ (Thin Film)

$3289,2919,2218,1723,1601,1493,1451,1375,1315,1278,1174 \mathrm{~cm}^{-1}$

HRMS $\quad$ HRMS-ESI (m/z): $[\mathrm{M}+\mathrm{H}]^{+}$calcd for $\mathrm{C}_{20} \mathrm{H}_{19} \mathrm{O}_{3}, 307.1334$; found, 307.1341

$\underline{\text { TLC }} \quad R_{f}=0.70$ (35\% ethyl acetate/hexanes); silica gel, UV, $p$-anisaldehyde

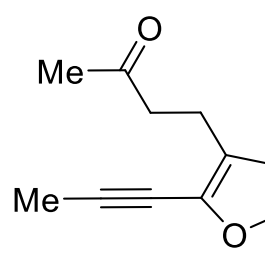

12

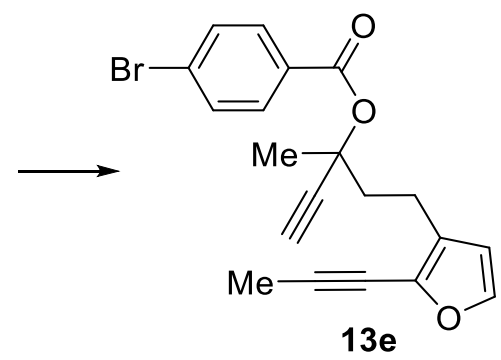


3-methyl-5-(2-(prop-1-yn-1-yl)furan-3-yl)pent-1-yn-3-yl 4-bromobenzoate (13e). This compound was prepared in a manner similar to that previously reported with some modifications. ${ }^{\mathbf{5}}$ A flame-dried, 2-necked, 50-mL, round-bottomed flask equipped with a magnetic stir bar, nitrogen inlet adapter, and septum was charged with 4-(2-(prop-1-yn-1-yl)furan-3-yl)butan-2-one (12) (208 $\mathrm{mg}, 1.18 \mathrm{mmol})$ and THF (3.9 mL). The solution was cooled by placing the flask in an ice-bath and ethynylmagnesium bromide (7.1 mL of a $0.5 \mathrm{M}$ solution in THF, $3.54 \mathrm{mmol}, 3.0$ equiv), was added dropwise via syringe over $5 \mathrm{~min}$. After allowing to warm to $\mathrm{rt}$ over $1 \mathrm{~h}$, TLC showed complete consumption of starting material. The flask was placed in an ice-bath and 4bromobenzoyl chloride (906 mg, $4.13 \mathrm{mmol}, 3.5$ equiv) was added by temporary removal of the septum. After $2 \mathrm{~h}$, TLC showed complete consumption of the propargyl alcohol intermediate. Diethyl ether $(5 \mathrm{~mL})$ and deionized water $(3 \mathrm{~mL})$ were added to the flask, the mixture was transferred to a separatory funnel, and the organic layer was separated. The aqueous layer was extracted with diethyl ether ( 3 x $5 \mathrm{~mL})$, dried over magnesium sulfate, filtered, and concentrated in vacuo. The crude residue was purified by silica gel flash chromatography (5-25\% ethyl acetate/hexanes) to afford $327 \mathrm{mg}$ (72\% yield) of the title compound as a brown oil.

\section{EDD6-487}

$\underline{{ }^{1} \mathrm{H} \mathrm{NMR}} \quad\left(400 \mathrm{MHz}, \mathrm{CDCl}_{3}\right)$

$7.85(\mathrm{~d}, J=8.8 \mathrm{~Hz}, 2 \mathrm{H}), 7.56(\mathrm{~d}, J=8.4 \mathrm{~Hz}, 2 \mathrm{H}), 7.23(\mathrm{~d}, J=2.0,1 \mathrm{H}), 6.29(\mathrm{~d}$, $J=2.0 \mathrm{~Hz}, 1 \mathrm{H}), 2.82-2.70(\mathrm{~m}, 2 \mathrm{H}), 2.66(\mathrm{~s}, 1 \mathrm{H}), 2.37-2.16(\mathrm{~m}, 2 \mathrm{H}), 2.09$ (s, 3

H), 1.87 (s, 3 H) ppm

${ }^{13} \mathrm{C} \mathrm{NMR} \quad\left(100 \mathrm{MHz}, \mathrm{CDCl}_{3}\right)$ 
$164.1,142.4,134.9,131.8,131.3,129.8,128.2,127.8,111.6,92.9,83.2,75.5$, $74.3,69.6,41.7,26.6,20.4,4.7 \mathrm{ppm}$

$\underline{\mathrm{IR}} \quad$ (Thin Film)

$2118,1724 \mathrm{~cm}^{-1}$

HRMS HRMS-ESI (m/z): $[\mathrm{M}+\mathrm{H}]^{+}$calcd for $\mathrm{C}_{20} \mathrm{H}_{18} \mathrm{O}_{3}{ }^{79} \mathrm{Br}, 385.0434$; found, 385.0427

TLC $\quad R_{f}=0.65$ (35\% ethyl acetate/hexanes); silica gel, UV, $p$-anisaldehyde

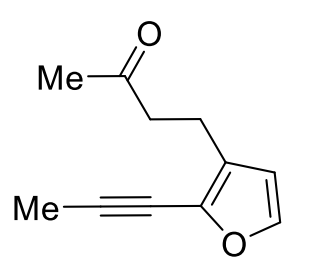

12

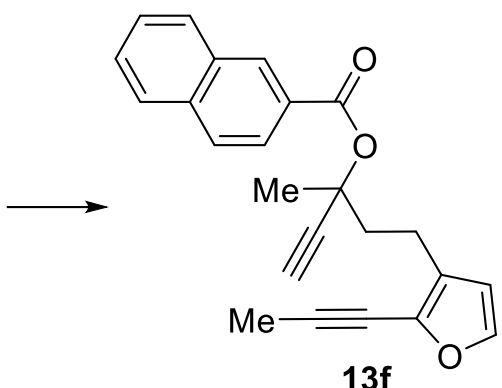

$13 f$

3-methyl-5-(2-(prop-1-yn-1-yl)furan-3-yl)pent-1-yn-3-yl 2-naphthoate (13f). This compound was prepared in a manner similar to that previously reported with some modifications. ${ }^{5}$ A flamedried, 2-necked, 10-mL, round bottomed flask equipped with a magnetic stir bar, nitrogen inlet adapter, and septum was charged with 4-(2-(prop-1-yn-1-yl)furan-3-yl)butan-2-one (12) (20 mg, $0.114 \mathrm{mmol})$ and THF $(0.38 \mathrm{~mL})$. The solution was cooled by placing the flask in an ice-bath and ethynylmagnesium bromide ( $0.34 \mathrm{~mL}$ of a $0.5 \mathrm{M}$ solution in THF, $0.171 \mathrm{mmol}, 1.5$ equiv), was added dropwise via syringe over $2 \mathrm{~min}$. After allowing to warm to rt over $1 \mathrm{~h}$, TLC showed complete consumption of starting material. The flask was placed in an ice-bath and 2-naphthoyl chloride ( $35 \mathrm{mg}, 0.182 \mathrm{mmol}, 1.6$ equiv) was added by temporary removal of the septum. After 4 h, TLC showed complete consumption of the propargyl alcohol intermediate. Diethyl ether (1 mL) 
and deionized water $(1 \mathrm{~mL})$ were added to the flask, the mixture was transferred to a separatory funnel, and the organic layer was separated. The aqueous layer was extracted with diethyl ether (3 x $5 \mathrm{~mL}$ ), dried over magnesium sulfate, filtered, and concentrated in vacuo. The crude residue was purified by silica gel flash chromatography (5\% ethyl acetate/hexanes). The title compound contained 2-naphthoyl chloride.

\section{EDD5-362}

${ }^{1} \mathrm{H} \mathrm{NMR} \quad\left(300 \mathrm{MHz}, \mathrm{CDCl}_{3}\right)$

$8.55(\mathrm{~s}, 1 \mathrm{H}), 8.04(\mathrm{dd}, J=\mathrm{Hz}, 1 \mathrm{H}), 7.96(\mathrm{~d}, J=6.0 \mathrm{~Hz}, 1 \mathrm{H}), 7.89-7.86(\mathrm{~m}, 2 \mathrm{H})$.

7.62-7.53 (m, $2 \mathrm{H}), 7.25(\mathrm{~d}, J=1.5 \mathrm{~Hz}), 6.33(\mathrm{~d}, J=1.5 \mathrm{~Hz}), 2.92-2.77(\mathrm{~m}, 2 \mathrm{H})$,

2.69 (s, $1 \mathrm{H}), 2.45-2.23(\mathrm{~m}, 2 \mathrm{H}), 2.08(\mathrm{~s}, 3 \mathrm{H}), 1.93$ (s, $3 \mathrm{H}) \mathrm{ppm}$; Impurities at:

$8.62(\mathrm{~s}), 6.99(\mathrm{~m}), 3.99(\mathrm{~s}), 1.43(\mathrm{~s}) \mathrm{ppm}$

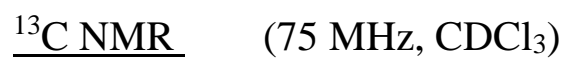

$165.0,142.4,135.7,135.0,132.6,131.3,129.5,128.4,128.2,128.2,128.0,127.9$, $126.8,125.4,111.7,93.0,83.6,75.3,74.2,69.6,41.8,26.8,20.5,4.8 \mathrm{ppm}$

$\underline{\text { IR }} \quad$ (Thin Film)

$1721,1281 \mathrm{~cm}^{-1}$

$\underline{\text { HRMS }}$ HRMS-ESI (m/z): $[\mathrm{M}+\mathrm{H}]^{+}$calcd for $\mathrm{C}_{24} \mathrm{H}_{21} \mathrm{O}_{3}, 357.1491$; found, 357.1475

$\underline{\text { TLC }} \quad R_{f}=0.60$ (35\% ethyl acetate/hexanes); silica gel, UV, $p$-anisaldehyde

Follows General Procedure A: 3-methyl-5-(2-(prop-1-yn-1-yl)furan-3-yl)pent-1-yn-3-yl 2naphthoate (13f) containing 2-naphthoyl chloride ( $28 \mathrm{mg}, 0.079 \mathrm{mmol}, 1$ equiv), methanol (0.79 $\mathrm{mL}, 0.1 \mathrm{M})$, triethylamine (17 $\mu \mathrm{L}, 0.119 \mathrm{mmol}, 1.5$ equiv), dimethylamino pyridine ( $3 \mathrm{mg}, 0.024$ 
mmol, 0.3 equiv) After $2 \mathrm{~h}$, saturated ammonium chloride $(0.5 \mathrm{~mL})$ was added and the solution transferred to a separatory funnel, extracted with diethyl ether $(5 \times 1 \mathrm{~mL})$, dried over magnesium sulfate, filtered, and concentrated in vacuo. The crude residue was purified by silica gel flash chromatography (10\% ethyl acetate/hexanes) to give $26 \mathrm{mg}$ (65\% from 12) of the title compound as a clear liquid.

\section{EDD5-363}

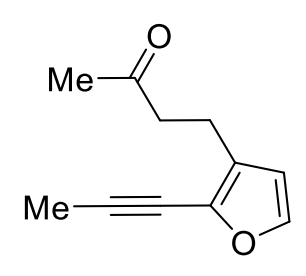

12

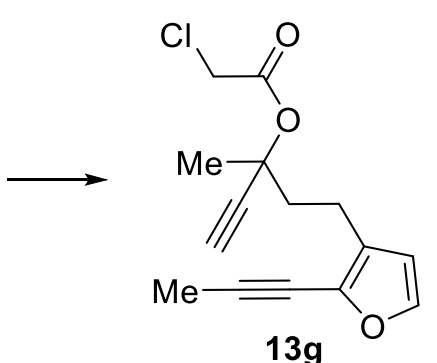

$13 \mathrm{~g}$

3-methyl-5-(2-(prop-1-yn-1-yl)furan-3-yl)pent-1-yn-3-yl $\quad$ 2-chloroacetate $\quad$ (13g). This compound was prepared in a manner similar to that previously reported with some modifications. ${ }^{5}$ A flame-dried, 2-necked, 25-mL, round-bottomed flask equipped with a magnetic stir bar, nitrogen inlet adapter, and septum was charged with 4-(2-(prop-1-yn-1-yl)furan-3-yl)butan-2-one (12) (125 $\mathrm{mg}, 0.709 \mathrm{mmol})$ and THF $(2.4 \mathrm{~mL})$. The solution was cooled by placing the flask in an ice-bath and ethynylmagnesium bromide ( $2.1 \mathrm{~mL}$ of a $0.5 \mathrm{M}$ solution in THF, $1.06 \mathrm{mmol}, 1.5$ equiv), was added dropwise via syringe over $5 \mathrm{~min}$. After allowing to warm to rt over $60 \mathrm{~min}$, the solution was cooled again by placing an ice-bath and additional ethynylmagnesium bromide $(1.4 \mathrm{~mL}$ of a 0.5 M solution in THF, $0.709 \mathrm{mmol}, 1$ equiv) was added via syringe over $3 \mathrm{~min}$. After allowing to warm to rt over $60 \mathrm{~min}$, TLC showed complete consumption of starting material. The flask was placed in an ice-bath and acetyl chloride $(0.11 \mathrm{~mL}, 1.42 \mathrm{mmol})$ was added dropwise via syringe over 1 min. After $2 \mathrm{~h}$, TLC showed complete consumption of the propargyl alcohol intermediate. 
Diethyl ether $(5 \mathrm{~mL})$ and deionized water $(5 \mathrm{~mL})$ were added to the flask, the mixture was transferred to a separatory funnel, and the organic layer was separated. The aqueous layer was extracted with diethyl ether ( $3 \times 15 \mathrm{~mL})$, washed with brine, the combined aqueous layers back extracted with diethyl ether ( $1 \times 15 \mathrm{~mL})$, dried over magnesium sulfate, filtered, and concentrated in vacuo. The crude residue was purified by silica gel flash chromatography (5\% ethyl acetate/hexanes) to give $103 \mathrm{mg}$ (52\%) of the title compound as a clear liquid. Product contained a small amount of an uncharacterizable byproduct.

\section{EDD5-344}

${ }^{1} \mathrm{HNMR} \quad\left(400 \mathrm{MHz}, \mathrm{CDCl}_{3}\right)$

$7.23(\mathrm{~s}, 1 \mathrm{H}), 6.26(\mathrm{~d}, J=1.2 \mathrm{~Hz}), 4.00(\mathrm{~s}, 2 \mathrm{H}), 2.71-2.66(\mathrm{~m}, 2 \mathrm{H}), 2.65(\mathrm{~s}, 1 \mathrm{H})$, 2.27-2.19 (m, $1 \mathrm{H}), 2.14-2.06(\mathrm{~m}, 1 \mathrm{H}), 2.11$ (s, $3 \mathrm{H}), 1.78$ (s, $3 \mathrm{H}) \mathrm{ppm}$

${ }^{13} \mathrm{C} \mathrm{NMR} \quad\left(100 \mathrm{MHz}, \mathrm{CDCl}_{3}\right)$

$165.4,142.4,134.9,127.6,111.6,93.0,82.5,76.9,74.8,69.5,41.5,41.2,26.4$, 20.2, $4.8 \mathrm{ppm}$

$\underline{\text { IR }} \quad$ (Thin Film)

$1762,1277 \mathrm{~cm}^{-1}$

HRMS HRMS-ESI (m/z): $[\mathrm{M}+\mathrm{H}]^{+}$calcd for $\mathrm{C}_{15} \mathrm{H}_{16} \mathrm{O}_{3} \mathrm{Cl}, 279.0788$; found, 279.0778

TLC $\quad R_{f}=0.56(35 \%$ ethyl acetate/hexanes); silica gel, UV, $p$-anisaldehyde 


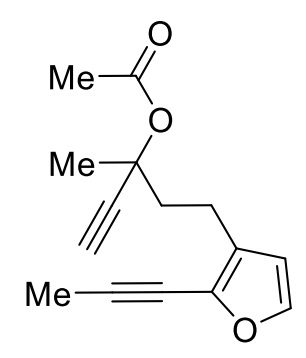

$13 a$

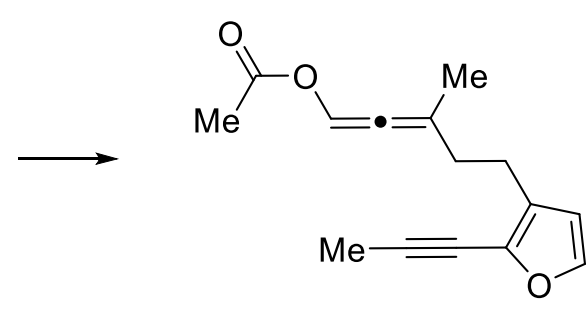

$4 \mathbf{a}$

\section{General Procedure B: Synthesis of allenyl esters via a formal [3,3]-sigmatropic} rearrangement ${ }^{5}$

3-methyl-5-(2-(prop-1-yn-1-yl)furan-3-yl)penta-1,2-dien-1-yl acetate (4a). A flame-dried, single-necked, 25-mL, round bottomed flask equipped with a magnetic stir bar was charged with rhodium(II) trifluoroacetate dimer $(0.05$ equiv, $12.1 \mathrm{mg}, 0.018 \mathrm{mmol})$ in a nitrogen filled glovebox and sealed with a septum before removal. The flask was placed under a nitrogen atmosphere using a nitrogen manifold and an inlet needle. A solution of 3-methyl-5-(2-(prop-1-yn-1-yl)furan-3yl)pent-1-yn-3-yl acetate (13a) (1 equiv, $90 \mathrm{mg}, 0.37 \mathrm{mmol})$ in toluene $(1.84 \mathrm{~mL}, 0.2 \mathrm{M})$ was added dropwise via syringe to the flask. The flask was lowered into a preheated $50{ }^{\circ} \mathrm{C}$ oil bath. After $1 \mathrm{~h}$, consumption of starting material was observed by TLC. The flask was allowed to cool to rt, the volatiles were removed in vacuo, and the crude residue immediately purified by silica gel flash chromatography ( $2 \%$ ethyl acetate/hexanes) to give $58 \mathrm{mg}(64 \%)$ of the title compound as a clear liquid.

\section{EDD2-107}

${ }^{1} \mathrm{H} \mathrm{NMR} \quad\left(400 \mathrm{MHz}, \mathrm{CDCl}_{3}\right)$ 
$7.34(\mathrm{q}, J=2.0 \mathrm{~Hz}, 1 \mathrm{H}), 7.22(\mathrm{~d}, J=1.6 \mathrm{~Hz}, 1 \mathrm{H}), 6.25(\mathrm{~d}, J=1.6 \mathrm{~Hz}, 1 \mathrm{H}), 2.61$ (t, $J=7.6 \mathrm{~Hz}, 2 \mathrm{H}), 2.40-2.25(\mathrm{~m}, 2 \mathrm{H}), 2.14(\mathrm{~s}, 3 \mathrm{H}), 2.11(\mathrm{~s}, 3 \mathrm{H}), 1.86(\mathrm{~d}, J=$ $1.6 \mathrm{~Hz}, 3 \mathrm{H}) \mathrm{ppm}$

${ }^{13} \mathrm{C} \mathrm{NMR} \quad\left(100 \mathrm{MHz}, \mathrm{CDCl}_{3}\right)$

189.7, 168.9, 142.3, 134.9, 128.0, 115.4, 111.6, 110.4, 92.8, 69.7, 35.2, 23.2, 21.1, $20.8,4.8 \mathrm{ppm}$

$\underline{\text { IR }} \quad$ (Thin Film) $1978,1747,1443,1368,1215,1048 \mathrm{~cm}^{-1}$

HRMS $\quad$ HRMS-ESI (m/z): $[\mathrm{M}+\mathrm{H}]^{+}$calcd for $\mathrm{C}_{15} \mathrm{H}_{17} \mathrm{O}_{3}, 245.1178$; found, 245.1176

TLC $\quad R_{f}=0.71$ (35\% ethyl acetate/hexanes); silica gel, UV, $p$-anisaldehyde

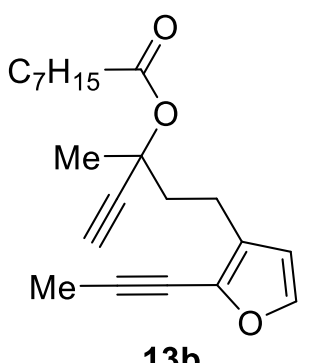

$13 b$

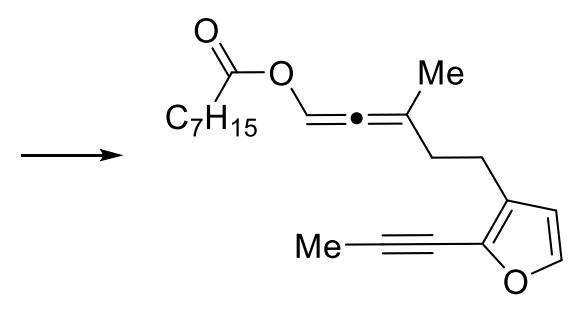

$4 b$

3-methyl-5-(2-(prop-1-yn-1-yl)furan-3-yl)penta-1,2-dien-1-yl octanoate (4b). Follows General Procedure B: Rhodium(II) trifluoroacetate dimer (7.9 mg, 0.012 mmol), 3-methyl-5-(2-(prop-1yn-1-yl)furan-3-yl)pent-1-yn-3-yl octanoate (13b)(77 mg, $0.23 \mathrm{mmol})$, toluene (1.2 mL, $0.2 \mathrm{M})$, the crude residue was purified by silica gel flash chromatography (1\% ethyl acetate/hexanes) to give $64 \mathrm{mg}(83 \%)$ of the title compound as a clear liquid. 
EDD5-329

${ }^{1} \mathrm{H} \mathrm{NMR} \quad\left(400 \mathrm{MHz}, \mathrm{CDCl}_{3}\right)$

7.36-7.35 (m, $1 \mathrm{H}), 7.22(\mathrm{~d}, J=2.0,1 \mathrm{H}), 6.25(\mathrm{~d} J=1.6 \mathrm{~Hz}, 1 \mathrm{H}), 2.61(\mathrm{t}, J=7.2$, $2 \mathrm{H}), 2.38(\mathrm{t}, J=7.6,2 \mathrm{H}), 2.34-2.27(\mathrm{~m}, 2 \mathrm{H}), 2.10(\mathrm{~s}, 3 \mathrm{H}), 1.85(\mathrm{~d}, J=2.0 \mathrm{~Hz}, 3$ H), 1.69-1.62 (m, $2 \mathrm{H}), 1.35-1.25(\mathrm{~m}, 8 \mathrm{H}), 0.89-0.86(\mathrm{~m}, 3 \mathrm{H}) \mathrm{ppm}$; Impurity at $1.35-1.25 \mathrm{ppm}$

${ }^{13} \mathrm{C} \mathrm{NMR} \quad\left(100 \mathrm{MHz}, \mathrm{CDCl}_{3}\right)$

$189.7,171.8,142.3,134.9,128.1,115.3,111.6,110.3,92.7,69.7,35.2,34.4,31.8$, $29.2,29.0,24.9,23.2,22.7,20.8,14.2,4.8 \mathrm{ppm}$

$\underline{\text { IR }} \quad$ (Thin Film)

$1745,1264 \mathrm{~cm}^{-1}$

$\underline{\text { HRMS }} \quad$ HRMS-ESI (m/z): $[\mathrm{M}+\mathrm{H}]^{+}$calcd for $\mathrm{C}_{15} \mathrm{H}_{16} \mathrm{O}_{3} \mathrm{Cl}, 279.0788$; found, 279.0781

TLC $\quad R_{f}=0.72(35 \%$ ethyl acetate/hexanes); silica gel, UV, $p$-anisaldehyde

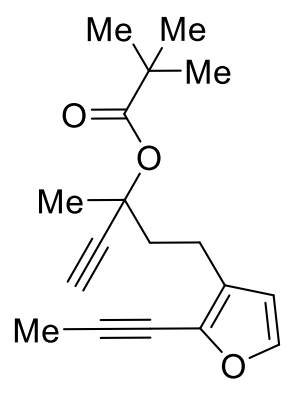

13c

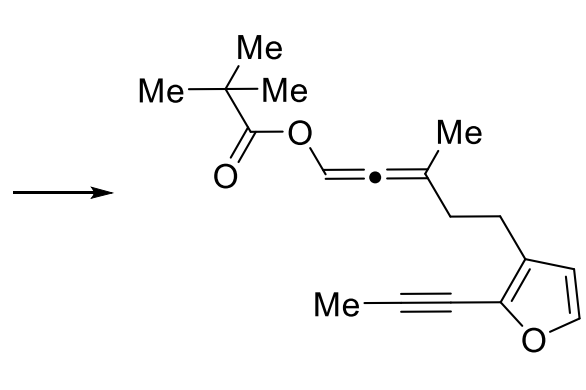

$4 c$

3-methyl-5-(2-(prop-1-yn-1-yl)furan-3-yl)penta-1,2-dien-1-yl pivalate (4c). Follows General Procedure B: Rhodium(II) trifluoroacetate dimer (11.8 mg, 0.018 mmol), 3-methyl-5-(2-(prop-1- 
yn-1-yl)furan-3-yl)pent-1-yn-3-yl pivalate (13c)(102 mg, $0.356 \mathrm{mmol})$, toluene (1.78 mL, 0.2 M), the crude residue was purified by silica gel flash chromatography (1-2\% ethyl acetate/hexanes) to give $78 \mathrm{mg}(76 \%)$ of the title compound as a clear liquid.

\section{EDD5-380}

${ }^{1} \mathrm{H} \mathrm{NMR} \quad\left(500 \mathrm{MHz}, \mathrm{CDCl}_{3}\right)$

7.32-7.31 (m, $1 \mathrm{H}), 7.21(\mathrm{~d}, J=2.0 \mathrm{~Hz}, 1 \mathrm{H}), 6.23(\mathrm{~d}, J, 1.5 \mathrm{~Hz}, 1 \mathrm{H}), 2.63-2.58$

(m, 2 H), 2.37-2.28 (m, 2 H), $2.10(\mathrm{~s}, 3 \mathrm{H}), 1.85(\mathrm{~d}, J=2.0 \mathrm{~Hz}, 3 \mathrm{H}), 1.24(\mathrm{~s}, 9 \mathrm{H})$ ppm

$\underline{{ }^{13} \mathrm{C} \mathrm{NMR}} \quad\left(125 \mathrm{MHz}, \mathrm{CDCl}_{3}\right)$

$189.9,176.5,142.2,134.9,128.1,114.8,111.7,110.5,92.7,69.7,39.2,35.2,27.2$, $26.7,25.8,23.1,20.8,4.8 \mathrm{ppm}$

$\underline{\text { IR }} \quad$ (Thin Film)

$1737,1278 \mathrm{~cm}^{-1}$

HRMS $\quad$ HRMS-ESI (m/z): $[\mathrm{M}+\mathrm{H}]^{+}$calcd for $\mathrm{C}_{18} \mathrm{H}_{23} \mathrm{O}_{3}, 287.1642$; found, 287.1642

TLC $\quad R_{f}=0.71$ (35\% ethyl acetate/hexanes); silica gel, UV, $p$-anisaldehyde

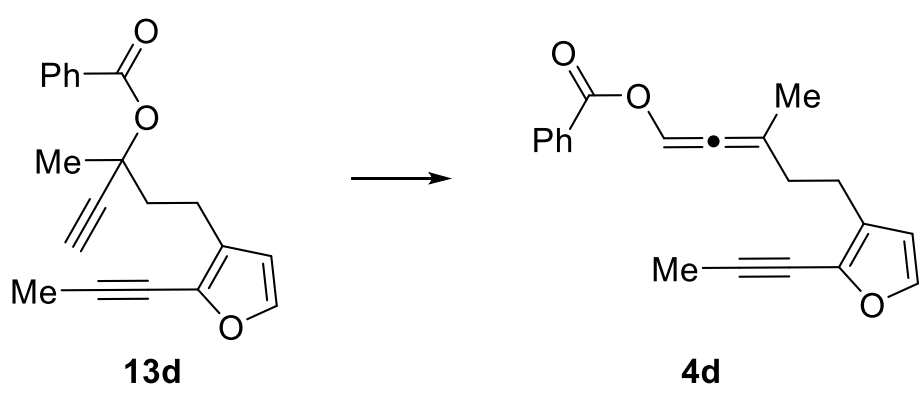


3-methyl-5-(2-(prop-1-yn-1-yl)furan-3-yl)penta-1,2-dien-1-yl benzoate (4d). Follows General Procedure B: Rhodium(II) trifluoroacetate dimer (4.6 mg, 0.007 mmol), 3-methyl-5(2-(prop-1-ynyl)furan-3-yl)pent-1-yn-3-yl benzoate (13d)(43 mg, $0.14 \mathrm{mmol})$, toluene $(0.7 \mathrm{~mL}, 0.2 \mathrm{M})$, the crude residue was purified by silica gel flash chromatography ( $2 \%$ ethyl acetate/hexanes) to give $34 \mathrm{mg}(79 \%)$ of the title compound as a clear liquid.

\section{EDD3-136}

$\underline{{ }^{1} \mathrm{H} \mathrm{NMR}} \quad\left(400 \mathrm{MHz}, \mathrm{CDCl}_{3}\right)$

8.10-8.07 (m, $2 \mathrm{H}), 7.61-7.57(\mathrm{~m}, 2 \mathrm{H}), 7.48-7.44(\mathrm{t}, J=7.9 \mathrm{~Hz}, 2 \mathrm{H}), 7.21(\mathrm{~d}, J=$ $1.6 \mathrm{~Hz}, 1 \mathrm{H}), 6.28(\mathrm{~d}, J=1.6 \mathrm{~Hz}, 1 \mathrm{H}), 2.68-2.64(\mathrm{~m}, 2 \mathrm{H}), 2.45-2.30(\mathrm{~m}, 2 \mathrm{H})$, $2.09(\mathrm{~s}, 3 \mathrm{H}), 1.90(\mathrm{~d}, J=2.0 \mathrm{~Hz}, 3 \mathrm{H}) \mathrm{ppm}$; Impurities at $3.48(\mathrm{q}, J=7.2 \mathrm{~Hz})$, $1.21(\mathrm{t}, J=6.8 \mathrm{~Hz})$ (diethyl ether) ppm

${ }^{13} \mathrm{C} \mathrm{NMR} \quad\left(100 \mathrm{MHz}, \mathrm{CDCl}_{3}\right)$

$190.4,164.6,142.3,139.8,135.0,133.4,130.1,129.7,128.6,128.0,115.4,111.7$, $110.7,92.8,69.7,35.2,23.2,20.8,4.8 \mathrm{ppm}$

$\underline{\text { IR }} \quad$ (Thin Film)

$3067,2917,2850,1976,1726,1601,1493,1451,1370,1269,1176,1070 \mathrm{~cm}^{-1}$

$\underline{\text { HRMS }} \quad$ HRMS-ESI $(\mathrm{m} / \mathrm{z}):[\mathrm{M}+\mathrm{H}]^{+}$calcd for $\mathrm{C}_{20} \mathrm{H}_{19} \mathrm{O}_{3}, 307.1334$; found, 307.1343

TLC $\quad R_{f}=0.71(35 \%$ ethyl acetate/hexanes); silica gel, UV, $p$-anisaldehyde 


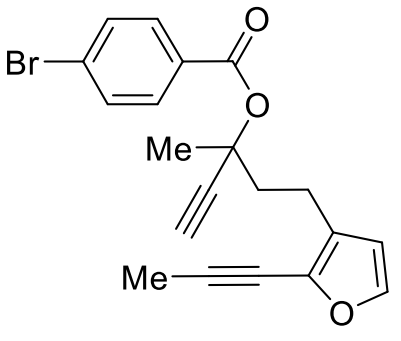

$13 e$

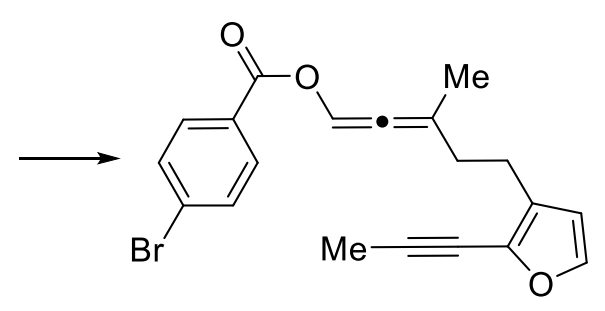

$4 e$

3-methyl-5-(2-(prop-1-yn-1-yl)furan-3-yl)penta-1,2-dien-1-yl 4-bromobenzoate (4e). Follows

General Procedure B: Rhodium(II) trifluoroacetate dimer (26 mg, $0.04 \mathrm{mmol})$, 3-methyl-5-(2(prop-1-yn-1-yl)furan-3-yl)pent-1-yn-3-yl 4-bromobenzoate (13e)(310 mg, $0.81 \mathrm{mmol})$, toluene (4.0 mL, $0.2 \mathrm{M})$, the crude residue was purified by silica gel flash chromatography (2-5\% ethyl acetate/hexanes) to give $227 \mathrm{mg}(73 \%)$ of the title compound as a yellow oil.

\section{EDD6-488}

$\underline{{ }^{1} \mathrm{H} \mathrm{NMR}} \quad\left(400 \mathrm{MHz}, \mathrm{CDCl}_{3}\right)$

$7.94(\mathrm{~d}, J=8.4 \mathrm{~Hz}, 2 \mathrm{H}), 7.60(\mathrm{~d}, J=8.4 \mathrm{~Hz}, 2 \mathrm{H}), 7.55-7.53(\mathrm{~m}, 1 \mathrm{H}), 7.21(\mathrm{~d}, J=$ $1.6 \mathrm{~Hz}, 1 \mathrm{H}), 6.27(\mathrm{~d}, J=2.0 \mathrm{~Hz}, 1 \mathrm{H}), 2.66(\mathrm{t}, J=6.8 \mathrm{~Hz}, 2 \mathrm{H}), 2.45-2.31(\mathrm{~m}, 2$ H), 2.08 (s, $3 \mathrm{H}), 1.90(\mathrm{~d}, J=1.6 \mathrm{~Hz}, 3 \mathrm{H}) \mathrm{ppm}$

$\stackrel{{ }^{13} \mathrm{C} \mathrm{NMR}}{ }\left(100 \mathrm{MHz}, \mathrm{CDCl}_{3}\right)$

$190.3,163.9,142.3,135.0,131.9,131.5,128.7,128.6,127.9,115.7,111.6,110.6$, $92.8,69.7,35.1,23.2,20.8,4.8 \mathrm{ppm}$

$\underline{\text { IR }} \quad$ (Thin Film)

$2095,1978,1725 \mathrm{~cm}^{-1}$

$\underline{\text { HRMS }} \quad$ HRMS-ESI (m/z): $[\mathrm{M}+\mathrm{H}]^{+}$calcd for $\mathrm{C}_{20} \mathrm{H}_{18} \mathrm{O}_{3} \mathrm{Br}, 385.0434$; found, 385.0427 
$\underline{\text { TLC }} \quad R_{f}=0.65$ (35\% ethyl acetate/hexanes); silica gel, UV, $p$-anisaldehyde

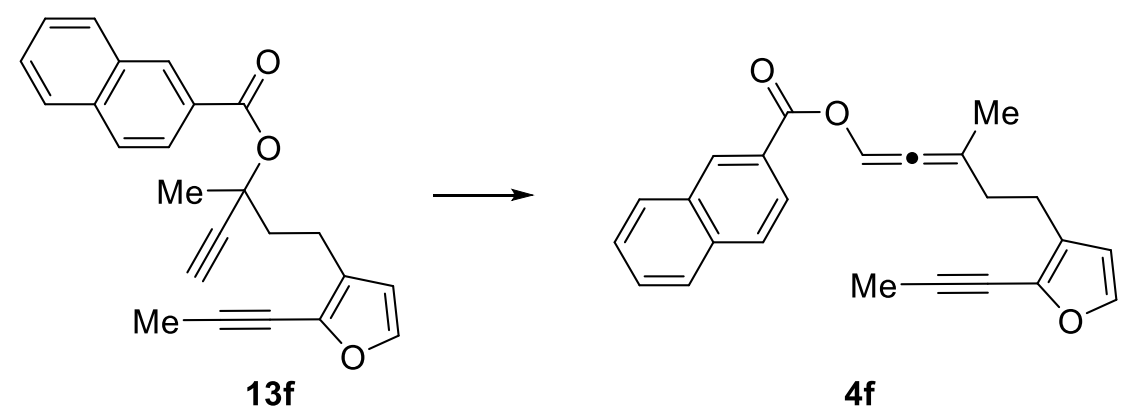

3-methyl-5-(2-(prop-1-yn-1-yl)furan-3-yl)penta-1,2-dien-1-yl 2-naphthoate (4f). Follows General Procedure B: Rhodium(II) trifluoroacetate dimer (3.9 mg, $0.006 \mathrm{mmol})$, 3-methyl-5-(2(prop-1-yn-1-yl)furan-3-yl)pent-1-yn-3-yl 2-naphthoate (13f) (44 mg, $0.123 \mathrm{mmol})$, toluene (0.62 $\mathrm{mL}, 0.2 \mathrm{M}$ ), he crude residue was purified by silica gel flash chromatography (2\% ethyl acetate/hexanes) to give $30 \mathrm{mg}(68 \%)$ of the title compound as a clear liquid.

\section{EDD5-364}

${ }^{1} \mathrm{H} \mathrm{NMR} \quad\left(300 \mathrm{MHz}, \mathrm{CDCl}_{3}\right)$

$8.66(\mathrm{~s}, 1 \mathrm{H}), 8.10(\mathrm{dd}, J=8.7 \mathrm{~Hz}, 1.5 \mathrm{~Hz}, 1 \mathrm{H}), 7.98(\mathrm{~d}, J=7.8 \mathrm{~Hz}, 1 \mathrm{H}), 7.91-$

7.89 (m, 2 H), 7.65-7.64 (m, $1 \mathrm{H}), 7.62-7.53(\mathrm{~m}, 2 \mathrm{H}), 7.23(\mathrm{~d}, J=1.5 \mathrm{~Hz}, 1 \mathrm{H})$,

$6.30(\mathrm{~d}, J=1.8 \mathrm{~Hz}, 1 \mathrm{H}), 2.70(\mathrm{t}, J=7.5 \mathrm{~Hz}, 2 \mathrm{H}), 2.50-2.31(\mathrm{~m}, 2 \mathrm{H}), 2.09$ (s, 3

H), $1.93(\mathrm{~d}, J=1.8 \mathrm{~Hz}, 3 \mathrm{H}) \mathrm{ppm}$; Impurities at: $8.62(\mathrm{~s}), 8.05(\mathrm{~d}, J=1.5 \mathrm{~Hz})$,

$3.99(\mathrm{~s}) \mathrm{ppm}$

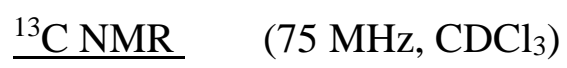


$190.4,164.8,142.3,135.8,135.0,132.6,131.7,129.6,128.6,128.4,128.0,127.9$, $127.0,126.9,125.5,115.5,111.7,110.8,92.8,69.8,35.2,23.2,20.8,4.8 \mathrm{ppm}$

$\underline{\text { IR }} \quad$ (Thin Film)

$3062,1974,1719,1221,1278 \mathrm{~cm}^{-1}$

HRMS $\quad$ HRMS-ESI (m/z): $[\mathrm{M}+\mathrm{H}]^{+}$calcd for $\mathrm{C}_{24} \mathrm{H}_{21} \mathrm{O}_{3}, 357.1491$; found, 357.1477

$\underline{\text { TLC }} \quad R_{f}=0.64$ (35\% ethyl acetate/hexanes); silica gel, UV, $p$-anisaldehyde

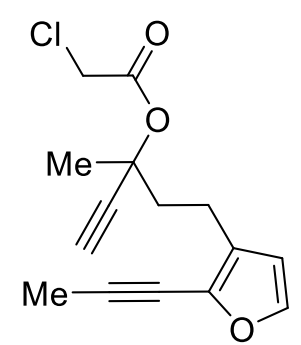

$13 \mathrm{~g}$

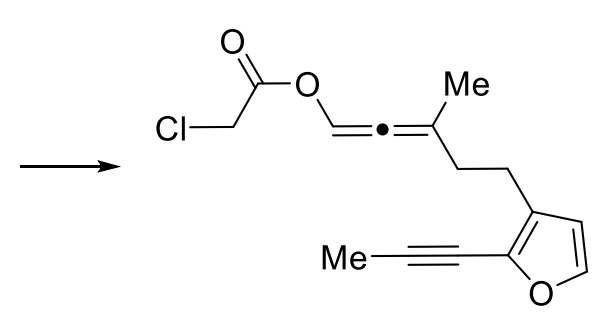

$4 g$

3-methyl-5-(2-(prop-1-yn-1-yl)furan-3-yl)penta-1,2-dien-1-yl 2-chloroacetate (4g). Follows

General Procedure B: Rhodium(II) trifluoroacetate dimer (1.4 mg, $0.002 \mathrm{mmol}), 3$-methyl-5-(2(prop-1-yn-1-yl)furan-3-yl)penta-1,2-dien-1-yl 2-chloroacetate $(\mathbf{1 3 g})(12 \quad \mathrm{mg}, \quad 0.043 \mathrm{mmol})$, toluene $(0.22 \mathrm{~mL}, 0.2 \mathrm{M})$, the crude residue was purified by silica gel flash chromatography ( $2 \%$ ethyl acetate/hexanes) to give $10 \mathrm{mg}(83 \%)$ of the title compound as a clear liquid.

\section{EDD5-336}

${ }^{1} \mathrm{H} \mathrm{NMR} \quad\left(400 \mathrm{MHz}, \mathrm{CDCl}_{3}\right)$

7.34-7.32 (m, $1 \mathrm{H}), 7.22(\mathrm{~d}, J=1.6 \mathrm{~Hz}, 1 \mathrm{H}), 6.24(\mathrm{~d}, J=1.6 \mathrm{~Hz}, 1 \mathrm{H}), 4.13(\mathrm{~s}, 2$

H), $2.62(\mathrm{t}, J=7.2 \mathrm{~Hz}, 2 \mathrm{H}), 2.42-2.27(\mathrm{~m}, 2 \mathrm{H}), 2.11(\mathrm{~s}, 3 \mathrm{H}), 1.87(\mathrm{~d}, J=1.6$

$\mathrm{Hz}) \mathrm{ppm}$ 
${ }^{13} \mathrm{C} \mathrm{NMR} \quad\left(100 \mathrm{MHz}, \mathrm{CDCl}_{3}\right)$

$189.8,165.3,142.3,135.0,127.8,116.6,111.5,110.8,92.8,69.7,40.9,35.0,23.1$, 20.7, $4.8 \mathrm{ppm}$

$\underline{\text { IR }} \quad$ (Thin Film)

$1979,1765 \mathrm{~cm}^{-1}$

HRMS $\quad$ HRMS-ESI (m/z): $[\mathrm{M}+\mathrm{H}]^{+}$calcd for $\mathrm{C}_{15} \mathrm{H}_{16} \mathrm{O}_{3} \mathrm{Cl}$, 279.0788; found, 279.0781

TLC $\quad R_{f}=0.73$ (35\% ethyl acetate/hexanes); silica gel, UV, $p$-anisaldehyde

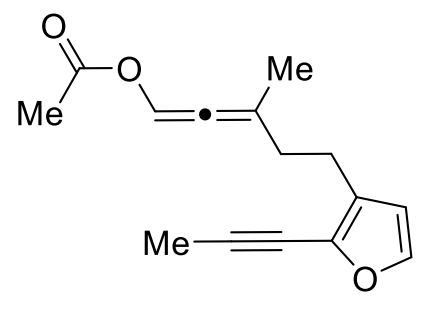

4a

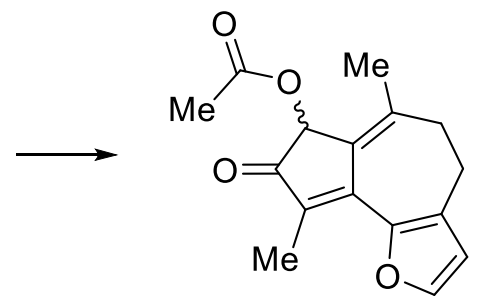

$3 a$

\section{General Procedure C: Synthesis of 5,7,5-ring system via racemic allenic Pauson-Khand reaction $^{5}$}

\section{6,9-dimethyl-8-oxo-4,5,7,8-tetrahydroazuleno[4,5-b]furan-7-yl acetate (3a). Bis(1,5-} cyclooctadiene)rhodium(I) tetrafluoroborate (0.1 equiv, $3.1 \mathrm{mg}, 0.0078 \mathrm{mmol}$ ) was weighed into a flame-dried 5-mL pear-shaped flask in a nitrogen glovebox and sealed with a septum. DCE (1.0 $\mathrm{mL}$ ) was added under nitrogen to the flask and the solution was transferred via syringe to a flamedried $25-\mathrm{mL}$ Schlenk tube equipped with magnetic stir bar and septum. In a separate flask, triphenylphosphine (0.15 equiv, $3.0 \mathrm{mg}, 0.012 \mathrm{mmol})$ was dissolved in DCE $(1.0 \mathrm{~mL})$ and added 
to the Schlenk tube via syringe. The resulting solution was stirred at rt under nitrogen for $30 \mathrm{~min}$. The Schlenk tube was evacuated via the inlet needle and refilled with $100 \%$ carbon monoxide (3 $\mathrm{x})$ using a separate inlet needle and stirred for $1 \mathrm{~h}$. Mesitylene (5.0 equiv, $54 \mu \mathrm{L}, 0.39 \mathrm{mmol}$ ) was added via syringe to the tube followed by a solution of 3-methyl-5-(2-(prop-1-yn-1-yl)furan-3yl)penta-1,2-dien-1-yl acetate (4a) (1.0 equiv, $18 \mathrm{mg}, 0.078 \mathrm{mmol})$ in DCE (1.9 mL) dropwise via syringe over $2 \mathrm{~min}$. The tube was lowered into a preheated $70{ }^{\circ} \mathrm{C}$ oil bath and the reaction stirred for $4 \mathrm{~h}$ until complete by TLC. The solution was transferred to a $100-\mathrm{mL}$ round-bottomed flask containing silica gel, the volatiles were removed in vacuo and the product was purified by silica gel flash chromatography (5\% to $20 \%$ ethyl acetate/hexanes) to give $14 \mathrm{mg}(70 \%)$ of the title compound as a yellow oil.

\section{EDD2-099}

$\underline{{ }^{1} \mathrm{H} \mathrm{NMR}} \quad\left(400 \mathrm{MHz}, \mathrm{CDCl}_{3}\right)$

$7.54(\mathrm{~d}, J=1.6 \mathrm{~Hz}, 1 \mathrm{H}), 6.39(\mathrm{~d}, J=1.6 \mathrm{~Hz}, 1 \mathrm{H}), 5.82(\mathrm{~s}, 1 \mathrm{H}), 2.88-2.73(\mathrm{~m}, 2$

H), $2.55(\mathrm{t}, J=5.2 \mathrm{~Hz}, 2 \mathrm{H}), 2.19(\mathrm{~s}, 3 \mathrm{H}), 2.15(\mathrm{~s}, 3 \mathrm{H}), 1.92(\mathrm{~s}, 3 \mathrm{H}) \mathrm{ppm}$

${ }^{13} \mathrm{C} \mathrm{NMR} \quad\left(100 \mathrm{MHz}, \mathrm{CDCl}_{3}\right)$

$199.8,170.0,149.8,147.4,145.0,138.7,132.4,132.2,127.7,113.8,71.8,34.6$,

$24.8,24.0,20.9,10.2 \mathrm{ppm}$

$\underline{\text { IR }} \quad$ (Thin Film)

$1744,1693,1602,1443,1224 \mathrm{~cm}^{-1}$

$\underline{\text { HRMS }} \quad$ HRMS-ESI (m/z): $[\mathrm{M}+\mathrm{H}]^{+}$calcd for $\mathrm{C}_{16} \mathrm{H}_{17} \mathrm{O}_{4}, 273.1127$; found, 273.1130

TLC $\quad R_{f}=0.4(35 \%$ ethyl acetate/hexanes); silica gel, UV, $p$-anisaldehyde 
Waters 600 HPLC, UV/PDA detector, 328 nm, Daicel CHIRALCEL-OD, 250 X 4.6 mm column, $1 \% \mathrm{iPrOH} / \mathrm{hexanes}$, Flow rate: $1 \mathrm{~mL} / \mathrm{min}$

\begin{tabular}{|c|c|c|}
\hline Peak & Retention Time (min) & Peak area (\%) \\
\hline Peak 1 & 33.09 & 49.98 \\
\hline Peak 2 & 46.68 & 50.02 \\
\hline
\end{tabular}

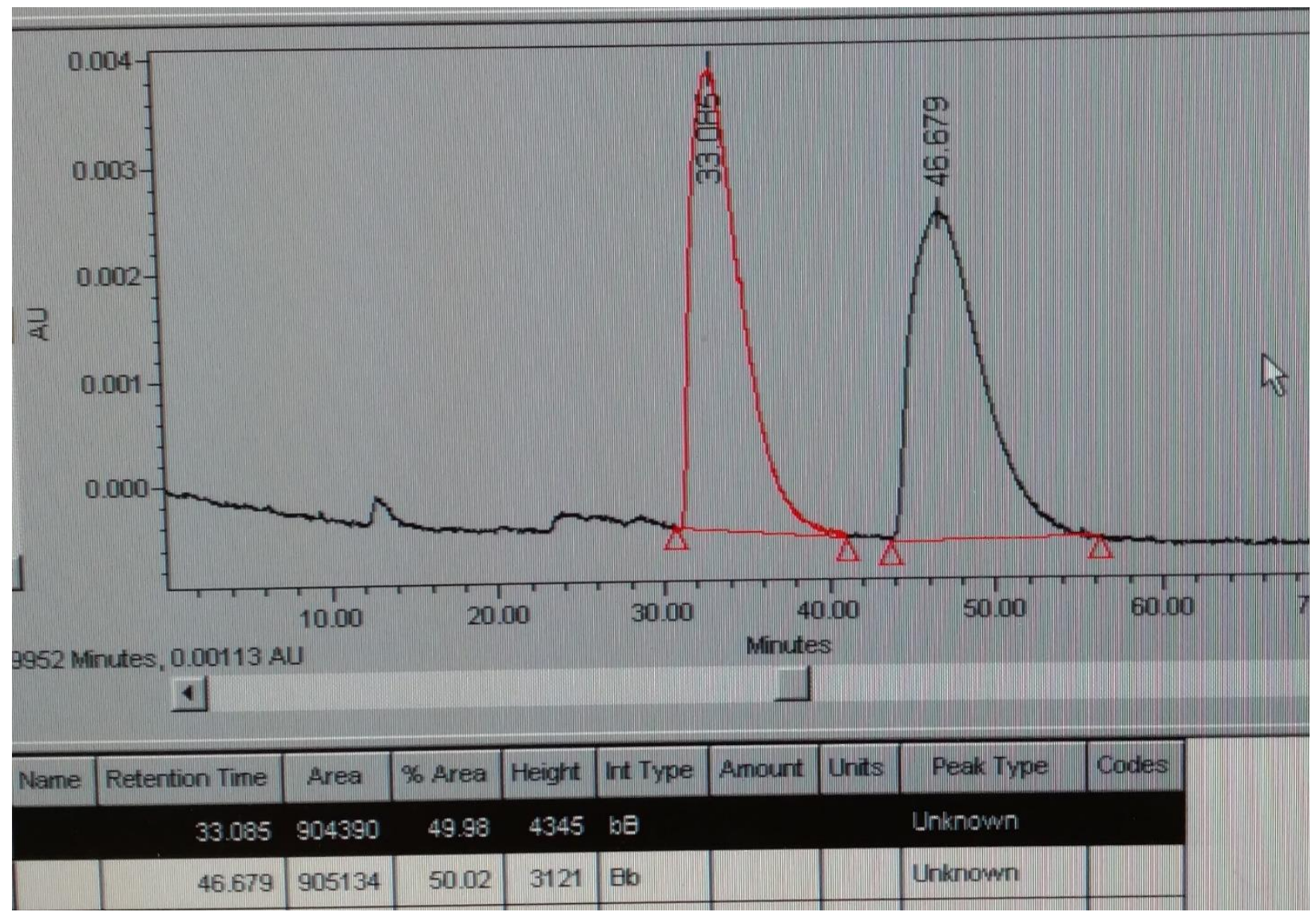


General Procedure D: Synthesis of 5,7,5-ring system via asymmetric allenic Pauson-Khand reaction $^{5}$

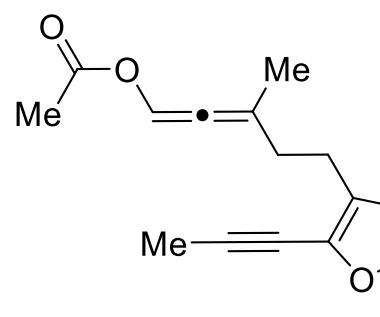

$4 a$

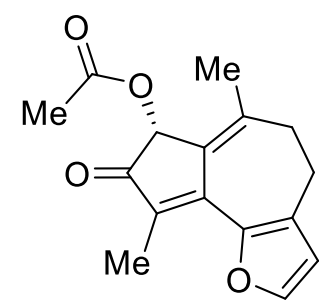

$3 \mathbf{a}$

\section{Table 1, entry 2}

\section{(R)-6,9-dimethyl-8-oxo-4,5,7,8-tetrahydroazuleno[4,5-b]furan-7-yl acetate (3a). Bis(1,5-}

cyclooctadiene)rhodium(I) tetrafluoroborate (0.1 equiv, $2.6 \mathrm{mg}, 0.0065 \mathrm{mmol})$ and $(S)$-MonoPhosalkene (8) (0.15 equiv, $4.0 \mathrm{mg}, 0.0098 \mathrm{mmol}$ ) were weighed into separate flame-dried 5-mL pearshaped flasks in a nitrogen glovebox and each sealed with a septum before removing. The flasks were placed under a nitrogen atmosphere via a Schlenk manifold and an inlet needle. Bis(1,5cyclooctadiene)rhodium(I) tetrafluoroborate and (S)-MonoPhos-alkene (8) were each dissolved in DCE ( $0.5 \mathrm{ml}$ each) and the resulting solutions transferred via syringe to a flame dried Schlenk tube and stirred under nitrogen for $30 \mathrm{~min}$. The tube was evacuated via the inlet needle for a few seconds and refilled $(3 \mathrm{x})$ with carbon monoxide (10\% in argon) using a separate inlet needle attached to a balloon of $\mathrm{CO}$ and stirred for $1 \mathrm{~h}$. Mesitylene ( 1 equiv, $9.0 \mu \mathrm{L}, 0.065 \mathrm{mmol}$ ) was added via syringe to the tube followed by 3-methyl-5-(2-(prop-1-yn-1-yl)furan-3-yl)penta-1,2-dien-1-yl acetate (4a) (1 equiv, $16 \mathrm{mg}, 0.065 \mathrm{mmol})$ in $\operatorname{DCE}(0.03 \mathrm{M}, 1.2 \mathrm{~mL})$ dropwise via syringe. The tube was lowered into a preheated $70{ }^{\circ} \mathrm{C}$ oil bath and stirred until TLC showed complete consumption of starting material $(24 \mathrm{~h})$. The solution was transferred to a $100-\mathrm{mL}$ round bottomed flask containing silica gel, the volatiles were removed in vacuo and the product purified using silica gel flash 
chromatography (5\% to $20 \%$ ethyl acetate/hexanes) to give the title compound (5 $\mathrm{mg}, 28 \%$ ) as a yellow oil in a 85:15 ratio of enantiomers and $8 \mathrm{mg}(62 \%)$ of the aldehyde product (15). TLC and ${ }^{1} \mathrm{H}$ NMR data match those of racemic 3a.

\section{EDD2-109}

Waters 600 HPLC, UV/PDA detector, 328 nm, Daicel CHIRALCEL-OD, 250 X 4.6 mm column, $1 \% \mathrm{iPrOH} /$ hexanes, Flow rate: $1 \mathrm{~mL} / \mathrm{min}$

\begin{tabular}{|l|c|c|}
\hline Peak & Retention Time (min) & Peak area (\%) \\
\hline Peak 1 & 34.23 & 15.37 \\
\hline Peak 2 & 47.41 & 84.63 \\
\hline
\end{tabular}

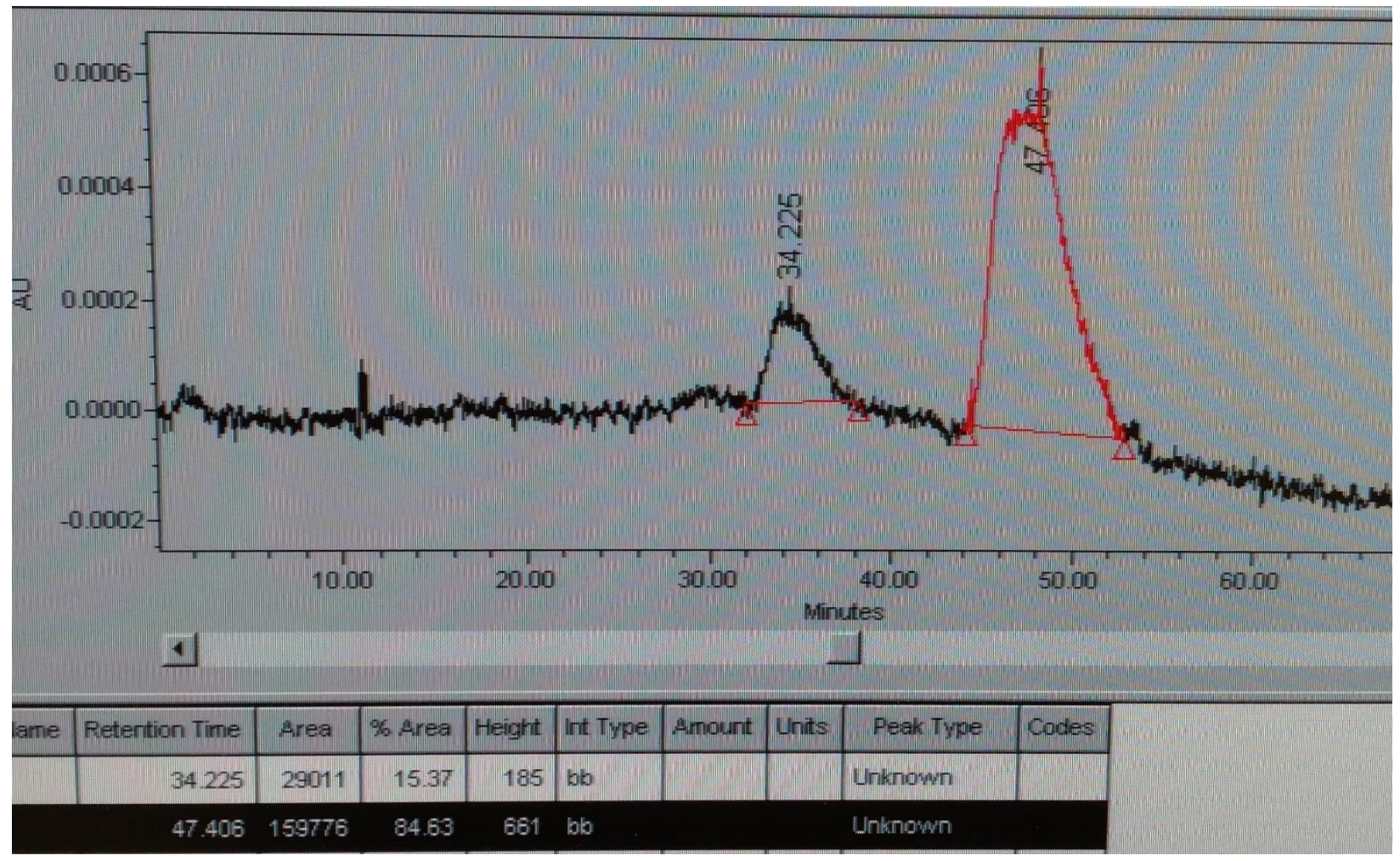


Table 1, entry 3

Follows General Procedure D using $100 \%$ carbon monoxide atmosphere

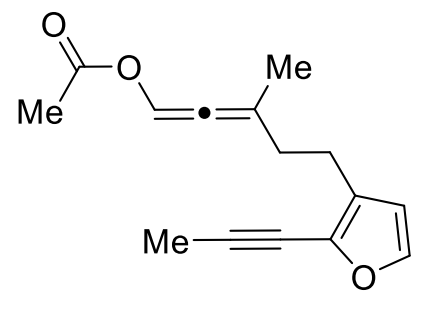

4a<smiles>CC(=O)O[C@H]1C(=O)C(C)=C2C1=C(C)CCc1ccoc12</smiles>

$3 a$

(R)-6,9-dimethyl-8-oxo-4,5,7,8-tetrahydroazuleno[4,5-b]furan-7-yl acetate (3a). Follows general procedure D: Bis(1,5-cyclooctadiene)rhodium(I) tetrafluoroborate $(1.7 \mathrm{mg}, 0.0041 \mathrm{mmol})$, (S)-MonoPhos-alkene (8) $(2.5 \mathrm{mg}, 0.0062 \mathrm{mmol})$, carbon monoxide $(100 \%)$, mesitylene $(5.7 \mu \mathrm{L}$, $0.041 \mathrm{mmol})$, (3-methyl-5-(2-(prop-1-yn-1-yl)furan-3-yl)penta-1,2-dien-1-yl acetate (4a) (10 mg, $0.041 \mathrm{mmol})$, DCE $(1.37 \mathrm{~mL}, 0.03 \mathrm{M})$. The reaction stirred for $4 \mathrm{~h}$ in an oil bath $\left(70{ }^{\circ} \mathrm{C}\right)$. The crude residue was purified via silica gel flash chromatography (5\% to $20 \%$ ethyl acetate/hexanes) to give the title compound ( $4 \mathrm{mg}, 36 \%$ ) as a yellow oil in a $83: 17$ ratio of enantiomers and $26 \%$ yield of the aldehyde byproduct (15) by crude NMR.

\section{EDD2-114}

Waters 600 HPLC, UV/PDA detector, 328 nm, Daicel CHIRALCEL-OD, 250 X 4.6 mm column, $1 \% \mathrm{iPrOH} /$ hexanes, Flow rate: $1 \mathrm{~mL} / \mathrm{min}$

\begin{tabular}{|c|c|c|}
\hline Peak & Retention Time (min) & Peak area (\%) \\
\hline Peak 1 & 33.65 & 16.68 \\
\hline Peak 2 & 45.24 & 83.32 \\
\hline
\end{tabular}


Supporting Information for Organic Letters

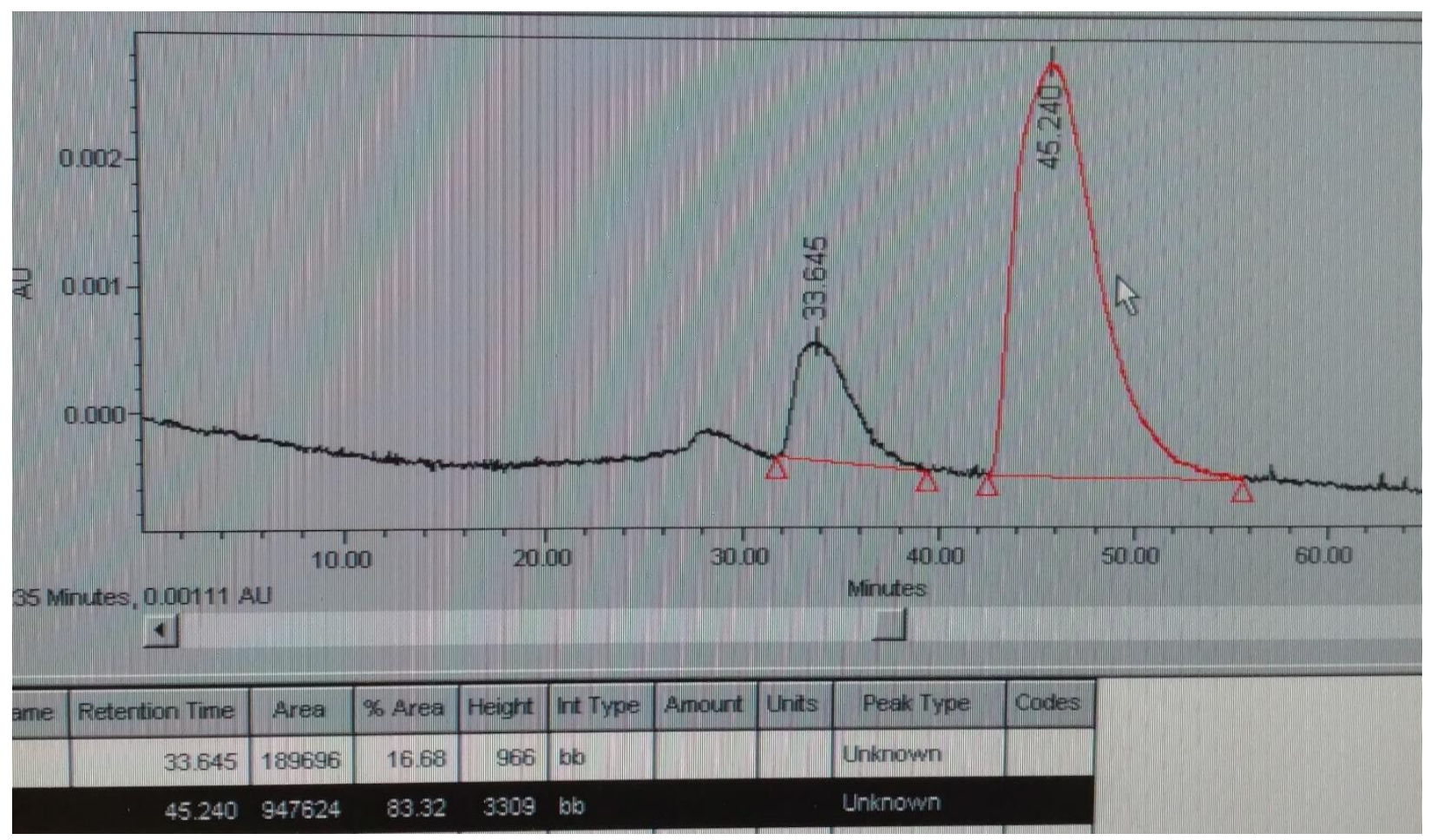




\section{Table 1, entry 4}

Follows General Procedure D using $100 \%$ carbon monoxide and reaction temp $50^{\circ} \mathrm{C}$

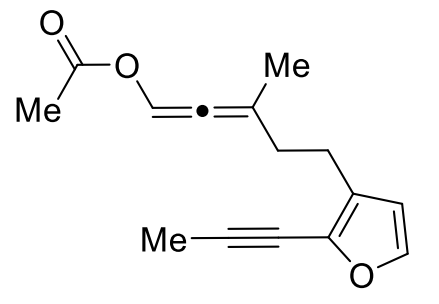

$4 a$

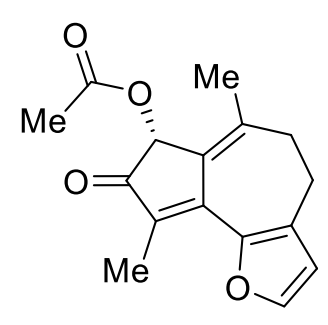

$3 a$

(R)-6,9-dimethyl-8-oxo-4,5,7,8-tetrahydroazuleno[4,5-b]furan-7-yl acetate (3a). Follows general procedure D: Bis(1,5-cyclooctadiene)rhodium(I) tetrafluoroborate $(1.7 \mathrm{mg}, 0.0041 \mathrm{mmol})$, (S)-MonoPhos-alkene (8) (2.5 mg, $0.0062 \mathrm{mmol})$, carbon monoxide (100\%), mesitylene (5.7 $\mu \mathrm{L}$, $0.041 \mathrm{mmol}),(3-\mathrm{methyl}-5$-(2-(prop-1-yn-1-yl)furan-3-yl)penta-1,2-dien-1-yl acetate (4a) (10 mg, $0.041 \mathrm{mmol})$, DCE $(1.37 \mathrm{~mL}, 0.03 \mathrm{M})$. The reaction was stirred for $20 \mathrm{~h}$ in an oil bath $\left(50{ }^{\circ} \mathrm{C}\right)$. The crude residue was purified via silica gel flash chromatography (5\% to $10 \%$ ethyl acetate/hexanes) to give the title compound ( $3 \mathrm{mg}, 27 \%$ ) as a yellow oil in an 81:19 ratio of enantiomers and $4 \mathrm{mg}(50 \%)$ of the aldehyde byproduct (15).

\section{EDD2-119}

Waters 600 HPLC, UV/PDA detector, 328 nm, Daicel CHIRALCEL-OD, 250 X 4.6 mm column, $1 \% \mathrm{iPrOH} / \mathrm{hexanes}$, Flow rate: $1 \mathrm{~mL} / \mathrm{min}$

\begin{tabular}{|c|c|c|}
\hline Peak & Retention Time (min) & Peak area (\%) \\
\hline Peak 1 & 33.49 & 19.03 \\
\hline Peak 2 & 45.41 & 80.97 \\
\hline
\end{tabular}




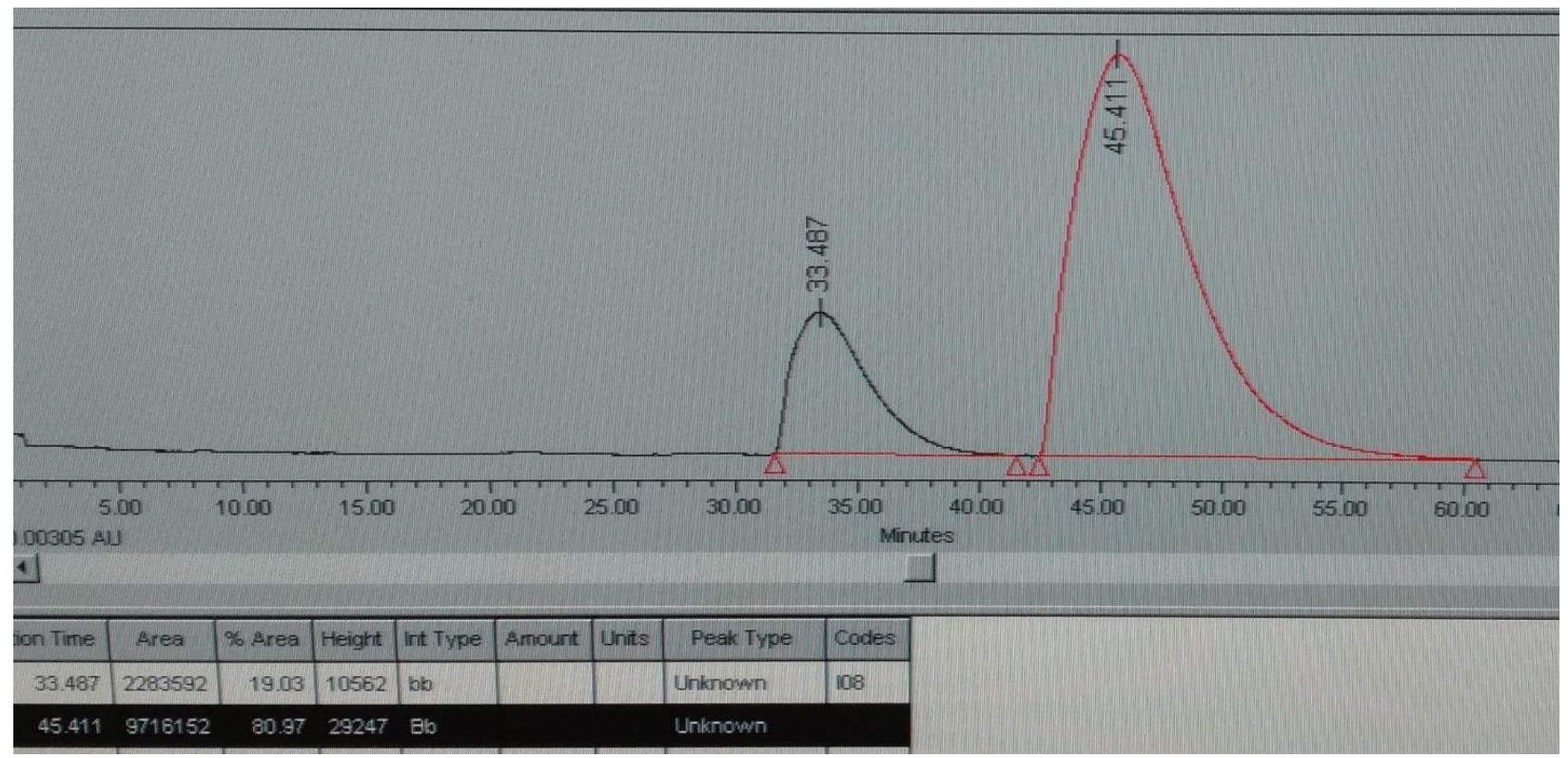

Table 1, entry 5

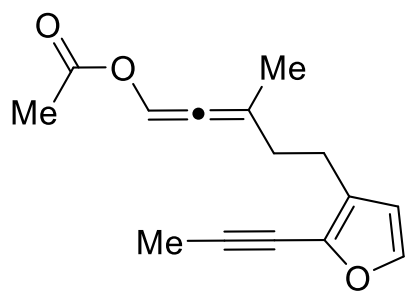

$4 a$<smiles>CC(=O)O[C@H]1C(=O)C(C)=C2C1=C(C)CCc1ccoc12</smiles>

$3 a$

General Procedure E: Asymmetric Allenic Pauson-Khand reaction under dilute conditions and removal of rhodium catalyst with polymer bound $\mathrm{PPh}_{3}$

(R)-6,9-dimethyl-8-oxo-4,5,7,8-tetrahydroazuleno[4,5-b]furan-7-yl acetate (3a). Run 1:

Bis(1,5-cyclooctadiene)rhodium(I) tetrafluoroborate $(0.1$ equiv, $1.7 \mathrm{mg}, 0.0041 \mathrm{mmol})$ and $(S)$ MonoPhos-alkene (8) (0.15 equiv, $2.5 \mathrm{mg}, 0.0062 \mathrm{mmol})$ were weighed into separate flame-dried 5-mL pear-shaped flasks in a nitrogen glovebox and each sealed with a septum. The flasks were placed under a nitrogen atmosphere via a Schlenk manifold and an inlet needle. Bis(1,5cyclooctadiene)rhodium(I) tetrafluoroborate and $(S)$-MonoPhos-alkene $(\mathbf{8})$ were each dissolved in 
DCE (0.75 mL each) and the resulting solutions transferred via syringe to a flame dried Schlenk tube and stirred under nitrogen for $30 \mathrm{~min}$. The tube was evacuated via the inlet needle for a few seconds and refilled with carbon monoxide (100\%) using a separate inlet needle attached to a balloon of $\mathrm{CO}$ and stirred for $1 \mathrm{~h}$. 3-Methyl-5-(2-(prop-1-yn-1-yl)furan-3-yl)penta-1,2-dien-1-yl acetate (4a) $(1.0$ equiv, $10 \mathrm{mg}, 0.041 \mathrm{mmol})$ in DCE $(0.01 \mathrm{M}, 2.6 \mathrm{~mL})$ was added dropwise via syringe. The tube was lowered into a preheated $70{ }^{\circ} \mathrm{C}$ oil bath and stirred until TLC showed complete consumption of starting material $(20.5 \mathrm{~h})$. The Schlenk tube was cooled to rt and polymer bound triphenylphosphine ( $14 \mathrm{mg}, 1.0 \mathrm{eq}, 3 \mathrm{mmol} / \mathrm{g}$ ) was added and stirred for $4 \mathrm{~h}$. The solution was vacuum filtered, concentrated in vacuo, and the product purified using silica gel flash chromatography (5\% to $20 \%$ ethyl acetate/hexanes) to give the title compound ( $7 \mathrm{mg}, 62 \%)$ as a yellow oil in a 80:20 ratio of enantiomers and $2 \mathrm{mg}(25 \%)$ of the aldehyde byproduct (15).

\section{EDD3-161}

$[\alpha]^{25} \mathrm{D}=-2.51\left(\mathrm{c} .=0.25, \mathrm{CH}_{2} \mathrm{Cl}_{2}\right)$

Waters 600 HPLC, UV/PDA detector, 328 nm, Daicel CHIRALCEL-OD, 250 X 4.6 mm column, $1 \% \mathrm{iPrOH} / \mathrm{hexanes}$, Flow rate: $1 \mathrm{~mL} / \mathrm{min}$

\begin{tabular}{|c|c|c|}
\hline Peak & Retention Time (min) & Peak area (\%) \\
\hline Peak 1 & 31.57 & 20.23 \\
\hline Peak 2 & 38.74 & 79.77 \\
\hline
\end{tabular}




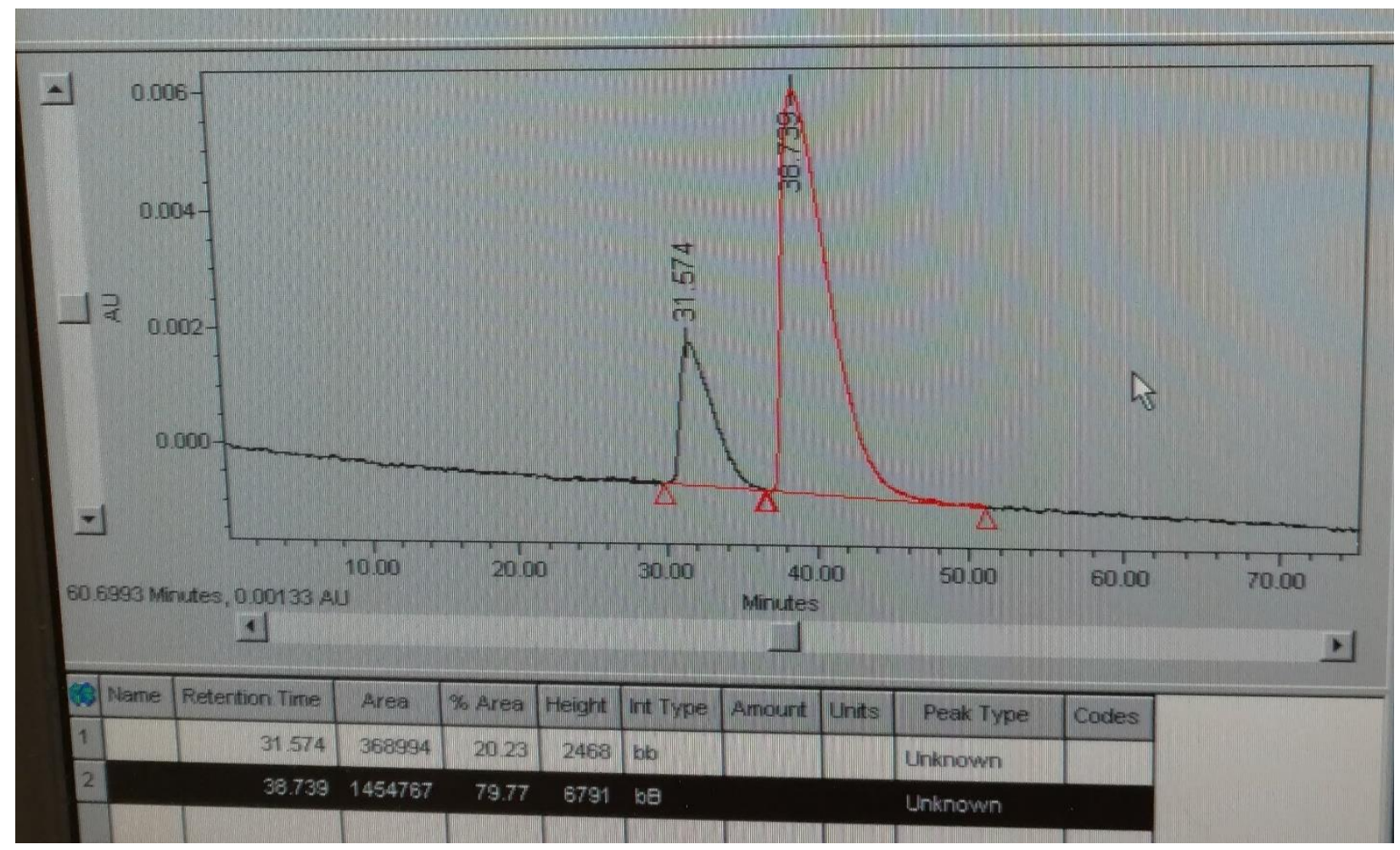

Run 2: Bis(1,5-cyclooctadiene)rhodium(I) tetrafluoroborate (0.1 equiv, $2.5 \mathrm{mg}, 0.0061 \mathrm{mmol})$ and (S)-MonoPhos-alkene (8) (0.15 equiv, $3.8 \mathrm{mg}, 0.0092 \mathrm{mmol})$ were weighed into separate flamedried 5-mL pear-shaped flasks in a nitrogen glovebox and each sealed with a septum. The flasks were placed under a nitrogen atmosphere via a Schlenk manifold and an inlet needle. Bis(1,5cyclooctadiene)rhodium(I) tetrafluoroborate and $(S)$-MonoPhos-alkene $(\mathbf{8})$ were each dissolved in DCE (1.0 mL each) and the resulting solution transferred via syringe to a flame dried Schlenk tube and stirred under nitrogen for $30 \mathrm{~min}$. The tube was evacuated via the inlet needle for a few seconds and refilled with carbon monoxide (100\%) using a separate inlet needle attached to a balloon of $\mathrm{CO}$ and stirred for 1 h. 3-Methyl-5-(2-(prop-1-yn-1-yl)furan-3-yl)penta-1,2-dien-1-yl acetate (4a) (1.0 equiv, $15 \mathrm{mg}, 0.061 \mathrm{mmol})$ in DCE $(0.01 \mathrm{M}, 6.1 \mathrm{~mL})$ was added dropwise via syringe. The tube was lowered into a preheated $70{ }^{\circ} \mathrm{C}$ oil bath and stirred until TLC showed complete 
consumption of starting material $(20 \mathrm{~h})$. The Schlenk tube was cooled to $\mathrm{rt}$ and polymer bound triphenylphosphine $(20 \mathrm{mg}, 1.0 \mathrm{eq}, 3 \mathrm{mmol} / \mathrm{g}$ ) was added and stirred for $4 \mathrm{~h}$. The solution was vacuum filtered, concentrated in vacuo, and the product purified using silica gel flash chromatography (5\% to $20 \%$ ethyl acetate/hexanes) to give the title compound (10 $\mathrm{mg}, 59 \%$ ) as a yellow oil in a 84:16 ratio of enantiomers and $2 \mathrm{mg}(17 \%)$ of the aldehyde byproduct (15).

\section{EDD5-318}

Waters 600 HPLC, UV/PDA detector, 328 nm, Daicel CHIRALCEL-OD, 250 X 4.6 mm column, $1 \% \mathrm{iPrOH} / \mathrm{hexanes}$, Flow rate: $1 \mathrm{~mL} / \mathrm{min}$

\begin{tabular}{|c|c|c|}
\hline Peak & Retention Time (min) & Peak area (\%) \\
\hline Peak 1 & 31.71 & 15.59 \\
\hline Peak 2 & 38.94 & 84.41 \\
\hline
\end{tabular}




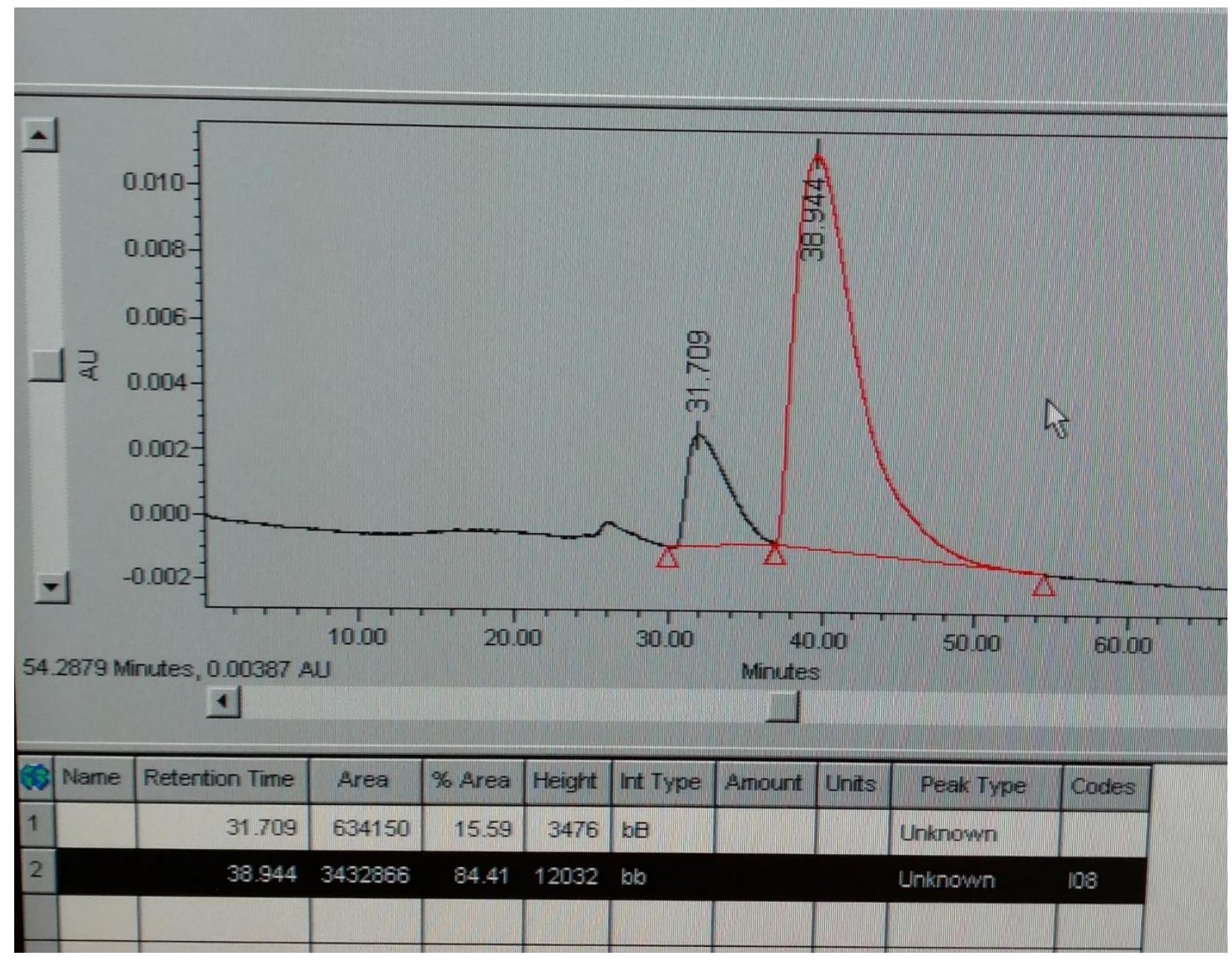

The asymmetric Allenic Pauson-Khand reaction under dilute conditions without removal

\section{of rhodium catalyst with polymer bound $\mathrm{PPh}_{3}$}

Follows General Procedure E: Bis(1,5-cyclooctadiene)rhodium(I) tetrafluoroborate (2.5 mg, $0.0061 \mathrm{mmol}),(S)-$ MonoPhos-alkene (8) (3.8 mg, $0.0092 \mathrm{mmol})$, carbon monoxide (100\%), 3Methyl-5-(2-(prop-1-yn-1-yl)furan-3-yl)penta-1,2-dien-1-yl acetate (4a) (15 mg, $0.061 \mathrm{mmol}$ ), DCE $(6.1 \mathrm{~mL}, 0.01 \mathrm{M})$. The reaction was stirred for $21 \mathrm{~h}$ in an oil bath $\left(70{ }^{\circ} \mathrm{C}\right)$. The crude residue was purified via silica gel flash chromatography (5\% to $20 \%$ ethyl acetate/hexanes) to give $11 \mathrm{mg}(65 \%)$ of the title compound as a yellow oil and $2 \mathrm{mg}(17 \%)$ of the aldehyde byproduct (15). 
EDD5-366

Synthesis of $[5,7,5]$ ring system via the asymmetric allenic Pauson-Khand reaction in $o$ DCB

Table 1, entry 6

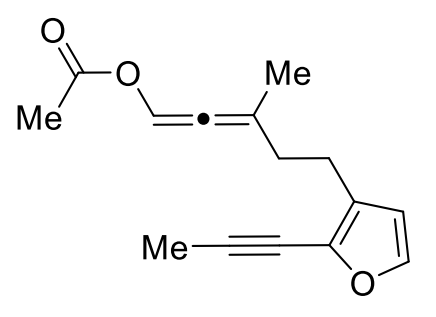

$4 a$

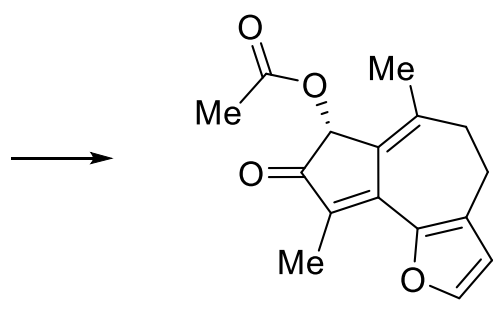

$3 a$

(R)-6,9-dimethyl-8-oxo-4,5,7,8-tetrahydroazuleno[4,5-b]furan-7-yl acetate (3a). Bis(1,5cyclooctadiene)rhodium(I) tetrafluoroborate (0.1 equiv, $1.7 \mathrm{mg}, 0.0041 \mathrm{mmol}$ ) and $(S)$-MonoPhosalkene (8) (0.15 equiv, $2.5 \mathrm{mg}, 0.0062 \mathrm{mmol})$ were weighed into separate flame-dried pear-shaped flasks in a nitrogen glovebox and each sealed with a septum. The flasks were placed under a nitrogen atmosphere via a Schlenk manifold and an inlet needle. Bis(1,5cyclooctadiene)rhodium(I) tetrafluoroborate and $(S)$-MonoPhos-alkene $(\mathbf{8})$ were each dissolved in $o$-DCB $(0.75 \mathrm{ml}$ each $)$ and the resulting solutions transferred via syringe to a flame dried Schlenk tube and stirred under nitrogen for $30 \mathrm{~min}$. The tube was evacuated via the inlet needle for a few seconds and refilled with carbon monoxide (100\%) using a separate inlet needle attached to a balloon of $\mathrm{CO}$ and stirred for $1 \mathrm{~h}$. 3-Methyl-5-(2-(prop-1-yn-1-yl)furan-3-yl)penta-1,2-dien-1-yl acetate $(4 \mathbf{a})(1.0$ equiv, $1.0 \mathrm{mg}, 0.041 \mathrm{mmol})$ in $o$-DCB $(0.01 \mathrm{M}, 2.6 \mathrm{~mL})$ was added dropwise via syringe. The tube was lowered into a preheated $70{ }^{\circ} \mathrm{C}$ oil bath and stirred for $44 \mathrm{~h}$. The Schlenk tube was cooled to $\mathrm{rt}$ and polymer bound triphenylphosphine $(14 \mathrm{mg}, 1.0 \mathrm{eq}, 3 \mathrm{mmol} / \mathrm{g})$ was added by temporary removal of the septum and stirred for $4 \mathrm{~h}$. The solution was vacuum filtered, 
concentrated in vacuo, and the product purified using silica gel flash chromatography (5\% to 20\% ethyl acetate/hexanes) to give the title compound ( $3 \mathrm{mg}, 27 \%, 34 \% \mathrm{brsm})$ as a yellow oil in a 74:26 ratio of enantiomers and $2.5 \mathrm{mg}(25 \%, 31 \% \mathrm{brsm})$ of the aldehyde byproduct (15). $2 \mathrm{mg}$ of starting material was also recovered.

Waters 600 HPLC, UV/PDA detector, 328 nm, Daicel CHIRALCEL-OD, 250 X 4.6 mm column, $1 \% \mathrm{iPrOH} / \mathrm{hexanes}$, Flow rate: $1 \mathrm{~mL} / \mathrm{min}$

\begin{tabular}{|c|c|c|}
\hline Peak & Retention Time (min) & Peak area (\%) \\
\hline Peak 1 & 32.50 & 25.73 \\
\hline Peak 2 & 39.87 & 74.27 \\
\hline
\end{tabular}




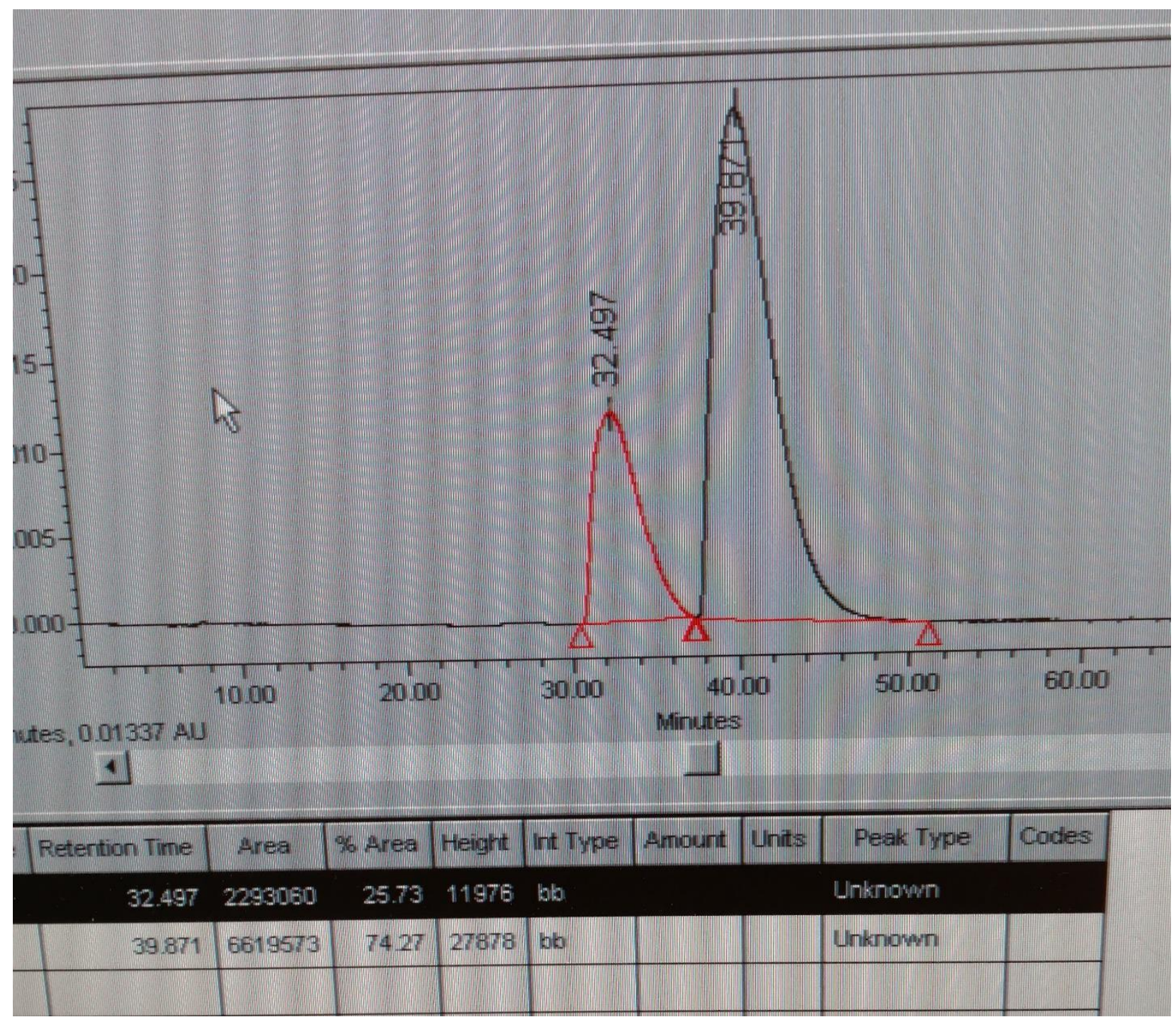

Aldehyde byproduct of the asymmetric allenic Pauson-Khand reaction

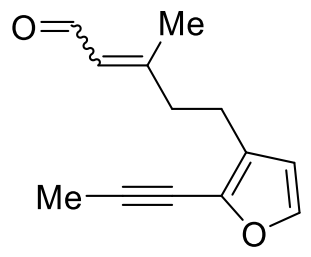

15

3-methyl-5-(2-(prop-1-yn-1-yl)furan-3-yl)pent-2-enal (15). Obtained as a 3:2 ratio of isomers as a byproduct of the asymmetric allenic Pauson-Khand reaction that were inseparable by column chromatography. 
$\underline{{ }^{1} \mathrm{H} \mathrm{NMR}} \quad\left(400 \mathrm{MHz}, \mathrm{CDCl}_{3}\right)$

$9.99(\mathrm{~d}, J=8.0 \mathrm{~Hz}, 1 \mathrm{H})^{*}, 9.84(\mathrm{~d}, J=8.0 \mathrm{~Hz}, 1 \mathrm{H})^{* *}, 7.23(\mathrm{~d}, J=1.6 \mathrm{~Hz}, 1 \mathrm{H})$,

$6.23(\mathrm{~d}, J=1.6 \mathrm{~Hz}, 1 \mathrm{H}), 5.90-5.87(\mathrm{~m}, 1 \mathrm{H}), 2.84-2.80(\mathrm{~m}, 2 \mathrm{H}) * *, 2.74-2.70(\mathrm{~m}$,

$2 \mathrm{H}), 2.47(\mathrm{t}, J=8.0 \mathrm{~Hz}, 2 \mathrm{H}), 2.20(\mathrm{~d}, J=0.4 \mathrm{~Hz}, 3 \mathrm{H})^{*}, 2.11(\mathrm{~s}, 3 \mathrm{H})^{*}, 2.10(\mathrm{~s}, 3$

$\mathrm{H})^{* *}, 2.01(\mathrm{~d}, J=1.2 \mathrm{~Hz}, 3 \mathrm{H})^{* *} \mathrm{ppm}$

Peaks designated $(*)$ and $(* *)$ appear in a 3:2 ratio respectively

$\stackrel{{ }^{13} \mathrm{C} \mathrm{NMR}}{ } \quad\left(100 \mathrm{MHz}, \mathrm{CDCl}_{3}\right)$

191.4, 190.8, 162.8, 142.7, 142.6, 135.0, 129.3, 127.9, 127.1, 127.0, 111.5, 111.4, $93.1,69.4,40.5,32.9,29.9,25.1,24.6,23.1,17.7,4.8,4.7 \mathrm{ppm}$

$\underline{\mathrm{IR}} \quad$ (Thin Film)

$2921,1671,1442,1197,1074 \mathrm{~cm}^{-1}$

HRMS HRMS-ESI (m/z): $[\mathrm{M}+\mathrm{H}]^{+}$calcd for $\mathrm{C}_{13} \mathrm{H}_{15} \mathrm{O}_{2}, 203.1072$; found, 203.1046

TLC $\quad R_{f}=0.55$ (35\% ethyl acetate/hexanes); silica gel, UV, $p$-anisaldehyde

\section{Hydrolysis of $\alpha$-acyloxy cyclopentenone}

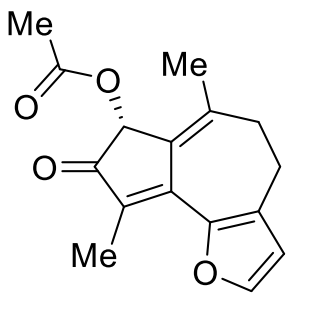

3a

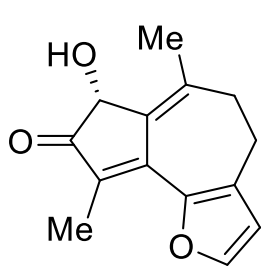

S4

7-hydroxy-6,9-dimethyl-5,7-dihydroazuleno[4,5-b]furan-8(4H)-one (S4). This compound was prepared in a manner similar to that previously reported with some modifications. ${ }^{6}$ An 8-mL, screw 
cap test tube equipped with a magnetic stir bar and needle attached to a nitrogen manifold was charged with scandium(III) triflate $(3.8 \mathrm{mg}, 0.0078 \mathrm{mmol}, 0.3$ equiv) dissolved in a $20 \%$ (v/v) water/methanol solution (0.5 mL, 0.0156 M). 6,9-Dimethyl-8-oxo-4,5,7,8-tetrahydroazuleno[4,5b]furan-7-yl acetate (3a) was dissolved in a $20 \%(\mathrm{v} / \mathrm{v})$ water/methanol solution $(0.5 \mathrm{~mL}, 0.052 \mathrm{M})$ and transferred to the test tube dropwise via syringe. The tube was lowered into a $30{ }^{\circ} \mathrm{C}$ oil bath and stirred for $44 \mathrm{~h}$ until no starting material was evident by TLC. The solution was diluted with deionized water $(5 \mathrm{~mL})$, transferred to a separatory funnel and extracted with DCM $(3 \times 5 \mathrm{~mL})$. The combined organic extracts were dried over magnesium sulfate, filtered, and concentrated. The crude residue was purified via silica gel flash chromatography (35\% ethyl acetate/hexanes) to give the title compound ( $3 \mathrm{mg}, 50 \%)$ as a yellow solid in a 87:13 ratio of enantiomers.

\section{EDD3-165}

${ }^{1} \mathrm{H} \mathrm{NMR} \quad\left(400 \mathrm{MHz}, \mathrm{CDCl}_{3}\right)$

$7.54(\mathrm{~d}, J=1.6 \mathrm{~Hz}, 1 \mathrm{H}), 6.38(\mathrm{~d}, J=1.6 \mathrm{~Hz}, 1 \mathrm{H}), 4.57(\mathrm{~s}, 1 \mathrm{H}), 2.86-2.73(\mathrm{~m}, 2$

H), 2.56-2.53 (m, 2 H), 2.19 (s, $3 \mathrm{H}), 2.14$ (s, $3 \mathrm{H}) \mathrm{ppm}$

${ }^{13} \mathrm{C} \mathrm{NMR} \quad\left(100 \mathrm{MHz}, \mathrm{CDCl}_{3}\right)$

$205.1,150.3,147.5,145.1,139.5,132.7,130.8,130.0,113.8,71.6,34.5,25.1$, 24.0, $10.1 \mathrm{ppm}$

$\underline{\text { IR }} \quad$ (Thin Film)

$3409,2924,1680,1598,1033 \mathrm{~cm}^{-1}$

$\underline{\text { HRMS }} \quad$ HRMS-ESI (m/z): $[\mathrm{M}+\mathrm{H}]^{+}$calcd for $\mathrm{C}_{14} \mathrm{H}_{14} \mathrm{O}_{3}, 231.1021$; found, 231.1014

TLC $\quad R_{f}=0.27$ (35\% ethyl acetate/hexanes); silica gel, UV, $p$-anisaldehyde 
Waters 600 HPLC, UV/PDA detector, 328 nm, Daicel CHIRALCEL-OD, 250 X 4.6 mm column, $1 \% \mathrm{iPrOH} / \mathrm{hexanes}$, Flow rate: $1 \mathrm{~mL} / \mathrm{min}$

\begin{tabular}{|c|c|c|}
\hline Peak & Retention Time (min) & Peak area (\%) \\
\hline Peak 1 & 41.78 & 13.02 \\
\hline Peak 2 & 47.12 & 86.98 \\
\hline
\end{tabular}

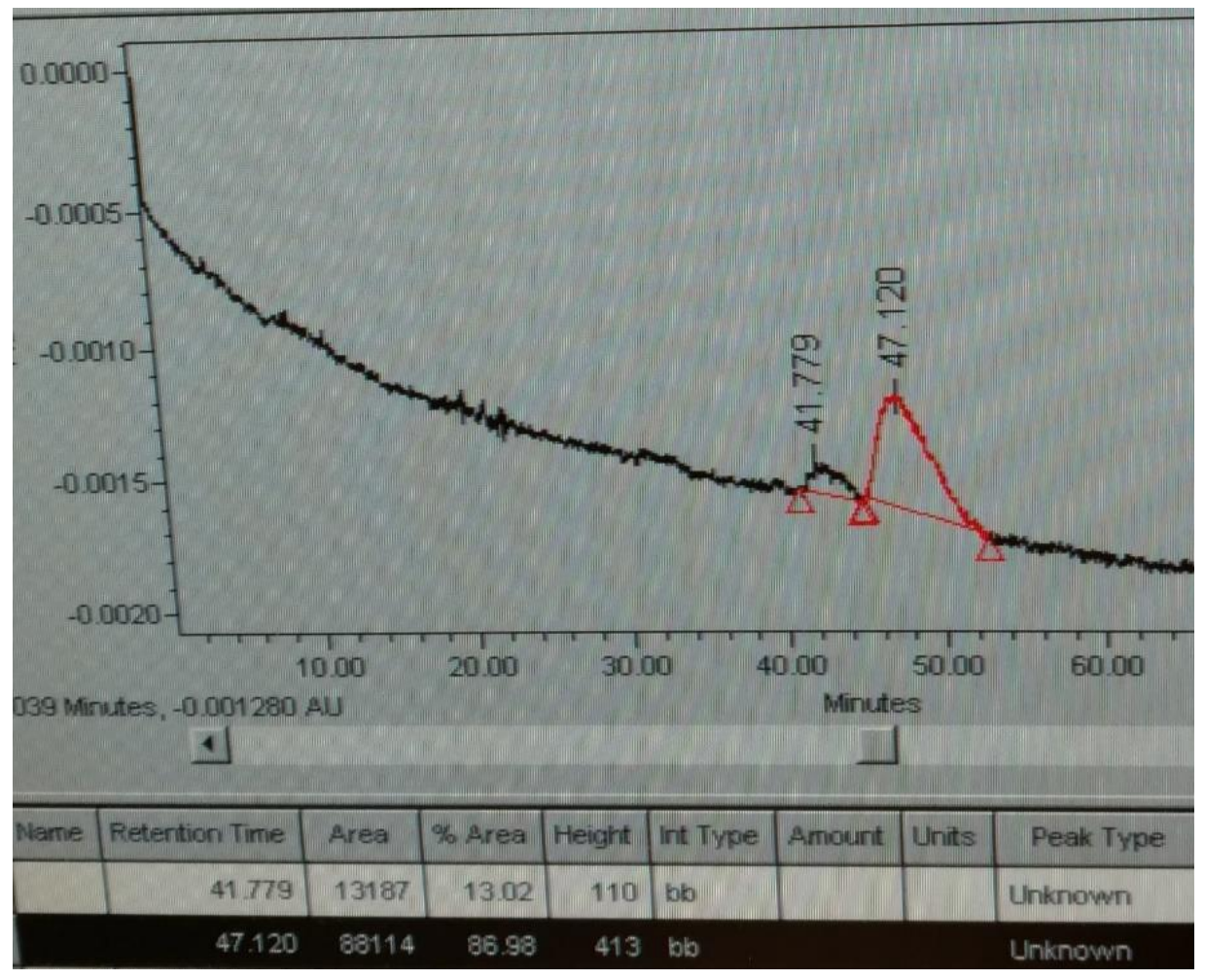

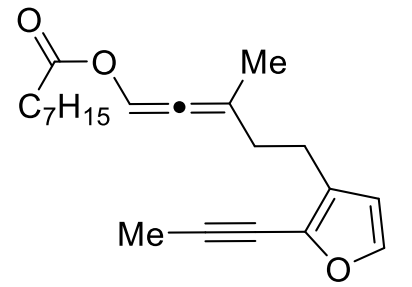

4b

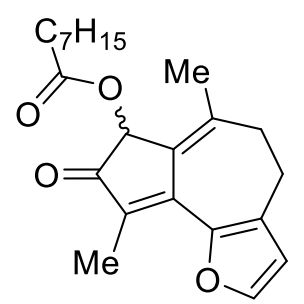

3b 
6,9-dimethyl-8-oxo-4,5,7,8-tetrahydroazuleno[4,5-b]furan-7-yl octanoate (3b). Follows

General Procedure C: Bis(1,5-cyclooctadiene)rhodium(I) tetrafluoroborate (1.9 mg, 0.0046 mmol), triphenyl phosphine $(1.8 \mathrm{mg}, 0.0069 \mathrm{mmol})$, carbon monoxide (100\%), 3-methyl-5-(2(prop-1-yn-1-yl)furan-3-yl)penta-1,2-dien-1-yl octanoate (4b) (15 mg, 0.046 mmol), DCE (2.3 $\mathrm{mL}, 0.02 \mathrm{M})$. The reaction was stirred for $26.5 \mathrm{~h}$ in an oil bath $\left(70{ }^{\circ} \mathrm{C}\right)$. The crude residue was purified via silica gel flash chromatography (5\% to $10 \%$ ethyl acetate/hexanes) to give the title compound (11 mg, 69\%) as a yellow oil.

\section{EDD5-331}

$\underline{{ }^{1} \mathrm{H} \mathrm{NMR}} \quad\left(400 \mathrm{MHz}, \mathrm{CDCl}_{3}\right)$

7.54 (b s, $1 \mathrm{H}), 6.38$ (b s, $1 \mathrm{H}), 5.81$ (s, $1 \mathrm{H}), 2.88-2.73$ (m, $2 \mathrm{H}), 2.56-2.55$ (m, 2

H), 2.41-2.37 (m, $2 \mathrm{H}), 2.19$ (s, $3 \mathrm{H}), 1.91(\mathrm{~s}, 3 \mathrm{H}), 1.66$ (p, J= 7.2 Hz, $2 \mathrm{H})$, 1.29-1.27 (m, $8 \mathrm{H}), 0.89-0.85(\mathrm{~m}, 3 \mathrm{H}) \mathrm{ppm}$

${ }^{13} \mathrm{C} \mathrm{NMR} \quad\left(100 \mathrm{MHz}, \mathrm{CDCl}_{3}\right)$

$199.9,172.8,149.7,147.4,145.0,138.6,132.4,132.1,127.8,113.8,71.7,34.6$, $34.3,31.8,29.2,29.1,25.1,24.9,24.0,22.7,14.2,10.2 \mathrm{ppm}$

$\underline{\text { IR }} \quad$ (Thin Film)

$1739,1693,1603 \mathrm{~cm}^{-1}$

$\underline{\text { HRMS }} \quad$ HRMS-ESI (m/z): $[\mathrm{M}+\mathrm{H}]^{+}$calcd for $\mathrm{C}_{22} \mathrm{H}_{29} \mathrm{O}_{4}, 357.2066$; found, 357.2047

TLC $\quad R_{f}=0.53(35 \%$ ethyl acetate/hexanes); silica gel, UV, $p$-anisaldehyde

Waters 600 HPLC, UV/PDA detector, 328 nm, Daicel CHIRALCEL-OD, 250 X 4.6 mm column, $1 \% \mathrm{iPrOH} /$ hexanes, Flow rate: $1 \mathrm{~mL} / \mathrm{min}$ 


\begin{tabular}{|c|c|c|}
\hline Peak & Retention Time (min) & Peak area (\%) \\
\hline Peak 1 & 17.86 & 49.98 \\
\hline Peak 2 & 23.90 & 50.02 \\
\hline
\end{tabular}

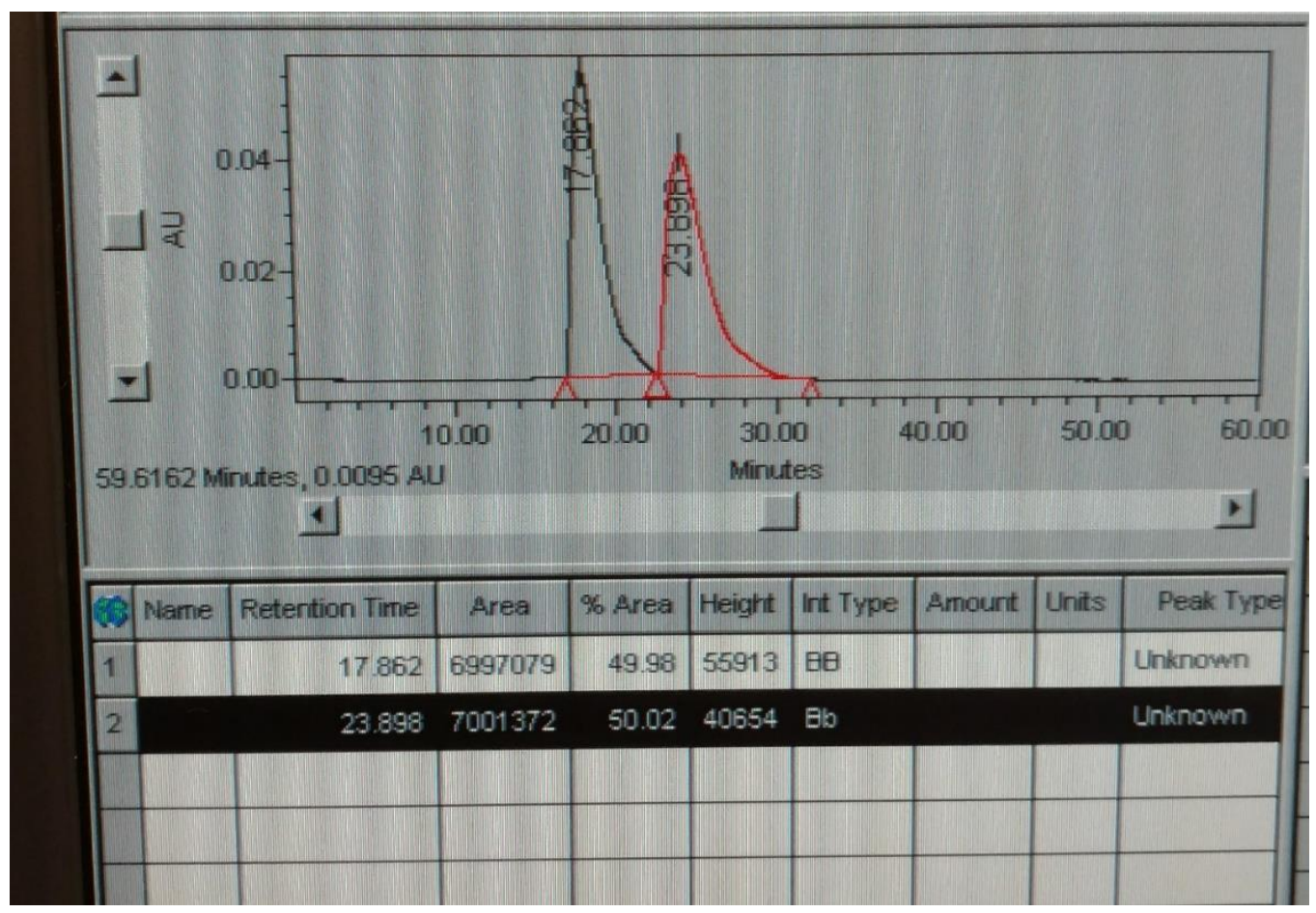

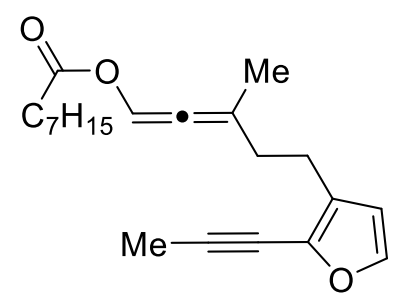

4b

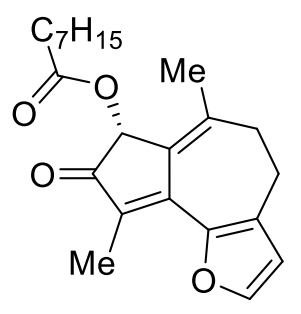

3b

(R)-6,9-dimethyl-8-oxo-4,5,7,8-tetrahydroazuleno[4,5-b]furan-7-yl octanoate (3b). Run 1:

Follows General Procedure E: Bis(1,5-cyclooctadiene)rhodium(I) tetrafluoroborate (1.9 mg, $0.0046 \mathrm{mmol}),(S)$-MonoPhos-alkene $(\mathbf{8})(2.8 \mathrm{mg}, 0.0069 \mathrm{mmol})$, carbon monoxide (100\%), 3methyl-5-(2-(prop-1-yn-1-yl)furan-3-yl)penta-1,2-dien-1-yl octanoate (4b) (15 mg, 0.046 mmol), 
DCE $(4.6 \mathrm{~mL}, 0.01 \mathrm{M})$. The reaction was stirred for $42.5 \mathrm{~h}$ in an oil bath $\left(70^{\circ} \mathrm{C}\right)$. The crude residue was purified via silica gel flash chromatography (2.5\% to 5\% ethyl acetate/hexanes) to give $9 \mathrm{mg}$ (56\%) of the title compound as a yellow oil in a 84:16 ratio of enantiomers and $0.7 \mathrm{mg}(8 \%)$ of the aldehyde byproduct (15).

\section{EDD5-338}

Waters 600 HPLC, UV/PDA detector, 328 nm, Daicel CHIRALCEL-OD, 250 X 4.6 mm column, $1.0 \% \mathrm{iPrOH} / \mathrm{hexanes,} \mathrm{Flow} \mathrm{rate:} 1 \mathrm{~mL} / \mathrm{min}$

\begin{tabular}{|l|c|c|}
\hline Peak & Retention Time (min) & Peak area (\%) \\
\hline Peak 1 & 18.03 & 15.89 \\
\hline Peak 2 & 22.46 & 84.11 \\
\hline
\end{tabular}




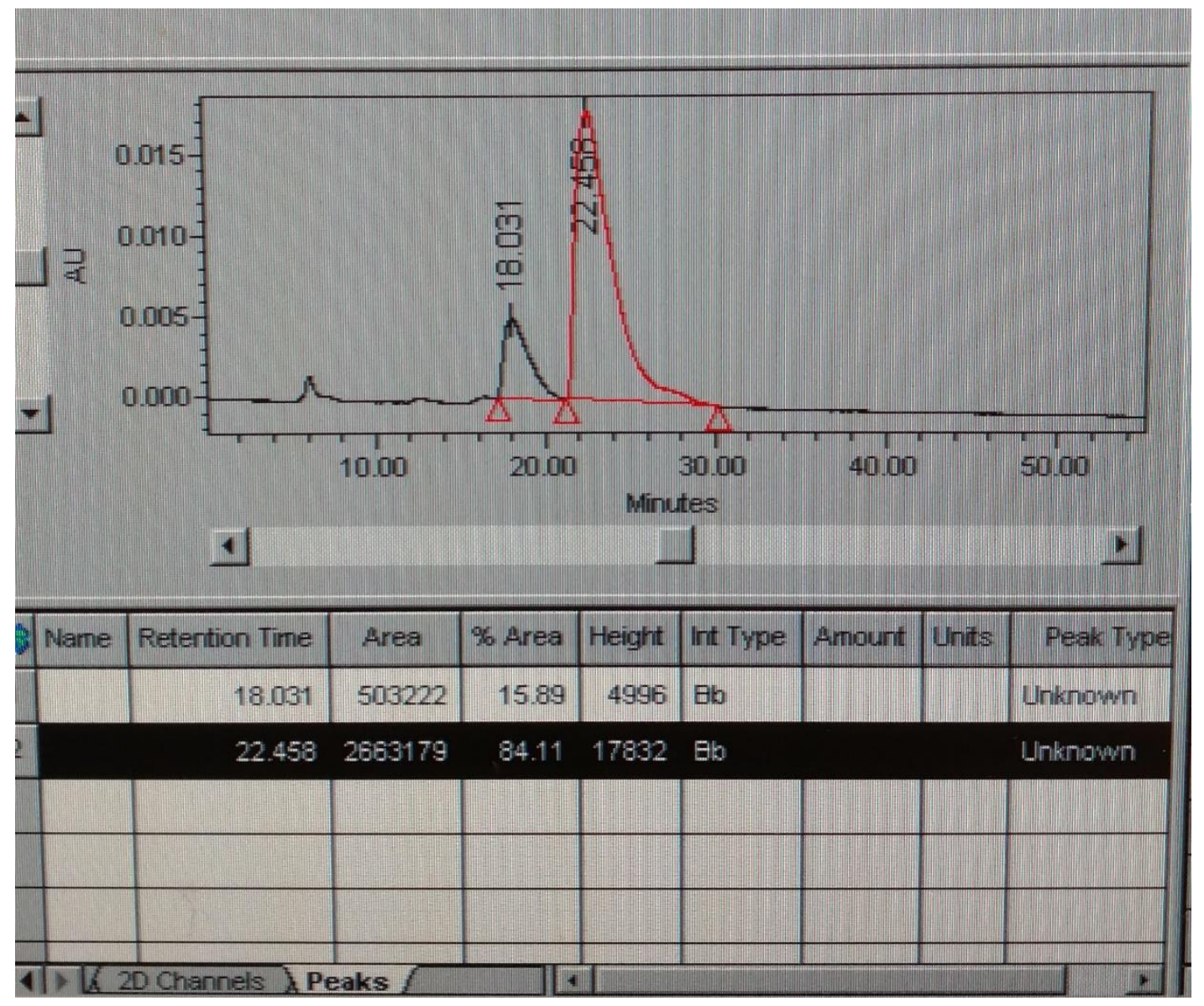

Run 2: Follows General Procedure E: Bis(1,5-cyclooctadiene)rhodium(I) tetrafluoroborate (1.9 mg, $0.0046 \mathrm{mmol}$ ), (S)-MonoPhos-alkene (8) (2.8 mg, $0.0069 \mathrm{mmol})$, carbon monoxide (100\%), 3-methyl-5-(2-(prop-1-yn-1-yl)furan-3-yl)penta-1,2-dien-1-yl octanoate (4b) (15 mg, 0.046 mmol), DCE (4.6 mL, 0.01 M). The reaction was stirred for $29 \mathrm{~h}$ in an oil bath $\left(70{ }^{\circ} \mathrm{C}\right)$. The crude residue was purified via silica gel flash chromatography (5\% ethyl acetate/hexanes) to give $6.4 \mathrm{mg}$ $(40 \%)$ of the title compound as a yellow oil in a $84: 16$ ratio of enantiomers and $0.6 \mathrm{mg}(7 \%)$ of the aldehyde byproduct (15).

This run (run 2) was not included in the percent yield calculation in Table 2 because of unforeseen complication.

\section{EDD5-334}


Waters 600 HPLC, UV/PDA detector, 328 nm, Daicel CHIRALCEL-OD, 250 X 4.6 mm column, $1.0 \% \mathrm{iPrOH} /$ hexanes, Flow rate: $1 \mathrm{~mL} / \mathrm{min}$

\begin{tabular}{|l|c|c|}
\hline Peak & Retention Time (min) & Peak area (\%) \\
\hline Peak 1 & 18.38 & 16.19 \\
\hline Peak 2 & 22.25 & 83.81 \\
\hline
\end{tabular}

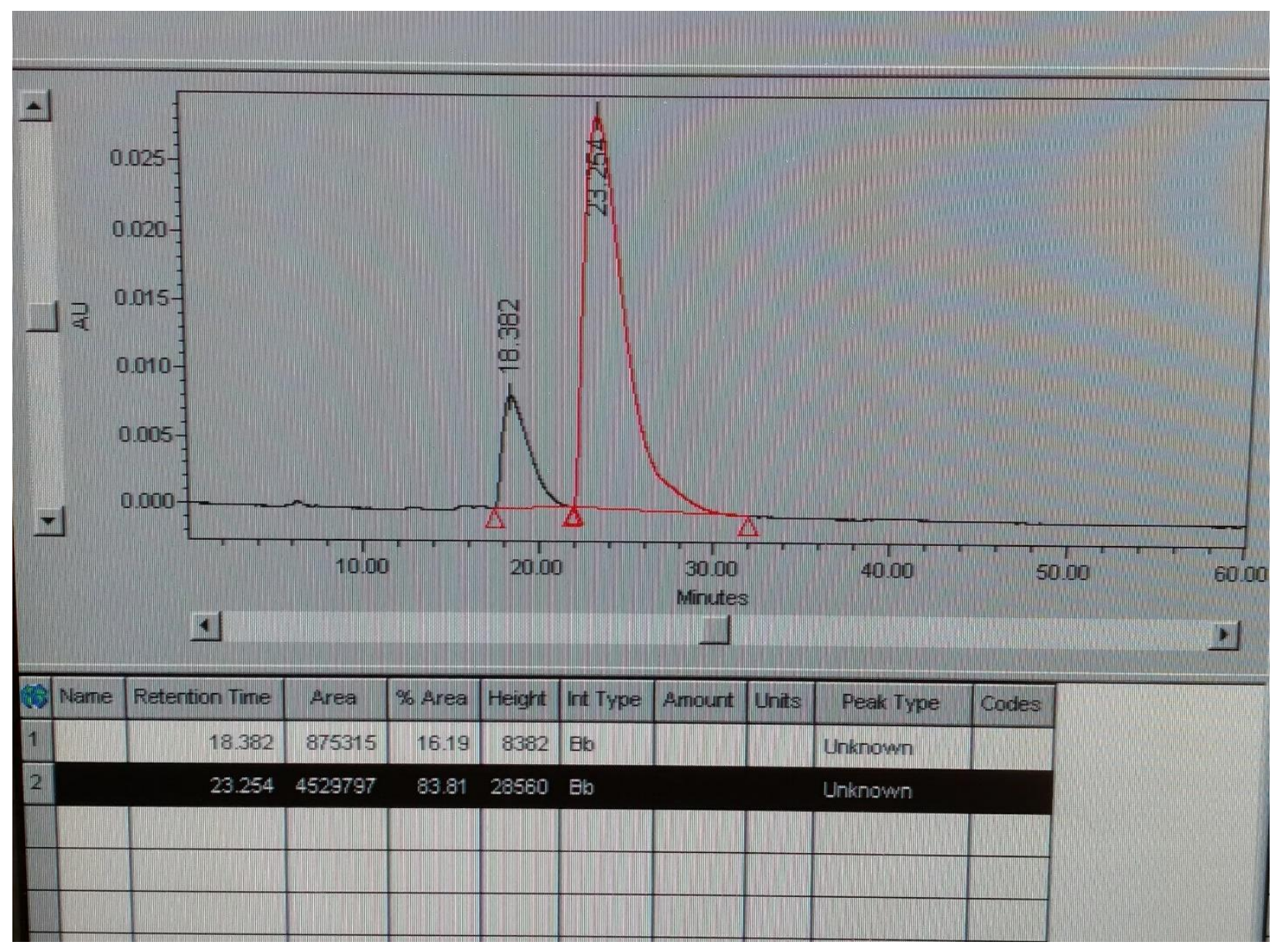

Run 3: Follows General Procedure E: Bis(1,5-cyclooctadiene)rhodium(I) tetrafluoroborate (3.1 mg, $0.0076 \mathrm{mmol}),(S)-$ MonoPhos-alkene (8) (4.5 mg, $0.011 \mathrm{mmol})$, carbon monoxide (100\%), 3methyl-5-(2-(prop-1-yn-1-yl)furan-3-yl)penta-1,2-dien-1-yl octanoate (4b) (25 mg, $0.076 \mathrm{mmol})$, DCE $(7.6 \mathrm{~mL}, 0.01 \mathrm{M})$. The reaction was stirred for $45 \mathrm{~h}$ in an oil bath $\left(70{ }^{\circ} \mathrm{C}\right)$. The crude residue 
was purified via silica gel flash chromatography (5\% ethyl acetate/hexanes) to give $18.9 \mathrm{mg}(70 \%)$ of the title compound as a yellow oil in a 16:84 ratio of enantiomers and $2.1 \mathrm{mg}(14 \%)$ of the aldehyde byproduct (15). The ${ }^{1} \mathrm{H}$ NMR matches that of racemic

\section{EDD6-505}

Waters 600 HPLC, UV/PDA detector, 328 nm, Daicel CHIRALCEL-OD, 250 X 4.6 mm column, $1.0 \% \mathrm{iPrOH} /$ hexanes, Flow rate: $1 \mathrm{~mL} / \mathrm{min}$

\begin{tabular}{|l|c|c|}
\hline Peak & Retention Time (min) & Peak area (\%) \\
\hline Peak 1 & 16.59 & 16.25 \\
\hline Peak 2 & 20.09 & 83.75 \\
\hline
\end{tabular}

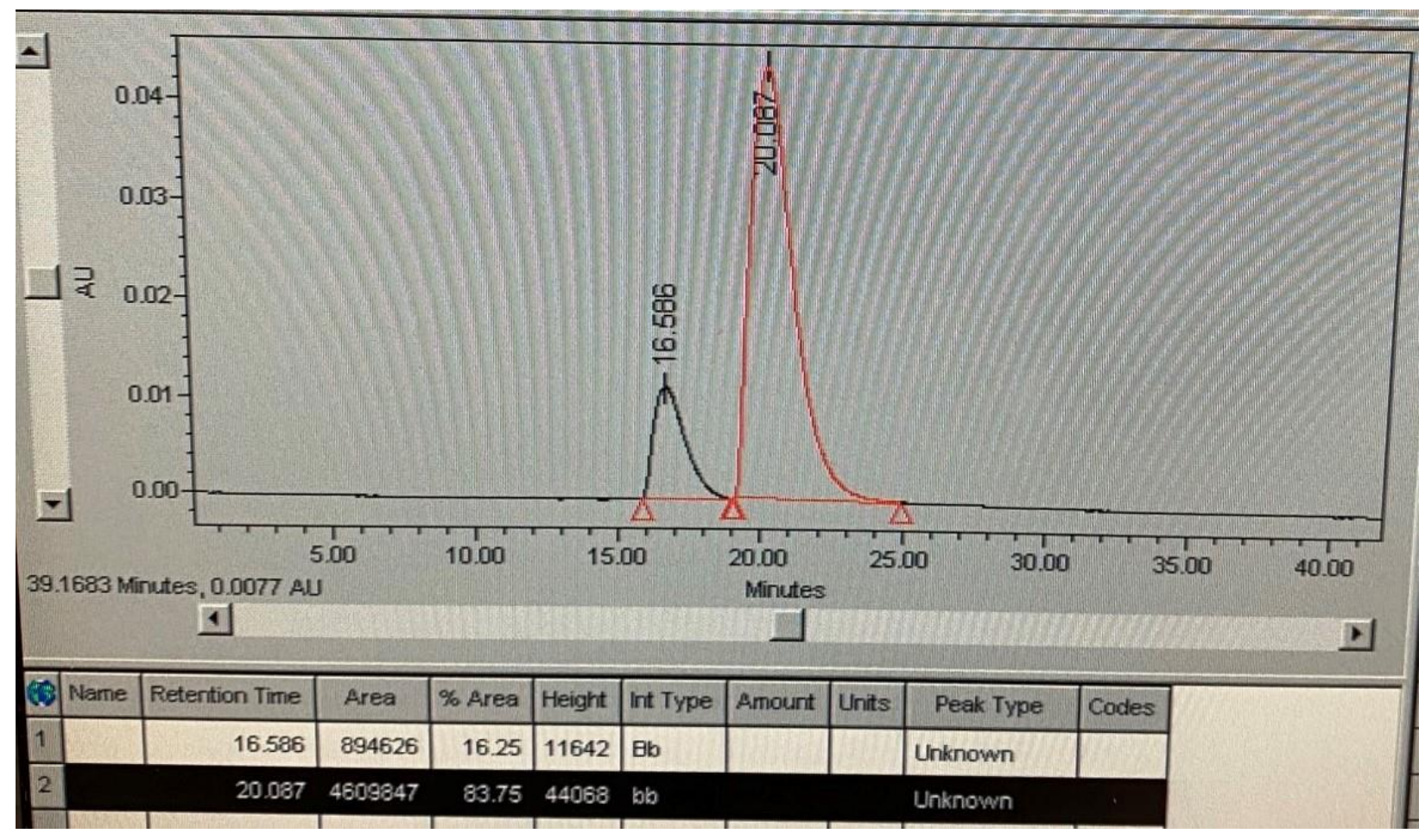


APKR with $(R)$-MonoPhos-alkene to give opposite enantiomer

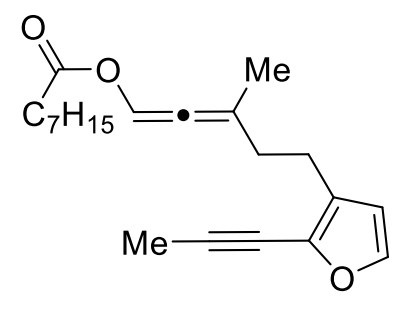

4b

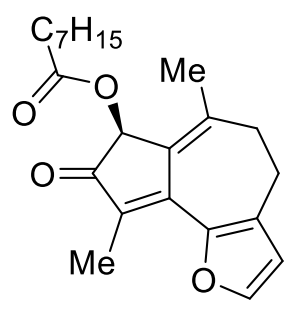

S-3b

(S)-6,9-dimethyl-8-oxo-4,5,7,8-tetrahydroazuleno[4,5-b]furan-7-yl octanoate (3b). Follows

General Procedure E: Bis(1,5-cyclooctadiene)rhodium(I) tetrafluoroborate $(2.5 \mathrm{mg}, 0.0061$ mmol), (R)-MonoPhos-alkene (8) (3.8 mg, $0.0092 \mathrm{mmol})$, carbon monoxide (100\%), 3-methyl-5(2-(prop-1-yn-1-yl)furan-3-yl)penta-1,2-dien-1-yl octanoate (4b) (20 mg, 0.061 mmol), DCE (6.1 $\mathrm{mL}, 0.01 \mathrm{M})$. The reaction was stirred for $45 \mathrm{~h}$ in an oil bath $\left(70{ }^{\circ} \mathrm{C}\right)$. The crude residue was purified via silica gel flash chromatography (2.5\% to 5\% ethyl acetate/hexanes) to give $14.4 \mathrm{mg}$ (65\%) of the title compound as a yellow oil in an 16:84 ratio of enantiomers and $1.7 \mathrm{mg}(14 \%)$ of the aldehyde byproduct 15 .

\section{EDD7-506}

Waters 600 HPLC, UV/PDA detector, 328 nm, Daicel CHIRALCEL-OD, 250 X 4.6 mm column, $1.0 \%$ iPrOH/hexanes, Flow rate: $1 \mathrm{~mL} / \mathrm{min}$

\begin{tabular}{|l|c|c|}
\hline Peak & Retention Time (min) & Peak area (\%) \\
\hline Peak 1 & 15.89 & 83.94 \\
\hline Peak 2 & 19.98 & 16.06 \\
\hline
\end{tabular}




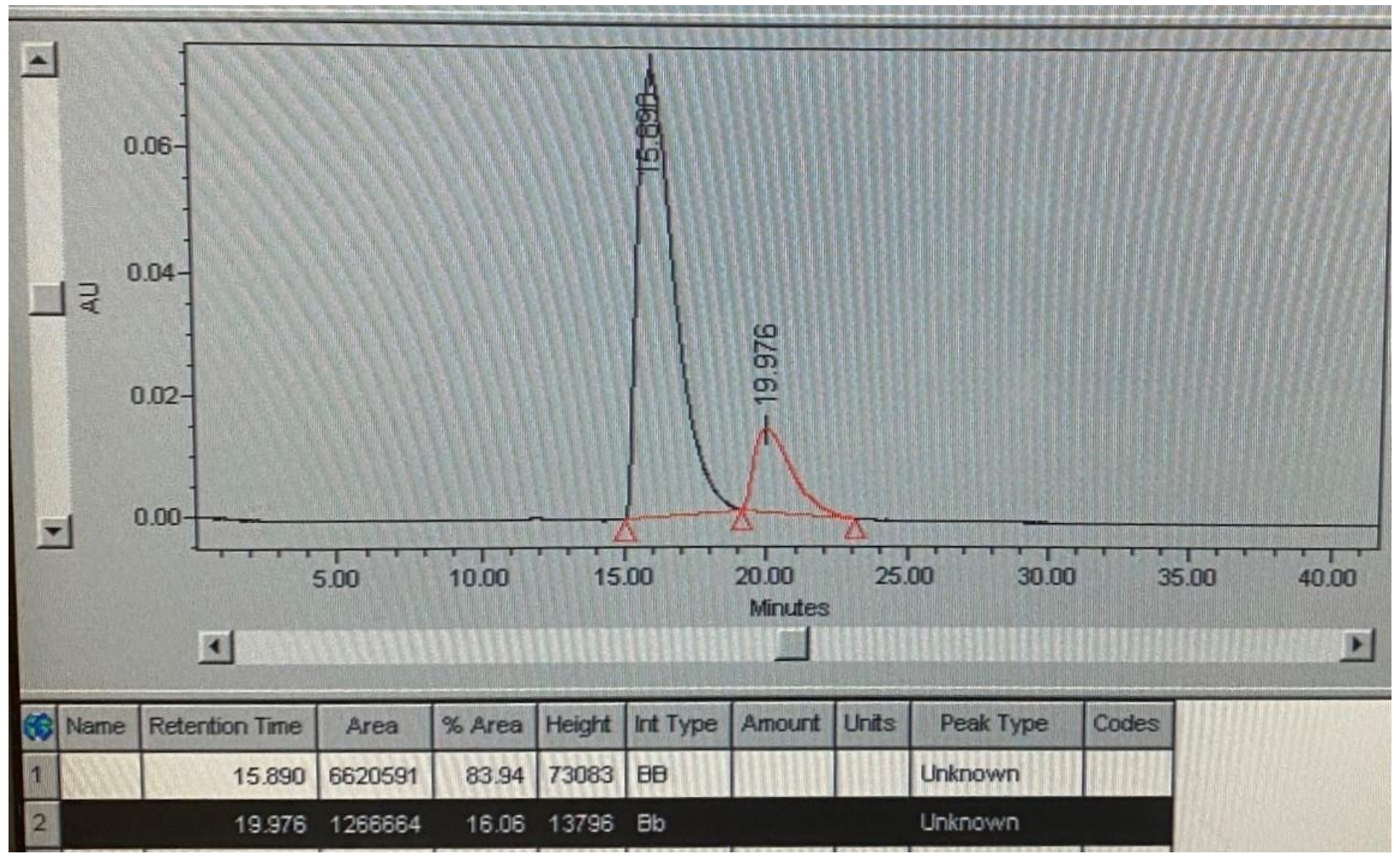

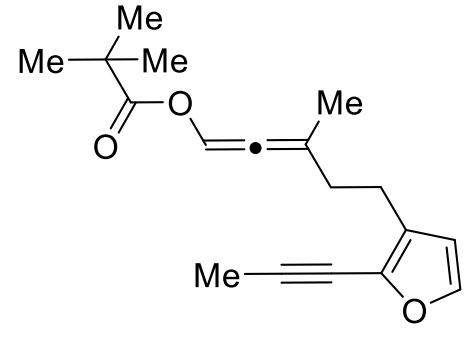

$4 \mathrm{c}$

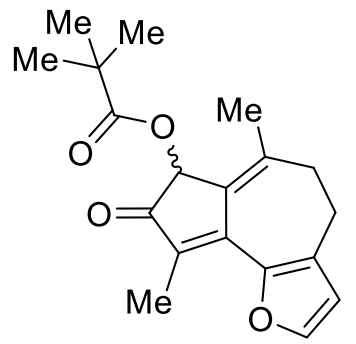

$3 c$

6,9-dimethyl-8-oxo-4,5,7,8-tetrahydroazuleno[4,5-b]furan-7-yl pivalate (3c). Follows General

Procedure C: Bis(1,5-cyclooctadiene)rhodium(I) tetrafluoroborate $(2.1 \mathrm{mg}, 0.0052 \mathrm{mmol})$, triphenyl phosphine(2.0 mg, $0.0078 \mathrm{mmol})$, carbon monoxide (100\%), 3-methyl-5-(2-(prop-1-yn1-yl)furan-3-yl)penta-1,2-dien-1-yl pivalate (4c) (15 mg, $0.052 \mathrm{mmol})$, DCE (2.6 mL, $0.02 \mathrm{M})$. The reaction was stirred for $45 \mathrm{~h}$ in an oil bath $\left(70^{\circ} \mathrm{C}\right)$. The crude residue was purified via silica 
gel flash chromatography (5\% ethyl acetate/hexanes) to give the title compound (11 mg, 69\%) as a yellow oil.

EDD5-381

$\underline{{ }^{1} \mathrm{H} \mathrm{NMR}} \quad\left(500 \mathrm{MHz}, \mathrm{CDCl}_{3}\right)$

$7.54(\mathrm{~d}, J=1.0 \mathrm{~Hz}, 1 \mathrm{H}), 6.38(\mathrm{~d}, J=1.5 \mathrm{~Hz}, 1 \mathrm{H}), 5.78(\mathrm{~s}, 1 \mathrm{H}), 2.87-2.74(\mathrm{~m}, 2$

H), 2.59-2.49 (m, 2 H), 2.19 (s, $3 \mathrm{H}), 1.90$ (s, $3 \mathrm{H}), 1.24$ (s, $9 \mathrm{H}) \mathrm{ppm}$

${ }^{13} \mathrm{C} \mathrm{NMR} \quad\left(125 \mathrm{MHz}, \mathrm{CDCl}_{3}\right)$

$199.9,177.5,149.5,147.4,144.9,138.4,132.2,132.1,127.8,113.8,71.8,39.1$, $34.5,27.3,24.9,24.0,10.2 \mathrm{ppm}$

$\underline{\mathrm{IR}}$

(Thin Film)

$1733,1603 \mathrm{~cm}^{-1}$

$\underline{\text { HRMS }}$ HRMS-ESI (m/z): $[\mathrm{M}+\mathrm{H}]^{+}$calcd for $\mathrm{C}_{19} \mathrm{H}_{23} \mathrm{O}_{4}, 315.1591$; found, 315.1592

$\underline{\text { TLC }} \quad R_{f}=0.35$ (35\% ethyl acetate/hexanes); silica gel, UV, $p$-anisaldehyde

Waters 600 HPLC, UV/PDA detector, 328 nm, Daicel CHIRALCEL-OD, 250 X 4.6 mm column, $1 \% \mathrm{iPrOH} / \mathrm{hexanes}$, Flow rate: $1 \mathrm{~mL} / \mathrm{min}$

\begin{tabular}{|l|c|c|}
\hline Peak & Retention Time (min) & Peak area (\%) \\
\hline Peak 1 & 11.54 & 50.00 \\
\hline Peak 2 & 14.71 & 50.00 \\
\hline
\end{tabular}




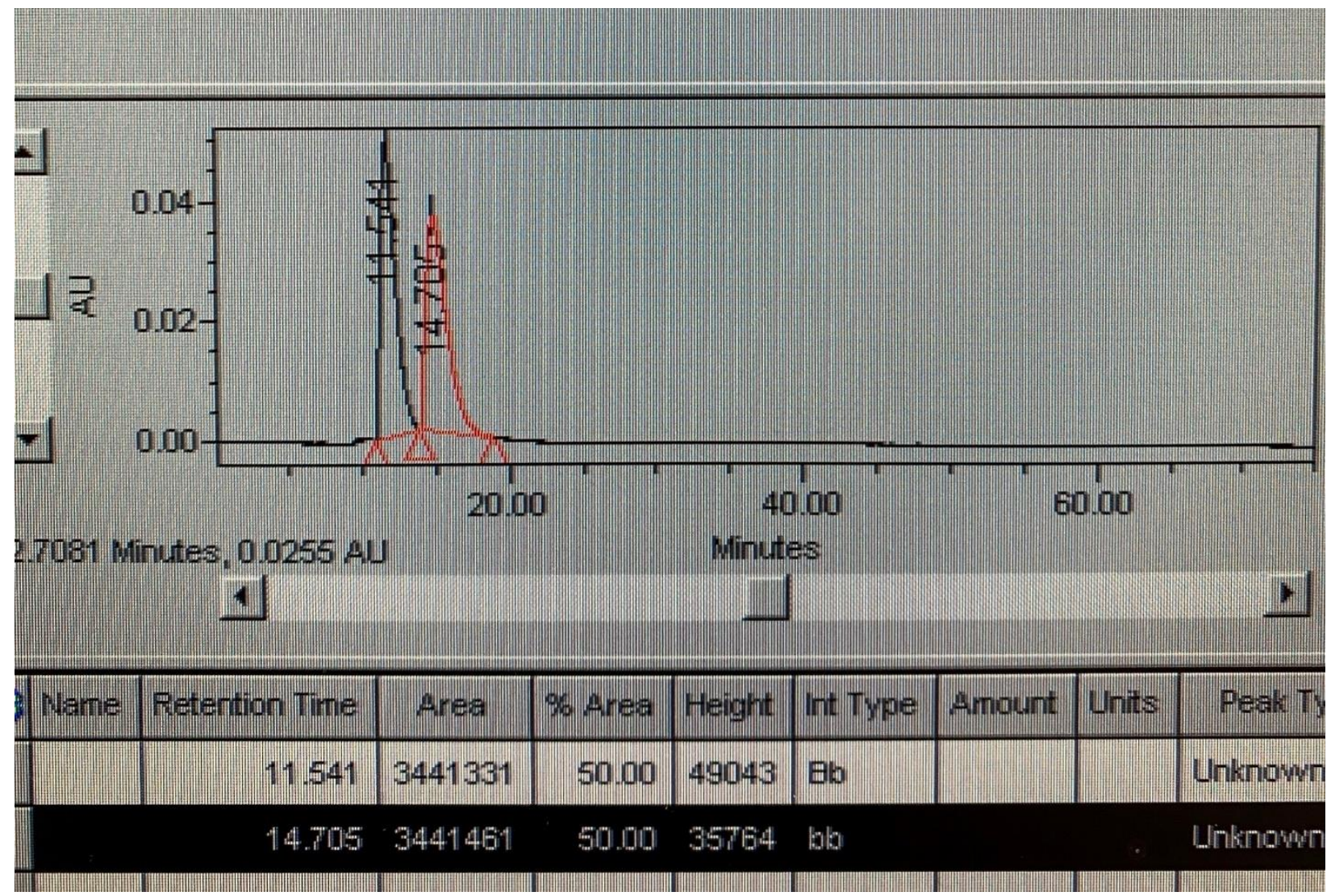

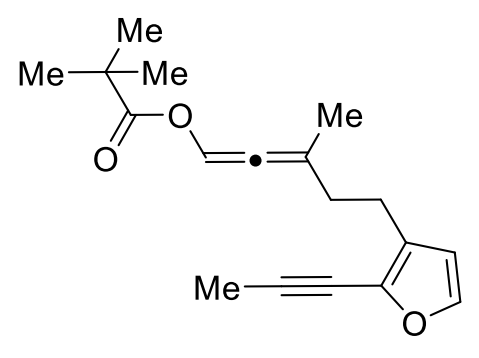

4c

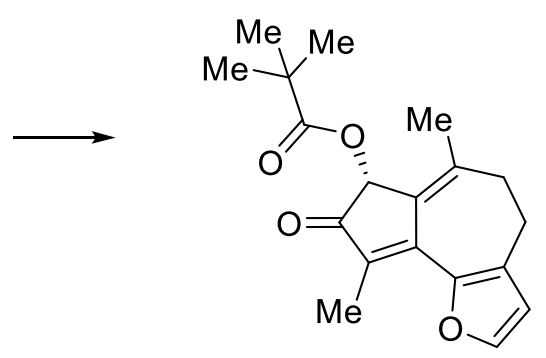

3c

(R)-6,9-dimethyl-8-oxo-4,5,7,8-tetrahydroazuleno[4,5-b]furan-7-yl pivalate (3c). Run 1:

Follows General Procedure E: Bis(1,5-cyclooctadiene)rhodium(I) tetrafluoroborate (2.1 mg, $0.0052 \mathrm{mmol}),(S)$-MonoPhos-alkene (8) (3.2 mg, $0.0078 \mathrm{mmol})$, carbon monoxide (100\%), 3methyl-5-(2-(prop-1-yn-1-yl)furan-3-yl)penta-1,2-dien-1-yl pivalate (4c) (15 mg, $0.052 \mathrm{mmol}$ ), DCE (5.2 mL, 0.01 M). The reaction was stirred for $45 \mathrm{~h}$ in an oil bath $\left(70{ }^{\circ} \mathrm{C}\right)$. The crude residue was purified via silica gel flash chromatography (5\% ethyl acetate/hexanes) to give $6.9 \mathrm{mg}(43 \%)$ 
of the title compound as a yellow oil in a 78:22 ratio of enantiomers and $2.1 \mathrm{mg}(20 \%)$ of the aldehyde byproduct (15).

\section{EDD5-384}

Waters 600 HPLC, UV/PDA detector, 328 nm, Daicel CHIRALCEL-OD, 250 X 4.6 mm column, $1 \% \mathrm{iPrOH} /$ hexanes, Flow rate: $1 \mathrm{~mL} / \mathrm{min}$

\begin{tabular}{|l|c|c|}
\hline Peak & Retention Time (min) & Peak area (\%) \\
\hline Peak 1 & 11.99 & 22.07 \\
\hline Peak 2 & 15.35 & 77.93 \\
\hline
\end{tabular}

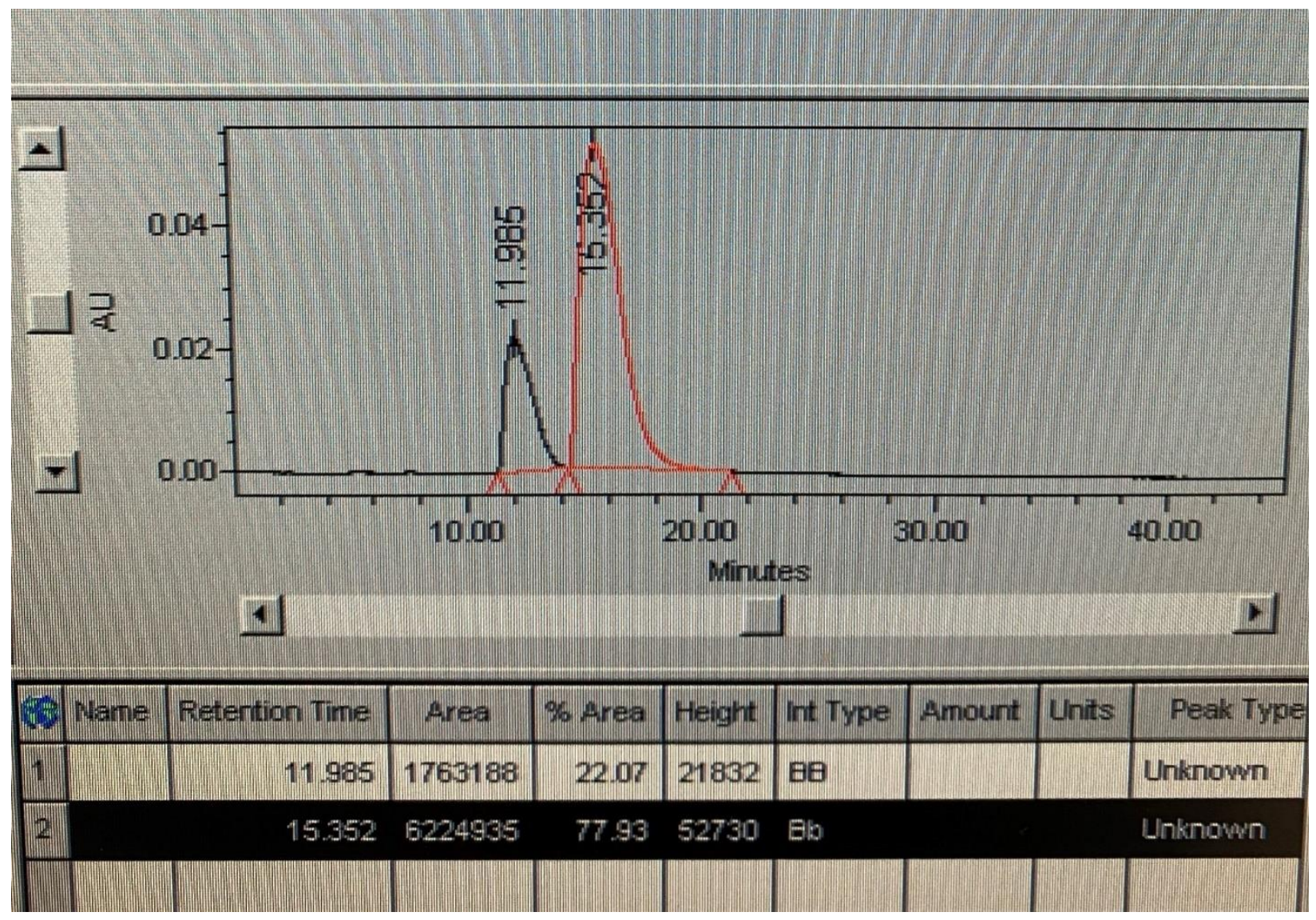


Run 2: Follows general procedure E: Bis(1,5-cyclooctadiene)rhodium(I) tetrafluoroborate (2.1 mg, $0.0052 \mathrm{mmol}$ ), (S)-MonoPhos-alkene (8) (3.2 mg, $0.0078 \mathrm{mmol})$, carbon monoxide (100\%), 3-methyl-5-(2-(prop-1-yn-1-yl)furan-3-yl)penta-1,2-dien-1-yl pivalate (4c) (15 mg, $0.052 \mathrm{mmol})$, DCE $(5.2 \mathrm{~mL}, 0.01 \mathrm{M})$. The reaction was stirred for $45 \mathrm{~h}$ in an oil bath $\left(70{ }^{\circ} \mathrm{C}\right)$. The crude residue was purified via silica gel flash chromatography (5\% ethyl acetate/hexanes) to give $6.8 \mathrm{mg}(43 \%)$ of the title compound as a yellow oil in a 77:23 ratio of enantiomers and $2.2 \mathrm{mg}(21 \%)$ of the aldehyde byproduct (15).

\section{EDD5-386}

Waters 600 HPLC, UV/PDA detector, 328 nm, Daicel CHIRALCEL-OD, 250 X 4.6 mm column, $1 \% \mathrm{iPrOH} / \mathrm{hexanes}$, Flow rate: $1 \mathrm{~mL} / \mathrm{min}$

\begin{tabular}{|l|c|c|}
\hline Peak & Retention Time (min) & Peak area (\%) \\
\hline Peak 1 & 12.11 & 23.15 \\
\hline Peak 2 & 15.36 & 76.85 \\
\hline
\end{tabular}




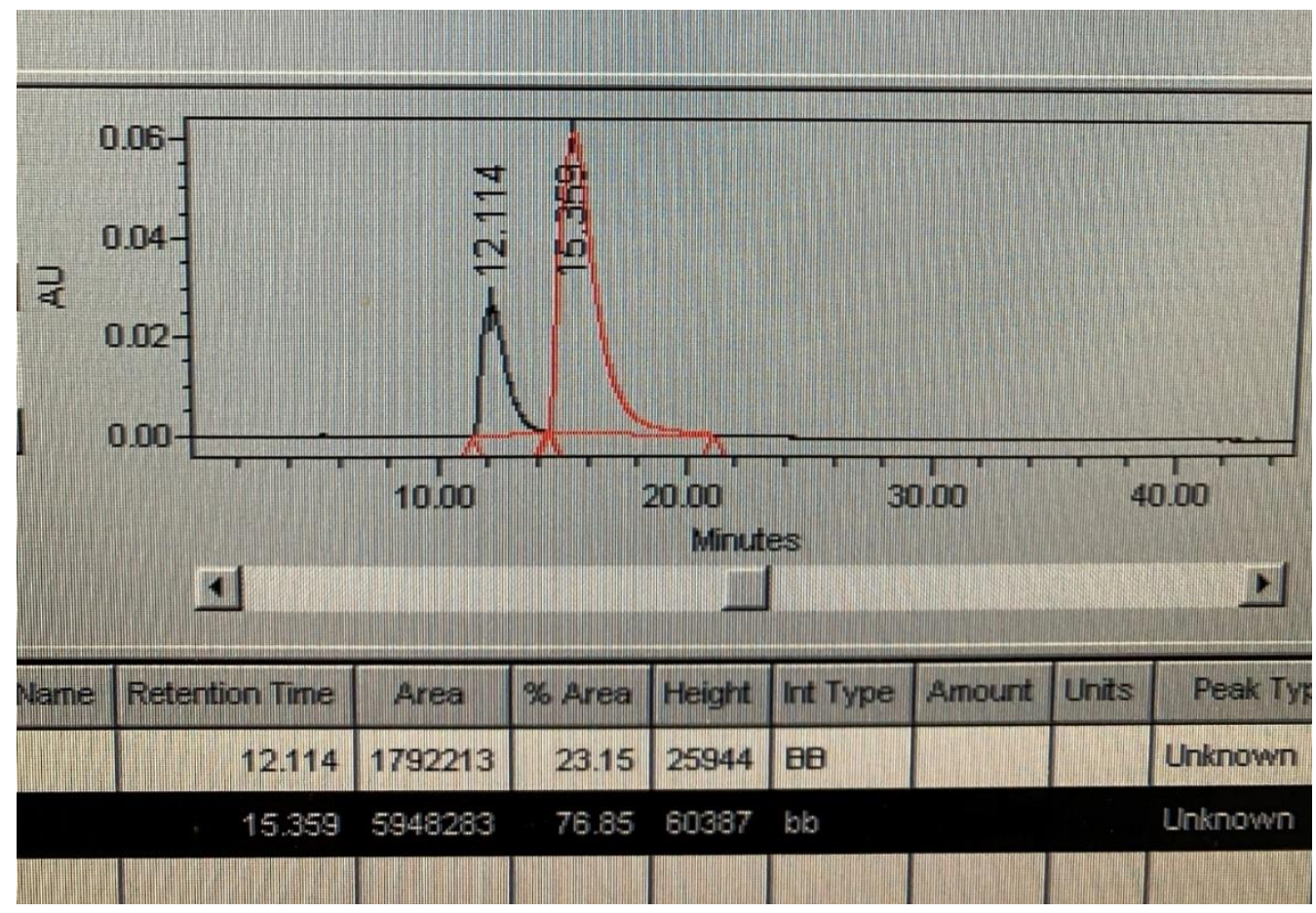

$[\alpha]^{25} \mathrm{D}=+64.9\left(\mathrm{c} .=0.2, \mathrm{CHCl}_{3}\right)$

Synthesis of $[5,7,5]$ ring system via racemic allenic Pauson-Khand reaction

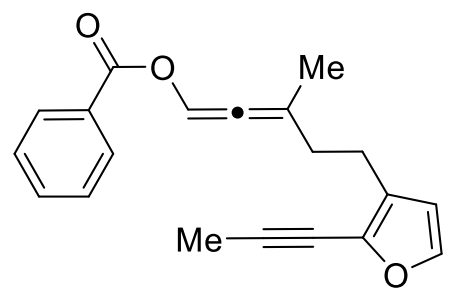

4d<smiles>CC1=C2C(=C(C)C(=O)C2OC(=O)c2ccccc2)c2occc2CC1</smiles>

3d

\section{6,9-dimethyl-8-oxo-4,5,7,8-tetrahydroazuleno[4,5-b]furan-7-yl benzoate $\quad(3 \mathrm{~d})$. Follows}

General Procedure C: Bis(1,5-cyclooctadiene)rhodium(I) tetrafluoroborate $(1.3 \mathrm{mg}, 0.0033$ mmol), triphenyl phosphine $(1.3 \mathrm{mg}, 0.0049 \mathrm{mmol})$, carbon monoxide $(100 \%)$, mesitylene $(23 \mu \mathrm{L}$, $0.16 \mathrm{mmol}), 3$-methyl-5-(2-(prop-1-yn-1-yl)furan-3-yl)penta-1,2-dien-1-yl benzoate (4d) (10 mg, 
$0.033 \mathrm{mmol})$, DCE $(1.63 \mathrm{~mL}, 0.02 \mathrm{M})$. The reaction was stirred for $21.5 \mathrm{~h}$ in an oil bath $\left(70^{\circ} \mathrm{C}\right)$. The crude residue was purified via silica gel flash chromatography (5\% to $15 \%$ ethyl acetate/hexanes) to give the title compound ( $9 \mathrm{mg}, 82 \%)$ as a yellow oil.

\section{EDD3-139}

${ }^{1} \mathrm{H} \mathrm{NMR} \quad\left(400 \mathrm{MHz}, \mathrm{CDCl}_{3}\right)$

8.08-8.06 (m, 2 H), 7.58-7.54 (m, 2 H), $7.43(\mathrm{t}, J=8.0 \mathrm{~Hz}, 2 \mathrm{H}), 6.40$ (d, $J=1.6$ Hz, 1 H), 6.00 (s, 1 H), 2.90-2.75 (m, 2 H), 2.57-2.54 (m, 2 H), 2.24 (s, 3 H), 1.95 (s, $3 \mathrm{H}) \mathrm{ppm}$;

Aldehyde impurity at 9.99 (d, $J=8.0 \mathrm{~Hz}), 9.85(\mathrm{~d}, J=8.0 \mathrm{~Hz}), 7.23$ (d), 6.24 (d), 5.90-5.89 (m), $2.11(\mathrm{~d}, J=2.0 \mathrm{~Hz}), 2.05(\mathrm{~s}) \mathrm{ppm}$ (Some aldehyde resonances could not be integrated or coupling constants calculated because of overlap with APKR product resonances or poor signal)

${ }^{13} \mathrm{C} \mathrm{NMR} \quad\left(100 \mathrm{MHz}, \mathrm{CDCl}_{3}\right)$

199.6, 165.6, 149.6, 147.4, 145.0, 138.9, 133.3, 132.4, 132.2, 130.1, 129.8, 128.5, $127.8,113.8,72.5,34.7,24.9,24.0,10.3 \mathrm{ppm}$

$\underline{\mathrm{IR}} \quad$ (Thin Film)

2924, 1722, 1693, 1647, 1601, 1541, 1444, 1383, 1325, 1257, 1177, 1111, 1067, $1026 \mathrm{~cm}^{-1}$

HRMS HRMS-ESI (m/z): $[\mathrm{M}+\mathrm{H}]^{+}$calcd for $\mathrm{C}_{21} \mathrm{H}_{29} \mathrm{O}_{4}, 335.1283$; found, 335.1301

$\underline{\text { TLC }} \quad R_{f}=0.54$ (35\% ethyl acetate/hexanes); silica gel, UV, $p$-anisaldehyde 
Waters 600 HPLC, UV/PDA detector, 328 nm, Daicel CHIRALCEL-OD, 250 X 4.6 mm column, $0.25 \%$ EtOH/hexanes, Flow rate: $1 \mathrm{~mL} / \mathrm{min}$

\begin{tabular}{|l|c|c|}
\hline Peak & Retention Time (min) & Peak area (\%) \\
\hline Peak 1 & 81.54 & 50.28 \\
\hline Peak 2 & 96.36 & 49.72 \\
\hline
\end{tabular}

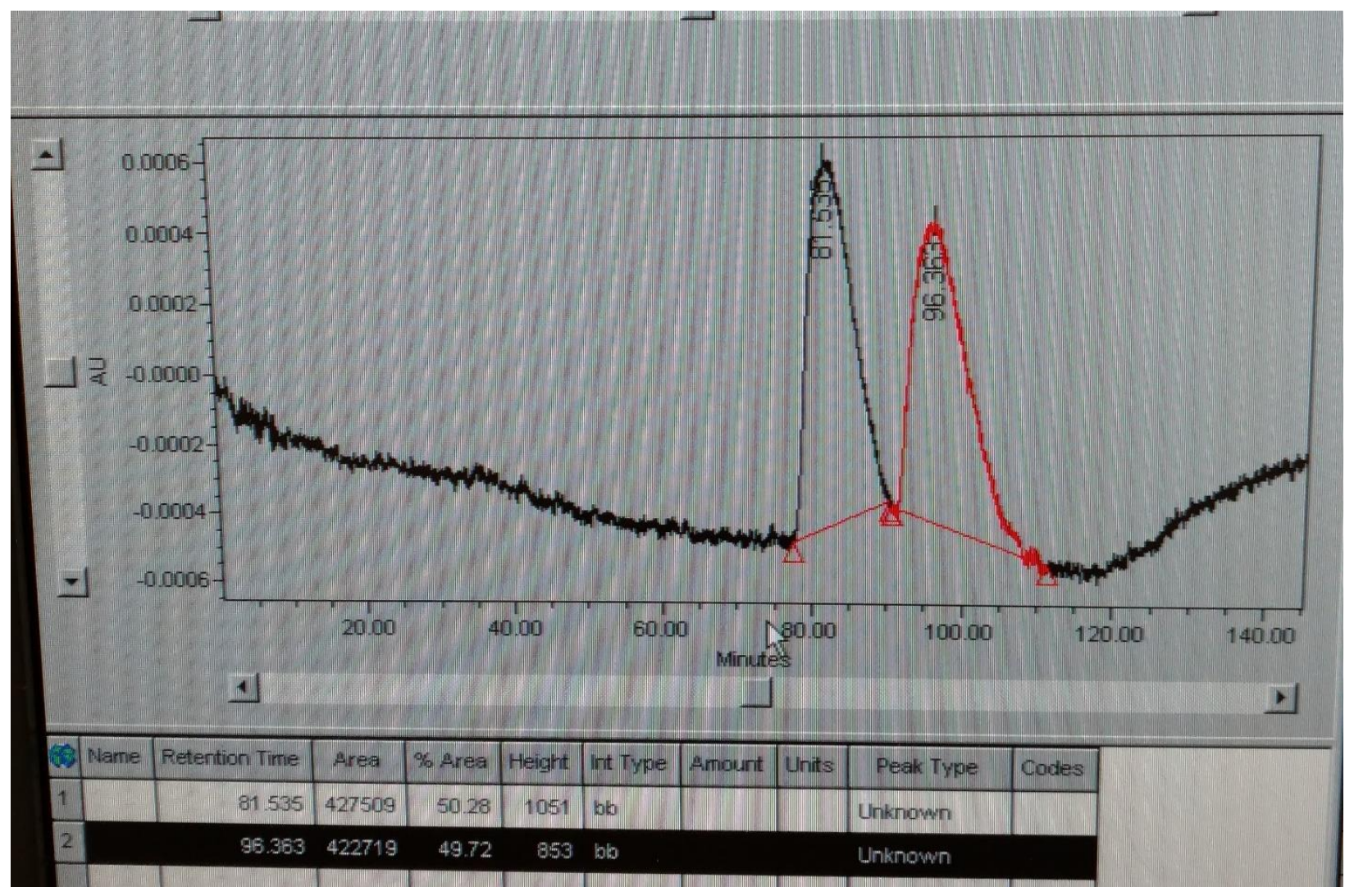


Synthesis of $[5,7,5]$ ring system via asymmetric allenic Pauson-Khand reaction under dilute conditions

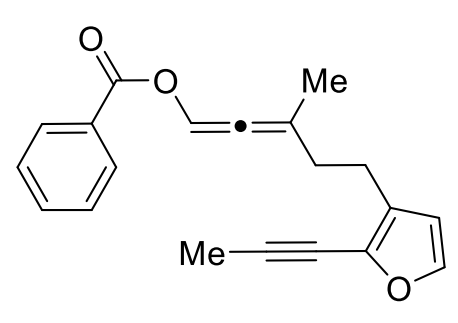

4d

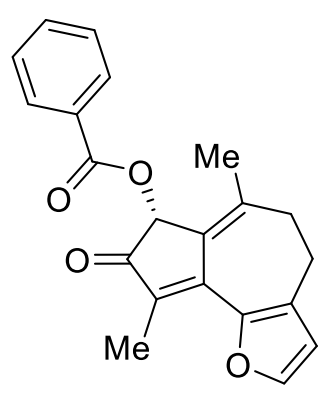

3d

\section{6,9-dimethyl-8-oxo-4,5,7,8-tetrahydroazuleno[4,5-b]furan-7-yl benzoate (3d). Run 1: Follows}

General Procedure E: Bis(1,5-cyclooctadiene)rhodium(I) tetrafluoroborate $(3.2 \mathrm{mg}, 0.0078$ mmol), (S)-MonoPhos-alkene (8) (4.8 mg, 0.012 mmol), carbon monoxide (100\%), 3-methyl-5(2-(prop-1-yn-1-yl)furan-3-yl)penta-1,2-dien-1-yl benzoate (4d) $(24 \mathrm{mg}, 0.078 \mathrm{mmol})$, DCE (7.8 $\mathrm{mL}, 0.01 \mathrm{M})$. The reaction was stirred for $22 \mathrm{~h}$ in an oil bath $\left(70{ }^{\circ} \mathrm{C}\right)$. The crude residue was purified via silica gel flash chromatography (5\% to $15 \%$ ethyl acetate/hexanes) to give $15 \mathrm{mg}$ (58\%) of the title compound as a yellow oil in a 95:5 ratio of enantiomers and $1.3 \mathrm{mg}(8 \%)$ of the aldehyde byproduct. The enantiomeric ratio was confirmed after hydrolysis of the benzoate ester.

\section{EDD5-320}

$[\alpha]^{20}{ }_{\mathrm{D}}=-3.6\left(\mathrm{c} .=0.3, \mathrm{CHCl}_{3}\right)$

Waters 600 HPLC, UV/PDA detector, 328 nm, Daicel CHIRALCEL-OD, 250 X 4.6 mm column, $0.2 \% \mathrm{EtOH} / \mathrm{hexanes,} \mathrm{Flow} \mathrm{rate:} 1 \mathrm{~mL} / \mathrm{min}$

\begin{tabular}{|l|c|c|}
\hline Peak & Retention Time (min) & Peak area (\%) \\
\hline Peak 1 & 116.79 & 94.84 \\
\hline Peak 2 & 146.22 & 5.16 \\
\hline
\end{tabular}




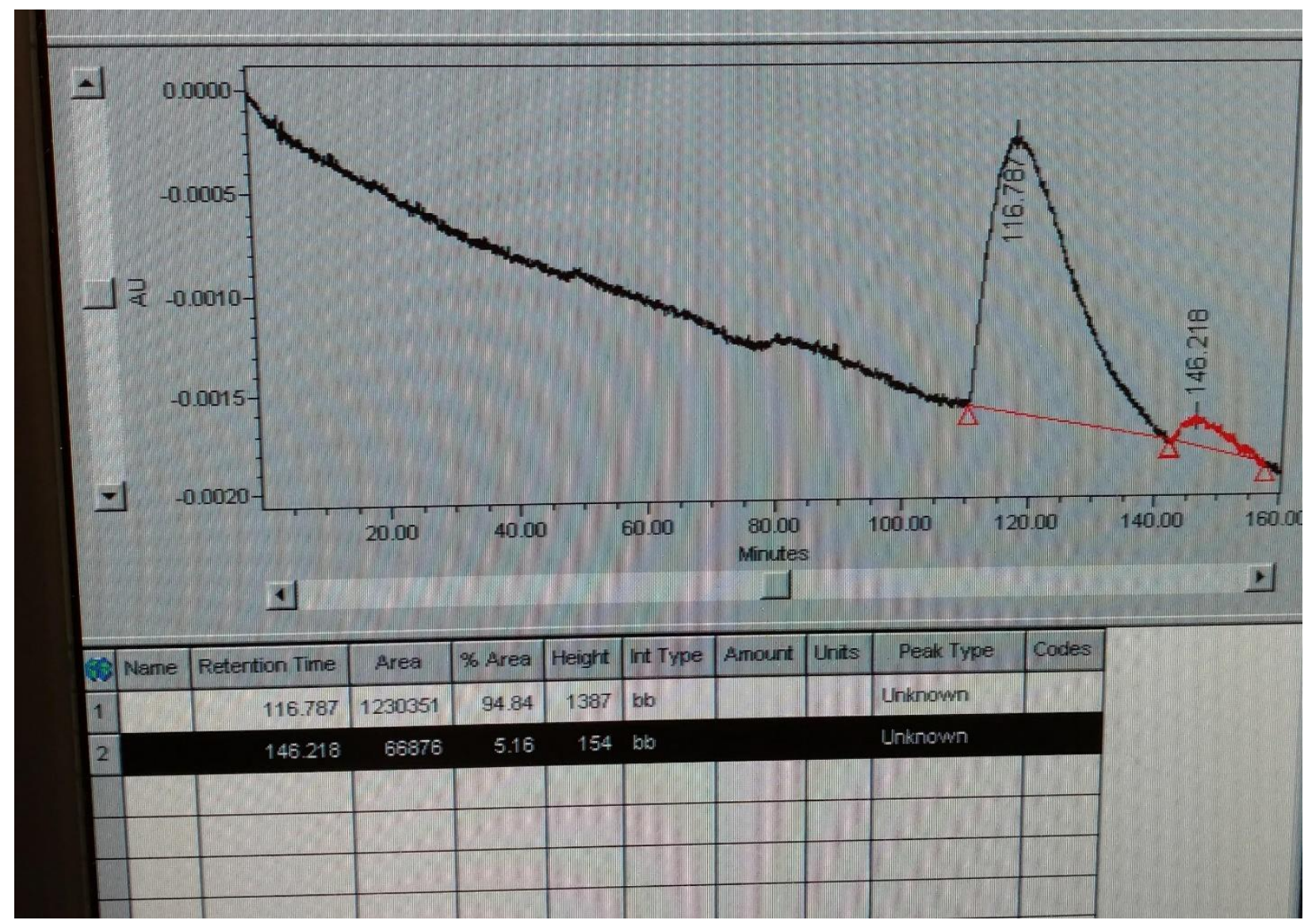

Run 2: Follows General Procedure E: Bis(1,5-cyclooctadiene)rhodium(I) tetrafluoroborate (2.6 mg, $0.0065 \mathrm{mmol}$ ), (S)-MonoPhos-alkene (8) (4.0 mg, $0.0098 \mathrm{mmol}$ ), carbon monoxide (100\%), 3-methyl-5-(2-(prop-1-yn-1-yl)furan-3-yl)penta-1,2-dien-1-yl benzoate (4d) (20 mg, 0.065 $\mathrm{mmol})$, DCE $(6.5 \mathrm{~mL}, 0.01 \mathrm{M})$. The reaction was stirred for $22 \mathrm{~h}$ in an oil bath $\left(70{ }^{\circ} \mathrm{C}\right)$. The crude residue was purified via silica gel flash chromatography (5\% to $15 \%$ ethyl acetate/hexanes) to give $11.7 \mathrm{mg}(52 \%)$ of the title compound as a yellow oil in a 91:9 ratio of enantiomers and $1.3 \mathrm{mg}$ (10\%) of the aldehyde byproduct.

\section{EDD5-330}


Waters 600 HPLC, UV/PDA detector, 328 nm, Daicel CHIRALCEL-OD, 250 X 4.6 mm column, $0.2 \% \mathrm{EtOH} / \mathrm{h}$ exanes, Flow rate: $1 \mathrm{~mL} / \mathrm{min}$

\begin{tabular}{|l|c|c|}
\hline Peak & Retention Time (min) & Peak area (\%) \\
\hline Peak 1 & 107.66 & 91.13 \\
\hline Peak 2 & 144.70 & 8.87 \\
\hline
\end{tabular}

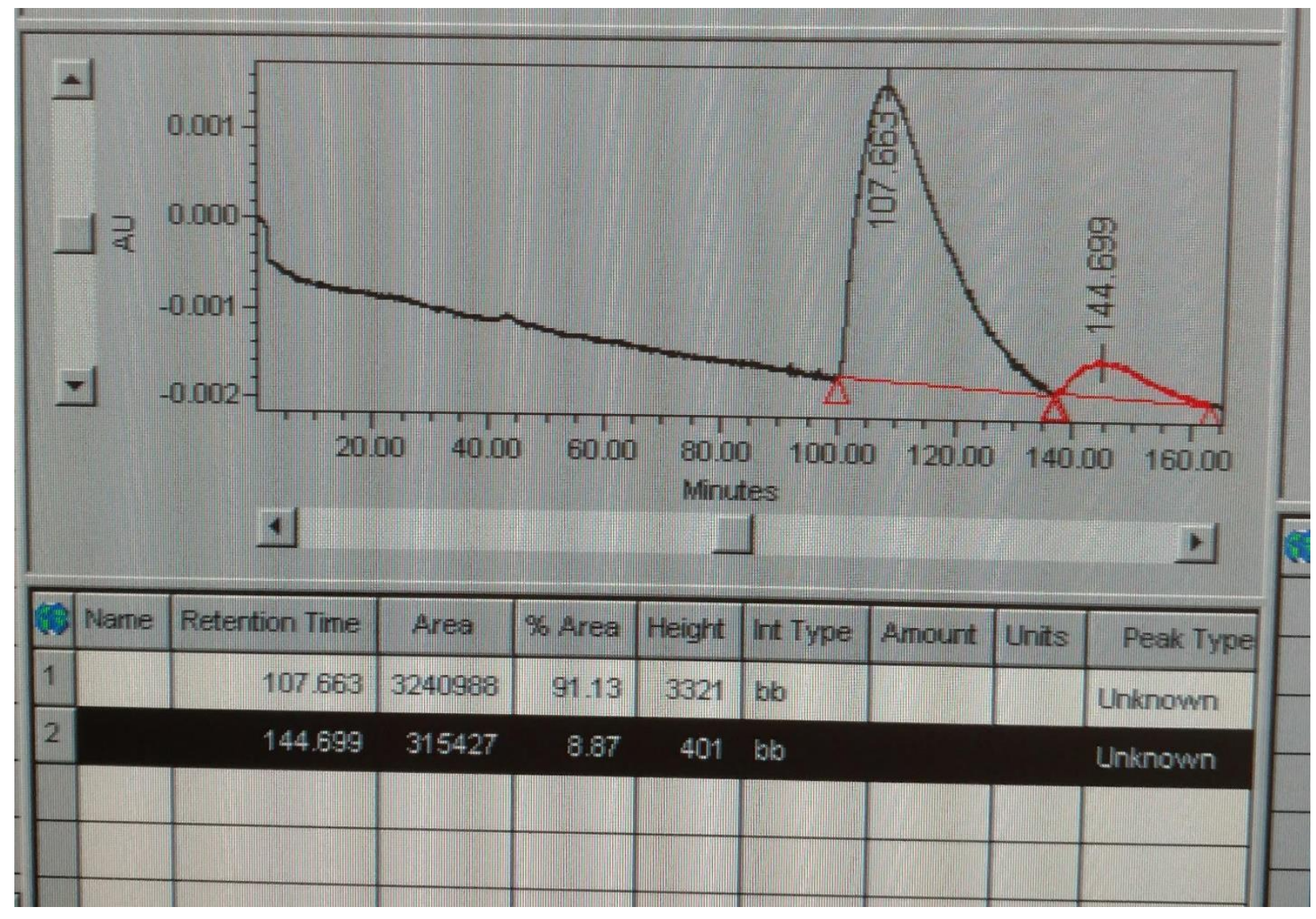




\section{Cleavage of the benzoate group to confirm enantiomeric ratio of the APKR}

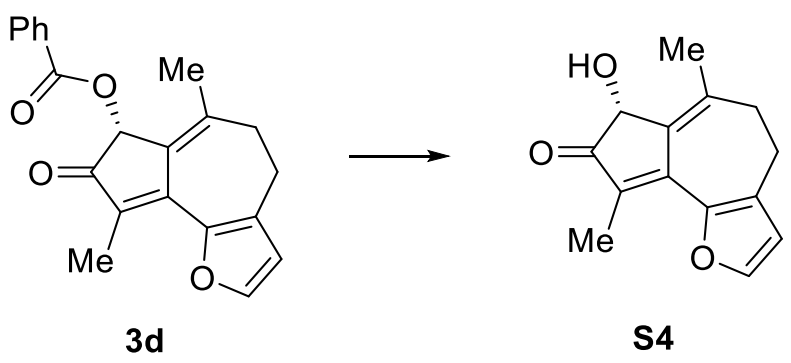

(R)-7-hydroxy-6,9-dimethyl-5,7-dihydroazuleno[4,5-b]furan-8(4H)-one (S4). A flame-dried, 1-necked,10 $\mathrm{mL}$ round bottomed flask equipped with a magnetic stir bar and needle attached to a nitrogen manifold was charged with scandium(III) triflate $(7.7 \mathrm{mg}, 0.0156 \mathrm{mmol}, 0.4$ equiv) in a nitrogen filled glove box and sealed with a septum. (R)-6,9-dimethyl-8-oxo-4,5,7,8tetrahydroazuleno[4,5-b]furan-7-yl benzoate (3d) (13 mg, $0.039 \mathrm{mmol}, 1$ equiv) was dissolved in methanol $(1.28 \mathrm{~mL}, 0.03 \mathrm{M})$ and transferred to the flask via syringe. Water $(0.32 \mathrm{~mL}, 0.12 \mathrm{M})$ was added dropwise via syringe. The tube was lowered into a $40{ }^{\circ} \mathrm{C}$ oil bath and stirred for $4 \mathrm{~d}$. The solution was diluted with deionized water $(3 \mathrm{~mL})$, transferred to a separatory funnel and extracted with DCM (5 x $3 \mathrm{~mL})$. The combined organic extracts were dried over magnesium sulfate, filtered, and concentrated. The crude residue was purified via silica gel flash chromatography (20 to $35 \%$ ethyl acetate/hexanes) to give the title compound ( $3 \mathrm{mg}, 33 \%, 50 \% \mathrm{brsm})$ as a yellow solid in a 95:5 ratio of enantiomers (and $4 \mathrm{mg}$ of recovered starting material).

\section{EDD5-343}

Waters 600 HPLC, UV/PDA detector, 332 nm, Daicel CHIRALCEL-OD, 250 X 4.6 mm column, $1.0 \% \mathrm{iPrOH} /$ hexanes, Flow rate: $1 \mathrm{~mL} / \mathrm{min}$

\begin{tabular}{|l|c|c|}
\hline Peak & Retention Time (min) & Peak area (\%) \\
\hline Peak 1 & 42.93 & 5.37 \\
\hline
\end{tabular}




\begin{tabular}{|l|l|l|}
\hline Peak 2 & 47.61 & 94.63 \\
\hline
\end{tabular}
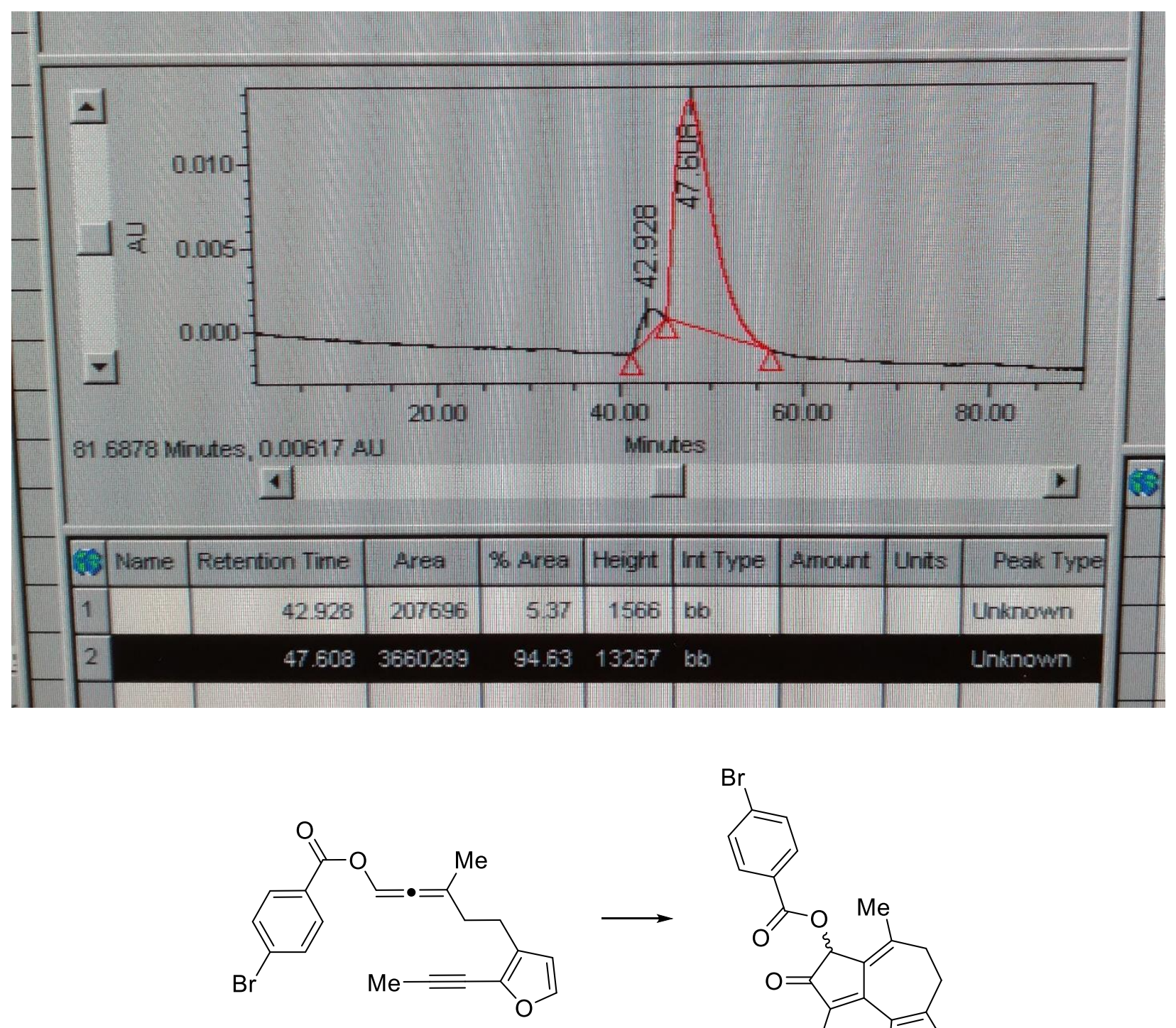

$4 e$

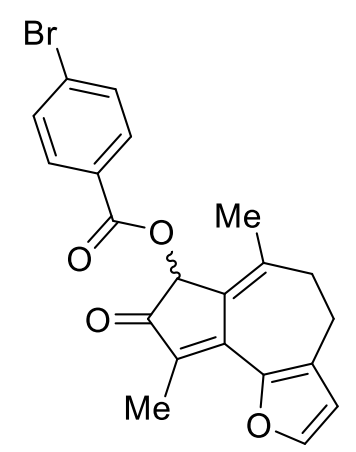

$3 e$

\section{6,9-dimethyl-8-oxo-4,5,7,8-tetrahydroazuleno[4,5-b]furan-7-yl 2-chloroacetate (3e). Follows}

General Procedure C: Bis(1,5-cyclooctadiene)rhodium(I) tetrafluoroborate (4.8 mg, $0.012 \mathrm{mmol})$, triphenyl phosphine $(4.7 \mathrm{mg}, 0.018 \mathrm{mmol})$, carbon monoxide (100\%), 3-methyl-5-(2-(prop-1-yn1-yl)furan-3-yl)penta-1,2-dien-1-yl 4-bromobenzoate (4e) (46 mg, $0.12 \mathrm{mmol})$, DCE (6.0 mL, 
$0.02 \mathrm{M})$. The reaction was stirred for $24 \mathrm{~h}$ in an oil bath $\left(70{ }^{\circ} \mathrm{C}\right)$. The crude residue was purified via silica gel flash chromatography (5\% to $15 \%$ ethyl acetate/hexanes) to give the title compound (31 mg, 63\%) as a brown solid containing $1.7 \mathrm{mg}(7 \%)$ of aldehyde byproduct 15.

\section{EDD6-489}

$\underline{{ }^{1} \mathrm{H} \mathrm{NMR}} \quad\left(500 \mathrm{MHz}, \mathrm{CDCl}_{3}\right)$

7.94-7.91 (m, 2 H), 7.58-7.56 (m, 3 H), 6.40 (d, 1.5 Hz, 1 H), 6.00 (s, 1 H), 2.89-

2.76 (m, $2 \mathrm{H}), 2.58-2.54(\mathrm{~m}, 2 \mathrm{H}), 2.23$ (s, $3 \mathrm{H}), 1.92$ (s, $3 \mathrm{H}) \mathrm{ppm}$

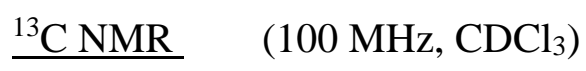

$199.4,164.9,149.8,147.3,145.1,139.1,132.5,132.2,131.8,131.6,128.7,128.5$, $127.6,113.9,72.7,34.6,24.9,24.0,10.3 \mathrm{ppm}$

$\underline{\text { IR }} \quad$ (Thin Film)

$1723,1692 \mathrm{~cm}^{-1}$

$\underline{\text { HRMS }} \quad$ HRMS-ESI (m/z): $[\mathrm{M}+\mathrm{H}]^{+}$calcd for $\mathrm{C}_{21} \mathrm{H}_{18} \mathrm{O}_{4} \mathrm{Br}, 413.0383$; found, 413.0378 and 415.0354

$\underline{\text { TLC }} \quad R_{f}=0.51$ (35\% ethyl acetate/hexanes); silica gel, UV, $p$-anisaldehyde

Waters 600 HPLC, UV/PDA detector, 328 nm, Daicel CHIRALCEL-OD, 250 X 4.6 mm column, $0.2 \% \mathrm{EtOH} /$ hexanes, Flow rate: $1 \mathrm{~mL} / \mathrm{min}$

\begin{tabular}{|l|c|c|}
\hline Peak & Retention Time (min) & Peak area (\%) \\
\hline Peak 1 & 95.65 & 49.69 \\
\hline Peak 2 & 131.40 & 50.31 \\
\hline
\end{tabular}



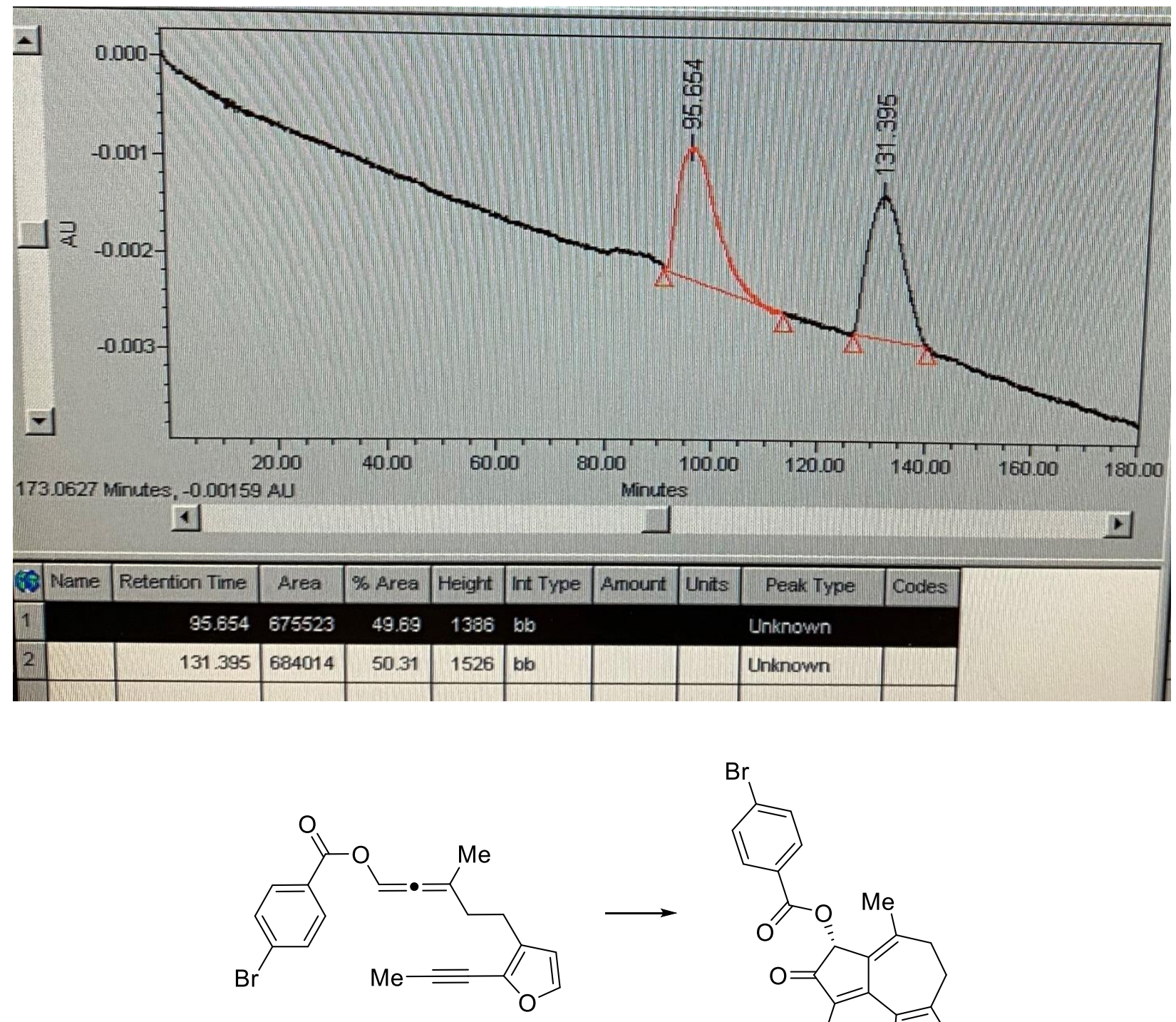

$4 e$

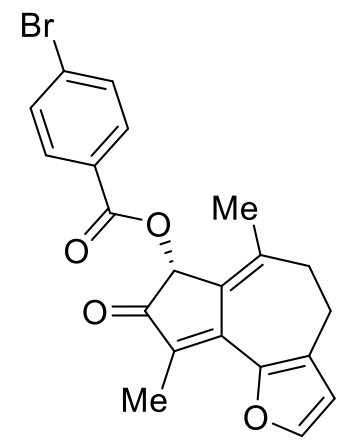

$3 e$

(R)-6,9-dimethyl-8-oxo-4,5,7,8-tetrahydroazuleno[4,5-b]furan-7-yl 2-chloroacetate (3e). Run

1: Follows General Procedure E: Bis(1,5-cyclooctadiene)rhodium(I) tetrafluoroborate (2.8 mg, $0.007 \mathrm{mmol}),(S)-$ MonoPhos-alkene $(8)(4.5 \mathrm{mg}, 0.011 \mathrm{mmol})$, carbon monoxide (100\%), 3methyl-5-(2-(prop-1-yn-1-yl)furan-3-yl)penta-1,2-dien-1-yl 4-bromobenzoate (4e) (27 mg, 0.07 mmol), DCE $(7.0 \mathrm{~mL}, 0.01 \mathrm{M})$. The reaction was stirred for $45 \mathrm{~h}$ in an oil bath $\left(70{ }^{\circ} \mathrm{C}\right)$. The crude residue was purified via silica gel flash chromatography (5\% to $10 \%$ ethyl acetate/hexanes) to give 
$13 \mathrm{mg}(45 \%)$ of the title compound as a yellow solid in an 84:16 er and $3 \mathrm{mg}(21 \%)$ of aldehyde

\section{5.}

\section{EDD6-490}

Waters 600 HPLC, UV/PDA detector, 328 nm, Daicel CHIRALCEL-OD, 250 X 4.6 mm column, $0.2 \% \mathrm{EtOH} / \mathrm{hexanes,} \mathrm{Flow} \mathrm{rate:} 1 \mathrm{~mL} / \mathrm{min}$

\begin{tabular}{|l|c|c|}
\hline Peak & Retention Time (min) & Peak area (\%) \\
\hline Peak 1 & 83.08 & 15.82 \\
\hline Peak 2 & 114.04 & 84.18 \\
\hline
\end{tabular}

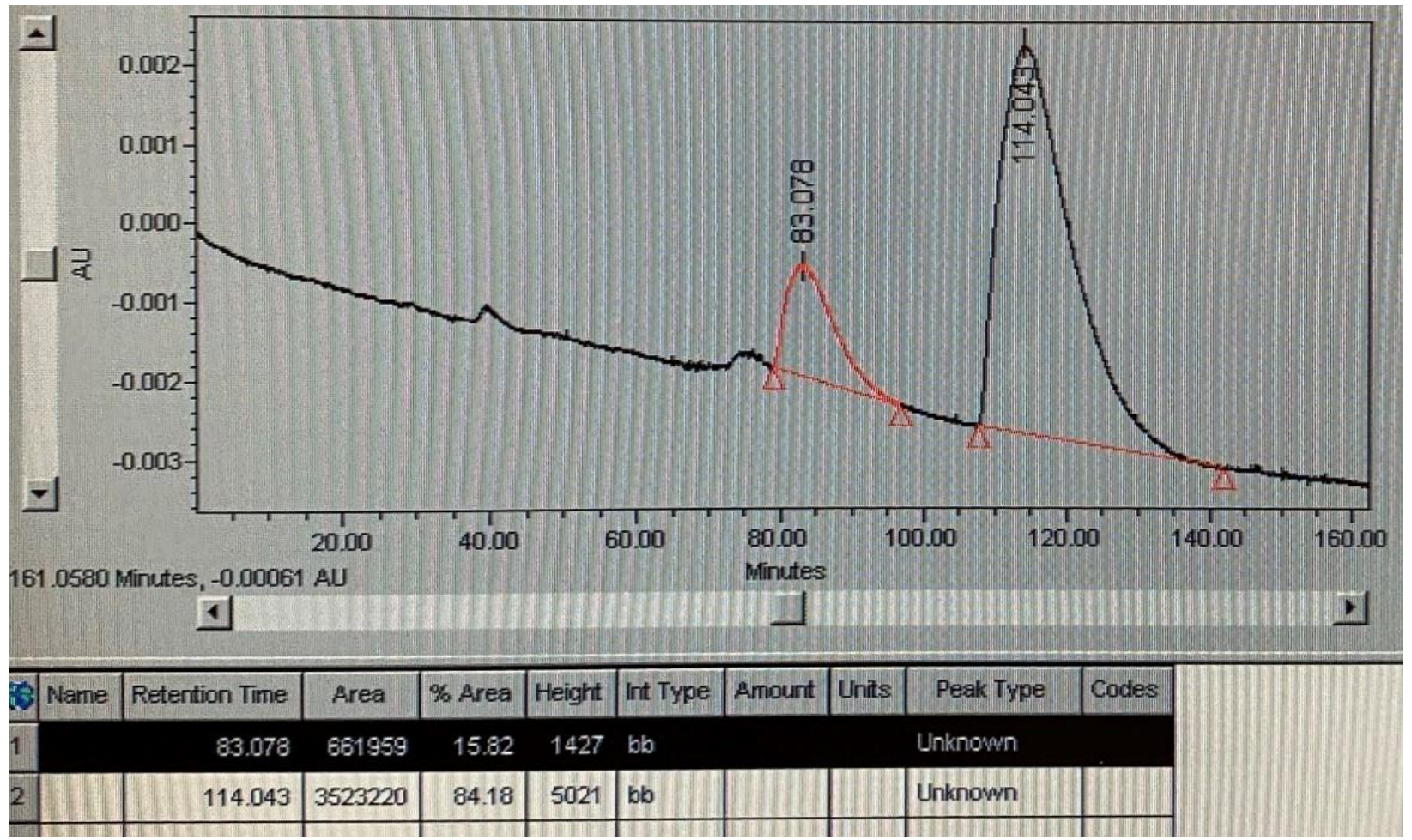

Run 2: Bis(1,5-cyclooctadiene)rhodium(I) tetrafluoroborate $(3.4 \mathrm{mg}, 0.0083 \mathrm{mmol}),(S)$ MonoPhos-alkene (8) (4.9 mg, 0.012 mmol), carbon monoxide (100\%), 3-methyl-5-(2-(prop-1- 
yn-1-yl)furan-3-yl)penta-1,2-dien-1-yl 4-bromobenzoate (4e) (32 mg, 0.083 mmol), DCE (8.3 mL, $0.01 \mathrm{M})$. The reaction was stirred for $42 \mathrm{~h}$ in an oil bath $\left(70{ }^{\circ} \mathrm{C}\right)$. The crude residue was purified via silica gel flash chromatography (10\% ethyl acetate/hexanes) to give $16 \mathrm{mg}(47 \%)$ of the title compound as a yellow solid in an 84:16 er and $3 \mathrm{mg}$ (18\%) of aldehyde 15.

\section{EDD6-491}

$[\alpha]^{25} \mathrm{D}=+91.3\left(\mathrm{c} .=0.3, \mathrm{CHCl}_{3}\right)$

Waters 600 HPLC, UV/PDA detector, 328 nm, Daicel CHIRALCEL-OD, 250 X 4.6 mm column, $0.2 \% \mathrm{EtOH} / \mathrm{h}$ xanes, Flow rate: $1 \mathrm{~mL} / \mathrm{min}$

\begin{tabular}{|l|c|c|}
\hline Peak & Retention Time (min) & Peak area (\%) \\
\hline Peak 1 & 90.26 & 16.05 \\
\hline Peak 2 & 123.97 & 83.95 \\
\hline
\end{tabular}

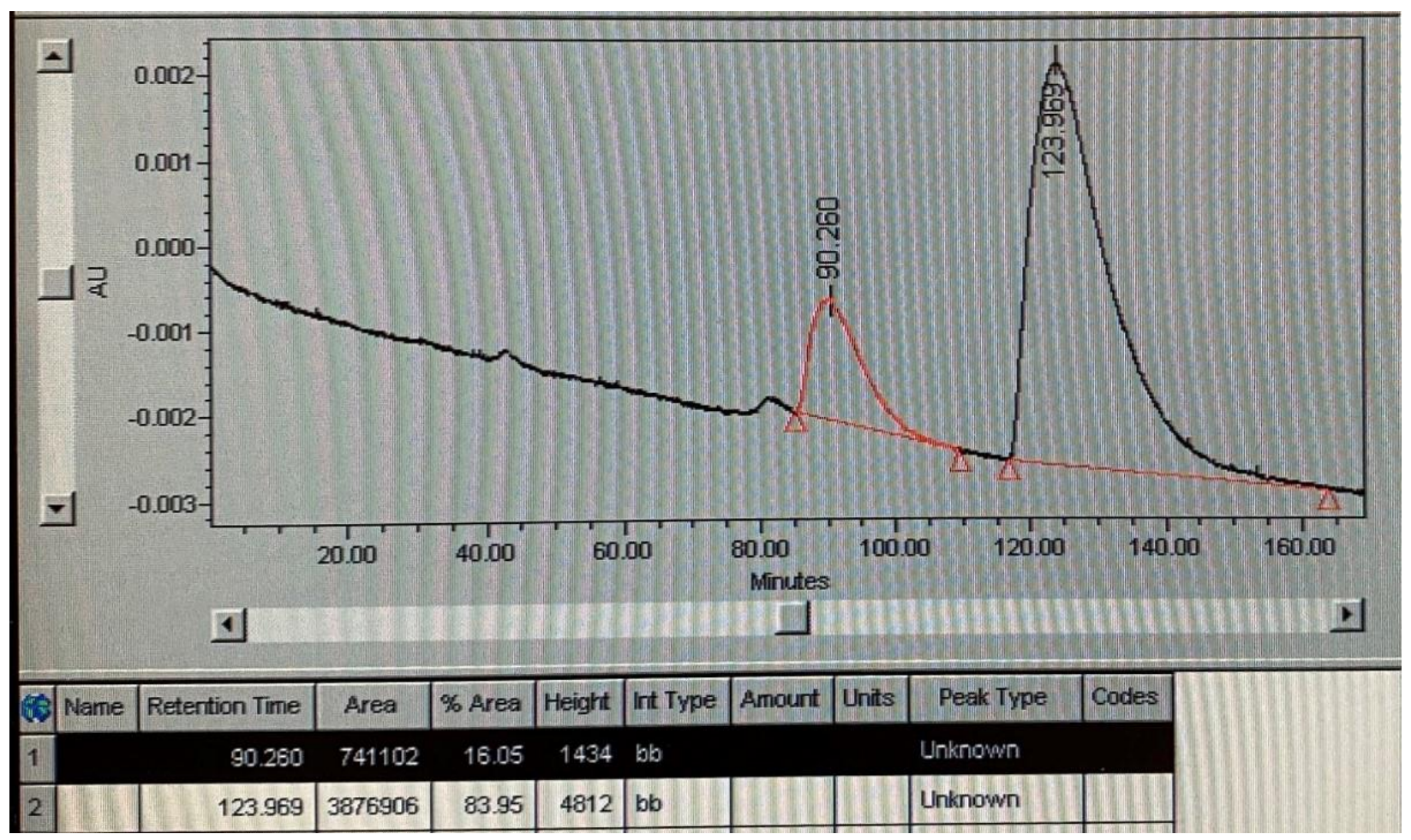




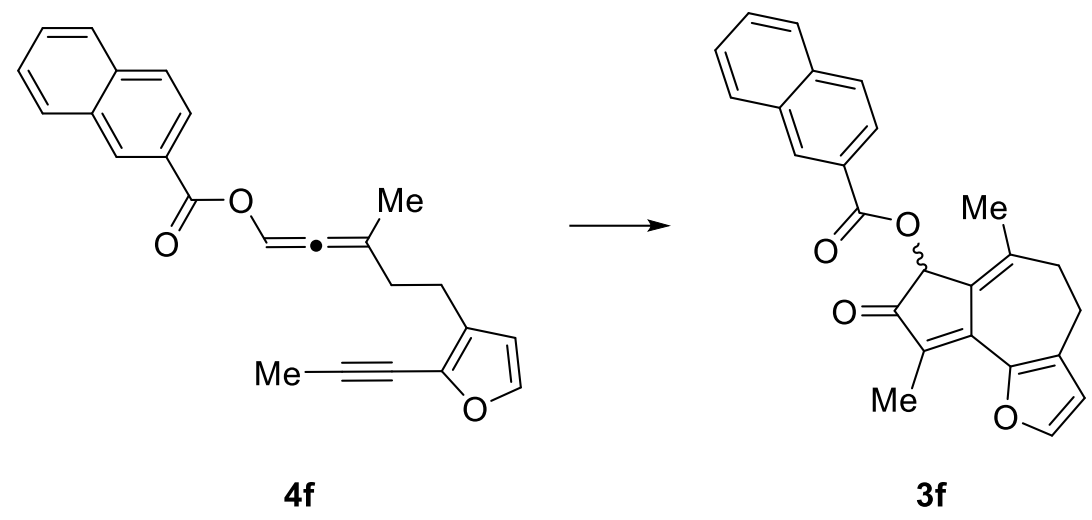

6,9-dimethyl-8-oxo-4,5,7,8-tetrahydroazuleno[4,5-b]furan-7-yl 2-naphthoate (3f). Follows

General Procedure C: Bis(1,5-cyclooctadiene)rhodium(I) tetrafluoroborate (1.5 mg, 0.0036 mmol), triphenyl phosphine (1.4 mg, $0.0054 \mathrm{mmol})$, carbon monoxide (100\%), 3-methyl-5-(2(prop-1-yn-1-yl)furan-3-yl)penta-1,2-dien-1-yl 2-naphthoate (4f) (13 mg, 0.036 mmol), DCE (1.8 $\mathrm{mL}, 0.02 \mathrm{M})$. The reaction was stirred for $45 \mathrm{~h}$ in an oil bath $\left(70{ }^{\circ} \mathrm{C}\right)$. The crude residue was purified via silica gel flash chromatography (5\% to $10 \%$ ethyl acetate/hexanes) to give the title compound $9 \mathrm{mg}$ (64\%) of the title compound as a yellow oil and $0.7 \mathrm{mg}(9 \%)$ of aldehyde $\mathbf{1 5}$.

\section{EDD5-365}

${ }^{1} \mathrm{H} \mathrm{NMR} \quad\left(400 \mathrm{MHz}, \mathrm{CDCl}_{3}\right)$

$8.64(\mathrm{~s}, 1 \mathrm{H}), 8.09-8.07$ (dd, $J=1.2 \mathrm{~Hz}, 8.4 \mathrm{~Hz}, 1 \mathrm{H}), 7.94(\mathrm{~d}, J=8.4 \mathrm{~Hz}, 1 \mathrm{H})$,

$7.87(\mathrm{~d}, J=8.4 \mathrm{~Hz}, 2 \mathrm{H}), 7.61-7.51(\mathrm{~m}, 3 \mathrm{H}), 6.41(\mathrm{~d}, J=1.6 \mathrm{~Hz}, 1 \mathrm{H}), 6.08(\mathrm{~s}, 1$

H), 2.92-2.76 (m, 2 H), 2.60-2.56 (m, 2 H), 2.26 (s, 3 H), 1.97 (s, 3 H) ppm;

Impurity at: $9.99(\mathrm{~d}, J=8.0 \mathrm{~Hz}, 0.08 \mathrm{H}), 9.84(\mathrm{~d}, J=8.4 \mathrm{~Hz}, 0.07 \mathrm{H})$,

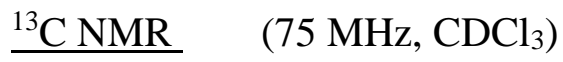


199.7, 165.8, 149.8, 147.5, 145.0, 139.0, 135.8, 132.6, 132.5, 132.2, 131.7, 129.6, $128.5,128.3,127.9,127.8,127.0,126.8,125.7,113.9,72.6,34.7,25.0,24.0,10.3$ ppm

$\underline{\text { IR }} \quad$ (Thin Film) $2924,1720,1693,1004 \mathrm{~cm}^{-1}$

HRMS HRMS-ESI (m/z): $[\mathrm{M}+\mathrm{H}]^{+}$calcd for $\mathrm{C}_{25} \mathrm{H}_{21} \mathrm{O}_{4}, 385.1440$; found, 385.1431

TLC $\quad R_{f}=0.54$ (35\% ethyl acetate/hexanes); silica gel, UV, $p$-anisaldehyde

Waters 600 HPLC, UV/PDA detector, 235 nm, Daicel CHIRALCEL-OD, 250 X 4.6 mm

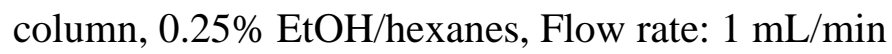

\begin{tabular}{|l|c|c|}
\hline Peak & Retention Time (min) & Peak area (\%) \\
\hline Peak 1 & 96.71 & 49.48 \\
\hline Peak 2 & 137.21 & 50.52 \\
\hline
\end{tabular}

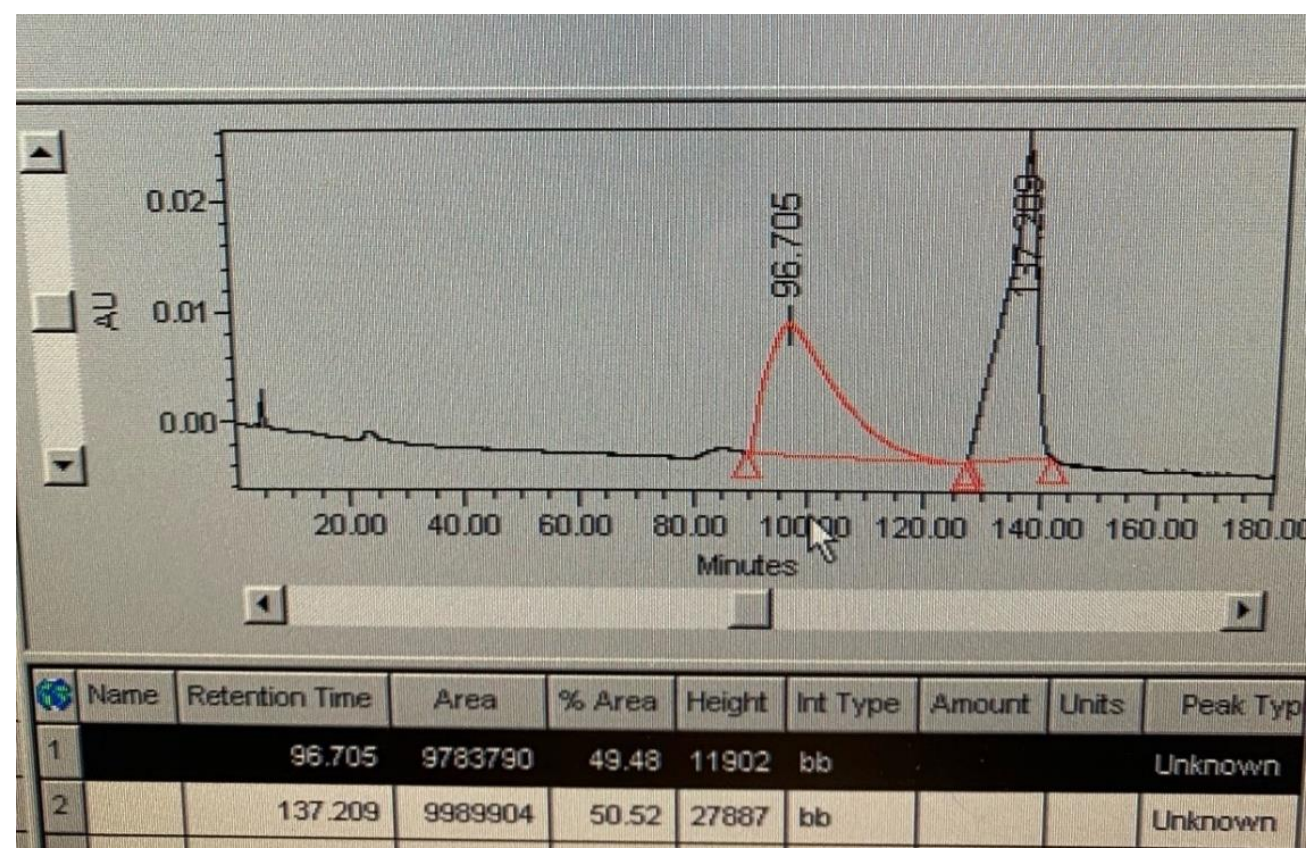




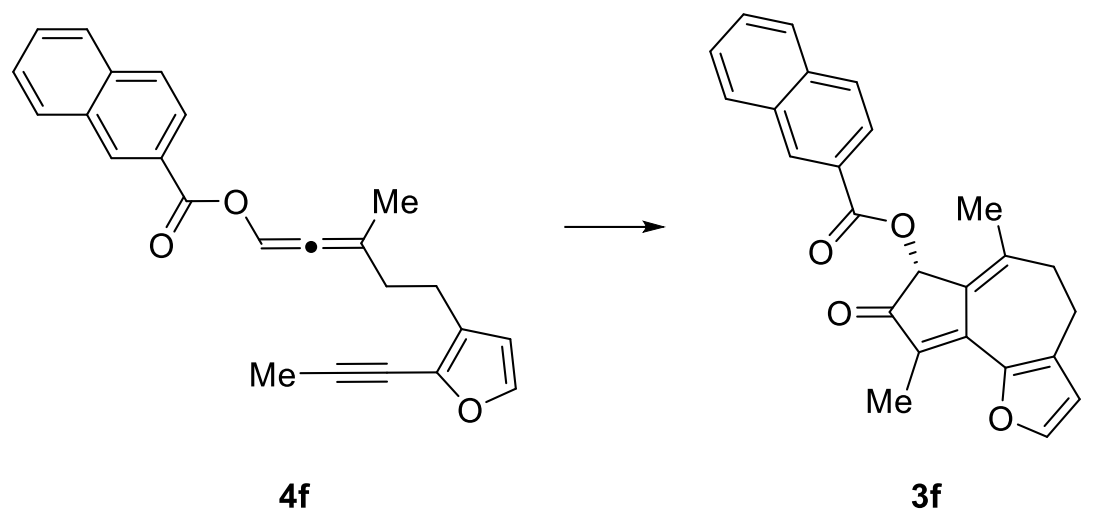

(R)-6,9-dimethyl-8-oxo-4,5,7,8-tetrahydroazuleno[4,5-b]furan-7-yl 2-naphthoate $\quad(3 f)$.

Follows General Procedure E: Bis(1,5-cyclooctadiene)rhodium(I) tetrafluoroborate (1.5 mg, $0.0036 \mathrm{mmol}),(S)$-MonoPhos-alkene (8) (2.2 $\mathrm{mg}, 0.0054 \mathrm{mmol})$, carbon monoxide (100\%), 3methyl-5-(2-(prop-1-yn-1-yl)furan-3-yl)penta-1,2-dien-1-yl 2-naphthoate (4f) (13 mg, 0.036 mmol), DCE $(3.6 \mathrm{~mL}, 0.01 \mathrm{M})$. The reaction was stirred for $25 \mathrm{~h}$ in an oil bath $\left(70{ }^{\circ} \mathrm{C}\right)$. The crude residue was purified via silica gel flash chromatography (5\% to 10\% ethyl acetate/hexanes) to give a $7 \mathrm{mg}$ mixture as a yellow oil containing $5.8 \mathrm{mg}(42 \%)$ of the title compound in a 90:10 er and $1.2 \mathrm{mg}(17 \%)$ of aldehyde $\mathbf{1 5}$.

\section{EDD5-369}

Waters 600 HPLC, UV/PDA detector, 235 nm, Daicel CHIRALCEL-OD, 250 X 4.6 mm column, $0.25 \%$ EtOH/hexanes, Flow rate: $1 \mathrm{~mL} / \mathrm{min}$

\begin{tabular}{|l|c|c|}
\hline Peak & Retention Time (min) & Peak area (\%) \\
\hline Peak 1 & 105.62 & 10.19 \\
\hline Peak 2 & 136.34 & 89.81 \\
\hline
\end{tabular}




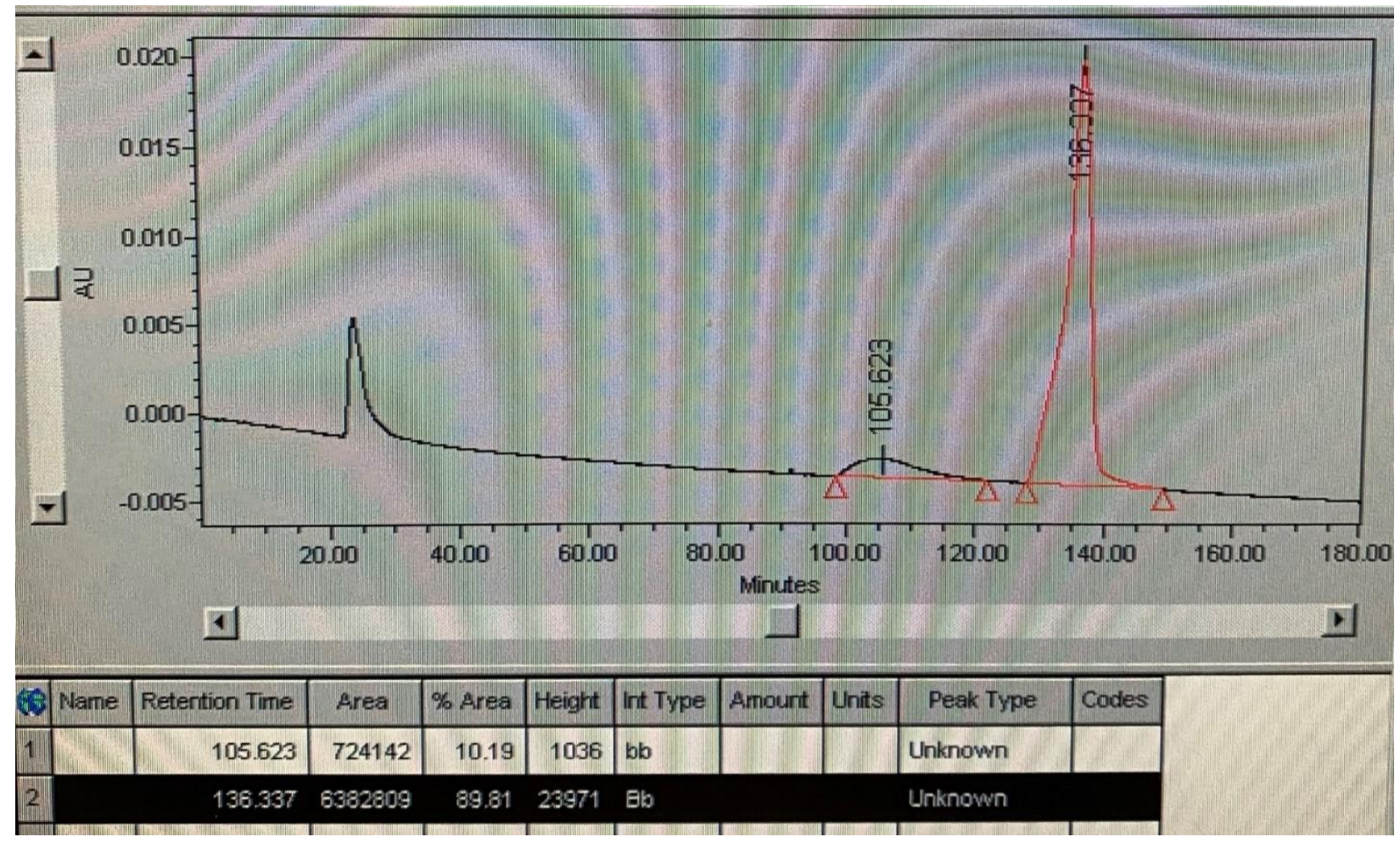

$[\alpha]^{25}=+137.6\left(\mathrm{c} .=0.25, \mathrm{CHCl}_{3}\right)$

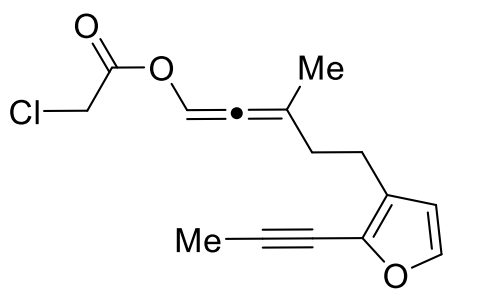

$4 \mathrm{~g}$

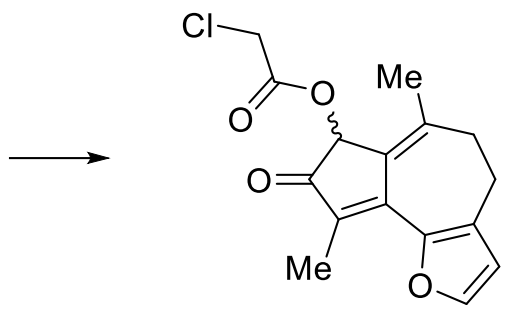

$3 \mathrm{~g}$

6,9-dimethyl-8-oxo-4,5,7,8-tetrahydroazuleno[4,5-b]furan-7-yl 2-chloroacetate (3g). Follows

General Procedure C: Bis(1,5-cyclooctadiene)rhodium(I) tetrafluoroborate (2.8 mg, 0.0068 mmol), triphenyl phosphine $(2.7 \mathrm{mg}, 0.0102 \mathrm{mmol})$, carbon monoxide (100\%), 3-methyl-5-(2(prop-1-yn-1-yl)furan-3-yl)penta-1,2-dien-1-yl 2-chloroacetate (4g) (19 mg, 0.068 mmol), DCE (3.4 mL, $0.02 \mathrm{M})$. The reaction was stirred for $90 \mathrm{~h}$ in an oil bath $\left(70^{\circ} \mathrm{C}\right)$. The crude residue was purified via silica gel flash chromatography (5\% to $10 \%$ ethyl acetate/hexanes) to give $15 \mathrm{mg}$ of the title compound (71\%) as a yellow solid. 
${ }^{1} \mathrm{H} \mathrm{NMR} \quad\left(500 \mathrm{MHz}, \mathrm{CDCl}_{3}\right)$

$7.56(\mathrm{~d}, J=1.5 \mathrm{~Hz}, 1 \mathrm{H}), 6.40(\mathrm{~d}, J=1.5 \mathrm{~Hz}, 1 \mathrm{H}), 5.86(\mathrm{~s}, 1 \mathrm{H}), 4.14(\mathrm{~d}, J=1.5$

Hz, 2 H), 2.88-2.75 (m, 2 H), 2.58-2.56 (m, 2 H), 2.20 (s, 3 H), 1.94 (s, 3 H) ppm

${ }^{13} \mathrm{C} \mathrm{NMR} \quad\left(125 \mathrm{MHz}, \mathrm{CDCl}_{3}\right)$

$198.7,166.5,150.0,147.2,145.2,139.6,132.8,132.1,126.9,113.9,73.3,40.8$, $34.6,25.0,23.9,10.2 \mathrm{ppm}$

$\underline{\text { IR }} \quad$ (Thin Film)

$1754,1692 \mathrm{~cm}^{-1}$

HRMS HRMS-ESI (m/z): $[\mathrm{M}+\mathrm{H}]^{+}$calcd for $\mathrm{C}_{16} \mathrm{H}_{16} \mathrm{O}_{4} \mathrm{Cl}, 307.0737$; found, 307.0744

$\underline{\text { TLC }} \quad R_{f}=0.42$ (35\% ethyl acetate/hexanes); silica gel, UV, $p$-anisaldehyde

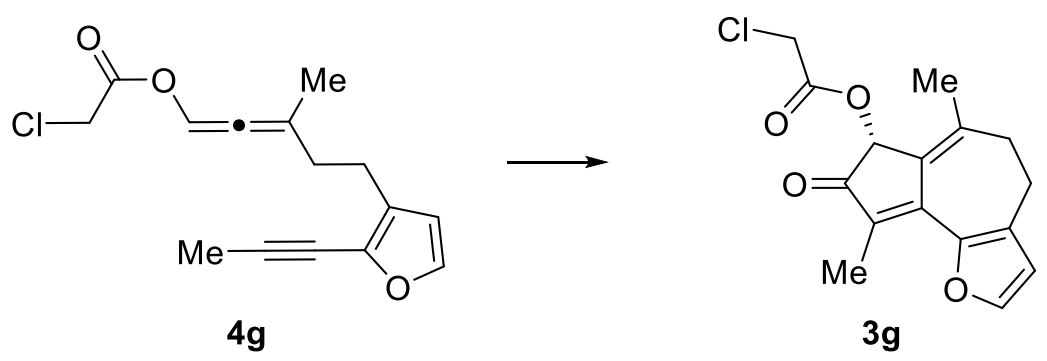

(R)-6,9-dimethyl-8-oxo-4,5,7,8-tetrahydroazuleno[4,5-b]furan-7-yl 2-chloroacetate $(3 \mathrm{~g})$.

Follows General Procedure E: Bis(1,5-cyclooctadiene)rhodium(I) tetrafluoroborate (2.2 mg, $0.0054 \mathrm{mmol}),(S)$-MonoPhos-alkene (8) (3.3 mg, $0.0081 \mathrm{mmol})$, carbon monoxide (100\%), 3methyl-5-(2-(prop-1-yn-1-yl)furan-3-yl)penta-1,2-dien-1-yl 2-chloroacetate (4g) (15 mg, 0.054 mmol), DCE $(5.4 \mathrm{~mL}, 0.01 \mathrm{M})$. The reaction was stirred for $115 \mathrm{~h}$ in an oil bath $\left(70{ }^{\circ} \mathrm{C}\right)$. The crude 
residue was purified via silica gel flash chromatography (10\% to $15 \%$ ethyl acetate/hexanes) to give $9.5 \mathrm{mg}(56 \%)$ of the title compound as a yellow solid and $1.5 \mathrm{mg}(14 \%)$ of aldehyde 15 . The enantiomers of $\mathbf{3 g}$ could not be separated by chiral HPLC and the enantiomeric ratio was determined after hydrolysis (96.5:3.5).

\section{Hydrolysis of APKR adduct for HPLC determination of enantiomeric ratio}

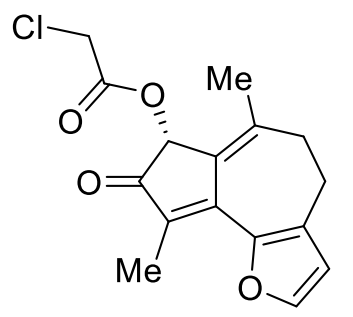

$3 g$

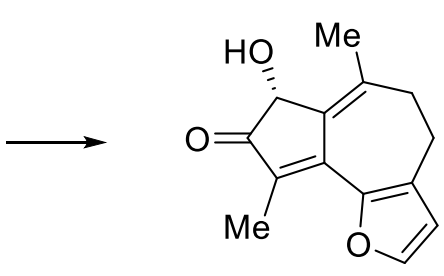

S4

(R)-7-hydroxy-6,9-dimethyl-5,7-dihydroazuleno[4,5-b]furan-8(4H)-one (S4). A flame-dried, 1-necked,10 $\mathrm{mL}$ round bottomed flask equipped with a magnetic stir bar and needle attached to a nitrogen manifold was charged with scandium(III) triflate ( $3.8 \mathrm{mg}, 0.0078 \mathrm{mmol}, 0.3$ equiv) in a nitrogen filled glove box. $(R)$-6,9-dimethyl-8-oxo-4,5,7,8-tetrahydroazuleno[4,5-b]furan-7-yl 2chloroacetate (3g) ( $8 \mathrm{mg}, 0.026 \mathrm{mmol}, 1$ equiv) was dissolved in methanol ( $0.83 \mathrm{~mL}, 0.03 \mathrm{M})$ and transferred to the flask via syringe. Water $(0.21 \mathrm{~mL}, 0.12 \mathrm{M})$ was added dropwise via syringe. The tube was lowered into a $30{ }^{\circ} \mathrm{C}$ oil bath and stirred for 90 min until no starting material was evident by TLC. The solution was diluted with deionized water $(3 \mathrm{~mL})$, transferred to a separatory funnel and extracted with DCM $(5 \times 3 \mathrm{~mL})$. The combined organic extracts were dried over magnesium sulfate, filtered, and concentrated. The crude residue was purified via silica gel flash chromatography (30\% ethyl acetate/hexanes) to give the title compound (4 mg, 67\%) as a yellow solid in a 96.5:3.5 ratio of enantiomers.

EDD5-349 
Waters 600 HPLC, UV/PDA detector, 332 nm, Daicel CHIRALCEL-OD, 250 X 4.6 mm column, $1.0 \% \mathrm{iPrOH} /$ hexanes, Flow rate: $1 \mathrm{~mL} / \mathrm{min}$

\begin{tabular}{|l|c|c|}
\hline Peak & Retention Time (min) & Peak area (\%) \\
\hline Peak 1 & 42.68 & 3.52 \\
\hline Peak 2 & 47.26 & 96.48 \\
\hline
\end{tabular}

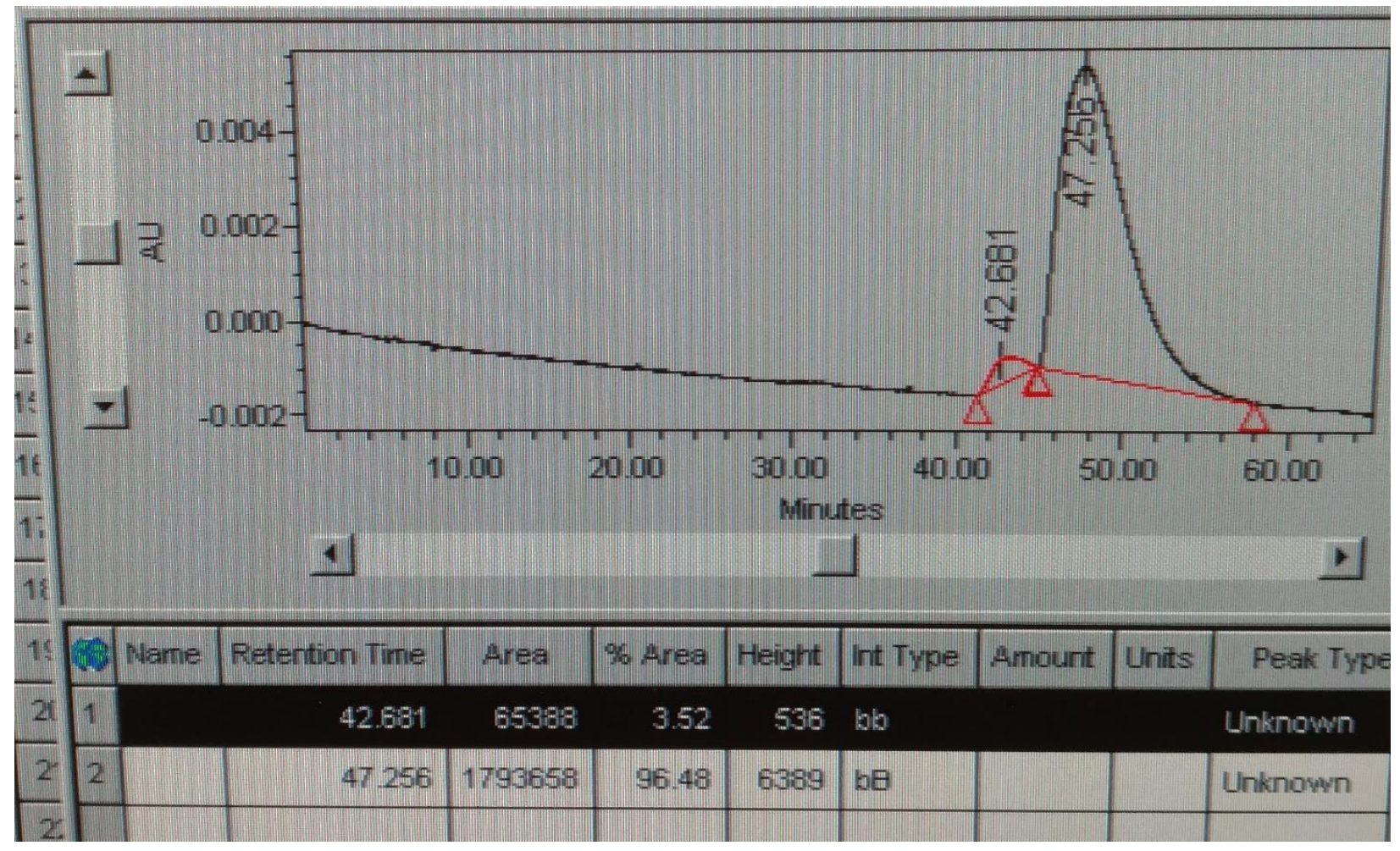

\section{EDD5-347}

APKR Run 2: Bis(1,5-cyclooctadiene)rhodium(I) tetrafluoroborate (2.2 mg, $0.0054 \mathrm{mmol}),(S)$ MonoPhos-alkene (8) (3.3 mg, $0.0081 \mathrm{mmol})$, carbon monoxide (100\%), 3-methyl-5-(2-(prop-1yn-1-yl)furan-3-yl)penta-1,2-dien-1-yl 2-chloroacetate (4g) (15 mg, $0.054 \mathrm{mmol}), \mathrm{DCE}$ (5.4 mL, $0.01 \mathrm{M})$. The reaction was stirred for $115 \mathrm{~h}$ in an oil bath $\left(70^{\circ} \mathrm{C}\right)$. The crude residue was purified via silica gel flash chromatography (10\% to $15 \%$ ethyl acetate/hexanes) to give $9.5 \mathrm{mg}(52 \%)$ of 
the title compound as a yellow solid and $2.1 \mathrm{mg}(19 \%)$ of aldehyde 15 . The enantiomers of $\mathbf{3 g}$ could not be separated by chiral HPLC and the enantiomeric ratio was determined after hydrolysis (99.8:0.2).

\section{EDD5-350}

$[\alpha]^{25} \mathrm{D}=-340.4\left(\mathrm{c} .=0.267, \mathrm{CH}_{2} \mathrm{Cl}_{2}\right)$

Hydrolysis Run 2: (R)-6,9-dimethyl-8-oxo-4,5,7,8-tetrahydroazuleno[4,5-b]furan-7-yl 2chloroacetate (3g) ( $8 \mathrm{mg}, 0.026 \mathrm{mmol}, 1$ equiv), methanol $(0.83 \mathrm{~mL}, 0.03 \mathrm{M})$, water $(0.21 \mathrm{~mL}$, 0.12 M), $90 \mathrm{~min}, \mathbf{S 4} 4 \mathrm{mg}, 67 \%$, 99.8:0.2 ratio of enantiomers.

\section{EDD5-352}

Waters 600 HPLC, UV/PDA detector, 332 nm, Daicel CHIRALCEL-OD, 250 X 4.6 mm column, $1.0 \% \mathrm{iPrOH} /$ hexanes, Flow rate: $1 \mathrm{~mL} / \mathrm{min}$

\begin{tabular}{|l|c|c|}
\hline Peak & Retention Time (min) & Peak area (\%) \\
\hline Peak 1 & 44.22 & 0.21 \\
\hline Peak 2 & 48.05 & 99.79 \\
\hline
\end{tabular}




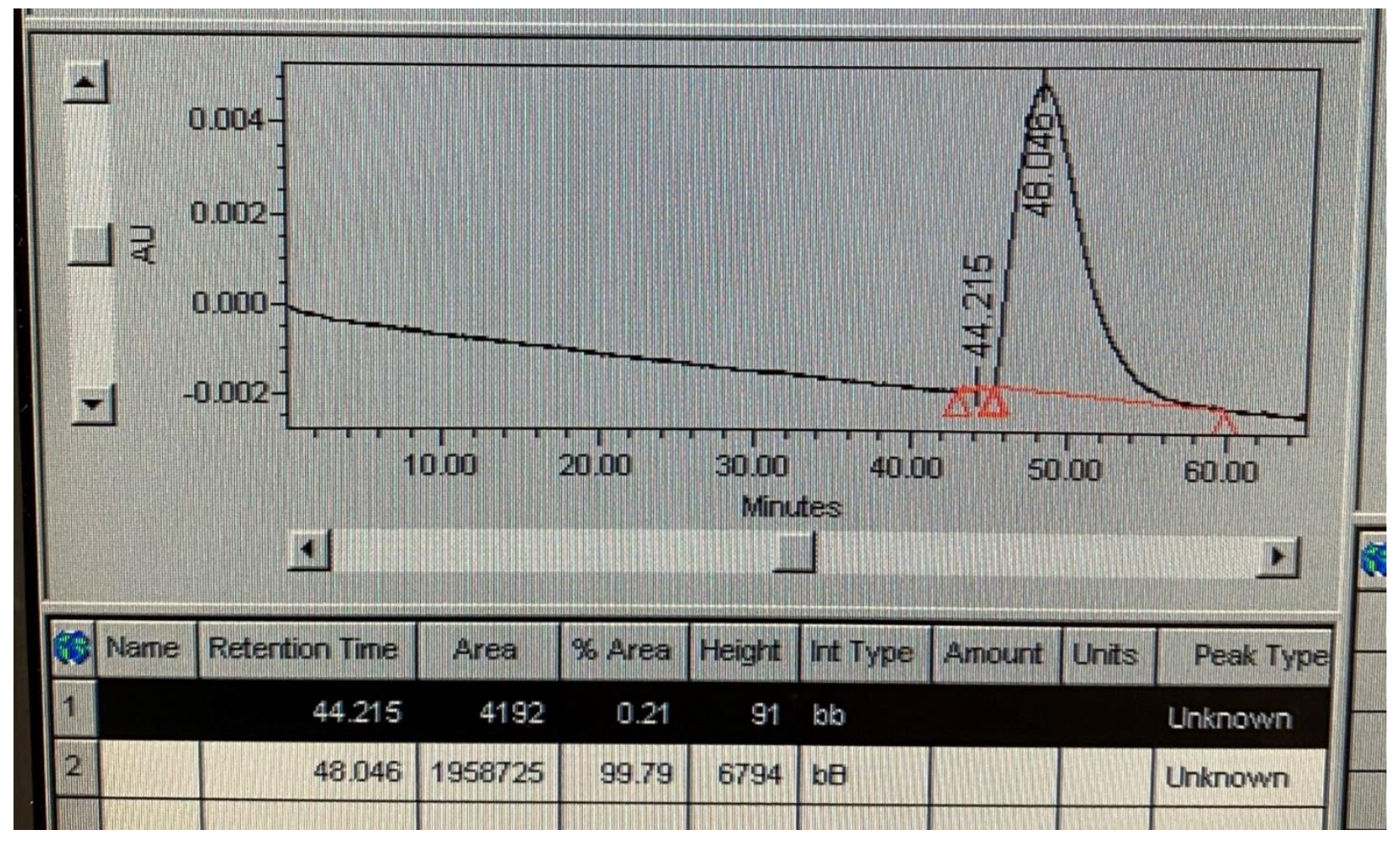

APKR Run 3: Bis(1,5-cyclooctadiene)rhodium(I) tetrafluoroborate (2.9 mg, $0.0072 \mathrm{mmol}),(S)$ MonoPhos-alkene (8) (4.5 mg, $0.011 \mathrm{mmol})$, carbon monoxide (100\%), 3-methyl-5-(2-(prop-1yn-1-yl)furan-3-yl)penta-1,2-dien-1-yl 2-chloroacetate (4g) (20 mg, $0.072 \mathrm{mmol})$, DCE (7.2 mL, $0.01 \mathrm{M})$. The reaction was stirred for $96 \mathrm{~h}$ in an oil bath $\left(70^{\circ} \mathrm{C}\right)$. The crude residue was purified via silica gel flash chromatography (5\% to $10 \%$ ethyl acetate/hexanes) to give $14 \mathrm{mg}(64 \%)$ of the title compound as a yellow solid and $5 \mathrm{mg}(34 \%)$ of aldehyde $\mathbf{1 5}$. The enantiomers of $\mathbf{3 g}$ could not be separated by chiral HPLC and the enantiomeric ratio was determined after hydrolysis (96:4).

Hydrolysis Run 3: (R)-6,9-dimethyl-8-oxo-4,5,7,8-tetrahydroazuleno[4,5-b]furan-7-yl 2chloroacetate (3g) (14 mg, $0.046 \mathrm{mmol}, 1$ equiv), methanol (1.47 mL, $0.03 \mathrm{M})$, water $(0.37 \mathrm{~mL}$, $0.12 \mathrm{M}), 90 \mathrm{~min}, \mathbf{S 4} 7 \mathrm{mg}, 67 \%, 96: 4$ ratio of enantiomers.

\section{EDD6-503}


Waters 600 HPLC, UV/PDA detector, 332 nm, Daicel CHIRALCEL-OD, 250 X 4.6 mm column, $1.0 \% \mathrm{iPrOH} / \mathrm{hexanes}$, Flow rate: $1 \mathrm{~mL} / \mathrm{min}$

\begin{tabular}{|l|c|c|}
\hline Peak & Retention Time (min) & Peak area (\%) \\
\hline Peak 1 & 39.76 & 4.38 \\
\hline Peak 2 & 44.20 & 95.62 \\
\hline
\end{tabular}

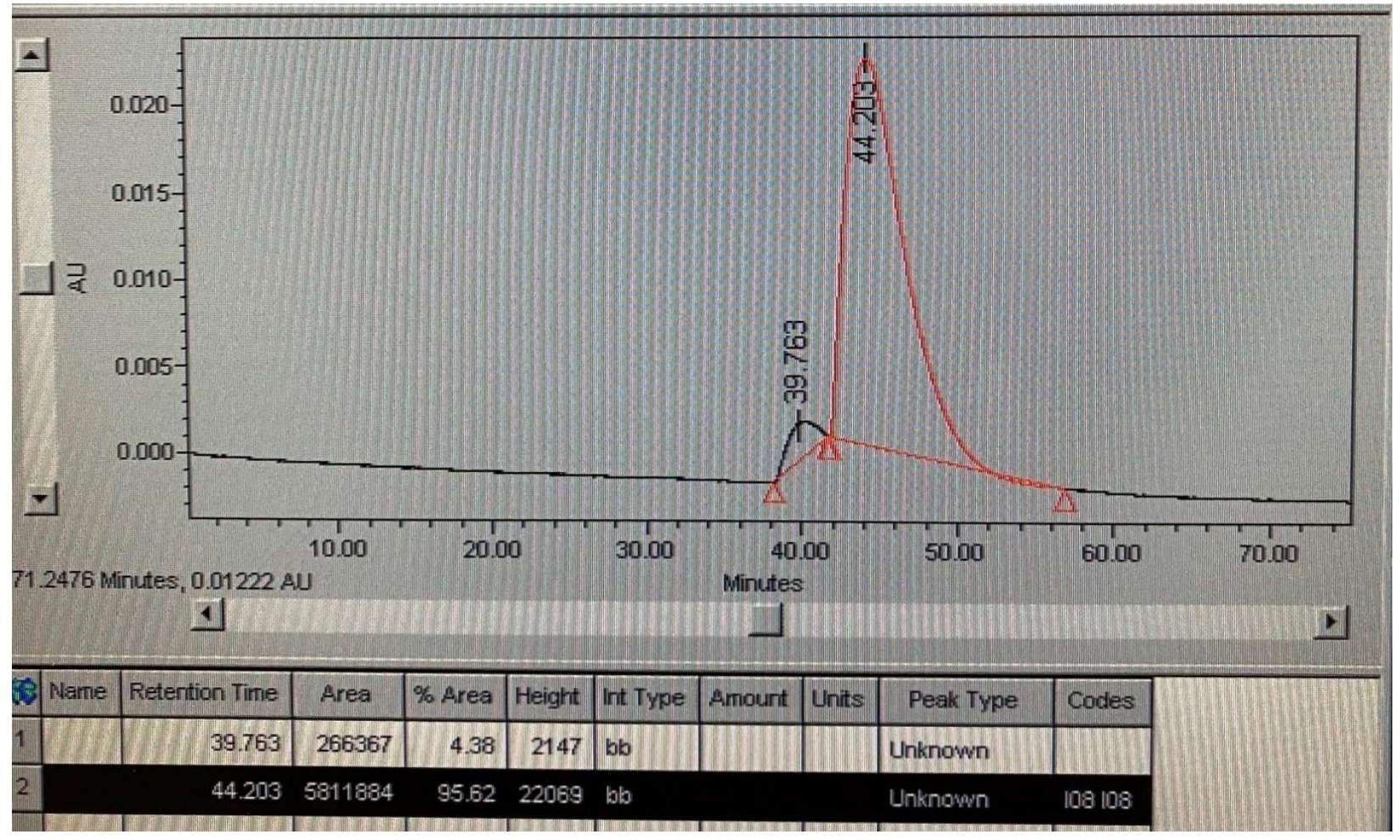

\section{Large scale APKR}<smiles>CC#Cc1occc1CCC(C)=C=COC(=O)CCl</smiles>

49<smiles>CC1=C2C(=C(C)C(=O)[C@@H]2OC(=O)CCl)c2occc2CC1</smiles>

$3 g$ 


\section{(R)-6,9-dimethyl-8-oxo-4,5,7,8-tetrahydroazuleno[4,5-b]furan-7-yl 2-chloroacetate $(3 g)$.}

Follows General Procedure E: Bis(1,5-cyclooctadiene)rhodium(I) tetrafluoroborate (13.8 mg, $0.0341 \mathrm{mmol}),(S)$-MonoPhos-alkene $(\mathbf{8})(21.0 \mathrm{mg}, 0.051 \mathrm{mmol})$, carbon monoxide (100\%), 3methyl-5-(2-(prop-1-yn-1-yl)furan-3-yl)penta-1,2-dien-1-yl 2-chloroacetate (4g) (95 mg, 0.341 mmol), DCE $(34.1 \mathrm{~mL}, 0.01 \mathrm{M})$. The reaction was stirred for $115 \mathrm{~h}$ in an oil bath $\left(70{ }^{\circ} \mathrm{C}\right)$. The crude residue was purified via silica gel flash chromatography (5\% to $15 \%$ ethyl acetate/hexanes) to give $59 \mathrm{mg}(56 \%)$ of the title compound as a yellow solid and $10 \mathrm{mg}$ (14\%) of aldehyde 15 . The enantiomers of $\mathbf{3 g}$ could not be separated by chiral HPLC and the enantiomeric ratio was determined after hydrolysis (90:10).

Hydrolysis: $(R)$-6,9-dimethyl-8-oxo-4,5,7,8-tetrahydroazuleno[4,5-b]furan-7-yl 2-chloroacetate (3g) (59 mg, $0.192 \mathrm{mmol}, 1$ equiv), methanol (6.2 mL, $0.03 \mathrm{M})$, water (1.5 mL, $0.12 \mathrm{M}), 60 \mathrm{~min}$, S4 $44 \mathrm{mg}, 100 \%, 90: 10$ ratio of enantiomers.

Waters 600 HPLC, UV/PDA detector, 332 nm, Daicel CHIRALCEL-OD, 250 X 4.6 mm column, $1.0 \%$ iPrOH/hexanes, Flow rate: $1 \mathrm{~mL} / \mathrm{min}$

\begin{tabular}{|l|c|c|}
\hline Peak & Retention Time (min) & Peak area (\%) \\
\hline Peak 1 & 39.47 & 10.16 \\
\hline Peak 2 & 43.89 & 89.84 \\
\hline
\end{tabular}




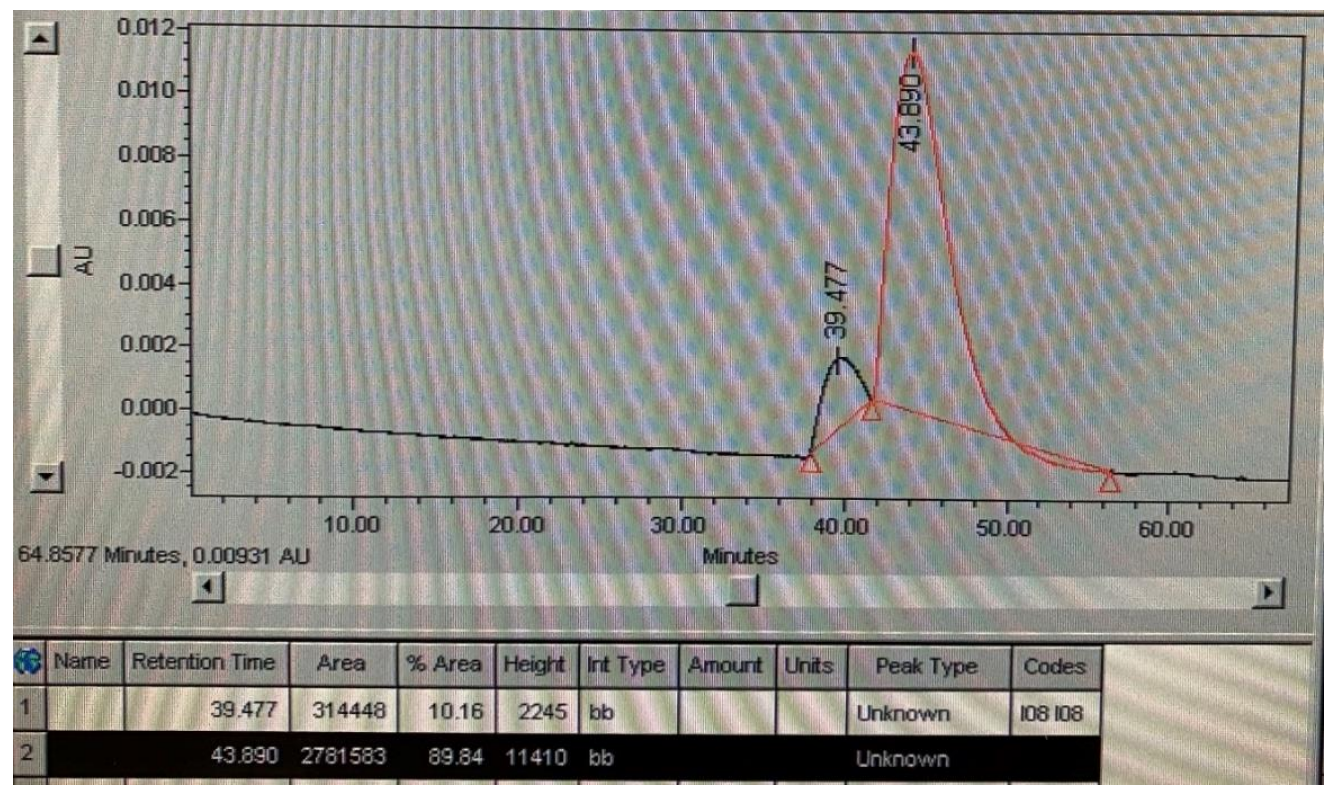

Synthesis of (S)-MonoPhos-alkene (8)<smiles>COP(Oc1ccc2ccccc2c1-c1cccc2ccccc12)N1c2ccccc2C=Cc2ccccc21</smiles>

8

5-((11bS)-dinaphtho[2,1-d:1',2'-f][1,3,2]dioxaphosphepin-4-yl)-5H-dibenzo[b,f]azepine (8).

This compound was prepared using a procedure entirely analogous to that previously reported. ${ }^{5}$ 
$\mathrm{X}$ ray quality crystals grown by dissolving $3 \mathrm{~g}$ in dichloromethane in a vial at room temperature and this vial was placed in a second larger vial with hexanes. The larger vial was capped and placed in a refrigerator $\left(0^{\circ} \mathrm{C}\right)$.

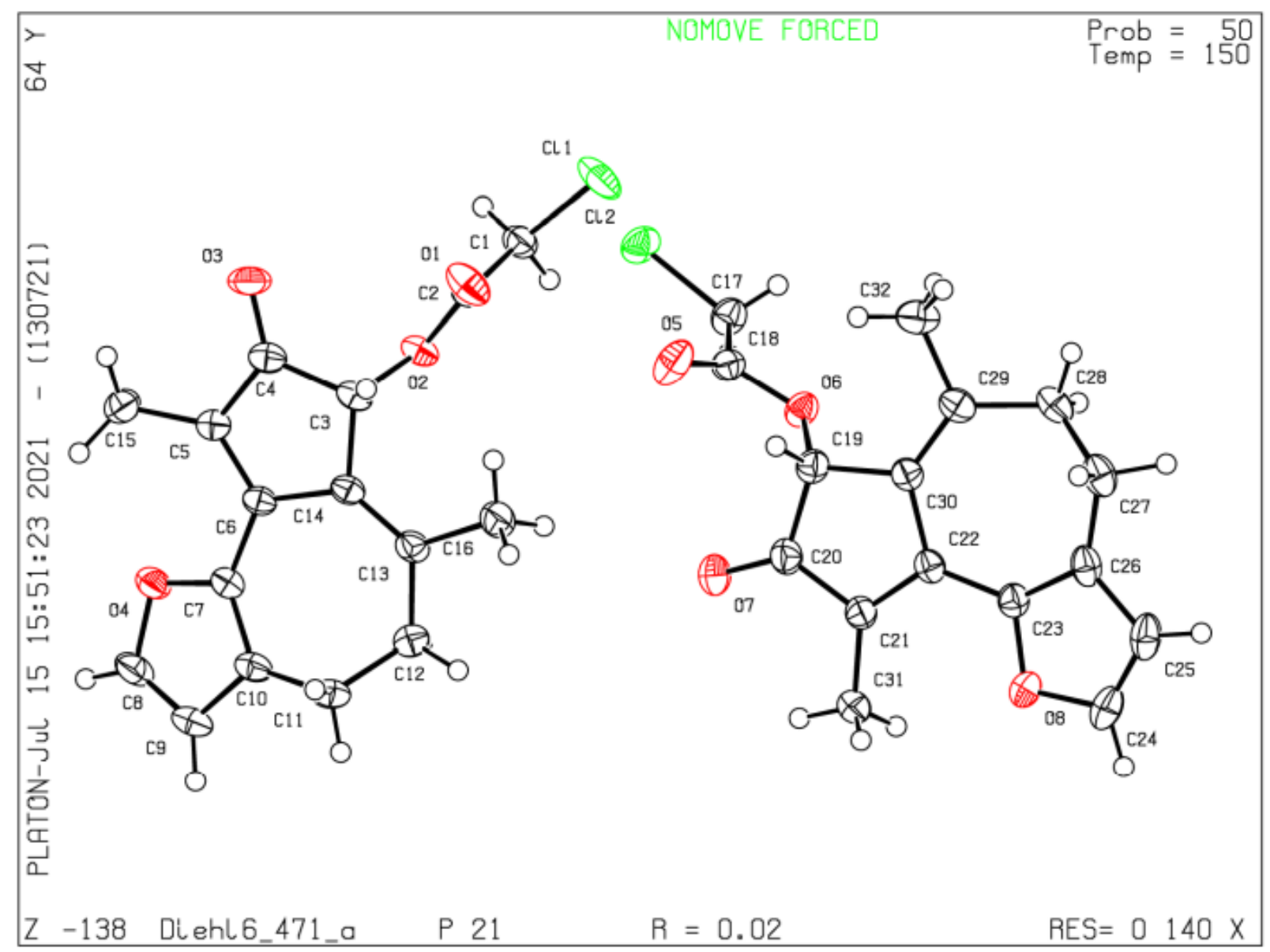

Probability level $=50 \%$

For hydrolysis of 3a, 3d, and 3g to give S4. See page S47, S68, and S79<smiles>[R]C(=O)O[C@H]1C(=O)C(C)=C2C1=C(C)CCc1ccoc12</smiles>

3a, 3d, 3g

$\mathrm{R}=\mathrm{Me}, \mathrm{Ph}, \mathrm{CH}_{2} \mathrm{Cl}$

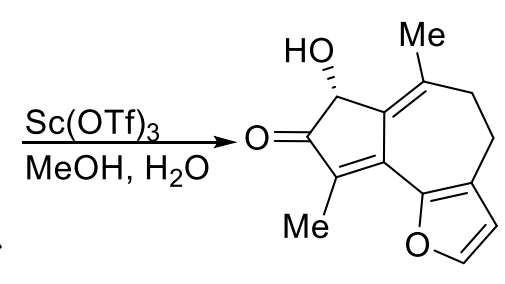

S4 


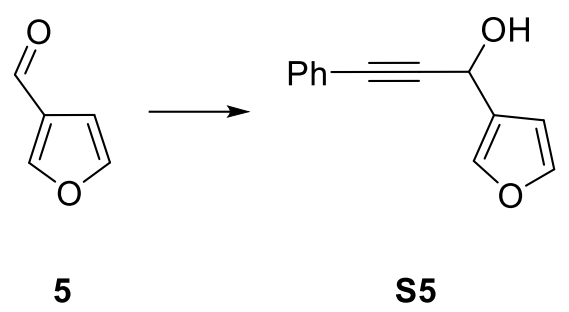

1-(furan-3-yl)-3-phenylprop-2-yn-1-ol (S5). This compound was prepared in a manner similar to that previously reported with some modifications. ${ }^{1}$ A flame-dried, 2-necked, $100-\mathrm{mL}$ roundbottomed flask equipped with a magnetic stir bar, nitrogen inlet adapter, and septum was charged with 3-furfural (5) $(0.8 \mathrm{~mL}, 9.24 \mathrm{mmol})$ and THF $(9.24 \mathrm{~mL})$. The solution was cooled by placing the flask in an ice-bath. Phenylethynylmagnesium bromide (13.9 $\mathrm{mL}$ of a $1.0 \mathrm{M}$ solution in THF, $13.86 \mathrm{mmol}$ ) was added dropwise via syringe over $5 \mathrm{~min}$. After $3 \mathrm{~h}$ at $0{ }^{\circ} \mathrm{C}$, TLC showed complete consumption of starting material. Saturated aqueous ammonium chloride $(3 \mathrm{~mL})$ was added all at once. The reaction mixture was transferred to a separatory funnel and the organic phase was separated. The aqueous phase was extracted with diethyl ether $(3 \times 10 \mathrm{~mL})$, dried over magnesium sulfate, filtered, and concentrated in vacuo. The crude residue was purified by running through a short plug of silica gel (15 to $20 \%$ ethyl acetate/hexanes) to afford $1.610 \mathrm{~g}$ ( $89 \%$ yield) of the title compound as a yellow oil.

\section{EDD5-387}

${ }^{1} \mathrm{H} \mathrm{NMR} \quad\left(400 \mathrm{MHz}, \mathrm{CDCl}_{3}\right)$

$7.61(\mathrm{~d}, J=0.8 \mathrm{~Hz}, 2 \mathrm{H}), 7.48-7.46(\mathrm{~m}, 2 \mathrm{H}), 7.43(\mathrm{t}, J=1.6 \mathrm{~Hz}, 1 \mathrm{H}), 7.35-7.31$

(m, 2 H), 6.582-6.578 (m, $1 \mathrm{H}), 5.63(\mathrm{~s}, 1 \mathrm{H}), 2.19$ (bs, $1 \mathrm{H}) \mathrm{ppm}$

${ }^{13} \mathrm{C} \mathrm{NMR} \quad\left(125 \mathrm{MHz}, \mathrm{CDCl}_{3}\right)$

$143.9,140.4,131.9,128.9,128.5,126.6,122.4,109.4,88.3,85.3,58.0 \mathrm{ppm}$ 
$\underline{\text { IR }} \quad$ (Thin Film)

$3345,1502,1019 \mathrm{~cm}^{-1}$

HRMS $\quad H R M S-E S I(m / z):[M+H]^{+}$calcd for $\mathrm{C}_{13} \mathrm{H}_{11} \mathrm{O}_{2}, 199.0759$; found, 199.0755

TLC $\quad R_{f}=0.4$ (35\% ethyl acetate/hexanes); silica gel, UV, $\mathrm{KMnO}_{4}$

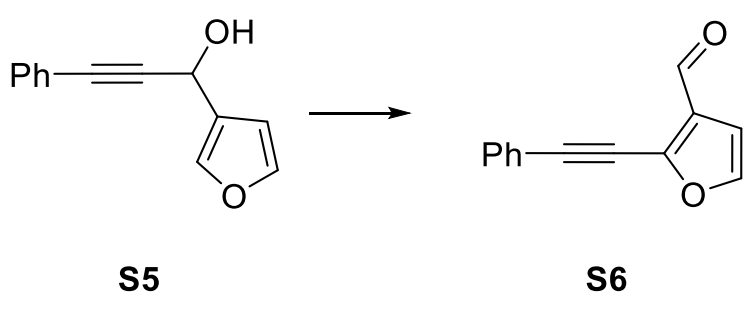

2-(phenylethynyl)furan-3-carbaldehyde (S6). This compound was prepared in a manner similar to that previously reported with some modifications. ${ }^{1}$ A flame-dried, 2-necked, $100-\mathrm{mL}$ roundbottomed flask equipped with a magnetic stir bar, nitrogen inlet adapter, and septum was charged with 1-(furan-3-yl)-3-phenylprop-2-yn-1-ol (S5) (1.082 g, $5.46 \mathrm{mmol})$, THF (21.8 mL, 0.25 M) and deionized water (5.46 mL, 1.0 M). $N$-bromosuccinimide $(972 \mathrm{mg}, 5.46 \mathrm{mmol}$ ) was added in two portions about $10 \mathrm{~min}$ apart by temporary removal of the septum. After $4 \mathrm{~h}, 1 \mathrm{M} \mathrm{HCl}(5.46$ $\mathrm{mL}$ ) was added dropwise over $1 \mathrm{~min}$. After $10 \mathrm{~min}$, the solution was transferred to a separatory funnel, extracted with diethyl ether $(3 \times 15 \mathrm{~mL})$, the combined organic layers washed with brine, dried over magnesium sulfate, filtered, and concentrated in vacuo. The crude residue was purified by silica gel flash chromatography (10\% ethyl acetate/hexanes) to afford $804 \mathrm{mg}$ (75\% yield) of the title compound as a yellow oil.

EDD5-388

${ }^{1} \mathrm{H} \mathrm{NMR} \quad\left(500 \mathrm{MHz}, \mathrm{CDCl}_{3}\right)$ 
$10.11(\mathrm{~s}, 1 \mathrm{H}), 7.60-7.58(\mathrm{~m}, 2 \mathrm{H}), 7.44-7.39(\mathrm{~m}, 4 \mathrm{H}), 6.81(\mathrm{~d}, J=1.5 \mathrm{~Hz}, 1 \mathrm{H})$ ppm

${ }^{13} \mathrm{C} \mathrm{NMR} \quad\left(100 \mathrm{MHz}, \mathrm{CDCl}_{3}\right)$

$184.8,145.2,144.8,132.0,130.1,129.5,128.8,121.0,108.1,98.9,76.8 \mathrm{ppm}$

$\underline{\text { IR }} \quad$ (Thin Film)

$2215,1680,1565,1270 \mathrm{~cm}^{-1}$

HRMS HRMS-ESI (m/z): $[\mathrm{M}+\mathrm{H}]^{+}$calcd for $\mathrm{C}_{13} \mathrm{H}_{9} \mathrm{O}_{2}, 197.0603$; found, 197.0601

TLC $\quad R_{f}=0.63$ (35\% ethyl acetate/hexanes); silica gel, UV, PAA

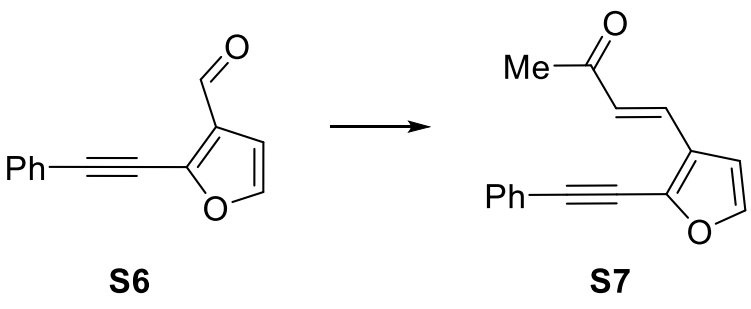

(E)-4-(2-(phenylethynyl)furan-3-yl)but-3-en-2-one (S7). This compound was prepared in a manner similar to that previously reported with some modifications. ${ }^{2}$ A flame-dried, 2-necked, 25$\mathrm{mL}$, round-bottomed flask equipped with a magnetic stir bar, nitrogen inlet adapter, and septum was charged with 2-(phenylethynyl)furan-3-carbaldehyde (S6)(693 mg, $3.48 \mathrm{mmol})$, deionized water $(2.93 \mathrm{~mL}, 1.2 \mathrm{M})$ and acetone $(2.56 \mathrm{~mL}, 34.8 \mathrm{mmol})$ via syringe. The solution was cooled in an ice-bath, and 33\% $\mathrm{NaOH}(0.17 \mathrm{~mL}, 1.39 \mathrm{mmol})$ was added dropwise over $1 \mathrm{~min}$. After allowing to warm to room temperature over $4 \mathrm{~h}$, TLC showed complete consumption of starting material. $10 \%$ Sulfuric acid was added until the solution reached a $\mathrm{pH} 4$ as determined using $\mathrm{pH}$ paper. The resulting solution was transferred to a separatory funnel and the organic layer separated. The 
aqueous layer was extracted with $\mathrm{CHCl}_{3}(5 \times 5 \mathrm{~mL})$, the combined organic layers were dried over magnesium sulfate, filtered, and concentrated in vacuo. The crude residue was purified by silica gel flash chromatography (10-15\% ethyl acetate/hexanes) to afford $634 \mathrm{mg}$ (77\% yield) of the title compound as a yellow solid.

EDD5-399

$\underline{{ }^{1} \mathrm{H} \mathrm{NMR}} \quad\left(500 \mathrm{MHz}, \mathrm{CDCl}_{3}\right)$

7.59-7.57 (m, $2 \mathrm{H}), 7.54(\mathrm{~d}, J=16.0 \mathrm{~Hz}, 1 \mathrm{H}), 7.42-7.38(\mathrm{~m}, 4 \mathrm{H}), 6.63(\mathrm{~d}, J=2.0$

$\mathrm{Hz}, 1 \mathrm{H}), 6.56(\mathrm{~d}, J=16.0,1 \mathrm{H}), 2.38(\mathrm{~s}, 3 \mathrm{H}) \mathrm{ppm}$

${ }^{13} \mathrm{C} \mathrm{NMR} \quad\left(100 \mathrm{MHz}, \mathrm{CDCl}_{3}\right)$

$198.3,144.7,139.0,133.0,131.8,129.6,128.7,128.2,126.3,121.7,108.6,99.2$, $77.9,27.6, \mathrm{ppm}$

$\underline{\text { IR }} \quad$ (Thin Film)

$2981,1667,1608 \mathrm{~cm}^{-1}$

HRMS $\quad$ HRMS-ESI (m/z): $[\mathrm{M}+\mathrm{H}]^{+}$calcd for $\mathrm{C}_{16} \mathrm{H}_{13} \mathrm{O}_{2}, 237.0916$; found, 237.0911

TLC $\quad R_{f}=0.48$ (35\% ethyl acetate/hexanes); silica gel, UV, PAA

M.P. $\quad 65.9-67.7^{\circ} \mathrm{C}$ 


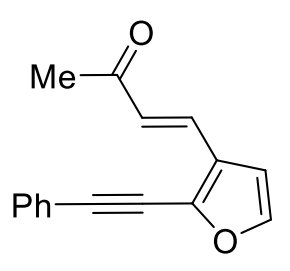

S7

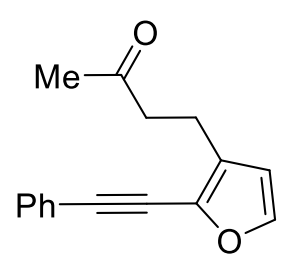

S8

4-(2-(phenylethynyl)furan-3-yl)butan-2-one (S8). This compound was prepared in a manner similar to that previously reported with some modifications. ${ }^{3,4}$ A flame-dried, 2-necked, 100-mL, round-bottomed flask equipped with a magnetic stir bar, condenser with septum and nitrogen inlet needle, and septum in the second arm was charged with $(E)-4-(2-($ phenylethynyl)furan-3-yl)but3-en-2-one (S7) (632 mg, $2.67 \mathrm{mmol})$ in toluene (26.7 mL, 0.1M) via syringe. Diethyl 2,6dimethyl-1,4-dihydropyridine-3,5-dicarboxylate (Hantzsch ester) (2.029 g, $8.01 \mathrm{mmol}$ ), then silica gel (4.005 g, $1.5 \mathrm{~g} / \mathrm{mmol})$ were added by temporary removal of the septum. The flask was lowered into a preheated oil bath $\left(110^{\circ} \mathrm{C}\right)$ and maintained for $27 \mathrm{~h}$, at which time the TLC showed complete consumption of starting material. The mixture was filtered using a glass funnel fitted with filter paper eluting with hexanes $(80 \mathrm{~mL})$. The solid was rinsed with additional hexanes $(75 \mathrm{~mL})$ and collected in a separate flask. The solid was further rinsed with ethyl acetate $(50 \mathrm{~mL})$ and collected in a third flask. The solutions in all three flasks were examined by TLC. Because all three solutions showed product and Hantzsch ester, all were combined, concentrated in vacuo, dissolved in ethyl acetate $(25 \mathrm{~mL})$, transferred to a separatory funnel, washed with $2 \mathrm{~N} \mathrm{HCl}(5 \times 10 \mathrm{~mL})$, brine, dried over magnesium sulfate, filtered and concentrated in vacuo. The crude residue was purified by silica gel flash chromatography (5-15\% ethyl acetate/hexanes) to afford $453 \mathrm{mg}$ ( $72 \%$ yield) of the title compound as a yellow oil.

\section{EDD5-401}

${ }^{1} \mathrm{H} \mathrm{NMR} \quad\left(400 \mathrm{MHz}, \mathrm{CDCl}_{3}\right)$ 
7.53-7.50 (m, 2 H), 7.37-7.33 (m, 3 H), $7.33(\mathrm{~d}, J=1.6 \mathrm{~Hz}, 1 \mathrm{H}), 6.32(\mathrm{~d}, J=1.6$

Hz, $1 \mathrm{H}), 2.86-2.75$ (m, $4 \mathrm{H}), 2.17$ (s, $3 \mathrm{H}) \mathrm{ppm}$

${ }^{13} \mathrm{C} \mathrm{NMR} \quad\left(100 \mathrm{MHz}, \mathrm{CDCl}_{3}\right)$

$207.8,143.4,134.3,131.5,129.5,128.8,128.6,122.6,112.2,96.1,78.8,43.6$,

$30.1,19.8 \mathrm{ppm}$

$\underline{\mathrm{IR}} \quad$ (Thin Film)

$2971,1714,1497 \mathrm{~cm}^{-1}$

HRMS $\quad$ HRMS-ESI (m/z): $[\mathrm{M}+\mathrm{H}]^{+}$calcd for $\mathrm{C}_{16} \mathrm{H}_{15} \mathrm{O}_{2}, 239.1072$; found, 239.1066

$\underline{\text { TLC }} \quad R_{f}=0.5$ (35\% ethyl acetate/hexanes); silica gel, UV, PAA

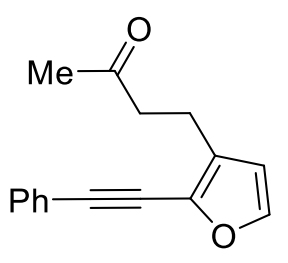

S8

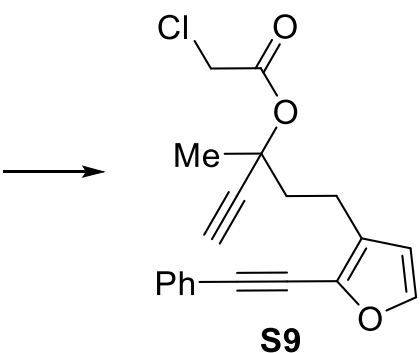

S9

3-methyl-5-(2-(phenylethynyl)furan-3-yl)pent-1-yn-3-yl 2-chloroacetate (S9). This compound was prepared in a manner similar to that previously reported with some modifications. ${ }^{5}$ A flamedried, 2-necked, 50-mL, round bottomed flask equipped with a magnetic stir bar, nitrogen inlet adapter, and septum was charged with 4-(2-(phenylethynyl)furan-3-yl)butan-2-one (S8) (350 mg, $1.47 \mathrm{mmol})$ and THF $(4.9 \mathrm{~mL})$. The solution was cooled by placing the flask in an ice-bath and ethynylmagnesium bromide ( $4.9 \mathrm{~mL}$ of a $0.5 \mathrm{M}$ solution in THF, $3.68 \mathrm{mmol}, 2.5$ equiv), was added dropwise via syringe over $5 \mathrm{~min}$. The solution was allowed to warm to rt over $60 \mathrm{~min}$. The flask 
was placed in an ice-bath and ethynylmagnesium bromide $(1.4 \mathrm{~mL}$ of a $0.5 \mathrm{M}$ solution in THF, $0.709 \mathrm{mmol}, 1$ equiv) was added via syringe over $3 \mathrm{~min}$. After warming to rt over $60 \mathrm{~min}$, TLC showed complete consumption of starting material. The flask was placed in an ice-bath and chloroacetyl chloride ( $0.35 \mathrm{~mL}, 4.41 \mathrm{mmol}, 3.0$ equiv) was added dropwise via syringe over $2 \mathrm{~min}$. After $2 \mathrm{~h}$, TLC showed complete consumption of the propargyl alcohol intermediate. Diethyl ether $(5 \mathrm{~mL})$ and deionized water $(5 \mathrm{~mL})$ were added to the flask, the mixture was transferred to a separatory funnel, and the organic layer was separated. The aqueous layer was extracted with diethyl ether ( $3 \times 15 \mathrm{~mL}$ ), the organic layers were combined, washed with brine, dried over magnesium sulfate, filtered, and concentrated in vacuo. The crude residue was purified by silica gel flash chromatography (10 to $30 \%$ ethyl acetate/hexanes) to give $312 \mathrm{mg}(62 \%)$ of the title compound as a clear liquid. Product contains a small amount of uncharacterized byproduct.

\section{EDD6-409}

$\underline{{ }^{1} \mathrm{H} \mathrm{NMR}} \quad\left(500 \mathrm{MHz}, \mathrm{CDCl}_{3}\right)$

7.54-7.51 (m, $2 \mathrm{H}), 7.36-7.35(\mathrm{~m}, 4 \mathrm{H}), 6.35(\mathrm{~d}, J=2.0,1 \mathrm{H}), 4.0(\mathrm{~s}, 2 \mathrm{H}), 2.82-$

2.79 (m, 2 H), 2.65 (s, $1 \mathrm{H}), 2.34-2.28$ (m, $1 \mathrm{H}), 2.19-2.13$ (m, $1 \mathrm{H}), 1.80(\mathrm{~s}, 3 \mathrm{H})$ ppm Impurities at: 4.13 (s, $0.15 \mathrm{H}), 3.98(\mathrm{~s}, 0.14 \mathrm{H})$

${ }^{13} \mathrm{C} \mathrm{NMR} \quad\left(125 \mathrm{MHz}, \mathrm{CDCl}_{3}\right)$

$165.5,143.5,134.5,131.4,129.6,128.8,128.6,122.7,112.1,96.2,82.5,78.8$, $74.9,41.5,41.3,26.5,20.5 \mathrm{ppm}$

IR (Thin Film)

$2978,1761,1743,1286 \mathrm{~cm}^{-1}$ 
HRMS HRMS-ESI (m/z): $[\mathrm{M}+\mathrm{H}]^{+}$calcd for $\mathrm{C}_{20} \mathrm{H}_{18} \mathrm{O}_{3} \mathrm{Cl}$, 341.0944; found, 341.0947

TLC $\quad R_{f}=0.56$ (35\% ethyl acetate/hexanes); silica gel, UV, PAA

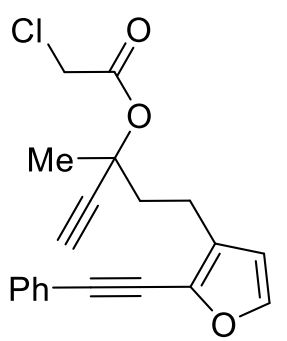

S9

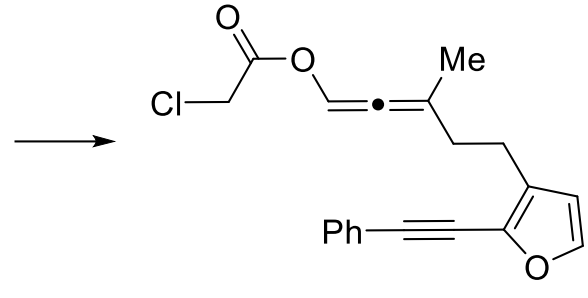

S10

3-methyl-5-(2-(phenylethynyl)furan-3-yl)penta-1,2-dien-1-yl 2-chloroacetate (S10). Follows

General Procedure B: Rhodium(II) trifluoroacetate dimer (29.6 mg, 0.012 mmol), 3-methyl-5-(2(phenylethynyl)furan-3-yl)pent-1-yn-3-yl 2-chloroacetate (S9)(307 mg, $0.23 \mathrm{mmol})$, toluene (4.5 $\mathrm{mL}, 0.2 \mathrm{M})$ The reaction was stirred for $1 \mathrm{~h}$ in an oil bath $\left(50^{\circ} \mathrm{C}\right)$ and the crude residue immediately loaded onto a packed column and purified by silica gel flash chromatography (2\% ethyl acetate/hexanes) to give $224 \mathrm{mg}$ (73\%) of the title compound as a clear liquid.

\section{EDD6-410}

${ }^{1} \mathrm{H} \mathrm{NMR} \quad\left(400 \mathrm{MHz}, \mathrm{CDCl}_{3}\right)$

7.52-7.50 (m, $2 \mathrm{H}), 7.36-7.33(\mathrm{~m}, 5 \mathrm{H}), 6.33(\mathrm{~d}, J=1.6 \mathrm{~Hz}, 1 \mathrm{H}), 4.08(\mathrm{~s}, 2 \mathrm{H}), 2.73$

(t, $J=7.6 \mathrm{~Hz}, 2 \mathrm{H}), 2.47-2.37(\mathrm{~m}, 2 \mathrm{H}), 1.89(\mathrm{~d}, J=1.2 \mathrm{~Hz}, 3 \mathrm{H}) \mathrm{ppm}$

${ }^{13} \mathrm{C} \mathrm{NMR} \quad\left(100 \mathrm{MHz}, \mathrm{CDCl}_{3}\right)$

$189.9,165.3,143.4,134.5,131.4,129.8,128.7,128.6,122.7,116.5,112.0,110.8$, $96.0,79.0,40.8,35.0,23.4,20.7 \mathrm{ppm}$ 
$\underline{\text { IR }} \quad$ (Thin Film)

$2920,1980,1766,1744 \mathrm{~cm}^{-1}$

HRMS $\quad$ HRMS-ESI (m/z): $[\mathrm{M}+\mathrm{H}]^{+}$calcd for $\mathrm{C}_{20} \mathrm{H}_{18} \mathrm{O}_{3} \mathrm{Cl}$, 341.0944; found, 341.0940

TLC $\quad R_{f}=0.64$ (35\% ethyl acetate/hexanes); silica gel, UV, PAA

\section{Racemic APKR}
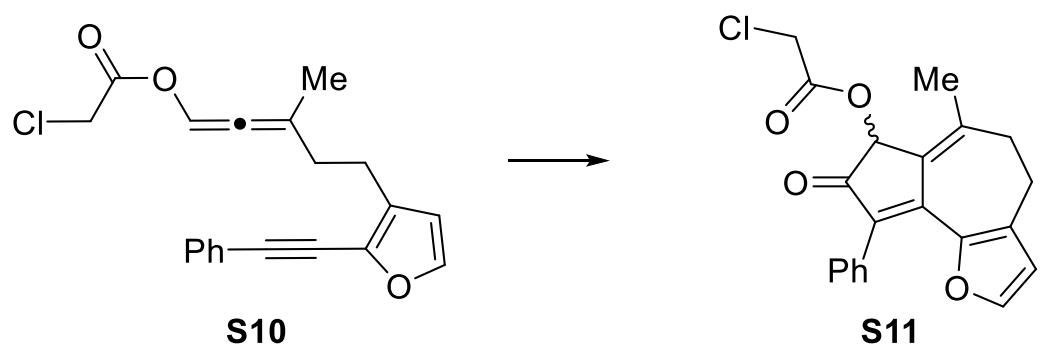

6-methyl-8-oxo-9-phenyl-4,5,7,8-tetrahydroazuleno[4,5-b]furan-7-yl 2-chloroacetate (S11).

Follows General Procedure C: Bis(1,5-cyclooctadiene)rhodium(I) tetrafluoroborate (2.4 mg, $0.0059 \mathrm{mmol})$, triphenyl phosphine (2.3 $\mathrm{mg}, 0.0089 \mathrm{mmol})$, carbon monoxide (100\%), 3-methyl5-(2-(phenylethynyl)furan-3-yl)penta-1,2-dien-1-yl 2-chloroacetate (S10) $(20 \mathrm{mg}, 0.059 \mathrm{mmol})$, DCE $(3.0 \mathrm{~mL}, 0.02 \mathrm{M})$. The reaction was stirred for $46 \mathrm{~h}$ in an oil bath $\left(70{ }^{\circ} \mathrm{C}\right)$. The crude residue was purified via silica gel flash chromatography (5\% to $20 \%$ ethyl acetate/hexanes) to give the title compound (13 mg, 59\%) as a yellow solid.

\section{EDD5-404}

${ }^{1} \mathrm{H} \mathrm{NMR} \quad\left(400 \mathrm{MHz}, \mathrm{CDCl}_{3}\right)$ 
7.38-7.28 (m, 5 H), 7.21 (s, $1 \mathrm{H}), 6.33$ (s, $1 \mathrm{H}), 5.99$ (s, $1 \mathrm{H}), 4.16$ (s, $2 \mathrm{H}), 2.94-$ 2.89 (m, $1 \mathrm{H}), 2.83-2.77(\mathrm{~m}, 1 \mathrm{H}), 2.65-2.63(\mathrm{~m}, 2 \mathrm{H}), 2.02$ (s, $3 \mathrm{H}) \mathrm{ppm}$

${ }^{13} \mathrm{C} \mathrm{NMR} \quad\left(100 \mathrm{MHz}, \mathrm{CDCl}_{3}\right)$

197.0, 166.5, 149.4, 146.1, 145.3, 142.1, 133.7, 132.1, 130.1, 128.0, 127.7, 127.0, $113.8,73.8,40.8,34.8,29.8,25.2,23.7 \mathrm{ppm}$

$\underline{\text { IR }} \quad$ (Thin Film)

$2915,1756,1687 \mathrm{~cm}^{-1}$

$\underline{\text { HRMS }}$ HRMS-ESI (m/z): $[\mathrm{M}+\mathrm{H}]^{+}$calcd for $\mathrm{C}_{21} \mathrm{H}_{18} \mathrm{O}_{4} \mathrm{Cl}, 369.0894$; found, 369.0887

TLC $\quad R_{f}=0.38$ (35\% ethyl acetate/hexanes); silica gel, UV, PAA

Waters 600 HPLC, UV/PDA detector, 332 nm, Daicel CHIRALCEL-OD, 250 X 4.6 mm column, $1.5 \%$ iPrOH/hexanes, Flow rate: $1 \mathrm{~mL} / \mathrm{min}$

\begin{tabular}{|c|c|c|}
\hline Peak & Retention Time (min) & Peak area (\%) \\
\hline Peak 1 & 66.84 & 50.21 \\
\hline Peak 2 & 96.18 & 49.79 \\
\hline
\end{tabular}




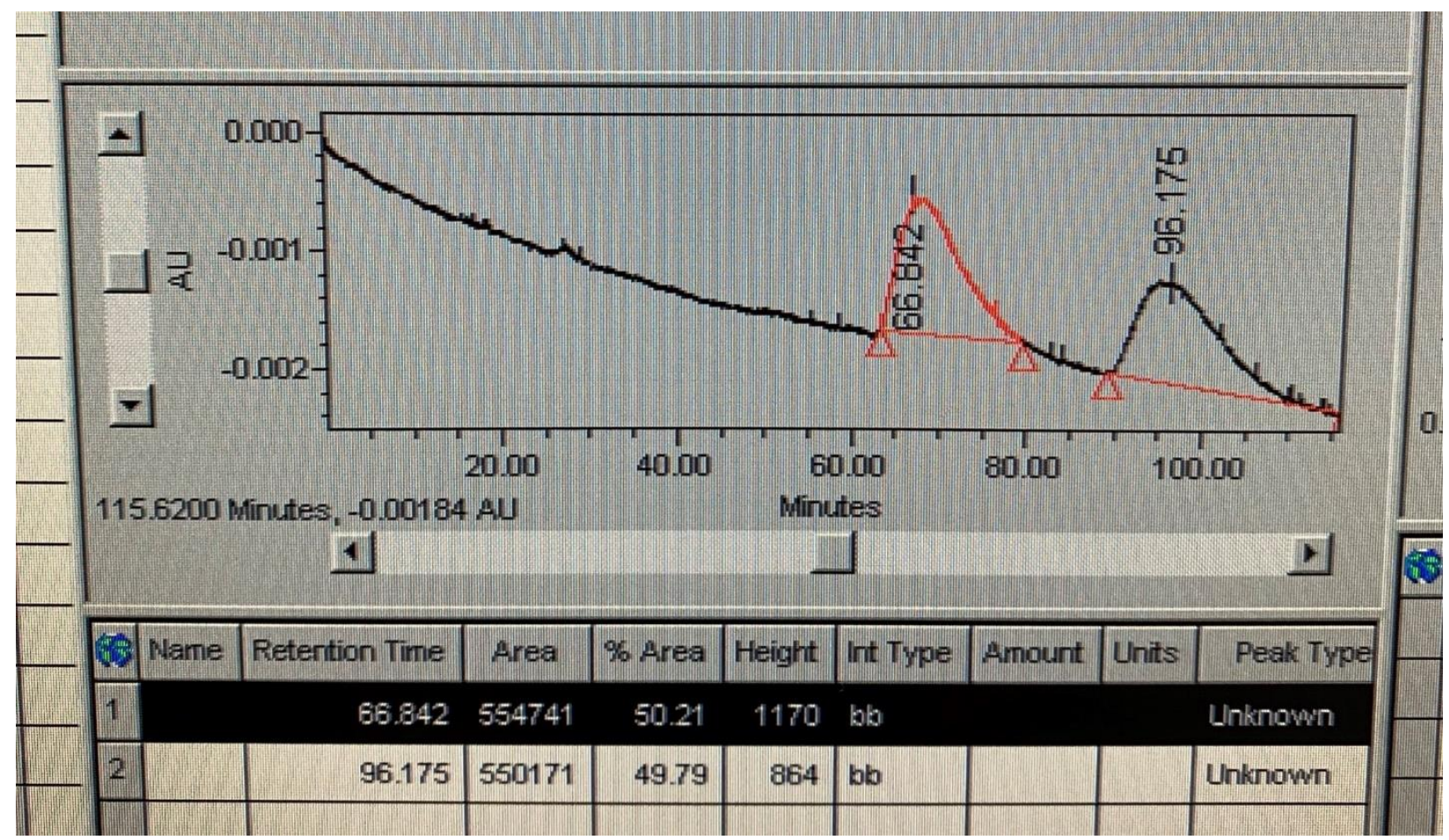

\section{Asymmetric APKR}

\section{Run 1:}

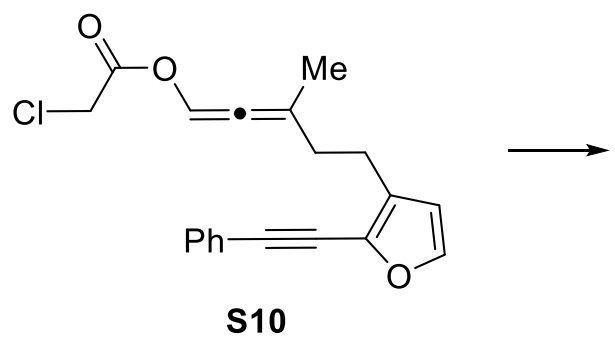

(R)-6-methyl-8-oxo-9-phenyl-4,5,7,8-tetrahydroazuleno[4,5-b]furan-7-yl

2-chloroacetate

(S11). Run 1: Follows General Procedure E: Bis(1,5-cyclooctadiene)rhodium(I) tetrafluoroborate (2.4 mg, $0.0059 \mathrm{mmol})$, (S)-MonoPhos-alkene (8) (3.7 mg, $0.0089 \mathrm{mmol}$ ), carbon monoxide (100\%), 3-methyl-5-(2-(phenylethynyl)furan-3-yl)penta-1,2-dien-1-yl 2-chloroacetate (S10) (20 $\mathrm{mg}, 0.059 \mathrm{mmol})$, DCE $(5.9 \mathrm{~mL}, 0.01 \mathrm{M})$. The reaction was stirred for $70.5 \mathrm{~h}$ in an oil bath $(70$ ${ }^{\circ} \mathrm{C}$ ). The crude residue was purified via silica gel flash chromatography (5\% to $20 \%$ ethyl 
acetate/hexanes) to give $11 \mathrm{mg}(50 \%)$ of the title compound in a 98:5:1.5 er as a yellow solid and $2 \mathrm{mg}(13 \%)$.

\section{EDD6-407}

Waters 600 HPLC, UV/PDA detector, 332 nm, Daicel CHIRALCEL-OD, 250 X 4.6 mm column, $1.5 \%$ iPrOH/hexanes, Flow rate: $1 \mathrm{~mL} / \mathrm{min}$

\begin{tabular}{|c|c|c|}
\hline Peak & Retention Time (min) & Peak area (\%) \\
\hline Peak 1 & 72.71 & 1.50 \\
\hline Peak 2 & 92.50 & 98.50 \\
\hline
\end{tabular}

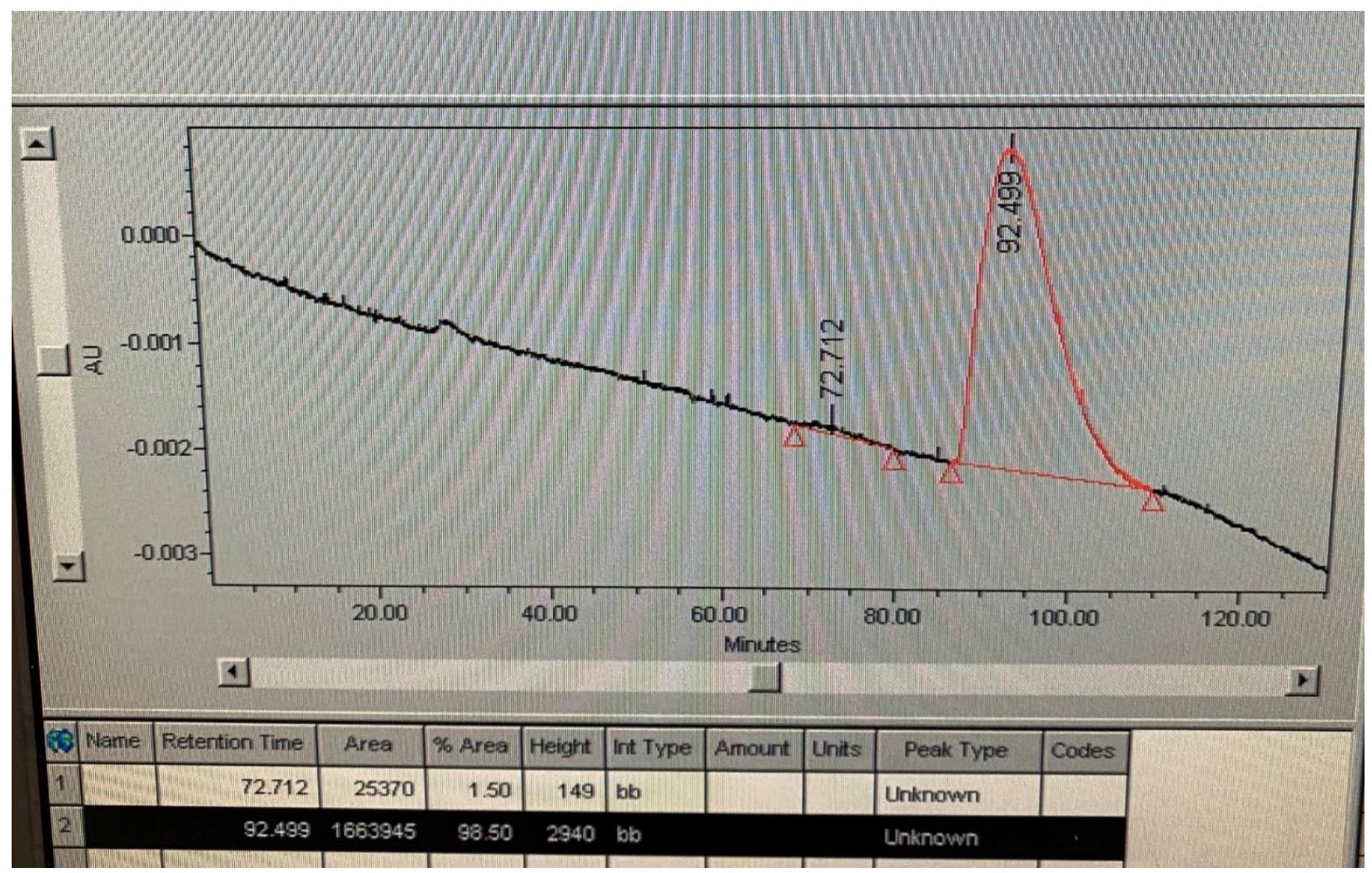

Run 2: Follows General Procedure E: Bis(1,5-cyclooctadiene)rhodium(I) tetrafluoroborate (3.0 $\mathrm{mg}, 0.0073 \mathrm{mmol}$ ), (S)-MonoPhos-alkene (8) (4.5 mg, $0.011 \mathrm{mmol})$, carbon monoxide (100\%), 3- 
methyl-5-(2-(phenylethynyl)furan-3-yl)penta-1,2-dien-1-yl 2-chloroacetate (S10) (25 mg, 0.073 mmol), DCE $(7.3 \mathrm{~mL}, 0.01 \mathrm{M})$. The reaction was stirred for $69 \mathrm{~h}$ in an oil bath $\left(70{ }^{\circ} \mathrm{C}\right)$. The crude residue was purified via silica gel flash chromatography (5\% to 20\% ethyl acetate/hexanes) to give $11 \mathrm{mg}(41 \%)$ of the title compound in 99:1 er as a yellow solid and $3 \mathrm{mg}(16 \%)$ of aldehyde byproduct.

$[\alpha]^{25} \mathrm{D}=+16.53\left(\mathrm{c} .=0.45, \mathrm{CHCl}_{3}\right)$

\section{EDD6-411}

Waters 600 HPLC, UV/PDA detector, 332 nm, Daicel CHIRALCEL-OD, 250 X 4.6 mm column, $1.5 \%$ iPrOH/hexanes, Flow rate: $1 \mathrm{~mL} / \mathrm{min}$

\begin{tabular}{|c|c|c|}
\hline Peak & Retention Time (min) & Peak area $(\%)$ \\
\hline Peak 1 & 70.39 & 1.10 \\
\hline Peak 2 & 98.13 & 98.90 \\
\hline
\end{tabular}

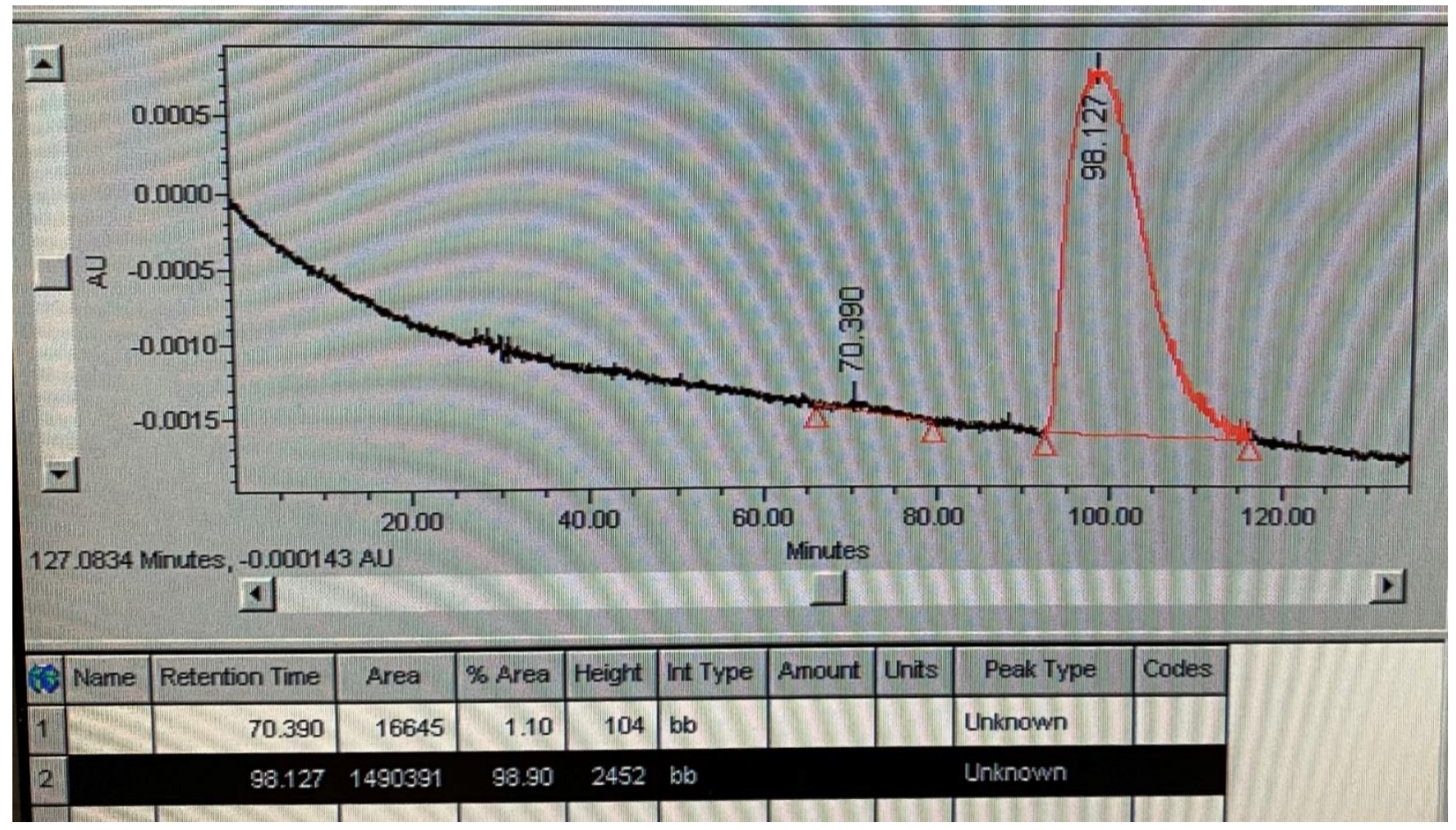




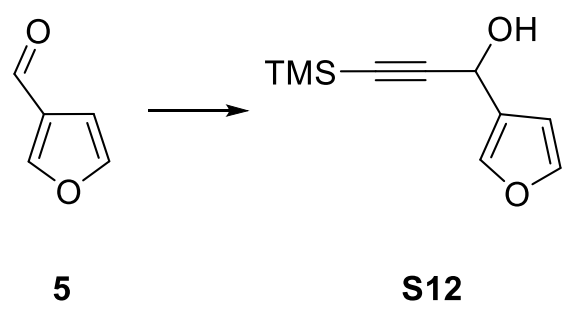

1-(furan-3-yl)-3-(trimethylsilyl)prop-2-yn-1-ol (S12). This compound was prepared in a manner similar to that previously reported with some modifications. ${ }^{7}$ A flame-dried, 2-necked, 25-mL, round bottomed flask equipped with a magnetic stir bar, nitrogen inlet adapter, and septum was charged with TMS-acetylene $(0.58 \mathrm{~mL}, 4.16 \mathrm{mmol})$ and THF $(3.48 \mathrm{~mL}, 1.0 \mathrm{M})$. The flask was cooled to $-78{ }^{\circ} \mathrm{C}$ by placing in a dry ice/acetone bath. $n$-BuLi $(2.6 \mathrm{~mL}$ of a $1.6 \mathrm{M}$ solution in hexanes, $4.16 \mathrm{mmol}$ ) was added dropwise over $3 \mathrm{~min}$ and allowed to stir for $1 \mathrm{~h}$. 3-Furfural (5) $(0.3 \mathrm{~mL}, 3.47 \mathrm{mmol})$ in THF $(1.72 \mathrm{~mL}, 2.0 \mathrm{M})$ was added dropwise over $3 \mathrm{~min}$. After $2 \mathrm{~h}$, TLC showed complete consumption of starting material. Aqueous saturated ammonium chloride (3 mL) was added and the solution was transferred to a separatory funnel and the layers separated. The aqueous layer was extracted with diethyl ether $(3 \times 3 \mathrm{~mL})$, the combined organic layers washed with water $(1 \times 3 \mathrm{~mL})$, brine $(1 \times 3 \mathrm{~mL})$, dried over magnesium sulfate, filtered, and concentrated in vacuo. The crude residue was purified by silica gel flash chromatography (5-15\% ethyl acetate/hexanes) to afford $546 \mathrm{mg}$ (81\% yield) of the title compound as a clear oil.

\section{EDD6-412}

$\underline{{ }^{1} \mathrm{H} \mathrm{NMR}} \quad\left(400 \mathrm{MHz}, \mathrm{CDCl}_{3}\right)$

$7.52(\mathrm{~d}, J=0.8 \mathrm{~Hz}, 1 \mathrm{H}), 7.40(\mathrm{t}, J=1.6 \mathrm{~Hz}, 1 \mathrm{H}), 6.50(\mathrm{~d}, J=0.8 \mathrm{~Hz}, 1 \mathrm{H}), 5.38$

(s, $1 \mathrm{H}), 2.10(\mathrm{bs}, 1 \mathrm{H}), 0.20(\mathrm{~s}, 9 \mathrm{H}) \mathrm{ppm}$ 
${ }^{13} \mathrm{C} \mathrm{NMR} \quad\left(100 \mathrm{MHz}, \mathrm{CDCl}_{3}\right)$

$143.8,140.5,126.3,109.4,104.5,90.2,57.8,0.1 \mathrm{ppm}$

$\underline{\mathrm{IR}} \quad$ (Thin Film)

$3352,2176 \mathrm{~cm}^{-1}$

HRMS HRMS-ESI (m/z): $[\mathrm{M}+\mathrm{H}]^{+}$calcd for $\mathrm{C}_{10} \mathrm{H}_{15} \mathrm{O}_{2} \mathrm{Si}$, 195.0836; found, 195.0836

TLC $\quad R_{f}=0.43$ (35\% ethyl acetate/hexanes); silica gel, UV, $p$-anisaldehyde

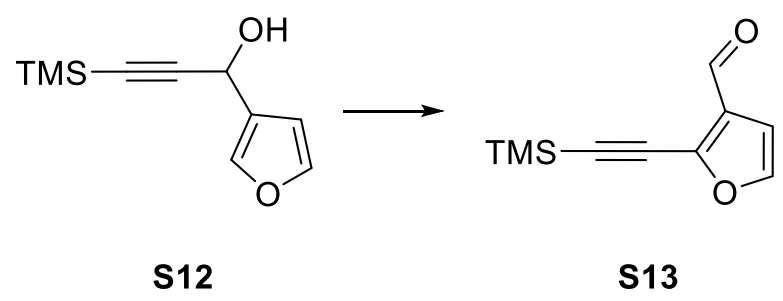

2-((trimethylsilyl)ethynyl)furan-3-carbaldehyde (S13). This compound was prepared in a manner similar to that previously reported with some modifications. ${ }^{1}$ A flame-dried, 2-necked, 50$\mathrm{mL}$ round-bottomed flask equipped with a magnetic stir bar, nitrogen inlet adapter, and septum was charged with 1-(furan-3-yl)-3-(trimethylsilyl)prop-2-yn-1-ol (S12)(536 mg, 2.76 mmol), THF $(11.0 \mathrm{~mL}, 0.25 \mathrm{M})$ and deionized water $(2.80 \mathrm{~mL}, 1.0 \mathrm{M}) . N$-bromosuccinimide $(491 \mathrm{mg}, 2.76$ mmol) was added in two portions about 10 min apart by temporary removal of the septum. After $3 \mathrm{~h}, 1 \mathrm{M} \mathrm{HCl}(2.80 \mathrm{~mL})$ was added dropwise over $2 \mathrm{~min}$. After $10 \mathrm{~min}$, the solution was transferred to a separatory funnel, extracted with diethyl ether $(3 \times 5 \mathrm{~mL})$, the combined organic layers washed with brine, dried over magnesium sulfate, filtered, and concentrated in vacuo. The crude residue was purified by silica gel flash chromatography (5\% ethyl acetate/hexanes) to afford $408 \mathrm{mg}$ (77\% yield) of the title compound as a light-yellow oil. 
EDD6-413

${ }^{1} \mathrm{H} \mathrm{NMR} \quad\left(400 \mathrm{MHz}, \mathrm{CDCl}_{3}\right)$

$10.01(\mathrm{~s}, 1 \mathrm{H}), 7.36(\mathrm{~d}, J=2.0 \mathrm{~Hz}, 1 \mathrm{H}), 6.75(\mathrm{~d}, J=2.0 \mathrm{~Hz}, 1 \mathrm{H}), 0.30(\mathrm{~s}, 9 \mathrm{H})$

ppm

${ }^{13} \mathrm{C} \mathrm{NMR} \quad\left(100 \mathrm{MHz}, \mathrm{CDCl}_{3}\right)$

$184.8,144.9,144.5,130.1,107.8,106.8,91.0,0.4 \mathrm{ppm}$

$\underline{\text { IR }} \quad$ (Thin Film)

$2161,1686 \mathrm{~cm}^{-1}$

$\underline{\text { HRMS }}$ HRMS-ESI (m/z): $[\mathrm{M}+\mathrm{H}]^{+}$calcd for $\mathrm{C}_{10} \mathrm{H}_{13} \mathrm{O}_{2} \mathrm{Si}, 193.0679$; found, 193.0683

$\underline{\text { TLC }} \quad R_{f}=0.68(35 \%$ ethyl acetate/hexanes $)$; silica gel, UV, $p$-anisaldehyde

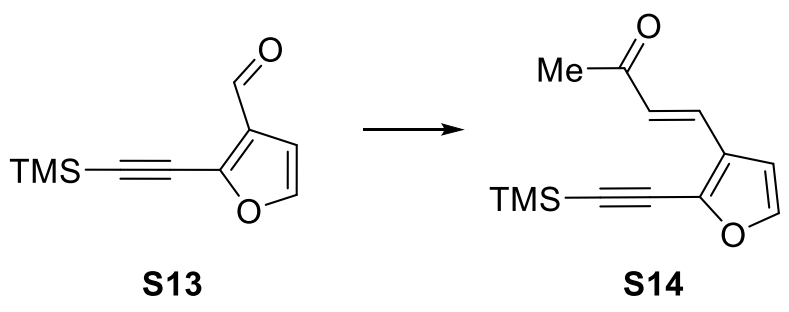

(E)-4-(2-((trimethylsilyl)ethynyl)furan-3-yl)but-3-en-2-one (S14). This compound was prepared in a manner similar to that previously reported with some modifications. ${ }^{8}$ A flame-dried, 2-necked, 10-mL round-bottomed flask equipped with a magnetic stir bar, nitrogen inlet adapter, and septum was charged with 2-((trimethylsilyl)ethynyl)furan-3-carbaldehyde (S13)(204 mg, 1.06 mmol), 1,4-dioxane (1.06 mL, 1.0 M), water (29 $\mu \mathrm{L}, 1.59)$, dimethyl-2-oxo-propyl phosphonate $(0.18 \mathrm{~mL}, 1.33 \mathrm{mmol})$, and cesium carbonate $(518 \mathrm{mg}, 1.25 \mathrm{mmol})$. After $1 \mathrm{~h}$ at room temperature, TLC showed complete consumption of starting material. The solution was filtered through a short 
plug of celite, eluting with DCM $(5 \times 1 \mathrm{~mL})$, and concentrated in vacuo. The crude residue was purified by silica gel flash chromatography (5-20\% ethyl acetate/ hexanes) to afford $189 \mathrm{mg}(77 \%)$ of the title compound as a white solid.

\section{EDD6-414}

M. P. $\quad 53.1-54.1{ }^{\circ} \mathrm{C}$

${ }^{1} \mathrm{H} \mathrm{NMR} \quad\left(400 \mathrm{MHz}, \mathrm{CDCl}_{3}\right)$

$7.44(\mathrm{~d}, J=16.4 \mathrm{~Hz}, 1 \mathrm{H}), 7.34(\mathrm{~d}, J=1.6 \mathrm{~Hz}, 1 \mathrm{H}), 6.56(\mathrm{~d}, J=2.0 \mathrm{~Hz}, 1 \mathrm{H})$,

$6.53(\mathrm{~d}, J=16.0 \mathrm{~Hz}, 1 \mathrm{H}), 2.36(\mathrm{~s}, 3 \mathrm{H}), 0.30(\mathrm{~s}, 9 \mathrm{H}) \mathrm{ppm}$

${ }^{13} \mathrm{C} \mathrm{NMR} \quad\left(100 \mathrm{MHz}, \mathrm{CDCl}_{3}\right)$

$198.3,144.4,138.6,132.9,128.3,126.9,108.4,106.5,92.5,27.4,0.2 \mathrm{ppm}$

$\underline{\text { IR }} \quad$ (Thin Film)

$2154,1693,1252 \mathrm{~cm}^{-1}$

HRMS HRMS-ESI (m/z): $[\mathrm{M}+\mathrm{H}]^{+}$calcd for $\mathrm{C}_{13} \mathrm{H}_{17} \mathrm{O}_{2} \mathrm{Si}$, 233.0992; found, 233.0994

TLC $\quad R_{f}=0.40(35 \%$ ethyl acetate/hexanes); silica gel, UV, $p$-anisaldehyde

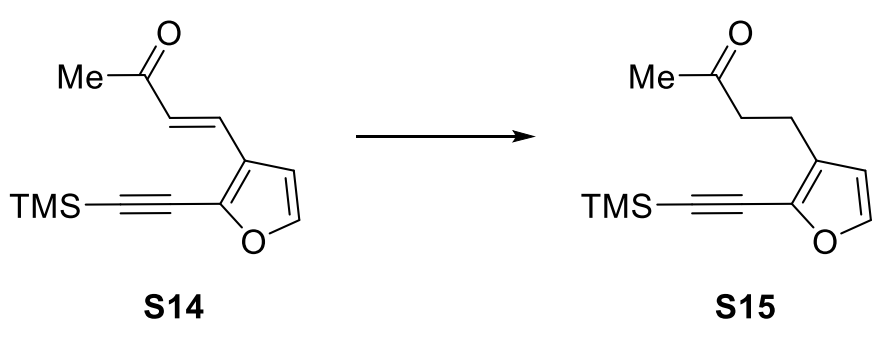


4-(2-((trimethylsilyl)ethynyl)furan-3-yl)butan-2-one (S15). A flame-dried, 2-necked, 50-mL round-bottomed flask equipped with a magnetic stir bar, nitrogen inlet adapter, and septum was charged with (E)-4-(2-((trimethylsilyl)ethynyl)furan-3-yl)but-3-en-2-one (S14) (358 mg, 1.54 $\mathrm{mmol})$, toluene $(15.4 \mathrm{~mL}, 0.01 \mathrm{M})$, Hantzsch ester (14)(1.170g, $4.62 \mathrm{mmol})$, and silica gel (2.31 g) and lowered into a preheated $110^{\circ} \mathrm{C}$ oil bath. After $48.5 \mathrm{~h}$, an NMR aliquot showed complete consumption of starting material. The solution was filtered through filter paper, eluting with ethyl acetate $(25 \mathrm{~mL})$, and concentrated in vacuo. The crude material was dissolved in ethyl acetate, transferred to a separatory funnel, extracted with $2 \mathrm{~N} \mathrm{HCl}(5 \times 5 \mathrm{~mL})$, and the organic layer was washed with brine ( $1 \times 5 \mathrm{~mL})$, dried over magnesium sulfate, filtered, and concentrated in vacuo. The crude residue was purified by silica gel flash chromatography (5-20\% ethyl acetate/ hexanes) to afford $199 \mathrm{mg}(55 \%)$ of the title compound as a light-yellow oil.

\section{EDD6-416}

$\underline{{ }^{1} \mathrm{H} \mathrm{NMR}} \quad\left(400 \mathrm{MHz}, \mathrm{CDCl}_{3}\right)$

$7.24(\mathrm{~d}, J=1.6 \mathrm{~Hz}, 1 \mathrm{H}), 6.25(\mathrm{~d}, J=2.0 \mathrm{~Hz}, 1 \mathrm{H}), 2.79-2.71(\mathrm{~m}, 4 \mathrm{H}), 2.16(\mathrm{~s}, 3$

H), 0.25 (s, $9 \mathrm{H}) \mathrm{ppm}$

${ }^{13} \mathrm{C} \mathrm{NMR} \quad\left(100 \mathrm{MHz}, \mathrm{CDCl}_{3}\right)$

$207.8,143.2,134.4,130.1,112.0,102.5,93.7,43.4,30.0,19.7,0.1 \mathrm{ppm}$

$\underline{\text { IR }} \quad$ (Thin Film)

$2152,1719 \mathrm{~cm}^{-1}$

HRMS HRMS-ESI (m/z): $[\mathrm{M}+\mathrm{H}]^{+}$calcd for $\mathrm{C}_{13} \mathrm{H}_{19} \mathrm{O}_{2} \mathrm{Si}$, 235.1154; found, 235.1140

$\underline{\text { TLC }} \quad R_{f}=0.39$ (35\% ethyl acetate/hexanes); silica gel, UV, $p$-anisaldehyde 

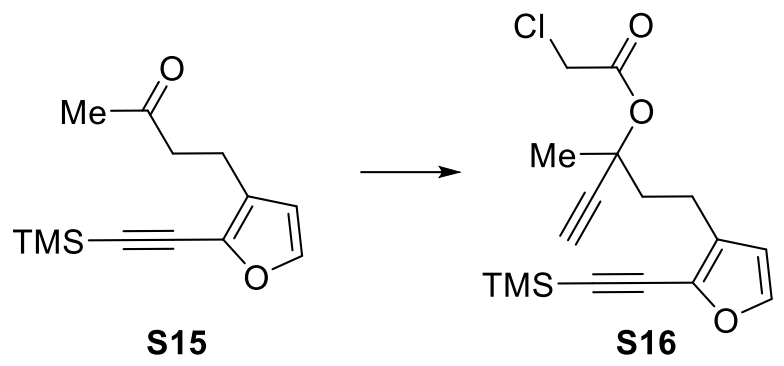

3-methyl-5-(2-((trimethylsilyl)ethynyl)furan-3-yl)pent-1-yn-3-yl 2-chloroacetate (S16). This compound was prepared in a manner similar to that previously reported with some modifications. ${ }^{5}$ A flame-dried, 2-necked, 25-mL, round bottomed flask equipped with a magnetic stir bar, nitrogen inlet adapter, and septum was charged with 4-(2-((trimethylsilyl)ethynyl)furan-3-yl)butan-2-one (S15) (187 mg, $0.798 \mathrm{mmol})$ and THF $(2.7 \mathrm{~mL})$. The solution was cooled by placing the flask in an ice-bath and ethynylmagnesium bromide $(4.0 \mathrm{~mL}$ of a $0.5 \mathrm{M}$ solution in THF, $2.0 \mathrm{mmol}, 2.5$ equiv), was added dropwise via syringe over $5 \mathrm{~min}$. After allowing to warm to rt over $60 \mathrm{~min}$, TLC showed complete consumption of starting material. The flask was placed in an ice-bath and chloroacetyl chloride $(0.19 \mathrm{~mL}, 2.39 \mathrm{mmol}, 3.0$ equiv) was added dropwise via syringe over $1 \mathrm{~min}$. After $2 \mathrm{~h}$, TLC showed complete consumption of the propargyl alcohol intermediate. Diethyl ether $(5 \mathrm{~mL})$ and deionized water $(3 \mathrm{~mL})$ were added to the flask, the mixture was transferred to a separatory funnel, and the organic layer was separated. The aqueous layer was extracted with diethyl ether ( $3 \times 5 \mathrm{~mL})$, washed with brine, dried over magnesium sulfate, filtered, and concentrated in vacuo. The crude residue was purified by silica gel flash chromatography (5 to $20 \%$ ethyl acetate/hexanes) to give $152 \mathrm{mg}(57 \%)$ of the title compound as a light-yellow oil.

\section{EDD6-417}

$\underline{{ }^{1} \mathrm{H} \mathrm{NMR}} \quad\left(400 \mathrm{MHz}, \mathrm{CDCl}_{3}\right)$ 
$7.27(\mathrm{~d}, J=1.6 \mathrm{~Hz}, 1 \mathrm{H}), 6.29(\mathrm{~d}, J=1.6 \mathrm{~Hz}, 1 \mathrm{H}), 4.01(\mathrm{~s}, 2 \mathrm{H}), 2.75-2.71(\mathrm{~m}, 2$ H), 2.64 (s, $1 \mathrm{H}), 2.29-2.05$ (m, $2 \mathrm{H}), 1.78(\mathrm{~s}, 3 \mathrm{H}), 0.25$ (s, 9H) ppm

${ }^{13} \mathrm{C} \mathrm{NMR} \quad\left(100 \mathrm{MHz}, \mathrm{CDCl}_{3}\right)$

$165.4,143.3,134.5,130.2,111.9,102.4,93.7,82.4,74.9,41.4,41.1,26.4,20.4$, $0.0 \mathrm{ppm}$

$\underline{\text { IR }} \quad$ (Thin Film)

$2153,1767 \mathrm{~cm}^{-1}$

HRMS HRMS-ESI (m/z): $[\mathrm{M}+\mathrm{H}]^{+}$calcd for $\mathrm{C}_{17} \mathrm{H}_{22} \mathrm{O}_{3} \mathrm{SiCl}, 337.1027$; found, 337.1033

TLC $\quad R_{f}=0.44$ (35\% ethyl acetate/hexanes); silica gel, UV, PAA

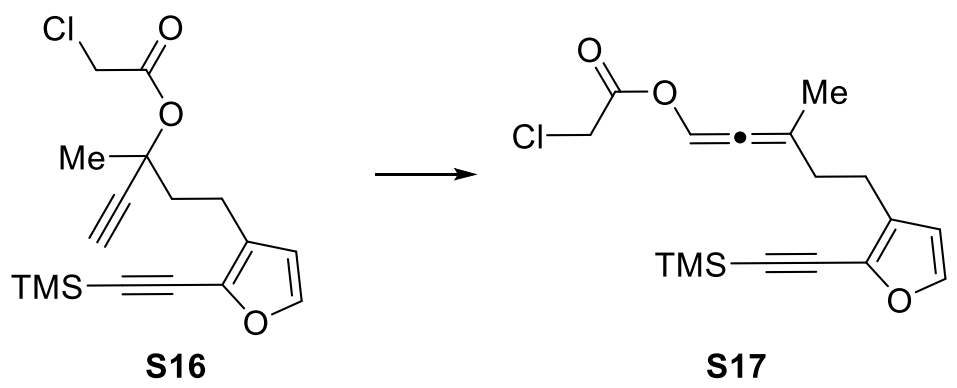

3-methyl-5-(2-((trimethylsilyl)ethynyl)furan-3-yl)penta-1,2-dien-1-yl 2-chloroacetate (S17).

Follows general procedure B: Rhodium(II) trifluoroacetate dimer (13.8 mg, $0.021 \mathrm{mmol})$, 3methyl-5-(2-((trimethylsilyl)ethynyl)furan-3-yl)pent-1-yn-3-yl 2-chloroacetate (S16) (144 mg, $0.427 \mathrm{mmol})$, toluene $(2.1 \mathrm{~mL}, 0.2 \mathrm{M})$ The reaction was stirred for $1 \mathrm{~h}$ in an oil bath $\left(50{ }^{\circ} \mathrm{C}\right)$ and the crude residue immediately loaded onto a packed column and purified by silica gel flash 
chromatography (2-4\% ethyl acetate/hexanes) to give $122 \mathrm{mg}(85 \%)$ of the title compound as a clear liquid.

EDD6-418

$\underline{{ }^{1} \mathrm{H} \mathrm{NMR}} \quad\left(400 \mathrm{MHz}, \mathrm{CDCl}_{3}\right)$

7.32-7.30 (m, $1 \mathrm{H}), 7.25(\mathrm{~d}, J=2.0 \mathrm{~Hz}, 1 \mathrm{H}), 6.26(\mathrm{~d}, J=2.0 \mathrm{~Hz}, 1 \mathrm{H}), 4.12(\mathrm{~s}, 2$

H), $2.65(\mathrm{t}, J=7.6 \mathrm{~Hz}, 2 \mathrm{H}), 2.43-2.33(\mathrm{~m}, 2 \mathrm{H}), 1.87(\mathrm{~d}, J=1.6 \mathrm{~Hz}, 3 \mathrm{H}), 0.25(\mathrm{~s}$,

9 H) ppm

${ }^{13} \mathrm{C} \mathrm{NMR} \quad\left(100 \mathrm{MHz}, \mathrm{CDCl}_{3}\right)$

$189.9,165.3,143.1,134.6,130.4,116.4,111.8,110.7,102.3,93.9,40.8,34.8$,

$23.3,20.5,0.0 \mathrm{ppm}$

$\underline{\text { IR }} \quad$ (Thin Film)

$2152,1980,1771 \mathrm{~cm}^{-1}$

$\underline{\text { HRMS }} \quad$ HRMS-ESI (m/z): $[\mathrm{M}+\mathrm{H}]^{+}$calcd for $\mathrm{C}_{17} \mathrm{H}_{22} \mathrm{O}_{3} \mathrm{SiCl}, 337.1027$; found, 337.1022

$\underline{\text { TLC }} \quad R_{f}=0.63(35 \%$ ethyl acetate/hexanes $)$; silica gel, UV, PAA

\section{Racemic APKR}
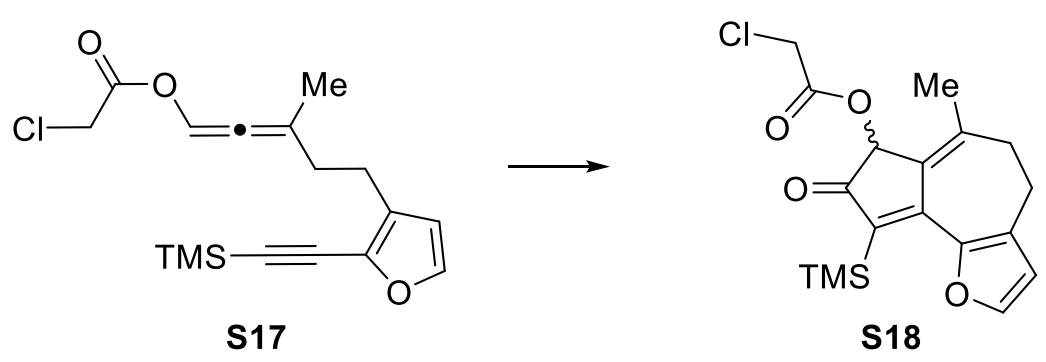
6-methyl-8-oxo-9-(trimethylsilyl)-4,5,7,8-tetrahydroazuleno[4,5-b]furan-7-yl

chloroacetate (S18). Follows General Procedure C: Bis(1,5-cyclooctadiene)rhodium(I) tetrafluoroborate $(2.4 \mathrm{mg}, 0.0059 \mathrm{mmol})$, triphenyl phosphine $(2.3 \mathrm{mg}, 0.0089 \mathrm{mmol})$, carbon monoxide (100\%), 3-methyl-5-(2-((trimethylsilyl)ethynyl)furan-3-yl)penta-1,2-dien-1-yl 2chloroacetate (S17) $(20 \mathrm{mg}, 0.059 \mathrm{mmol})$, DCE (3.0 mL, 0.02 M). The reaction was stirred for 46 $\mathrm{h}$ in an oil bath $\left(70^{\circ} \mathrm{C}\right)$. The crude residue was purified via silica gel flash chromatography $(5 \%$ ethyl acetate/hexanes) to give the title compound (10 $\mathrm{mg}, 45 \%)$ as a yellow solid.

\section{EDD6-421}

$\underline{{ }^{1} \mathrm{H} \mathrm{NMR}} \quad\left(400 \mathrm{MHz}, \mathrm{CDCl}_{3}\right)$

$7.52(\mathrm{~d}, J=1.6 \mathrm{~Hz}, 1 \mathrm{H}), 6.43(\mathrm{~d}, J=1.6 \mathrm{~Hz}, 1 \mathrm{H}), 5.91(\mathrm{~s}, 1 \mathrm{H}), 4.15(\mathrm{~d}, J=1.2$

Hz, 2 H), 2.89-2.74 (m, 2 H), 2.60-2.56 (m, 2 H), 1.95 (s, 3 H), 0.26 (s, 9 H) ppm

${ }^{13} \mathrm{C} \mathrm{NMR} \quad\left(100 \mathrm{MHz}, \mathrm{CDCl}_{3}\right)$

202.1, 166.6, 161.3, 147.3, 144.5, 141.5, 134.1, 132.7, 128.7, 114.6, 74.5, 40.9, $34.7,25.3,23.6,1.0 \mathrm{ppm}$

$\underline{\text { IR }} \quad$ (Thin Film)

$1762,1686,1641 \mathrm{~cm}^{-1}$

$\underline{\text { HRMS }} \quad$ HRMS-ESI (m/z): $[\mathrm{M}+\mathrm{H}]^{+}$calcd for $\mathrm{C}_{18} \mathrm{H}_{22} \mathrm{O}_{4} \mathrm{SiCl}, 365.0976$; found, 365.0971

$\underline{\text { TLC }} \quad R_{f}=0.34(35 \%$ ethyl acetate/hexanes $)$; silica gel, UV, PAA

Waters 600 HPLC, UV/PDA detector, 332 nm, Daicel CHIRALCEL-OD, 250 X 4.6 mm column, $1 \% \mathrm{iPrOH} /$ hexanes, Flow rate: $1 \mathrm{~mL} / \mathrm{min}$ 


\begin{tabular}{|c|c|c|}
\hline Peak & Retention Time (min) & Peak area (\%) \\
\hline Peak 1 & 13.00 & 50.05 \\
\hline Peak 2 & 16.53 & 49.95 \\
\hline
\end{tabular}

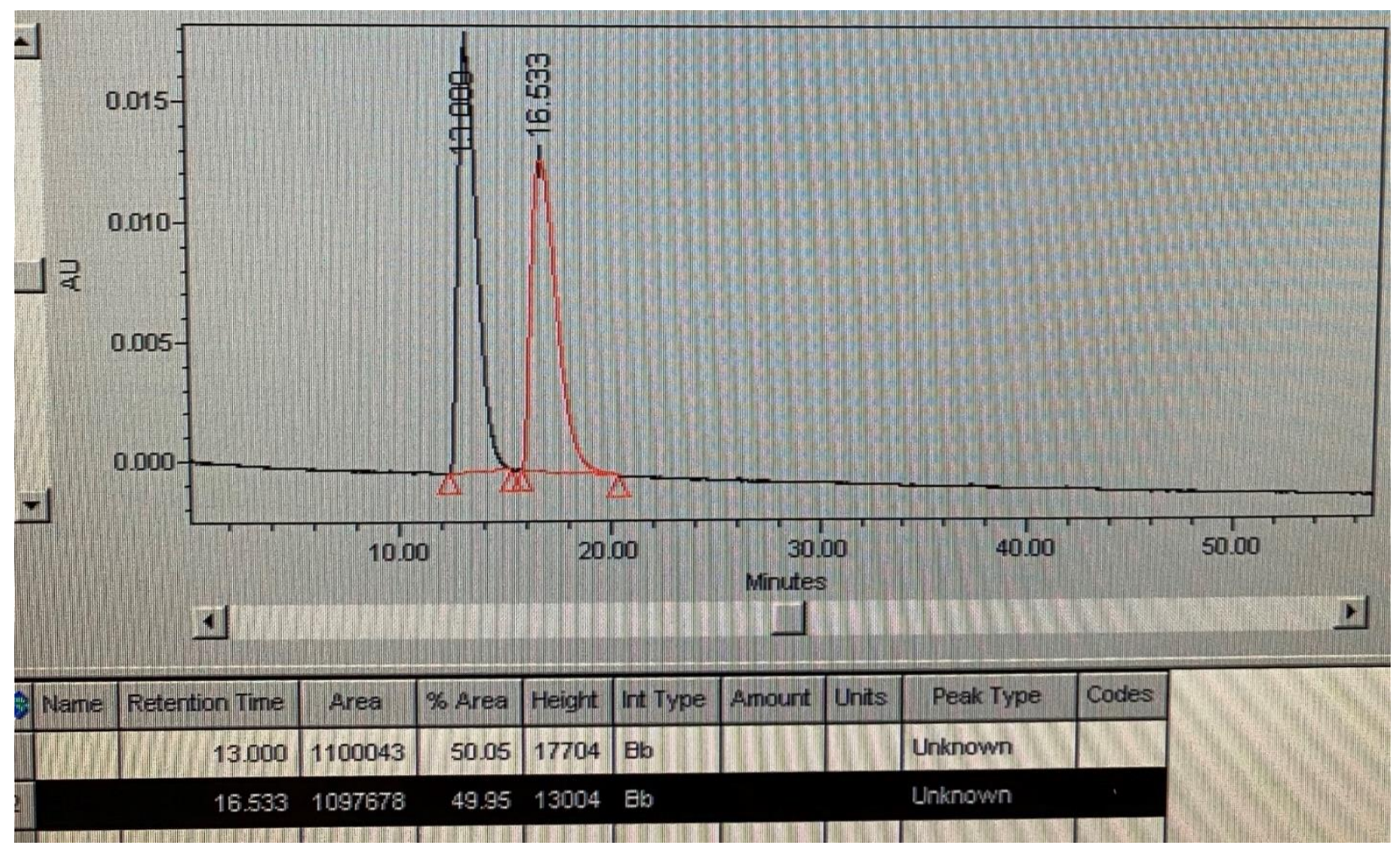

Asymmetric APKR

EDD6-420

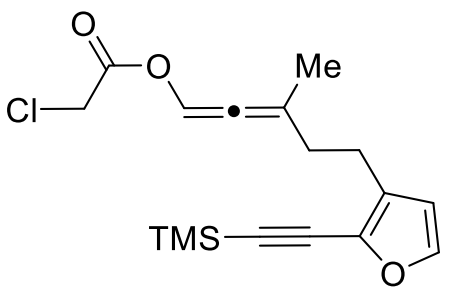

S17<smiles>CC1=C2C(=C(S(C)(=O)=O)C(=O)[C@@H]2OC(=O)CCl)c2occc2CC1</smiles>

S18

chloroacetate (S18). Run 1: Follows General Procedure E: Bis(1,5-cyclooctadiene)rhodium(I) 
tetrafluoroborate (3.0 mg, $0.0074 \mathrm{mmol}),(S)$-MonoPhos-alkene $(\mathbf{8})$ (4.5 mg, $0.011 \mathrm{mmol})$, carbon monoxide (100\%), 3-methyl-5-(2-((trimethylsilyl)ethynyl)furan-3-yl)penta-1,2-dien-1-yl 2chloroacetate $(\mathbf{S 1 7})$ (25 mg, $0.074 \mathrm{mmol})$, DCE (7.4 mL, 0.01 M). The reaction was stirred for 69 $\mathrm{h}$ in an oil bath $\left(70^{\circ} \mathrm{C}\right)$. The crude residue was purified via silica gel flash chromatography $(5 \%$ ethyl acetate/hexanes) to give $13 \mathrm{mg}(48 \%)$ of the title compound in 79:21 er as a yellow solid and $1 \mathrm{mg}(5 \%)$ of aldehyde byproduct.

\section{EDD6-420}

$[\alpha]^{25} \mathrm{D}=+8.7\left(\mathrm{c} .=0.2, \mathrm{CHCl}_{3}\right)$

Waters 600 HPLC, UV/PDA detector, 332 nm, Daicel CHIRALCEL-OD, 250 X 4.6 mm column, $1 \% \mathrm{iPrOH} /$ hexanes, Flow rate: $1 \mathrm{~mL} / \mathrm{min}$

\begin{tabular}{|c|c|c|}
\hline Peak & Retention Time (min) & Peak area (\%) \\
\hline Peak 1 & 13.12 & 78.81 \\
\hline Peak 2 & 16.93 & 21.19 \\
\hline
\end{tabular}




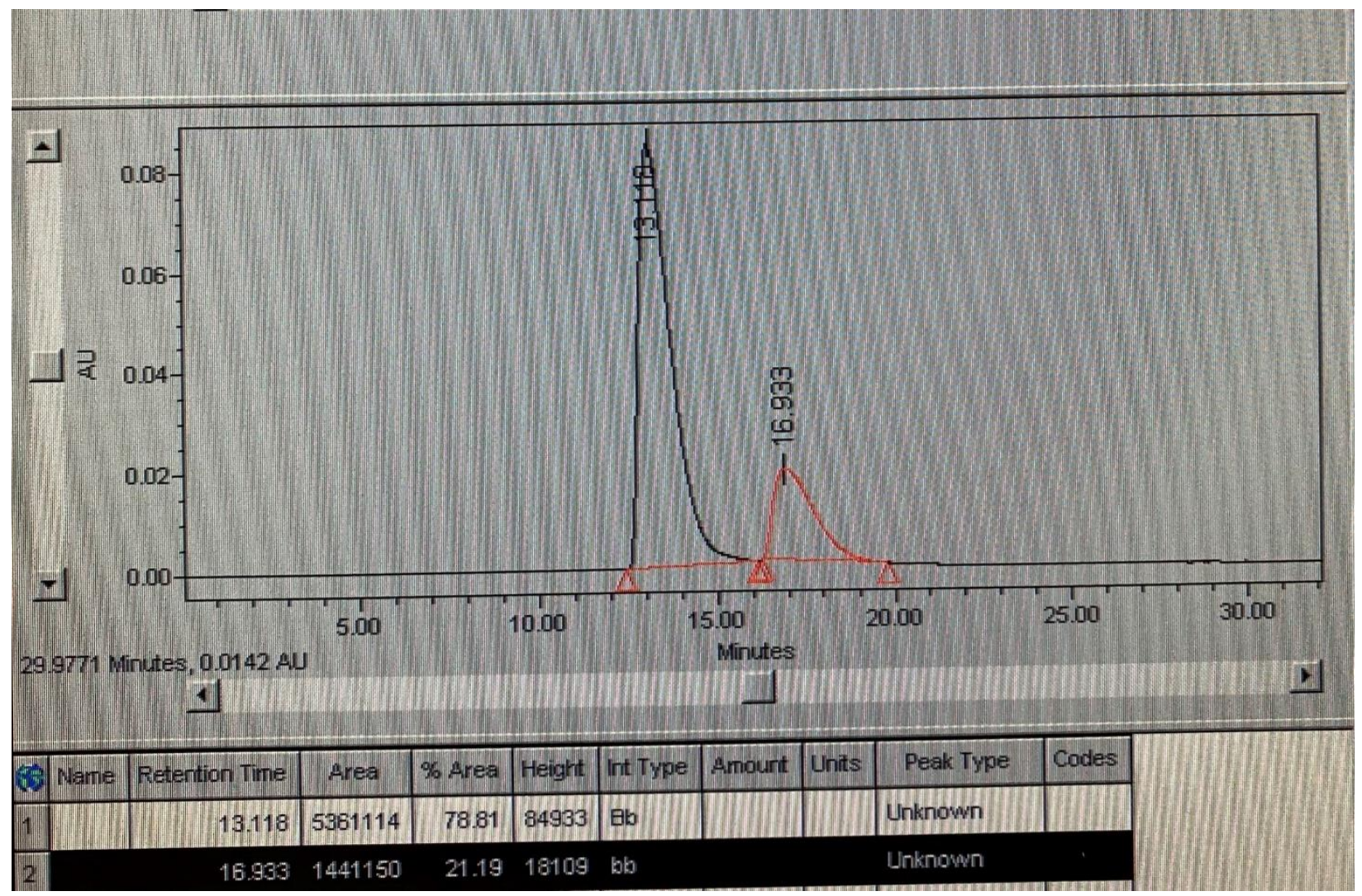

(R)-6-methyl-8-oxo-9-(trimethylsilyl)-4,5,7,8-tetrahydroazuleno[4,5-b]furan-7-yl

2-

chloroacetate (S18). Run 2: Follows general procedure E: Bis(1,5-cyclooctadiene)rhodium(I) tetrafluoroborate (3.0 mg, $0.0074 \mathrm{mmol}),(S)$-MonoPhos-alkene (8) (4.5 mg, $0.011 \mathrm{mmol})$, carbon monoxide (100\%), 3-methyl-5-(2-((trimethylsilyl)ethynyl)furan-3-yl)penta-1,2-dien-1-yl 2chloroacetate (S17) $(25 \mathrm{mg}, 0.074 \mathrm{mmol})$, DCE (7.4 mL, 0.01 M). The reaction was stirred for 68 $\mathrm{h}$ in an oil bath $\left(70^{\circ} \mathrm{C}\right)$. The crude residue was purified via silica gel flash chromatography $(5 \%$ ethyl acetate/hexanes) to give $14 \mathrm{mg}(52 \%)$ of the title compound in 77: 23 er as a yellow solid and $1 \mathrm{mg}(5 \%)$ of aldehyde byproduct.

Waters 600 HPLC, UV/PDA detector, 332 nm, Daicel CHIRALCEL-OD, 250 X 4.6 mm column, $1 \% \mathrm{iPrOH} /$ hexanes, Flow rate: $1 \mathrm{~mL} / \mathrm{min}$

\begin{tabular}{|c|l|l|}
\hline Peak & Retention Time (min) & Peak area (\%) \\
\hline
\end{tabular}




\begin{tabular}{|l|l|l|}
\hline Peak 1 & 12.46 & 76.93 \\
\hline Peak 2 & 15.93 & 23.07 \\
\hline
\end{tabular}

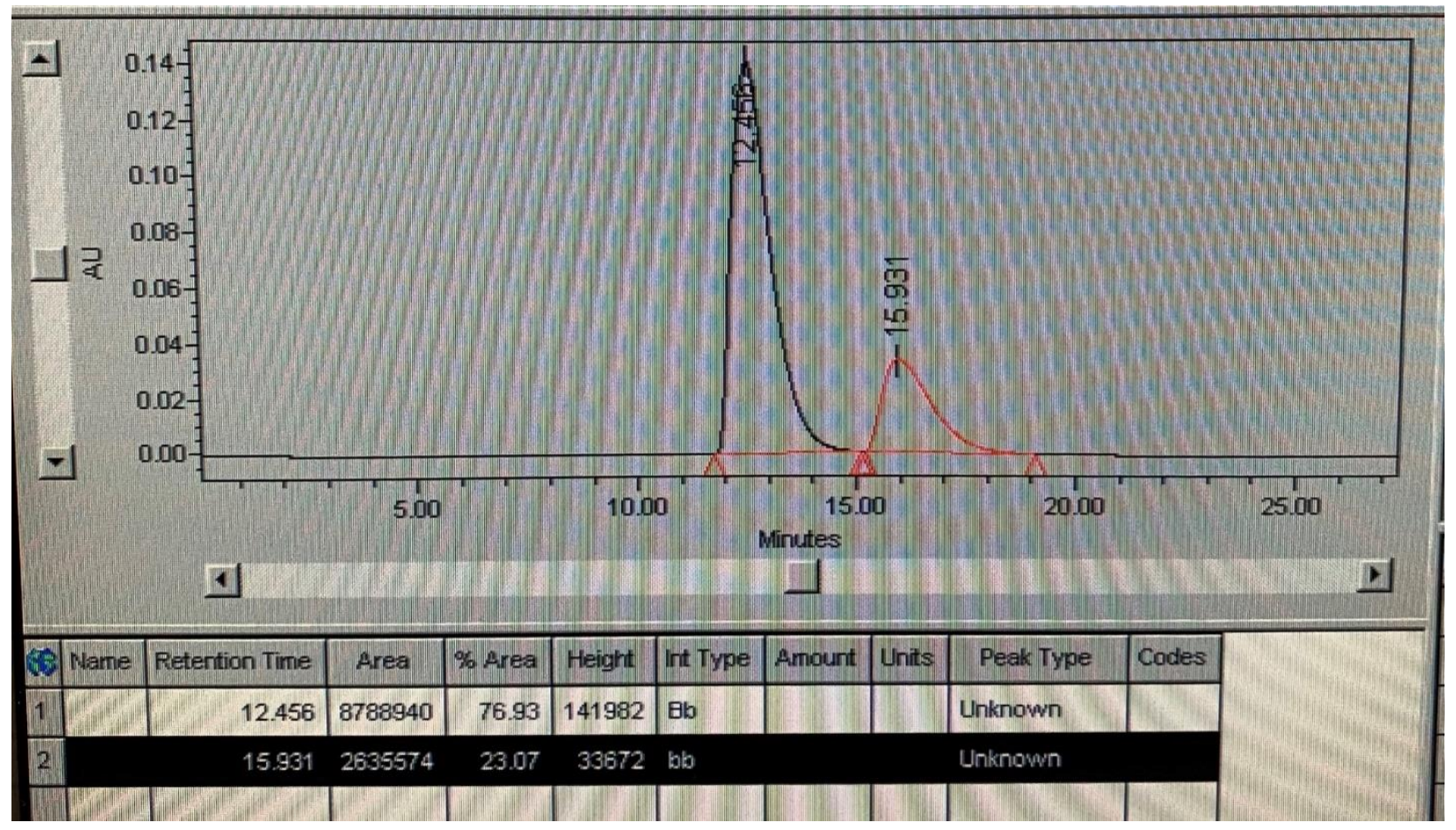

\section{EDD6-422}

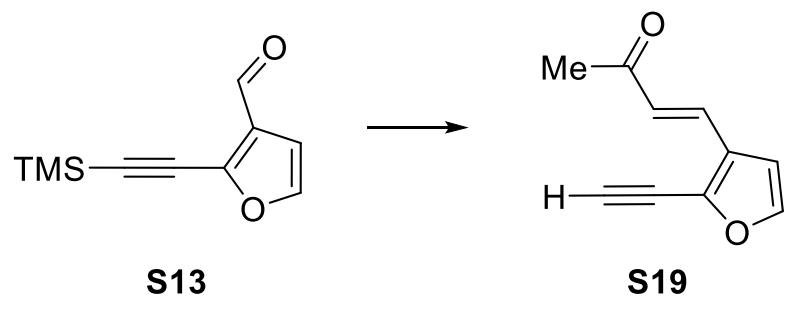

(E)-4-(2-ethynylfuran-3-yl)but-3-en-2-one (S19). This compound was prepared in a manner similar to that previously reported with some modifications. ${ }^{2}$ A flame-dried, single-necked, 10$\mathrm{mL}$, round-bottomed flask equipped with a magnetic stir bar, nitrogen inlet adapter, and septum was charged with 2-((trimethylsilyl)ethynyl)furan-3-carbaldehyde (S13)(311 mg, $1.62 \mathrm{mmol})$, deionized water $(1.36 \mathrm{~mL}, 1.2 \mathrm{M})$ and acetone $(0.30 \mathrm{~mL}, 4.04 \mathrm{mmol})$ via syringe. The solution was 
cooled in an ice-bath, and $33 \% \mathrm{NaOH}(0.04 \mathrm{~mL}, 0.32 \mathrm{mmol})$ was added dropwise. After allowing to warm to room temperature for $3.5 \mathrm{~h}$, TLC showed complete consumption of starting material. $10 \%$ Sulfuric acid was added until the solution reached a $\mathrm{pH} 4$ as determined using $\mathrm{pH}$ paper. The resulting solution was transferred to a separatory funnel. The aqueous layer was extracted with $\mathrm{CHCl}_{3}(5 \times 3 \mathrm{~mL})$, the combined organic layers were dried over magnesium sulfate, filtered, and concentrated in vacuo. The crude residue was purified by silica gel flash chromatography (7.5$15 \%$ ethyl acetate/hexanes) to afford $170 \mathrm{mg}$ (66\% yield) of the title compound as a brown oil.

\section{EDD6-452}

${ }^{1} \mathrm{H} \mathrm{NMR} \quad\left(400 \mathrm{MHz}, \mathrm{CDCl}_{3}\right)$

$7.45(\mathrm{~d}, J=16.0 \mathrm{~Hz}, 1 \mathrm{H}), 7.37(\mathrm{~d}, J=1.6 \mathrm{~Hz}, 1 \mathrm{H}), 6.59$ (d, $J=2.0 \mathrm{~Hz}, 1 \mathrm{H})$,

$6.52(\mathrm{~d}, J=16.4 \mathrm{~Hz}, 1 \mathrm{H}), 3.74(\mathrm{~s}, 1 \mathrm{H}), 2.37$ (s, $3 \mathrm{H}) \mathrm{ppm}$

$\stackrel{{ }^{13} \mathrm{C} \mathrm{NMR}}{ } \quad\left(100 \mathrm{MHz}, \mathrm{CDCl}_{3}\right)$

$198.3,144.8,137.8,132.5,128.8,127.5,108.4,87.5,72.5,27.5 \mathrm{ppm}$

$\underline{\text { IR }} \quad$ (Thin Film)

$3250,1689,1610 \mathrm{~cm}^{-1}$

$\underline{\text { HRMS }} \quad$ HRMS-ESI (m/z): $[\mathrm{M}+\mathrm{H}]^{+}$calcd for $\mathrm{C}_{10} \mathrm{H}_{9} \mathrm{O}_{2}, 161.0597$; found, 161.0600

$\underline{\text { TLC }} \quad R_{f}=0.43(35 \%$ ethyl acetate/hexanes $)$; silica gel, UV, PAA 


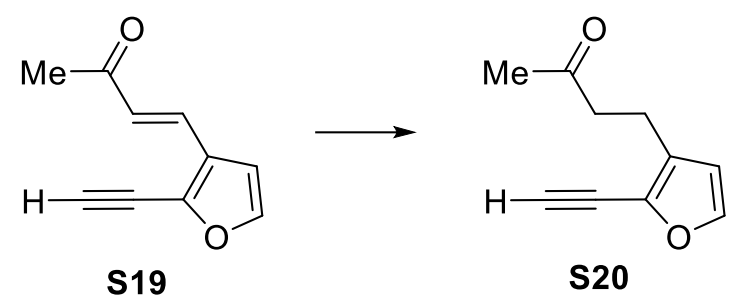

4-(2-ethynylfuran-3-yl)butan-2-one (S20). A flame-dried, 2-necked, 100-mL round-bottomed flask equipped with a magnetic stir bar, nitrogen inlet adapter, and septum was charged with $(E)$ 4-(2-ethynylfuran-3-yl)but-3-en-2-one (S19)(330 mg, $2.06 \mathrm{mmol})$, toluene (20.6 mL, $0.1 \mathrm{M})$, Hantzsch ester (14)(1.565g, $6.18 \mathrm{mmol})$, and silica gel (3.09 g) and lowered into a preheated 110 ${ }^{\circ} \mathrm{C}$ oil bath. After $20 \mathrm{~h}$, an NMR aliquot showed complete consumption of starting material. The solution was filtered using a glass funnel fitted with filter paper, eluting with ethyl acetate $(25 \mathrm{~mL})$, concentrated in vacuo. The crude material was dissolved in ethyl acetate $(15 \mathrm{~mL})$, transferred to a separatory funnel, extracted with $2 \mathrm{~N} \mathrm{HCl}(5 \times 5 \mathrm{~mL})$, the organic layer washed with brine $(1 \times 5$ $\mathrm{mL}$ ), dried over magnesium sulfate, filtered, and concentrated in vacuo. The crude residue was purified by silica gel flash chromatography (10\% ethyl acetate/ hexanes) to afford $220 \mathrm{mg}(66 \%)$ of the title compound as a yellow oil.

\section{EDD6-458}

$\underline{{ }^{1} \mathrm{H} \mathrm{NMR}} \quad\left(400 \mathrm{MHz}, \mathrm{CDCl}_{3}\right)$

$7.27(\mathrm{~d}, J=1.6 \mathrm{~Hz}, 1 \mathrm{H}), 6.27(\mathrm{~d}, J=1.6 \mathrm{~Hz}, 1 \mathrm{H}), 3.54(\mathrm{~s}, 1 \mathrm{H}), 2.80-2.70(\mathrm{~m}, 4$ $\mathrm{H}), 2.16(\mathrm{~s}, 3 \mathrm{H}) \mathrm{ppm}$

$\underline{{ }^{13} \mathrm{C} \mathrm{NMR}} \quad\left(100 \mathrm{MHz}, \mathrm{CDCl}_{3}\right)$

207.6, 143.5, 133.5, 130.6, 112.0, 84.5, 73.5, 43.4, 30.0, 19.5 ppm

$\underline{\text { IR }} \quad$ (Thin Film) 
$3287,2108,1711 \mathrm{~cm}^{-1}$

HRMS HRMS-ESI (m/z): $[\mathrm{M}+\mathrm{H}]^{+}$calcd for $\mathrm{C}_{10} \mathrm{H}_{11} \mathrm{O}_{2}, 163.0754$; found, 163.0755

TLC $\quad R_{f}=0.45$ (35\% ethyl acetate/hexanes); silica gel, UV, PAA

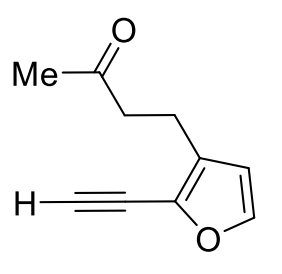

S20

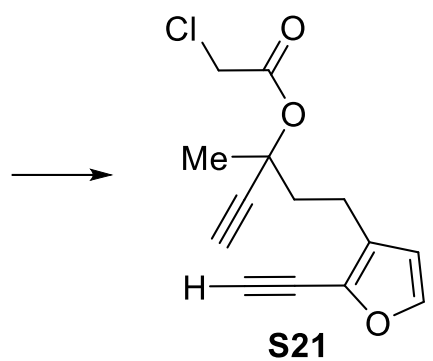

S21

5-(2-ethynylfuran-3-yl)-3-methylpent-1-yn-3-yl 2-chloroacetate (S21). This compound was prepared in a manner similar to that previously reported with some modifications. ${ }^{5}$ A flame-dried, 2-necked, 25-mL, round bottomed flask equipped with a magnetic stir bar, nitrogen inlet adapter, and septum was charged with 4-(2-ethynylfuran-3-yl)butan-2-one (S20) $(82 \mathrm{mg}, 0.51 \mathrm{mmol})$ and THF (1.69 mL). The solution was cooled by placing the flask in an ice-bath and ethynylmagnesium bromide (3.04 $\mathrm{mL}$ of a $0.5 \mathrm{M}$ solution in THF, $1.52 \mathrm{mmol}, 3.0$ equiv), was added dropwise via syringe over $5 \mathrm{~min}$. After allowing to warm to rt over $60 \mathrm{~min}$, TLC showed complete consumption of starting material. The flask was placed in an ice-bath and chloroacetyl chloride $(0.14 \mathrm{~mL}, 1.77$ mmol, 3.5 equiv) was added dropwise via syringe over $1 \mathrm{~min}$. After $2 \mathrm{~h}$, TLC showed complete consumption of the propargyl alcohol intermediate. Diethyl ether $(5 \mathrm{~mL})$ and deionized water $(3$ $\mathrm{mL}$ ) were added to the flask, the mixture was transferred to a separatory funnel, and the organic layer was separated. The aqueous layer was extracted with diethyl ether $(3 \times 5 \mathrm{~mL})$, washed with brine, dried over magnesium sulfate, filtered, and concentrated in vacuo. The crude residue was 
purified by silica gel flash chromatography (5-7.5\% ethyl acetate/hexanes) to give $63 \mathrm{mg}$ (47\%) of the title compound as a light-yellow oil containing an uncharacterized byproduct.

\section{EDD6-455}

${ }^{1} \mathrm{H} \mathrm{NMR} \quad\left(400 \mathrm{MHz}, \mathrm{CDCl}_{3}\right)$

$7.30(\mathrm{~d}, J=1.6 \mathrm{~Hz}, 1 \mathrm{H}), 6.31(\mathrm{~d}, J=2.0 \mathrm{~Hz}, 1 \mathrm{H}), 4.01(\mathrm{~s}, 2 \mathrm{H}), 3.55(\mathrm{~s}, 1 \mathrm{H})$, 2.76-2.71 (m, $2 \mathrm{H}), 2.65$ (s, $1 \mathrm{H}), 2.28-2.07$ (m, $2 \mathrm{H}), 1.78$ (s, $3 \mathrm{H})$; Contained impurities at: 4.13 (s, $0.4 \mathrm{H}), 3.98(\mathrm{~s}, 0.4 \mathrm{H}), 2.79$ (s, $0.4 \mathrm{H}) \mathrm{ppm}$

${ }^{13} \mathrm{C} \mathrm{NMR} \quad\left(100 \mathrm{MHz}, \mathrm{CDCl}_{3}\right)$

$165.4,143.6,133.6,130.6,111.8,84.5,82.4,76.2,74.9,73.4,41.5,41.1,26.4$, 20.2; contained impurities at: 164.4, 67.6, 49.7, $40.8 \mathrm{ppm}$

$\underline{\mathrm{IR}} \quad$ (Thin Film)

$3290,2117,1757 \mathrm{~cm}^{-1}$

HRMS HRMS-ESI (m/z): $[\mathrm{M}+\mathrm{H}]^{+}$calcd for $\mathrm{C}_{14} \mathrm{H}_{14} \mathrm{O}_{3} \mathrm{Cl}$, 265.0626; found, 265.0634

$\underline{\text { TLC }} \quad R_{f}=0.62$ (35\% ethyl acetate/hexanes); silica gel, UV, PAA

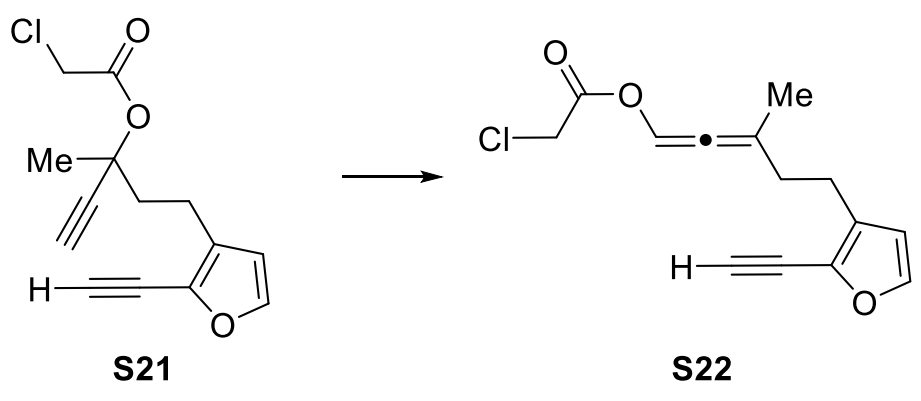


5-(2-ethynylfuran-3-yl)-3-methylpenta-1,2-dien-1-yl 2-chloroacetate (S22). Follows general procedure B: Rhodium(II) trifluoroacetate dimer (24 mg, $0.036 \mathrm{mmol}$ ) 5-(2-ethynylfuran-3-yl)-3methylpent-1-yn-3-yl 2-chloroacetate (S21)(191 mg, $0.72 \mathrm{mmol})$, toluene (3.6 mL, 0.2 M) The reaction was stirred for $2 \mathrm{~h}$ in an oil bath $\left(50^{\circ} \mathrm{C}\right)$ and the crude residue immediately loaded onto a packed column and purified by silica gel flash chromatography (2-7.5\% ethyl acetate/hexanes) to give $99 \mathrm{mg}(52 \%)$ of the title compound as a light brown liquid.

\section{EDD6-460}

$\underline{{ }^{1} \mathrm{H} \mathrm{NMR}} \quad\left(400 \mathrm{MHz}, \mathrm{CDCl}_{3}\right)$

$7.32(\mathrm{~d}, J=2.0 \mathrm{~Hz}, 1 \mathrm{H}), 7.28(\mathrm{~d}, J=1.6 \mathrm{~Hz}, 1 \mathrm{H}), 6.28(\mathrm{~d}, J=1.6 \mathrm{~Hz}, 1 \mathrm{H}), 4.12$

(s, $2 \mathrm{H}), 3.53(\mathrm{~s}, 1 \mathrm{H}), 2.66$ (t, $J=7.6 \mathrm{~Hz}, 2 \mathrm{H}), 2.42-2.32(\mathrm{~m}, 2 \mathrm{H}), 1.87$ (d, $J=$

$1.2 \mathrm{~Hz}, 3 \mathrm{H}) \mathrm{ppm}$

${ }^{13} \mathrm{C} \mathrm{NMR} \quad\left(100 \mathrm{MHz}, \mathrm{CDCl}_{3}\right)$

189.9, 165.3, 143.5, 133.7, 130.8, 116.4, 111.7, 110.8, 84.4, 73.6, 40.9, 34.9, 23.1, $20.7 \mathrm{ppm}$

$\underline{\text { IR }} \quad$ (Thin Film)

$3290,2108,1979,1763 \mathrm{~cm}^{-1}$

HRMS HRMS-ESI (m/z): $[\mathrm{M}+\mathrm{H}]^{+}$calcd for $\mathrm{C}_{14} \mathrm{H}_{14} \mathrm{O}_{3} \mathrm{Cl}$, 265.0626; found, 265.0635

$\underline{\text { TLC }} \quad R_{f}=0.63$ (35\% ethyl acetate/hexanes); silica gel, UV, PAA 


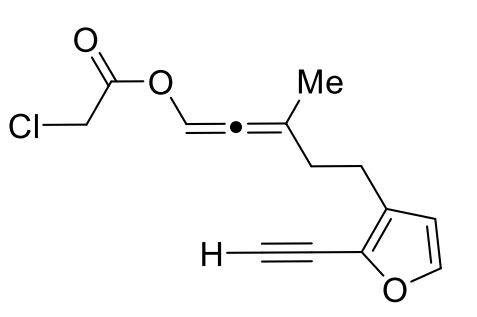

S22

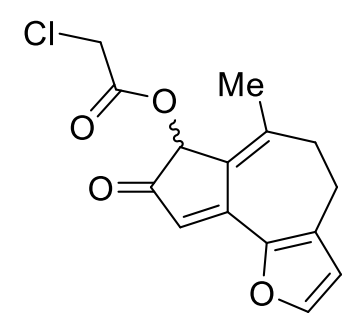

S23

6-methyl-8-oxo-4,5,7,8-tetrahydroazuleno[4,5-b]furan-7-yl 2-chloroacetate (S23). Follows

General Procedure C: Bis(1,5-cyclooctadiene)rhodium(I) tetrafluoroborate $(3.1 \mathrm{mg}, 0.0076$ mmol), triphenyl phosphine(3.0 mg, $0.0114 \mathrm{mmol}$ ), carbon monoxide (100\%), 5-(2-ethynylfuran3-yl)-3-methylpenta-1,2-dien-1-yl 2-chloroacetate (S22) (20 mg, 0.076 mmol), DCE (3.8 mL, 0.02 M). The reaction was stirred for $21.5 \mathrm{~h}$ in an oil bath $\left(70{ }^{\circ} \mathrm{C}\right)$. The crude residue was purified via silica gel flash chromatography (7.5-20\% ethyl acetate/hexanes) to give the title compound ( $7 \mathrm{mg}$, $32 \%)$ as a yellow solid.

\section{EDD6-461}

${ }^{1} \mathrm{H} \mathrm{NMR} \quad\left(400 \mathrm{MHz}, \mathrm{CDCl}_{3}\right)$

$7.51(\mathrm{~d}, J=1.2 \mathrm{~Hz}, 1 \mathrm{H}), 6.39$ (s, $1 \mathrm{H}), 5.87$ (s, $1 \mathrm{H}), 4.19-4.11$ (m, $2 \mathrm{H}), 2.89-$

$2.76(\mathrm{~m}, 2 \mathrm{H}), 2.68-2.54(\mathrm{~m}, 2 \mathrm{H}), 1.98$ (s, $3 \mathrm{H}) \mathrm{ppm}$

${ }^{13} \mathrm{C} \mathrm{NMR} \quad\left(100 \mathrm{MHz}, \mathrm{CDCl}_{3}\right)$

$197.8,166.5,156.0,146.1,145.8,142.9,133.5,127.6,120.4,114.3,73.8,40.7$, $34.4,24.9,23.5 \mathrm{ppm}$

$\underline{\text { IR }} \quad$ (Thin Film)

$1760,1694 \mathrm{~cm}^{-1}$

HRMS HRMS-ESI (m/z): $[\mathrm{M}+\mathrm{H}]^{+}$calcd for $\mathrm{C}_{15} \mathrm{H}_{14} \mathrm{O}_{4} \mathrm{Cl}$, 293.0575; found, 293.0588 
TLC $\quad R_{f}=0.29$ (35\% ethyl acetate/hexanes); silica gel, UV, PAA

Waters 600 HPLC, UV/PDA detector, 328 nm, Daicel CHIRALPak IA-3, 250 X 4.6 mm column, $1 \% \mathrm{iPrOH} / \mathrm{hexanes}$, Flow rate: $1 \mathrm{~mL} / \mathrm{min}$

\begin{tabular}{|c|c|c|}
\hline Peak & Retention Time (min) & Peak area (\%) \\
\hline Peak 1 & 114.08 & 50.30 \\
\hline Peak 2 & 133.64 & 49.70 \\
\hline
\end{tabular}

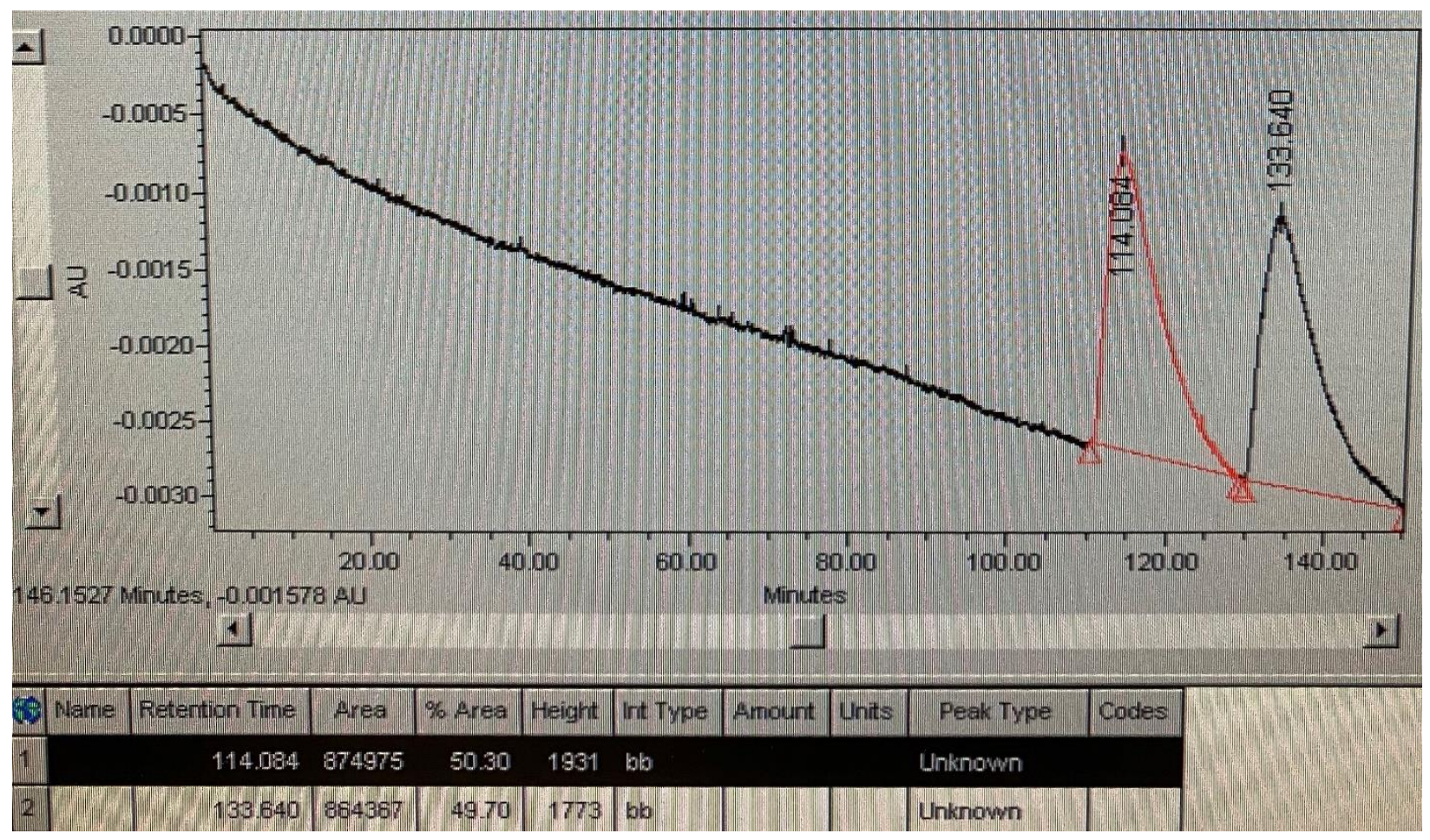<smiles>C#Cc1occc1CCC(C)=C=COC(=O)CCl</smiles>

S22<smiles>CC1=C2C(=CC(=O)[C@@H]2OC(=O)CCl)c2occc2CC1</smiles>

S23 


\section{(R)-6-methyl-8-oxo-4,5,7,8-tetrahydroazuleno[4,5-b]furan-7-yl 2-chloroacetate}

(S23).

Follows general procedure E: Bis(1,5-cyclooctadiene)rhodium(I) tetrafluoroborate (3.1 mg, $0.0076 \mathrm{mmol}),(S)-$ MonoPhos-alkene $(\mathbf{8})$ (4.7 mg, $0.114 \mathrm{mmol})$, carbon monoxide (100\%), 5-(2ethynylfuran-3-yl)-3-methylpenta-1,2-dien-1-yl 2-chloroacetate (S22) (20 mg, 0.076 mmol), DCE $(7.6 \mathrm{~mL}, 0.01 \mathrm{M})$. The reaction was stirred for $43 \mathrm{~h}$ in an oil bath $\left(70^{\circ} \mathrm{C}\right)$. The crude residue was purified via silica gel flash chromatography (10\% to $15 \%$ ethyl acetate/hexanes) to give $7 \mathrm{mg}$ (32\%) of the title compound in 77: 23 er as a yellow solid.

\section{EDD6-462}

Waters 600 HPLC, UV/PDA detector, 328 nm, Daicel CHIRALPak IA-3, 250 X 4.6 mm column, $1 \%$ PrOH/hexanes, Flow rate: $1 \mathrm{~mL} / \mathrm{min}$

\begin{tabular}{|c|c|c|}
\hline Peak & Retention Time (min) & Peak area (\%) \\
\hline Peak 1 & 121.10 & 23.32 \\
\hline Peak 2 & 136.94 & 76.68 \\
\hline
\end{tabular}




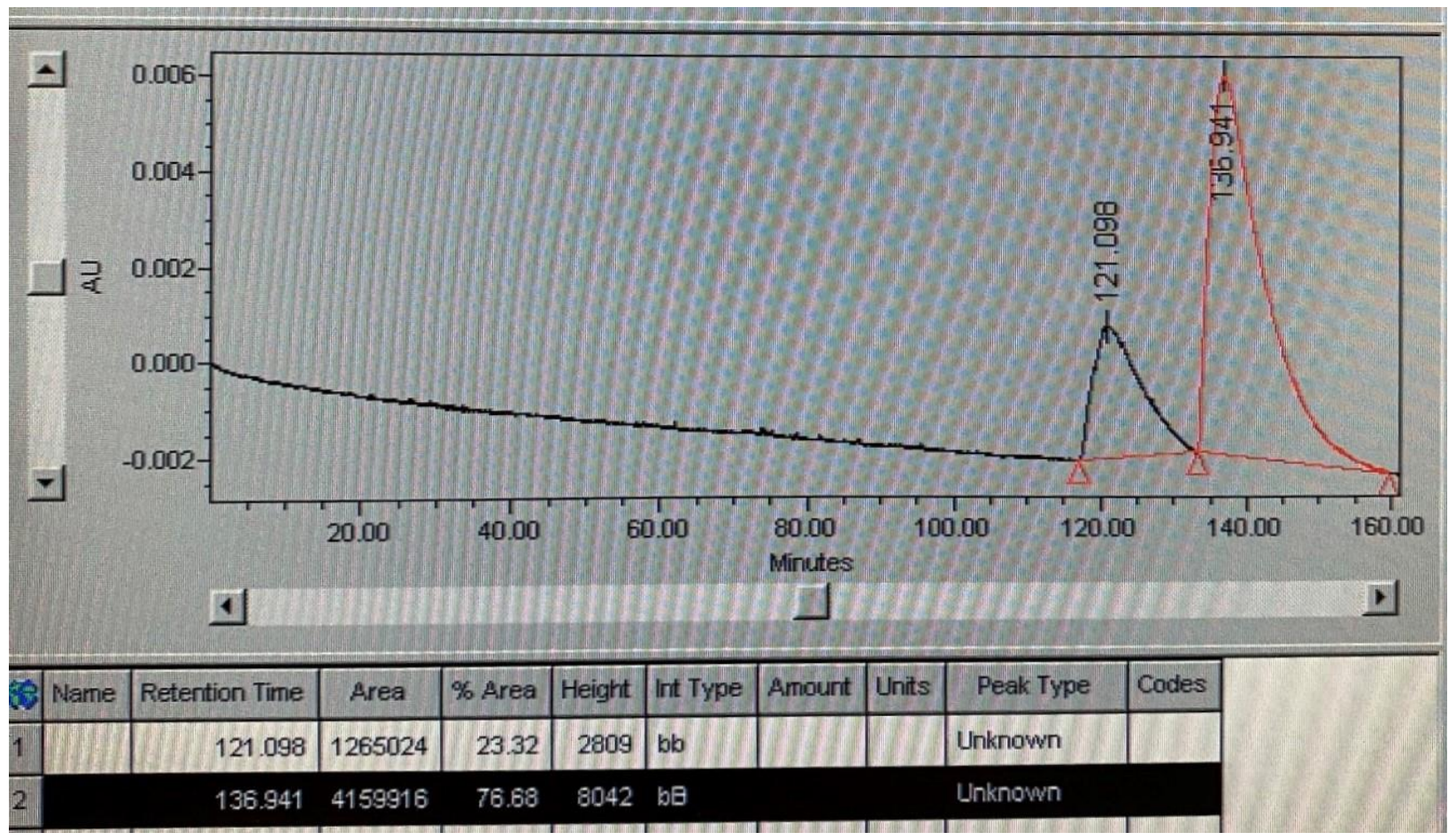

\section{Table S1. Influence of alkyne group on yield and er}<smiles>[R]C#Cc1occc1CCC(C)=C=COC(=O)CCl</smiles>

S10, S17, s22

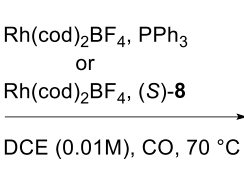

racemic yield ${ }^{b}$

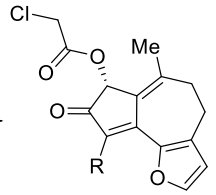

S11, S18, S23

$\begin{array}{ccccc}\text { R } & \text { racemic yield }^{\mathbf{b}} & \text { asymmetric yield }^{\mathbf{b}} & \text { er } & {\text { aldehyde } \text { yield }^{\mathbf{b}}}_{\text {Ph }} \\ \text { S10 } & 59 & 46^{\mathrm{a}} & 99: 1^{\mathrm{a}} & 15^{\mathrm{a}} \\ \text { TMS } & 45 & 50^{\mathrm{a}} & 78: 22^{\mathrm{a}} & 5^{\mathrm{a}} \\ \text { S17 } & 32 & 32 & 77: 23 & 0 \\ \text { H } & & & \end{array}$

a Average of two runs. ${ }^{\mathrm{b}}$ Isolated yield 


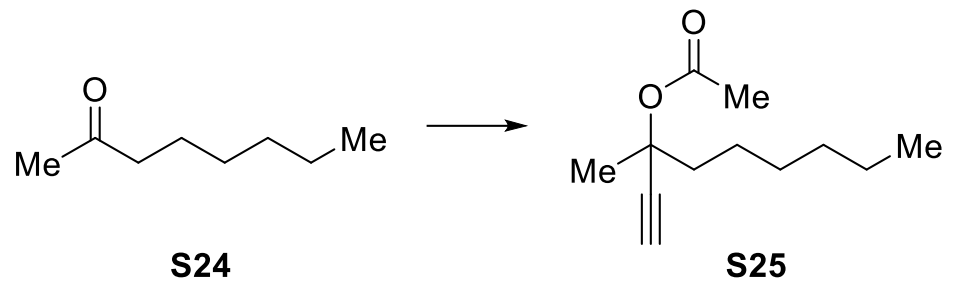

3-methylnon-1-yn-3-yl acetate (S25). A flame-dried, 2-necked, 100-mL, round bottomed flask equipped with a magnetic stir bar, nitrogen inlet adapter, and septum was charged with 2-octanone (S24) (328 mg, $2.56 \mathrm{mmol})$ and THF ( $8.5 \mathrm{~mL})$. The solution was cooled by placing the flask in an ice-bath and ethynylmagnesium bromide $(15.4 \mathrm{~mL}$ of a $0.5 \mathrm{M}$ solution in THF, $7.68 \mathrm{mmol}, 3$ equiv), was added dropwise via syringe over $5 \mathrm{~min}$. After allowing to warm to rt over $1 \mathrm{~h}$, TLC showed complete consumption of starting material. The flask was placed in an ice-bath and acetyl chloride ( $0.64 \mathrm{~mL}, 8.96 \mathrm{mmol})$ was added dropwise via syringe over $2 \mathrm{~min}$. After $2 \mathrm{~h}$, TLC showed complete consumption of the propargyl alcohol intermediate. Diethyl ether $(10 \mathrm{~mL})$ and deionized water $(10 \mathrm{~mL})$ were added to the flask, the mixture was transferred to a separatory funnel, and the organic layer was separated. The aqueous layer was extracted with diethyl ether $(3 \times 20 \mathrm{~mL})$, the combined organic layers washed with brine, dried over magnesium sulfate, filtered, and concentrated in vacuo. The crude residue was purified by silica gel flash chromatography $(5-15 \%$ ethyl acetate/hexanes) to give $336 \mathrm{mg}(67 \%)$ of the title compound as a clear liquid.

\section{EDD6-482}

$\underline{{ }^{1} \mathrm{H} \mathrm{NMR}} \quad\left(500 \mathrm{MHz}, \mathrm{CDCl}_{3}\right)$

2.54 (s, 1H), 2.03 (s, $3 \mathrm{H}), 1.94-1.90(\mathrm{~m}, 1 \mathrm{H}), 1.83-1.77$ (m, $1 \mathrm{H}), 1.66(\mathrm{~s}, 3 \mathrm{H})$, 1.51-1.41 (m, $2 \mathrm{H}), 1.33-1.30(\mathrm{~m}, 6 \mathrm{H}), 0.90-0.87$ (m, $3 \mathrm{H}) \mathrm{ppm}$

${ }^{13} \mathrm{C} \mathrm{NMR} \quad\left(125 \mathrm{MHz}, \mathrm{CDCl}_{3}\right)$ 
$169.6,84.2,75.1,73.2,41.5,31.8,29.3,26.5,24.1,22.7,22.1,14.2 \mathrm{ppm}$

$\underline{\mathrm{IR}} \quad$ (Thin Film)

$1743 \mathrm{~cm}^{-1}$

HRMS $\quad H R M S-E S I(m / z):[M+H]^{+}$calcd for $\mathrm{C}_{12} \mathrm{H}_{21} \mathrm{O}_{2}, 197.1536$; found, 197.1534

TLC $\quad R_{f}=0.54$ (35\% ethyl acetate/hexanes); silica gel, UV, PAA

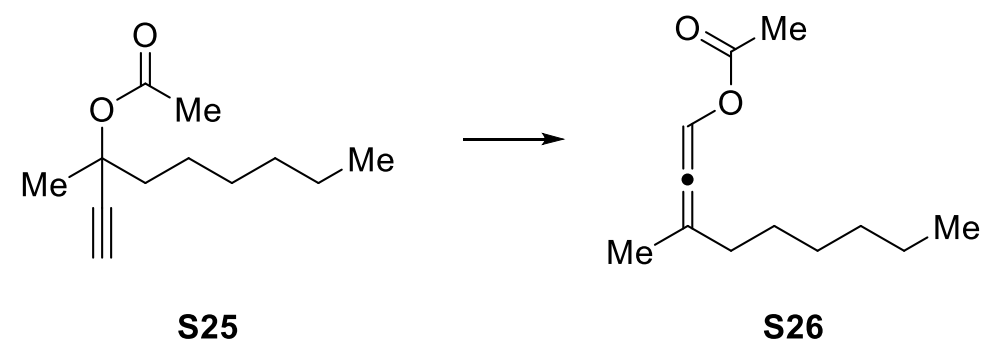

3-methylnona-1,2-dien-1-yl acetate (S26). Follows general procedure B: Rhodium(II) trifluoroacetate dimer (27.6 mg, $0.042 \mathrm{mmol})$, 3-methylnon-1-yn-3-yl acetate (S25)(164 mg, $0.835 \mathrm{mmol})$, toluene $(4.2 \mathrm{~mL}, 0.2 \mathrm{M})$ The reaction was stirred for $1 \mathrm{~h}$ in an oil bath $\left(50{ }^{\circ} \mathrm{C}\right)$. and the crude residue immediately loaded onto a packed column and purified by silica gel flash chromatography (2\% ethyl acetate/hexanes) to give $148 \mathrm{mg}(90 \%)$ of the title compound as a lightyellow oil.

\section{EDD6-483}

$\underline{{ }^{1} \mathrm{H} \mathrm{NMR}} \quad\left(400 \mathrm{MHz}, \mathrm{CDCl}_{3}\right)$

7.29-7.28 (m, $1 \mathrm{H}), 2.13(\mathrm{~s}, 3 \mathrm{H}), 2.09-2.03(\mathrm{~m}, 2 \mathrm{H}), 1.82(\mathrm{~d}, J=2.0 \mathrm{~Hz}, 3 \mathrm{H})$, $1.46-1.41(\mathrm{~m}, 2 \mathrm{H}), 1.32-1.28(\mathrm{~m}, 6 \mathrm{H}), 0.90-0.86(\mathrm{~m}, 3 \mathrm{H}) \mathrm{ppm}$ 
${ }^{13} \mathrm{C} \mathrm{NMR} \quad\left(100 \mathrm{MHz}, \mathrm{CDCl}_{3}\right)$

$189.4,169.0,116.4,109.7,35.3,31.8,28.9,27.3,22.8,21.1,20.7,14.2 \mathrm{ppm}$

$\underline{\mathrm{IR}} \quad$ (Thin Film)

$1977,1749 \mathrm{~cm}^{-1}$

HRMS HRMS-ESI (m/z): $[\mathrm{M}+\mathrm{H}]^{+}$calcd for $\mathrm{C}_{12} \mathrm{H}_{21} \mathrm{O}_{2}, 197.1536$; found, 197.1535

TLC $\quad R_{f}=0.65$ (35\% ethyl acetate/hexanes); silica gel, UV, PAA

a)

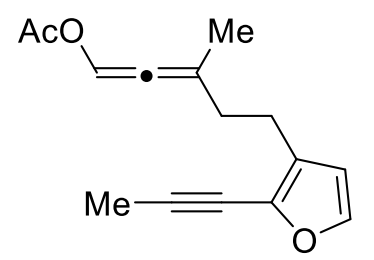

(S)-MonoPhos-Alkene,

$0.01 \mathrm{M} \mathrm{DCE}$,

$\stackrel{\mathrm{N}_{2}, 70^{\circ} \mathrm{C}, 19.5 \mathrm{hr}}{\longrightarrow}$ Recovered SM

4a

b) $\quad 4 a$

$\mathrm{Rh}(\operatorname{cod})_{2} \mathrm{BF}_{4}$,

(S)-MonoPhos-Alkene,

furfuryl alcohol

$0.01 \mathrm{M} \mathrm{DCE}$,

$\mathrm{N}_{2}, 70{ }^{\circ} \mathrm{C}, 22 \mathrm{hr}$

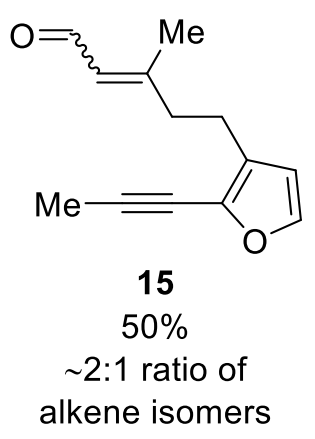

c)<smiles>CCCCCCC(C)=C=COC(C)=O</smiles>

$\mathrm{Rh}(\operatorname{cod})_{2} \mathrm{BF}_{4}$ $\underset{(S) \text {-MonoPhos-alkene }}{\longrightarrow}$ $\stackrel{\mathrm{CO}, \mathrm{DCE}, 70{ }^{\circ} \mathrm{C}}{\longrightarrow}$

$\mathrm{D}_{2} \mathrm{O}$ (5 equiv)<smiles>CCCCCC/C(C)=C(/[OH2+])C=O</smiles>

S26

S27

$44 \%$ yield $25 \%$ deuterium incorporation 


\section{Scheme S1. Aldehyde formation mechanistic studies}

Entry S1a: $(S)$-MonoPhos-alkene (8) (0.15 equiv, $0.5 \mathrm{mg}, 0.0012 \mathrm{mmol})$ was weighed into a flame-dried 5-mL pear-shaped flasks in a nitrogen glovebox and sealed with a septum. The flask was placed under a nitrogen atmosphere via a Schlenk manifold and an inlet needle. $(S)$ MonoPhos-alkene $(\mathbf{8})$ in DCE $(0.2 \mathrm{~mL})$ was added via syringe to a flame dried 8-mL screwcap vial. 3-Methyl-5-(2-(prop-1-yn-1-yl)furan-3-yl)penta-1,2-dien-1-yl acetate (4a) (2.0 mg, 0.0082 mmol) in DCE (0.01 M, $0.82 \mathrm{~mL})$ was added dropwise via syringe. The tube was lowered into a preheated $70{ }^{\circ} \mathrm{C}$ oil bath and stirred until TLC showed complete consumption of starting material (19.5 h). The solution was concentrated in vacuo acetate/hexanes to give recovered starting material 4a and no aldehyde byproduct (15).

Entry S1b: Bis(1,5-cyclooctadiene)rhodium(I) tetrafluoroborate (0.1 equiv, $2.5 \mathrm{mg}, 0.0061$ $\mathrm{mmol}$ ) and (S)-MonoPhos-alkene (8) (0.15 equiv, $3.8 \mathrm{mg}, 0.0092 \mathrm{mmol})$ were weighed into separate flame-dried 5-mL pear-shaped flasks in a nitrogen glovebox and each sealed with a septum. The flasks were placed under a nitrogen atmosphere via a Schlenk manifold and an inlet needle. Bis(1,5-cyclooctadiene)rhodium(I) tetrafluoroborate and $(S)$-MonoPhos-alkene $(\mathbf{8})$ were each dissolved in DCE (1.0 mL each) and the resulting solutions transferred via syringe to a flame dried $50 \mathrm{~mL}$ Schlenk tube and stirred for 30 minutes. Furfuryl alcohol $(6 \mathrm{mg}, 0.061 \mathrm{mmol})$ was added via syringe then (3-Methyl-5-(2-(prop-1-yn-1-yl)furan-3-yl)penta-1,2-dien-1-yl acetate (4a) $(15 \mathrm{mg}, 0.061 \mathrm{mmol})$ in DCE $(0.01 \mathrm{M}, 4.1 \mathrm{~mL})$ was added dropwise via syringe. The tube was lowered into a preheated $70{ }^{\circ} \mathrm{C}$ oil bath and stirred until TLC showed complete consumption of starting material $(22 \mathrm{~h})$. The solution was concentrated in vacuo, and the product purified using silica gel flash chromatography (5\% to $15 \%$ ethyl acetate/hexanes) to give aldehyde $\mathbf{1 5}$ (6 mg, $50 \%)$ 


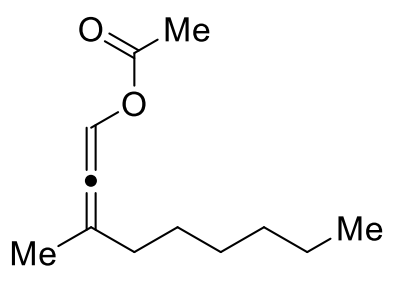

S26

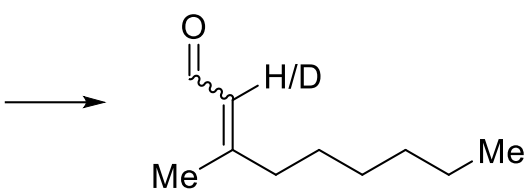

S27

Scheme S1c: 3-methylnon-2-enal (S27). Bis(1,5-cyclooctadiene)rhodium(I) tetrafluoroborate (4.1 mg, $0.0102 \mathrm{mmol}, 0.1$ equiv) and $(S)$-MonoPhos-alkene (8) (6.2 mg, $0.015 \mathrm{mmol}, 0.15$ equiv) were weighed into separate flame-dried round-bottomed flasks in a nitrogen glovebox and each sealed with a septum before removing. The flasks were placed under a nitrogen atmosphere via a Schlenk manifold and an inlet needle. Bis(1,5-cyclooctadiene)rhodium(I) tetrafluoroborate and (S)-MonoPhos-alkene $(\mathbf{8})$ were each dissolved in DCE $(0.8 \mathrm{ml}$ each) and the resulting solutions transferred via syringe to a flame dried Schlenk tube. The tube was evacuated via the inlet needle for a few seconds and refilled $(3 \mathrm{x})$ with $100 \%$ carbon monoxide using a separate inlet needle attached to a balloon of CO and stirred for $1 \mathrm{~h}$. 3-methylnona-1,2-dien-1-yl acetate (S26) (20 mg, $0.102 \mathrm{mmol})$ in DCE $(1.8 \mathrm{~mL}, 0.03 \mathrm{M})$ dropwise via syringe followed by $\mathrm{D}_{2} \mathrm{O}(9 \mu \mathrm{L}, 0.51 \mathrm{mmol}$, 5 equiv). The tube was lowered into a preheated $70{ }^{\circ} \mathrm{C}$ oil bath and stirred for $21.5 \mathrm{~h}$ until TLC showed complete consumption of starting material. The solution was concentrated in vacuo and the product purified using silica gel flash chromatography (5\% to $20 \%$ ethyl acetate/hexanes) to give the title compound $(7 \mathrm{mg}, 44 \%)$ as a yellow oil in a 2:1 ratio of diastereomers and approximately $25 \%$ deuterium incorporation.

\section{EDD6-492}

${ }^{1} \mathrm{H} \mathrm{NMR} \quad\left(400 \mathrm{MHz}, \mathrm{CDCl}_{3}\right)$ 
$9.9(\mathrm{~d}, J=8.4 \mathrm{~Hz}, 1 \mathrm{H})^{*}, 9.9(\mathrm{~s}, 1 \mathrm{H})^{* * *}, 9.95(\mathrm{~d}, J=8.0 \mathrm{~Hz}, 1 \mathrm{H})^{* *}, 9.95(\mathrm{~s}, 1$ $\mathrm{H})^{* * *}, 5.87(\mathrm{~d}, J=8.0 \mathrm{~Hz}, 1 \mathrm{H}), 2.56(\mathrm{t}, J=7.6 \mathrm{~Hz}, 2 \mathrm{H})^{* *}, 2.2(\mathrm{t}, J=7.6 \mathrm{~Hz}, 2$ H), $2.16(\mathrm{~s}, 3 \mathrm{H})^{*}, 1.97(\mathrm{~s}, 3 \mathrm{H})^{* *}, 1.56-1.46(\mathrm{~m}, 2 \mathrm{H}), 1.29-1.25$ (m, $\left.6 \mathrm{H}\right), 0.90-$ $0.87(\mathrm{~m}, 3 \mathrm{H}) \mathrm{ppm}$

*major isomer **minor isomer $* * *$ deuterated compound

${ }^{13} \mathrm{C} \mathrm{NMR} \quad\left(100 \mathrm{MHz}, \mathrm{CDCl}_{3}\right)$

191.5, 191.0, 128.5, 127.4, 40.8, 40.7, 32.7, 31.7, 29.8, 29.2, 29.0, 27.2, 25.1, $22.7,17.7,14.2 \mathrm{ppm}$

$\underline{\mathrm{IR}}$

(Thin Film)

$1674 \mathrm{~cm}^{-1}$

$\underline{\text { HRMS }}$ HRMS-ESI (m/z): $[\mathrm{M}+\mathrm{H}]^{+}$calcd for $\mathrm{C}_{10} \mathrm{H}_{19} \mathrm{O}, 155.1430$; found, 155.1431

HRMS-ESI (m/z): $[\mathrm{M}+\mathrm{H}]^{+}$calcd for $\mathrm{C}_{10} \mathrm{H}_{18} \mathrm{DO}, 156.1493$; found, 156.1494

$\underline{\text { TLC }} \quad R_{f}=0.58$ (35\% ethyl acetate/hexanes); silica gel, UV, PAA 
Supporting Information for Organic Letters

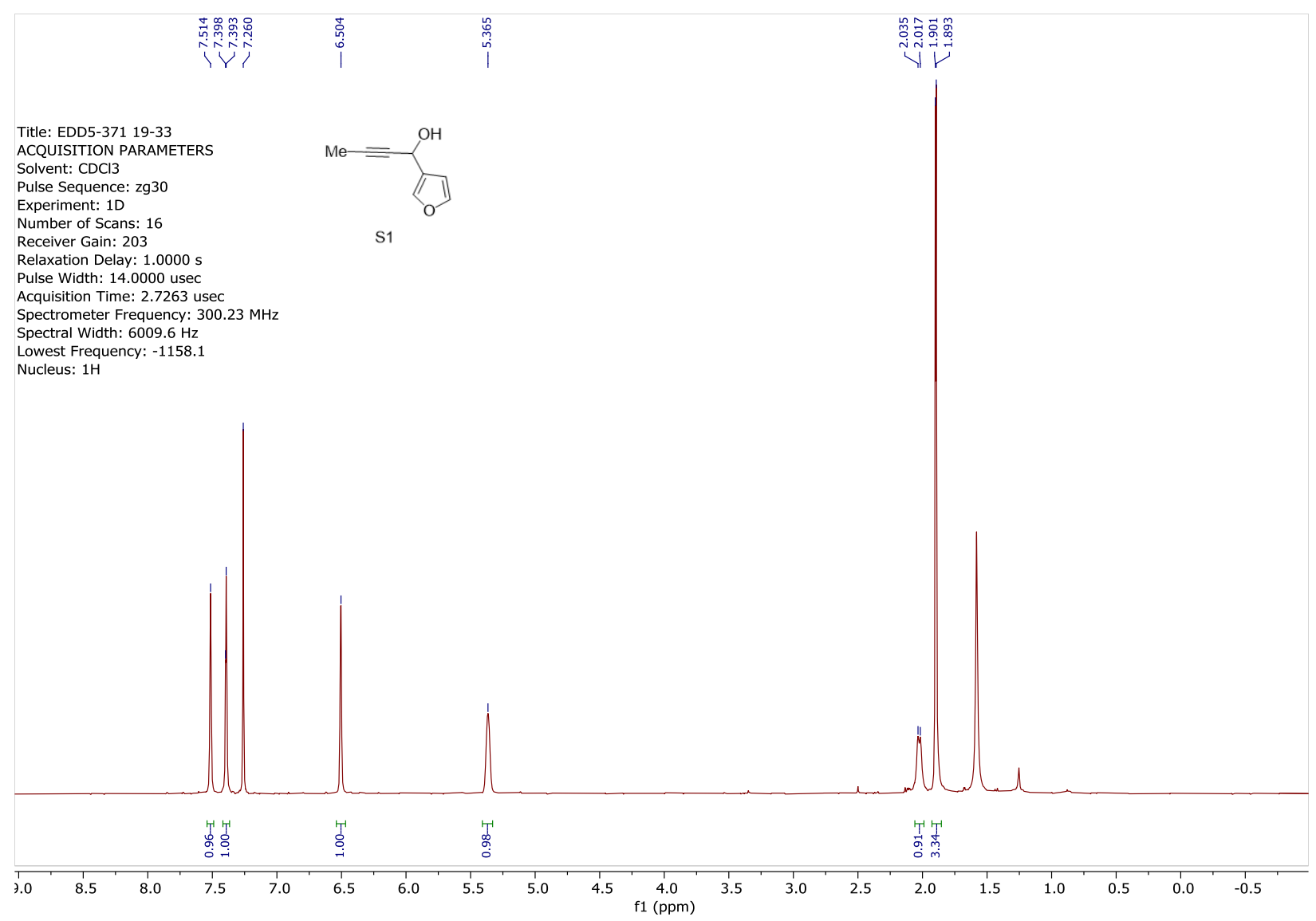


Supporting Information for Organic Letters

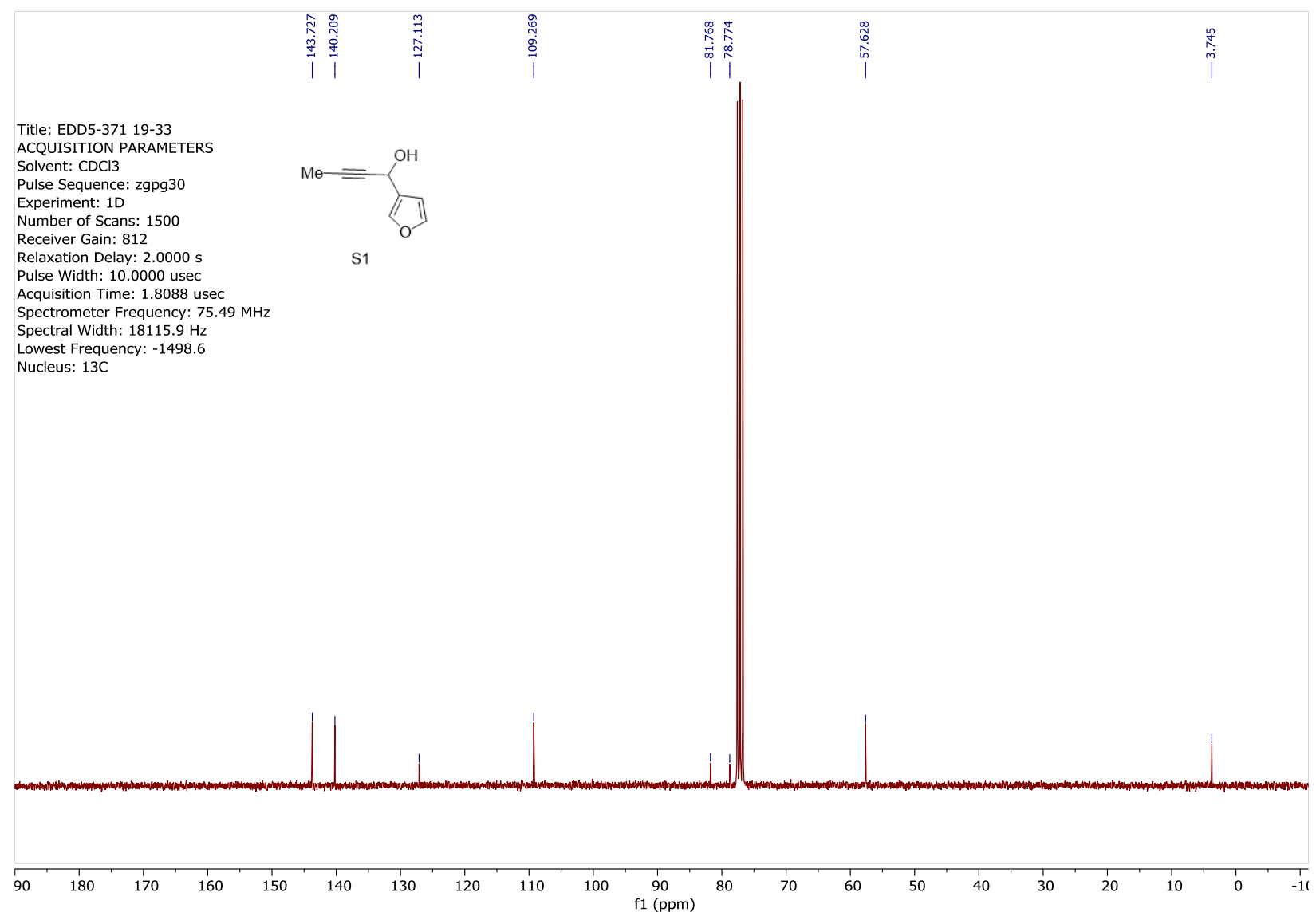


Supporting Information for Organic Letters

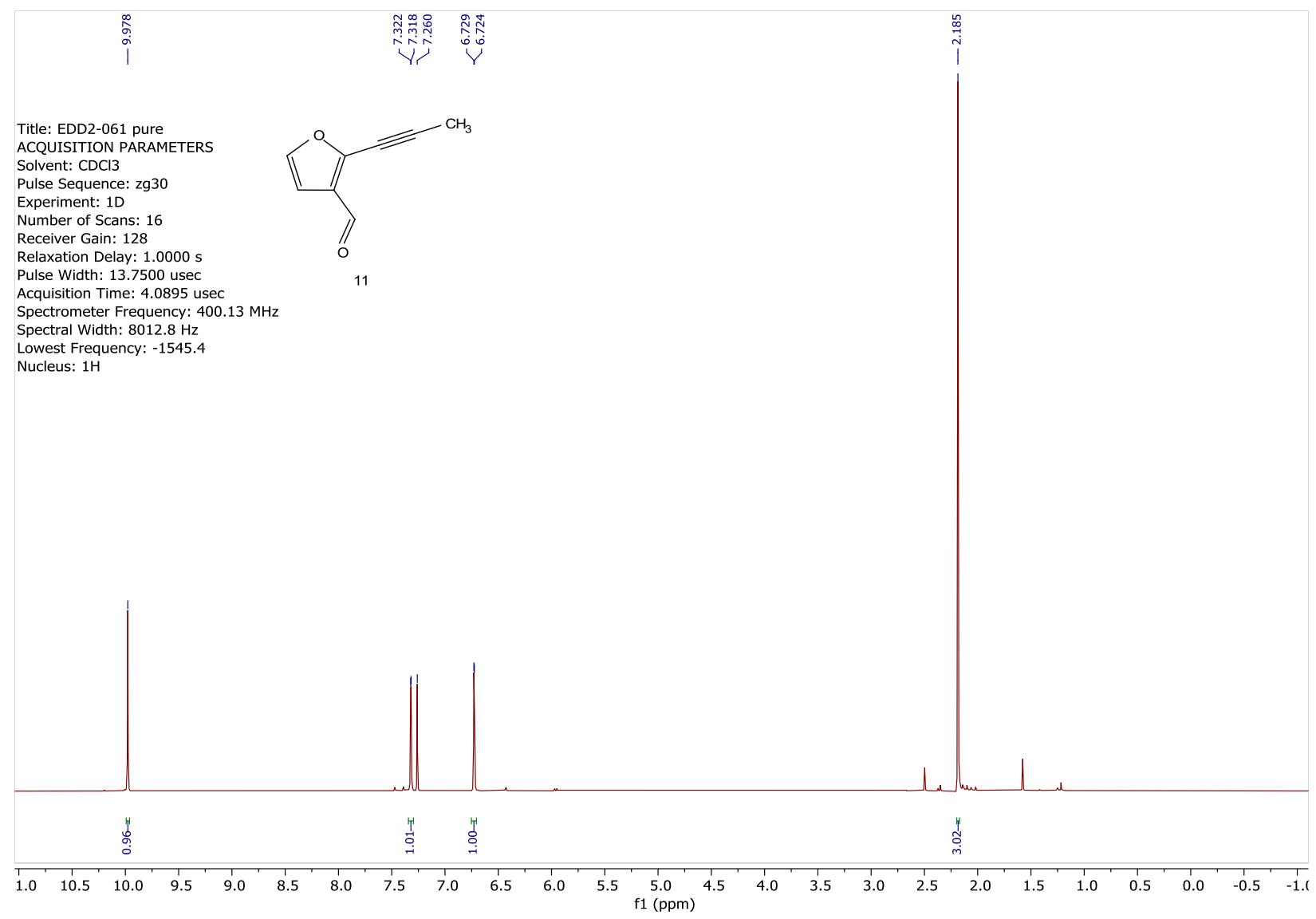


Supporting Information for Organic Letters

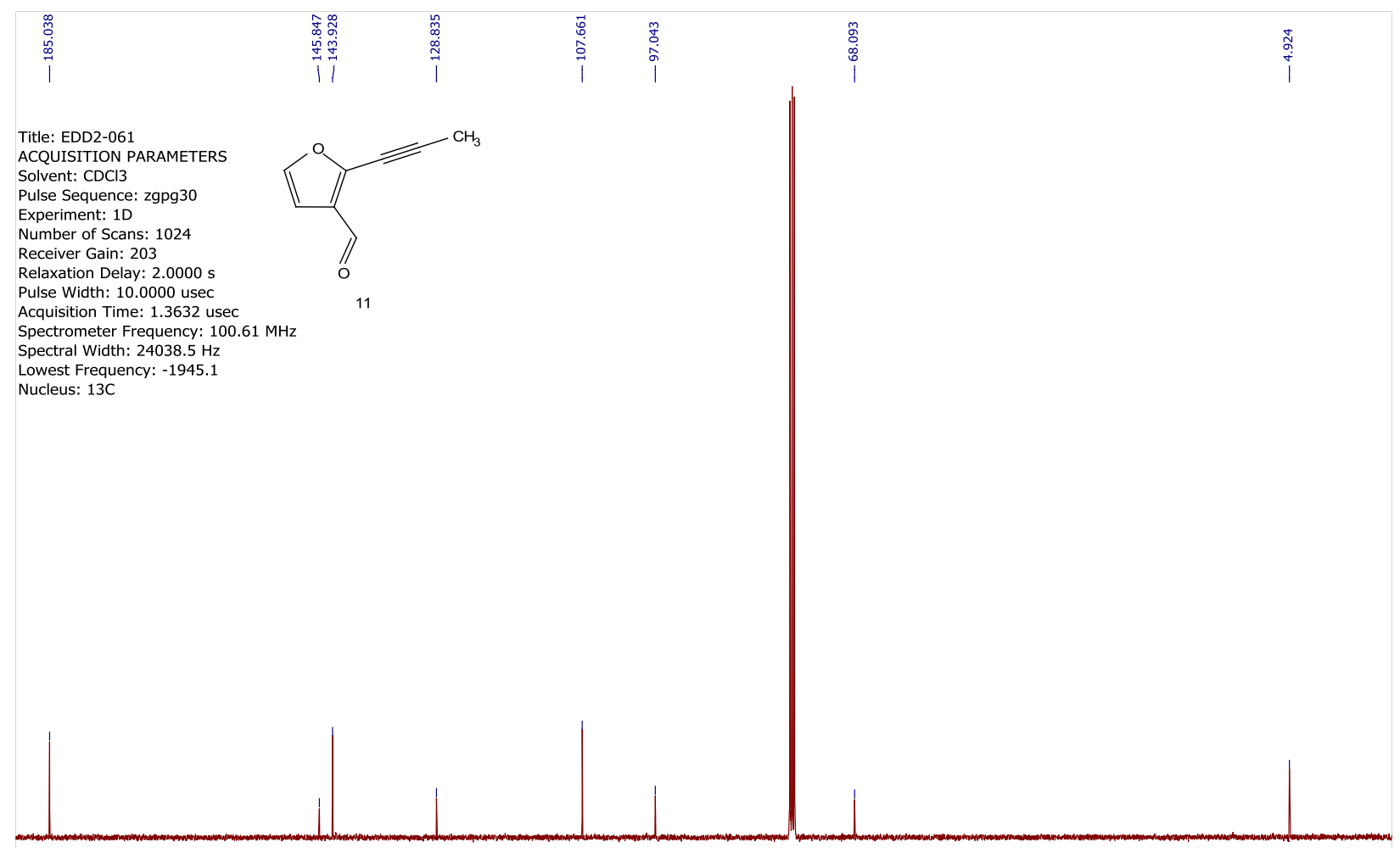

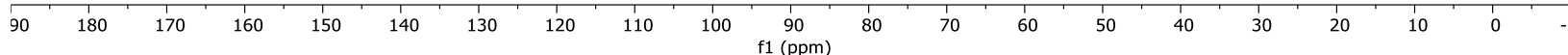




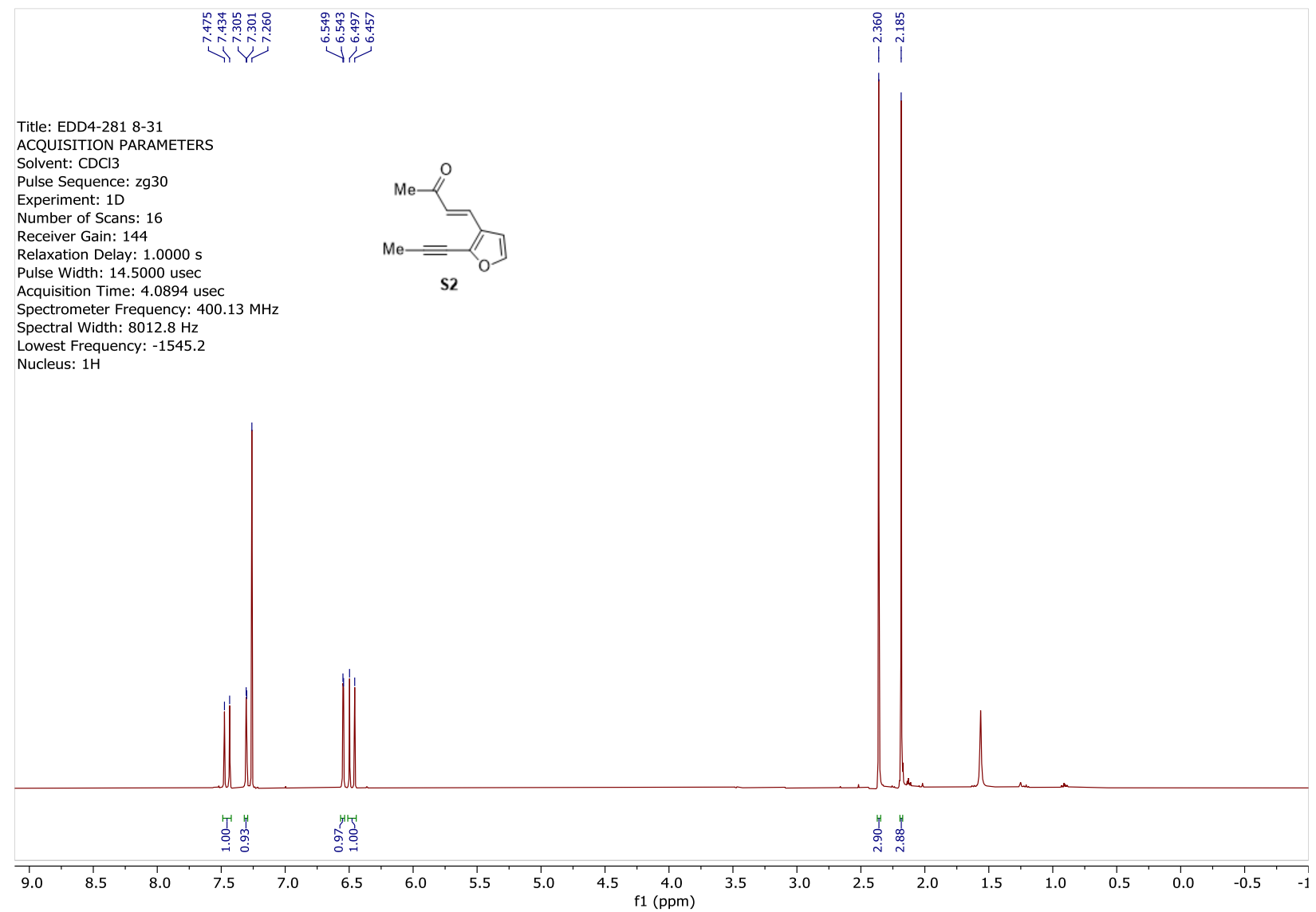


Supporting Information for Organic Letters
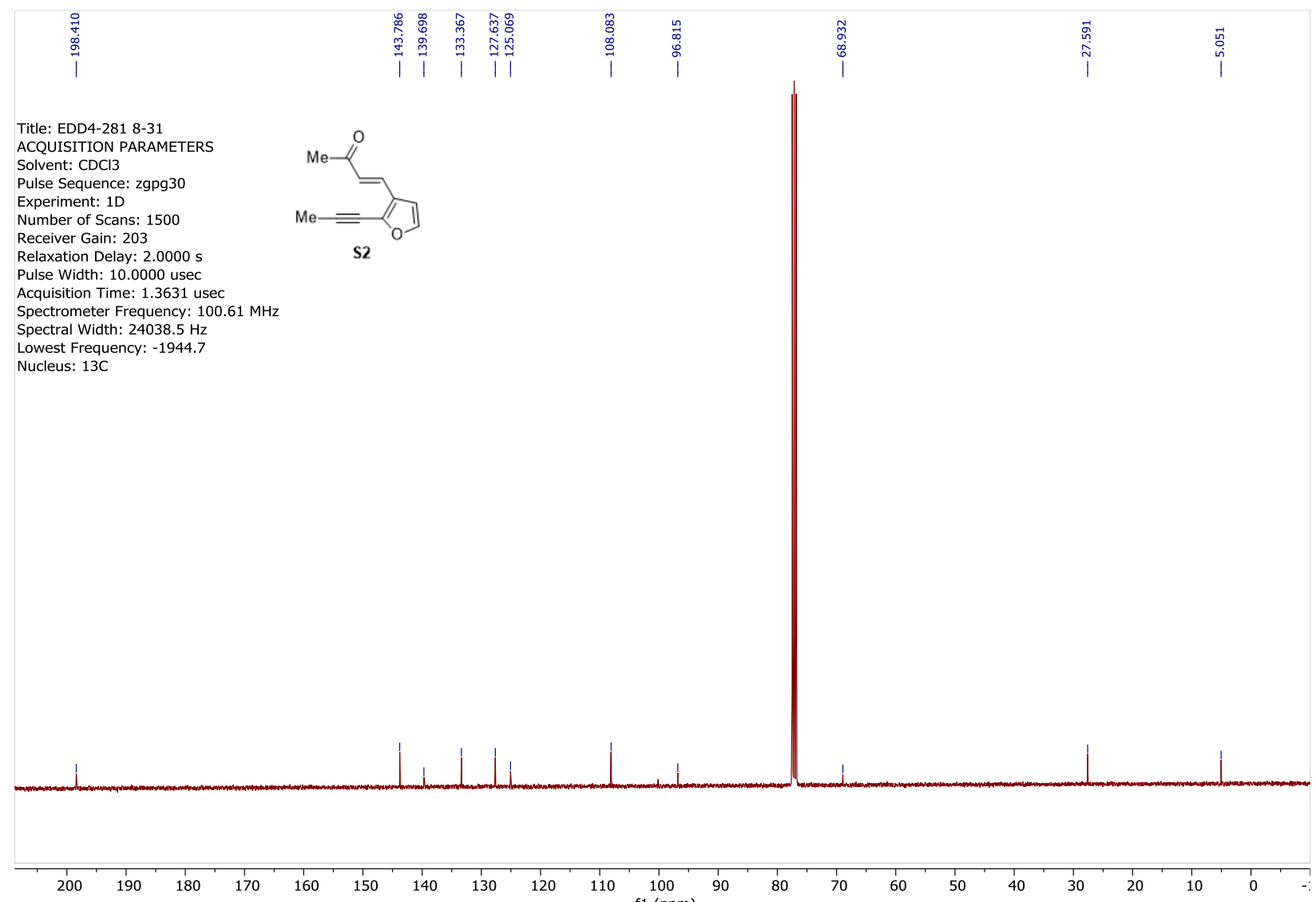

f1 (ppm) 


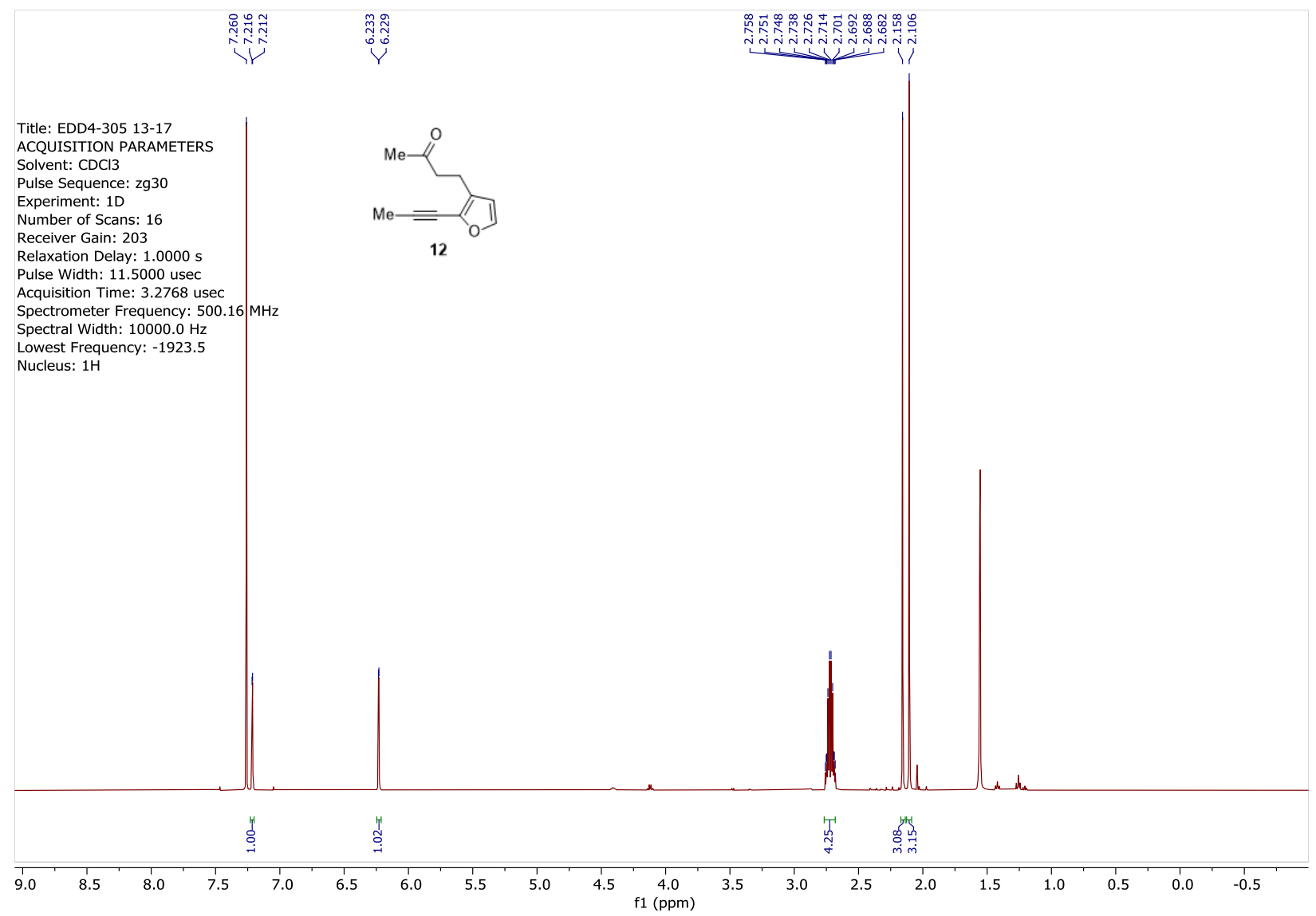




\section{i̊n.}

Title: EDD-ketone

ACQUISITION PARAMETERS

Solvent: $\mathrm{CDCl} 3$

Pulse Sequence: zgpg30

Experiment: 1D

Number of Scans: 1024

Receiver Gain: 203

Relaxation Delay: $2.0000 \mathrm{~s}$

Pulse Width: 10.0000 usec

Acquisition Time: 1.3632 usec

Spectrometer Frequency: $100.61 \mathrm{MHz}$

Spectral Width: $24038.5 \mathrm{~Hz}$
Lowest Frequency: -1945.4

Nucleus: $13 \mathrm{C}$

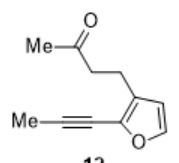

12

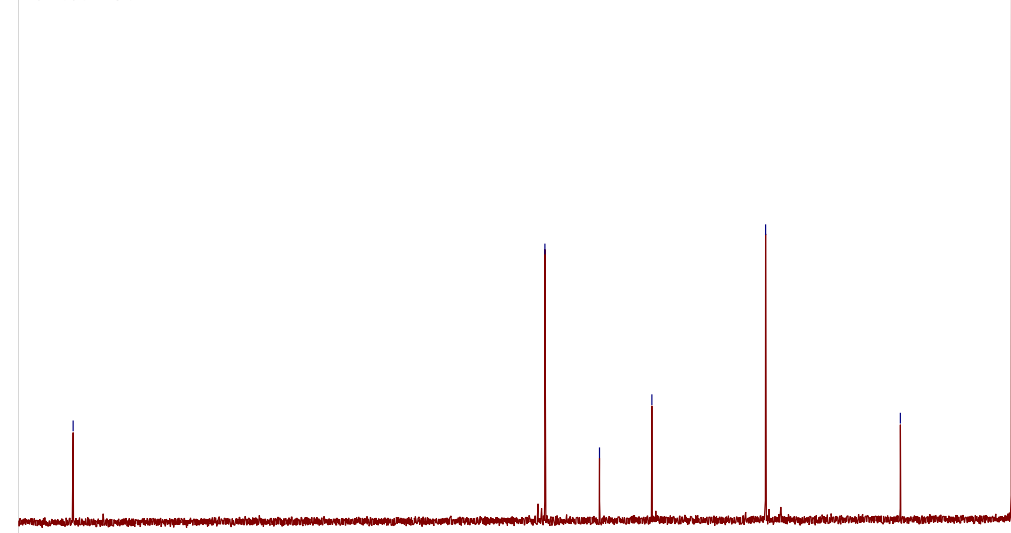

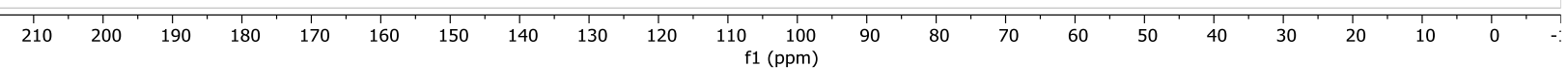


Supporting Information for Organic Letters

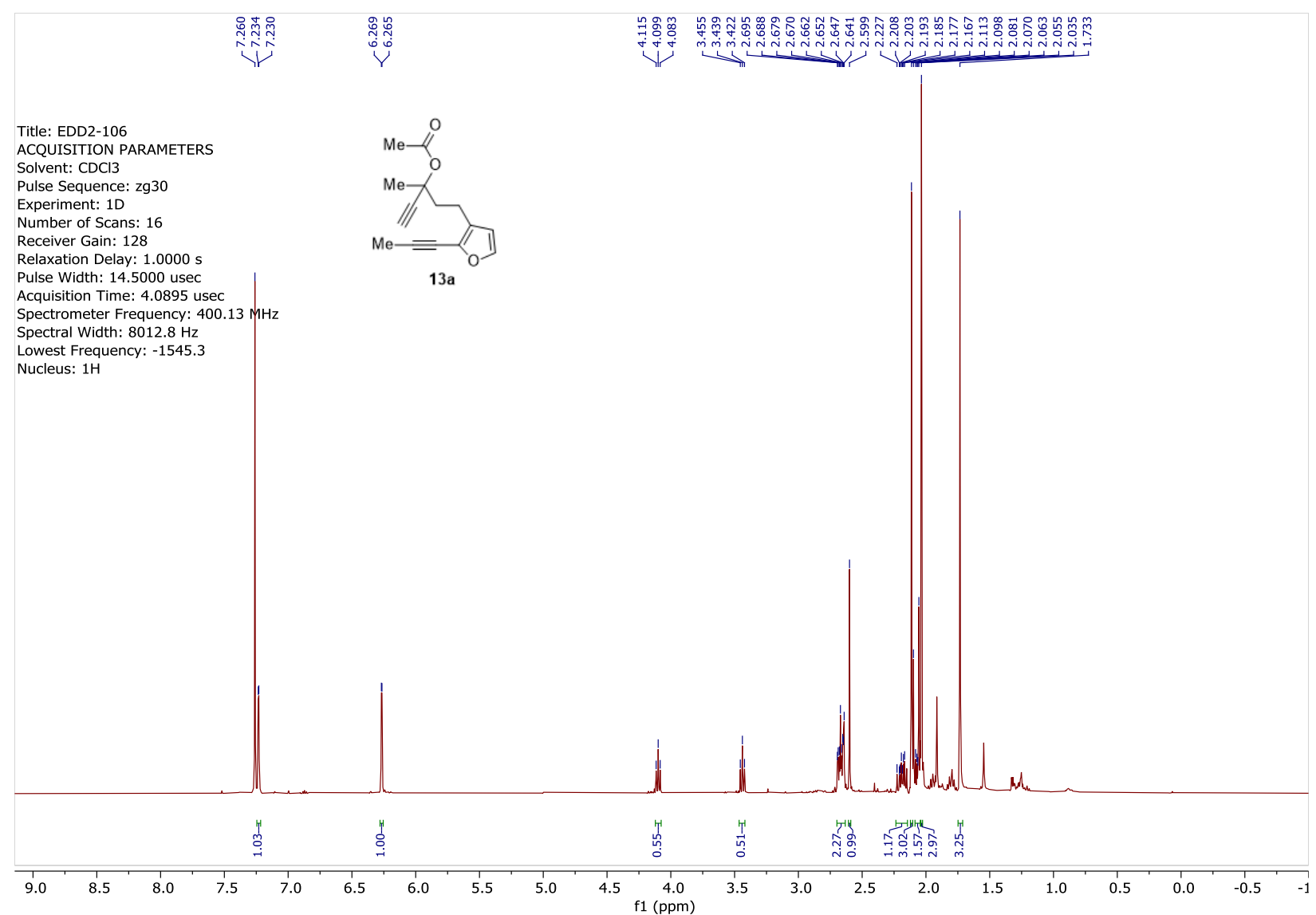


Supporting Information for Organic Letters

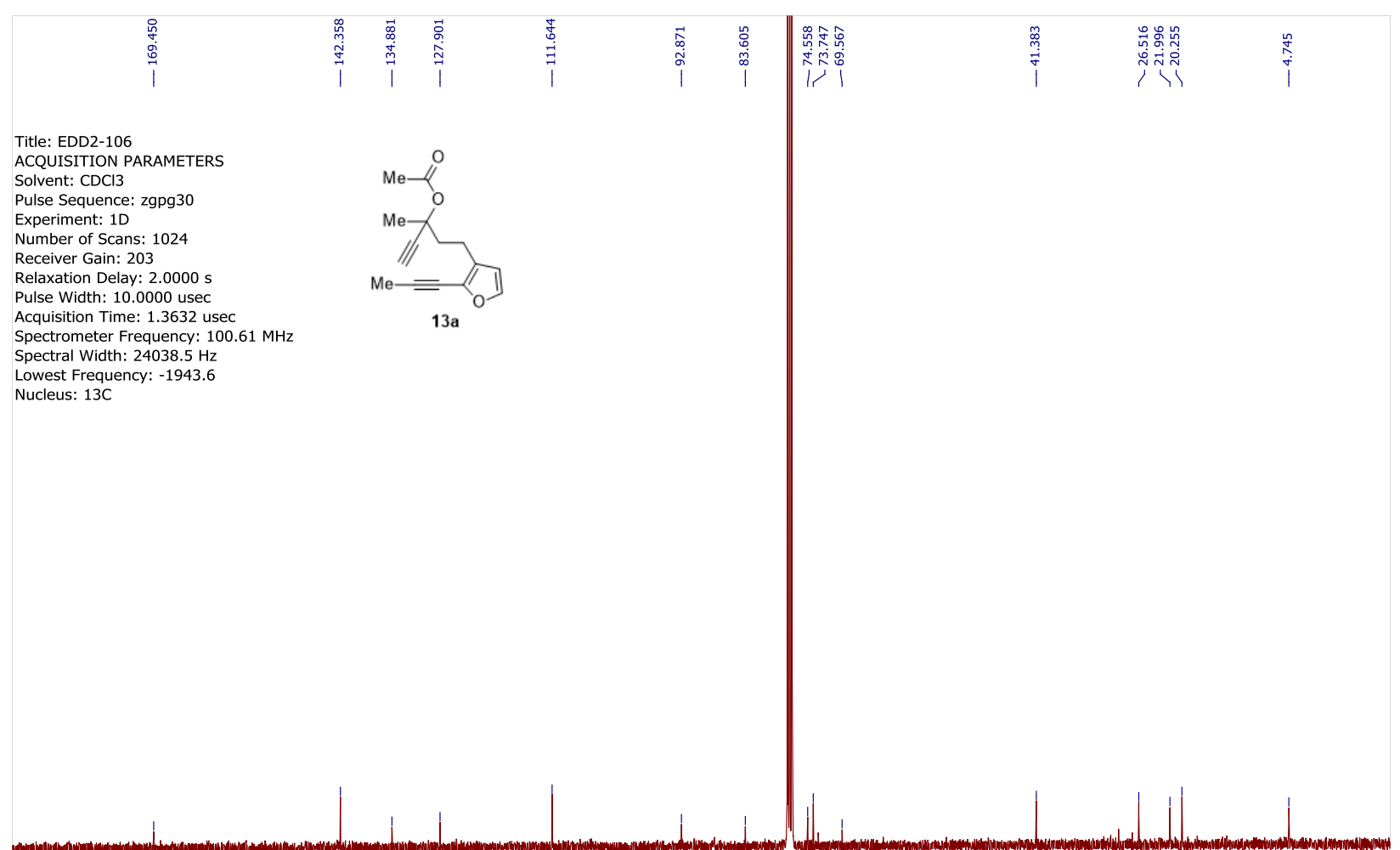

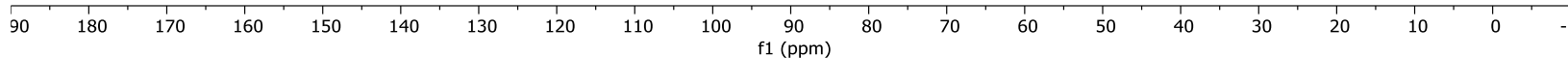


Supporting Information for Organic Letters

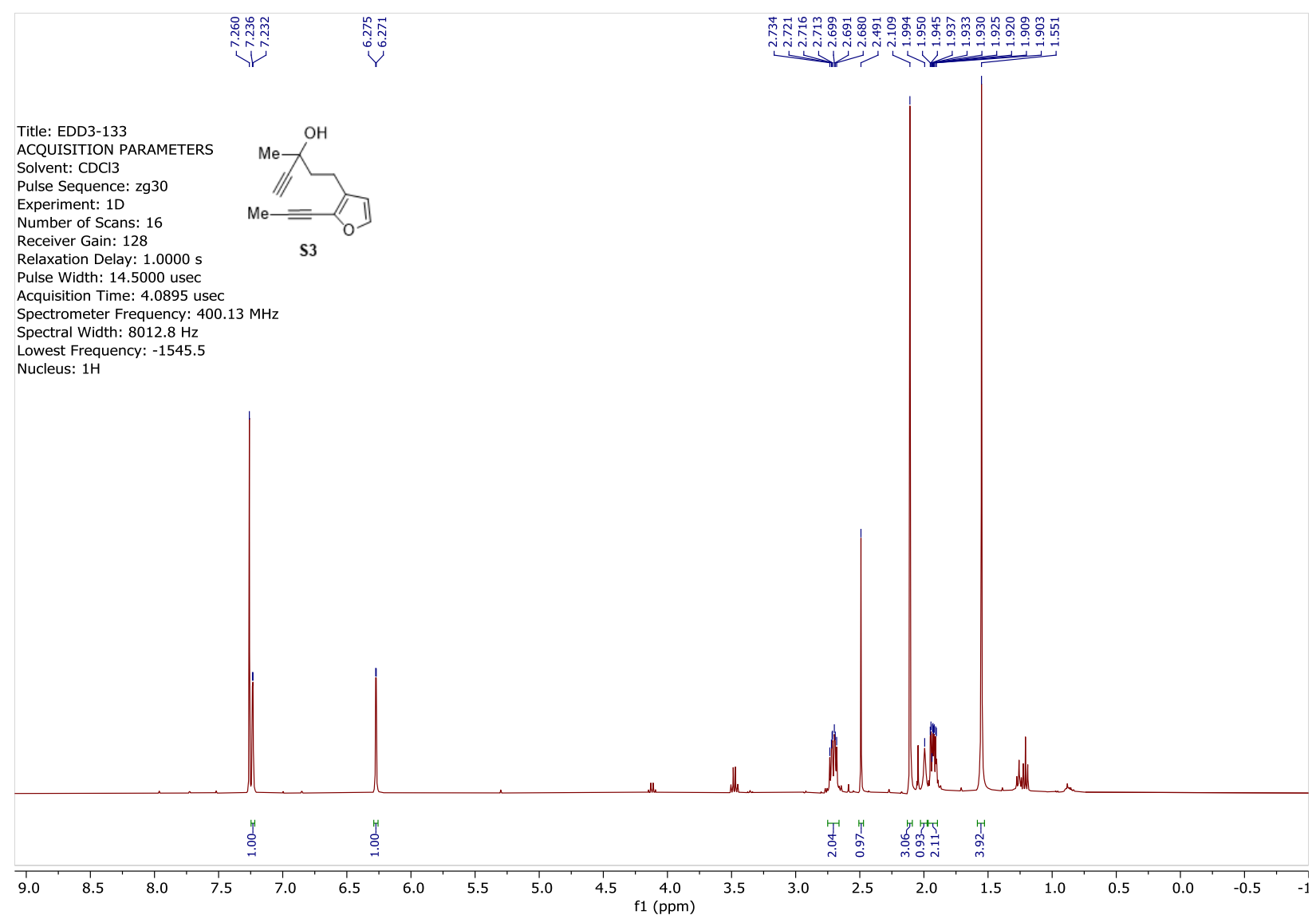


Supporting Information for Organic Letters

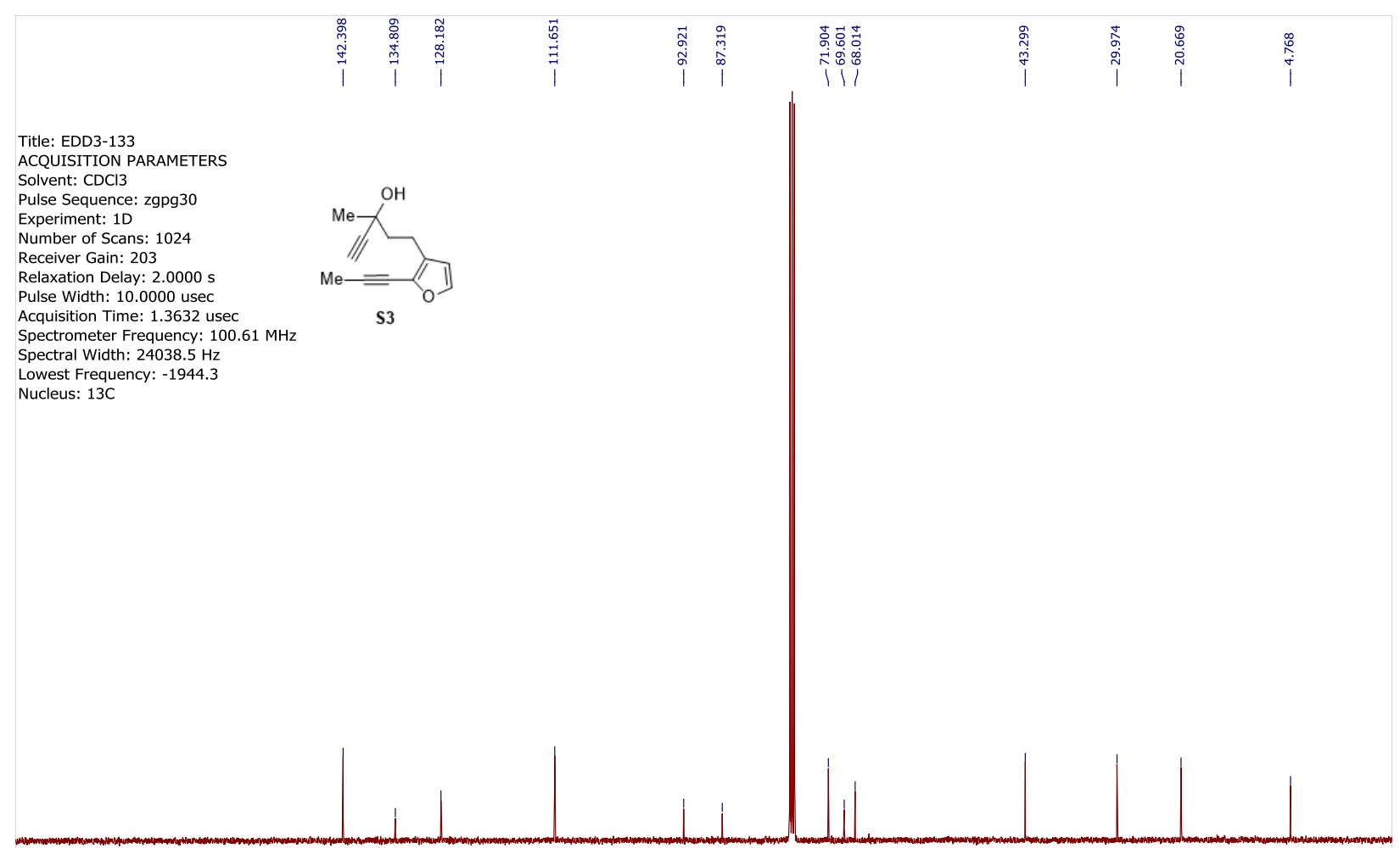

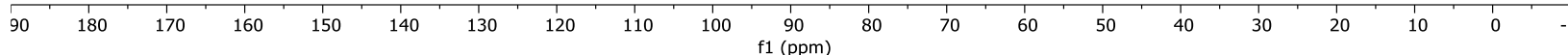


Supporting Information for Organic Letters

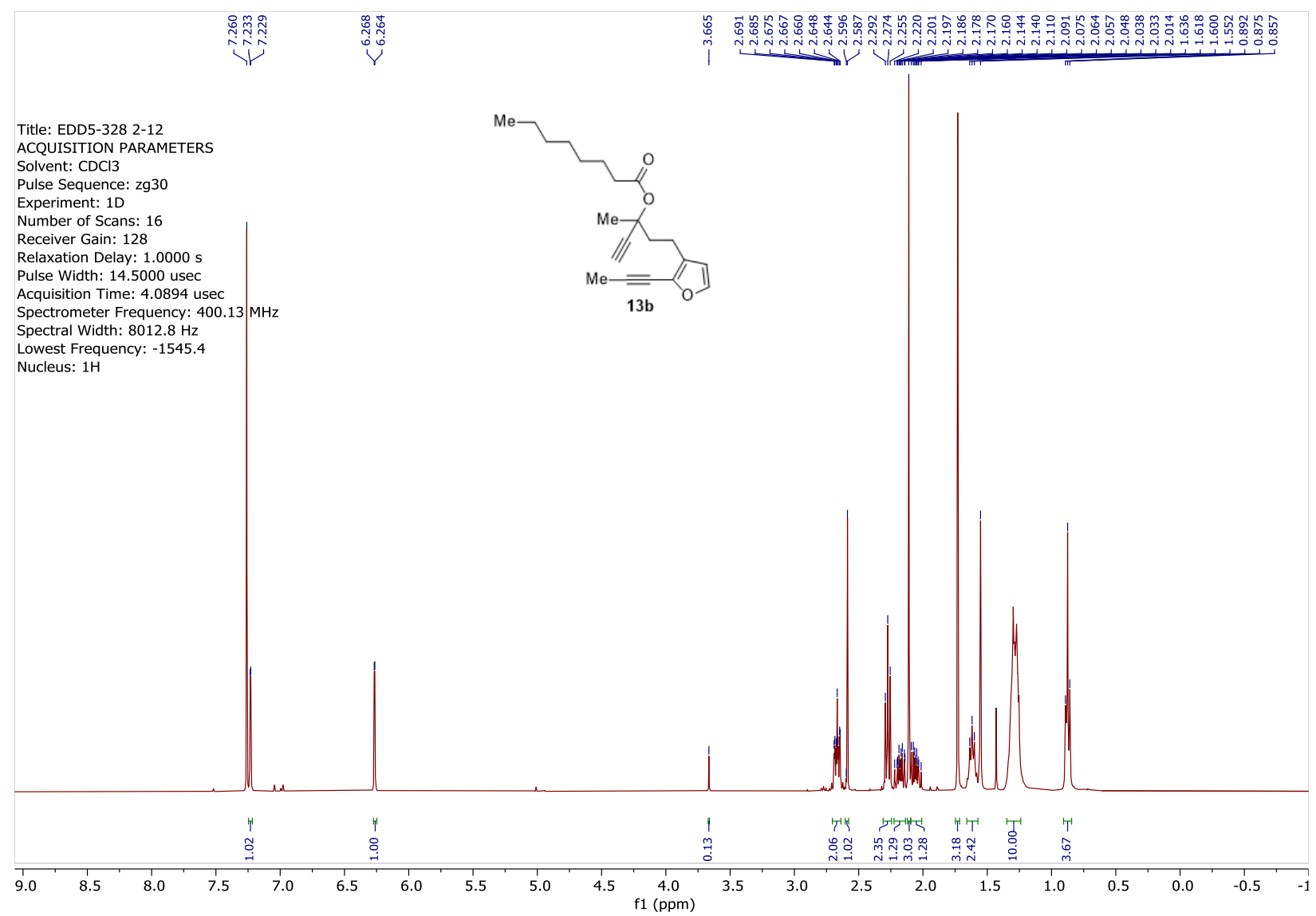


Supporting Information for Organic Letters

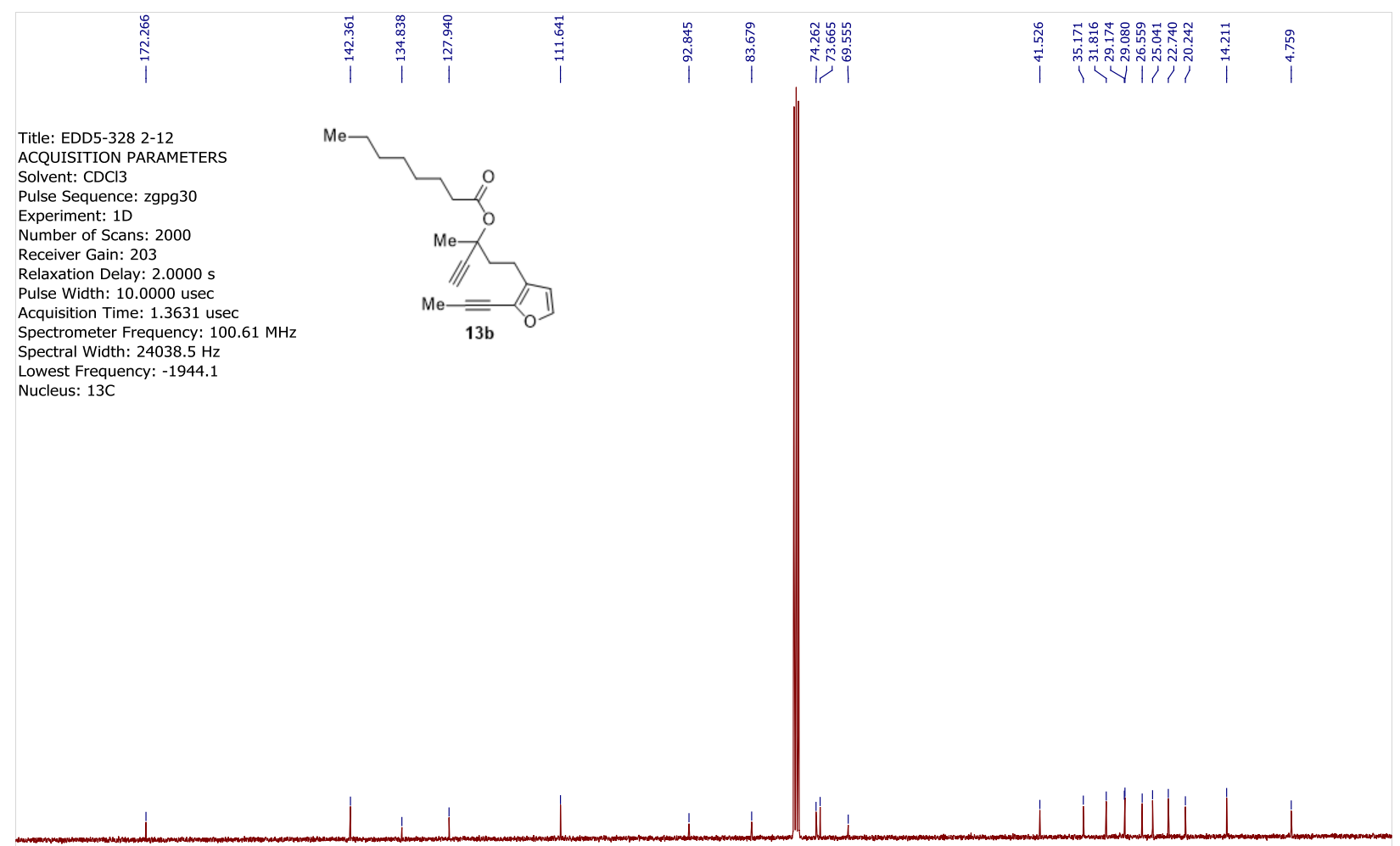

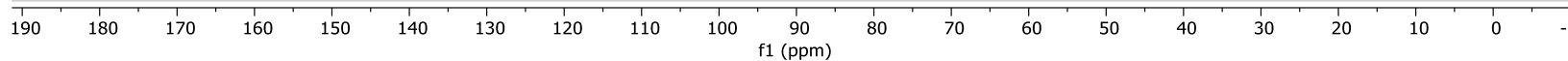


Supporting Information for Organic Letters

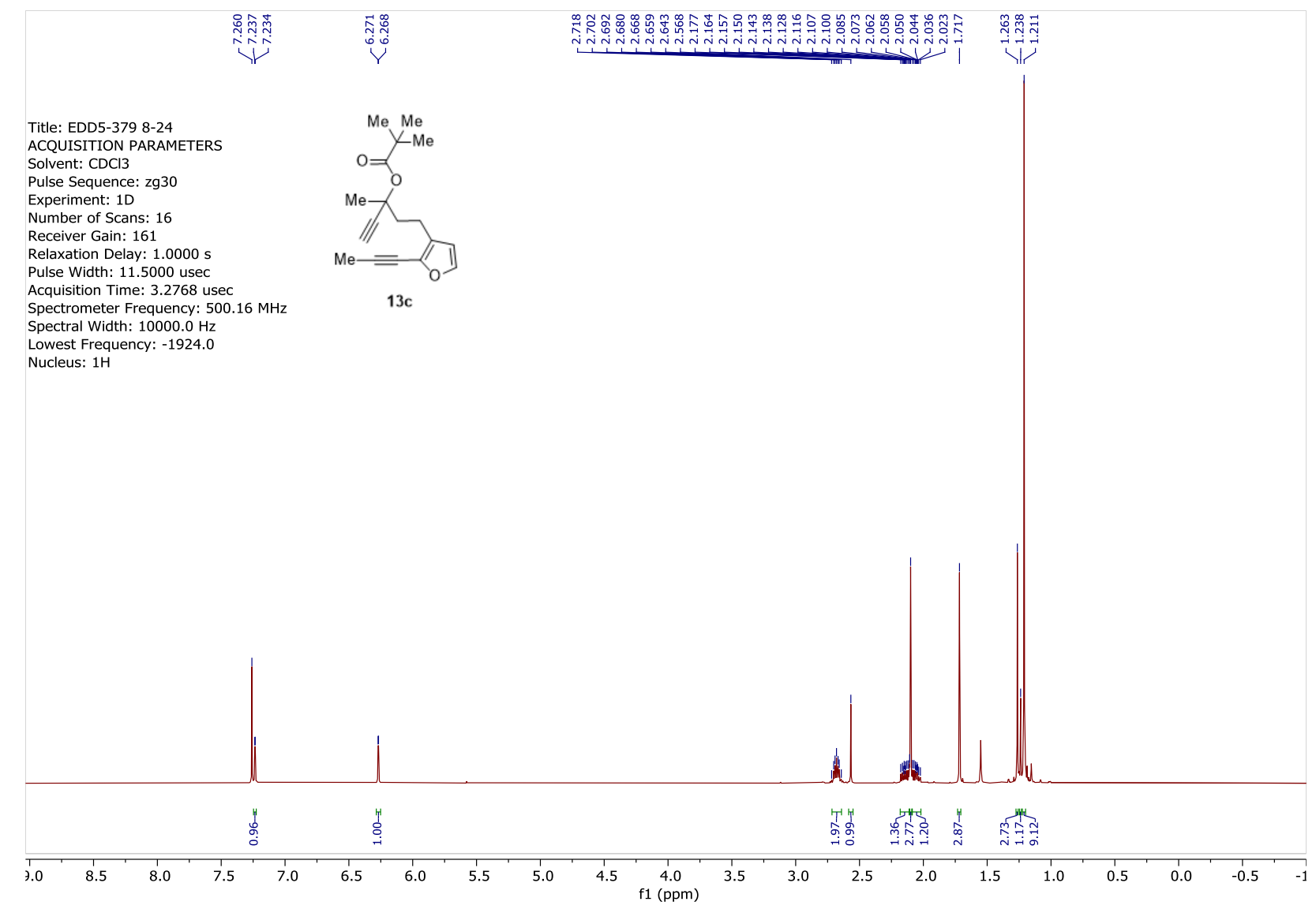


Supporting Information for Organic Letters

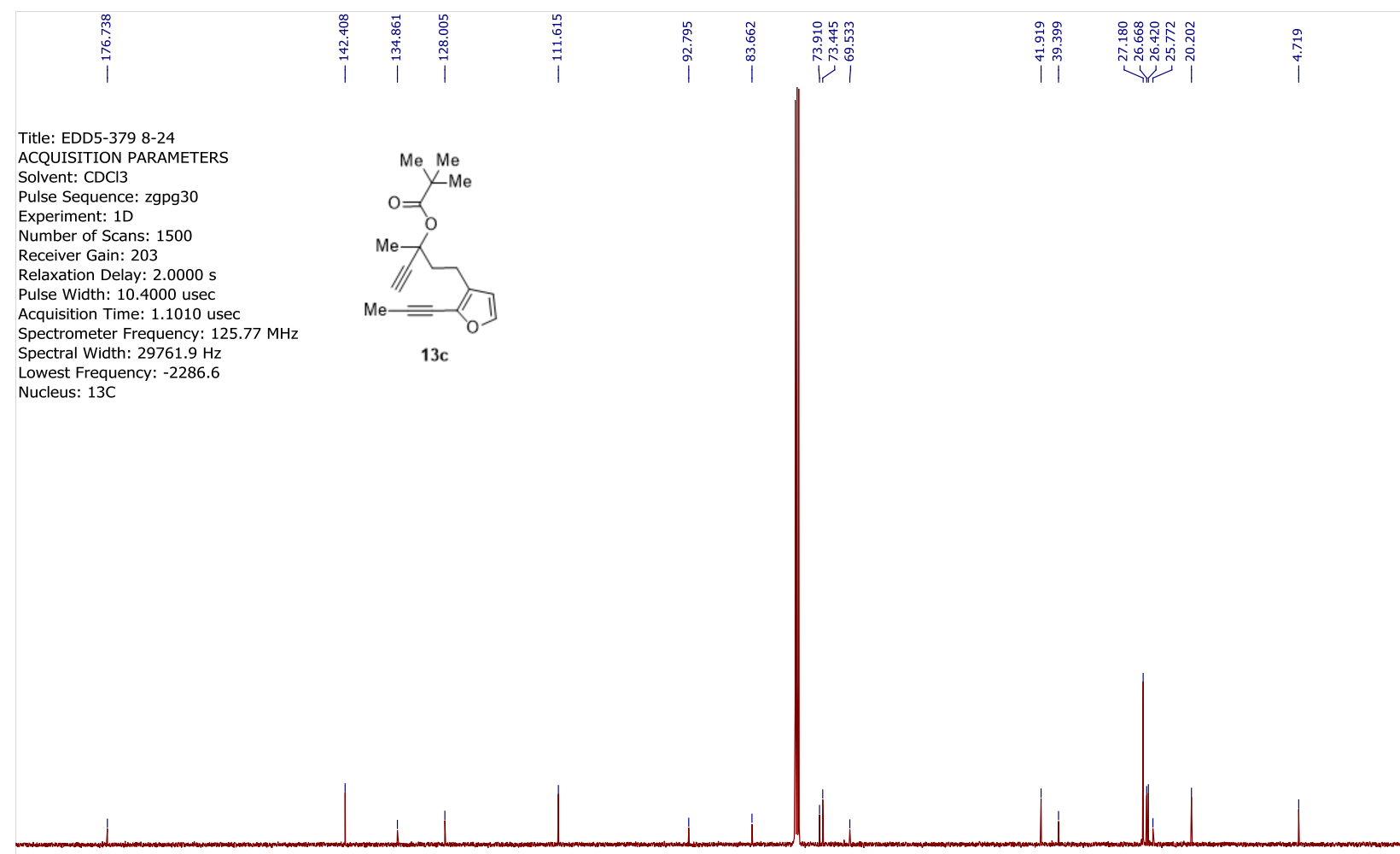

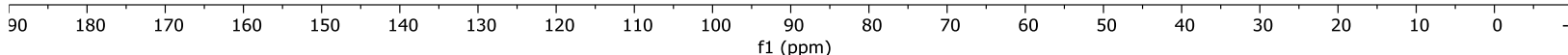


Supporting Information for Organic Letters

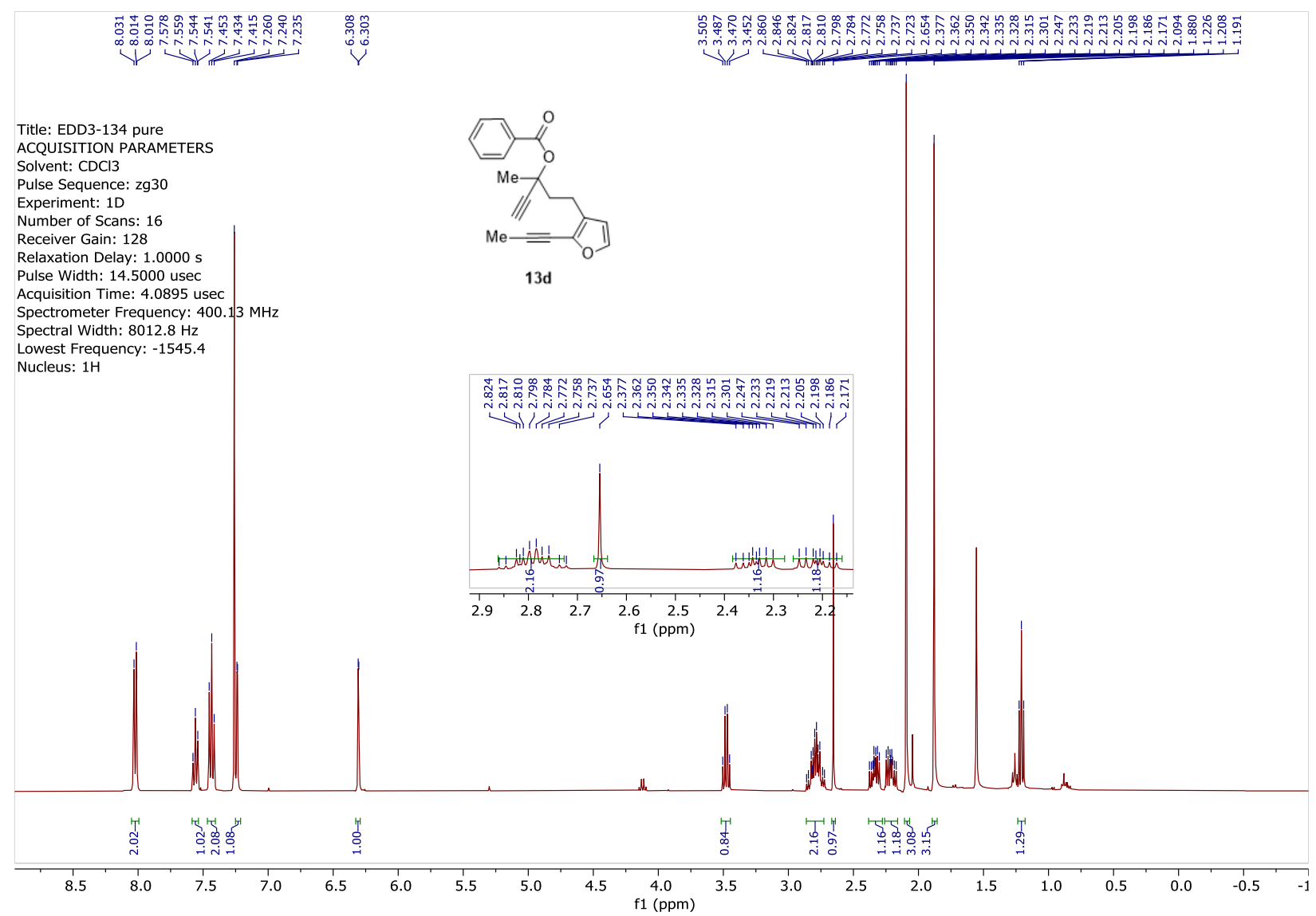


Supporting Information for Organic Letters

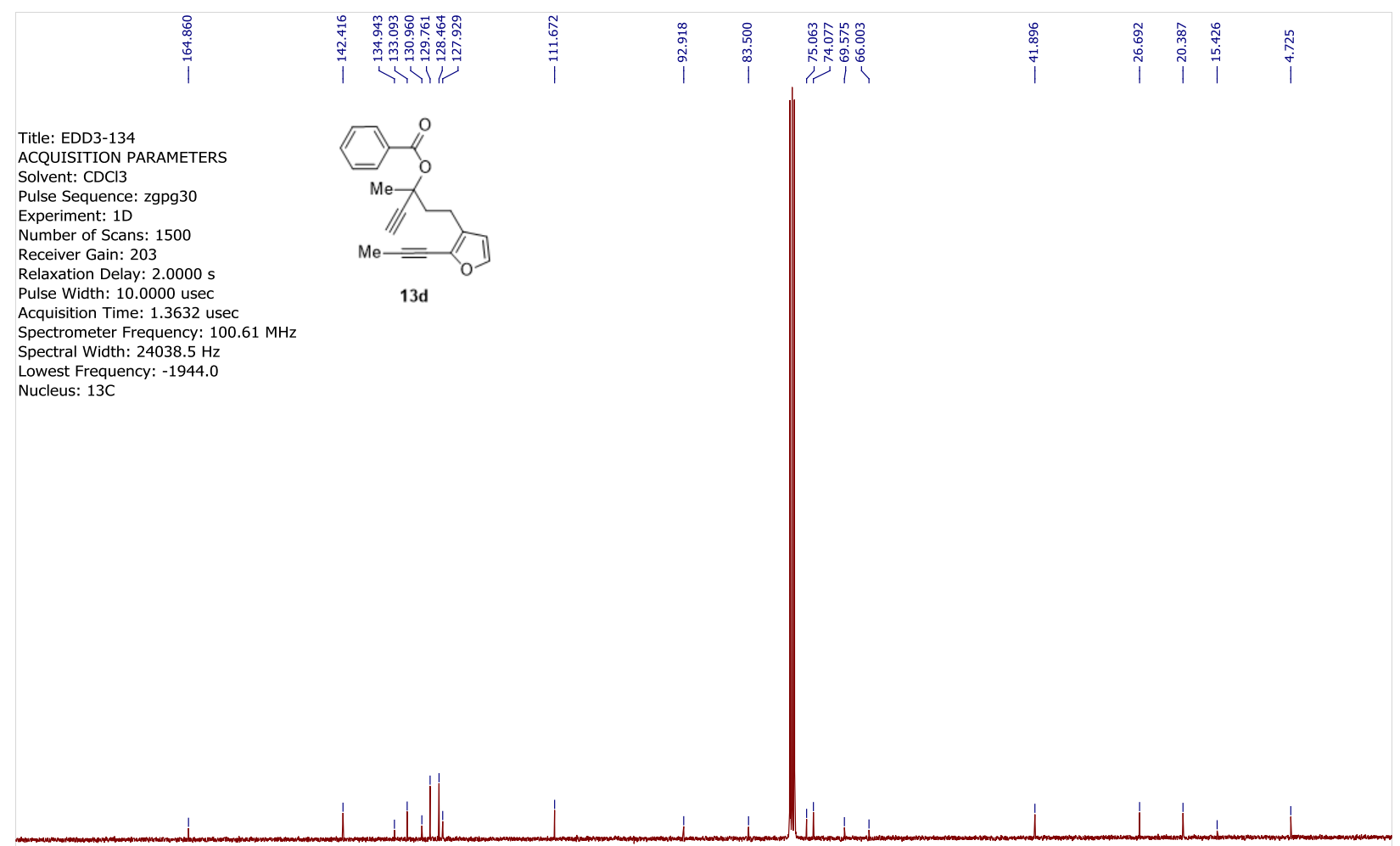

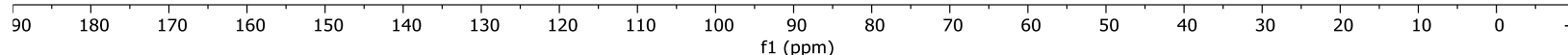


Supporting Information for Organic Letters

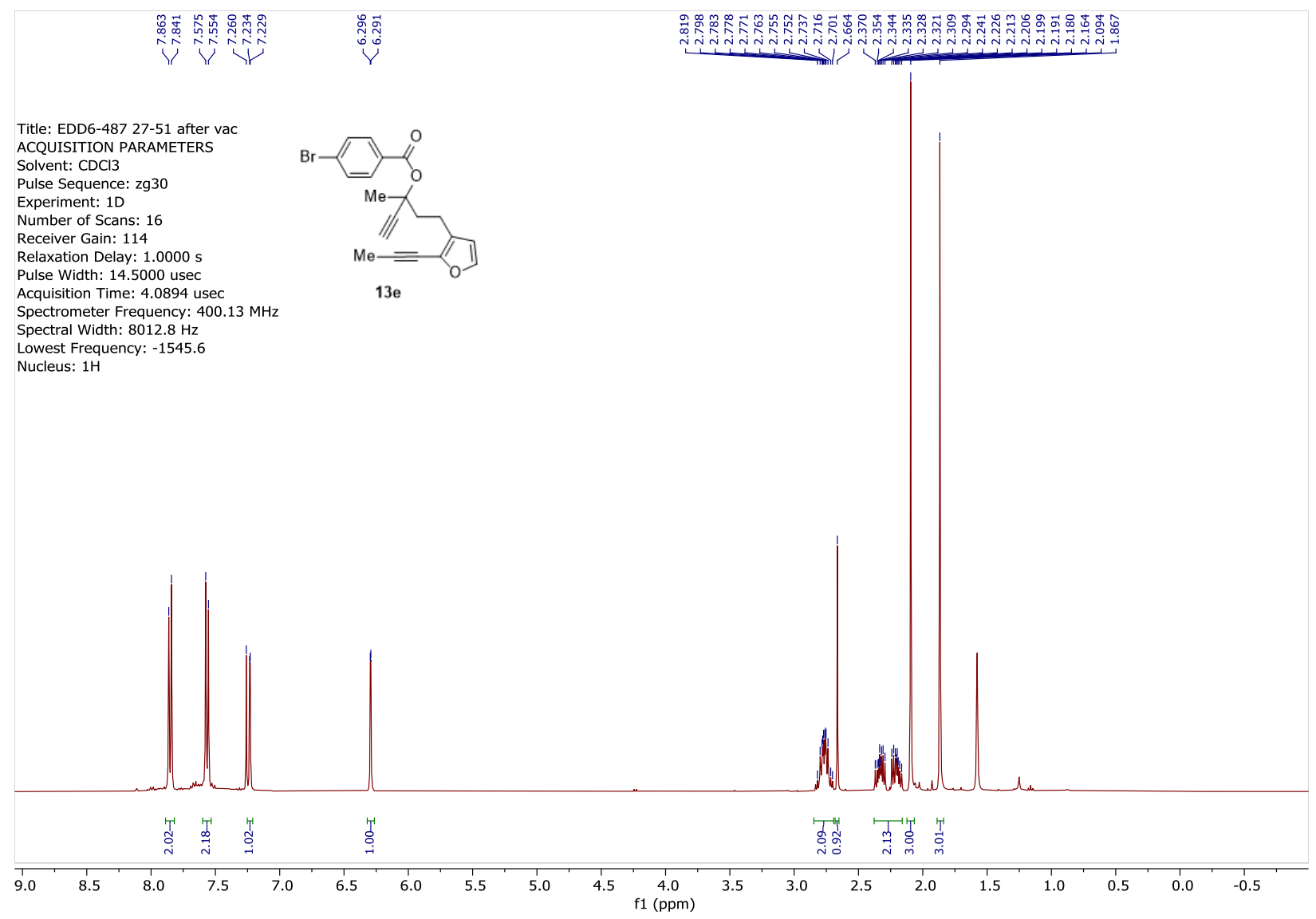


Supporting Information for Organic Letters

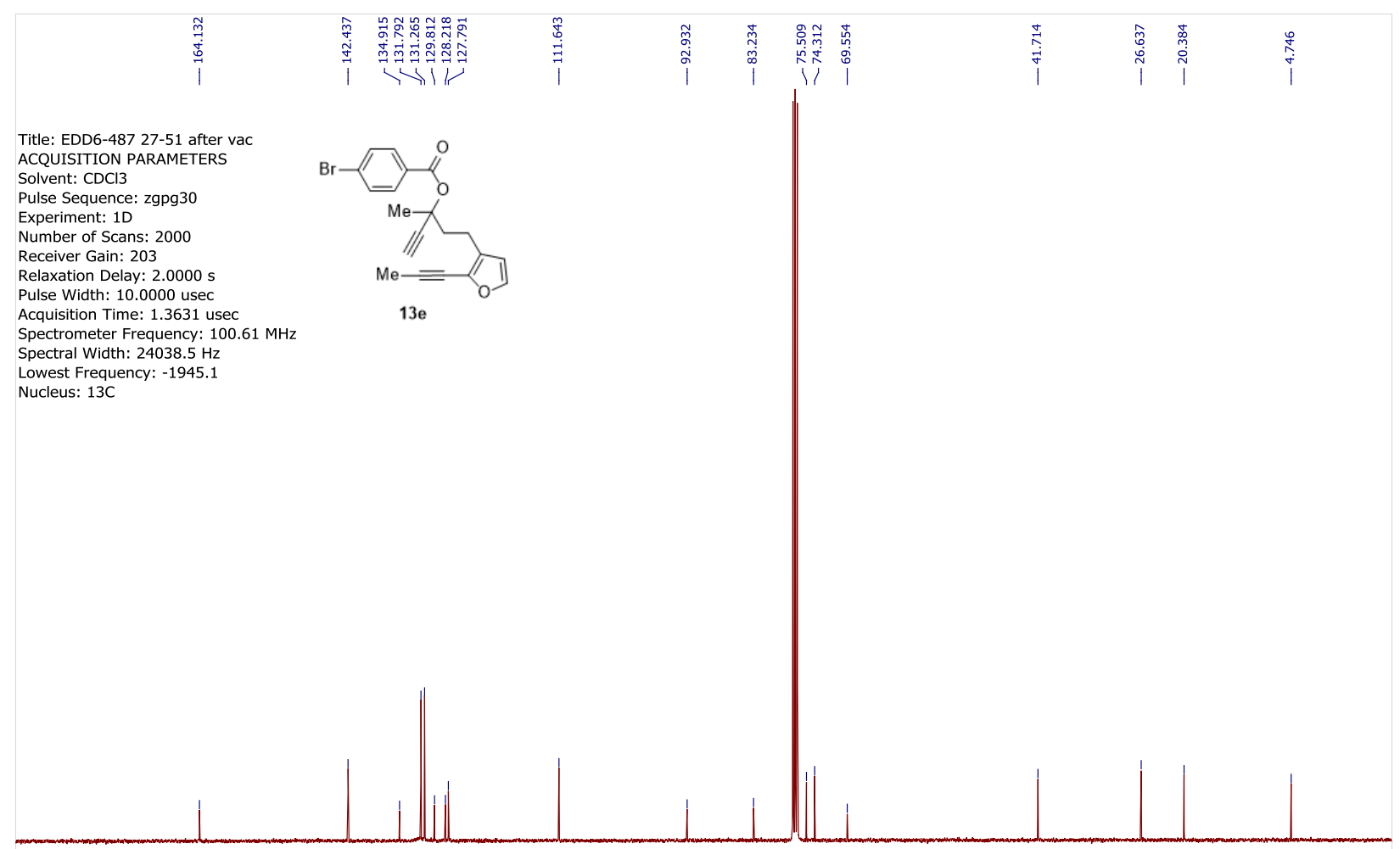

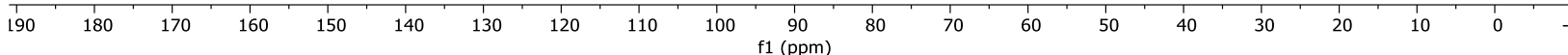


Supporting Information for Organic Letters

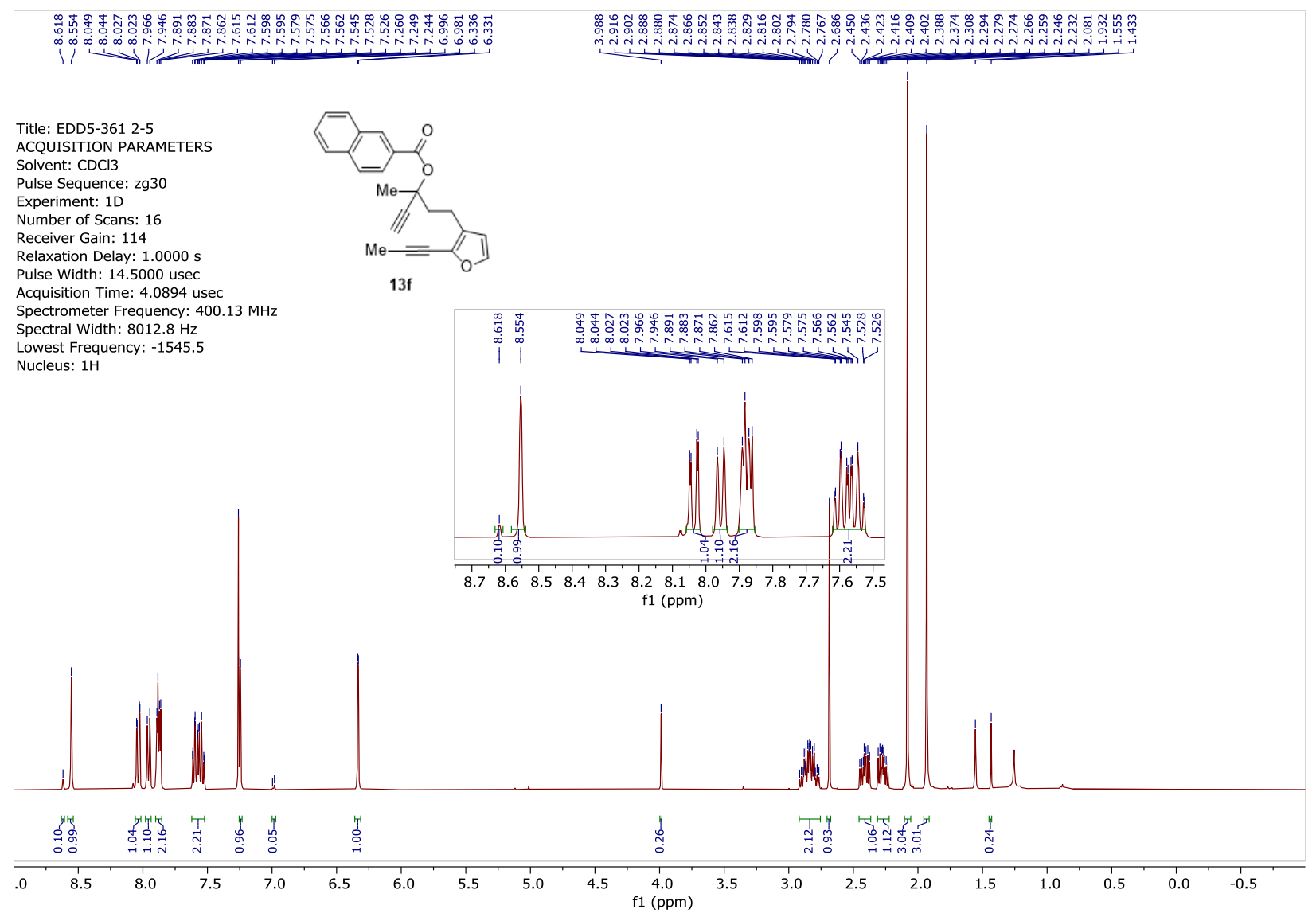


Supporting Information for Organic Letters
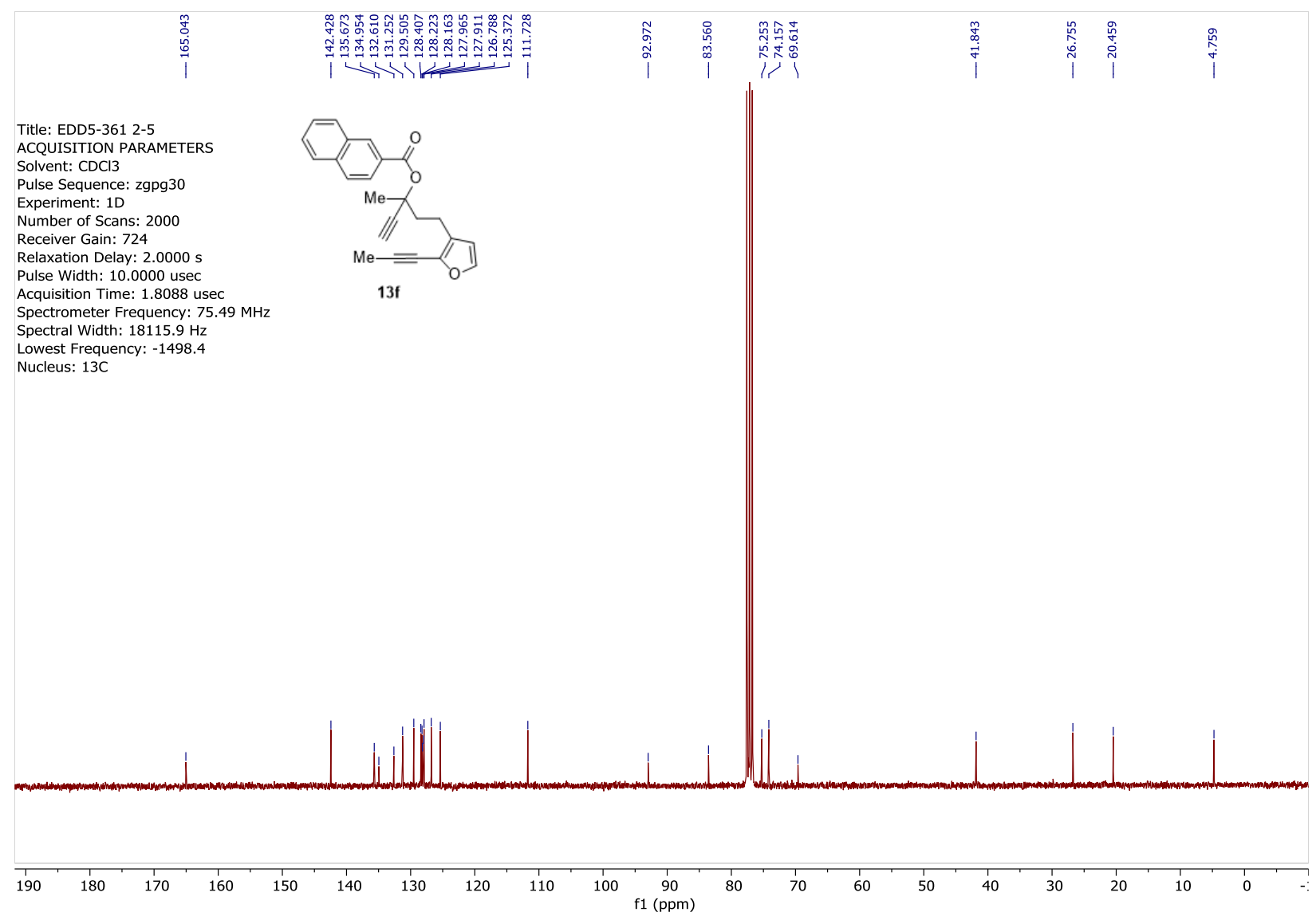
Supporting Information for Organic Letters

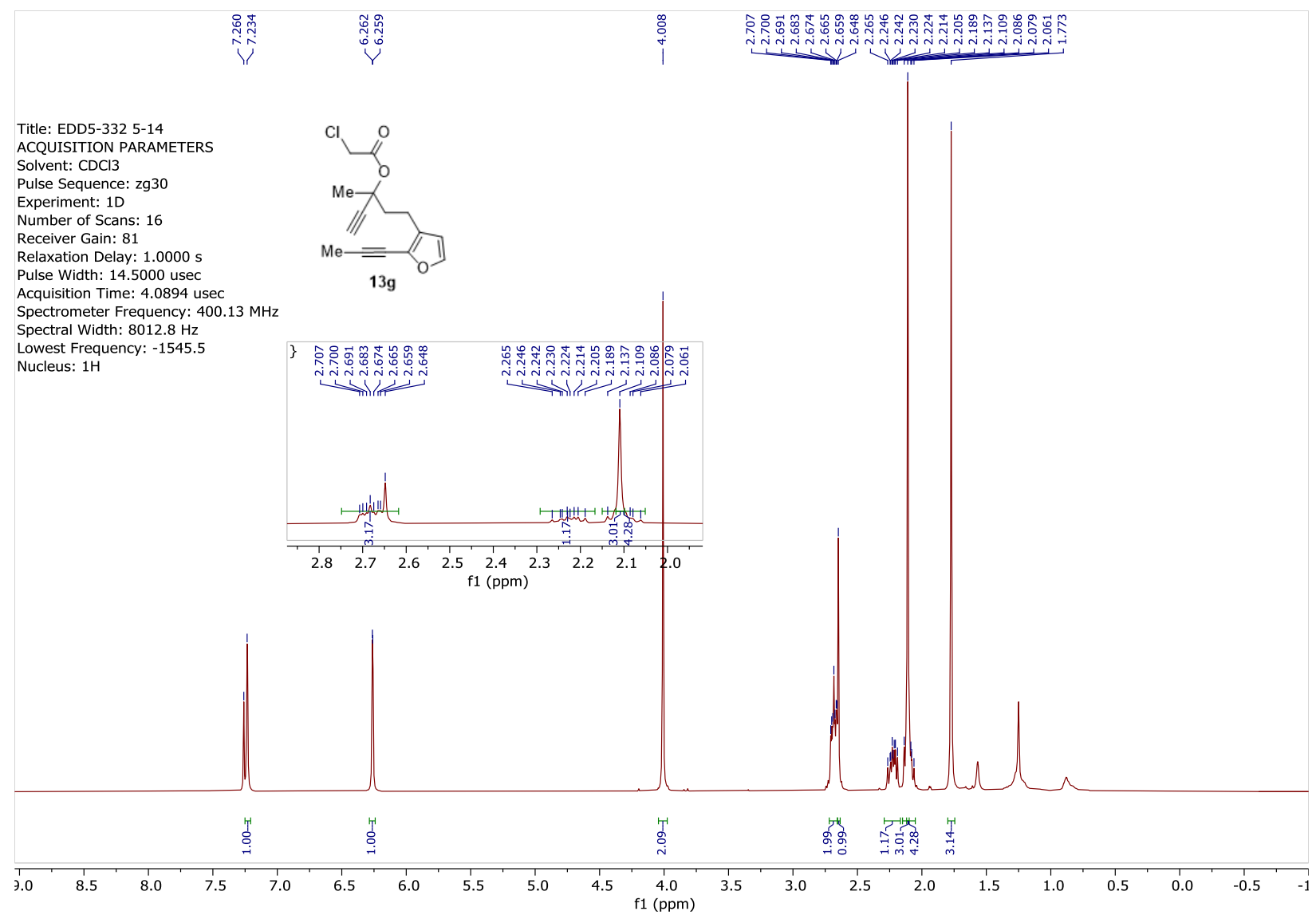


Supporting Information for Organic Letters

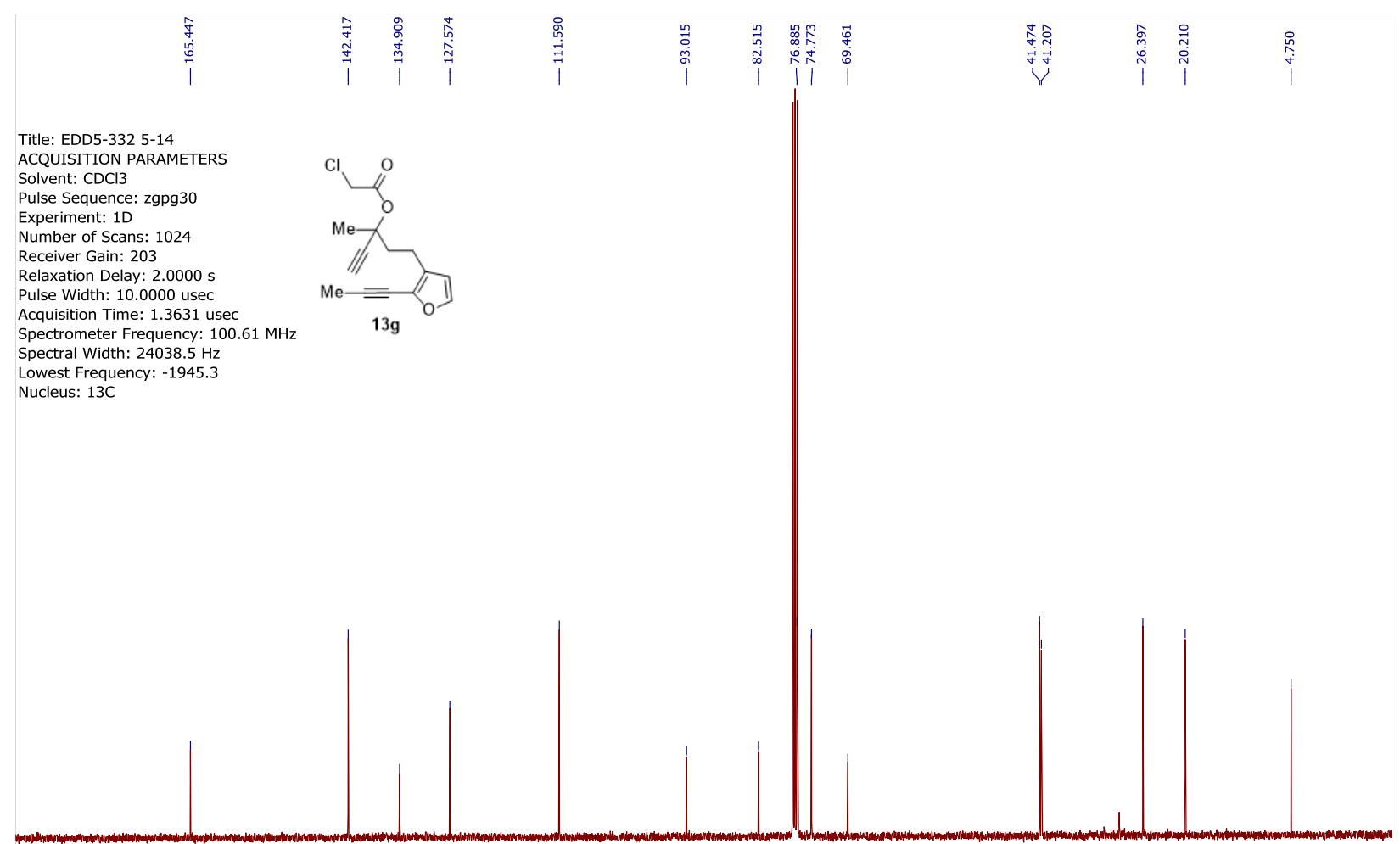

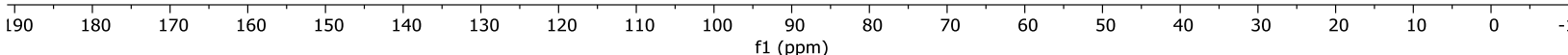




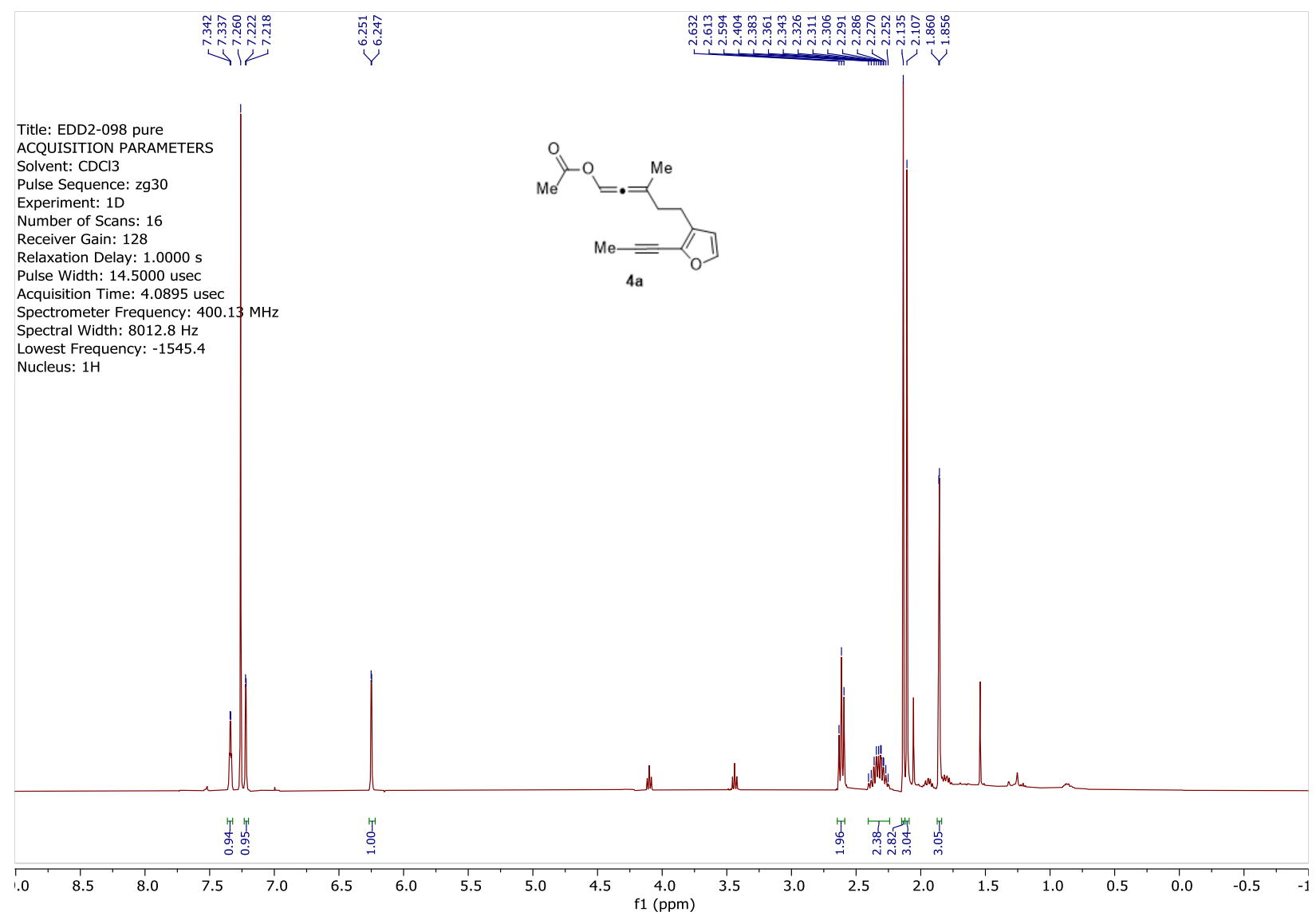


Supporting Information for Organic Letters

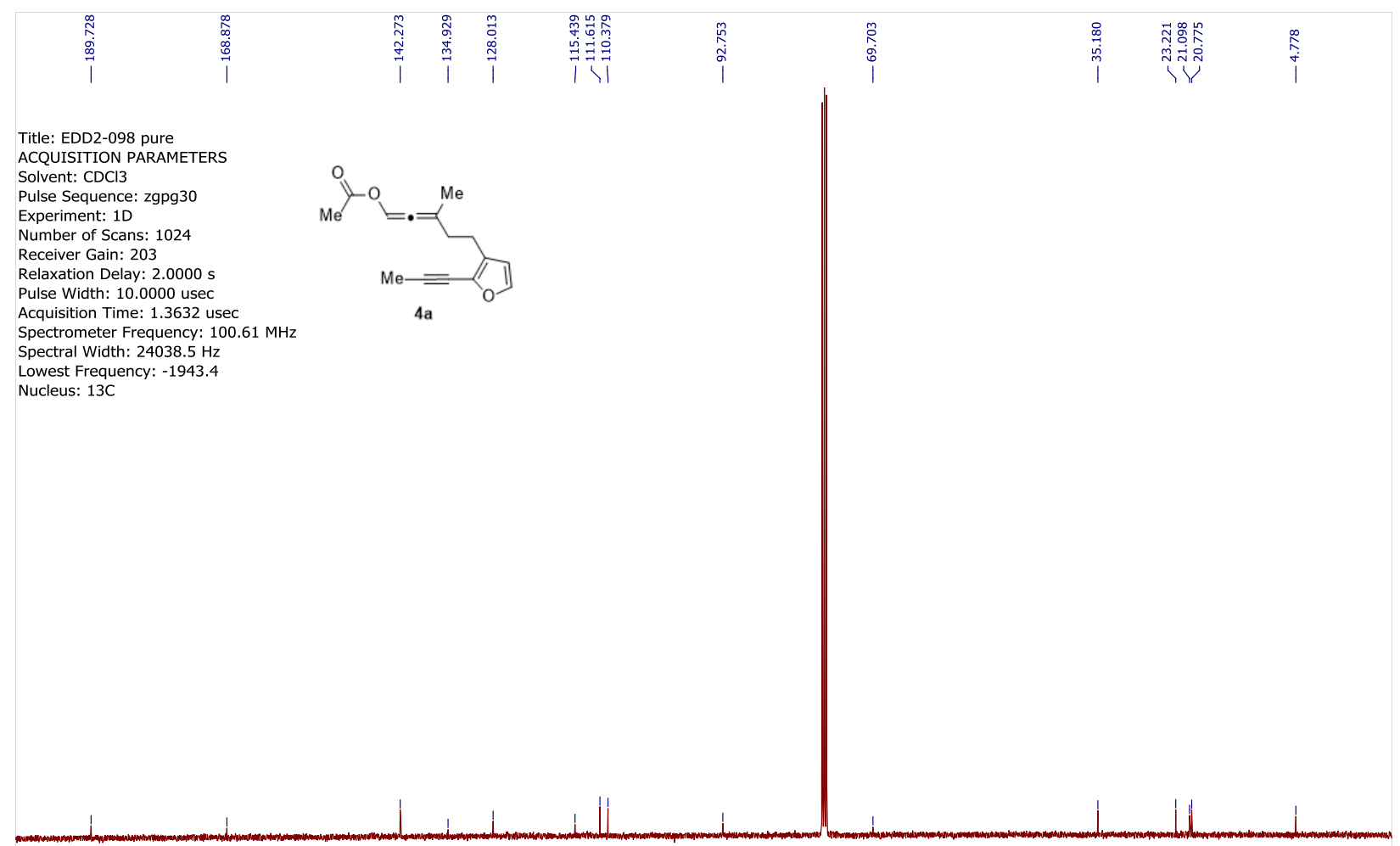

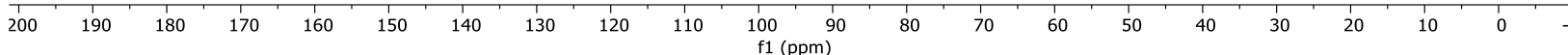


Supporting Information for Organic Letters

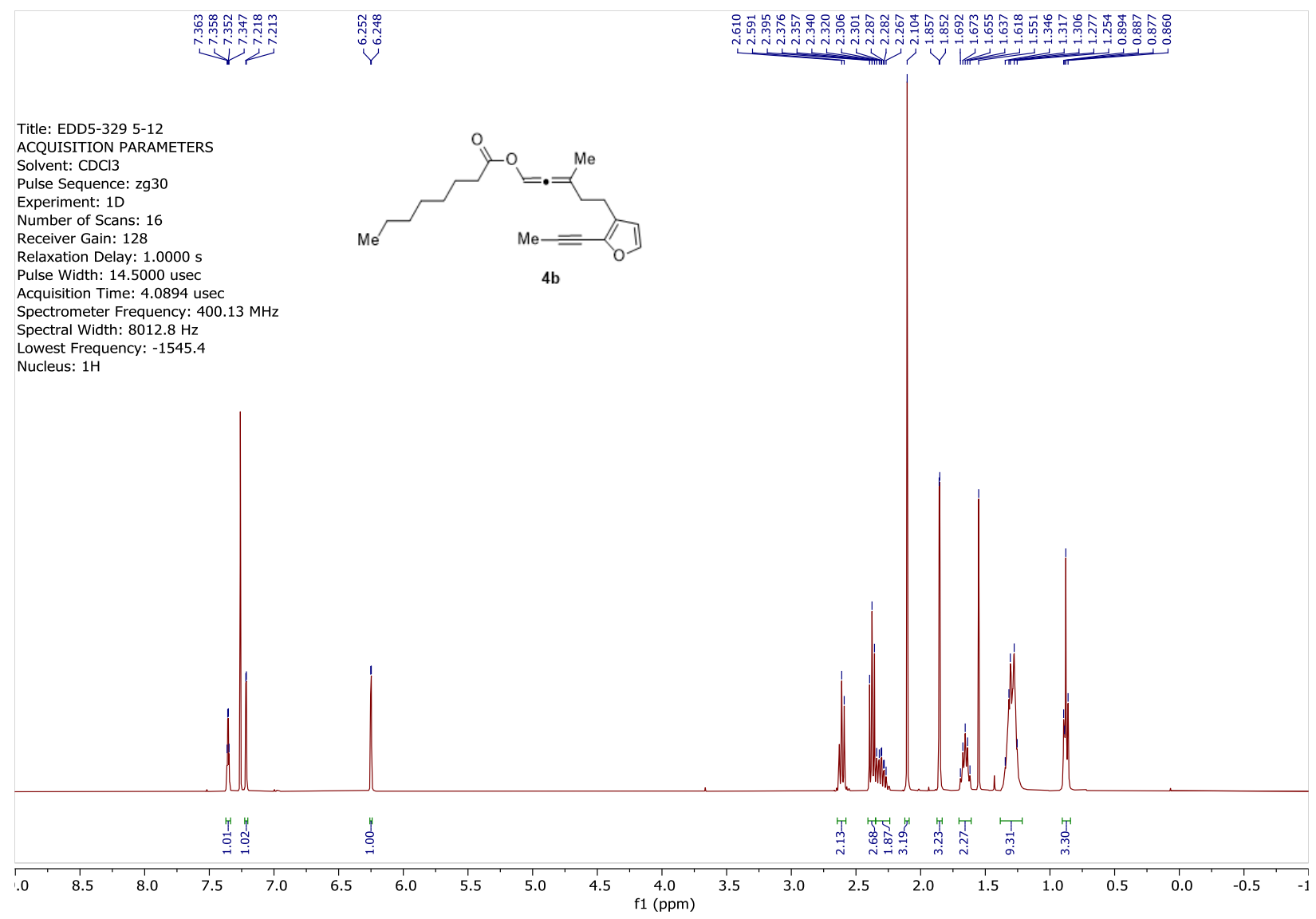


Supporting Information for Organic Letters

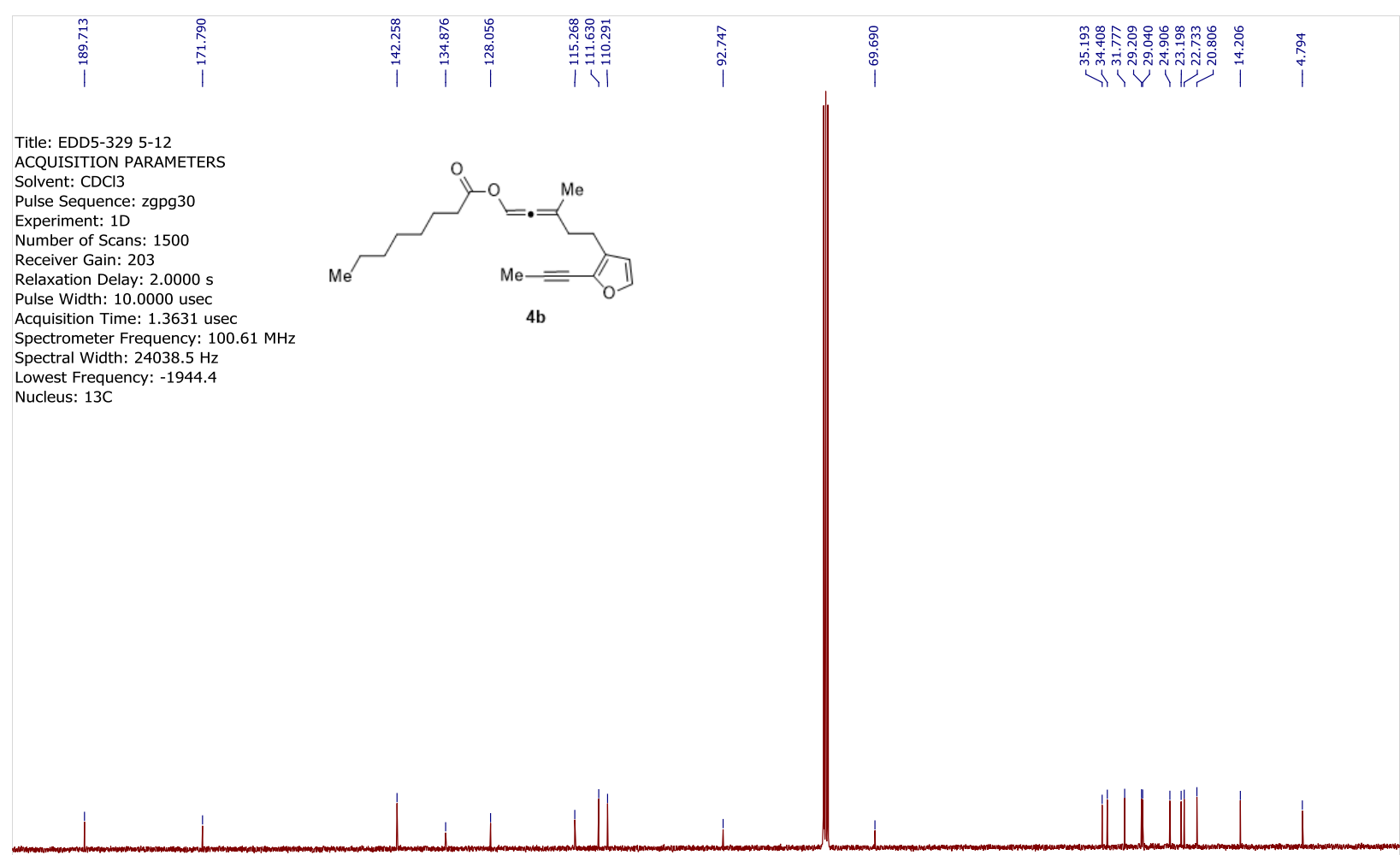

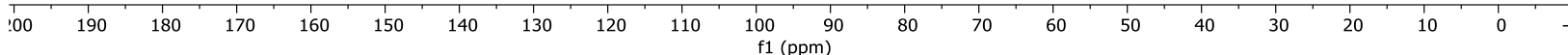




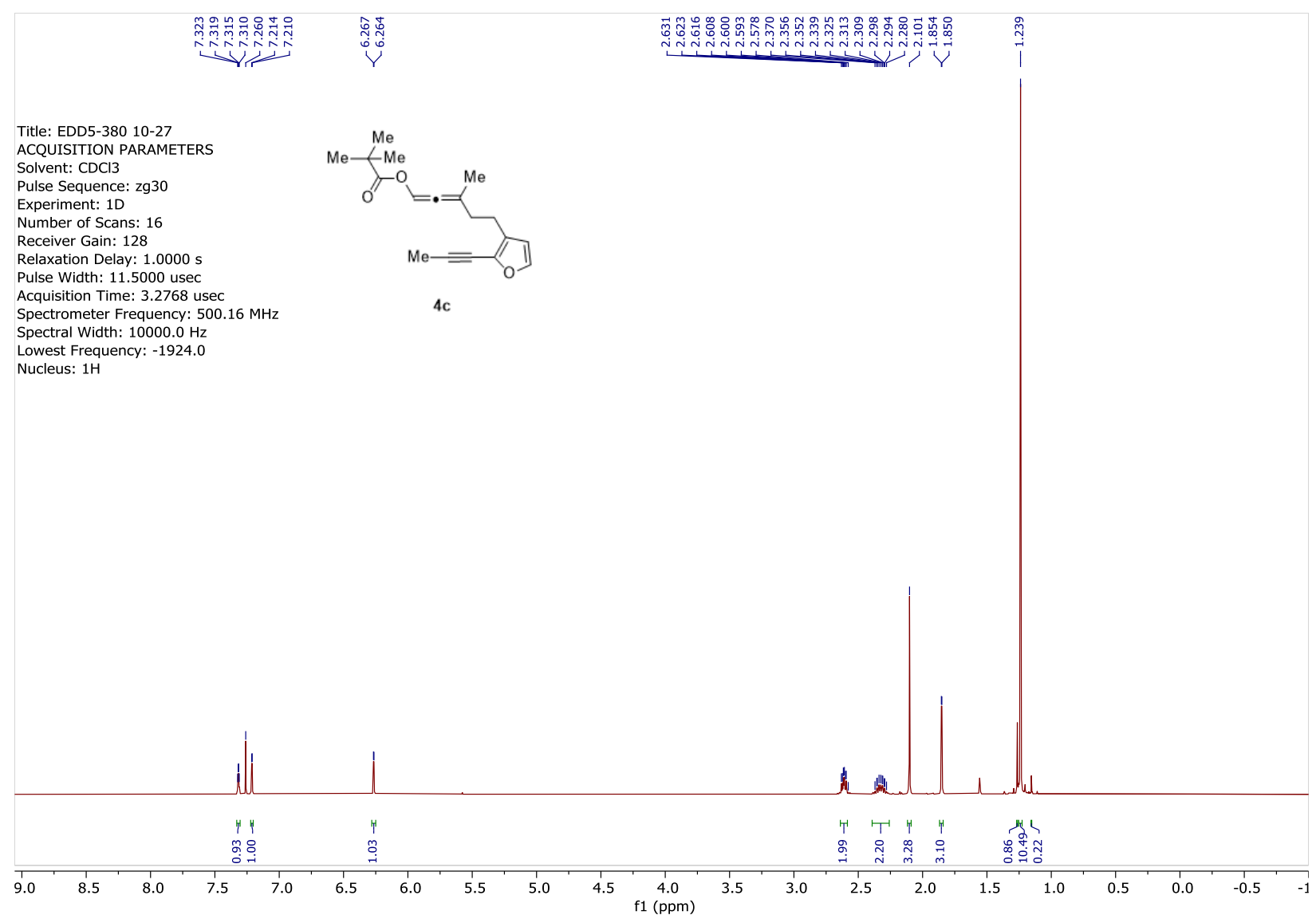


Supporting Information for Organic Letters

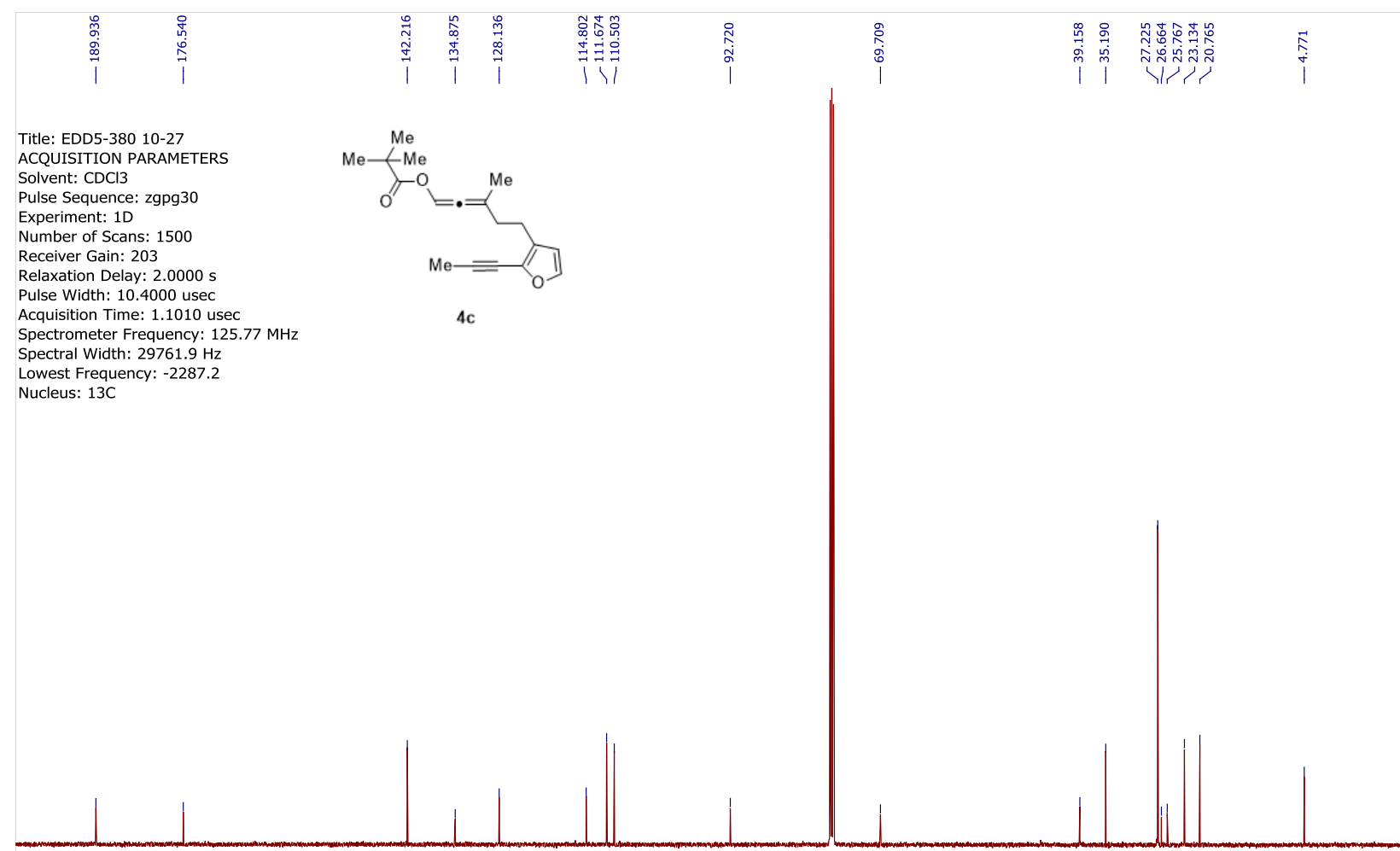

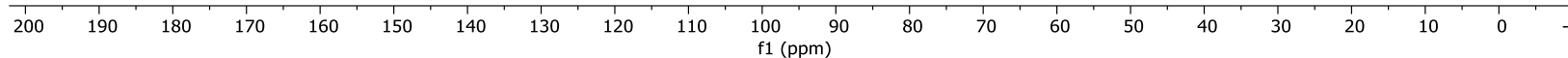


Supporting Information for Organic Letters

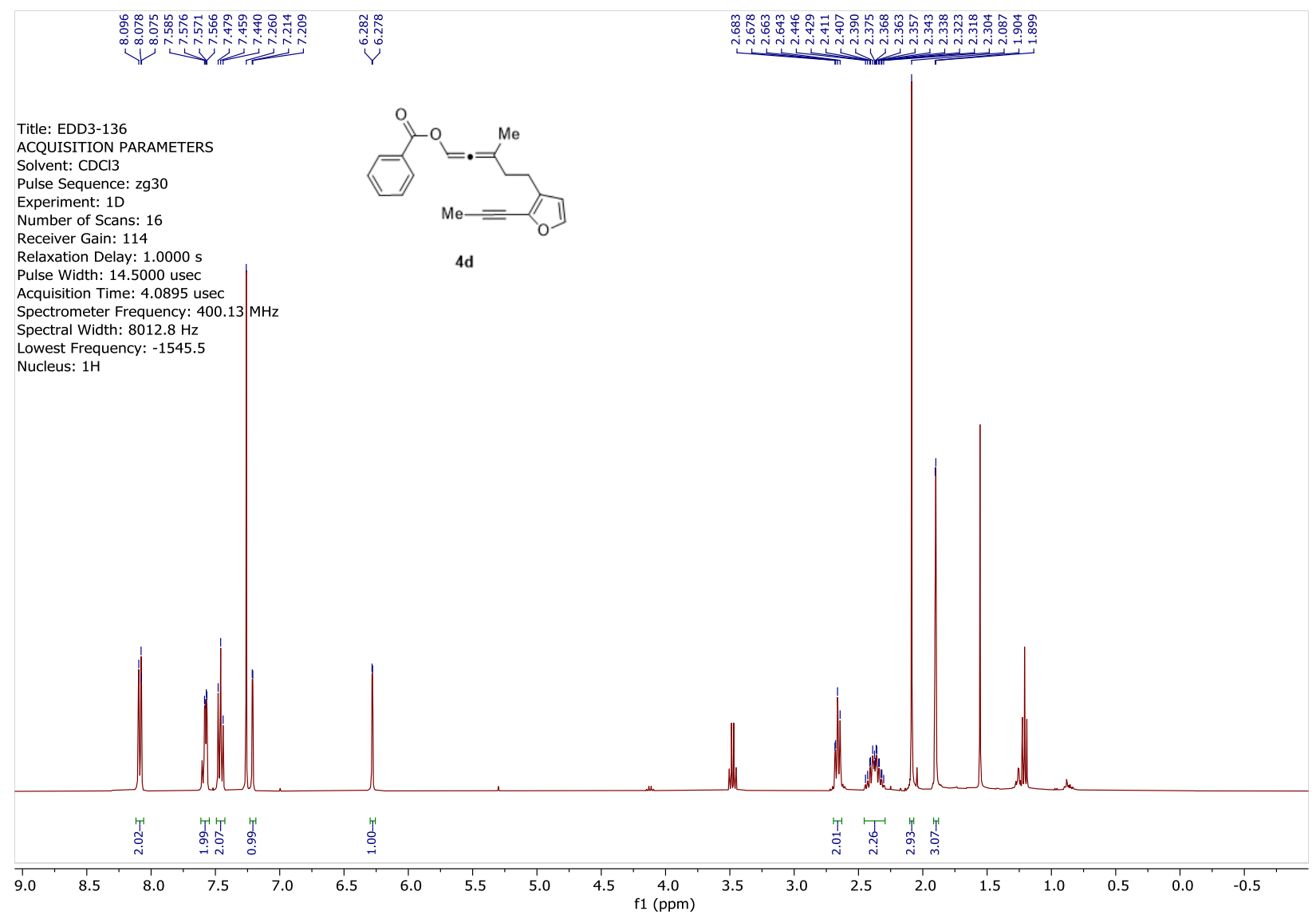


Supporting Information for Organic Letters

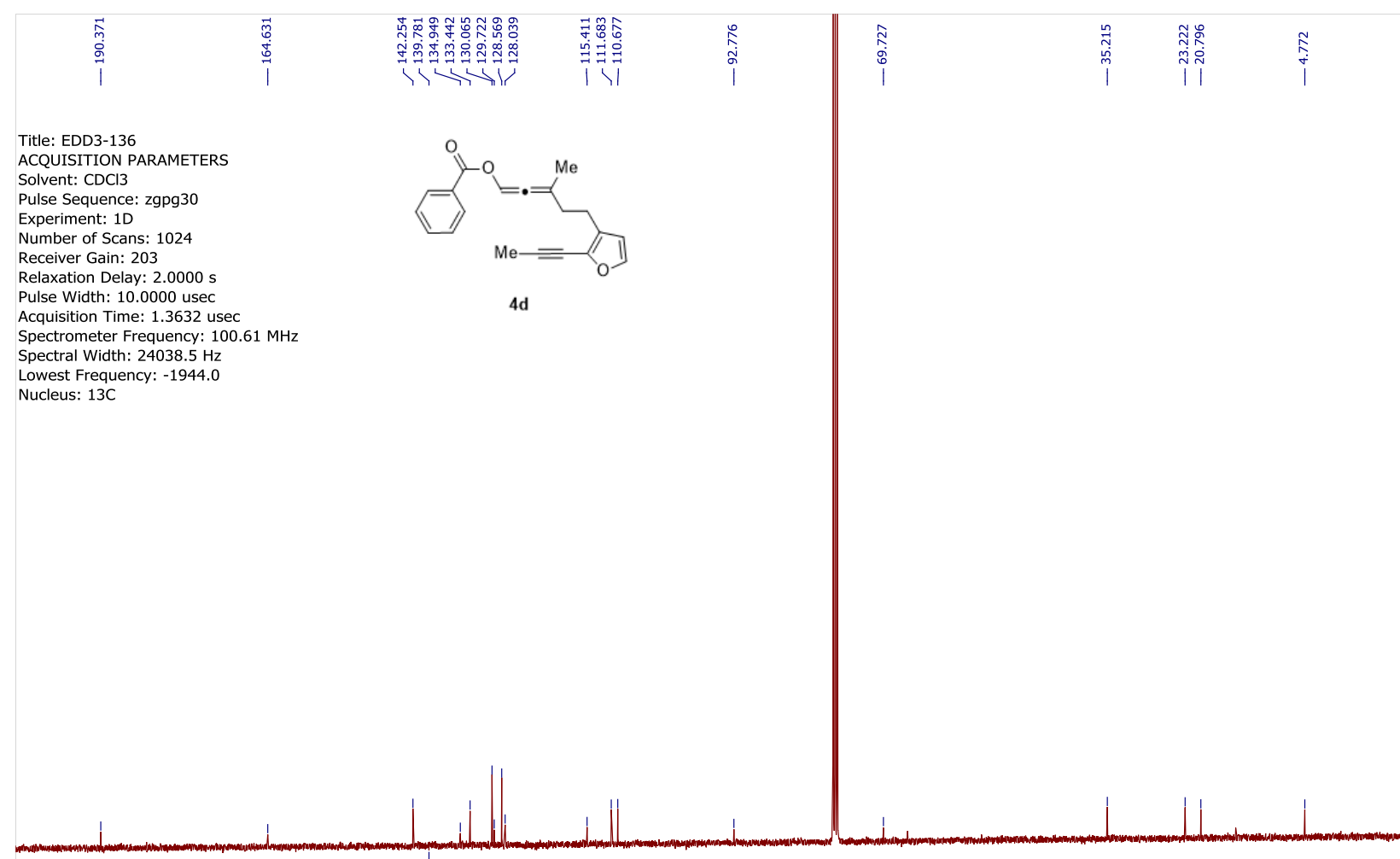

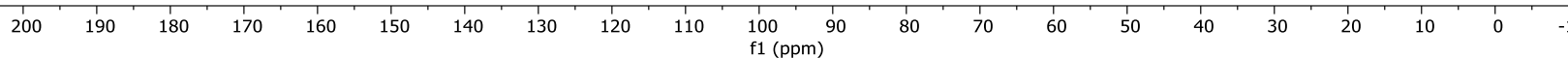


Supporting Information for Organic Letters

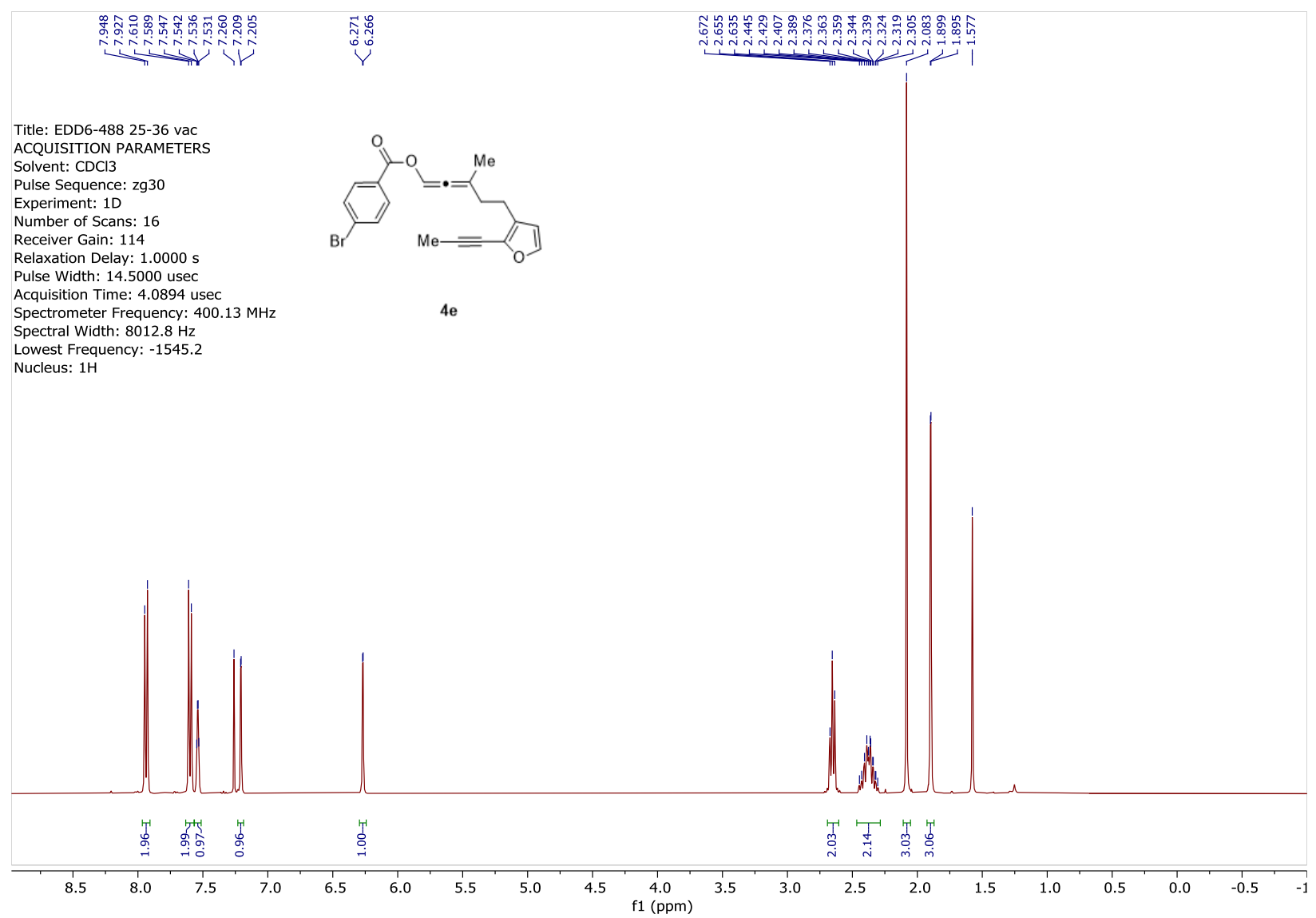


Supporting Information for Organic Letters

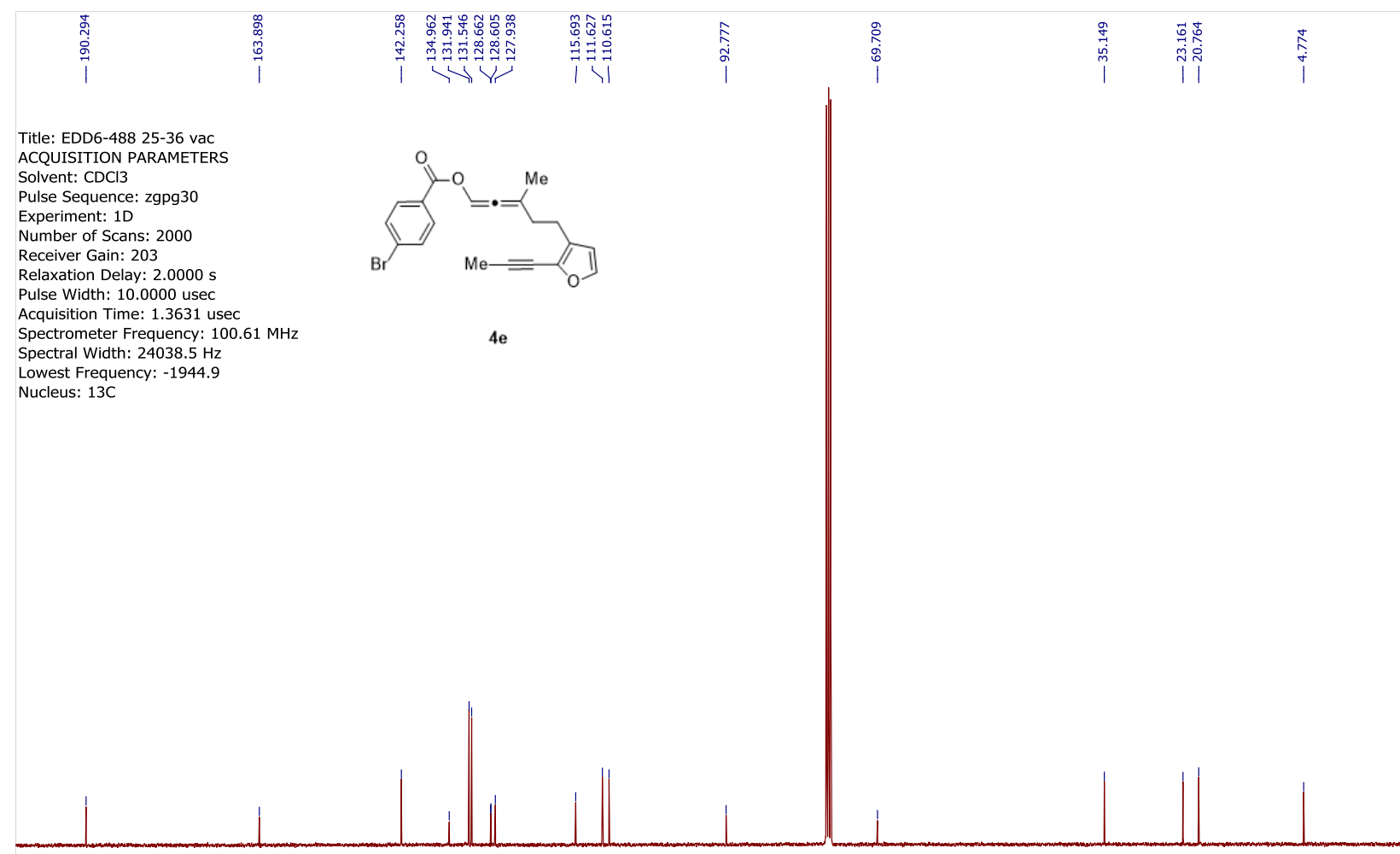

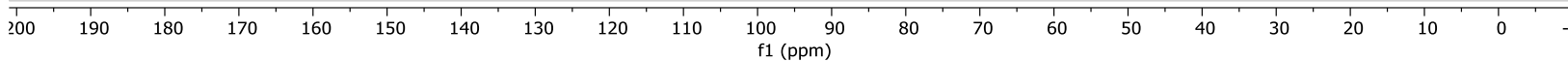




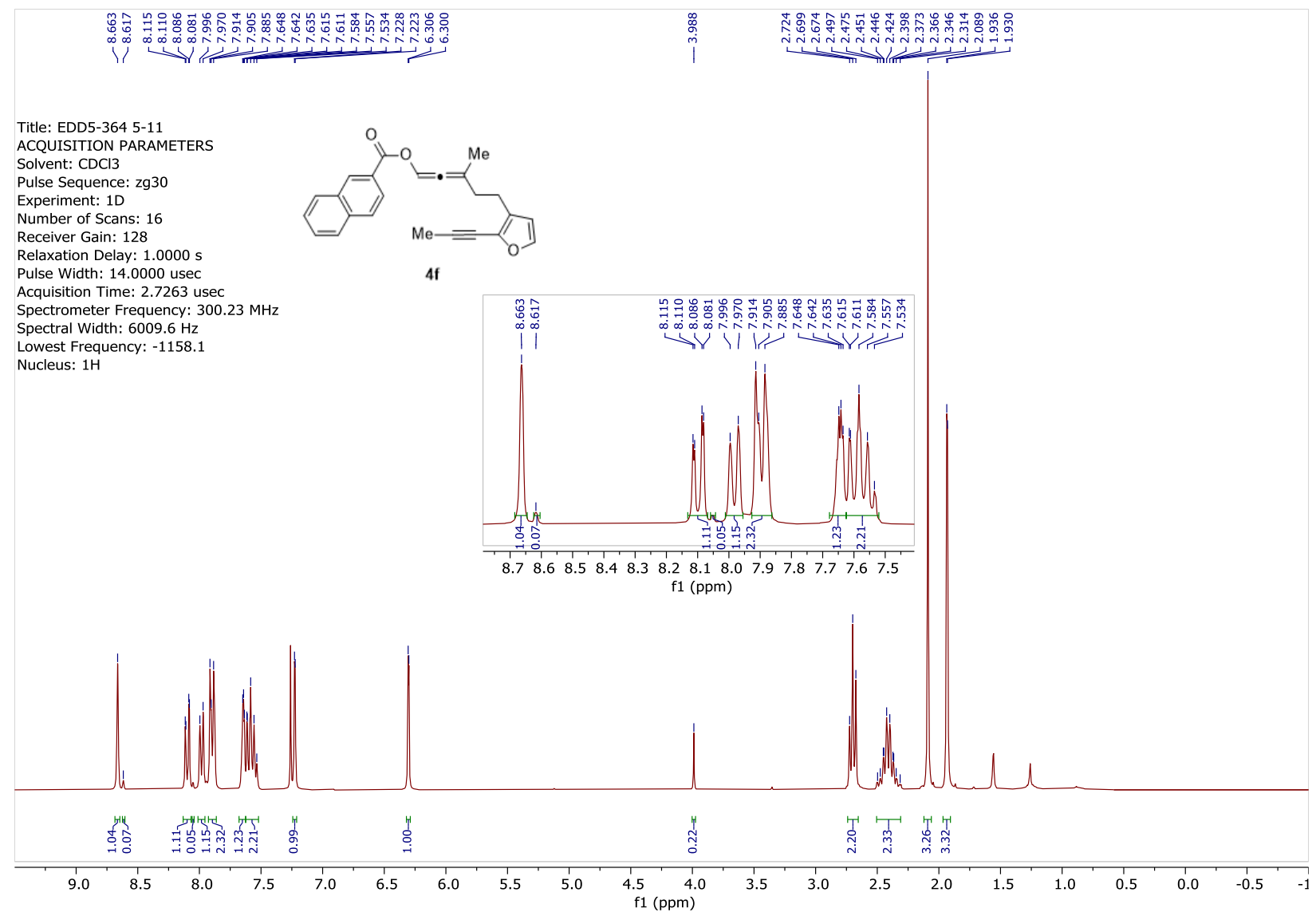


Supporting Information for Organic Letters

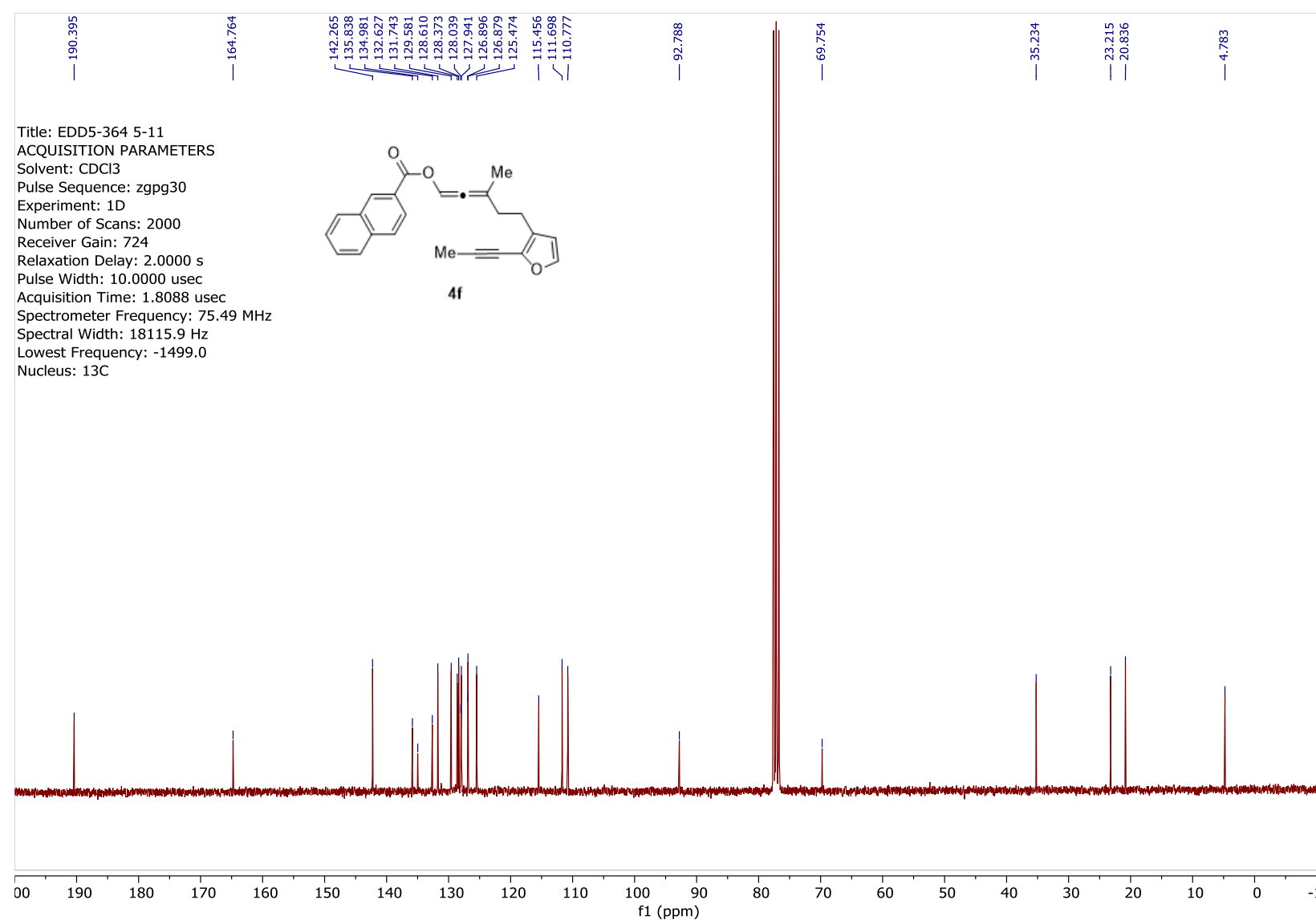


Supporting Information for Organic Letters

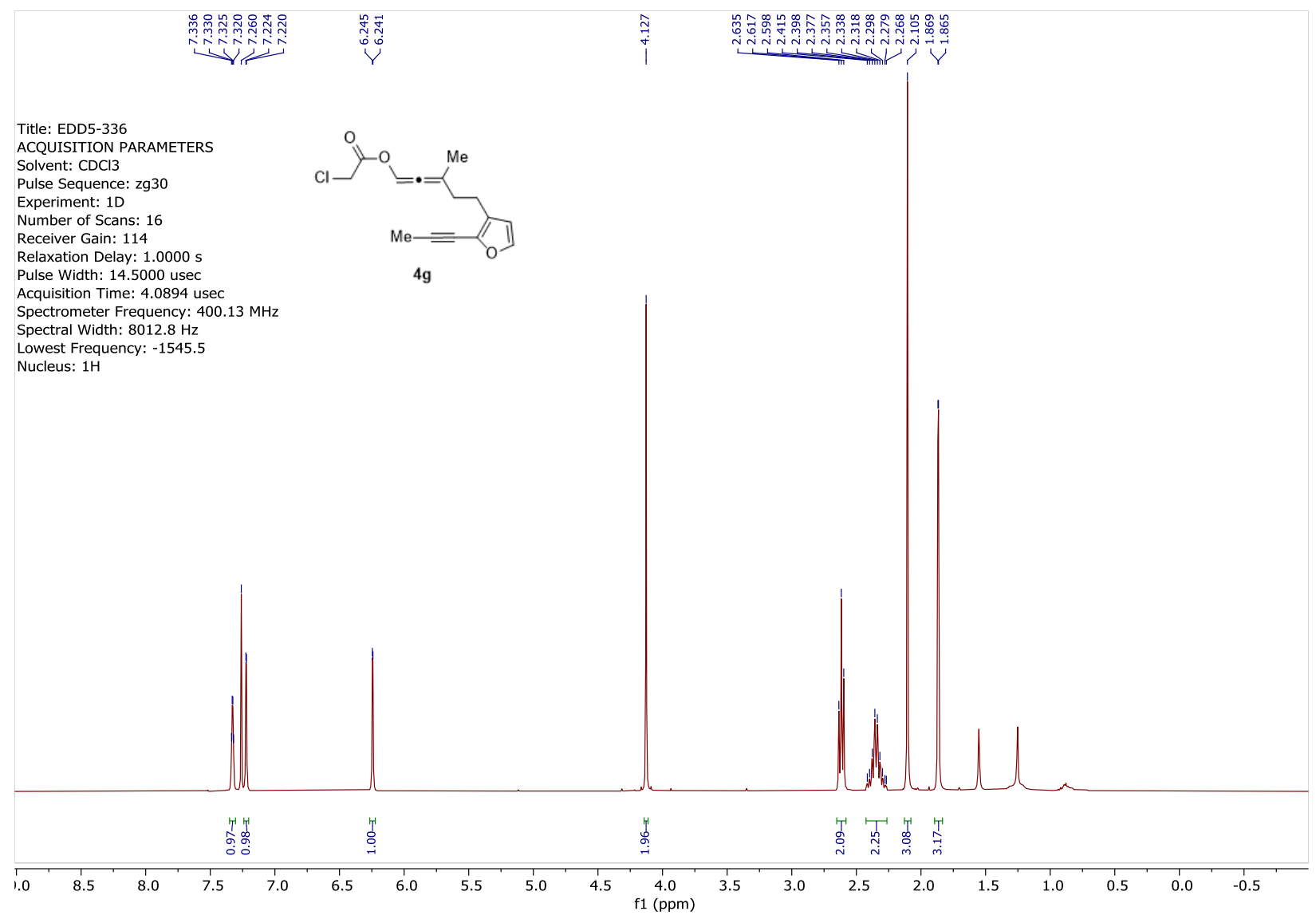


Supporting Information for Organic Letters

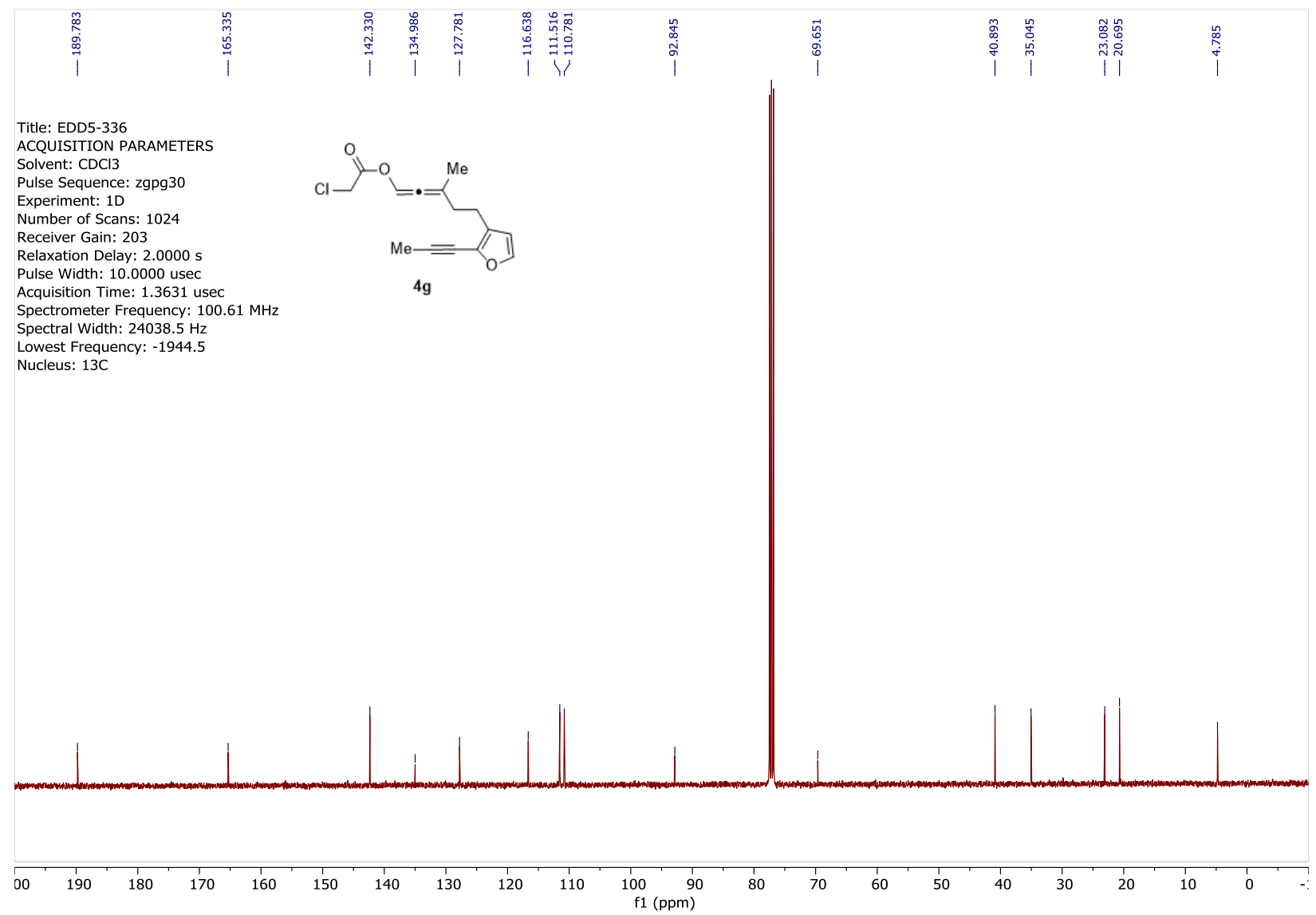




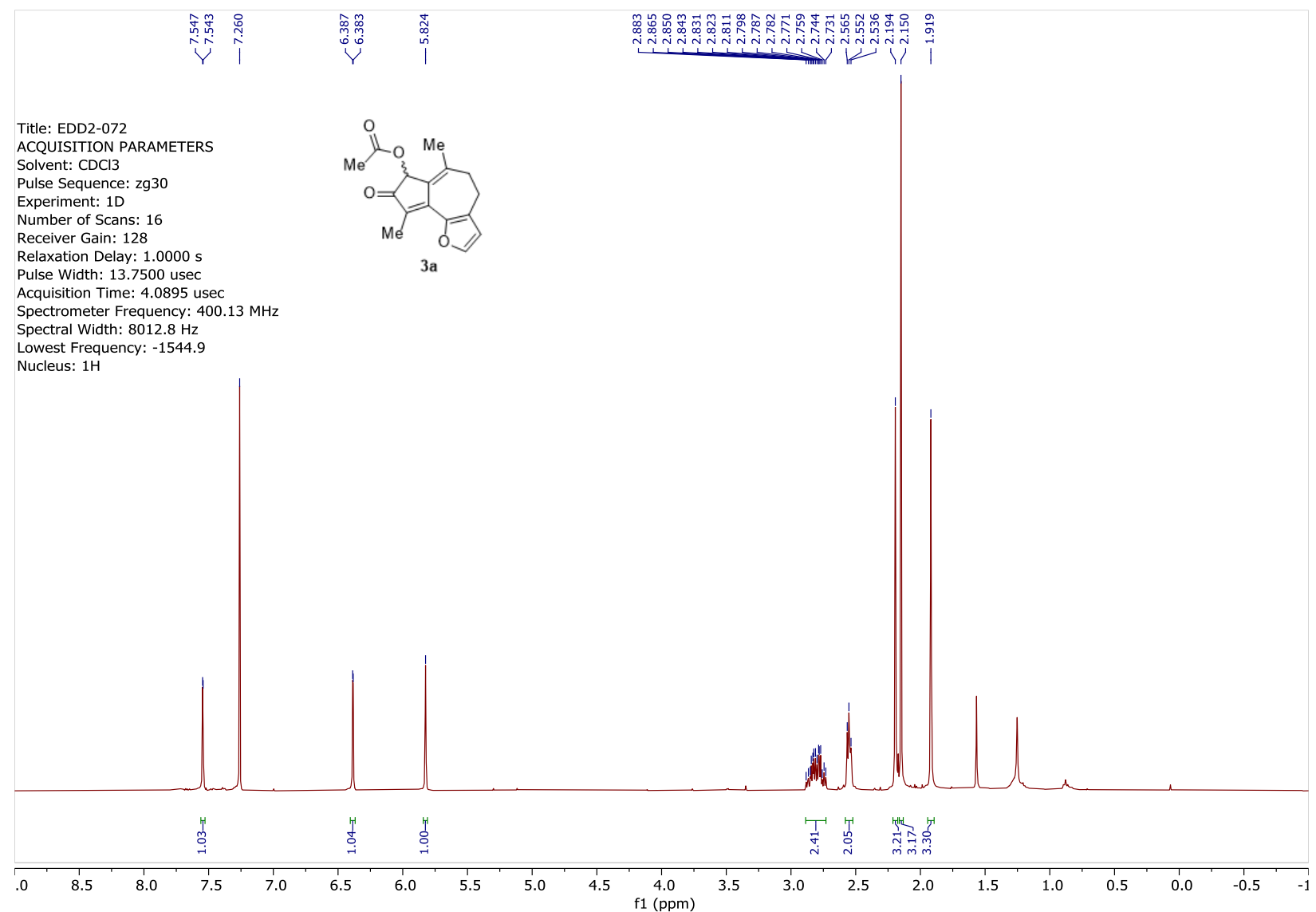


Supporting Information for Organic Letters

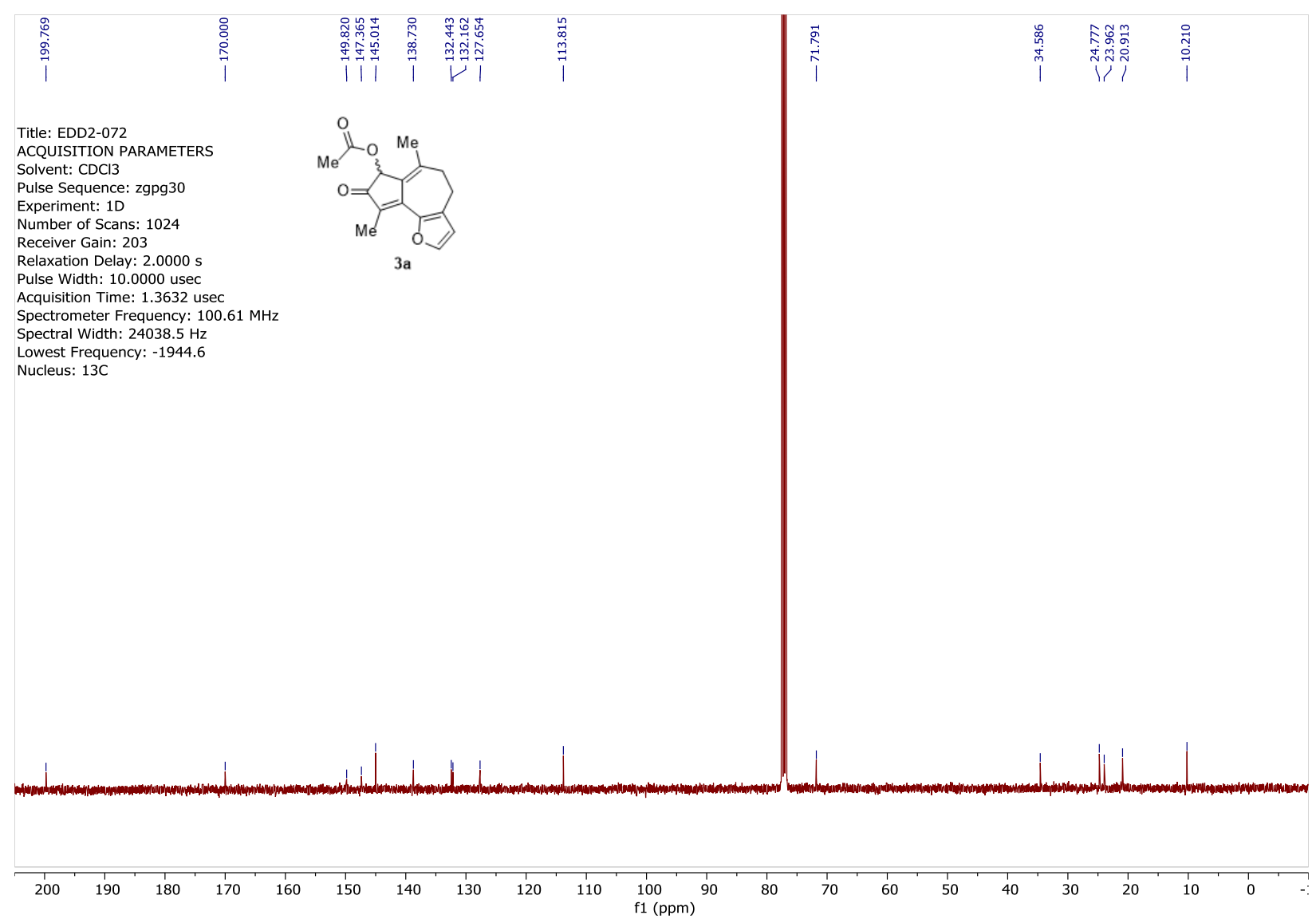


Supporting Information for Organic Letters

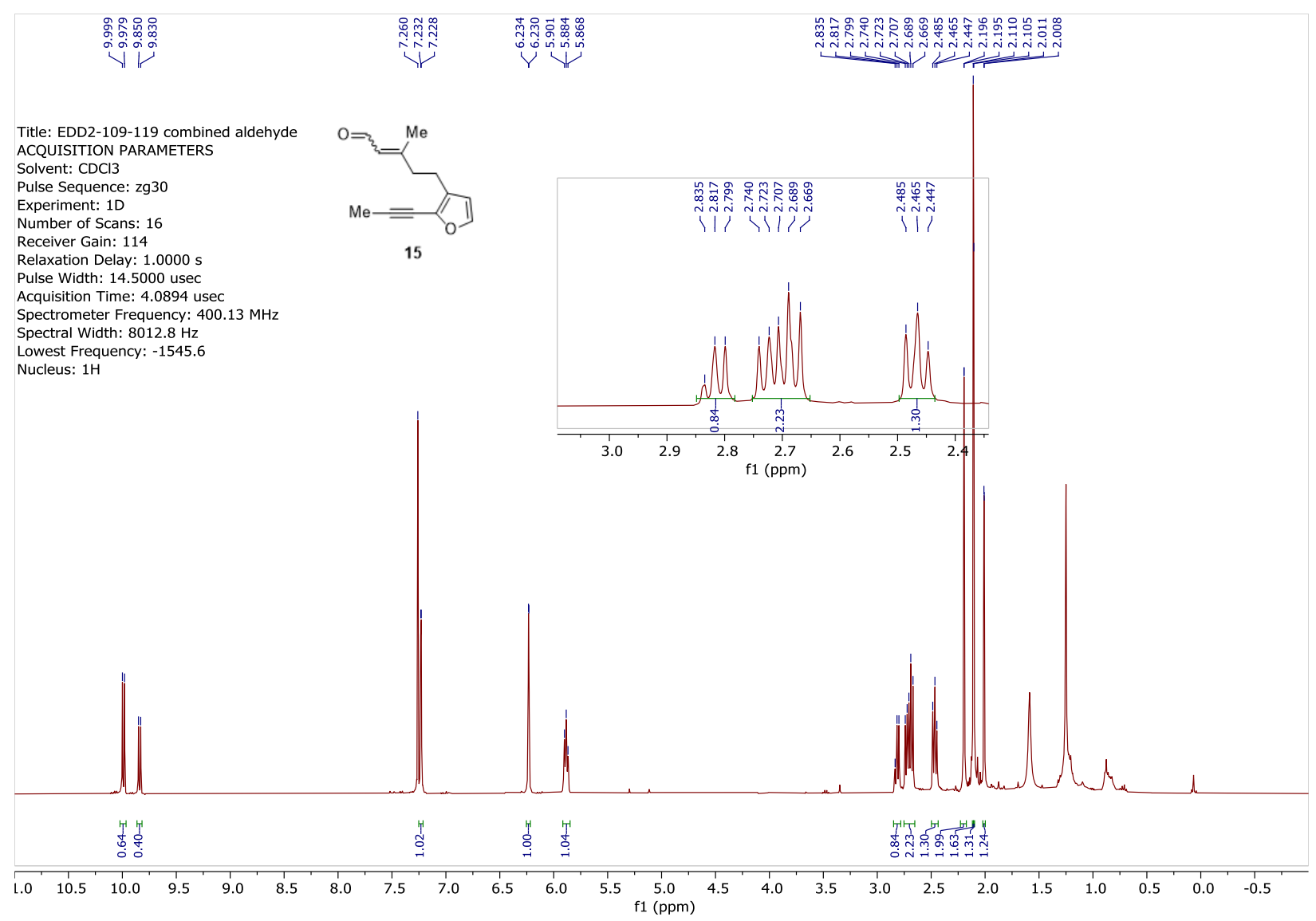


Supporting Information for Organic Letters

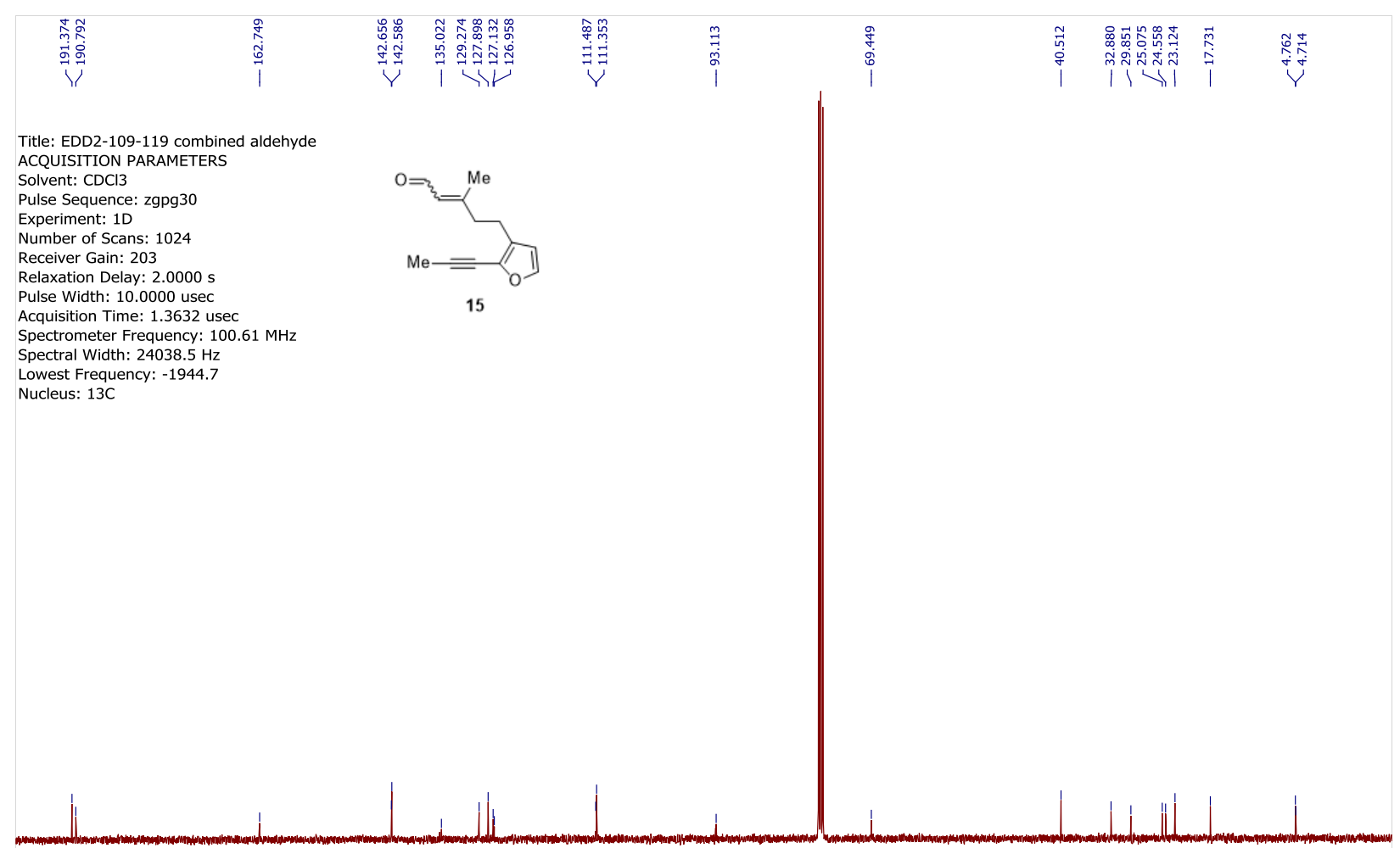

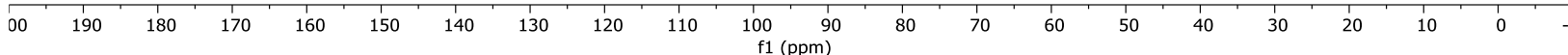


Supporting Information for Organic Letters

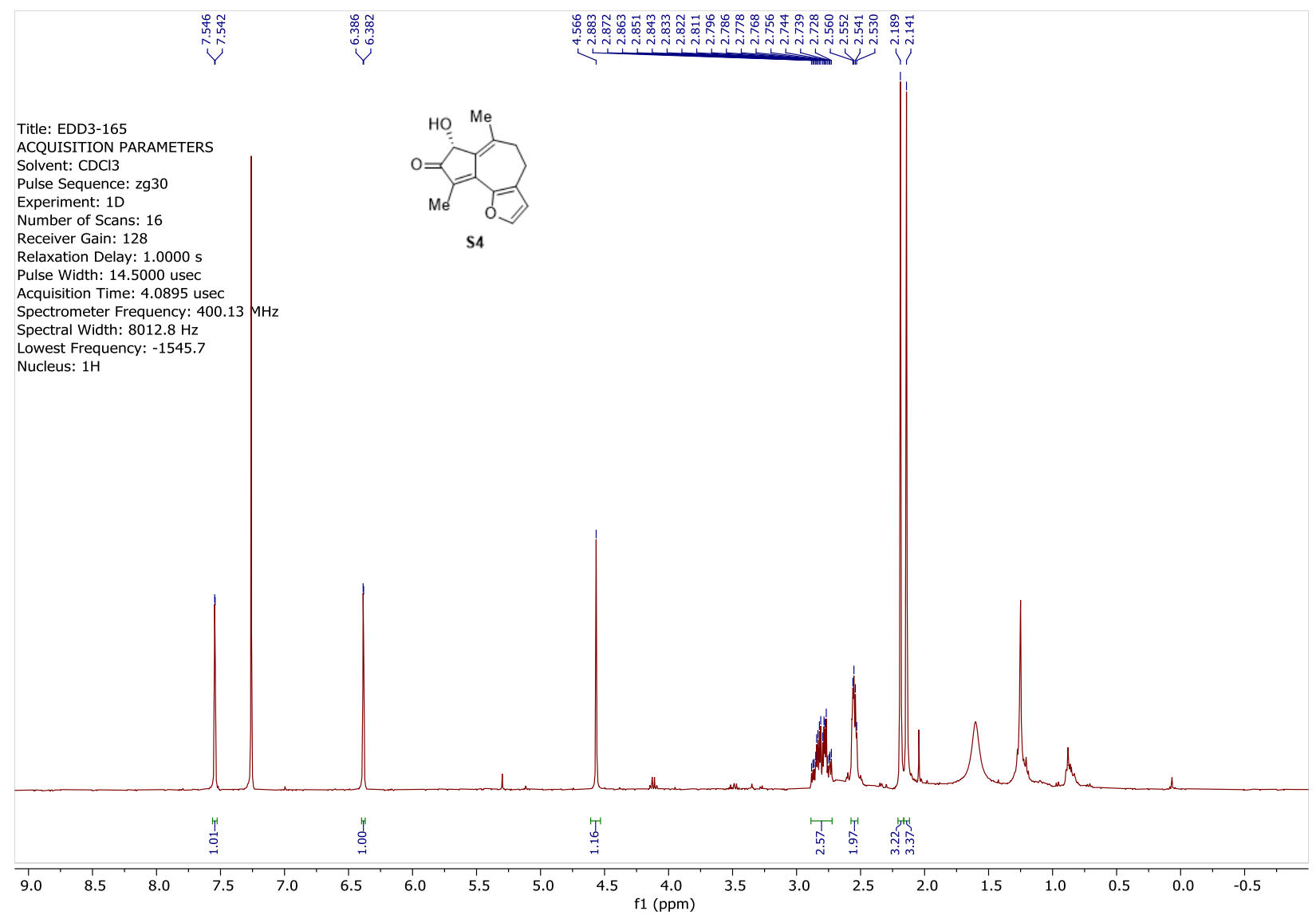


Supporting Information for Organic Letters

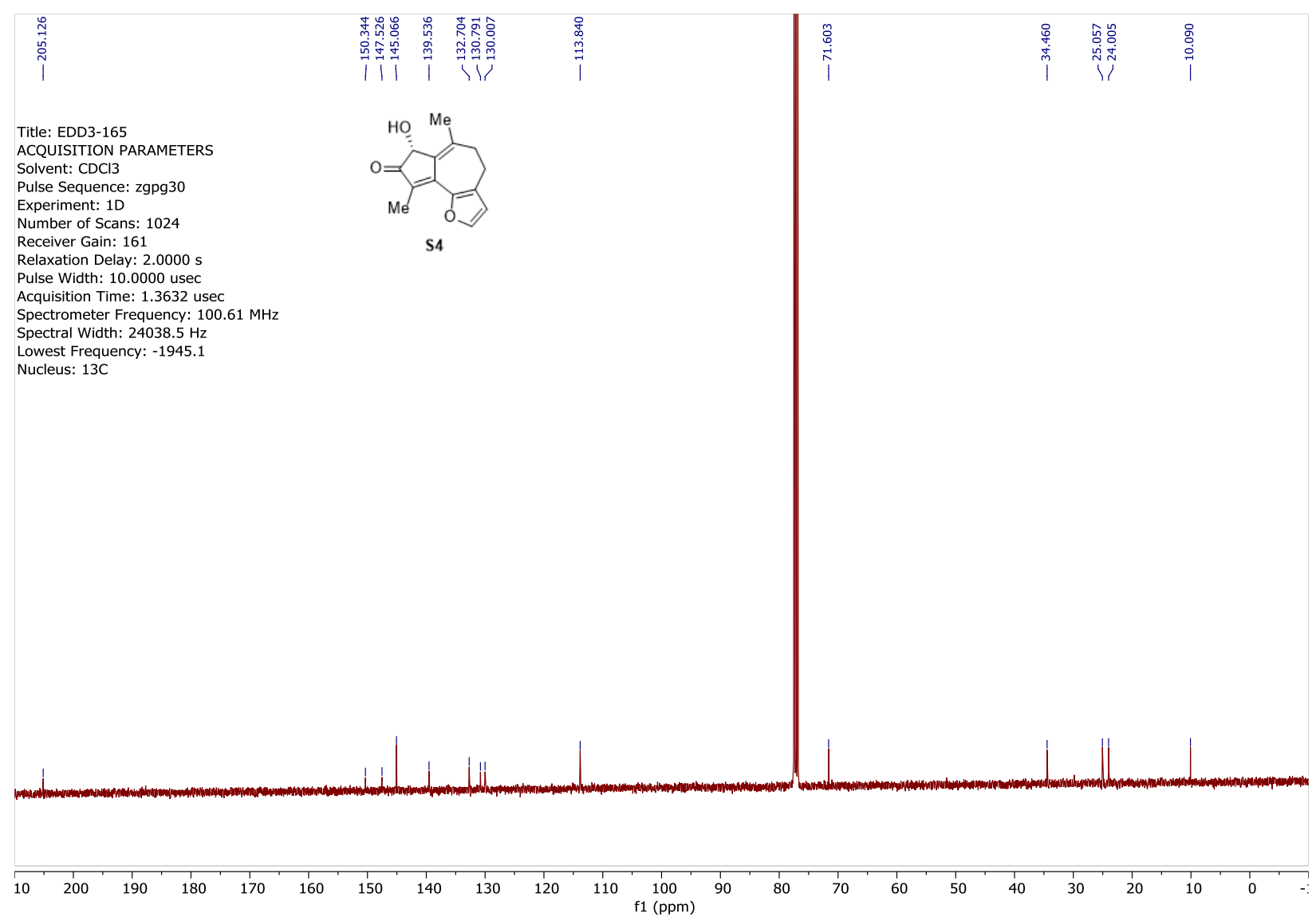


Supporting Information for Organic Letters

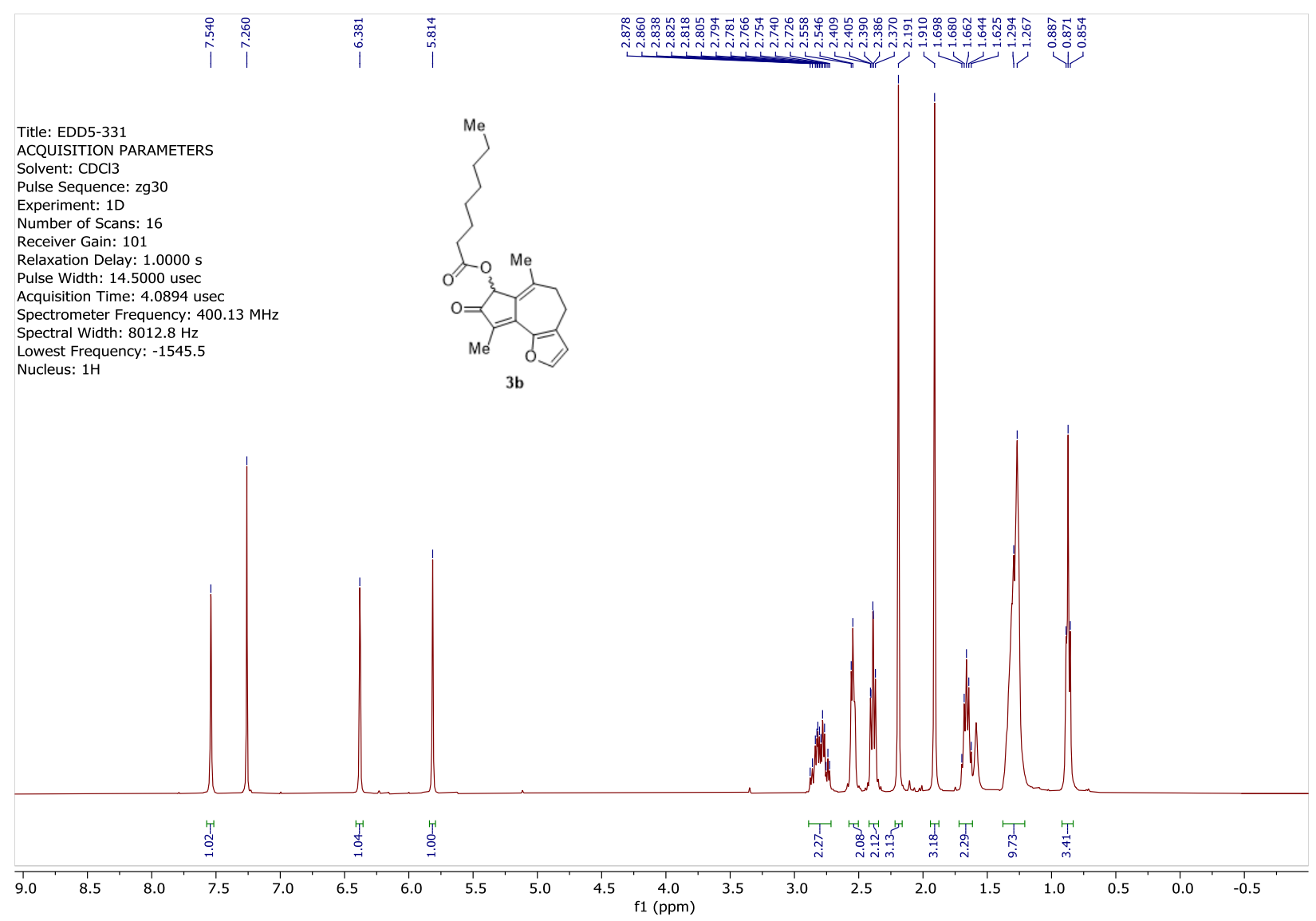


Supporting Information for Organic Letters

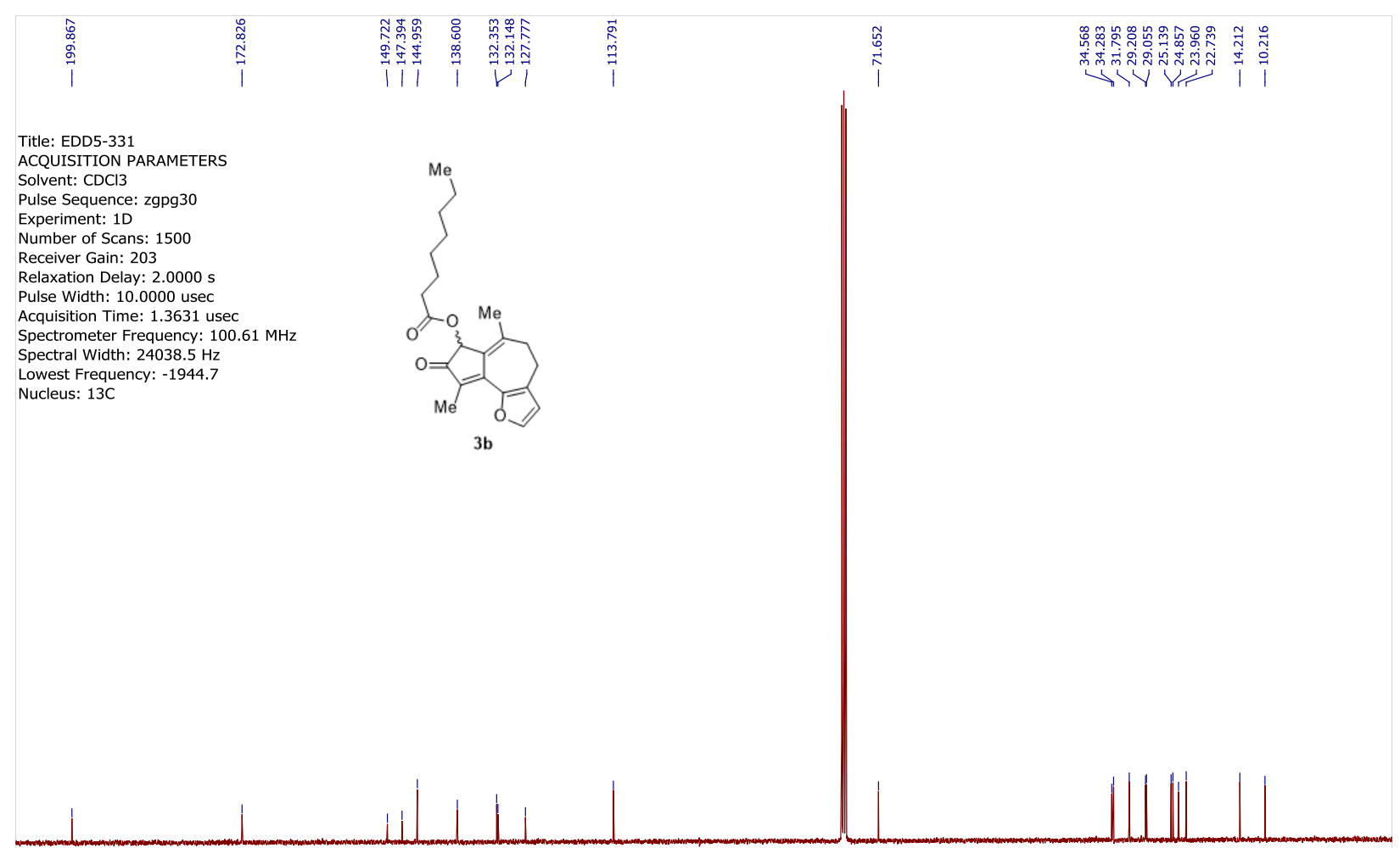

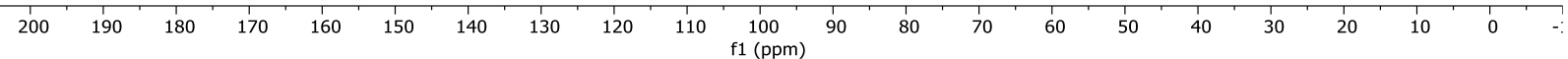


Supporting Information for Organic Letters

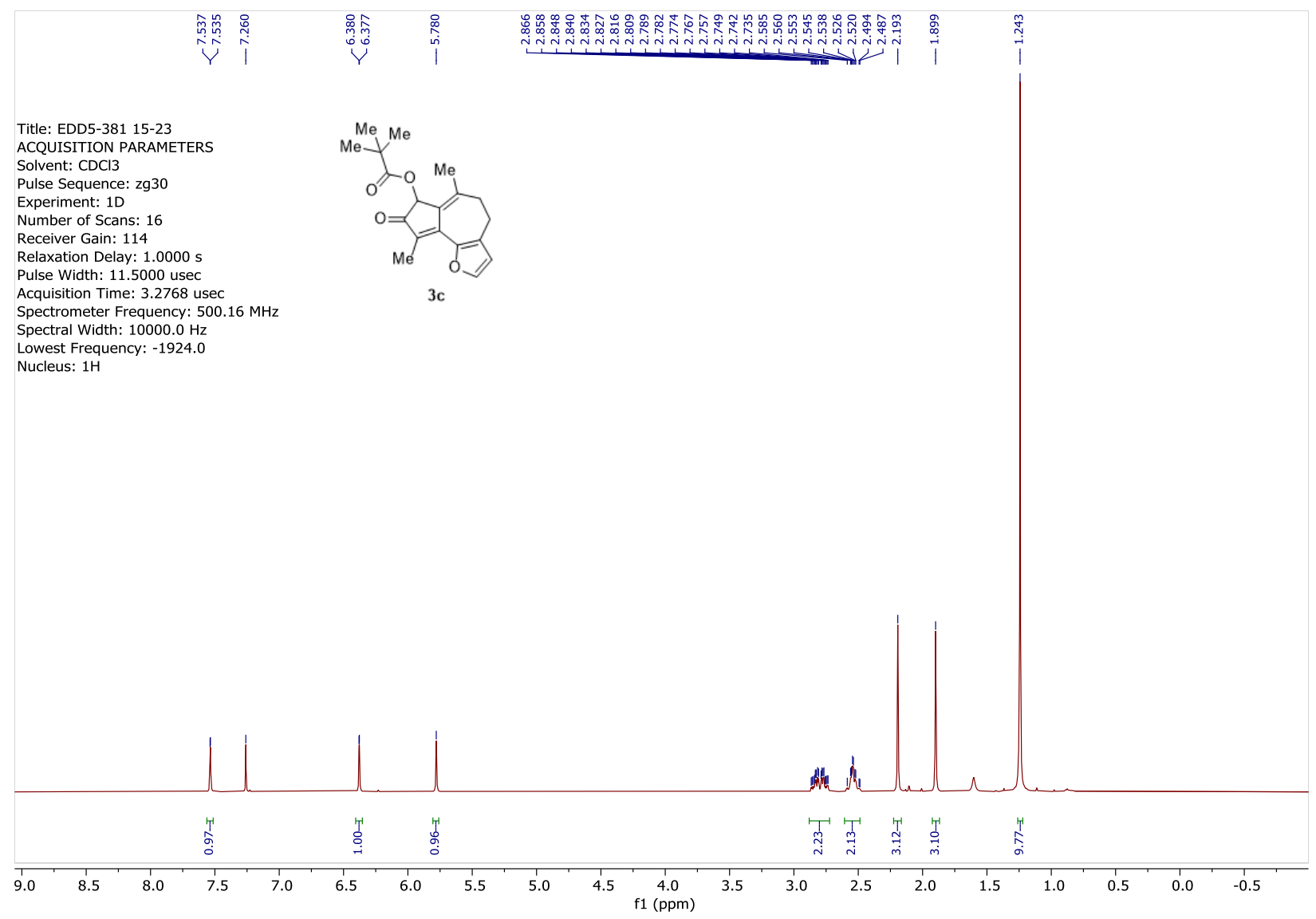


Supporting Information for Organic Letters

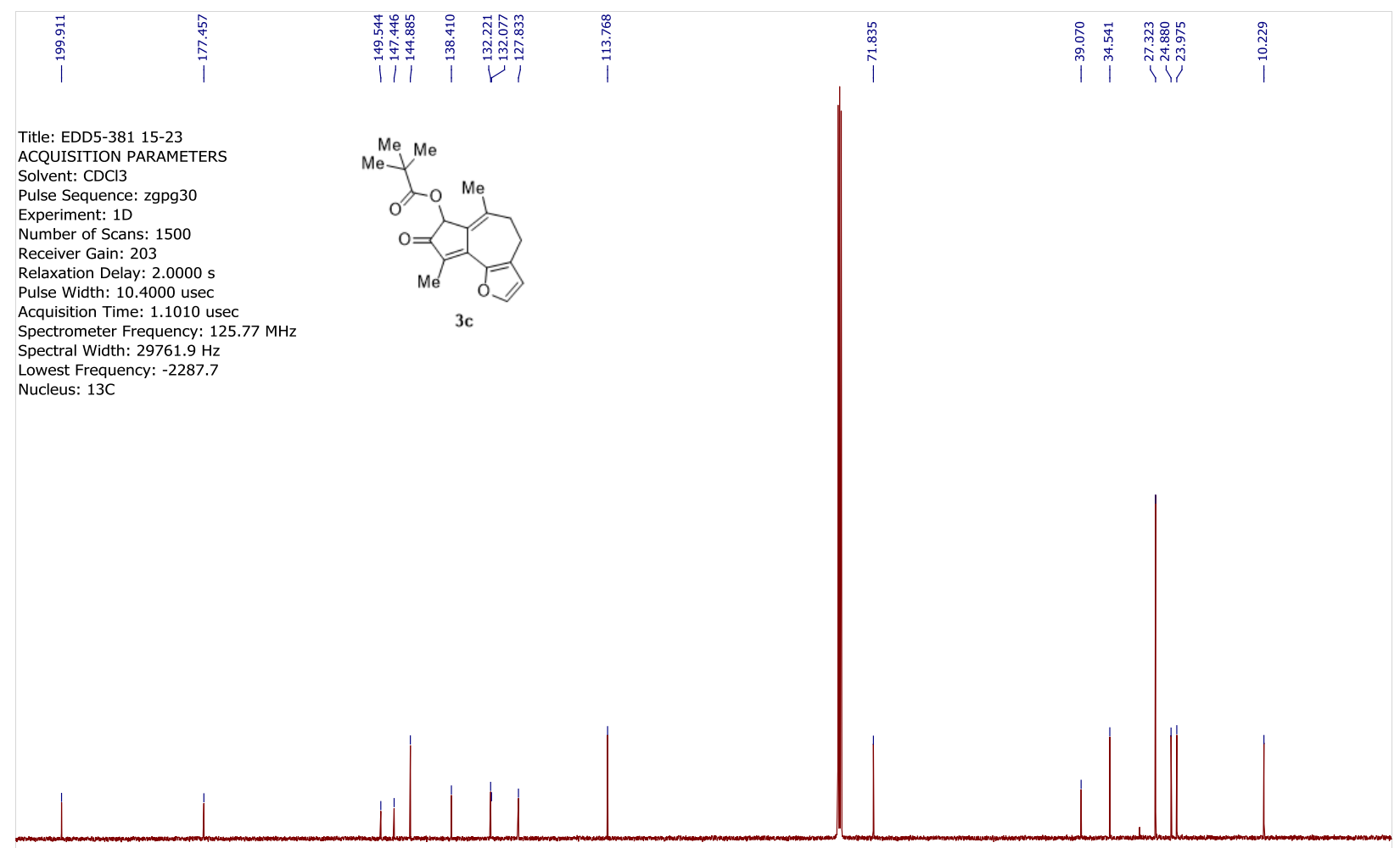

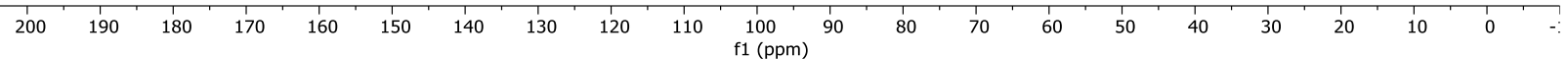


Supporting Information for Organic Letters

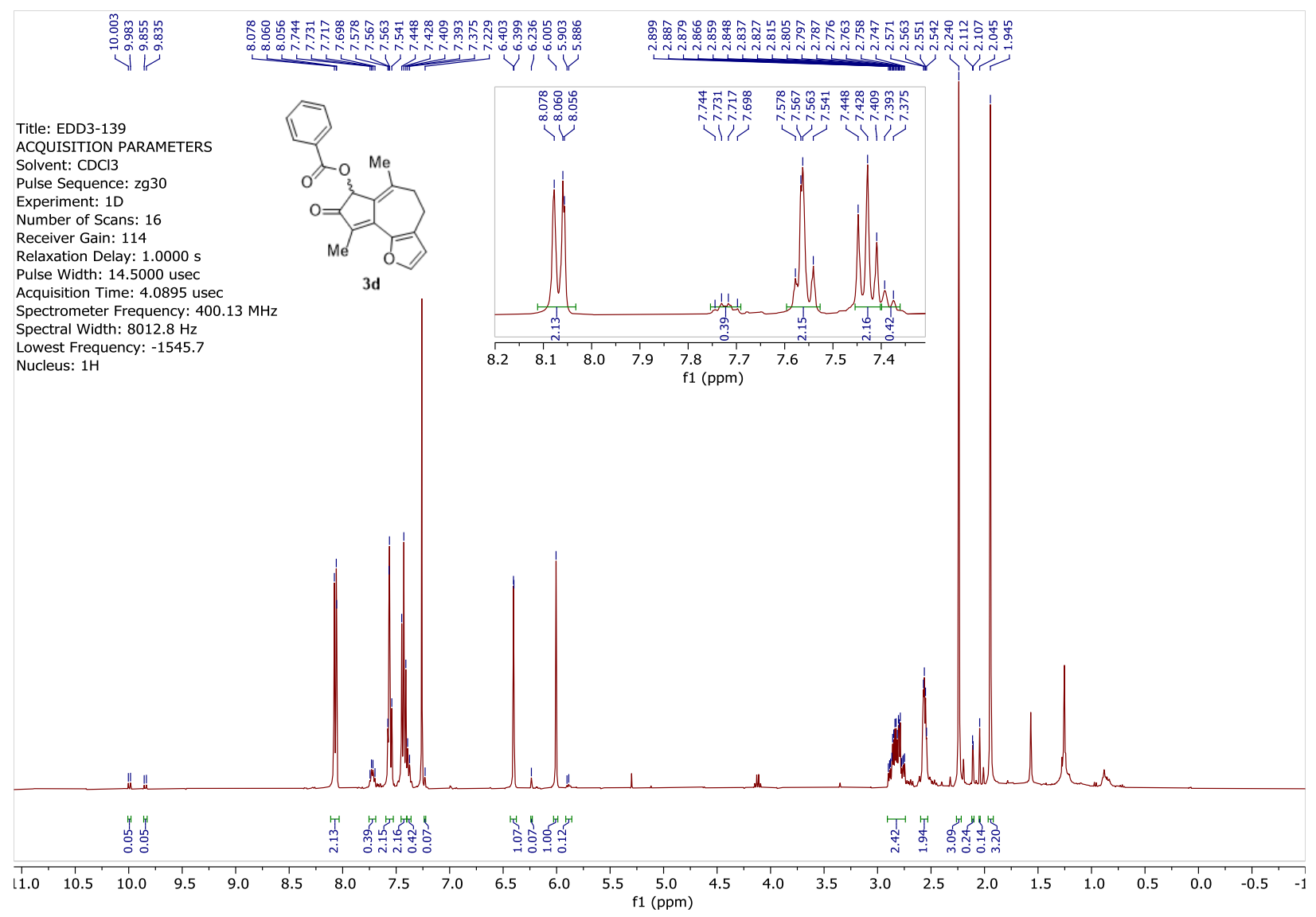


Supporting Information for Organic Letters

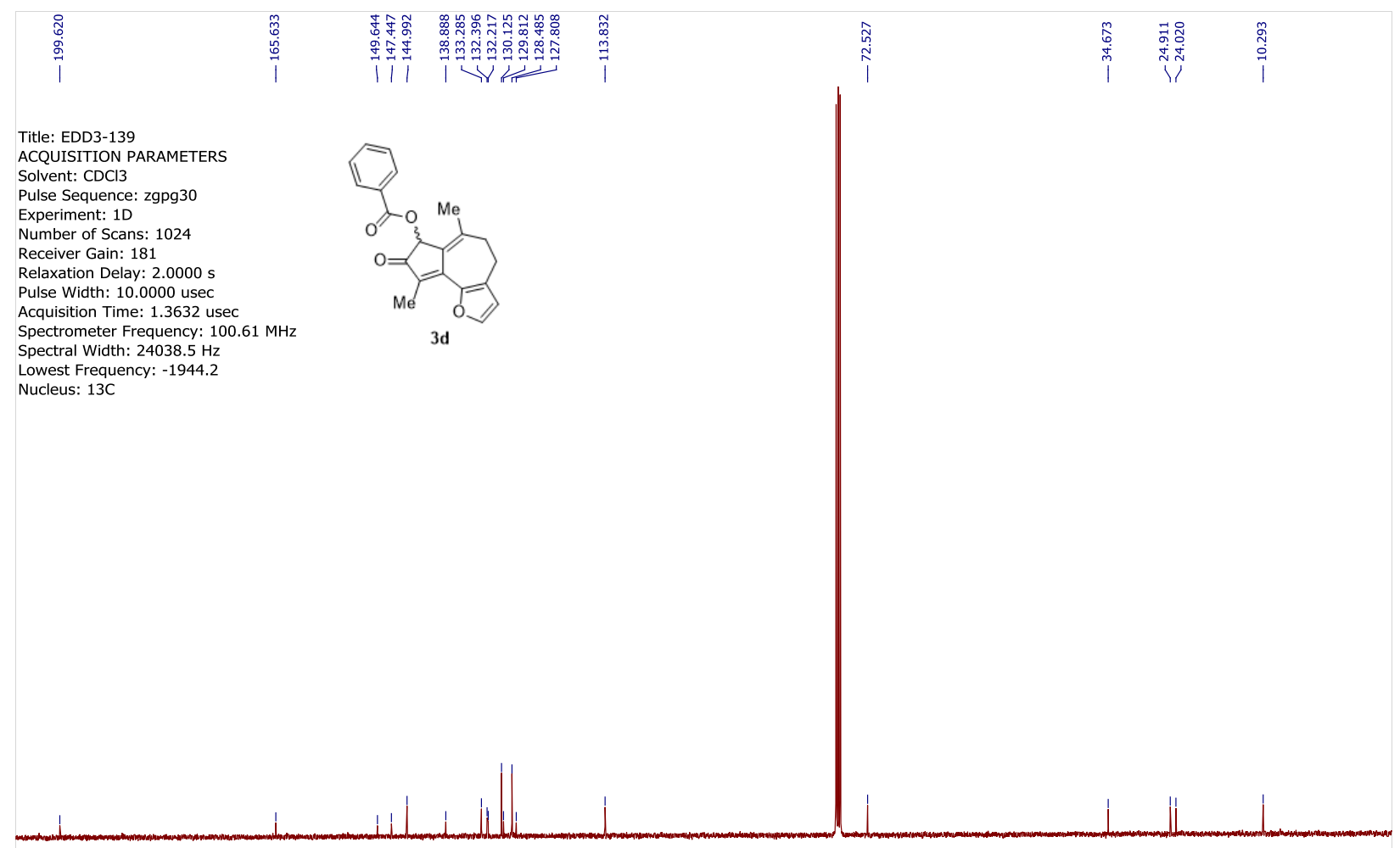

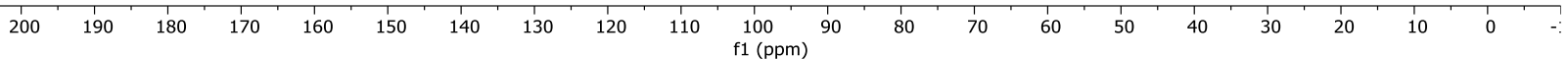


Supporting Information for Organic Letters

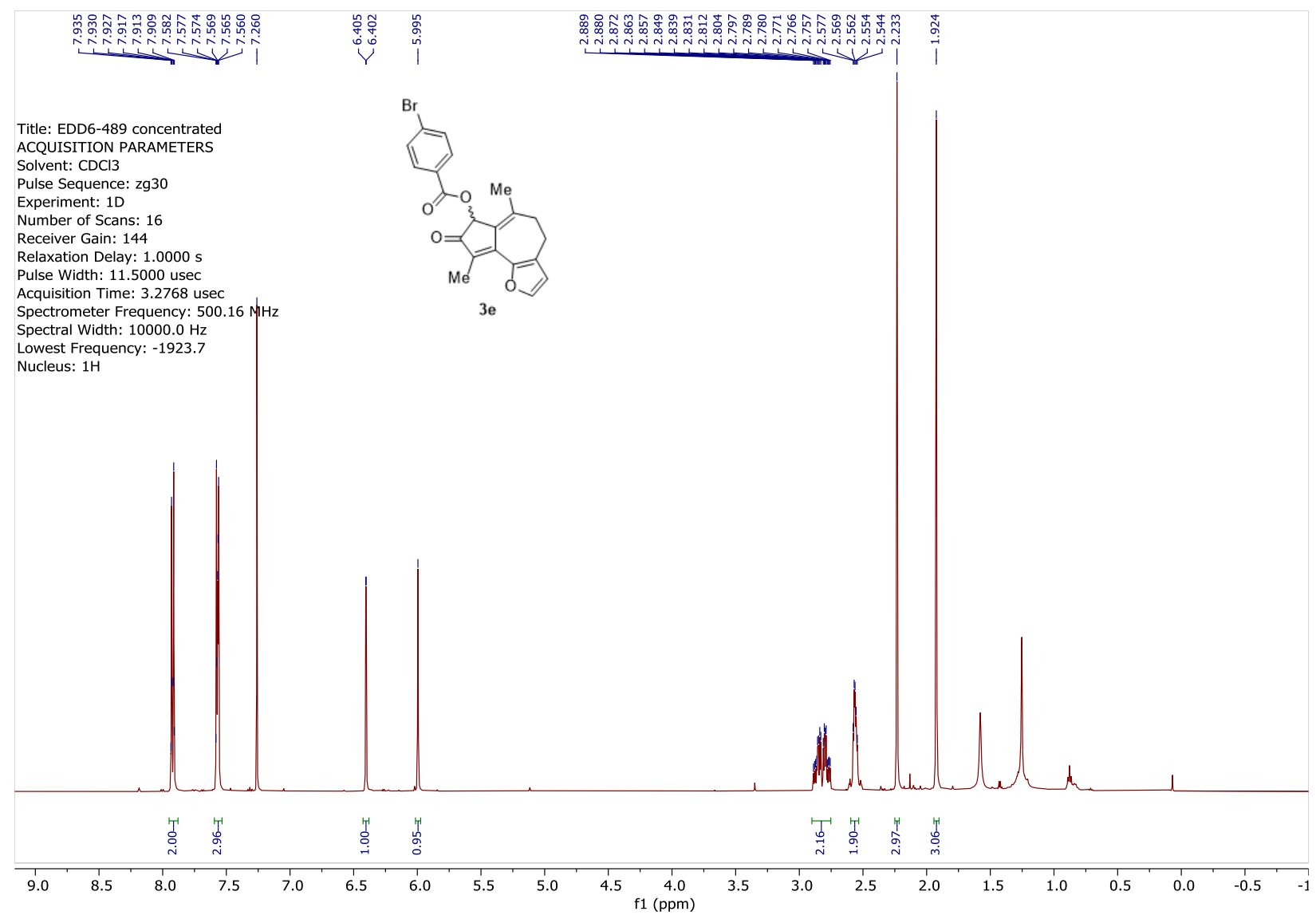


Supporting Information for Organic Letters

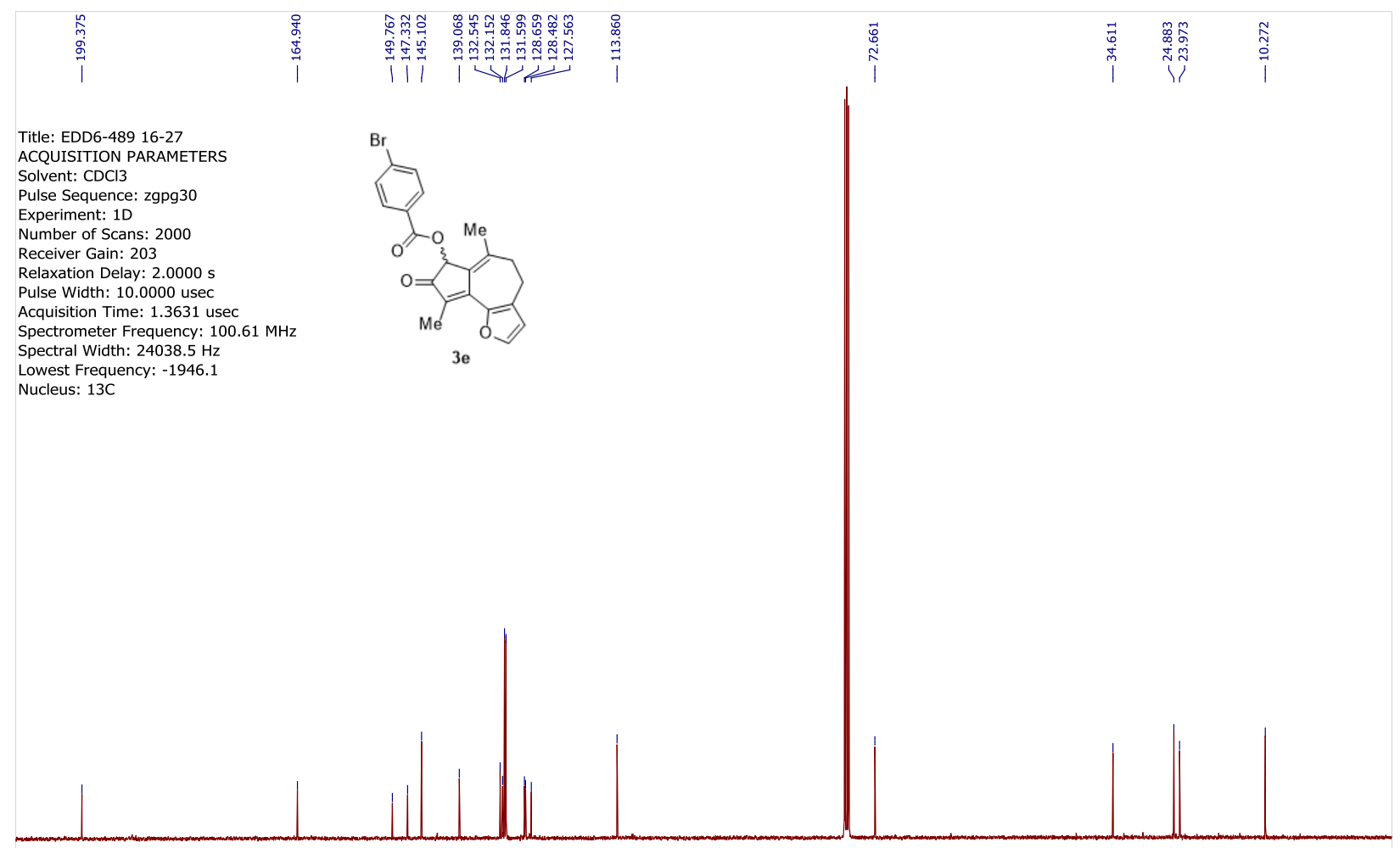

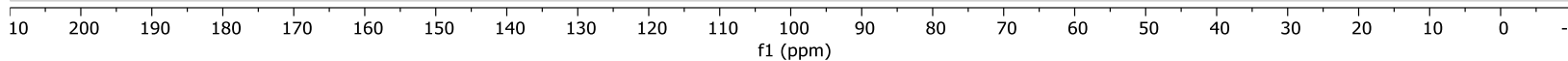


Supporting Information for Organic Letters

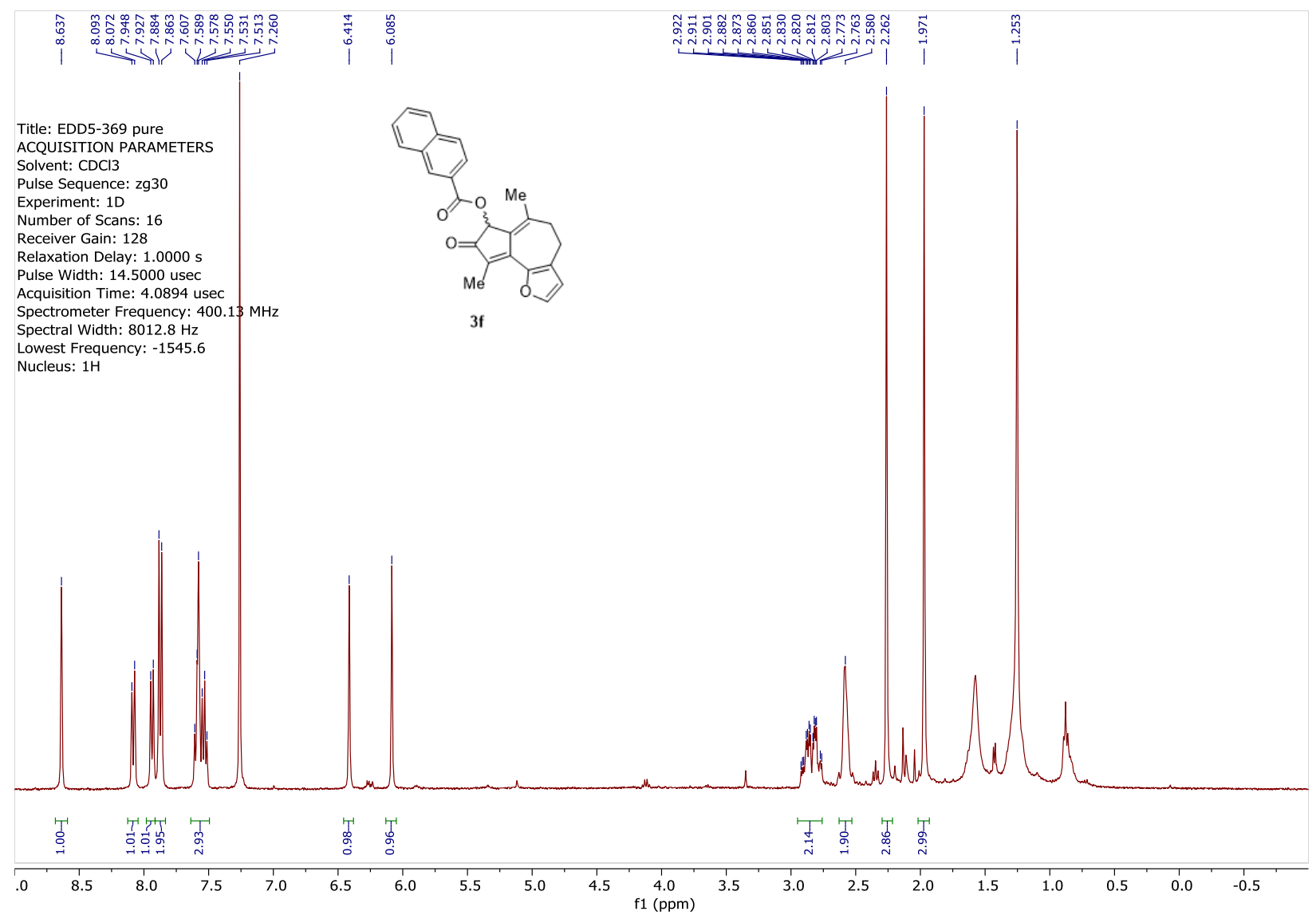


Supporting Information for Organic Letters

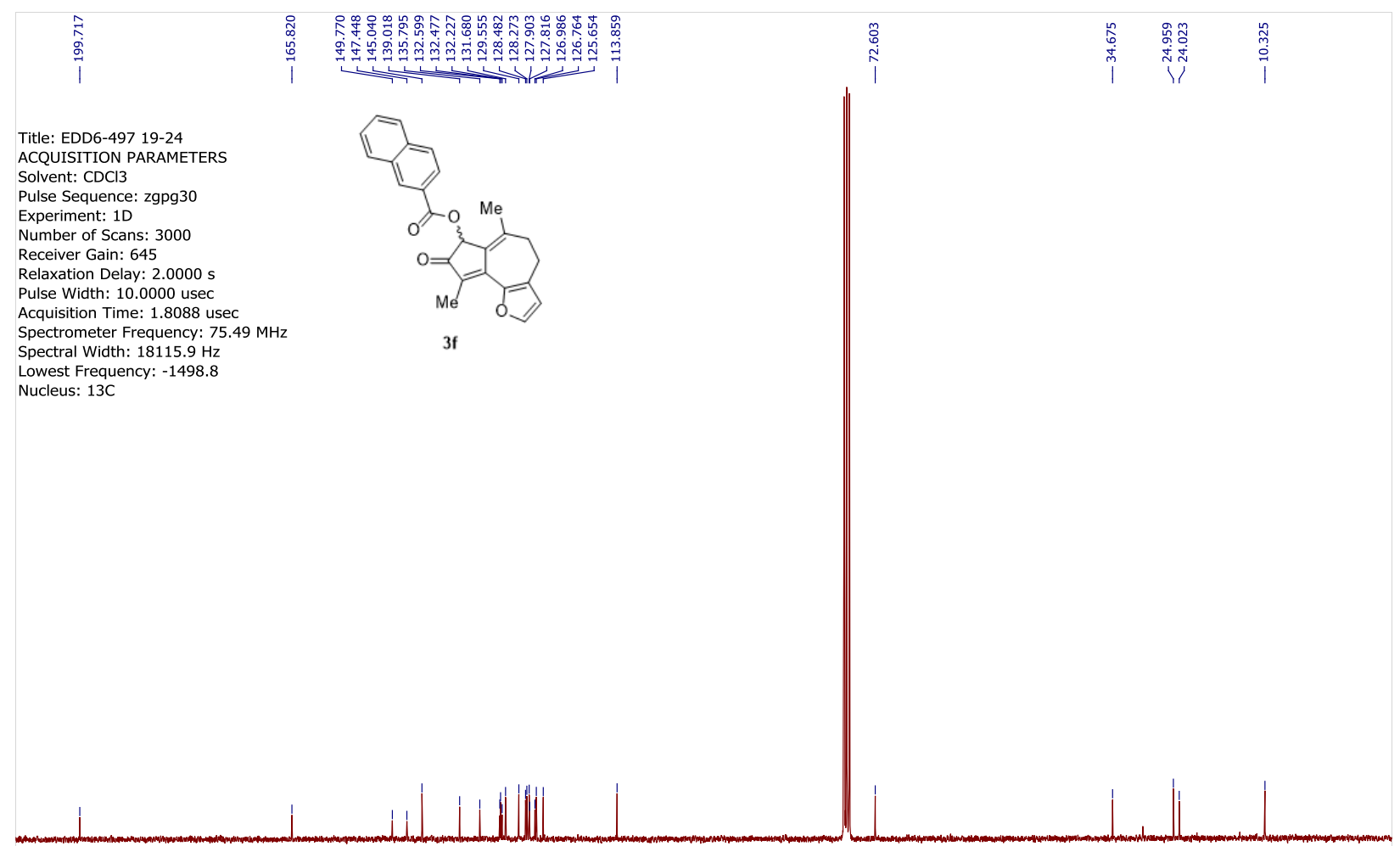

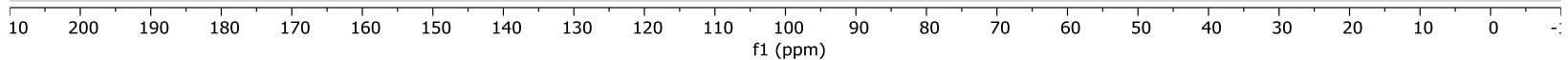


Supporting Information for Organic Letters

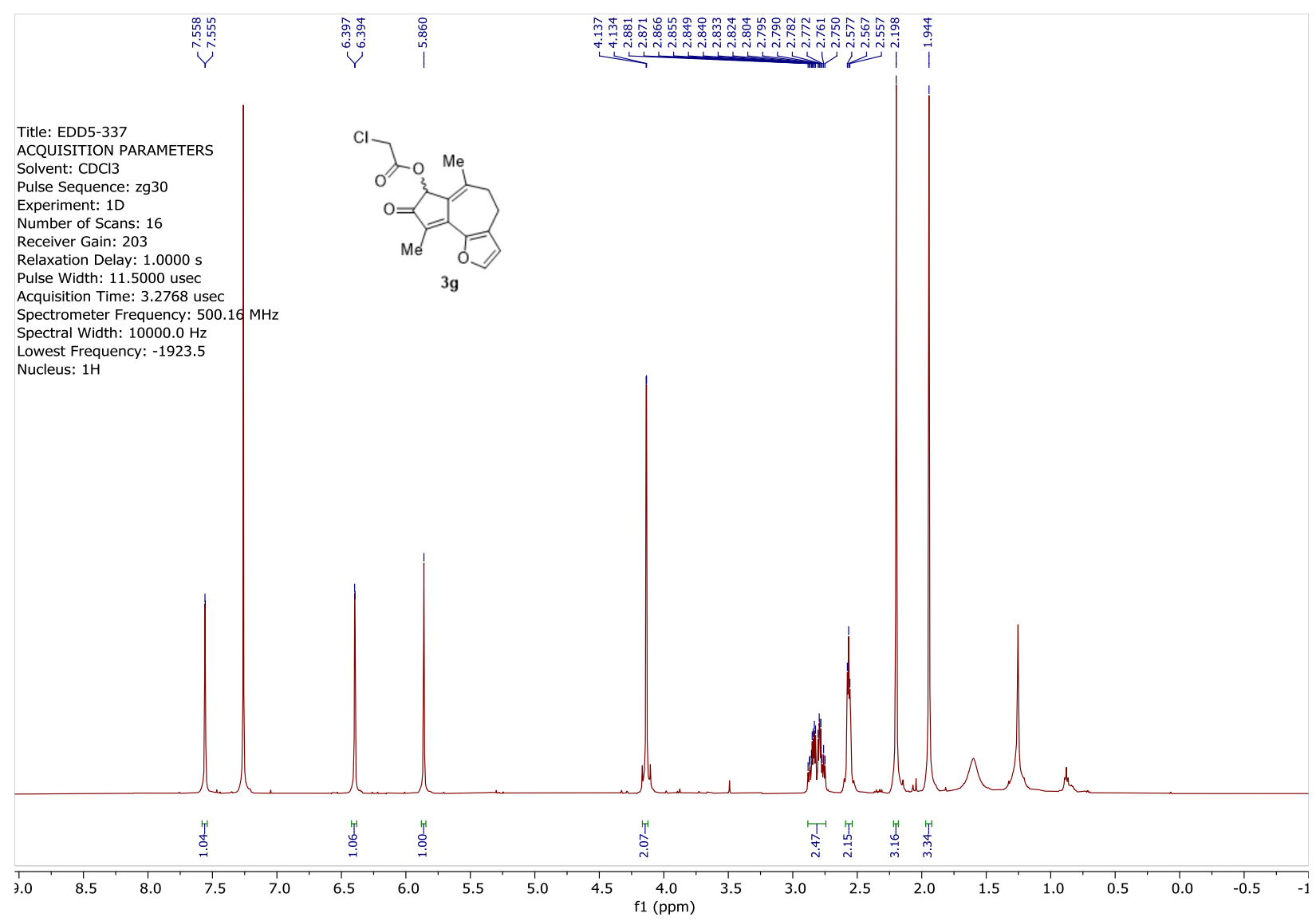


Supporting Information for Organic Letters

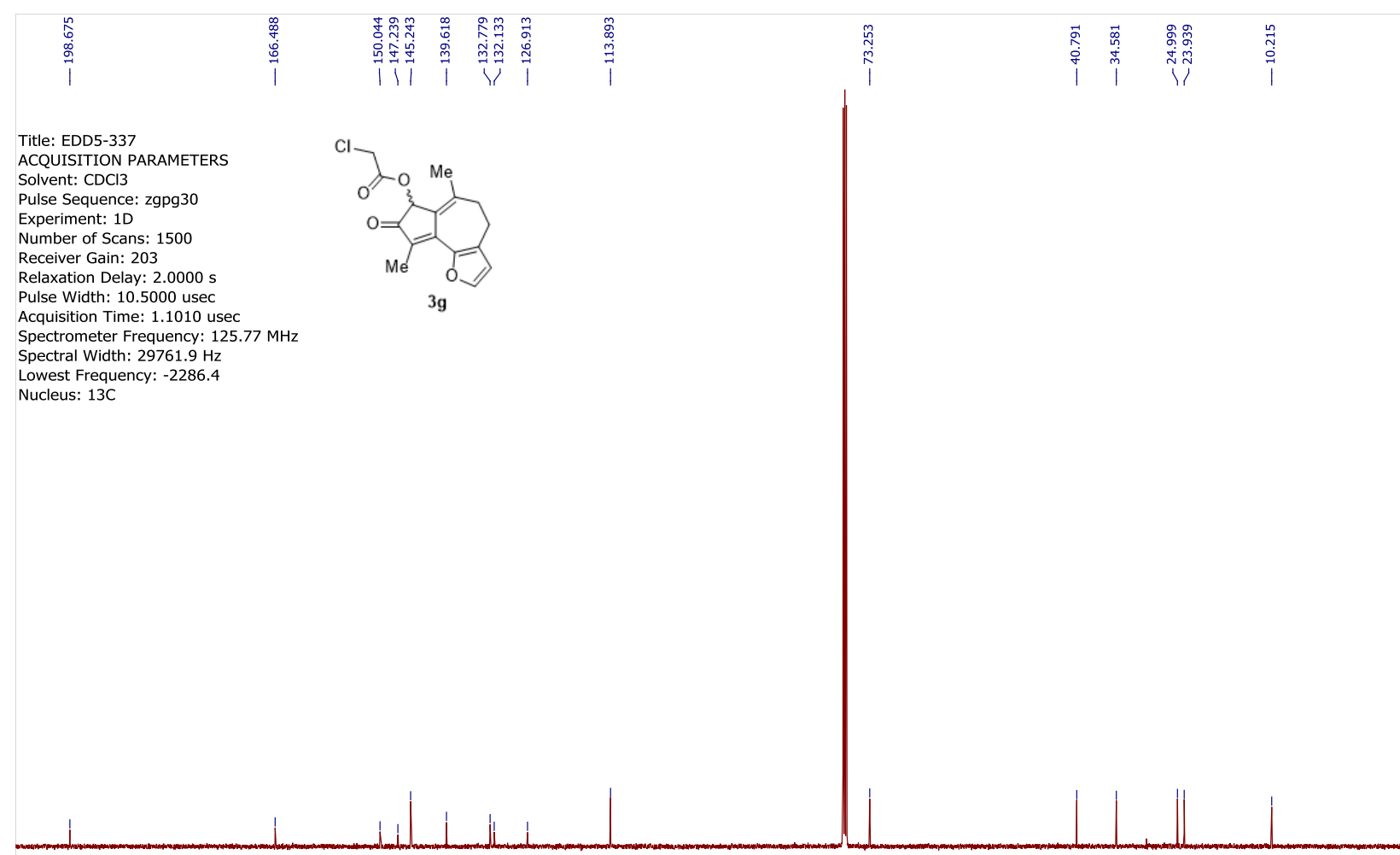

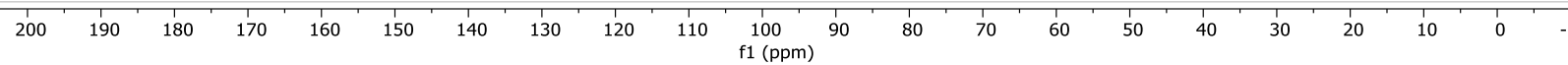


Supporting Information for Organic Letters

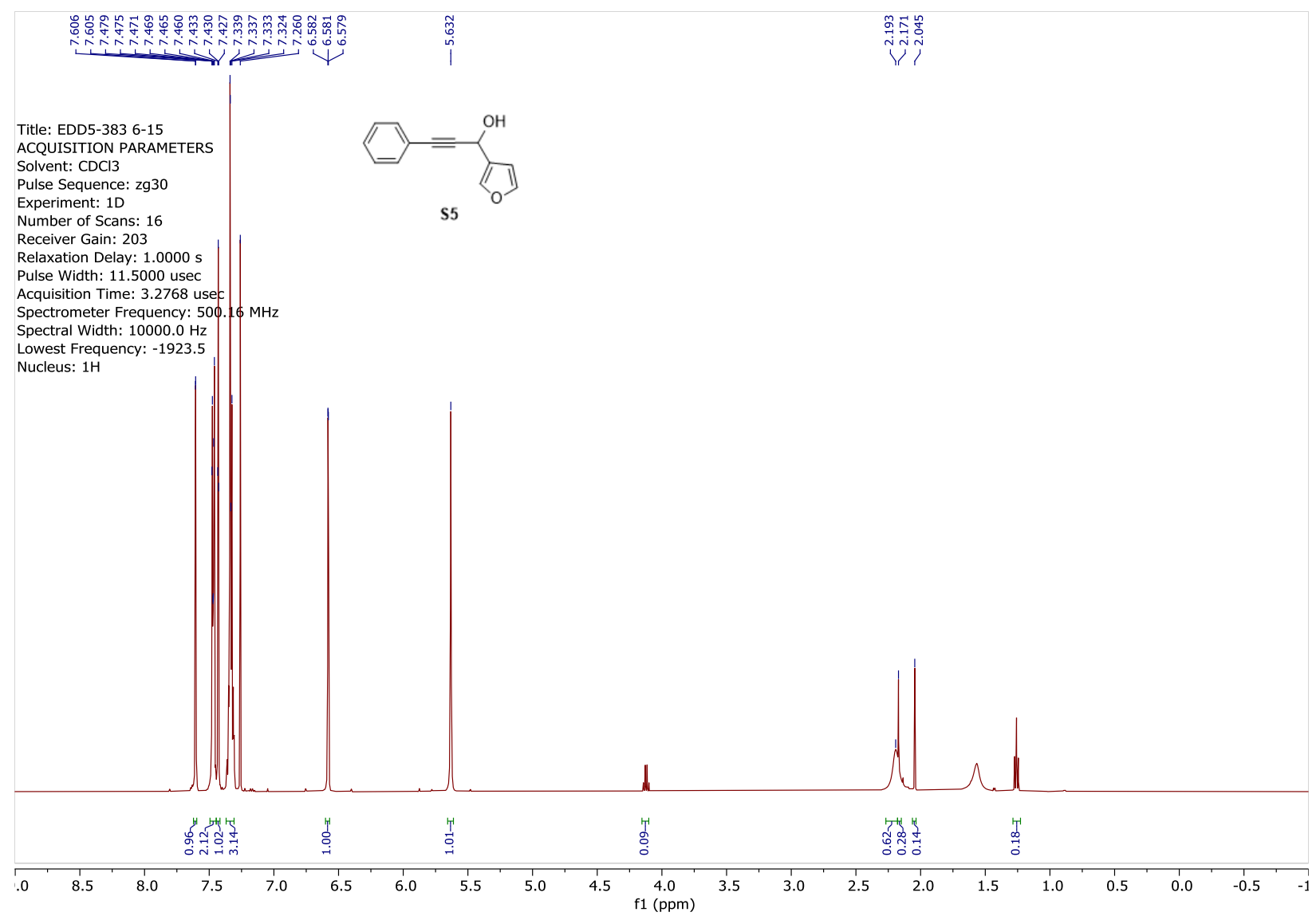


Supporting Information for Organic Letters

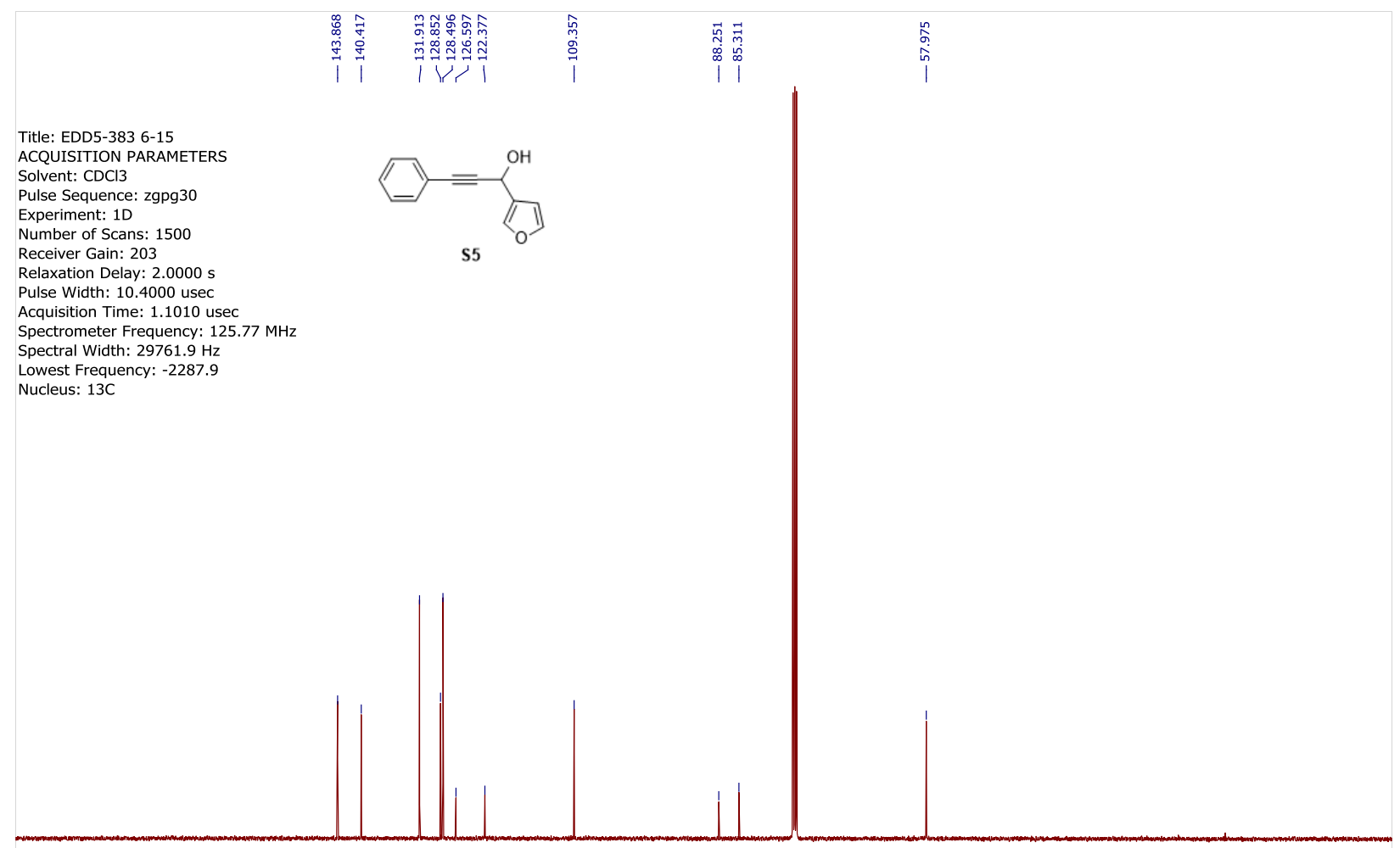

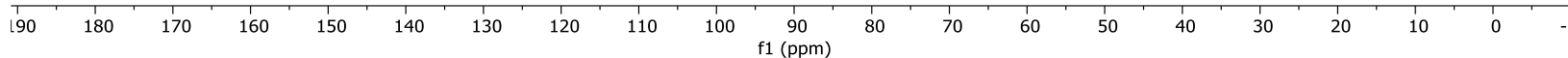


Supporting Information for Organic Letters

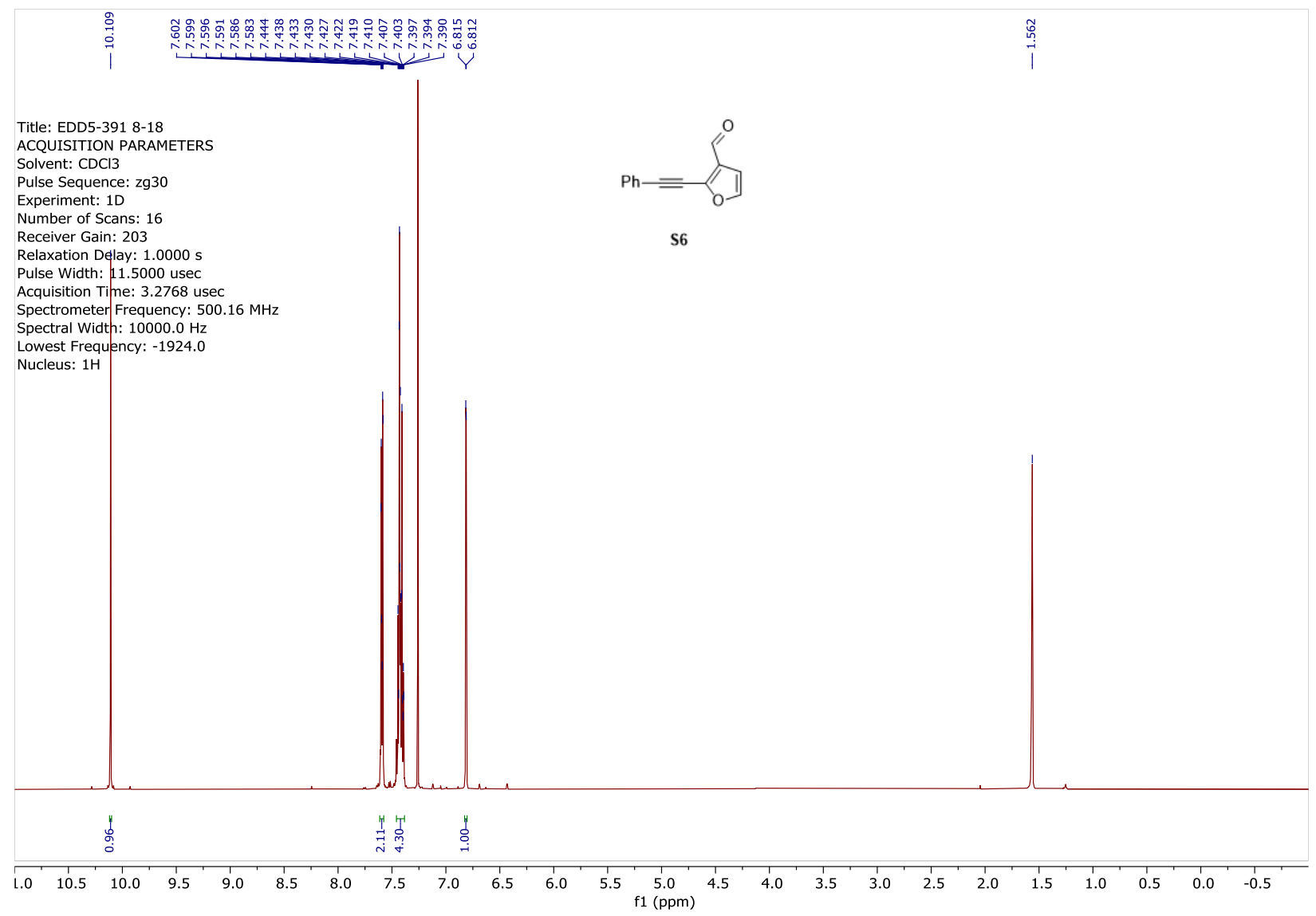


Supporting Information for Organic Letters

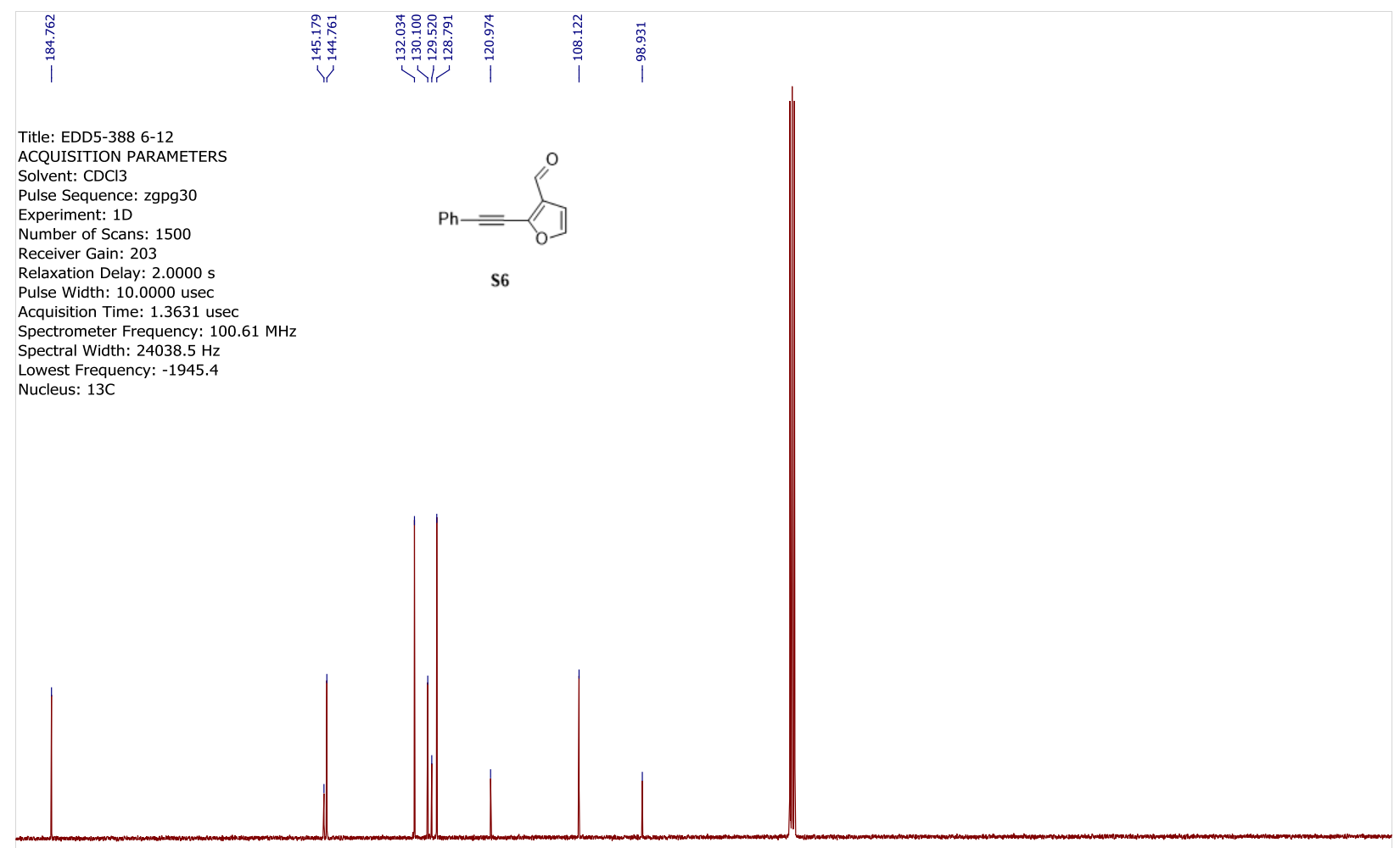

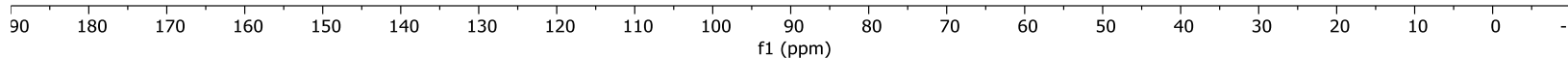


Supporting Information for Organic Letters

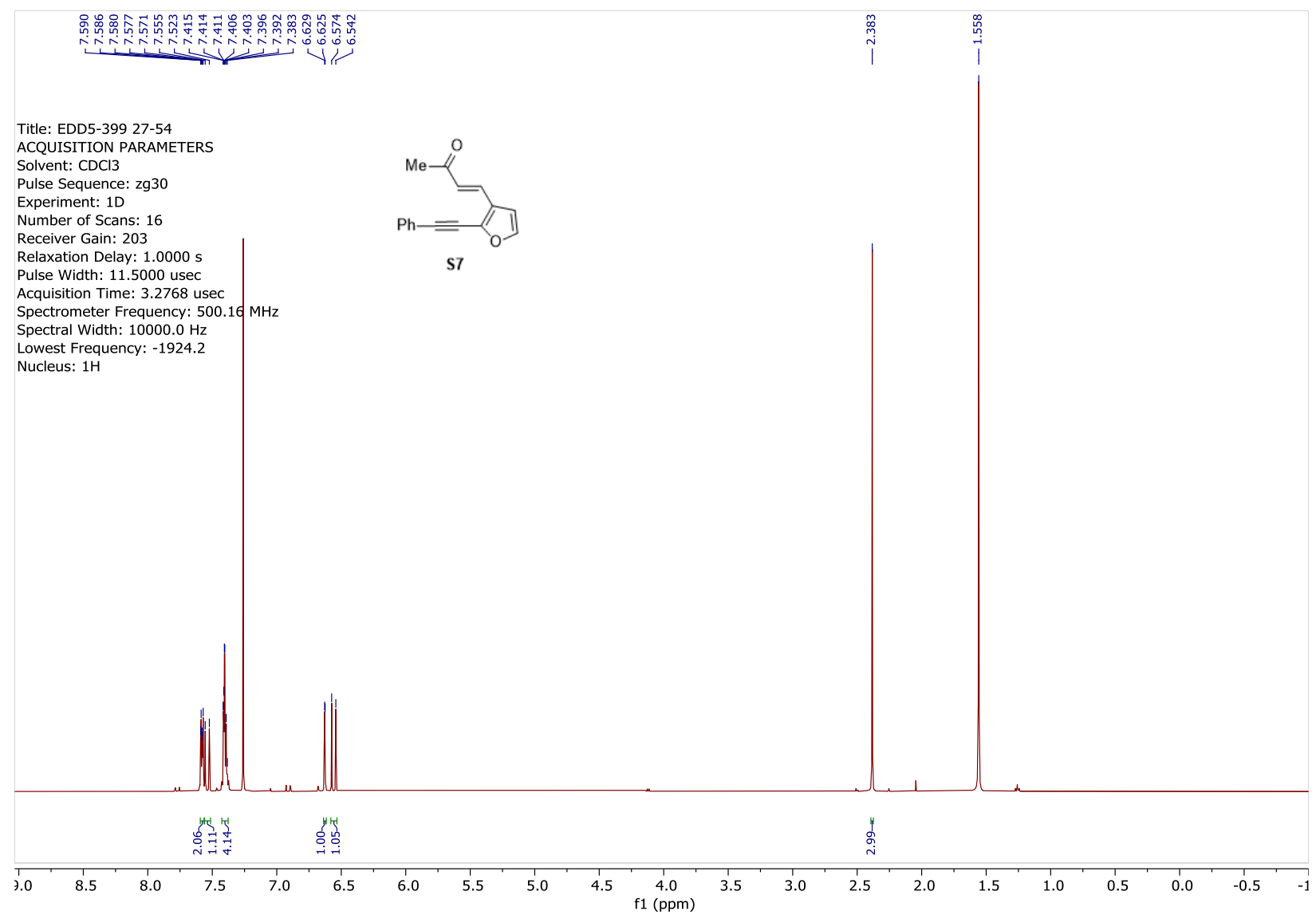


Supporting Information for Organic Letters

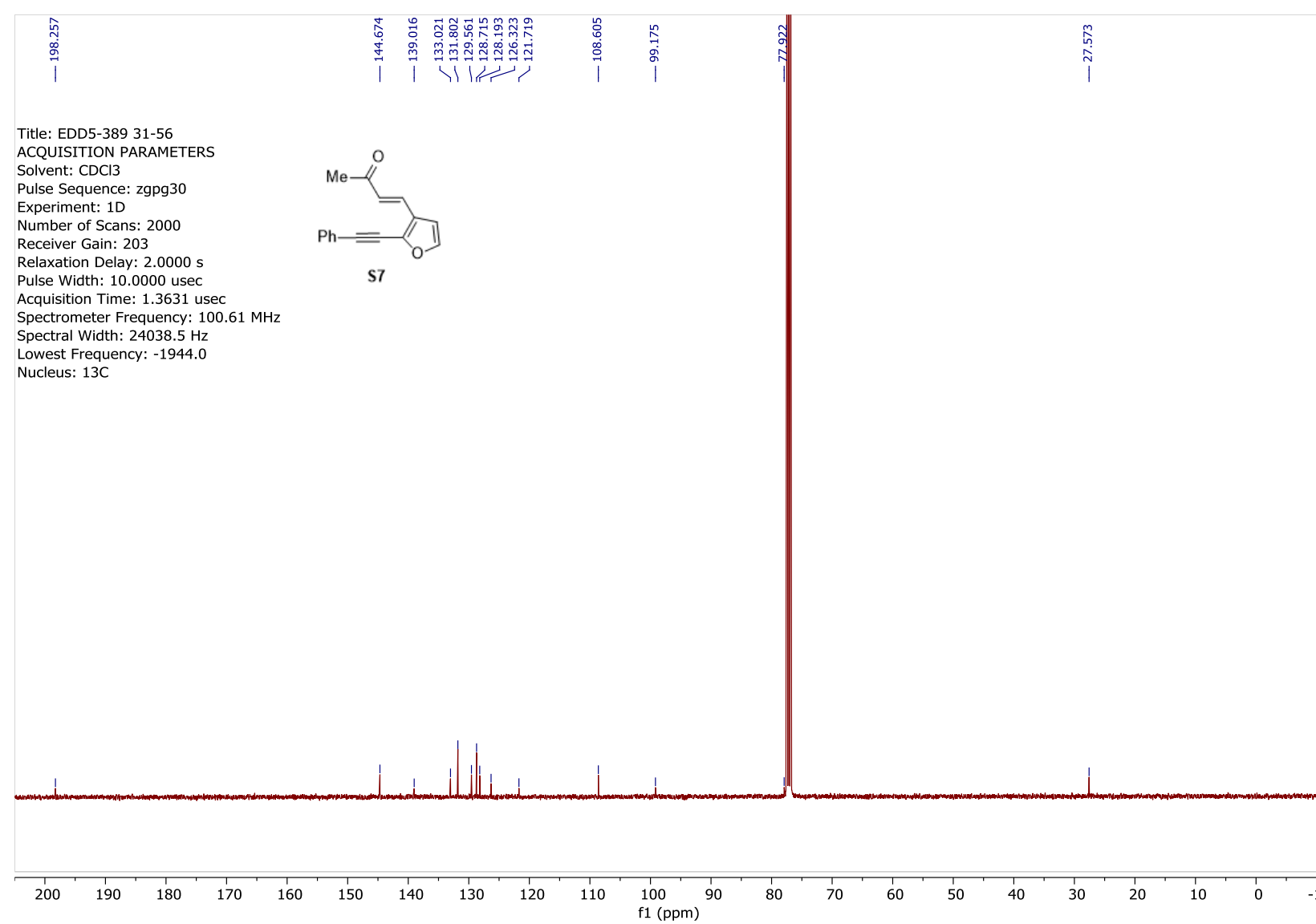




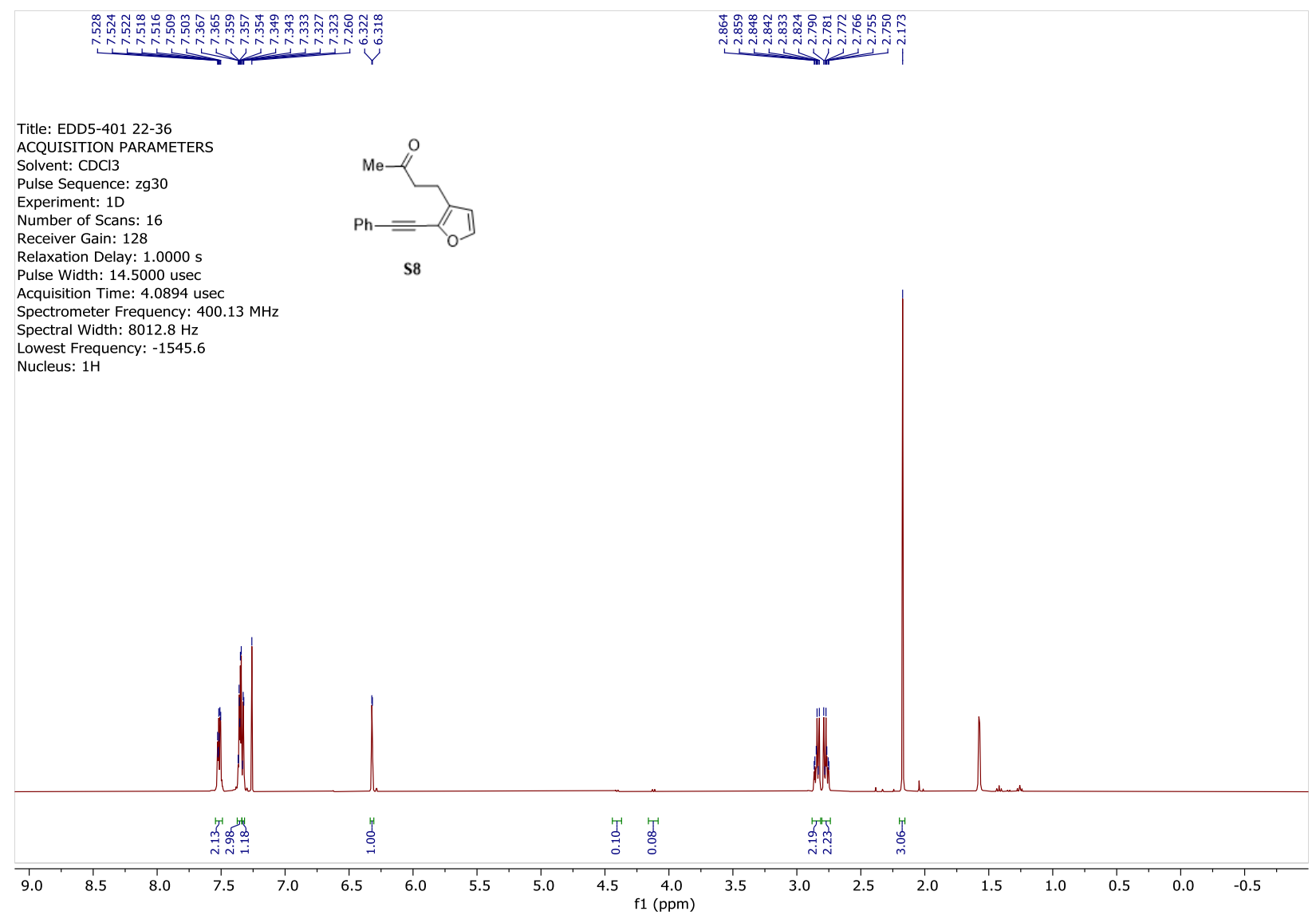


Supporting Information for Organic Letters

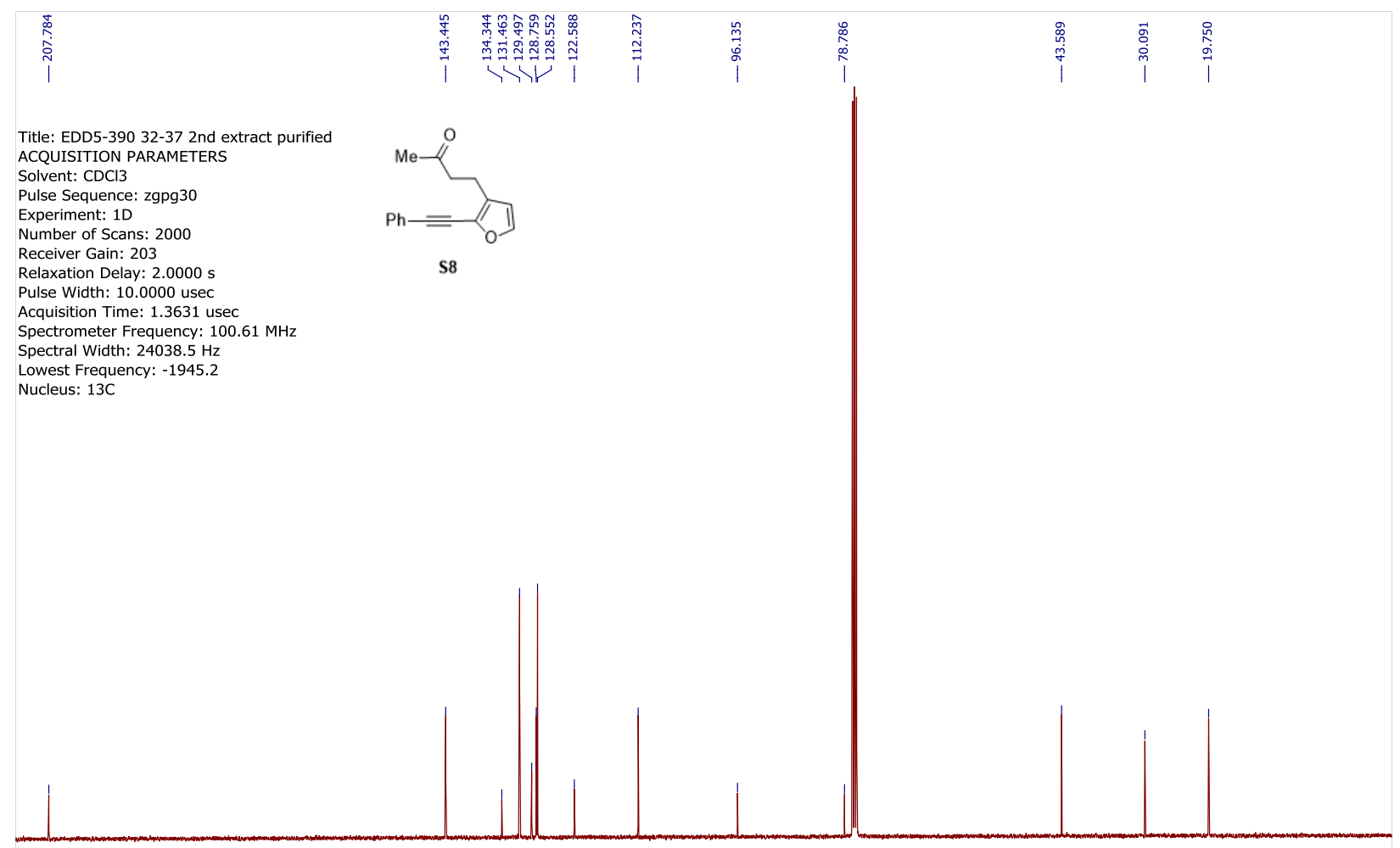

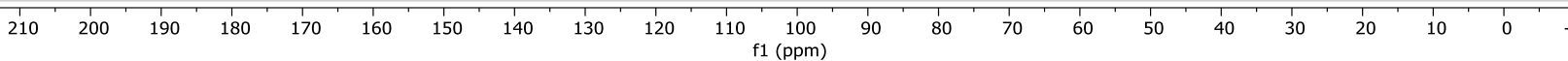


Supporting Information for Organic Letters

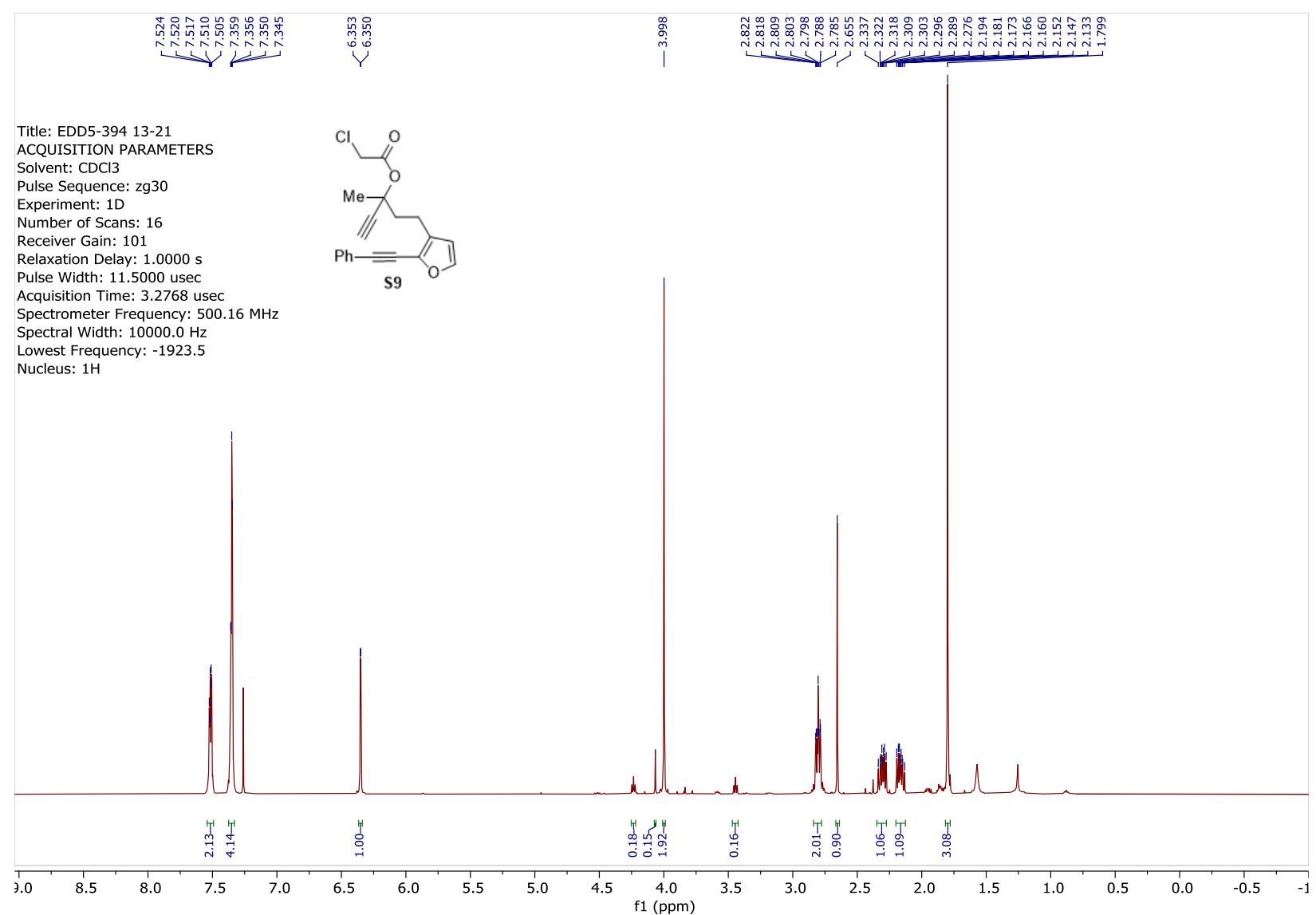


Supporting Information for Organic Letters

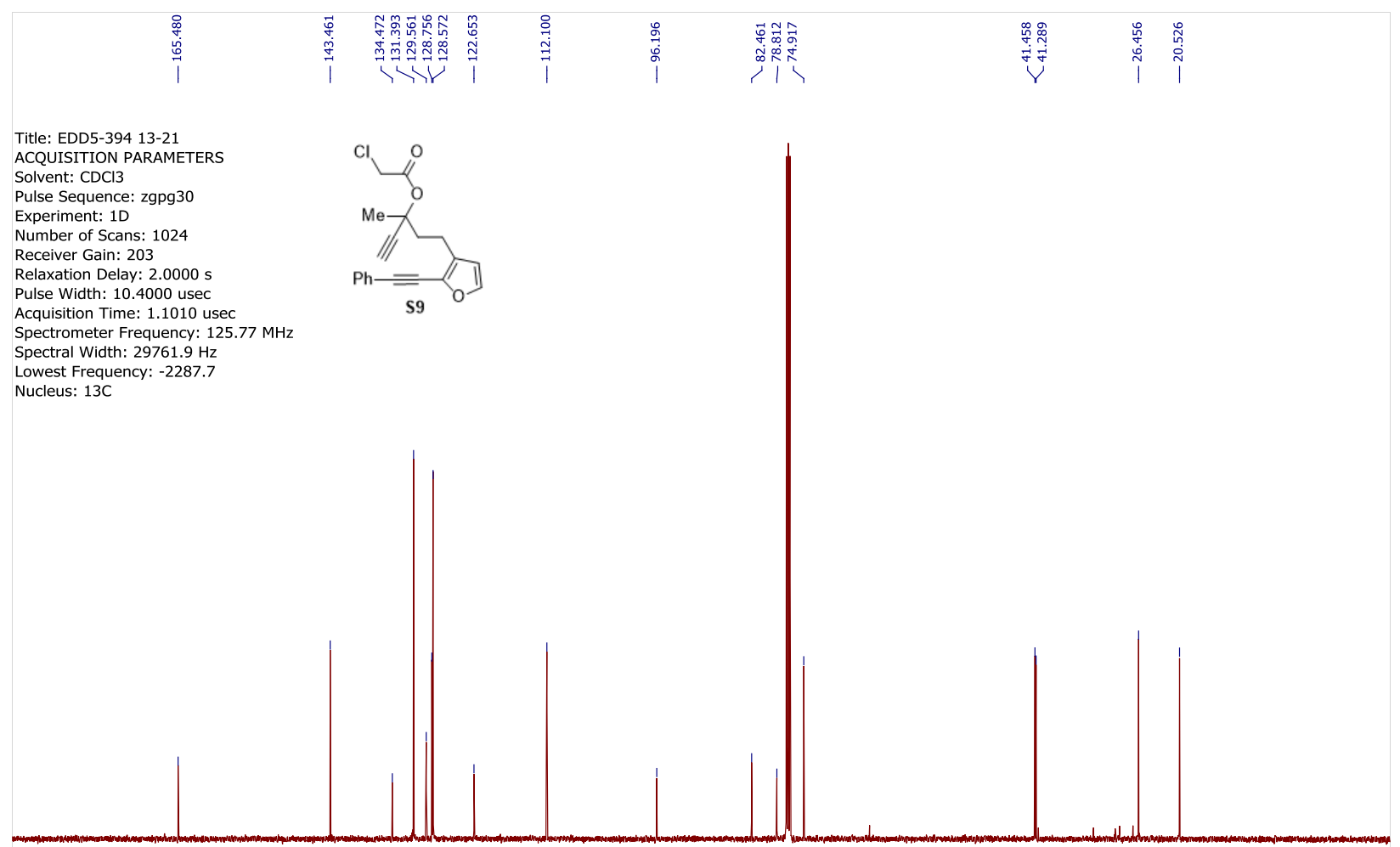

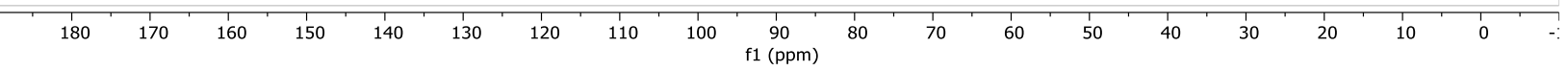


Supporting Information for Organic Letters

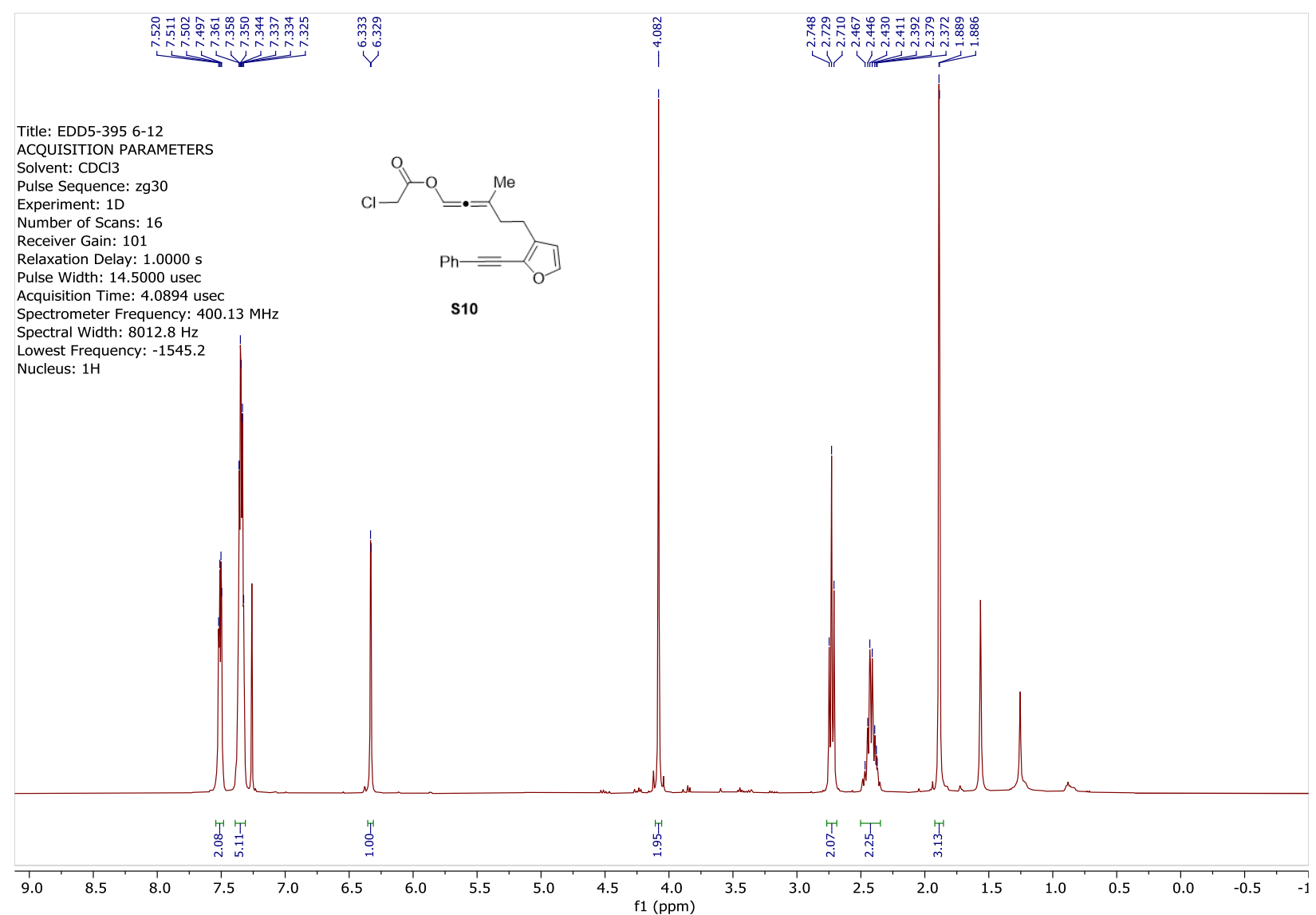


Supporting Information for Organic Letters

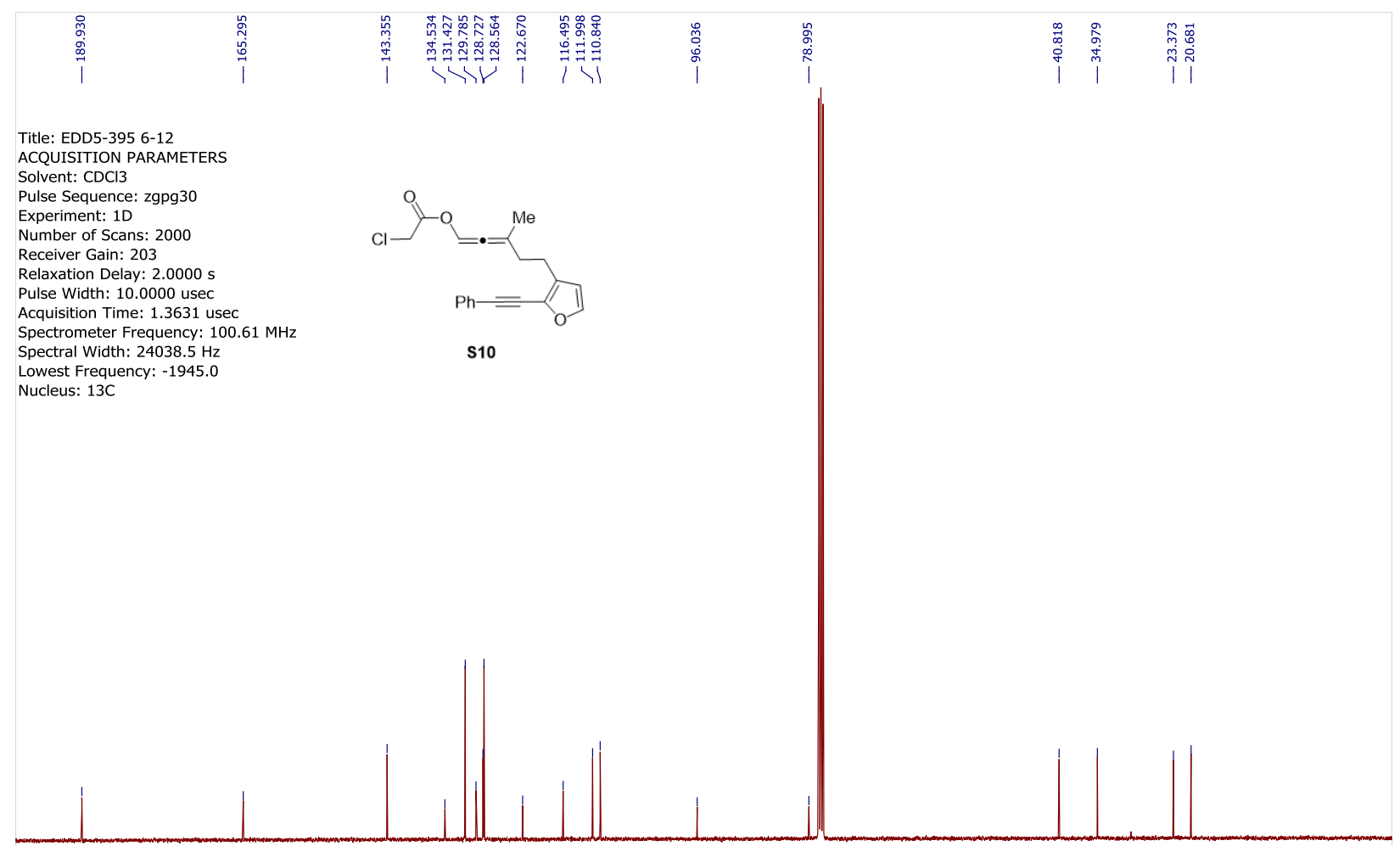

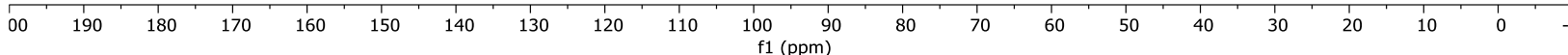


Supporting Information for Organic Letters

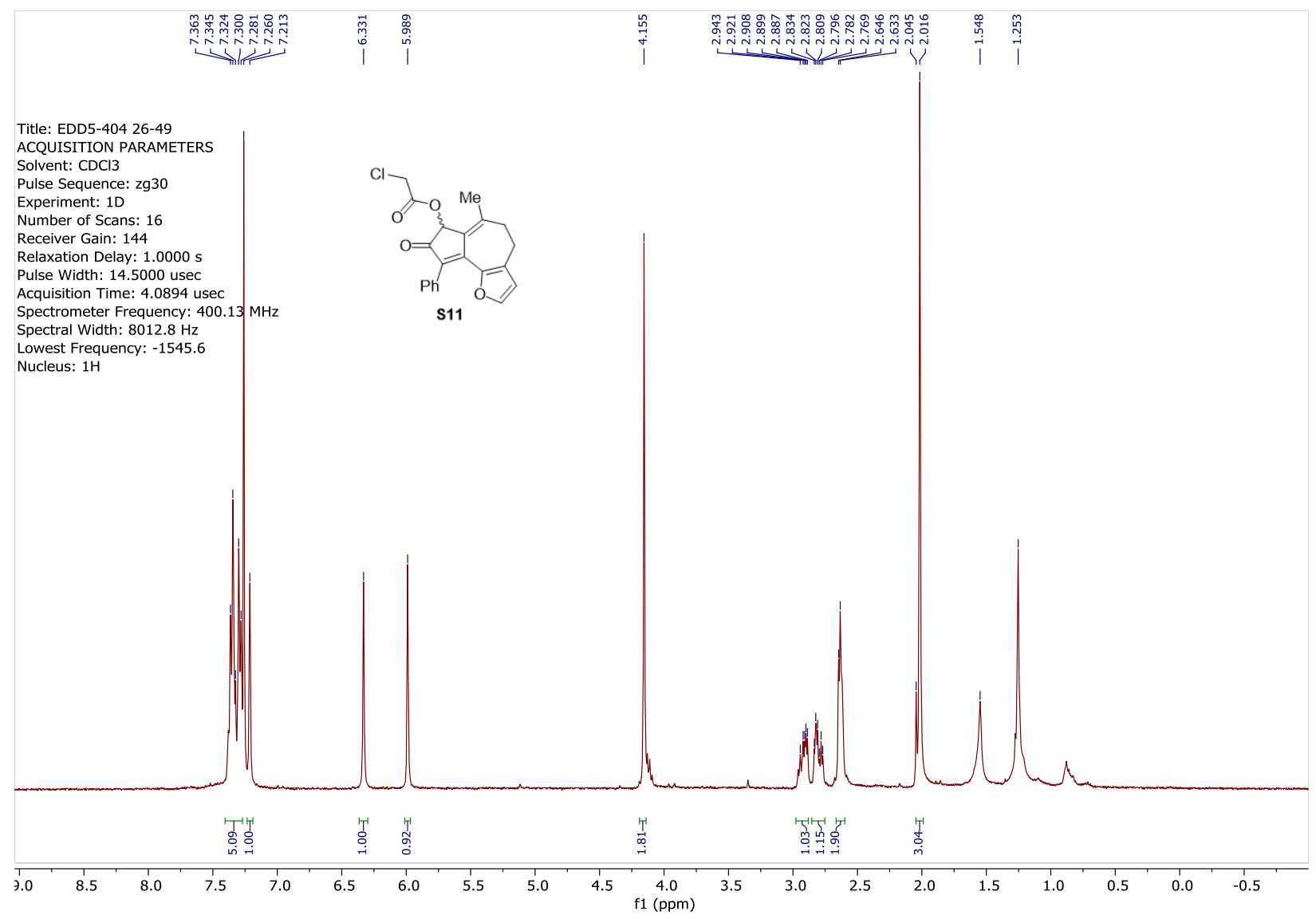


Supporting Information for Organic Letters
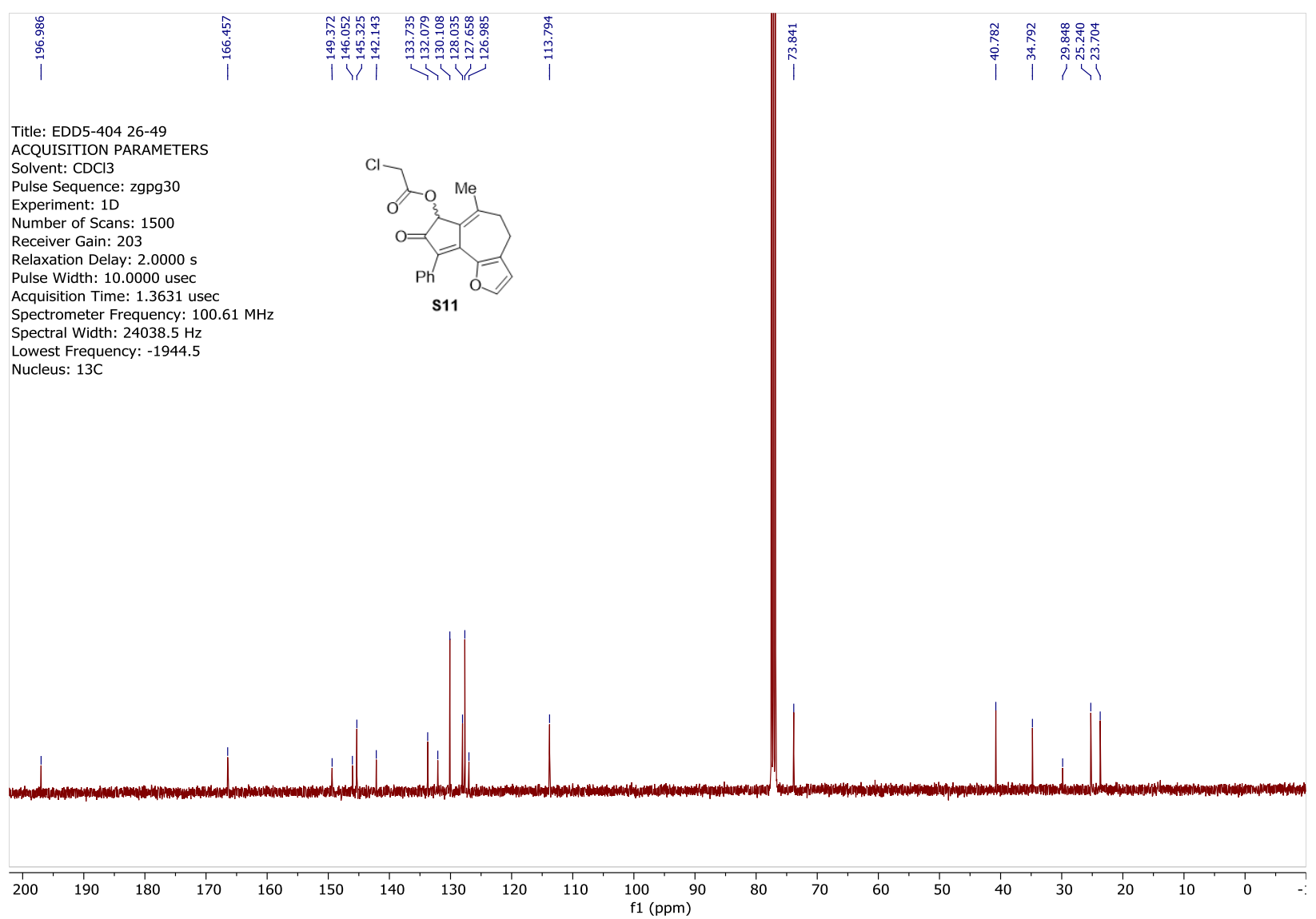
Supporting Information for Organic Letters

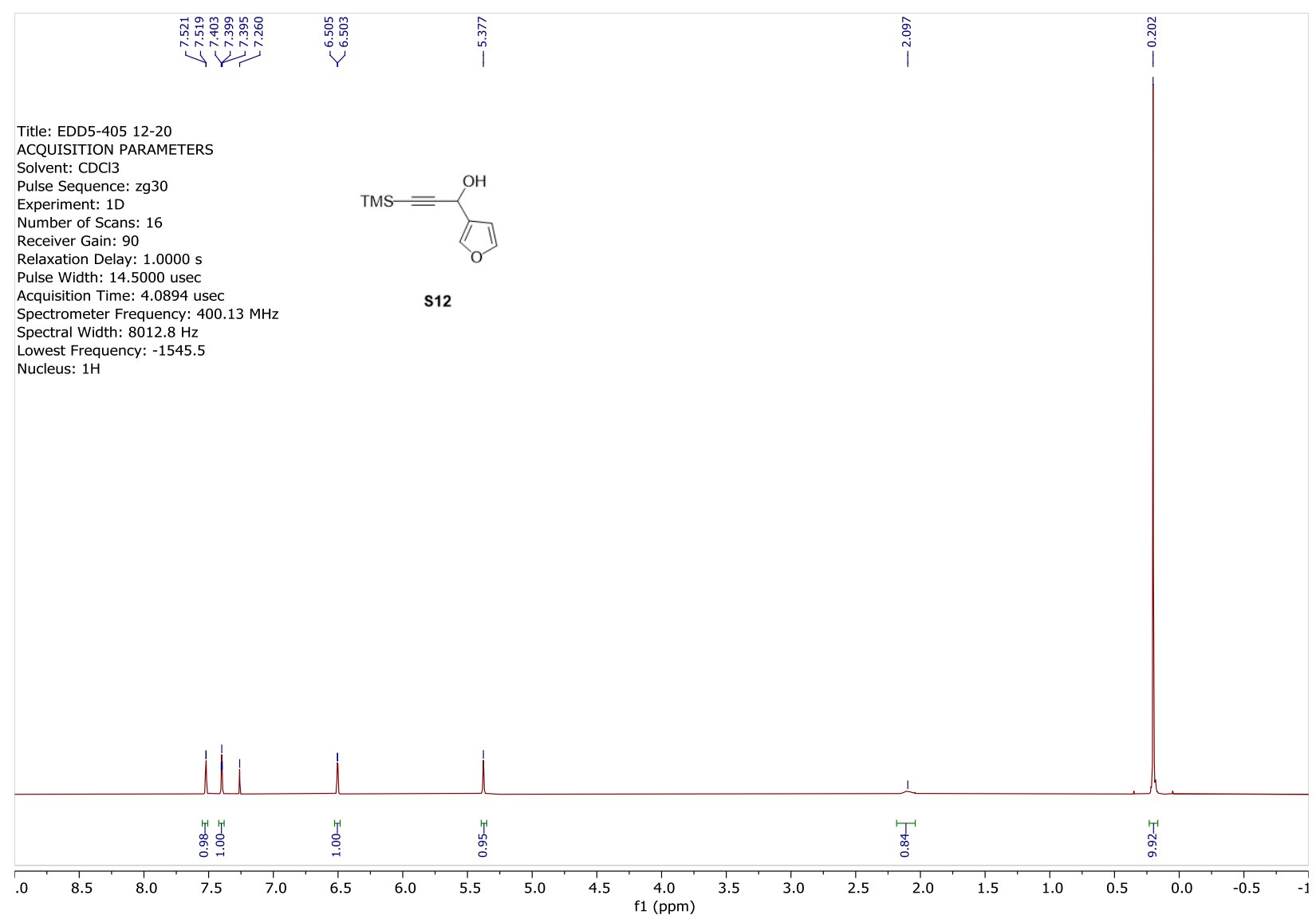


Supporting Information for Organic Letters

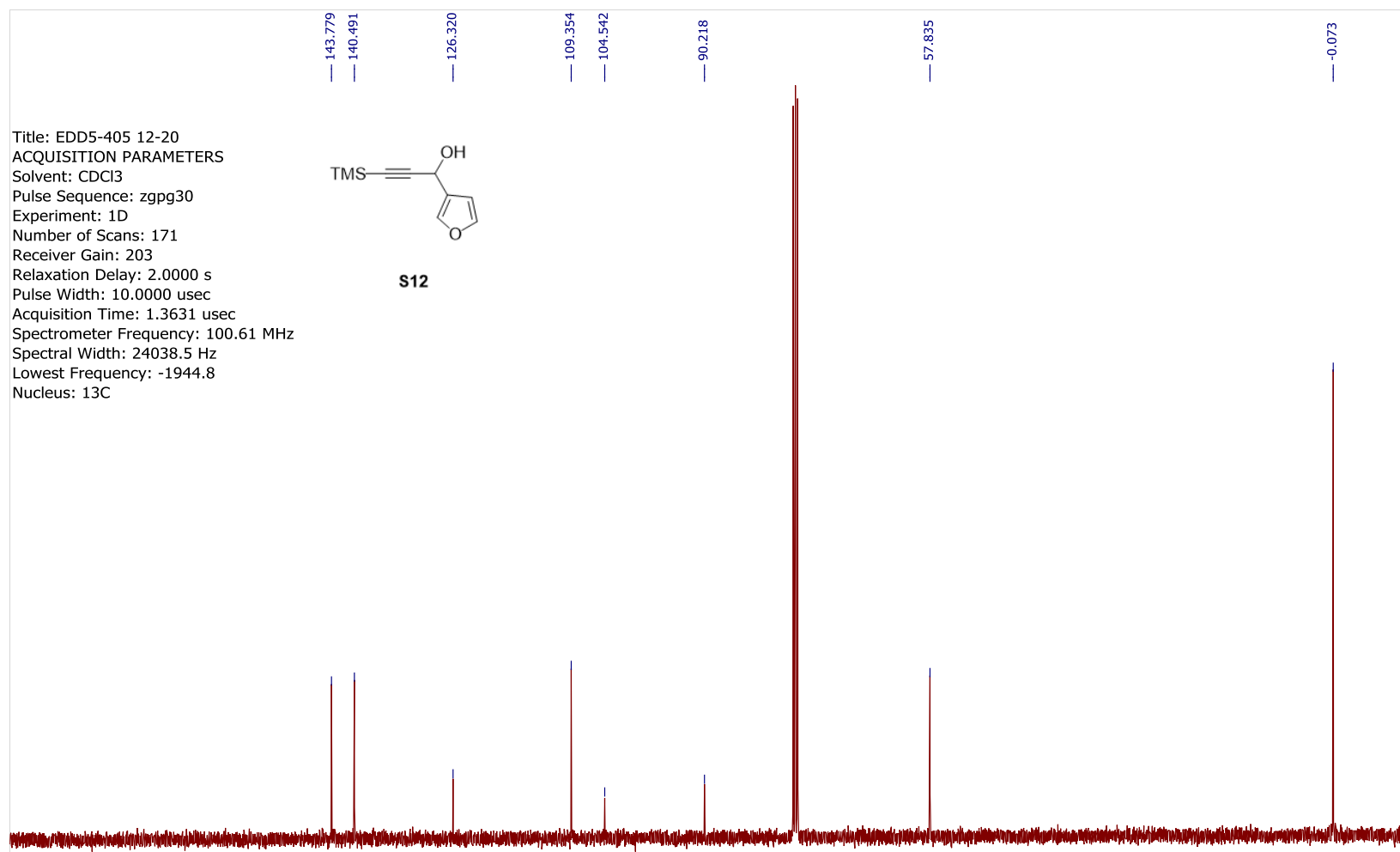

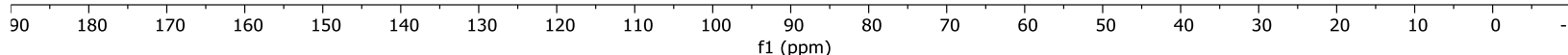


Supporting Information for Organic Letters

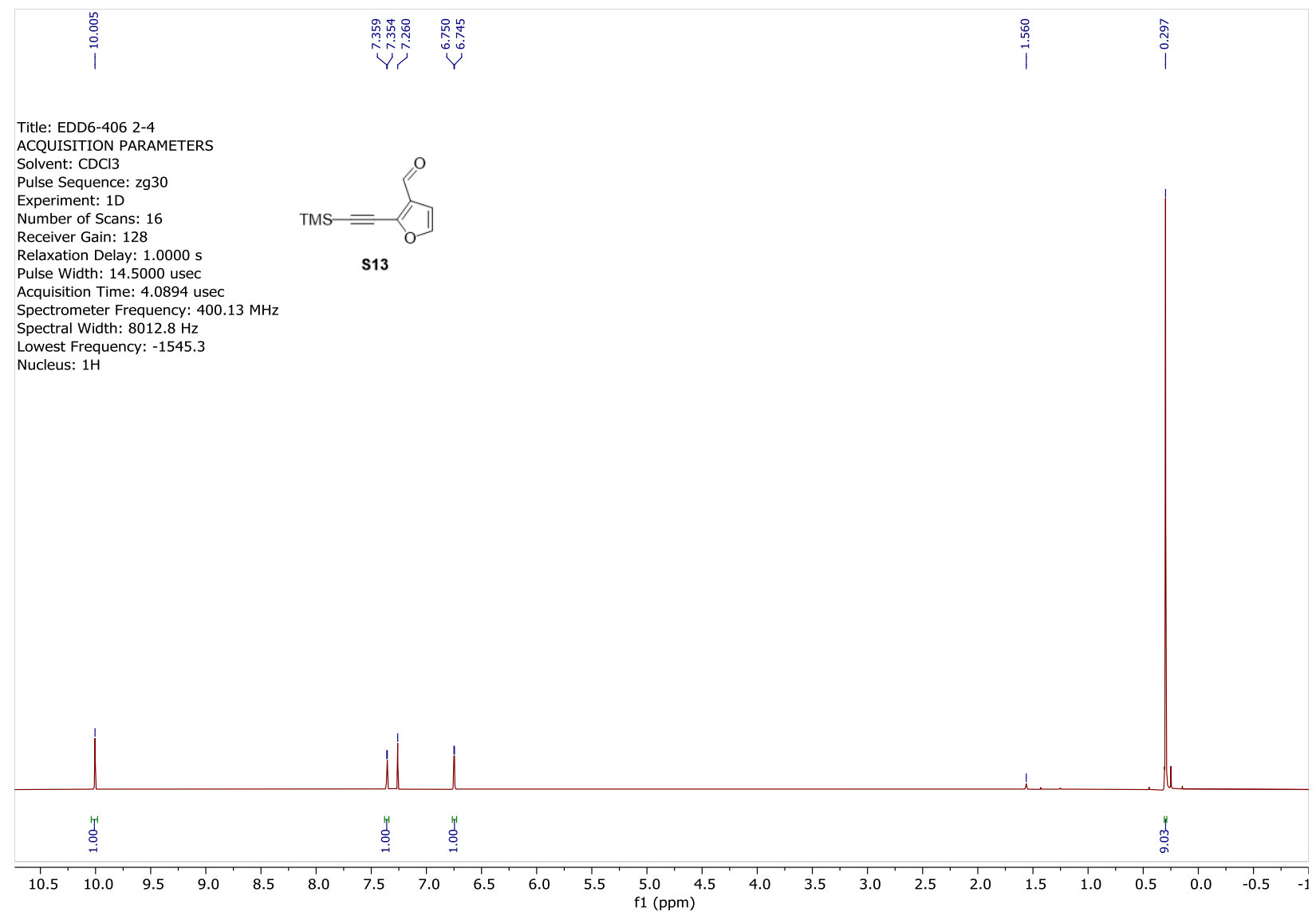


Supporting Information for Organic Letters

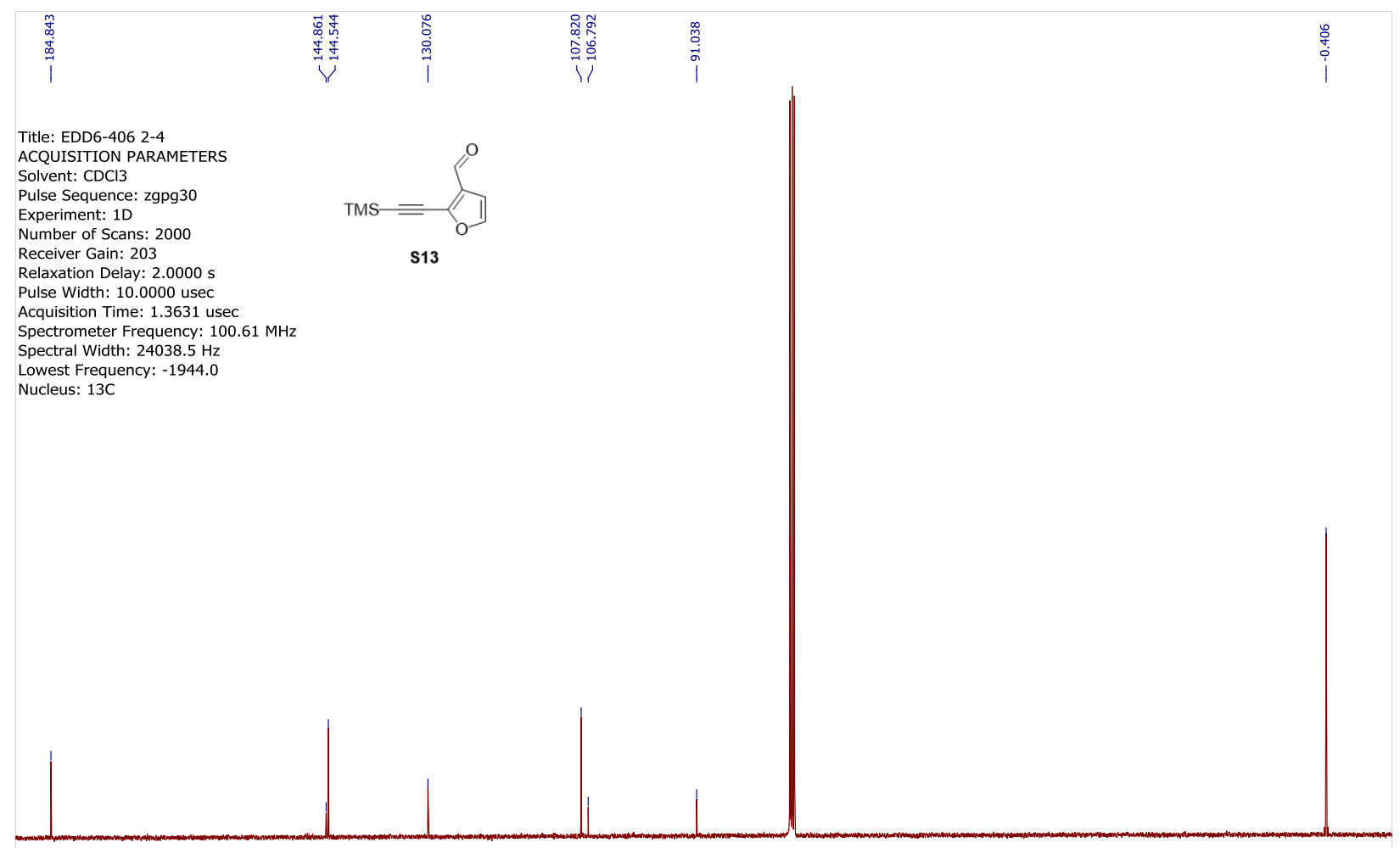

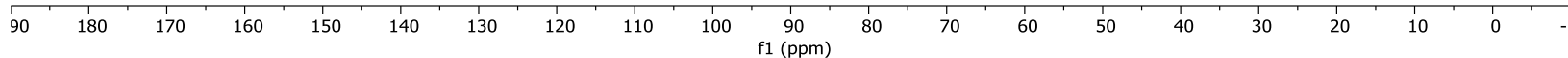


Supporting Information for Organic Letters

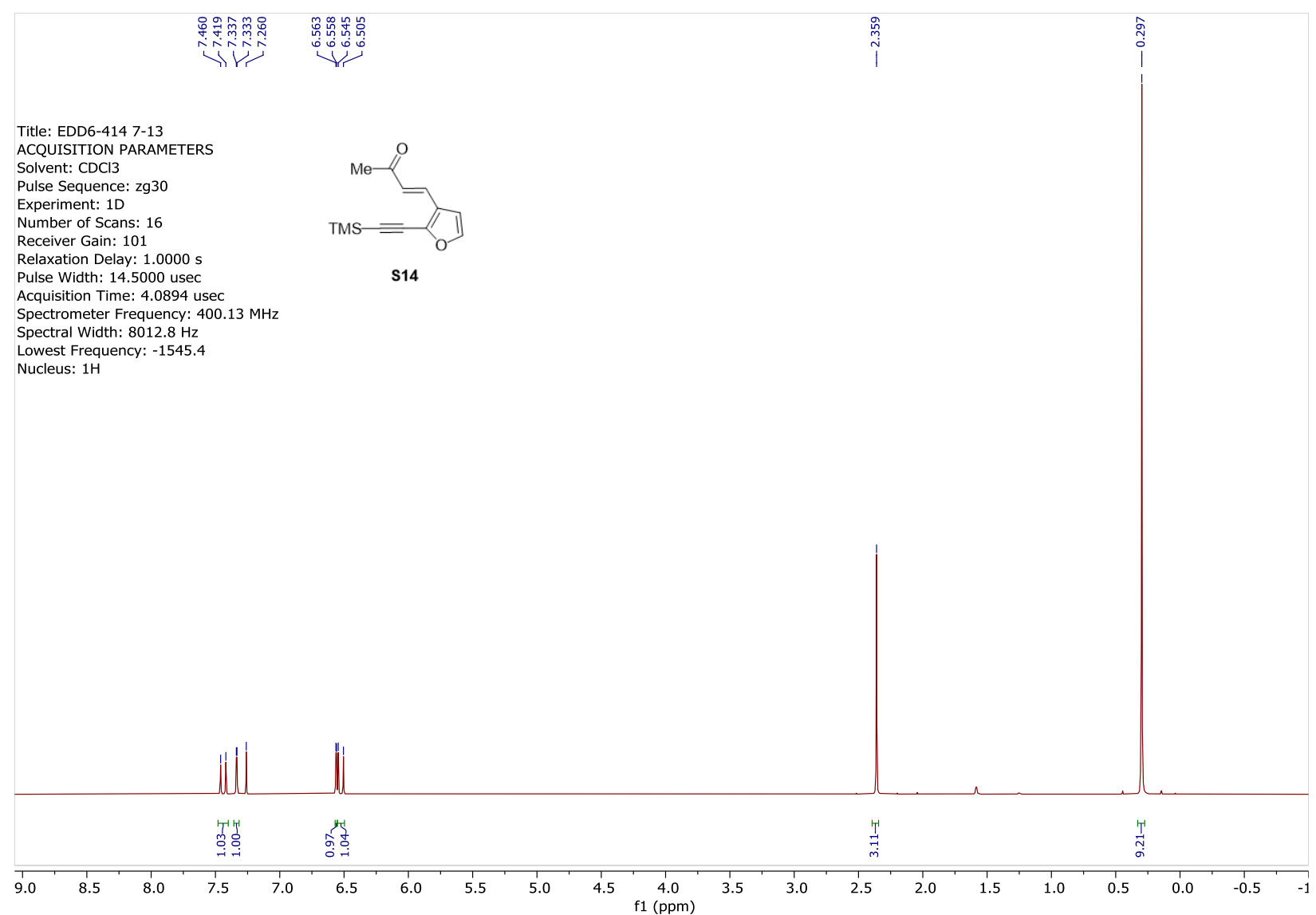


Supporting Information for Organic Letters

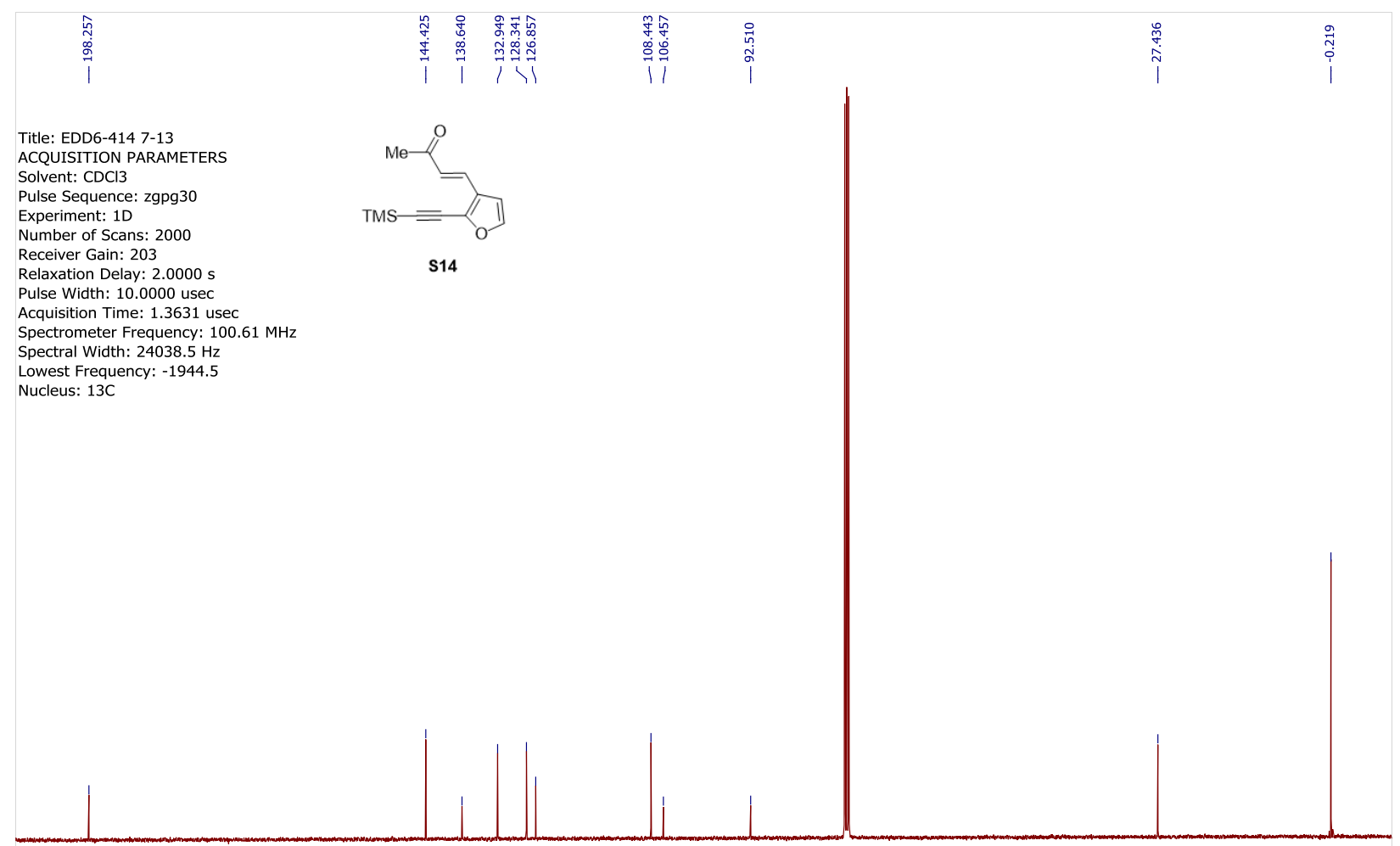

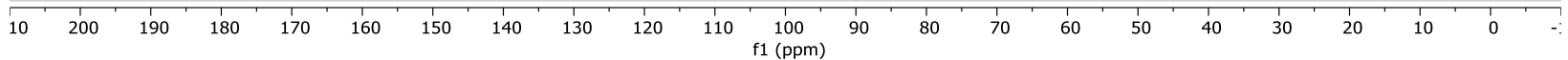




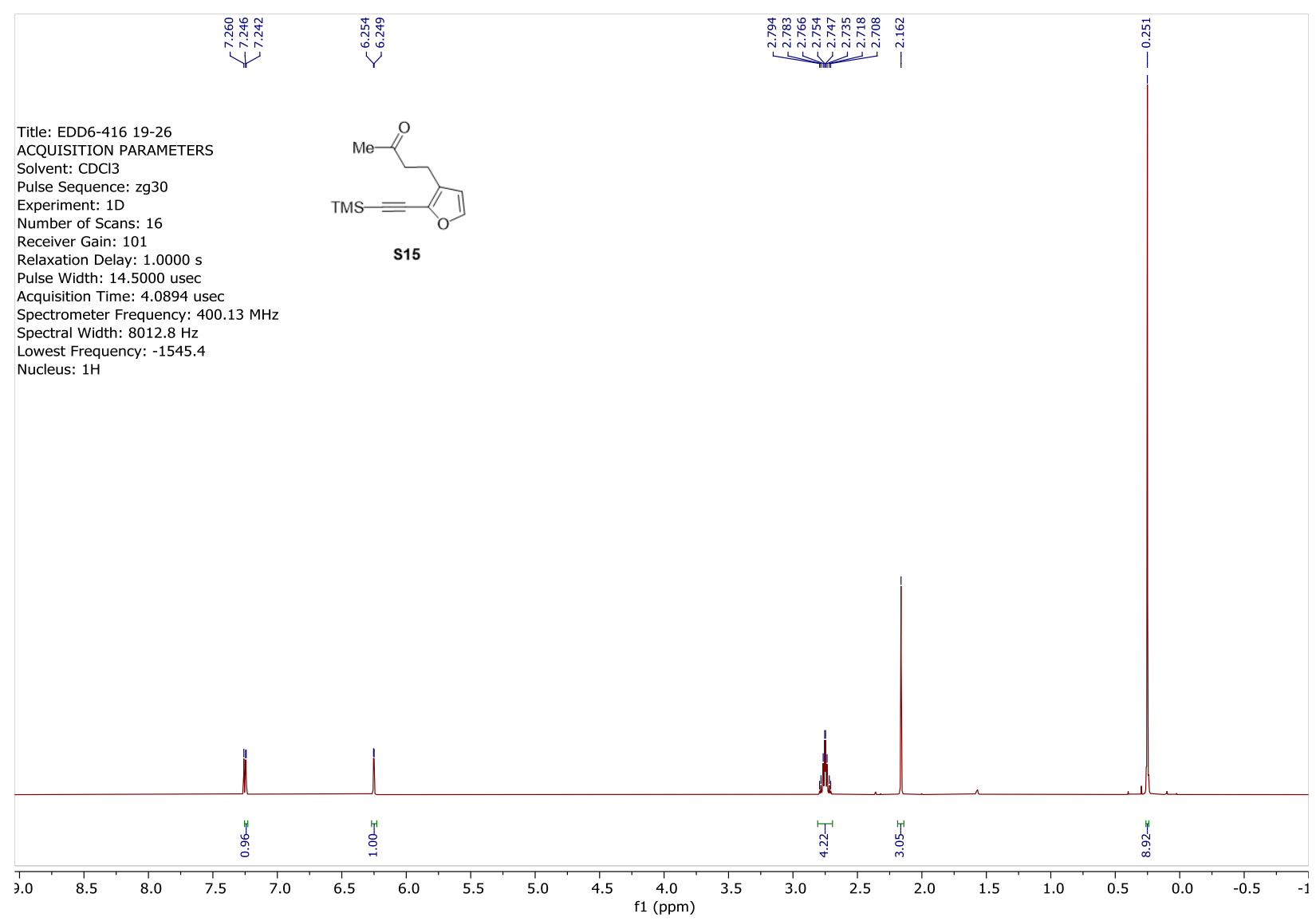


Supporting Information for Organic Letters

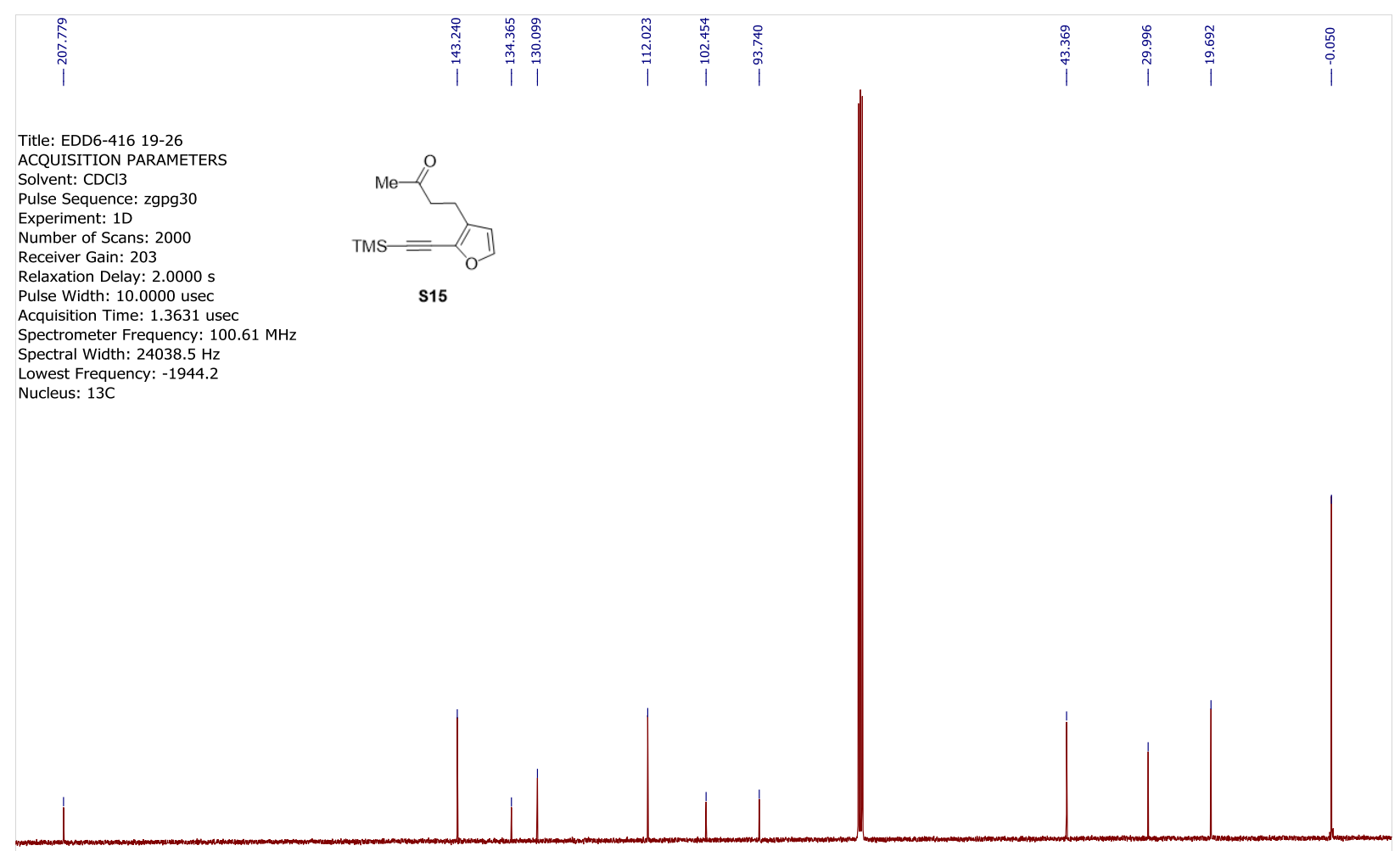

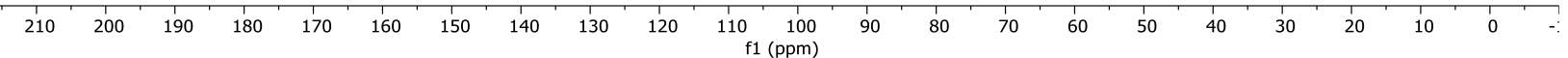


Supporting Information for Organic Letters

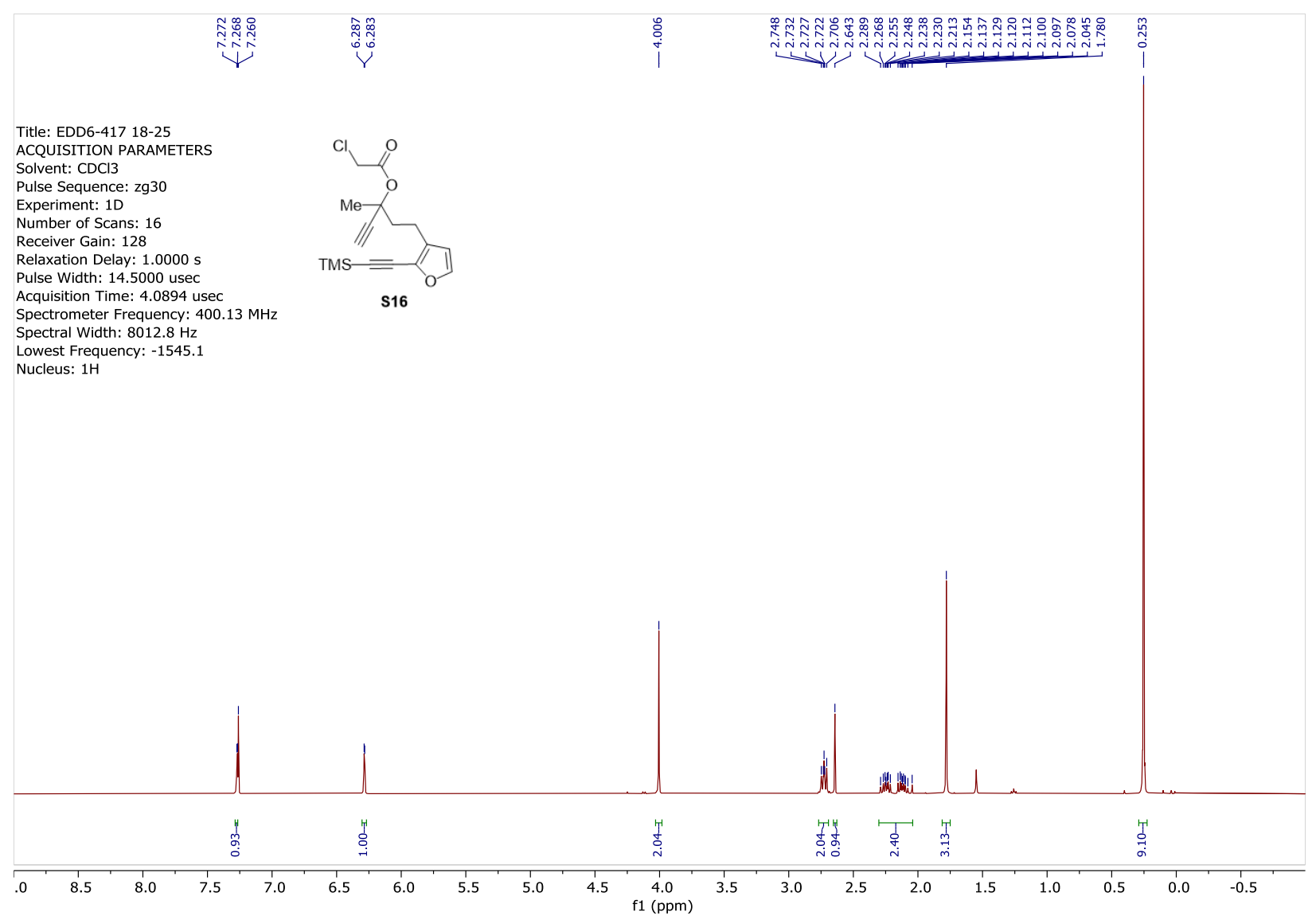


Supporting Information for Organic Letters

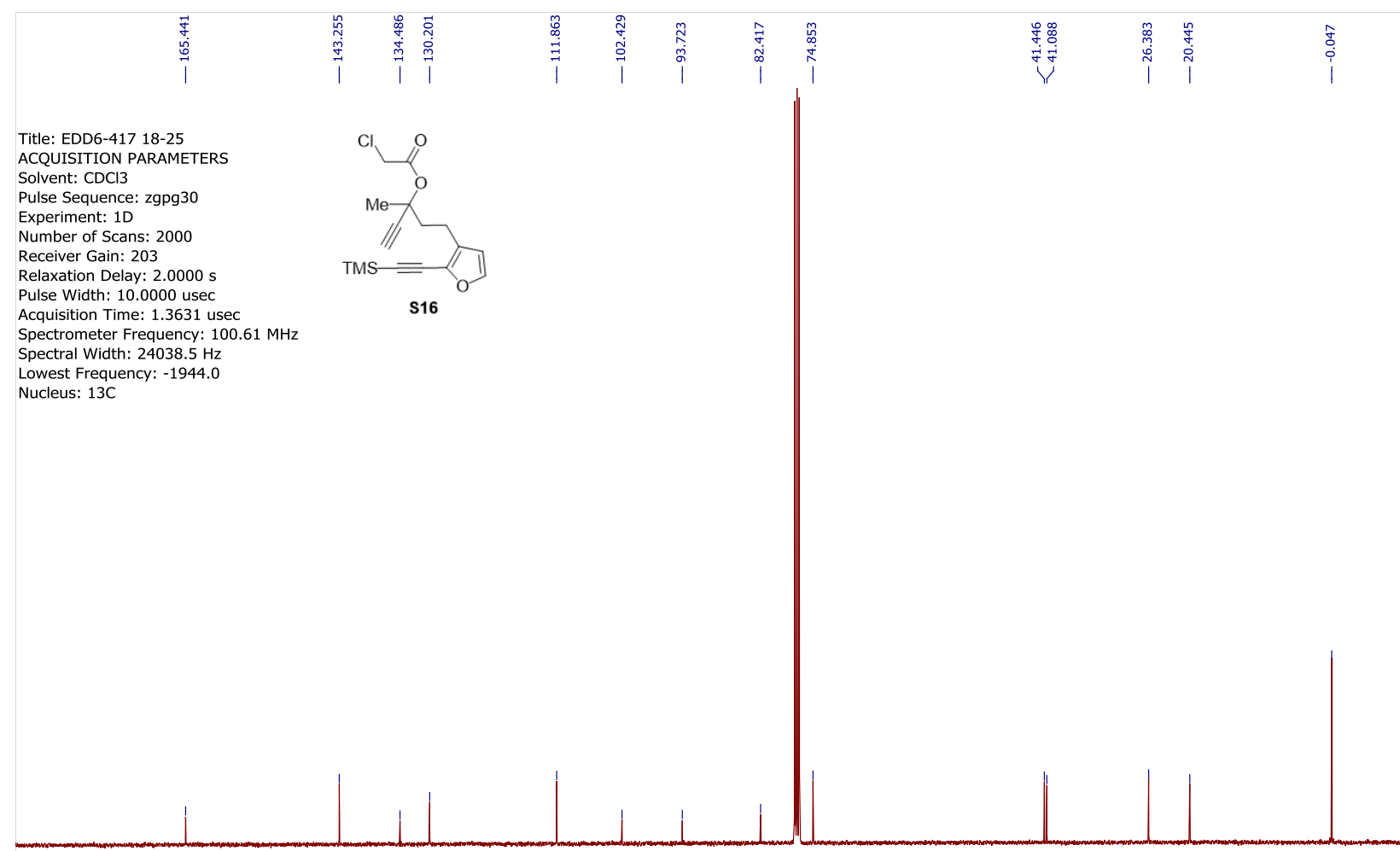

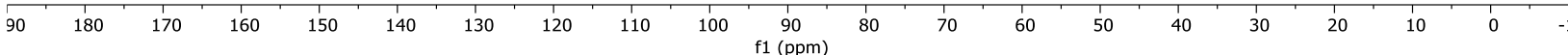


Supporting Information for Organic Letters

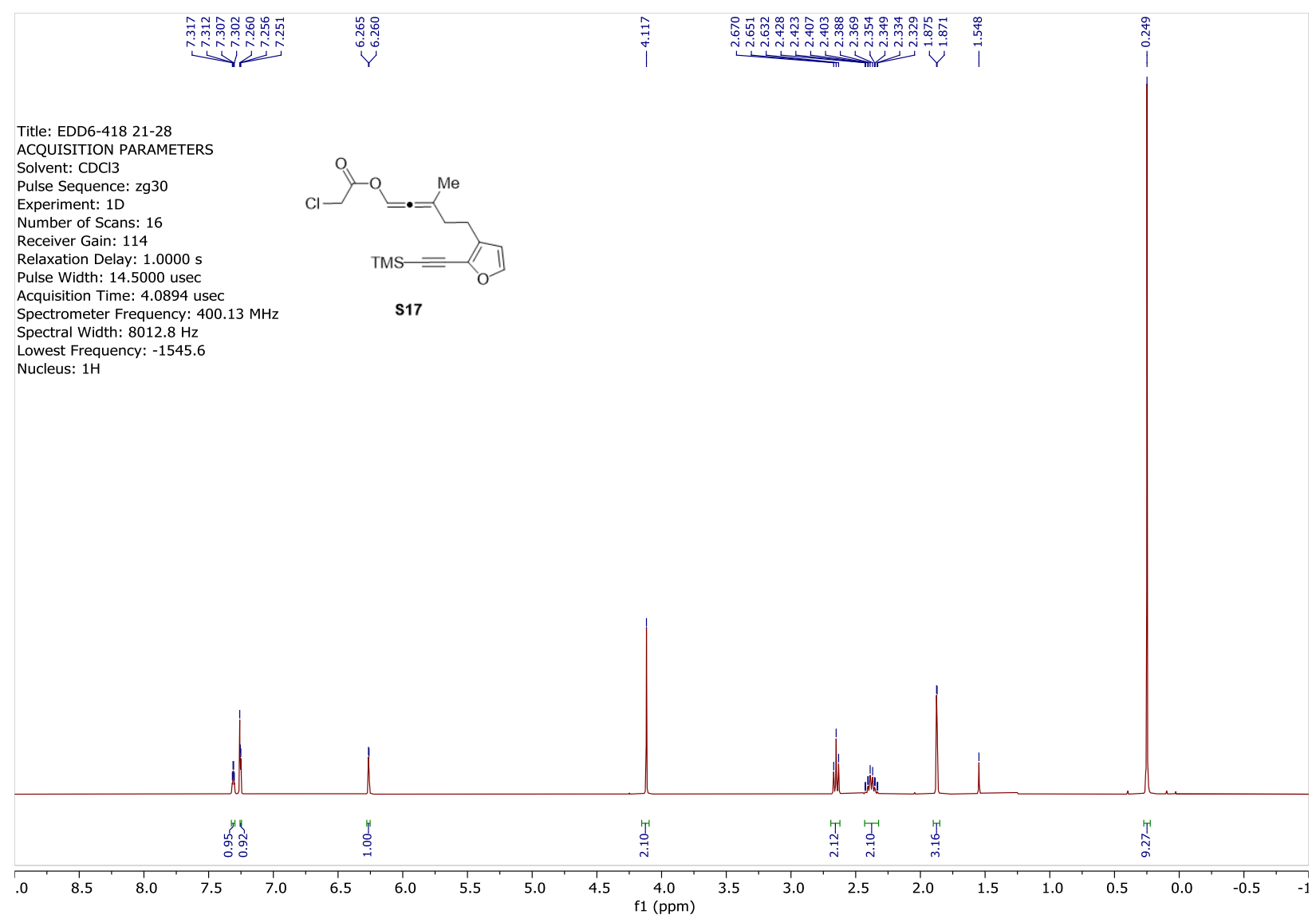


Supporting Information for Organic Letters

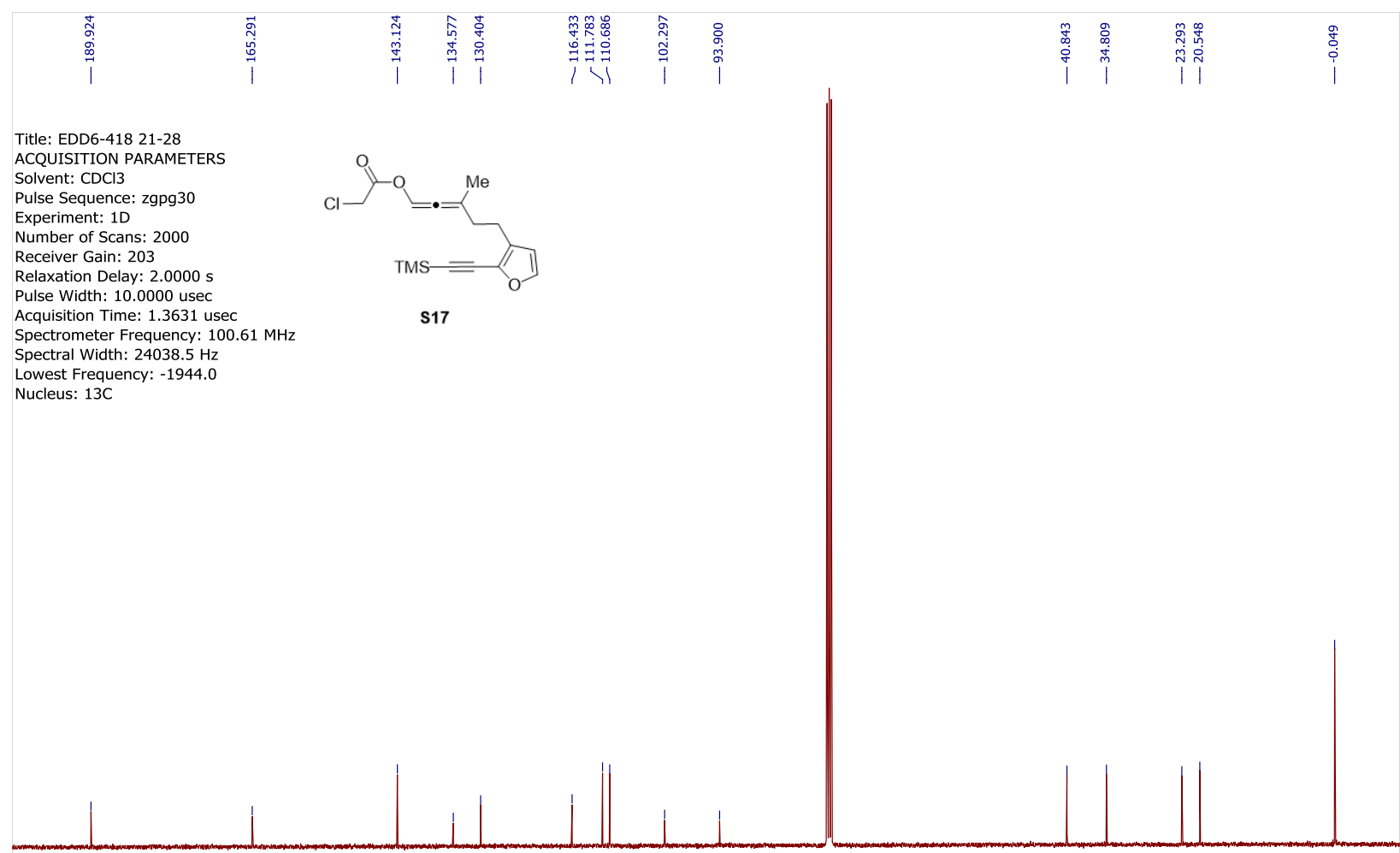

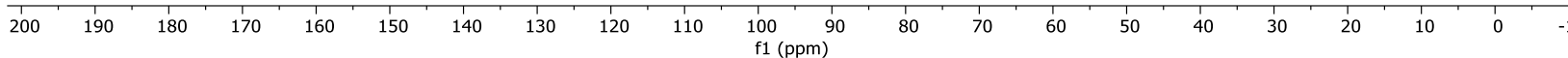


Supporting Information for Organic Letters

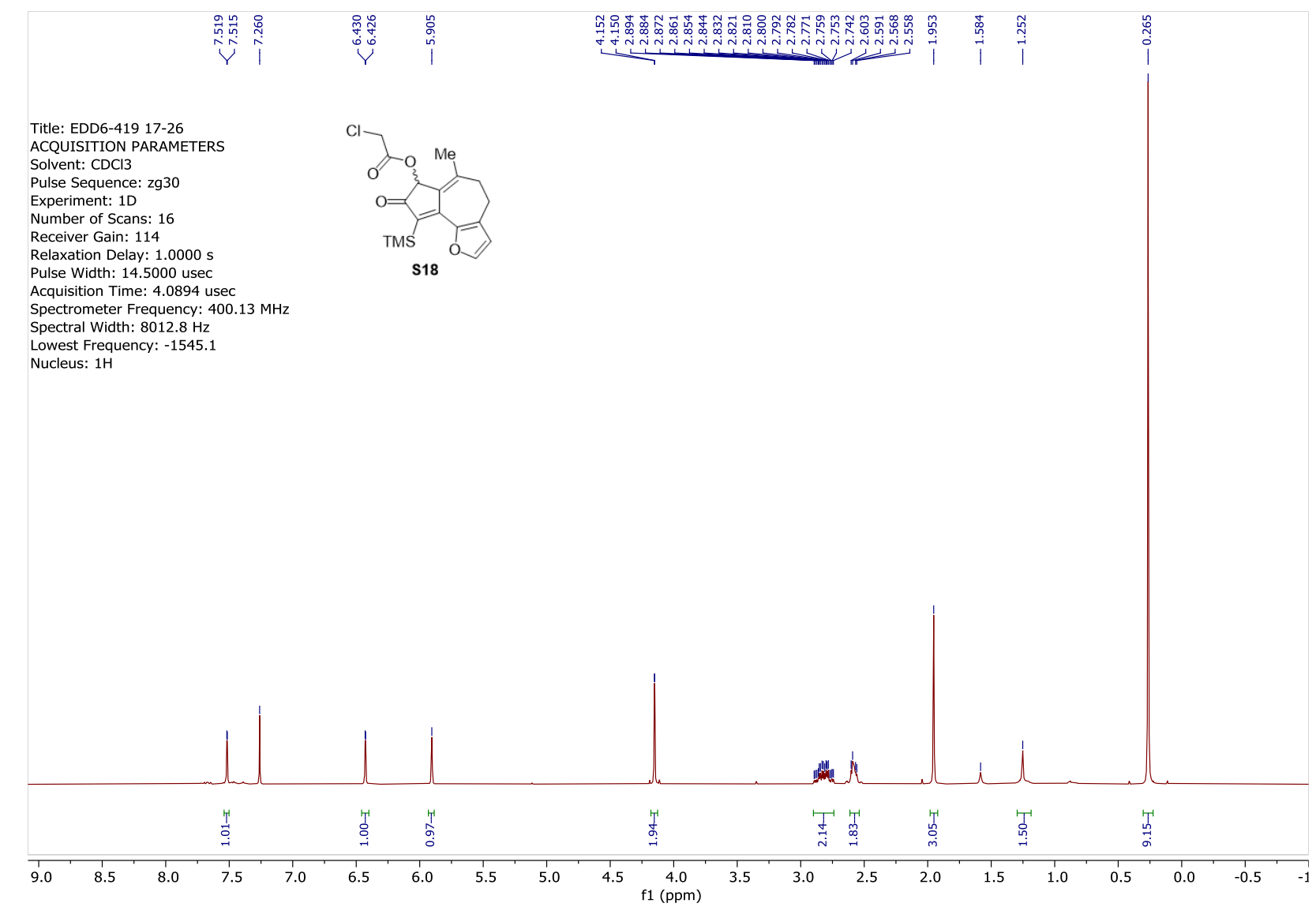


Supporting Information for Organic Letters

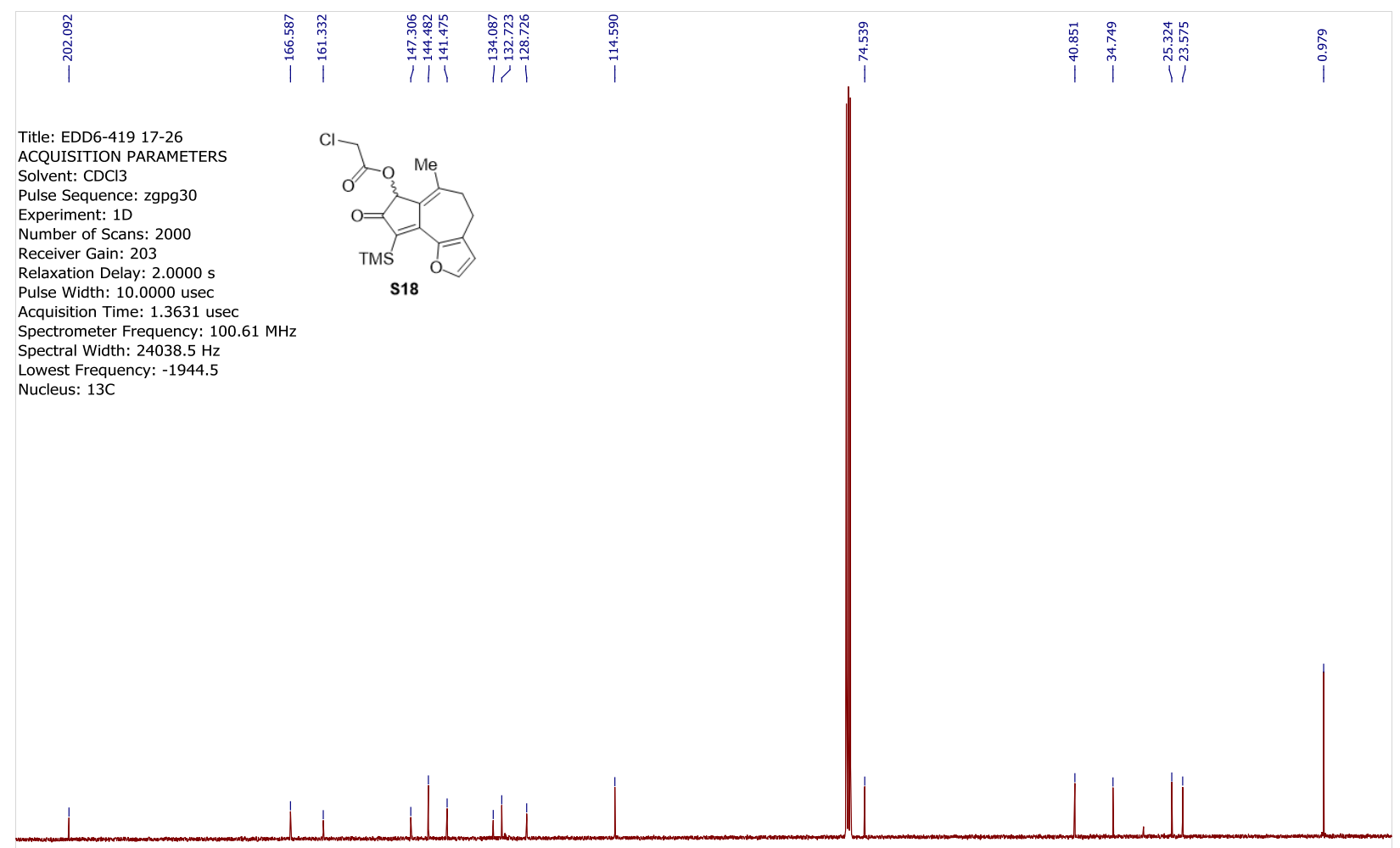

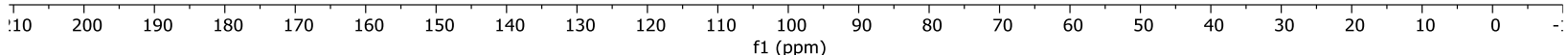


Supporting Information for Organic Letters

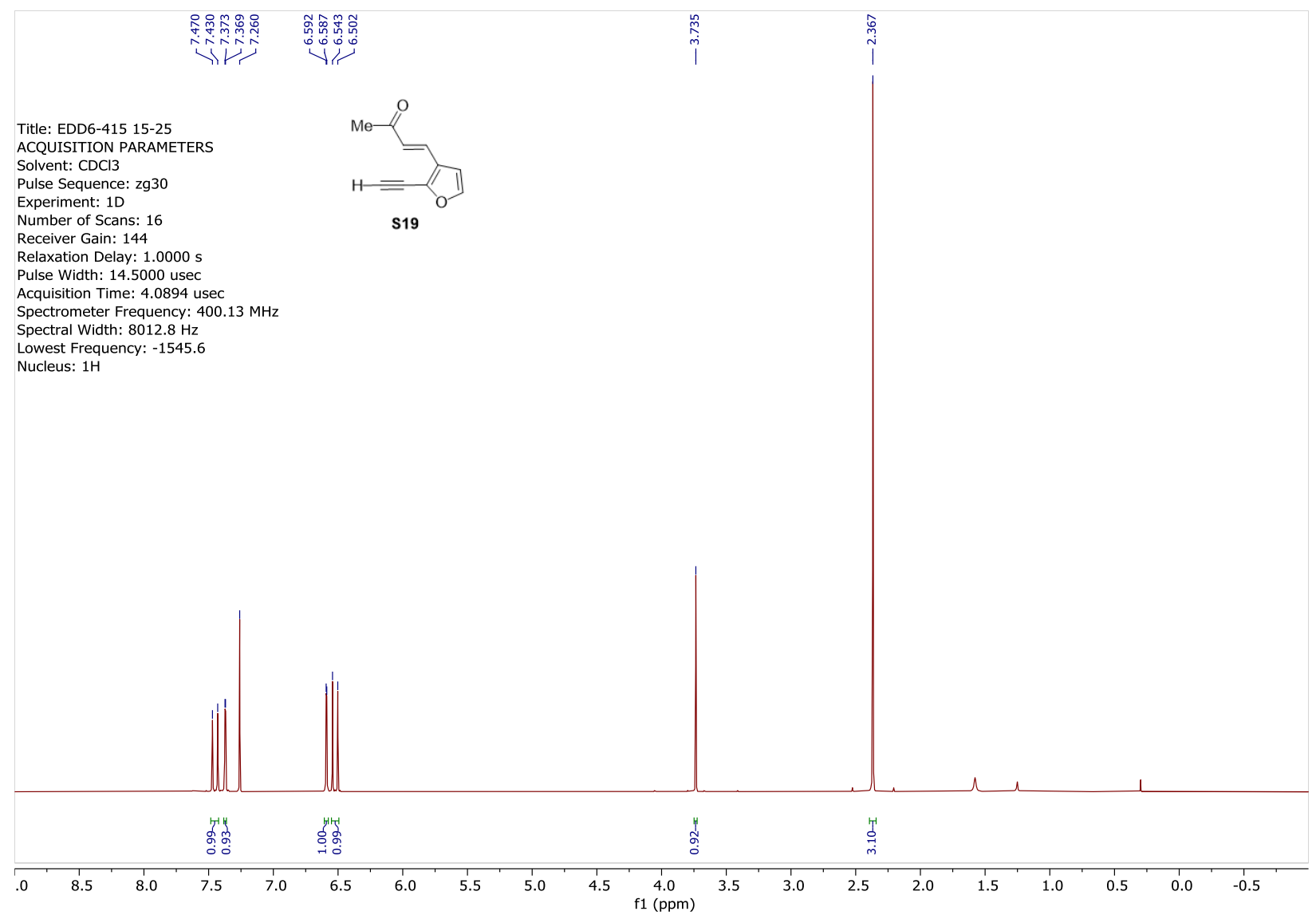


Supporting Information for Organic Letters

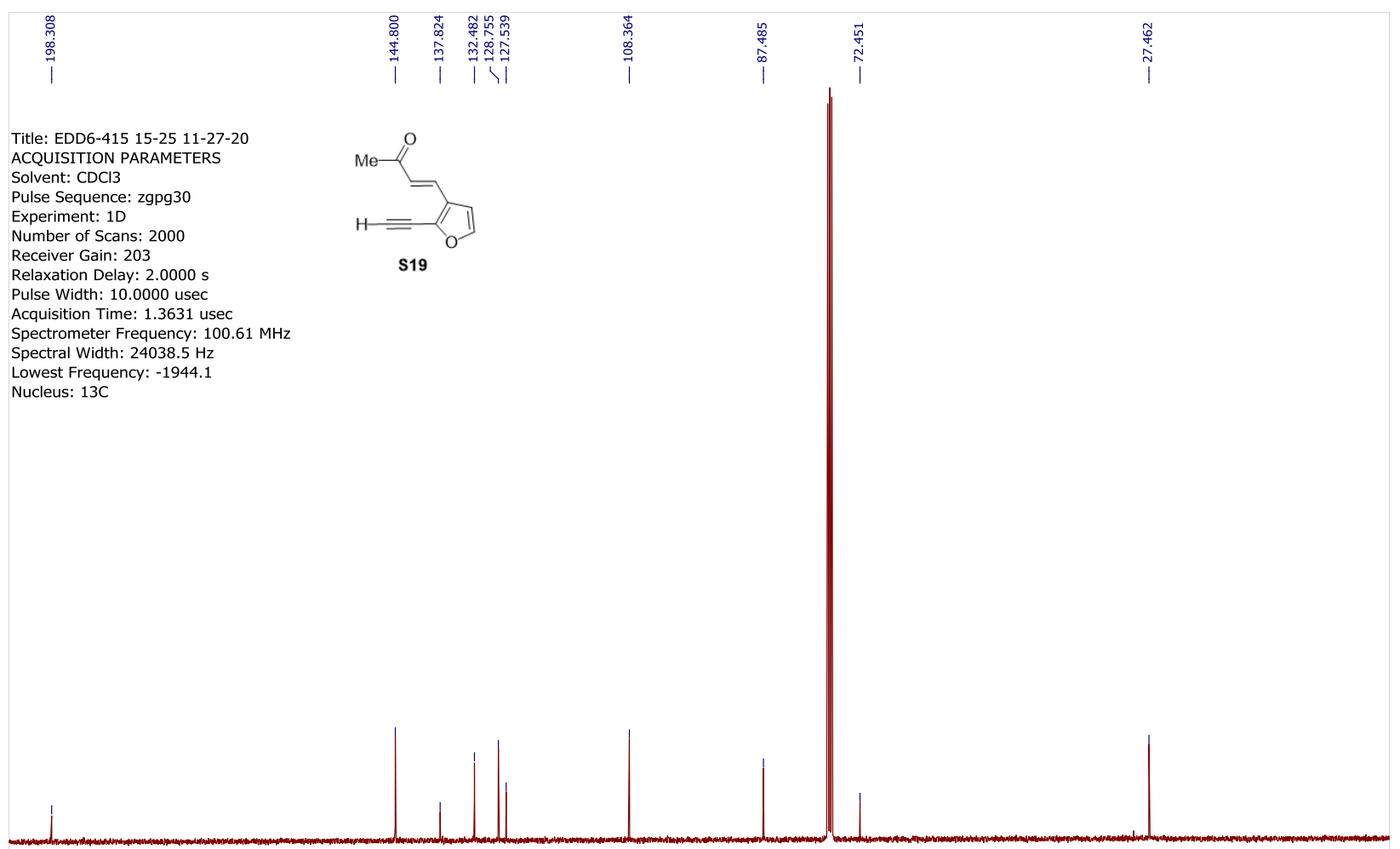

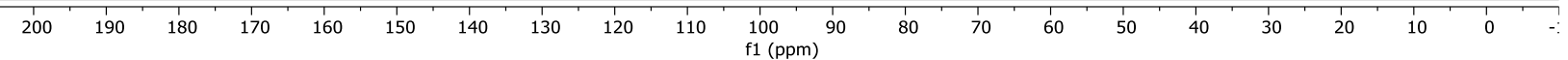


Supporting Information for Organic Letters

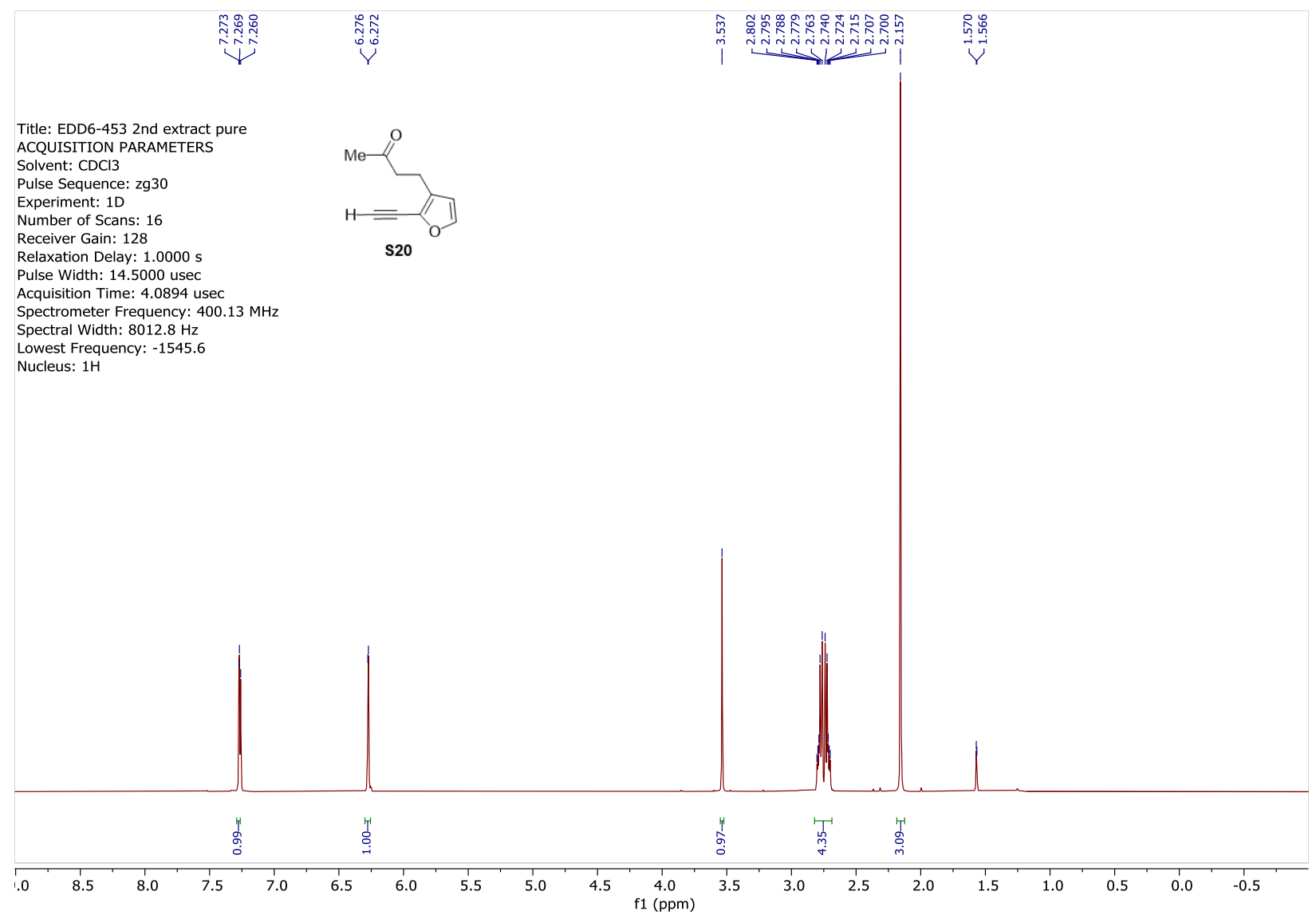


Supporting Information for Organic Letters

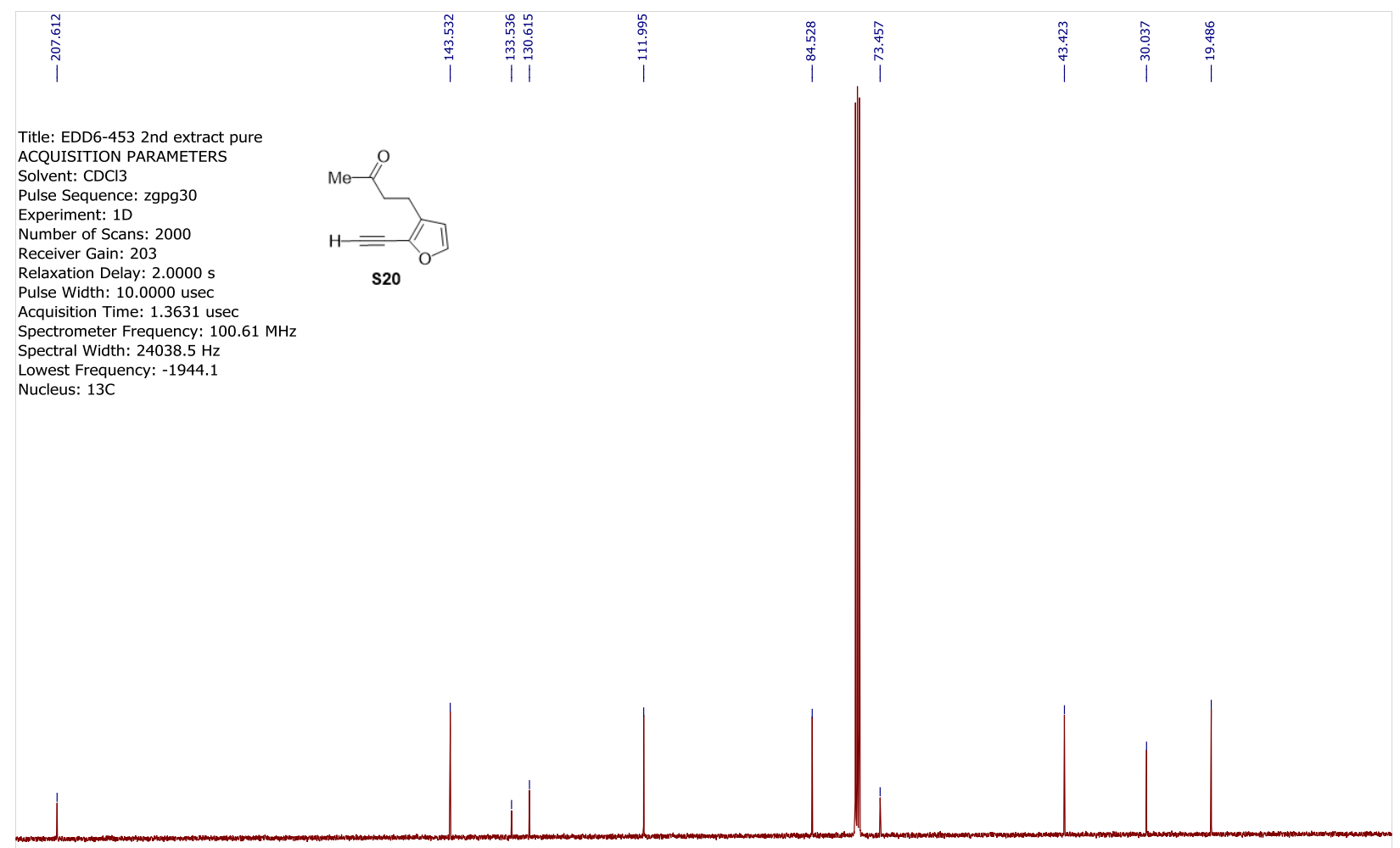

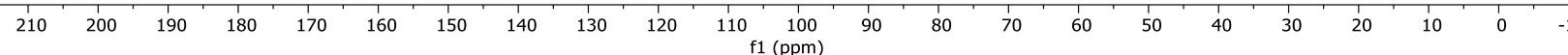


Supporting Information for Organic Letters

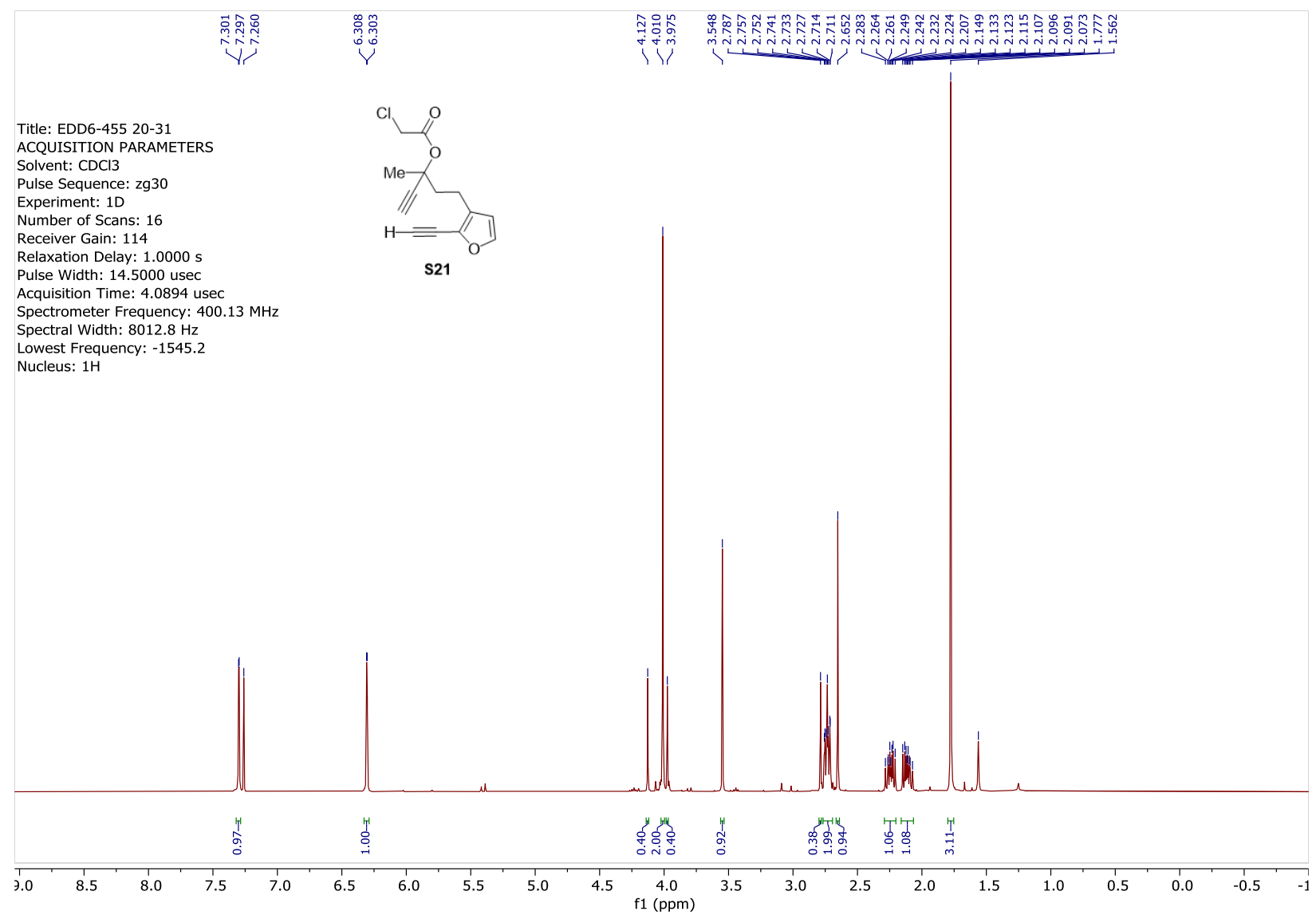


Supporting Information for Organic Letters

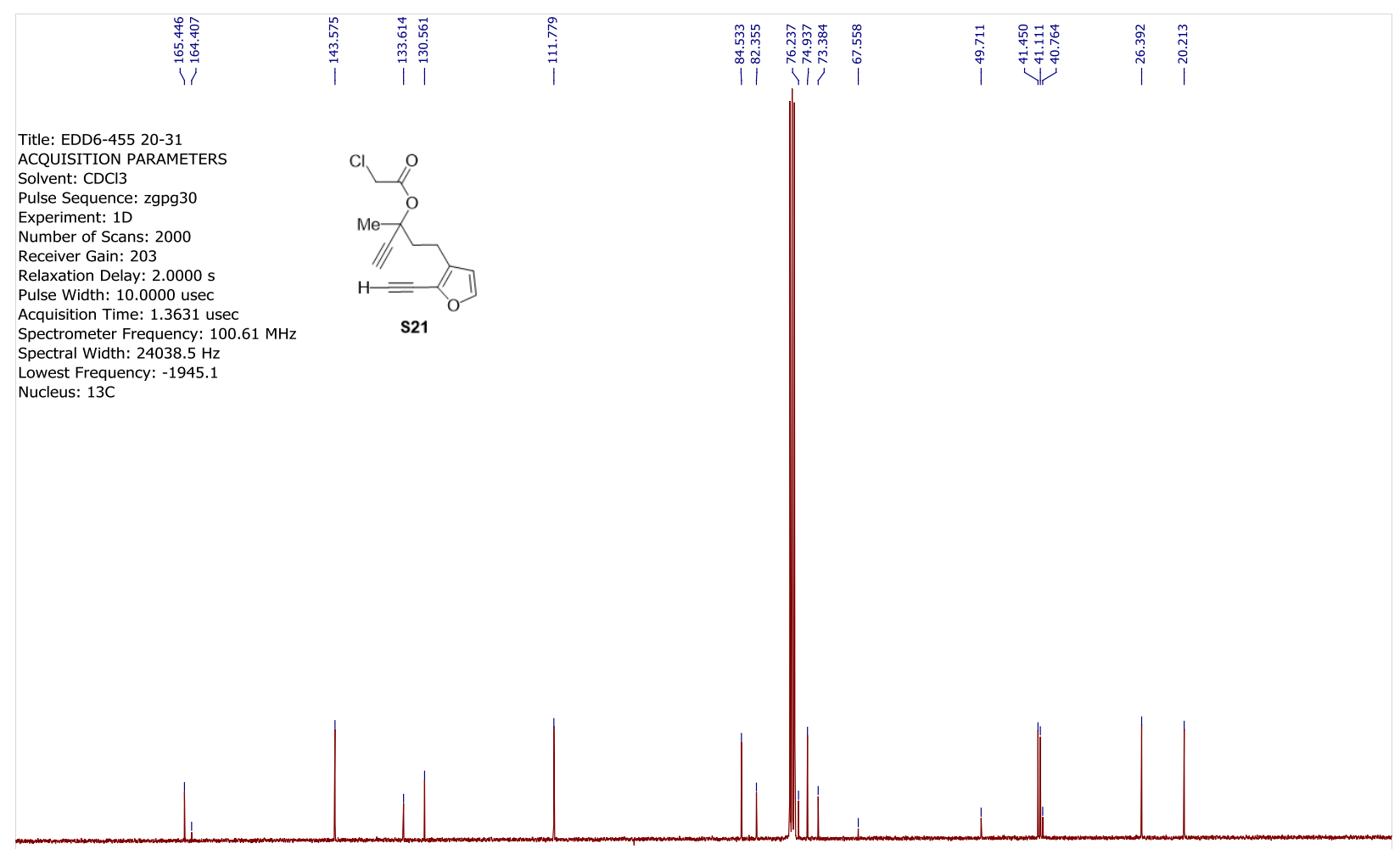

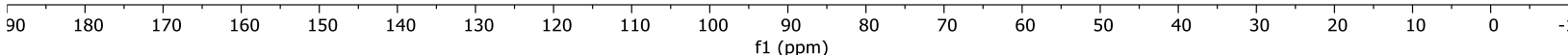


Supporting Information for Organic Letters

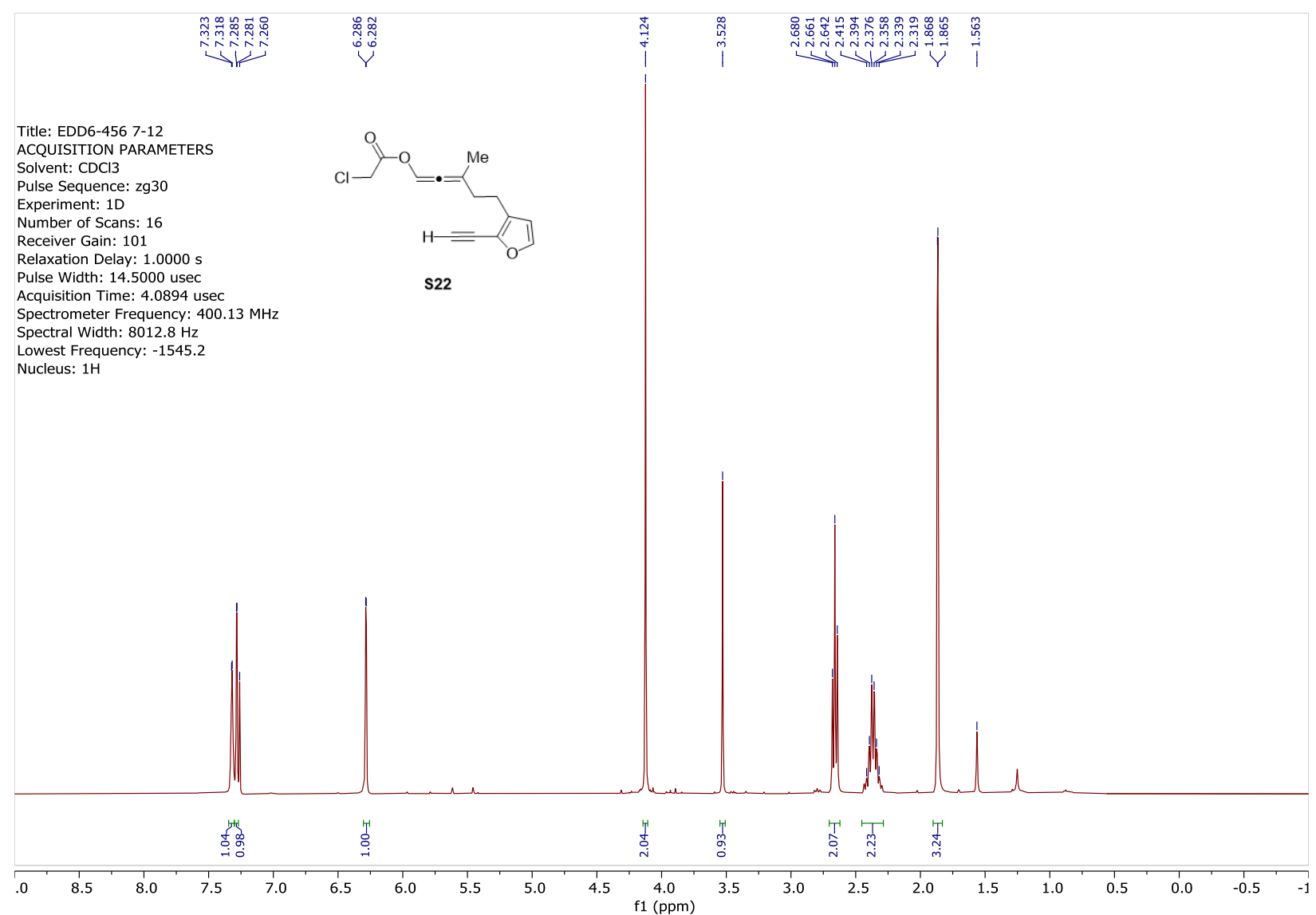


Supporting Information for Organic Letters

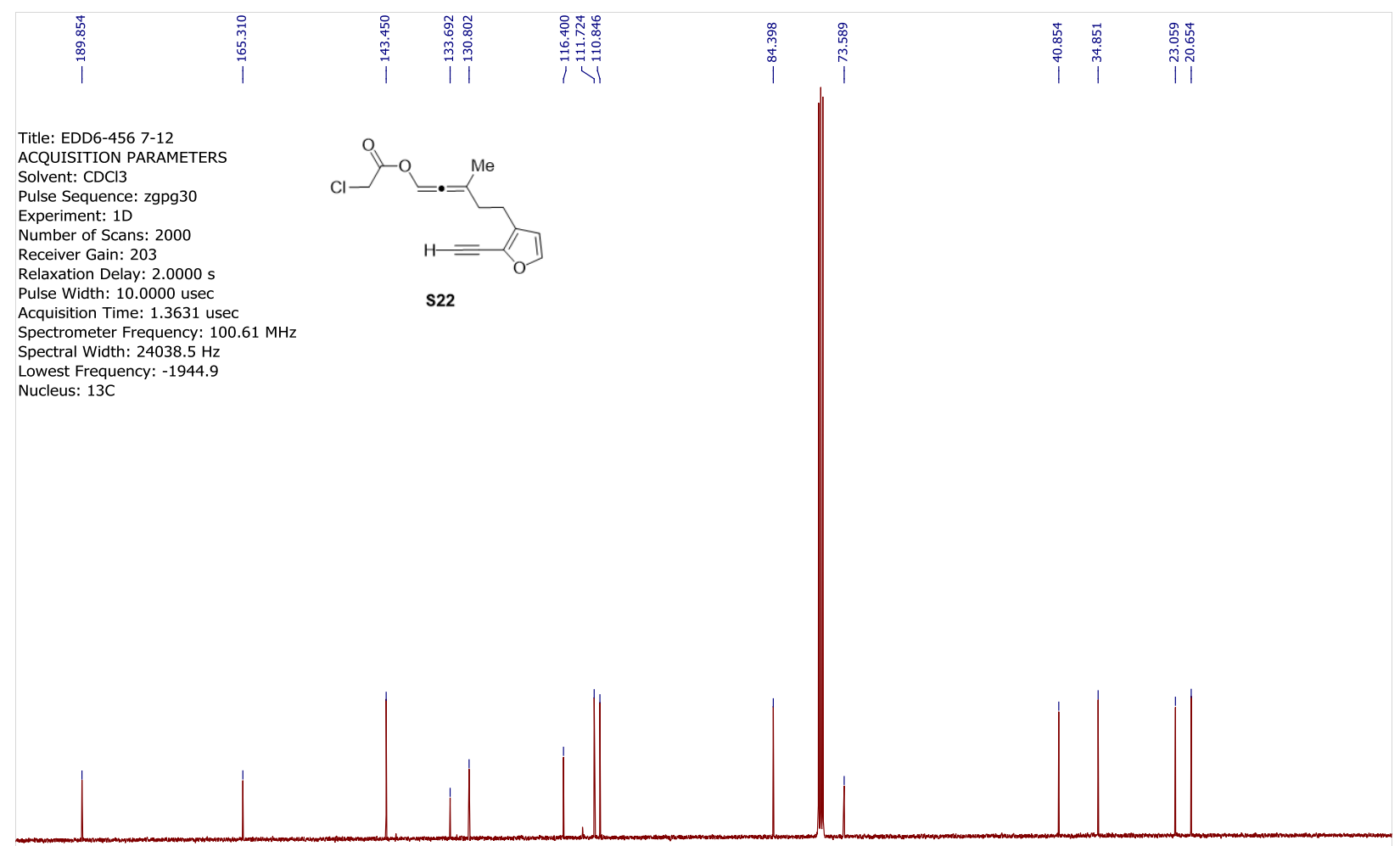

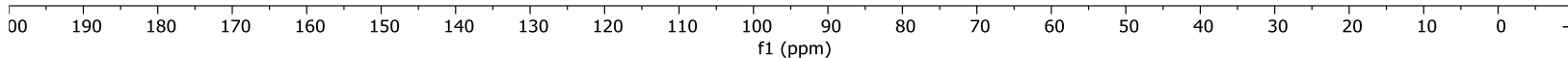


Supporting Information for Organic Letters

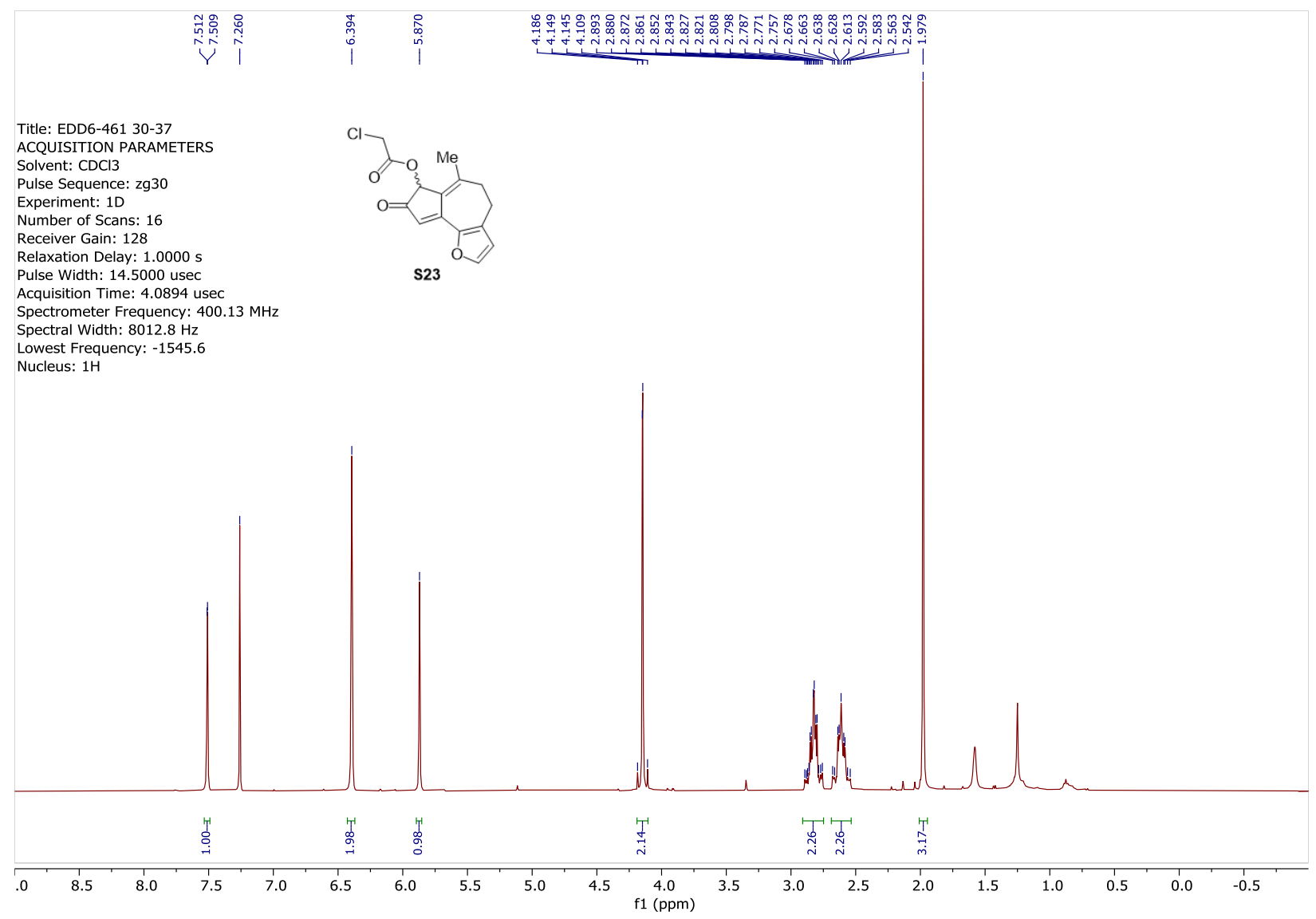


Supporting Information for Organic Letters
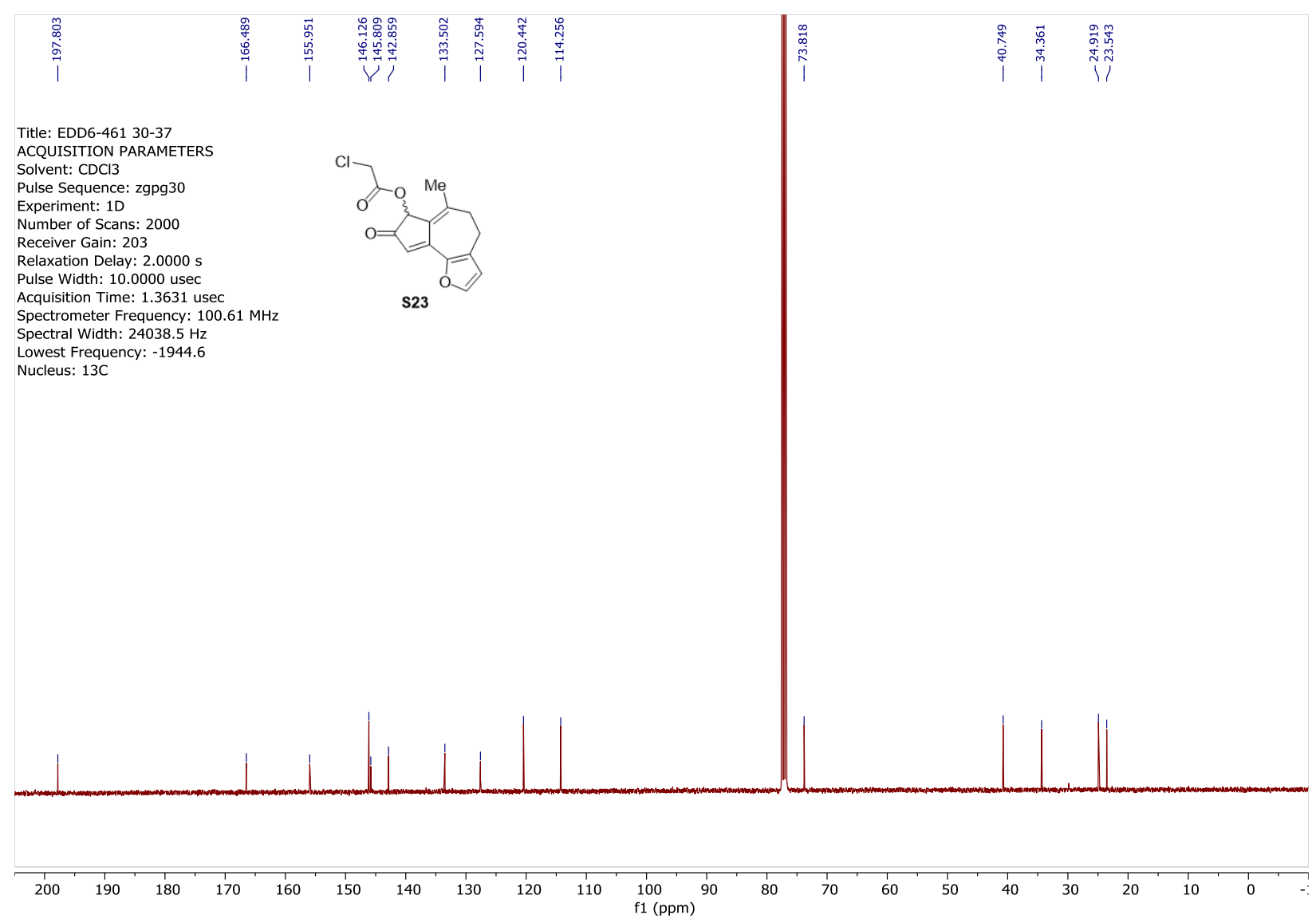
Supporting Information for Organic Letters

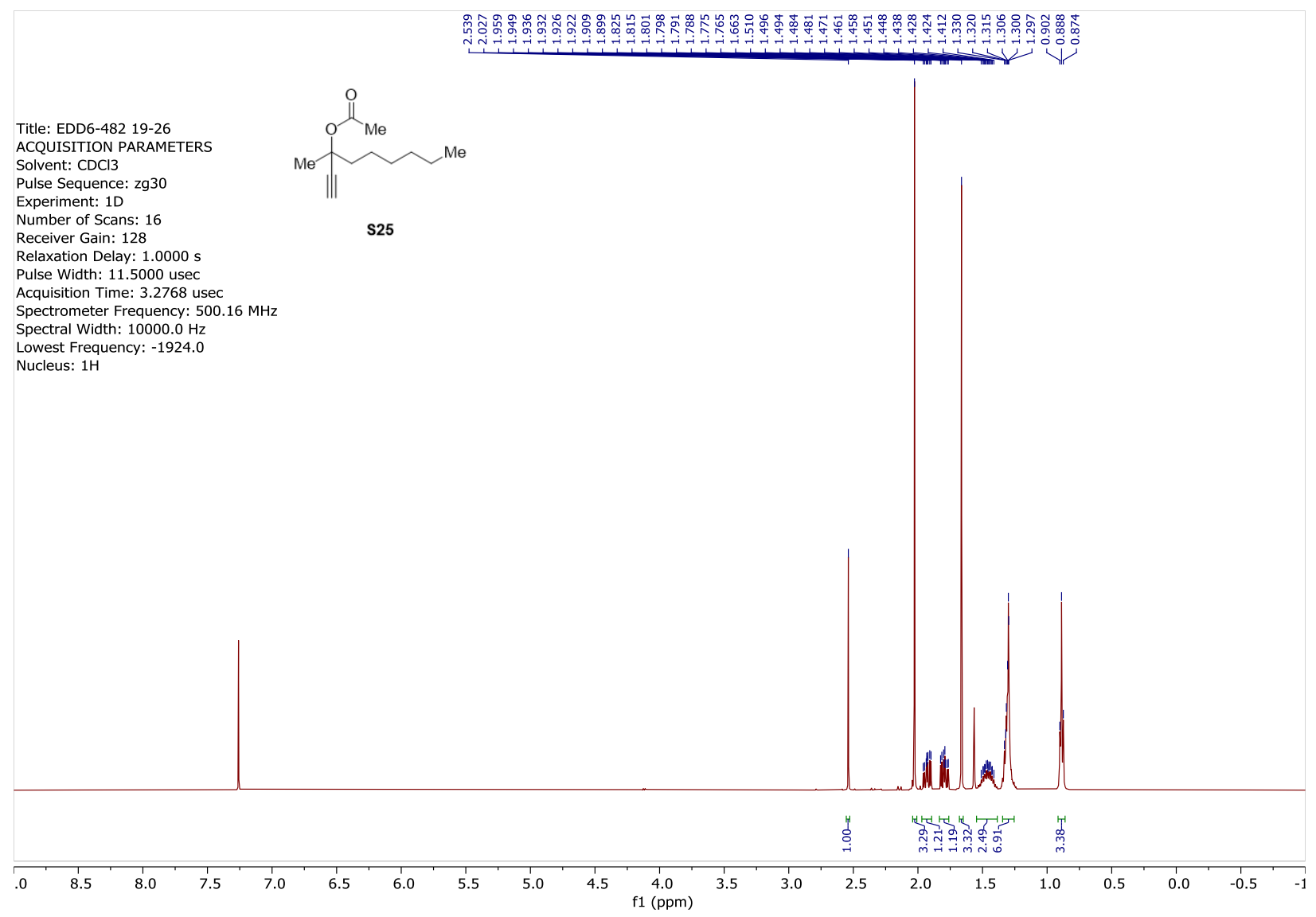


Supporting Information for Organic Letters

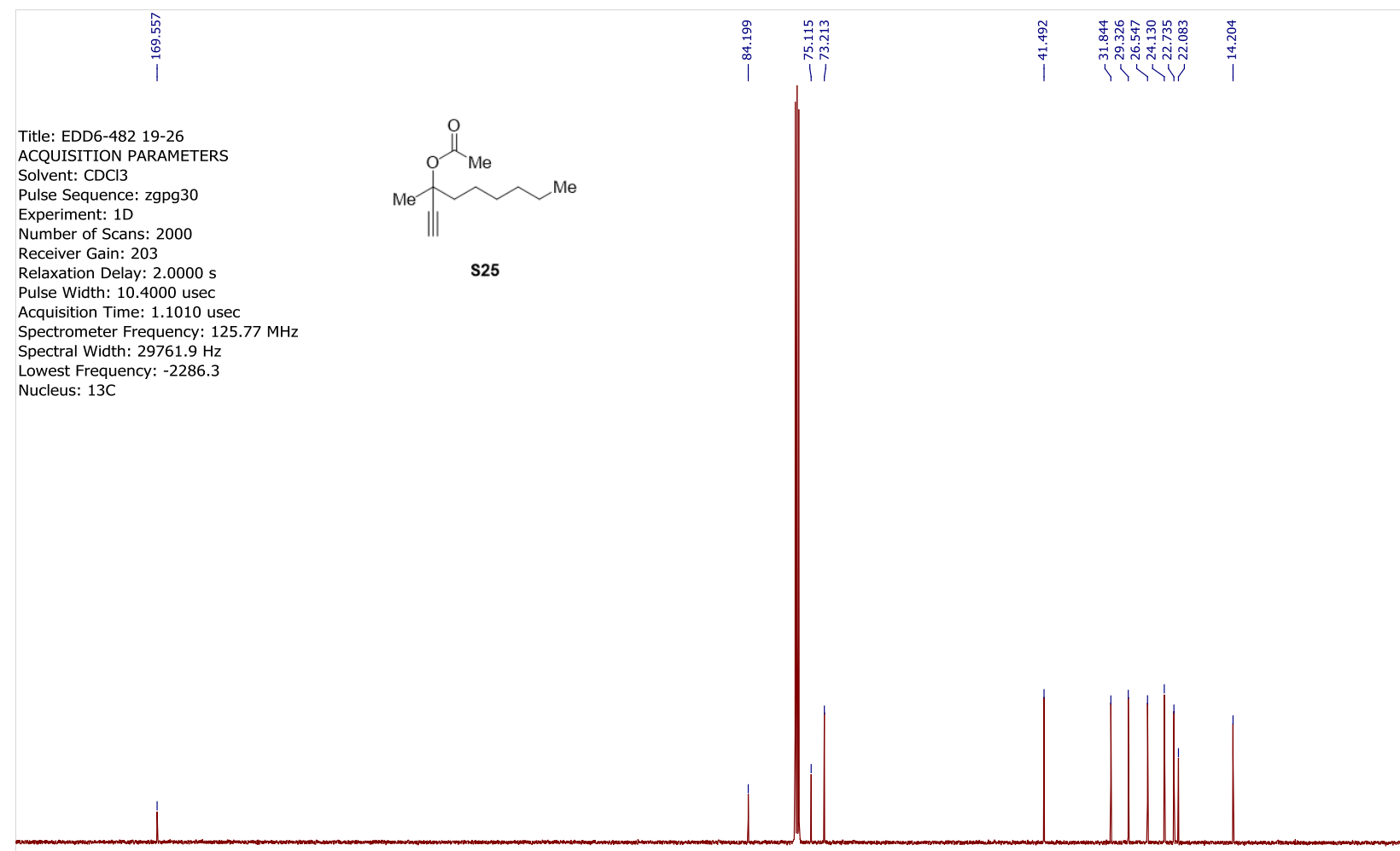

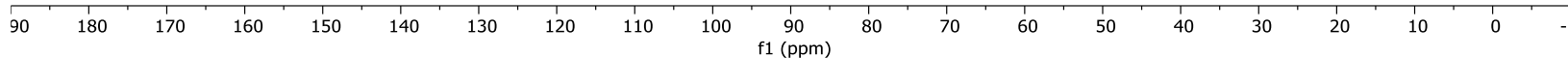


Supporting Information for Organic Letters

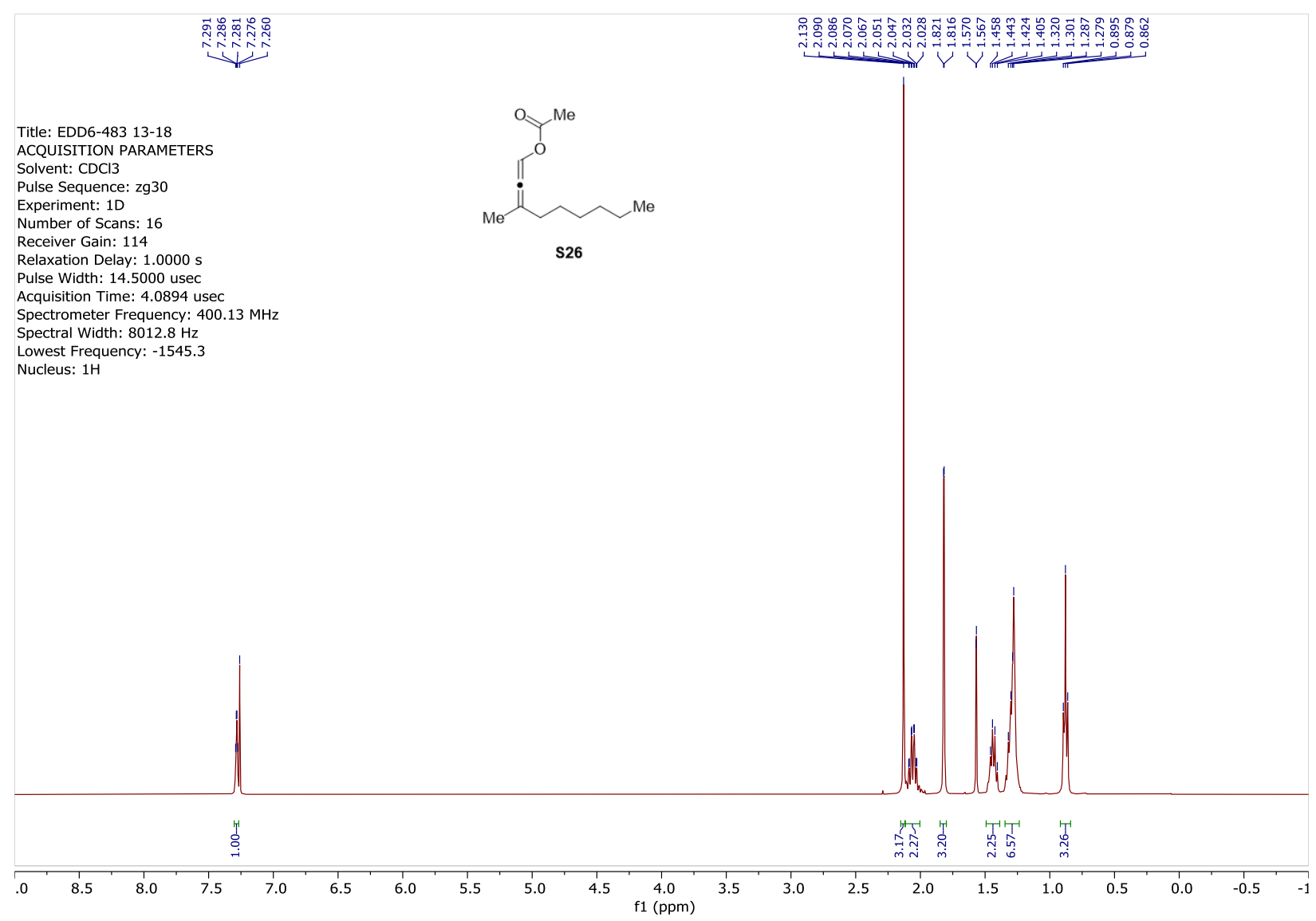


Supporting Information for Organic Letters

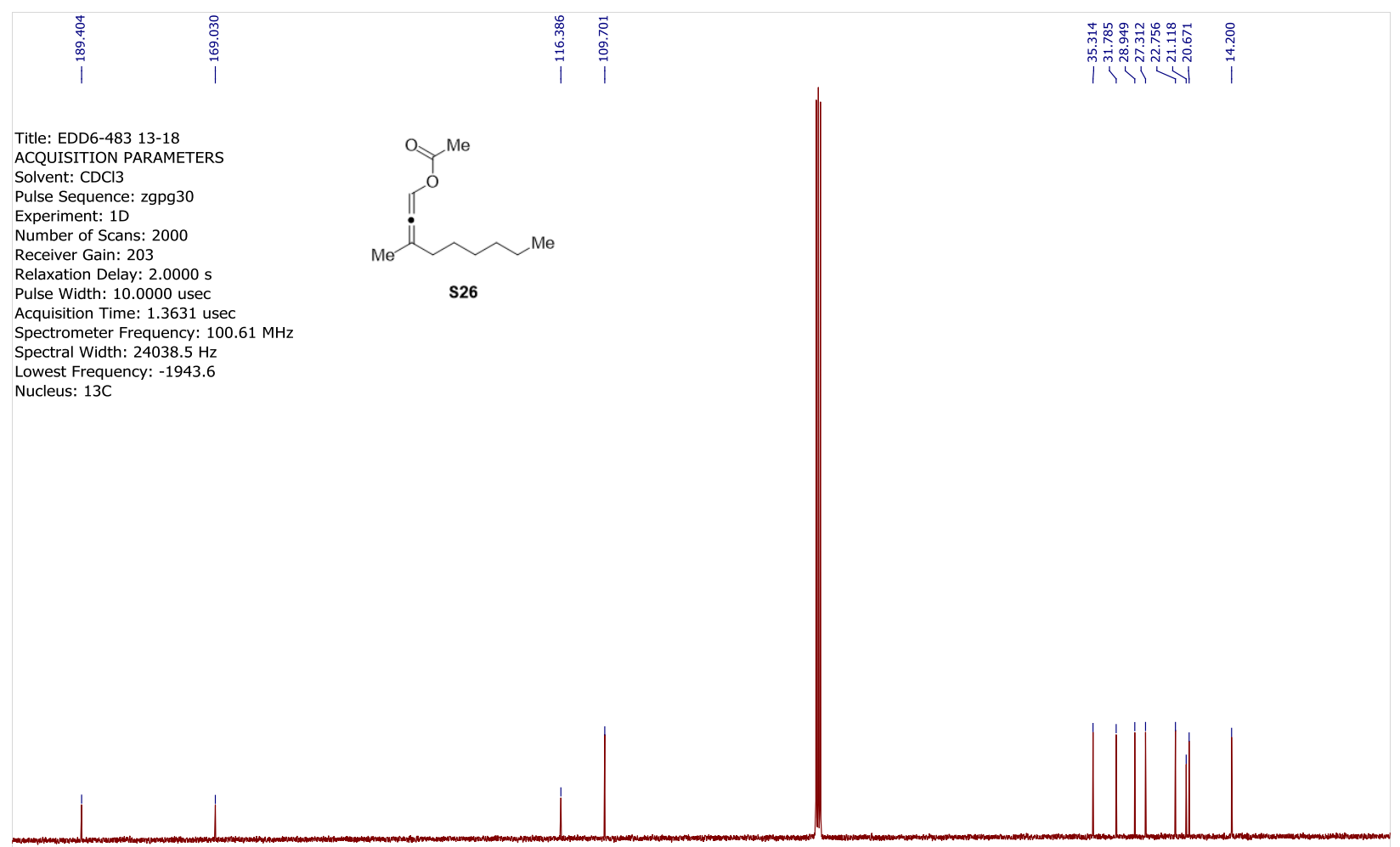

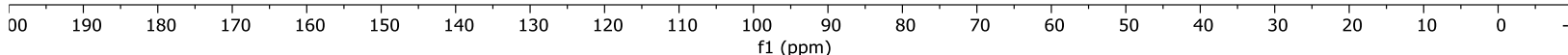


Supporting Information for Organic Letters

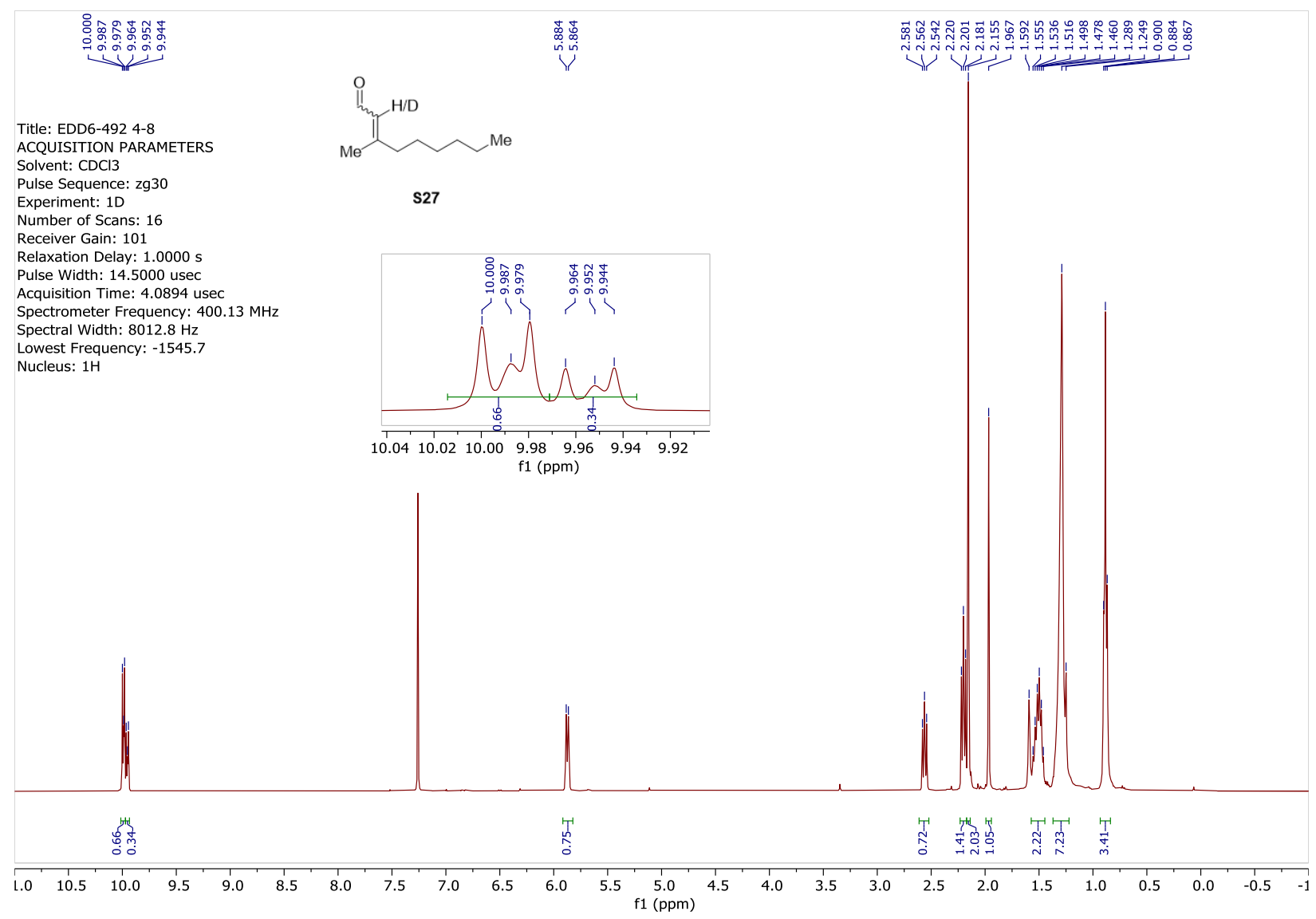


Supporting Information for Organic Letters

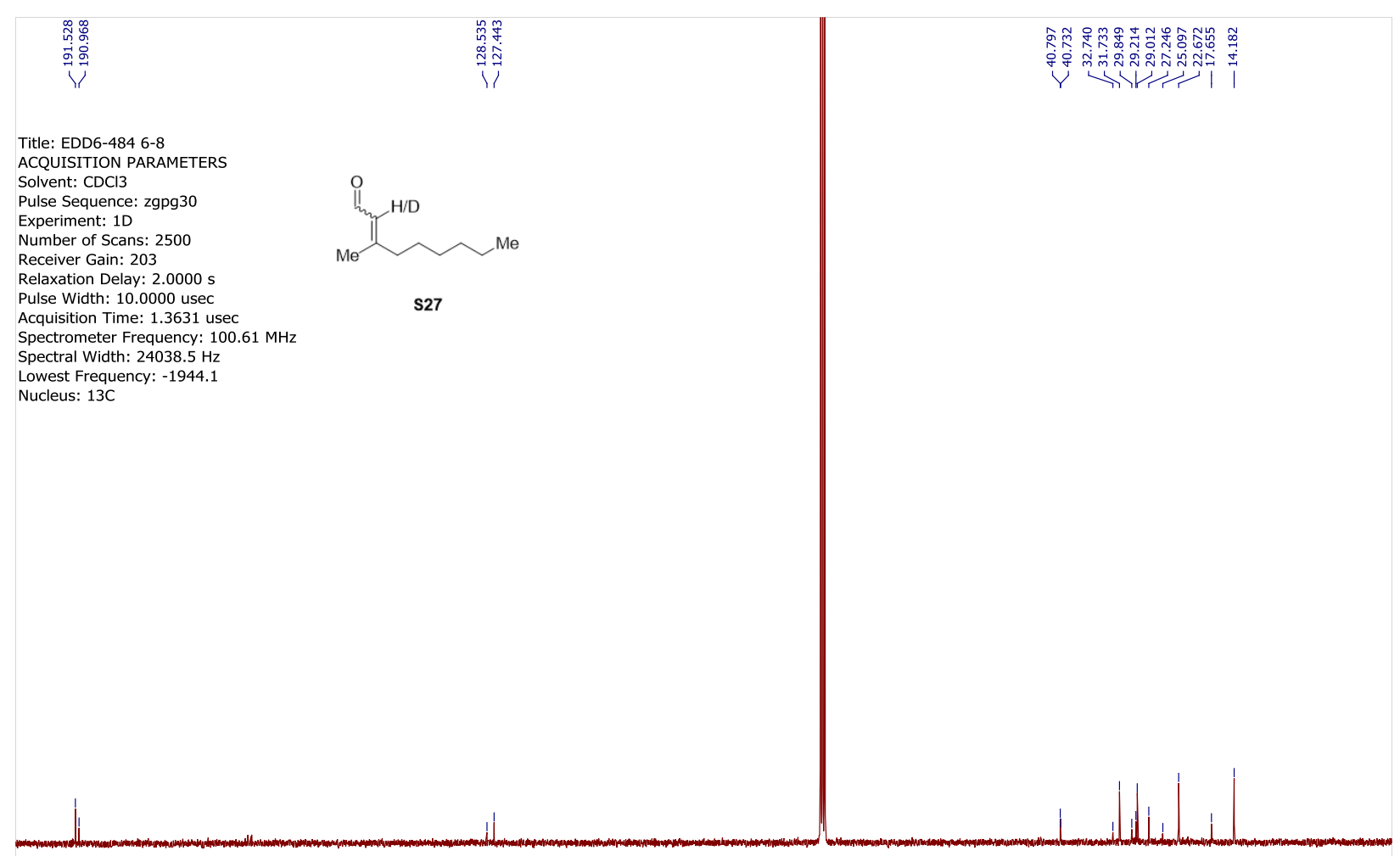

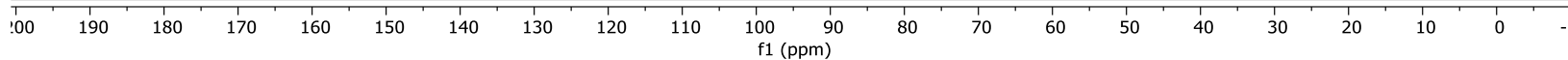




\section{Computational Details}

All DFT calculations were performed using Gaussian $16^{9}$ on Pitt CRC and XSEDE ${ }^{10}$ supercomputers. Molecular geometries were optimized using the B3LYP functional ${ }^{11}$ and the 6$31 \mathrm{G}(\mathrm{d})$ basis set for all non-metal atoms and LANL2DZ for rhodium in the gas phase. Single point energy calculations were performed using the M06 functional ${ }^{12,13}$ and 6-311+G(d,p) for all nonmetal atoms and SDD for rhodium with the SMD solvation model ${ }^{14}$ in dichloroethane (DCE). The computed Gibbs free energy values for all optimized transition state and intermediate structures were computed at $298 \mathrm{~K}$.

Possible isomers and conformers of the oxidative cyclization transition states TS1 and TS2 were systematically investigated. The Gibbs free energies and enthalpies of all TS isomers are provided in Figure S1. The lowest energy isomers (TS1 and TS2) were reported in the main text. The computed reaction energy profile of the enantioselectivity-determining oxidative cyclization step of the reaction with the choloroacetate substituted substrate $(\mathbf{4 g})$ is shown in Figure S2. In addition to the two pathways with the $(S)$-MonoPhos-alkene-supported Rh catalyst leading to the major (blue) and minor (red) enantiomeric products, a competing process in which the chiral phosphoramidite ligand is replaced by a CO ligand (black) is depicted. In all three pathways, the oxidative cyclization step is irreversible and thus enantioselectivity-determining. The competing oxidative cyclization transition state without the phosphoramidite ligand (TS3) is $8.7 \mathrm{kcal} / \mathrm{mol}$ higher in energy than the lowest energy transition state with the phosphoramidite ligand (TS1) making it unlikely that the CO-only pathway contributes significantly to the reaction.

In order to probe the effects of the chloro substitution on the substrate on the enantioselectivity of the reaction, calculations were performed on substrate $\mathbf{4 a}$, which demonstrated a slightly lower $e e$ experimentally. The partial reaction profile for this reaction is shown in Figure S3. The computed $\Delta \Delta G^{\ddagger}$ for the reaction with $4 a$ between the two enantioselectivity-determining transition states (TS4 vs. TS5) is $3.9 \mathrm{kcal} / \mathrm{mol}$, which is very close to the computed $\Delta \Delta G^{*}$ for the reaction with $\mathbf{4 g}$. In addition, the pathway in which the phosphoramidite ligand is replaced by a CO ligand (TS6) was similarly unfavorable being 8.8 $\mathrm{kcal} / \mathrm{mol}$ higher in energy than TS4. These computational results suggest that the lower enantioselectivity observed for the acetate substrate $\mathbf{4 a}$ compared to chloroacetate $\mathbf{4 g}$ is not due to the activation energy difference in the main reaction pathways catalyzed by the phosphoramiditesupported Rh catalyst. Instead, an unidentified background reaction, potentially catalyzed by an achiral $\mathrm{Rh}$ catalyst or the product-bound $\mathrm{Rh}$ complex, may be responsible for the lower enantioselectivity in the reaction with acetate $\mathbf{4 a}$. 


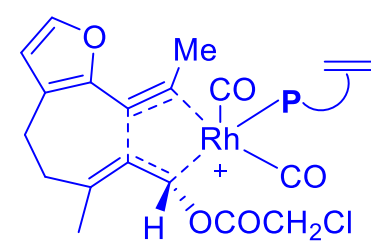

TS1

24.5

(15.1)

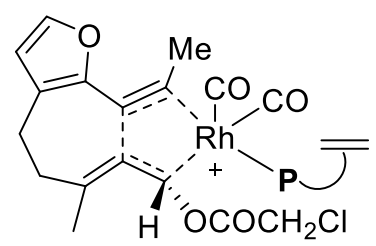

TS1b

28.6

(17.2)

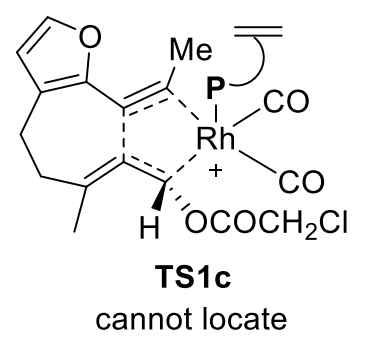<smiles></smiles>

TS1-rot

26.2

(16.0)

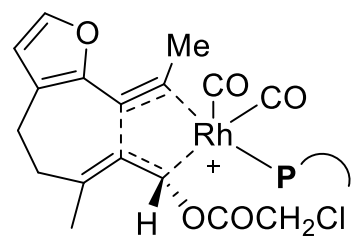

TS1b-rot

28.1

(17.5)

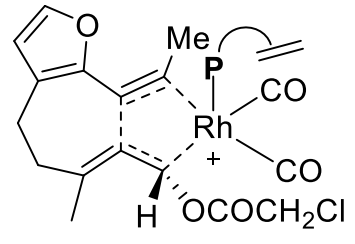

TS1c-rot

29.8

(19.3)

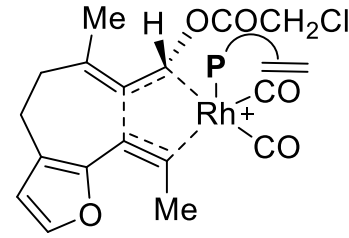

TS2b

28.9

(18.9)

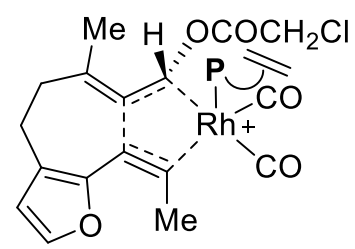

TS2b-rot

30.3

(20.0)

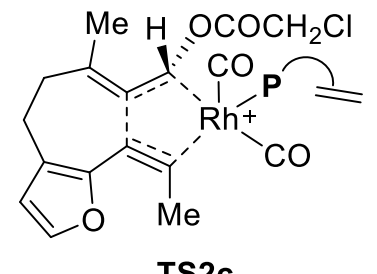

TS2C

29.4

(18.9)

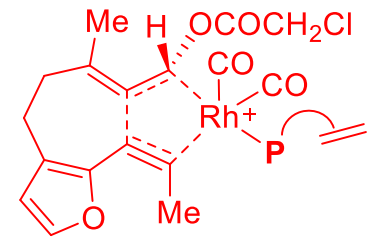

TS2

28.0

(18.5)

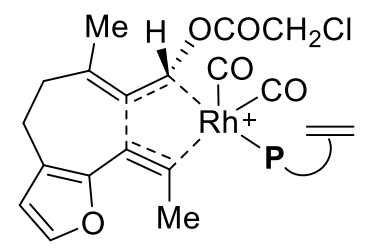

TS2-rot

33.6

(24.1)

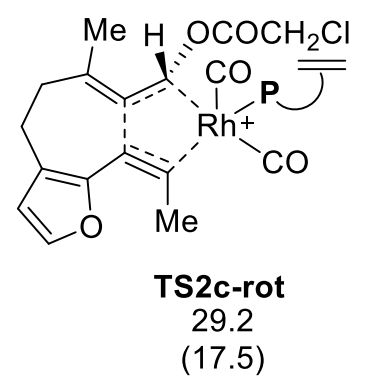

Figure S1. Higher energy isomers of the oxidative cyclization transition state. Gibbs free energies and enthalpies (in parenthesis) are in $\mathrm{kcal} / \mathrm{mol}$ with respect to the starting materials ( $\mathbf{S 2 8}$ and $\mathbf{4 g}$ ). 


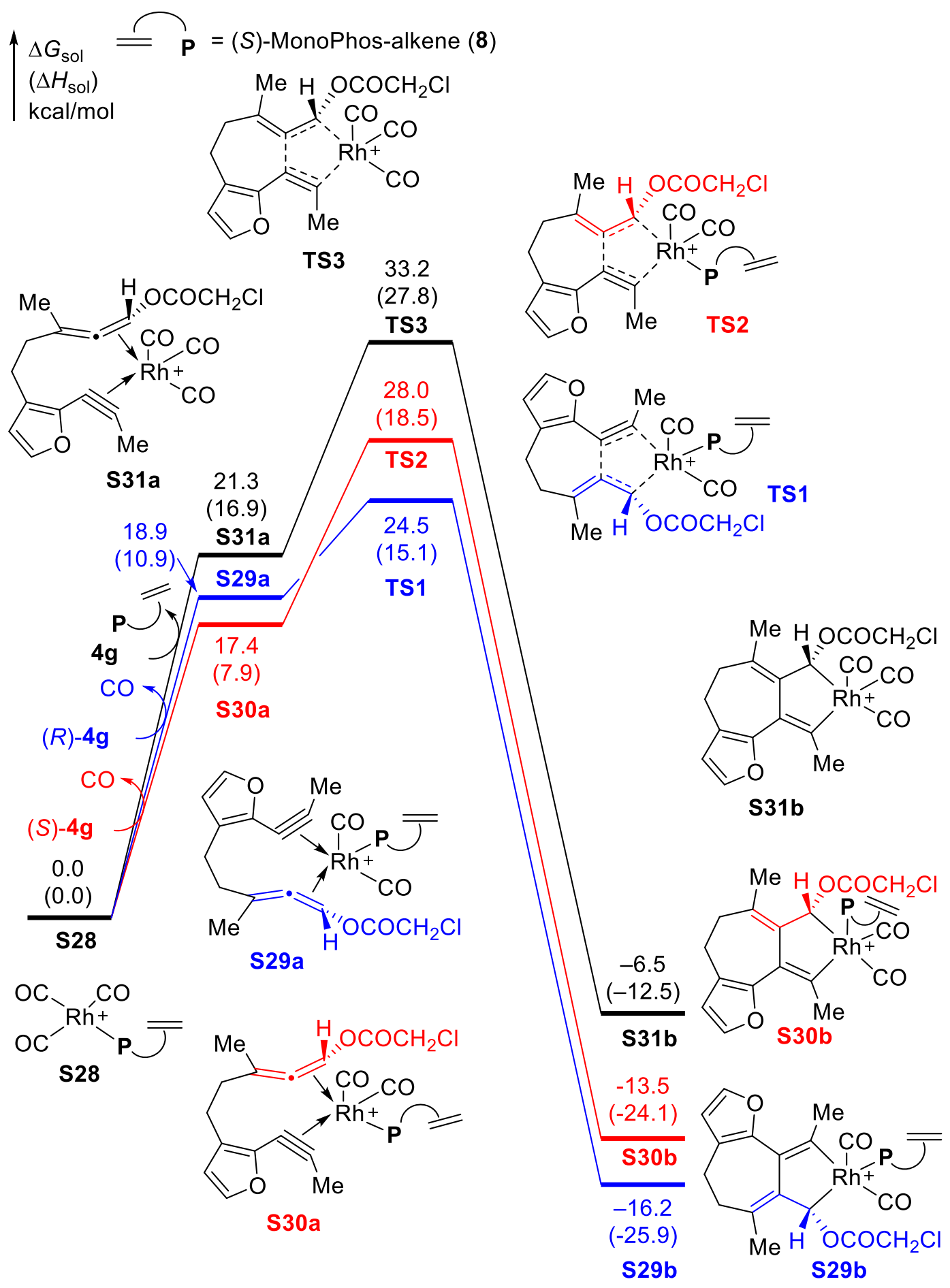

Figure S2. Computed enantioselectivity-determining oxidative cyclization transition states with chloroacetate substrate $\mathbf{4 g}$. 
$\underset{\substack{\left(\Delta H_{\text {sol }}\right) \\ \mathrm{kcal} / \mathrm{mol}}}{\Delta G_{\text {sol }}}=($ S $=$-MonoPhos-Alkene (8)

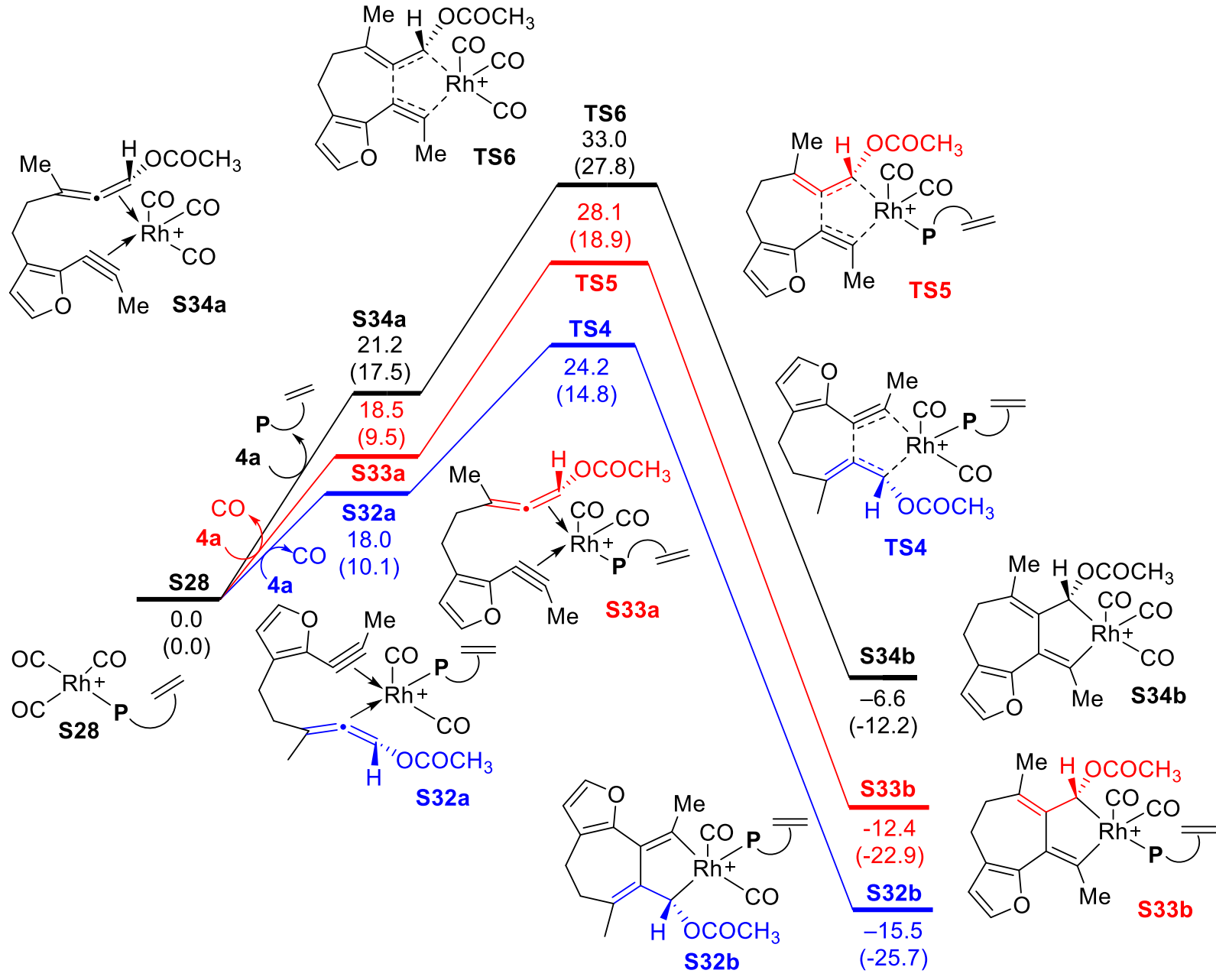

Figure S3. Computed enantioselectivity-determining oxidative cyclization transition states with acetate substrate $\mathbf{4 a}$.

\section{Cartesian Coordinates of All Optimized Structures **}

$4 \mathbf{a}$

M06-2X/6-31+G(d) SCF energy: $\quad-806.76511275$ a.u.

M06-2X/6-31+G(d) enthalpy: $\quad-806.470723$ a.u.

M06-2X/6-31+G(d) free energy: -806.542622 a.u.

M06-2X/6-311++G(d,p) SCF energy in solution: -806.45599020 a.u.

M06-2X/6-311++G(d,p) enthalpy: $\quad-806.161600$ a.u.

M06-2X/6-311++G(d,p) free energy: $\quad-806.233499$ a.u.

Cartesian coordinates 


$\begin{array}{lrcc}\text { ATOM } & \text { X } & \text { Y } & \text { Z } \\ \text { C } & 0.690422 & -2.479869 & -0.370287 \\ \text { C } & -0.524138 & -3.254573 & -0.601759 \\ \text { C } & 1.700551 & -1.835071 & -0.173961 \\ \text { C } & 2.817015 & -1.000725 & 0.035113 \\ \text { C } & 0.857525 & 1.614879 & 0.496863 \\ \text { C } & -0.384344 & 2.306781 & -0.048705 \\ \text { C } & -1.450987 & 1.590803 & -0.317852 \\ \text { C } & -2.448791 & 0.800906 & -0.611494 \\ \text { H } & -2.566510 & 0.277783 & -1.554276 \\ \text { O } & -3.490083 & 0.580310 & 0.305023 \\ \text { C } & -4.154521 & -0.602355 & 0.197660 \\ \text { O } & -3.854447 & -1.477866 & -0.584276 \\ \text { C } & 2.970751 & 0.358771 & -0.139522 \\ \text { C } & -0.313663 & 3.797077 & -0.301254 \\ \text { H } & -0.114423 & 4.333849 & 0.635768 \\ \text { H } & 0.508742 & 4.037154 & -0.987950 \\ \text { H } & -1.243137 & 4.180029 & -0.729866 \\ \text { C } & 4.317642 & 0.651679 & 0.248102 \\ \text { O } & 3.990510 & -1.550612 & 0.500622 \\ \text { C } & 4.881487 & -0.532638 & 0.620297 \\ \text { H } & 5.862886 & -0.809801 & 0.974609 \\ \text { H } & 4.799133 & 1.620533 & 0.240317 \\ \text { C } & -5.301570 & -0.651693 & 1.177652 \\ \text { H } & -1.407092 & -2.604560 & -0.641776 \\ \text { H } & -0.473339 & -3.808380 & -1.547860 \\ \text { H } & -0.684467 & -3.984238 & 0.201721 \\ \text { C } & 1.894389 & 1.293092 & -0.613541 \\ \text { H } & 1.357498 & 0.827521 & -1.448567 \\ \text { H } & 2.341105 & 2.218309 & -0.996186 \\ \text { H } & 1.327189 & 2.241242 & 1.267266 \\ \text { H } & 0.564949 & 0.675221 & 0.972677 \\ \text { H } & -4.940022 & -0.460812 & 2.193118 \\ \text { H } & -5.779673 & -1.630432 & 1.127175 \\ \text { H } & -6.031300 & 0.129540 & 0.939008\end{array}$

\section{$4 \mathrm{~g}$}

M06-2X/6-31+G(d) SCF energy: $\quad-1266.34730124$ a.u.

M06-2X/6-31+G(d) enthalpy: $\quad-1266.060448$ a.u.

M06-2X/6-31+G(d) free energy: -1266.135881 a.u.

M06-2X/6-311++G(d,p) SCF energy in solution: -1266.04160951 a.u.

M06-2X/6-311++G(d,p) enthalpy: $\quad-1265.754756$ a.u.

M06-2X/6-311++G(d,p) free energy: $\quad-1265.830189$ a.u.

Cartesian coordinates

$\begin{array}{lrrr}\text { ATOM } & \text { X } & \text { Y } & \text { Z } \\ \text { C } & 1.548420 & -2.496081 & -0.375740 \\ \text { C } & 0.377911 & -3.324551 & -0.644874 \\ \text { C } & 2.522473 & -1.807355 & -0.148957\end{array}$




$\begin{array}{lrrr}\mathrm{C} & 3.595962 & -0.926838 & 0.093255 \\ \mathrm{C} & 1.514011 & 1.601488 & 0.462059 \\ \mathrm{C} & 0.270217 & 2.248390 & -0.131216 \\ \mathrm{C} & -0.761771 & 1.496374 & -0.433966 \\ \mathrm{C} & -1.725185 & 0.677548 & -0.758246 \\ \mathrm{H} & -1.804600 & 0.146783 & -1.700388 \\ \mathrm{O} & -2.790062 & 0.434187 & 0.131595 \\ \mathrm{C} & -3.408196 & -0.758724 & 0.021504 \\ \mathrm{O} & -3.059435 & -1.663152 & -0.704243 \\ \mathrm{C} & 3.701203 & 0.436577 & -0.086636 \\ \mathrm{C} & 0.297047 & 3.738849 & -0.389367 \\ \mathrm{H} & 0.446456 & 4.284716 & 0.551450 \\ \mathrm{H} & 1.133424 & 4.003439 & -1.049371 \\ \mathrm{H} & -0.630190 & 4.087710 & -0.850311 \\ \mathrm{C} & 5.020397 & 0.786311 & 0.346174 \\ \mathrm{O} & 4.772958 & -1.425653 & 0.604395 \\ \mathrm{C} & 5.617590 & -0.371895 & 0.747434 \\ \mathrm{H} & 6.596016 & -0.606933 & 1.138450 \\ \mathrm{H} & 5.462631 & 1.773701 & 0.347697 \\ \mathrm{C} & -4.604676 & -0.835789 & 0.954918 \\ \mathrm{H} & -0.531989 & -2.714979 & -0.709611 \\ \mathrm{H} & 0.481436 & -3.871819 & -1.590420 \\ \mathrm{H} & 0.227439 & -4.063807 & 0.151674 \\ \mathrm{C} & 2.606439 & 1.324164 & -0.606199 \\ \mathrm{H} & 2.123797 & 0.836695 & -1.461655 \\ \mathrm{H} & 3.028834 & 2.267435 & -0.971665 \\ \mathrm{H} & 1.927140 & 2.243273 & 1.251694 \\ \mathrm{H} & 1.239085 & 0.649616 & 0.923916 \\ \mathrm{H} & -4.336228 & -0.499911 & 1.957892 \\ \mathrm{H} & -4.972061 & -1.860257 & 0.971726 \\ \mathrm{Cl} & -5.953525 & 0.225323 & 0.381417\end{array}$

\section{S28}

M06-2X/6-31+G(d) SCF energy: $\quad-2304.81449089$ a.u.

M06-2X/6-31+G(d) enthalpy: $\quad-2304.284182$ a.u.

M06-2X/6-31+G(d) free energy: -2304.395313 a.u.

M06-2X/6-311++G(d,p) SCF energy in solution: -2305.14670655 a.u.

M06-2X/6-311++G(d,p) enthalpy: $\quad-2304.616398$ a.u.

M06-2X/6-311++G(d,p) free energy: $\quad-2304.727529$ a.u.

Cartesian coordinates

\begin{tabular}{lccc} 
ATOM & X & Y & \multicolumn{2}{l}{ Z } \\
Rh & 2.243082 & -1.904619 & -0.434389 \\
P & 0.792465 & -0.094114 & 0.186092 \\
O & -0.293279 & -0.285195 & 1.388755 \\
O & -0.151849 & 0.145505 & -1.125073 \\
N & 1.641952 & 1.247055 & 0.688372 \\
C & -1.333769 & -1.223789 & 1.199201 \\
C & -1.368918 & 0.862602 & -1.088257
\end{tabular}




\begin{tabular}{|c|c|c|c|}
\hline & 84049 & 29964 & \\
\hline & 1.026062 & .515584 & \\
\hline & 0963 & 140 & \\
\hline & -2.360720 & -0.953440 & 706 \\
\hline & -2.44 & 098 & \\
\hline & -1.418703 & 475 & 8394 \\
\hline & 3.843143 & 0.527166 & 485 \\
\hline & 3.703979 & 378 & -0.5 \\
\hline & & & \\
\hline & 80 & 15 & \\
\hline & $-0.4 \xi$ & & \\
\hline & -2.238648 & & 365 \\
\hline & -3.322043 & -2.0 & 7026 \\
\hline & -3.6 & & \\
\hline & -0.5 & & \\
\hline & -2.5 & & \\
\hline & 3.3 & & \\
\hline & 5.22 & & \\
\hline & 5.10 & & \\
\hline & $2.9^{\circ}$ & & \\
\hline & 1.86 & & \\
\hline & 7 & & \\
\hline & 5 & & \\
\hline & 1 & & \\
\hline & -2.2 & & \\
\hline & -3.26 & & \\
\hline & -4.3 & -1. & -0 \\
\hline & -3 & & \\
\hline & & & \\
\hline & -2.6 & & 47 \\
\hline & & & \\
\hline & 5.85 & & \\
\hline & & & \\
\hline & & & \\
\hline & & & \\
\hline & 0. & & -0.2 \\
\hline & -0.2 ? & & \\
\hline & -0.8 & & \\
\hline & -4.21 & -4.2 & 326 \\
\hline & & & \\
\hline & & -1. & -1 . \\
\hline & -4.8 & & -1. \\
\hline & -5.8 & & \\
\hline & -4.69 & & 866 \\
\hline & 6.930461 & 381 & 379 \\
\hline & -0.72 & & \\
\hline & & & \\
\hline $\mathrm{C}$ & -5.174939 & -4.11 & -0.3 \\
\hline & -5.953659 & -2.863294 & -1.928421 \\
\hline $\mathrm{H}$ & -4.897369 & 4.096259 & -1.655579 \\
\hline C & -5.919479 & 2.859239 & -0.234560 \\
\hline
\end{tabular}




$\begin{array}{lrrc}\mathrm{H} & -6.669070 & 1.448599 & 1.230911 \\ \mathrm{H} & -5.895828 & -4.905225 & -0.499806 \\ \mathrm{H} & -6.806427 & 3.484805 & -0.200992 \\ \mathrm{C} & 2.218138 & -2.558639 & 1.408499 \\ \mathrm{O} & 2.232380 & -2.940018 & 2.481578 \\ \mathrm{C} & 2.163405 & -1.193257 & -2.269852 \\ \mathrm{O} & 2.138938 & -0.804029 & -3.337463 \\ \mathrm{C} & 3.354777 & -3.408896 & -1.011885 \\ \mathrm{O} & 3.993917 & -4.286890 & -1.354062\end{array}$

\section{S29a}

M06-2X/6-31+G(d) SCF energy: $\quad-3457.82036930$ a.u.

M06-2X/6-31+G(d) enthalpy: $\quad-3457.013211$ a.u.

M06-2X/6-31+G(d) free energy: -3457.164563 a.u.

M06-2X/6-311++G(d,p) SCF energy in solution: -3457.88919848 a.u.

M06-2X/6-311++G(d,p) enthalpy: $\quad-3457.082040$ a.u.

M06-2X/6-311++G(d,p) free energy: $\quad-3457.233392$ a.u.

Cartesian coordinates

\begin{tabular}{lccc} 
ATOM & X & Y & \multicolumn{2}{l}{ Z } \\
C & -1.212972 & 1.759060 & 1.459885 \\
C & -1.681892 & 0.886921 & 2.228960 \\
C & -0.679829 & 3.080036 & 1.328902 \\
C & -0.202535 & 3.946729 & 0.363488 \\
C & -0.856330 & 2.812970 & -1.904159 \\
H & -0.755679 & 3.036195 & -2.974065 \\
C & -2.313716 & 2.853618 & -1.512607 \\
C & -2.852818 & 1.911467 & -0.757488 \\
C & -3.890577 & 1.327725 & -0.065747 \\
H & -4.159985 & 1.602510 & 0.949265 \\
O & -4.923244 & 0.733086 & -0.793368 \\
C & -5.984799 & 0.254400 & -0.072125 \\
O & -6.007420 & 0.204810 & 1.132016 \\
H & -0.459311 & 1.810403 & -1.740646 \\
C & 0.003130 & 3.834798 & -1.126976 \\
H & -0.154013 & 4.835550 & -1.546806 \\
H & 1.057115 & 3.597471 & -1.324939 \\
Rh & -1.995530 & 0.082580 & 0.126712 \\
C & -3.085642 & -1.437064 & 1.027515 \\
O & -3.883419 & -2.114543 & 1.480520 \\
C & -3.136942 & 4.051255 & -1.935406 \\
H & -4.176798 & 3.971753 & -1.610854 \\
H & -2.712500 & 4.971737 & -1.515742 \\
H & -3.120010 & 4.151600 & -3.027661 \\
C & -2.126887 & -0.459182 & -1.727278 \\
O & -2.238656 & -0.771226 & -2.822070 \\
P & 0.301775 & -0.878219 & 0.112835 \\
O & 1.442664 & -0.464514 & 1.207165 \\
O & 0.911103 & -0.273104 & -1.304855
\end{tabular}




\begin{tabular}{|c|c|c|c|}
\hline & 511808 & -2.551020 & \\
\hline & 186269 & 0.718987 & \\
\hline & 35204 & -0.521429 & -1693919 \\
\hline & 612017 & -3.184731 & 8000 \\
\hline & 713 & 272 & 366 \\
\hline & 2.088334 & 1.526052 & \\
\hline & 033552 & 1.0 & 056 \\
\hline & 284506 & 507 & 8959 \\
\hline & 165 & 784 & -2 \\
\hline & 94 & -3.2 & \\
\hline & 83 & & \\
\hline & 1.328277 & 698 & 8814 \\
\hline & .753173 & -3.6 & 7723 \\
\hline & 696 & & \\
\hline & 617 & & \\
\hline & 33 & 2.3 & \\
\hline & 95 & -0.3 & \\
\hline & 922 & -1.6 & 706 \\
\hline & 372 & -1.6 & \\
\hline & 545 & -2.9 & 994 \\
\hline & 41 & -3.8 & \\
\hline & 74 & -4.2 & \\
\hline & 8 & -3 . & \\
\hline & 39 & -3.5 & \\
\hline & -2.3 & -4.9 & \\
\hline & 81 & & 190 \\
\hline & -0.1 & -3.1 & 260 \\
\hline & & & \\
\hline & 5 & 3.1 & \\
\hline 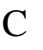 & 35 & & \\
\hline & & & 366 \\
\hline & 360 & -0.0 & \\
\hline & & & 376 \\
\hline & & -3.8 & \\
\hline & 36 & -4.2 & \\
\hline & -4 & -4.5 & \\
\hline & 0.0 & & \\
\hline & 1.98 & -4.1 & 743 \\
\hline & -2.99 & -5.4 & 463 \\
\hline & -2.60 & -5.1 & \\
\hline & -1.9 & -4.6 & 707 \\
\hline & 4.2 & 4.3 & \\
\hline $\mathrm{C}$ & 5.00 & 4.0 & 40 \\
\hline $\mathrm{H}$ & 4.455048 & 968 & 811 \\
\hline 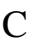 & 127114 & 053 & 969 \\
\hline & & -0.4 & \\
\hline & & & \\
\hline 2 & 4.58 & -4.6 & 53 \\
\hline $\mathrm{H}$ & -3.404891 & -5.756302 & -1.410720 \\
\hline 11 & & 5.0 & 884 \\
\hline $\mathrm{C}$ & 4.939535 & 4.848559 & 0.228077 \\
\hline
\end{tabular}




$\begin{array}{lrrc}\mathrm{H} & 5.535819 & 4.424781 & -1.813037 \\ \mathrm{H} & 6.253276 & -2.210070 & -3.765176 \\ \mathrm{C} & 7.215769 & -1.215832 & -2.128141 \\ \mathrm{H} & 7.884740 & -0.162722 & -0.355170 \\ \mathrm{H} & 5.435093 & 5.814514 & 0.255659 \\ \mathrm{H} & 8.215492 & -1.526887 & -2.416265 \\ \mathrm{C} & -2.015513 & 0.214106 & 3.496970 \\ \mathrm{H} & -3.092749 & 0.039218 & 3.593599 \\ \mathrm{H} & -1.503940 & -0.750715 & 3.586723 \\ \mathrm{H} & -1.696730 & 0.855554 & 4.327309 \\ \mathrm{O} & -0.641483 & 3.701273 & 2.567528 \\ \mathrm{C} & 0.142529 & 5.148388 & 1.068177 \\ \mathrm{C} & -0.137248 & 4.942770 & 2.381864 \\ \mathrm{H} & 0.549678 & 6.047173 & 0.625564 \\ \mathrm{H} & -0.052927 & 5.554475 & 3.267774 \\ \mathrm{C} & -7.098813 & -0.214143 & -0.985966 \\ \mathrm{H} & -6.728526 & -0.964406 & -1.688292 \\ \mathrm{H} & -7.898613 & -0.625913 & -0.372711 \\ \mathrm{Cl} & -7.770510 & 1.150123 & -1.954771\end{array}$

\section{S29b}

M06-2X/6-31+G(d) SCF energy: $\quad-3457.88490144$ a.u.

M06-2X/6-31+G(d) enthalpy: $\quad-3457.075818$ a.u.

M06-2X/6-31+G(d) free energy: -3457.224411 a.u.

M06-2X/6-311++G(d,p) SCF energy in solution: -3457.94970978 a.u.

M06-2X/6-311++G(d,p) enthalpy: $\quad-3457.140626$ a.u.

M06-2X/6-311++G(d,p) free energy: $\quad-3457.289219$ a.u.

Cartesian coordinates

\begin{tabular}{lcccc}
\multicolumn{2}{l}{ ATOM } & X & \multicolumn{2}{l}{ Y } \\
C & -2.241297 & 1.657665 & 1.484008 \\
C & -1.862305 & 0.433351 & 1.909279 \\
C & -2.208069 & 2.949715 & 2.137726 \\
C & -1.991328 & 4.204258 & 1.613819 \\
C & -1.096469 & 3.327059 & -0.617017 \\
H & -0.632442 & 3.696027 & -1.536231 \\
C & -2.172637 & 2.328014 & -0.968009 \\
C & -2.735073 & 1.577476 & 0.053543 \\
C & -3.769946 & 0.551028 & -0.021278 \\
H & -4.359557 & 0.376264 & 0.871636 \\
O & -4.516464 & 0.411468 & -1.186115 \\
C & -5.569956 & -0.460178 & -1.130511 \\
O & -5.803248 & -1.161747 & -0.179222 \\
H & -0.312605 & 2.851448 & -0.020082 \\
C & -1.649549 & 4.539579 & 0.186831 \\
H & -2.514569 & 4.967698 & -0.338742 \\
H & -0.878169 & 5.319145 & 0.181816 \\
Rh & -1.949860 & -0.618873 & 0.126295 \\
C & -2.788617 & -2.086511 & 1.007468
\end{tabular}




\begin{tabular}{|c|c|c|c|}
\hline $\mathrm{O}$ & -3.332516 & -2.906566 & 1.589622 \\
\hline $\mathrm{C}$ & -2.648518 & 2.382340 & -2.389068 \\
\hline $\mathrm{H}$ & -3.474362 & 1.715809 & -2.620556 \\
\hline $\mathrm{H}$ & -2.960345 & 3.412922 & -2.614347 \\
\hline $\mathrm{H}$ & -1.807061 & 2.174783 & -3.063527 \\
\hline $\mathrm{C}$ & -2.037570 & -1.308804 & -1.804941 \\
\hline $\mathrm{O}$ & -2.137944 & -1.661203 & -2.884103 \\
\hline $\mathrm{P}$ & 0.541130 & -1.043532 & 0.147979 \\
\hline $\mathrm{O}$ & 1.597014 & -0.279274 & 1.148411 \\
\hline $\mathrm{O}$ & 0.957254 & -0.422285 & -1.337862 \\
\hline $\mathrm{N}$ & 1.137514 & -2.605437 & 0.368866 \\
\hline $\mathrm{C}$ & 1.894615 & 1.078984 & 1.005035 \\
\hline $\mathrm{C}$ & 2.295856 & -0.314307 & -1.738140 \\
\hline $\mathrm{C}$ & 2.357441 & -2.931514 & 1.078447 \\
\hline $\mathrm{C}$ & 0.284589 & -3.717348 & 0.036277 \\
\hline $\mathrm{C}$ & 1.507476 & 1.914310 & 2.075736 \\
\hline $\mathrm{C}$ & 2.602173 & 1.541155 & -0.095150 \\
\hline $\mathrm{C}$ & 3.135697 & 0.595868 & -1.118178 \\
\hline $\mathrm{C}$ & 2.705792 & -1.140683 & -2.806215 \\
\hline $\mathrm{C}$ & 3.598787 & -2.772638 & 0.462244 \\
\hline $\mathrm{C}$ & 2.264765 & -3.387723 & 2.410463 \\
\hline $\mathrm{C}$ & -0.360210 & -4.434344 & 1.068805 \\
\hline $\mathrm{C}$ & 0.084613 & -4.059709 & -1.305050 \\
\hline $\mathrm{H}$ & 1.018342 & 1.466973 & 2.933091 \\
\hline $\mathrm{C}$ & 1.776308 & 3.259130 & 2.018706 \\
\hline $\mathrm{C}$ & 2.789680 & 2.967659 & -0.210889 \\
\hline $\mathrm{C}$ & 4.529744 & 0.574593 & -1.488416 \\
\hline $\mathrm{H}$ & 1.973410 & -1.783514 & -3.283402 \\
\hline $\mathrm{C}$ & 4.012382 & -1.098265 & -3.227923 \\
\hline $\mathrm{H}$ & 3.642423 & -2.444055 & -0.569590 \\
\hline $\mathrm{C}$ & 4.771002 & -3.020186 & 1.175553 \\
\hline $\mathrm{C}$ & 3.463062 & -3.608434 & 3.115791 \\
\hline $\mathrm{C}$ & 0.994057 & -3.692095 & 3.065366 \\
\hline $\mathrm{C}$ & -0.139120 & -4.155664 & 2.487387 \\
\hline $\mathrm{C}$ & -1.243685 & -5.466435 & 0.695811 \\
\hline $\mathrm{C}$ & -0.784990 & -5.094791 & -1.647611 \\
\hline $\mathrm{H}$ & 0.624353 & -3.512624 & -2.071817 \\
\hline $\mathrm{H}$ & 1.494425 & 3.908691 & 2.842652 \\
\hline $\mathrm{C}$ & 2.388101 & 3.825200 & 0.868667 \\
\hline $\mathrm{C}$ & 3.325679 & 3.578108 & -1.379619 \\
\hline $\mathrm{C}$ & 4.959328 & -0.271552 & -2.566728 \\
\hline $\mathrm{C}$ & 5.520547 & 1.327836 & -0.799237 \\
\hline $\mathrm{H}$ & 4.340824 & -1.718138 & -4.057719 \\
\hline $\mathrm{H}$ & 5.733399 & -2.882280 & 0.692114 \\
\hline $\mathrm{C}$ & 4.702138 & -3.427875 & 2.510225 \\
\hline $\mathrm{H}$ & 3.407764 & -3.947630 & 4.146869 \\
\hline $\mathrm{H}$ & 1.014034 & -3.657915 & 4.153244 \\
\hline $\mathrm{H}$ & -0.946117 & -4.462199 & 3.150345 \\
\hline $\mathrm{H}$ & -1.757755 & -6.021057 & 1.476231 \\
\hline $\mathrm{C}$ & -1.455451 & -5.795148 & -0.639843 \\
\hline $\mathrm{H}$ & -0.936096 & -5.353861 & -2.691302 \\
\hline
\end{tabular}




$\begin{array}{lrrr}\mathrm{C} & 2.587288 & 5.228673 & 0.760405 \\ \mathrm{C} & 3.486252 & 4.944384 & -1.460951 \\ \mathrm{H} & 3.601824 & 2.956849 & -2.223204 \\ \mathrm{C} & 6.329882 & -0.286360 & -2.942596 \\ \mathrm{C} & 6.844659 & 1.275666 & -1.176797 \\ \mathrm{H} & 5.229871 & 1.940043 & 0.045997 \\ \mathrm{H} & 5.611959 & -3.611606 & 3.073858 \\ \mathrm{H} & -2.134438 & -6.603097 & -0.895770 \\ \mathrm{H} & 2.296507 & 5.859284 & 1.596932 \\ \mathrm{C} & 3.128528 & 5.781053 & -0.377663 \\ \mathrm{H} & 3.891594 & 5.383619 & -2.367963 \\ \mathrm{H} & 6.634103 & -0.922607 & -3.769743 \\ \mathrm{C} & 7.256194 & 0.473722 & -2.267130 \\ \mathrm{H} & 7.581895 & 1.853760 & -0.627112 \\ \mathrm{H} & 3.274537 & 6.854713 & -0.450963 \\ \mathrm{H} & 8.302047 & 0.450781 & -2.558501 \\ \mathrm{C} & -1.445573 & -0.046388 & 3.257700 \\ \mathrm{H} & -0.523898 & -0.637012 & 3.211167 \\ \mathrm{H} & -1.303349 & 0.795442 & 3.944196 \\ \mathrm{H} & -2.216938 & -0.696064 & 3.693075 \\ \mathrm{C} & -2.036045 & 5.104280 & 2.729937 \\ \mathrm{O} & -2.363548 & 3.025544 & 3.498802 \\ \mathrm{C} & -2.262680 & 4.341962 & 3.835151 \\ \mathrm{H} & -2.385121 & 4.567860 & 4.883534 \\ \mathrm{H} & -1.911365 & 6.178344 & 2.704582 \\ \mathrm{C} & -6.370377 & -0.514858 & -2.418235 \\ \mathrm{H} & -5.939815 & -1.285604 & -3.065009 \\ \mathrm{H} & -7.389770 & -0.803848 & -2.162918 \\ \mathrm{Cl} & -6.423106 & 1.016349 & -3.353721\end{array}$

\section{S30a}

M06-2X/6-31+G(d) SCF energy: $\quad-3457.82663049$ a.u.

M06-2X/6-31+G(d) enthalpy: $\quad-3457.018983$ a.u.

M06-2X/6-31+G(d) free energy: -3457.167866 a.u.

M06-2X/6-311++G(d,p) SCF energy in solution: -3457.89445733 a.u.

M06-2X/6-311++G(d,p) enthalpy: $\quad-3457.086810$ a.u.

M06-2X/6-311++G(d,p) free energy: $\quad-3457.235693$ a.u.

Cartesian coordinates

\begin{tabular}{|c|c|c|c|}
\hline & $X$ & Y & Z \\
\hline C & -4.043227 & -0.171172 & 1.345515 \\
\hline C & -2.850988 & -0.038460 & 1.725709 \\
\hline 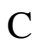 & -5.320546 & -0.384507 & 0.882205 \\
\hline$C$ & -6.306662 & 0.456581 & 0.351733 \\
\hline C & -5.136120 & 2.329762 & -0.958452 \\
\hline H & -5.574719 & 3.070725 & -1.636815 \\
\hline C & -3.898345 & 2.933367 & -0.329639 \\
\hline 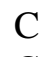 & -2.758338 & 2.282321 & -0.142362 \\
\hline C & -1.469261 & 2.311579 & 0.401540 \\
\hline
\end{tabular}




\begin{tabular}{|c|c|c|c|}
\hline & -1.288502 & 2.379678 & \\
\hline & .427115 & 2.922398 & \\
\hline & 00369 & & \\
\hline & 585777 & & \\
\hline & -4.873172 & & 6018 \\
\hline & -6.224666 & 1.934438 & 6881 \\
\hline & 366 & 078 & 265 \\
\hline & -7.208028 & 2.270369 & 439 \\
\hline & -1.930626 & 567 & -0 \\
\hline & -1.168688 & 060 & \\
\hline & & & \\
\hline & -4.021028 & & \\
\hline & & & \\
\hline & & 254 & \\
\hline & -4.1 & 5.0 & -0 . \\
\hline & & & \\
\hline & & & \\
\hline & & -1.0 & \\
\hline & & & \\
\hline & & & \\
\hline & & & \\
\hline & & & \\
\hline & & & \\
\hline & & & \\
\hline & & & \\
\hline & & & \\
\hline & & & \\
\hline & & & \\
\hline C & & & \\
\hline & & & \\
\hline & & -4.2 & \\
\hline & & -4.2 & 357 \\
\hline & & & \\
\hline & & & \\
\hline 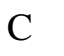 & & & \\
\hline $\mathrm{C}$ & & 14 & \\
\hline $\mathrm{C}$ & & & \\
\hline $\mathrm{H}$ & & -1.9 & 631 \\
\hline $\mathrm{C}$ & & -1.7 & -2.286624 \\
\hline 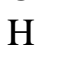 & & & \\
\hline $\mathrm{C}$ & 5 & -4.1 & \\
\hline $\mathrm{C}$ & -2.2 & -4.9 & -1 . \\
\hline $\mathrm{C}$ & & -4.4 & 395 \\
\hline $\mathrm{C}$ & 1.370720 & -4.4 & 897 \\
\hline $\mathrm{C}$ & 3.162691 & -4.9 & 790 \\
\hline 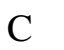 & & & \\
\hline-7 & & & \\
\hline $\mathrm{H}$ & & 3.449549 & 3.496 \\
\hline $\mathrm{C}$ & & & \\
\hline $\mathrm{C}$ & 4.319702 & 2.796465 & -0.1 \\
\hline $\mathrm{C}$ & 5.288175 & -1.229726 & -1.34978 \\
\hline
\end{tabular}




$\begin{array}{lrrr}\mathrm{C} & 5.741598 & 0.087144 & 0.663073 \\ \mathrm{H} & 4.747041 & -2.392121 & -3.096396 \\ \mathrm{H} & -3.985203 & -4.135321 & 1.653773 \\ \mathrm{C} & -3.306947 & -4.902007 & -0.250219 \\ \mathrm{H} & -2.350847 & -5.538005 & -2.067713 \\ \mathrm{H} & -0.222891 & -4.691413 & -2.883886 \\ \mathrm{H} & 2.062698 & -4.727699 & -2.343904 \\ \mathrm{H} & 3.617311 & -5.571201 & -0.708359 \\ \mathrm{C} & 3.741506 & -4.892104 & 1.327248 \\ \mathrm{H} & 3.585558 & -4.068394 & 3.317830 \\ \mathrm{C} & 3.479272 & 4.518470 & 1.844475 \\ \mathrm{C} & 4.804721 & 4.085328 & -0.124929 \\ \mathrm{H} & 4.642487 & 2.151264 & -1.007863 \\ \mathrm{C} & 6.674350 & -1.532203 & -1.423035 \\ \mathrm{C} & 7.076954 & -0.242058 & 0.575483 \\ \mathrm{H} & 5.395261 & 0.695879 & 1.490047 \\ \mathrm{H} & -4.215343 & -5.463962 & -0.446799 \\ \mathrm{H} & 4.653409 & -5.444142 & 1.534177 \\ \mathrm{H} & 3.139119 & 5.179822 & 2.637219 \\ \mathrm{C} & 4.396491 & 4.953312 & 0.914939 \\ \mathrm{H} & 5.507244 & 4.437576 & -0.874747 \\ \mathrm{H} & 7.024151 & -2.168378 & -2.232184 \\ \mathrm{C} & 7.555392 & -1.047246 & -0.484190 \\ \mathrm{H} & 7.768716 & 0.117272 & 1.332052 \\ \mathrm{H} & 4.797753 & 5.960999 & 0.969298 \\ \mathrm{H} & 8.612071 & -1.290183 & -0.545963 \\ \mathrm{C} & -2.106533 & 0.059454 & 3.016013 \\ \mathrm{H} & -2.723412 & -0.297043 & 3.847063 \\ \mathrm{H} & -1.179636 & -0.517903 & 2.982048 \\ \mathrm{H} & -1.835848 & 1.101405 & 3.215804 \\ \mathrm{C} & -7.393472 & -0.386897 & 0.020245 \\ \mathrm{O} & -5.782292 & -1.700343 & 0.860339 \\ \mathrm{C} & -7.021075 & -1.666173 & 0.357040 \\ \mathrm{H} & -8.335984 & -0.086215 & -0.415926 \\ \mathrm{H} & -7.534955 & -2.615277 & 0.297157 \\ \mathrm{C} & 0.955607 & 4.776119 & -0.907823 \\ \mathrm{H} & 1.239434 & 5.756239 & -0.530253 \\ \mathrm{H} & 1.825833 & 4.121675 & -0.972378 \\ \mathrm{Cl} & 0.308112 & 4.986319 & -2.583264\end{array}$

\section{S30b}

M06-2X/6-31+G(d) SCF energy: $\quad-3457.88091845$ a.u.

M06-2X/6-31+G(d) enthalpy: $\quad-3457.072149$ a.u.

M06-2X/6-31+G(d) free energy: -3457.219380 a.u.

M06-2X/6-311++G(d,p) SCF energy in solution: -3457.94650619 a.u.

M06-2X/6-311++G(d,p) enthalpy: $\quad-3457.137737$ a.u.

M06-2X/6-311++G(d,p) free energy: $\quad-3457.284968$ a.u.

Cartesian coordinates 


\begin{tabular}{|c|c|c|c|}
\hline & & Y & \\
\hline $\mathrm{C}$ & -3.493901 & Vד & \\
\hline & 466 & & \\
\hline & 6372 & 499 & \\
\hline C & -5.7 & 7348 & 1601 \\
\hline & -5.612807 & -0.069920 & \\
\hline & -6.218485 & 2114 & \\
\hline & -4.222196 & 567 & 735 \\
\hline & 423 & & \\
\hline C & 8138 & -1.0 & \\
\hline$H$ & -1.3 & & \\
\hline D & -1.373232 & -1.3 & \\
\hline C & -0.608562 & -2.43 & \\
\hline D & -0.4 & -3.2 & \\
\hline & -5.5 & 0.7 & \\
\hline & 641 & -1.3 & \\
\hline$H$ & -6.270529 & & \\
\hline t. & -7.3 & -1.0 & \\
\hline h & -1.776257 & & \\
\hline 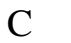 & -1.1 & & \\
\hline O & -0.8 & & \\
\hline & -4.1 & -0.5 & \\
\hline I & -3.1 & & \\
\hline $\mathrm{I}$ & -4.7 & -1.3 & \\
\hline I & -4.4 & & \\
\hline C & -2.5 & & \\
\hline $\mathrm{O}$ & -3.121510 & & \\
\hline & 0.4 & 1.0 & \\
\hline ) & & & \\
\hline O & & & \\
\hline $\mathrm{J}$ & & & \\
\hline C & 0.86 & -1.2 & \\
\hline C & & -0.2 & \\
\hline C & 0.4 & & \\
\hline 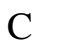 & 2.62 & & \\
\hline $\mathrm{C}$ & 86 & $-1 . \varepsilon$ & \\
\hline & 58 & & \\
\hline $\mathrm{C}$ & 2.858847 & -1.2 & 69 \\
\hline $\mathrm{C}$ & 3.500808 & & \\
\hline $\mathrm{C}$ & -0.1 & & \\
\hline & & 4.7 & \\
\hline 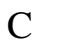 & 7 & 3.5 & \\
\hline C & & 2.1 & -1. \\
\hline $\mathrm{H}$ & -0.733159 & -1.2 & 7778 \\
\hline $\mathrm{C}$ & -0.156287 & -3.2 & 0595 \\
\hline & & & \\
\hline & & & \\
\hline 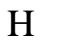 & 3.171242 & & \\
\hline $\mathrm{C}$ & 4.802229 & -0.193121 & \\
\hline $\mathrm{H}$ & 0.006037 & 537 & -2.9 \\
\hline G & -0.864485 & 4.866472 & -2.659423 \\
\hline
\end{tabular}




\begin{tabular}{|c|c|c|c|}
\hline & -0.423604 & 859448 & \\
\hline & 1.040954 & 4.763239 & \\
\hline & 2.258068 & 29843 & 33087 \\
\hline & 125 & & \\
\hline & 4.853050 & & \\
\hline & 3.058608 & 528354 & 1643 \\
\hline & 998 & 76 & 5904 \\
\hline & 0.68 & -4.0 & \\
\hline & 2.41 & -4.2 & \\
\hline & & & \\
\hline & 4.72 & & 979 \\
\hline & 5.535923 & & 0129 \\
\hline & -1.3 & & 932 \\
\hline & -1.0 & & \\
\hline & 34 & & \\
\hline & & & \\
\hline & 2.7 & & \\
\hline & 4.92 & & \\
\hline & $5.3^{\prime}$ & & \\
\hline & 5.5 & & \\
\hline & 0.5 & -5. & \\
\hline & & & \\
\hline & & & \\
\hline & & & \\
\hline & 6 & & \\
\hline & 4.0 & -2.8 & 733 \\
\hline & -1.5 & & 23 \\
\hline & & & 96 \\
\hline & -0.1 & & 050 \\
\hline & & & \\
\hline & & & \\
\hline & 7.31 & & \\
\hline & & & \\
\hline & & -3 . & \\
\hline & 1.2 & & \\
\hline & 8.0 & -2.5 & 215 \\
\hline & -2.54 & & 251 \\
\hline & -1.5 & & 9201 \\
\hline & -3.1 & & \\
\hline & -2.8 & & 944 \\
\hline & 0.0 & -2.5 & 63 \\
\hline & -6.47 & -2.3 & 8452 \\
\hline $\mathrm{O}$ & $-4.4 \varepsilon$ & 895 & 4325 \\
\hline & -5.6 & 851 & 4765 \\
\hline & & & 605 \\
\hline & -7.4 & -2.8 & -1.9 \\
\hline & & -2.054829 & 3.595805 \\
\hline 11 & 0.185015 & -3.581301 & 3.907992 \\
\hline & -0.02032 & -1.738547 & 5.02 \\
\hline
\end{tabular}




\section{S31a}

M06-2X/6-31+G(d) SCF energy: $\quad-1715.68222047$ a.u.

M06-2X/6-31+G(d) enthalpy: $\quad-1715.361155$ a.u.

M06-2X/6-31+G(d) free energy: -1715.453635 a.u.

M06-2X/6-311++G(d,p) SCF energy in solution: -1716.40548622 a.u.

M06-2X/6-311++G(d,p) enthalpy: $\quad-1716.084421$ a.u.

M06-2X/6-311++G(d,p) free energy: $\quad-1716.176901$ a.u.

Cartesian coordinates

$\begin{array}{lccc}\text { ATOM } & \text { X } & \text { Y } & \text { Z } \\ \text { C } & 2.157813 & -0.588200 & -1.152543 \\ \text { C } & 1.035238 & -0.983534 & -1.577227 \\ \text { C } & 3.370767 & -0.279007 & -0.596033 \\ \text { C } & 3.958670 & 0.909696 & -0.130566 \\ \text { C } & 2.103067 & 2.399033 & 0.836531 \\ \text { H } & 2.196782 & 3.320115 & 1.422646 \\ \text { C } & 0.811207 & 2.453074 & 0.051654 \\ \text { C } & -0.015106 & 1.428393 & -0.115463 \\ \text { C } & -1.108653 & 0.952937 & -0.840368 \\ \text { H } & -1.043975 & 0.756796 & -1.905903 \\ \text { O } & -2.410473 & 1.272387 & -0.426706 \\ \text { C } & -3.415813 & 0.619527 & -1.086834 \\ \text { O } & -3.220236 & -0.185246 & -1.965160 \\ \text { H } & 2.089177 & 1.568382 & 1.547965 \\ \text { C } & 3.360843 & 2.286474 & -0.095442 \\ \text { H } & 3.097053 & 2.598032 & -1.113057 \\ \text { H } & 4.133929 & 2.979681 & 0.247200 \\ \text { Rh } & -0.174352 & -0.678200 & 0.269307 \\ \text { C } & -1.224242 & -0.157441 & 1.837442 \\ \text { O } & -1.795396 & 0.156875 & 2.770147 \\ \text { C } & 0.490270 & 3.784613 & -0.599185 \\ \text { H } & -0.395471 & 3.730822 & -1.236150 \\ \text { H } & 1.333115 & 4.139323 & -1.206297 \\ \text { H } & 0.311495 & 4.544085 & 0.172875 \\ \text { C } & 1.201660 & -1.651644 & 1.456334 \\ \text { O } & 1.980159 & -2.176592 & 2.101236 \\ \text { C } & 0.432348 & -1.457279 & -2.861853 \\ \text { H } & 0.372696 & -2.552203 & -2.839221 \\ \text { H } & -0.583996 & -1.081634 & -3.002626 \\ \text { H } & 1.050884 & -1.167443 & -3.716712 \\ \text { C } & 5.211844 & 0.535892 & 0.400797 \\ \text { O } & 4.233905 & -1.344898 & -0.331870 \\ \text { C } & 5.322939 & -0.827635 & 0.239527 \\ \text { H } & 5.945569 & 1.189505 & 0.851866 \\ \text { H } & 6.111126 & -1.527898 & 0.479926 \\ \text { C } & -4.773365 & 1.024591 & -0.553846 \\ \text { C } & -1.355153 & -2.328168 & -0.288218 \\ \text { O } & -2.041554 & -3.176828 & -0.604579 \\ \text { H } & -5.544215 & 0.668583 & -1.235165 \\ \text { H } & -4.841248 & 2.103949 & -0.411757\end{array}$




\section{S31b}

M06-2X/6-31+G(d) SCF energy: $\quad-1715.72986085$ a.u.

M06-2X/6-31+G(d) enthalpy: $\quad-1715.407528$ a.u.

M06-2X/6-31+G(d) free energy: -1715.497304 a.u.

M06-2X/6-311++G(d,p) SCF energy in solution: -1716.45352689 a.u.

M06-2X/6-311++G(d,p) enthalpy: $\quad-1716.131194$ a.u.

M06-2X/6-311++G(d,p) free energy: $\quad-1716.220970$ a.u.

Cartesian coordinates

\begin{tabular}{lrrr} 
ATOM & X & Y & \multicolumn{2}{l}{ Z } \\
C & -1.813392 & 0.002861 & -0.422238 \\
C & -1.478245 & 1.296341 & -0.598729 \\
C & -3.066729 & -0.696656 & -0.600525 \\
C & -3.601938 & -1.743974 & 0.116806 \\
C & -1.957091 & -1.500447 & 2.066742 \\
H & -1.782777 & -1.905062 & 3.067570 \\
C & -0.647728 & -1.438186 & 1.318764 \\
C & -0.615856 & -0.761318 & 0.101325 \\
C & 0.500934 & -0.581806 & -0.812736 \\
H & 0.266857 & -0.344206 & -1.844386 \\
O & 1.638225 & -1.351275 & -0.677984 \\
C & 2.618432 & -1.165042 & -1.635179 \\
O & 2.538174 & -0.326983 & -2.493091 \\
H & -2.380464 & -0.495910 & 2.181141 \\
C & -3.012364 & -2.391158 & 1.340656 \\
H & -2.566282 & -3.366344 & 1.102114 \\
H & -3.817348 & -2.597999 & 2.055552 \\
Rh & 0.402648 & 1.305763 & 0.305225 \\
C & 2.176221 & 0.988043 & 1.365930 \\
O & 3.138373 & 0.831513 & 1.948650 \\
C & 0.453500 & -2.281580 & 1.883302 \\
H & 1.392671 & -2.235129 & 1.338769 \\
H & 0.112658 & -3.328254 & 1.903307 \\
H & 0.621991 & -2.010887 & 2.933492 \\
C & -0.306133 & 2.708526 & 1.637886 \\
O & -0.726429 & 3.528155 & 2.304774 \\
C & -2.228202 & 2.444135 & -1.179697 \\
H & -1.763799 & 2.791461 & -2.111917 \\
H & -3.255760 & 2.144939 & -1.412934 \\
H & -2.256762 & 3.302438 & -0.498111 \\
C & 3.755220 & -2.151380 & -1.474882 \\
C & -4.884904 & -1.998341 & -0.468127 \\
O & -3.944318 & -0.286657 & -1.568727 \\
C & -5.037008 & -1.094409 & -1.475866 \\
H & -5.821366 & -0.904807 & -2.192721 \\
H & -5.600373 & -2.751249 & -0.167239 \\
C & 1.163859 & 2.354840 & -1.139548
\end{tabular}




$\begin{array}{lrrr}\mathrm{O} & 1.569693 & 2.994262 & -1.990752 \\ \mathrm{H} & 4.555668 & -1.863309 & -2.154658 \\ \mathrm{H} & 3.408270 & -3.160310 & -1.714071 \\ \mathrm{Cl} & 4.408615 & -2.181736 & 0.203100\end{array}$

\section{S32a}

M06-2X/6-31+G(d) SCF energy: $\quad-2998.24142710$ a.u.

M06-2X/6-31+G(d) enthalpy: $\quad-2997.426733$ a.u.

M06-2X/6-31+G(d) free energy: -2997.574848 a.u.

M06-2X/6-311++G(d,p) SCF energy in solution: -2998.30472594 a.u.

M06-2X/6-311++G(d,p) enthalpy: $\quad$-2997.490032 a.u.

M06-2X/6-311++G(d,p) free energy: $\quad-2997.638147$ a.u.

Cartesian coordinates

$\begin{array}{lccc}\text { ATOM X } & \text { Y } & \text { Z } \\ \text { C } & -1.391140 & 1.974048 & 1.221650 \\ \text { C } & -1.998731 & 1.177452 & 1.978075 \\ \text { C } & -0.716604 & 3.230503 & 1.105853 \\ \text { C } & -0.111599 & 4.029066 & 0.153889 \\ \text { C } & -0.760181 & 2.904745 & -2.117790 \\ \text { H } & -0.584619 & 3.084385 & -3.186440 \\ \text { C } & -2.227584 & 3.084906 & -1.809783 \\ \text { C } & -2.897695 & 2.211824 & -1.076401 \\ \text { C } & -4.025611 & 1.742718 & -0.445032 \\ \text { H } & -4.315510 & 2.045088 & 0.556451 \\ \text { O } & -5.055084 & 1.211901 & -1.209669 \\ \text { C } & -6.192990 & 0.811841 & -0.521142 \\ \text { O } & -6.261698 & 0.846252 & 0.681189 \\ \text { H } & -0.463448 & 1.876874 & -1.906283 \\ \text { C } & 0.147372 & 3.869314 & -1.323547 \\ \text { H } & 0.093302 & 4.870191 & -1.768679 \\ \text { H } & 1.185176 & 3.540717 & -1.470505 \\ \text { Rh } & -2.228499 & 0.336217 & -0.105149 \\ \text { C } & -3.523670 & -1.050767 & 0.736464 \\ \text { O } & -4.396524 & -1.648729 & 1.161953 \\ \text { C } & -2.908162 & 4.346006 & -2.297987 \\ \text { H } & -3.967515 & 4.371147 & -2.032651 \\ \text { H } & -2.424087 & 5.231008 & -1.866799 \\ \text { H } & -2.819673 & 4.424217 & -3.388682 \\ \text { C } & -2.275949 & -0.226683 & -1.959348 \\ \text { O } & -2.342964 & -0.550857 & -3.054750 \\ \text { P } & -0.048770 & -0.829235 & 0.067504 \\ \text { O } & 1.051193 & -0.491178 & 1.227499 \\ \text { O } & 0.709778 & -0.327431 & -1.318723 \\ \text { N } & -0.005620 & -2.510597 & 0.233500 \\ \text { C } & 1.905944 & 0.615051 & 1.215461 \\ \text { C } & 2.026392 & -0.710635 & -1.609372 \\ \text { C } & 0.981029 & -3.222728 & 1.024622 \\ \text { C } & -1.094973 & -3.313501 & -0.257119\end{array}$




\begin{tabular}{|c|c|c|c|}
\hline & 09653 & 225 & \\
\hline & 2.846968 & 0.796865 & \\
\hline & 76015 & 91 & 395 \\
\hline & 200794 & -1.565712 & 8284 \\
\hline & 2.263189 & 420 & \\
\hline & 0.624186 & -3.670322 & 3907 \\
\hline & .010845 & -3.879683 & 8679 \\
\hline & .239619 & -3.522045 & 578 \\
\hline & 1477 & 1.2 & 316 \\
\hline & 626169 & 2.5 & \\
\hline & 487 & 2.02 & \\
\hline & 4.388123 & -0.76 & \\
\hline & .333033 & -1.8 & 7165 \\
\hline & 3.462284 & -2.0 & \\
\hline & 2.503609 & -3.1 & \\
\hline & 229420 & -4.0 & \\
\hline & 140 & -4.2 & \\
\hline . & 818 & $-3 .($ & \\
\hline 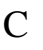 & 5039 & -3.7 & \\
\hline & 490 & -4.6 & 01 \\
\hline & -2.3 & -4.2 & -2 . \\
\hline & 397 & -3.1 & -2 \\
\hline & & & \\
\hline 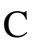 & 35 & & \\
\hline & 4.4 & & \\
\hline & 804 & -1.6 & 0397 \\
\hline & 5.510403 & -0.5 & -0 \\
\hline & 3.6 & -2.6 & 27 \\
\hline & 65 & -4.2 & 60 \\
\hline$y$ & 35 & -4.4 & \\
\hline & & & 720 \\
\hline & -0.835613 & -3.6 & \\
\hline & & & \\
\hline & -3.810404 & -5.0 & 504 \\
\hline & -3.2 & -4.8 & -1.2 \\
\hline & -2.4 & -4. & 320 \\
\hline & 4.28 & & 586 \\
\hline & 5.167217 & 3.6 & 5168 \\
\hline & 4.508332 & 1.8 & 3138 \\
\hline & 301 & -2.1 & 540 \\
\hline & 73 & -1.0 & -0.5 \\
\hline & 10 & & \\
\hline 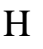 & 3.66 & -4.9 & 172 \\
\hline $\mathrm{H}$ & -4.079170 & -5.391575 & -1.610372 \\
\hline 1 & 4.214822 & 4.71 & 8360 \\
\hline & & & \\
\hline & & & \\
\hline$\ldots$ & 5.993226 & -2.828808 & -3.360809 \\
\hline $\mathrm{C}$ & 935578 & -1.882544 & -1.684076 \\
\hline $\mathrm{H}$ & 7.582843 & -0.846372 & 0.106881 \\
\hline $\mathrm{H}$ & 5.688226 & 5.348198 & 0.408208 \\
\hline
\end{tabular}




$\begin{array}{lrrc}\mathrm{H} & 7.917840 & -2.294487 & -1.895520 \\ \mathrm{C} & -2.498963 & 0.604847 & 3.241052 \\ \mathrm{H} & -3.592690 & 0.539334 & 3.251663 \\ \mathrm{H} & -2.096879 & -0.399797 & 3.413683 \\ \mathrm{H} & -2.185835 & 1.251968 & 4.069102 \\ \mathrm{O} & -0.674402 & 3.866409 & 2.336757 \\ \mathrm{C} & 0.318082 & 5.203493 & 0.858518 \\ \mathrm{C} & -0.041846 & 5.049417 & 2.159536 \\ \mathrm{H} & 0.832836 & 6.049855 & 0.424651 \\ \mathrm{H} & 0.060963 & 5.665383 & 3.040520 \\ \mathrm{C} & -7.255142 & 0.363319 & -1.484368 \\ \mathrm{H} & -6.875755 & -0.446304 & -2.116502 \\ \mathrm{H} & -8.128588 & 0.025499 & -0.926424 \\ \mathrm{H} & -7.533138 & 1.190378 & -2.146267\end{array}$

\section{S32b}

M06-2X/6-31+G(d) SCF energy: $\quad$-2998.30565621 a.u.

M06-2X/6-31+G(d) enthalpy: $\quad-2997.488867$ a.u.

M06-2X/6-31+G(d) free energy: -2997.633266 a.u.

M06-2X/6-311++G(d,p) SCF energy in solution: -2998.36384037 a.u.

M06-2X/6-311++G(d,p) enthalpy: $\quad-2997.547051$ a.u.

M06-2X/6-311++G(d,p) free energy: $\quad-2997.691450$ a.u.

Cartesian coordinates

\begin{tabular}{|c|c|c|c|}
\hline & $X$ & Y & $\mathrm{Z}$ \\
\hline 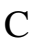 & -2.414566 & 1.987289 & - \\
\hline & & 306 & \\
\hline & 0 & 64 & \\
\hline & -1 & & \\
\hline & -0.866617 & & \\
\hline & -0.2 & 3.72 & \\
\hline & -1.9 & 2.537 & -1 \\
\hline & -2.7 & & \\
\hline & -3.8 & & \\
\hline & -4.5 & & \\
\hline & -4.5 & & \\
\hline & -5.6 & & \\
\hline & -6.0 & & \\
\hline & -0.208945 & 2.9 & -0. \\
\hline & -1.3 & 4.7 & \\
\hline & -2.1 & 33 & \\
\hline & -0.5 & & -0. \\
\hline & -2.206340 & -0.360558 & 656 \\
\hline & -3.300404 & -1.688902 & \\
\hline & -3.98 & -2.4 & \\
\hline & -2.284643 & 2.56 & -2.8 \\
\hline & -3.123099 & & \\
\hline & -2.489898 & 3.602401 & -3.1 \\
\hline & -1.387258 & 2.265418 & -3.438 \\
\hline
\end{tabular}




\begin{tabular}{|c|c|c|c|}
\hline & -2.165840 & -1.117899 & \\
\hline & -2.193753 & -1.498356 & \\
\hline & 0.209774 & -1.031094 & \\
\hline & 1.228283 & -0.337463 & \\
\hline & 0.852977 & -0.522838 & \\
\hline & 0.609904 & -2.635017 & 2467 \\
\hline & 1.678900 & 6504 & \\
\hline & 2.231036 & -0.567540 & 106 \\
\hline & 701912 & -3.0 & \\
\hline & 77 & & \\
\hline & & & \\
\hline & 2.547312 & & \\
\hline & 3.08 & & -0. \\
\hline & 2.668209 & & \\
\hline & 28 & & \\
\hline & & & \\
\hline & -1.1 & & \\
\hline & -0.4 & & -1 . \\
\hline & 92 & & \\
\hline & 1.6 & & \\
\hline & & & \\
\hline & & & \\
\hline & & & \\
\hline & & & \\
\hline & 3.2 & & -0. \\
\hline & 4.0 & & \\
\hline & 2.45 & & \\
\hline & & & \\
\hline & 4 & & \\
\hline & $-2 .($ & & \\
\hline & -1.3 & & \\
\hline & 245 & & -2. \\
\hline & & & \\
\hline & & & \\
\hline & & & \\
\hline & 3 & & \\
\hline & & & -0.7 \\
\hline & & -2.2 & -3 \\
\hline & & & \\
\hline & & & \\
\hline & 73 & & \\
\hline & -0.0 & -3 & 96 \\
\hline 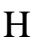 & -1.95 & -4.1 & 826 \\
\hline $\mathrm{H}$ & -2.739181 & -5.6 & 1.300643 \\
\hline & -2.181995 & -5.579367 & -0.772599 \\
\hline & & & \\
\hline & & & \\
\hline & 3.927324 & 4.553755 & -1.35 \\
\hline $\mathrm{H}$ & 3.916663 & 2.533913 & -2.036639 \\
\hline$C$ & 354495 & -0.996534 & -2.335561 \\
\hline$C^{2}$ & 6.831702 & 0.582844 & -0.574731 \\
\hline
\end{tabular}




$\begin{array}{lrrc}\mathrm{H} & 5.170149 & 1.458362 & 0.433606 \\ \mathrm{H} & 4.625095 & -3.978376 & 3.661252 \\ \mathrm{H} & -2.911959 & -6.321832 & -1.081102 \\ \mathrm{H} & 2.512402 & 5.713086 & 1.518931 \\ \mathrm{C} & 3.542881 & 5.467552 & -0.345280 \\ \mathrm{H} & 4.473503 & 4.910885 & -2.222967 \\ \mathrm{H} & 6.679946 & -1.696122 & -3.101210 \\ \mathrm{C} & 7.274988 & -0.303639 & -1.584144 \\ \mathrm{H} & 7.560101 & 1.108060 & 0.036389 \\ \mathrm{H} & 3.807889 & 6.516945 & -0.434786 \\ \mathrm{H} & 8.338451 & -0.443920 & -1.753326 \\ \mathrm{C} & -1.985978 & 0.287707 & 2.931789 \\ \mathrm{H} & -1.130597 & -0.393285 & 3.003282 \\ \mathrm{H} & -1.830224 & 1.138677 & 3.604048 \\ \mathrm{H} & -2.861546 & -0.263755 & 3.300436 \\ \mathrm{C} & -1.987373 & 5.443837 & 2.192831 \\ \mathrm{O} & -2.618689 & 3.443396 & 2.979662 \\ \mathrm{C} & -2.417094 & 4.755614 & 3.286263 \\ \mathrm{H} & -2.631941 & 5.036285 & 4.306152 \\ \mathrm{H} & -1.746756 & 6.497476 & 2.150377 \\ \mathrm{C} & -6.285089 & 0.148471 & -3.237724 \\ \mathrm{H} & -5.578901 & -0.184306 & -4.006002 \\ \mathrm{H} & -7.171191 & -0.486337 & -3.249129 \\ \mathrm{H} & -6.563806 & 1.181185 & -3.472476\end{array}$

\section{S33a}

M06-2X/6-31+G(d) SCF energy: $\quad$-2998.24513084 a.u.

M06-2X/6-31+G(d) enthalpy: $\quad-2997.430066$ a.u.

M06-2X/6-31+G(d) free energy: -2997.576158 a.u.

M06-2X/6-311++G(d,p) SCF energy in solution: -2998.30617912 a.u.

M06-2X/6-311++G(d,p) enthalpy: $\quad$-2997.491114 a.u.

M06-2X/6-311++G(d,p) free energy: $\quad-2997.637206$ a.u.

Cartesian coordinates

\begin{tabular}{|c|c|c|c|}
\hline & $X$ & Y & Z \\
\hline C & -4.023921 & 0.052539 & 1.274535 \\
\hline$C$ & -2.833001 & 0.241059 & 1.632103 \\
\hline 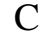 & -5.301635 & -0.226834 & 0.846087 \\
\hline & -6.313080 & 0.544329 & 0.261365 \\
\hline V & -5.201814 & 2.327881 & -1.215064 \\
\hline H & -5.664272 & 3.002668 & -1.944918 \\
\hline $\mathrm{C}$ & -3.969652 & 3.004574 & -0.6 \\
\hline$y$ & -2.812893 & 2.395845 & -0.429716 \\
\hline 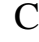 & -1.518144 & 2.507948 & 0.089129 \\
\hline $\mathrm{H}$ & -1.330384 & 2.662551 & 1.146130 \\
\hline $\mathrm{O}$ & -0.506112 & 3.076944 & -0.730431 \\
\hline C & -0.158949 & 4.371109 & -0.419397 \\
\hline $\mathrm{O}$ & -0.662132 & 4.975773 & 0.498477 \\
\hline U & -4.927831 & 1.416193 & -1.754025 \\
\hline
\end{tabular}




\begin{tabular}{|c|c|c|c|}
\hline & -6.269827 & . & \\
\hline $\mathrm{H}$ & -6.080485 & 2.604010 & \\
\hline & -7.264563 & 2.279221 & -0.474650 \\
\hline & -1.942880 & 0.452163 & -0.43728 \\
\hline & -1.199980 & 0.856764 & \\
\hline J & 653 & & \\
\hline & -4.117235 & 4.477269 & \\
\hline & -3.215899 & 4.88 & 503 \\
\hline & $-4.9 c$ & & 3541 \\
\hline & -4.3 & 5.05 & \\
\hline E & -2.9 & -1.1 & \\
\hline 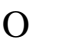 & -3.6 & -1.8 & \\
\hline & 0.22 & $-0.7 \xi$ & \\
\hline 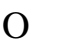 & & -0.2 & \\
\hline J & & & \\
\hline & 0.2 & -2.4 & \\
\hline & & & \\
\hline & 2.5 & -0.6 & \\
\hline & 08 & -3.2 & \\
\hline & & & \\
\hline & & & \\
\hline & 2.8 & 1.2 & \\
\hline & & & \\
\hline $\mathrm{C}$ & & -1.5 & \\
\hline & & & \\
\hline & 17 & & \\
\hline & & & \\
\hline & & & \\
\hline & & & \\
\hline $\mathrm{C}$ & & & \\
\hline & & & \\
\hline & & -0.2 & \\
\hline 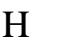 & & & \\
\hline & & & 411 \\
\hline I & & & \\
\hline 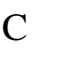 & -3.1 & -3.9 & \\
\hline $\mathrm{C}$ & -2.1 & -4.8 & 01 \\
\hline 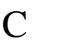 & & -4.3 & 30 \\
\hline $\mathrm{C}$ & & -4.3 & \\
\hline $\mathrm{C}$ & & -4.7 & \\
\hline & & & \\
\hline 2 & & -2.3 & \\
\hline $\mathrm{H}$ & 20 & 35 & \\
\hline $\mathrm{C}$ & & 466 & \\
\hline $\mathrm{C}$ & 4.363020 & 2.956309 & 57 \\
\hline 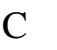 & & -1.1 & \\
\hline 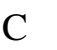 & & & \\
\hline $\mathrm{H}$ & & & \\
\hline $\mathrm{H}$ & & -3.866291 & 1.86614 \\
\hline $\mathrm{C}$ & -3.226953 & -4.777974 & 0.0327 \\
\hline 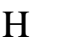 & -2.257952 & -5.547392 & -1.72540 \\
\hline
\end{tabular}




$\begin{array}{lrrr}\mathrm{H} & -0.141688 & -4.736832 & -2.608871 \\ \mathrm{H} & 2.142850 & -4.692245 & -2.063087 \\ \mathrm{H} & 3.702853 & -5.373640 & -0.357957 \\ \mathrm{C} & 3.809037 & -4.524876 & 1.613969 \\ \mathrm{H} & 3.632721 & -3.540100 & 3.528214 \\ \mathrm{C} & 3.532257 & 4.834868 & 1.382618 \\ \mathrm{C} & 4.863753 & 4.240876 & -0.539278 \\ \mathrm{H} & 4.682579 & 2.249668 & -1.278555 \\ \mathrm{C} & 6.674876 & -1.463626 & -1.424637 \\ \mathrm{C} & 7.085169 & -0.037177 & 0.477414 \\ \mathrm{H} & 5.409584 & 0.977535 & 1.318722 \\ \mathrm{H} & -4.125319 & -5.370027 & -0.115786 \\ \mathrm{H} & 4.727758 & -5.044465 & 1.869162 \\ \mathrm{H} & 3.193961 & 5.557567 & 2.120666 \\ \mathrm{C} & 4.460780 & 5.188280 & 0.430686 \\ \mathrm{H} & 5.575380 & 4.528019 & -1.308156 \\ \mathrm{H} & 7.020628 & -2.158769 & -2.185624 \\ \mathrm{C} & 7.558520 & -0.919770 & -0.521201 \\ \mathrm{H} & 7.778820 & 0.369899 & 1.207621 \\ \mathrm{H} & 4.874128 & 6.192394 & 0.412887 \\ \mathrm{H} & 8.613331 & -1.174818 & -0.563527 \\ \mathrm{C} & -2.074873 & 0.462899 & 2.898336 \\ \mathrm{H} & -2.676968 & 0.172919 & 3.765365 \\ \mathrm{H} & -1.139987 & -0.102520 & 2.903615 \\ \mathrm{H} & -1.817054 & 1.521953 & 3.001447 \\ \mathrm{C} & -7.384835 & -0.347869 & 0.018403 \\ \mathrm{O} & -5.733398 & -1.549199 & 0.942904 \\ \mathrm{C} & -6.979224 & -1.585266 & 0.456539 \\ \mathrm{H} & -8.339543 & -0.105437 & -0.427397 \\ \mathrm{H} & -7.471627 & -2.547092 & 0.484877 \\ \mathrm{C} & 0.880458 & 4.887911 & -1.376842 \\ \mathrm{H} & 1.188610 & 5.886810 & -1.068210 \\ \mathrm{H} & 1.746334 & 4.220372 & -1.402331 \\ \mathrm{H} & 0.460566 & 4.926859 & -2.388219\end{array}$

\section{S33b}

M06-2X/6-31+G(d) SCF energy: $\quad-2998.30042414$ a.u.

M06-2X/6-31+G(d) enthalpy: $\quad-2997.484024$ a.u.

M06-2X/6-31+G(d) free energy: -2997.627839 a.u.

M06-2X/6-311++G(d,p) SCF energy in solution: -2998.35911364 a.u.

M06-2X/6-311++G(d,p) enthalpy: $\quad-2997.542713$ a.u.

M06-2X/6-311++G(d,p) free energy: $\quad-2997.686529$ a.u.

Cartesian coordinates

\begin{tabular}{lrrr} 
ATOM X & \multicolumn{2}{l}{ Y Z } \\
C & -3.489734 & -0.774960 & -0.555115 \\
C & -2.687568 & 0.129563 & -1.152012 \\
C & -4.541355 & -1.623974 & -1.081170 \\
C & -5.751928 & -1.970953 & -0.528379
\end{tabular}




\begin{tabular}{|c|c|c|c|}
\hline C & -5.640393 & -0.154060 & \\
\hline & .266480 & 0.291434 & 1602 \\
\hline & 49359 & -0.381874 & 1733 \\
\hline & .242749 & -0.754876 & \\
\hline & .834809 & & \\
\hline & -1.321536 & -1.699350 & $94 / 1$ \\
\hline & 7884 & 4317 & 2 \\
\hline & -0.436675 & -2.136989 & \\
\hline & 493 & -2.8 & \\
\hline $\mathrm{H}$ & 569 & & \\
\hline C & -6.3 & & \\
\hline & -6.251850 & -2.2 & 0075 \\
\hline & -7.3 & -1.2 & \\
\hline $\mathrm{Rh}$ & -1.8 & 744 & \\
\hline & -1.2 & 1.7 & \\
\hline $\mathrm{O}$ & -0.8 & & \\
\hline $\mathrm{C}$ & -4.1 & & \\
\hline I & -3.1 & & \\
\hline 1 & -4.8 & & \\
\hline 1 & -4.5 & & \\
\hline E & -2.6 & & 48 \\
\hline 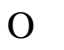 & -3.2 & & \\
\hline$P$ & 0.3 & & \\
\hline D & 0.8 & & \\
\hline O & 1.2 & & \\
\hline $\mathrm{N}$ & & & \\
\hline$C$ & $0.8 ?$ & -1.4 & \\
\hline 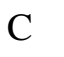 & 2.5 & -0.2 & \\
\hline $\mathrm{C}$ & 0.3 & & \\
\hline $\mathrm{C}$ & 2.5 & & -0 \\
\hline $\mathrm{C}$ & -0.11 & $-2 .($ & \\
\hline 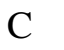 & $1.7 \xi$ & -2.0 & -0. \\
\hline $\mathrm{G}$ & & -1.3 & \\
\hline $\mathrm{C}$ & & & \\
\hline 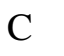 & 0.2 & & -2. \\
\hline $\mathrm{C}$ & 0.1 & & 62 \\
\hline $\mathrm{C}$ & & & \\
\hline $\mathrm{C}$ & 3.37 & & -1.4 \\
\hline $\mathrm{H}$ & -0.77 & & -2.8 \\
\hline 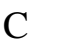 & -0.1 & & \\
\hline $\mathrm{C}$ & 1.6 & -3.5 & -0.6 \\
\hline $\mathrm{C}$ & 4.2 & -1.6 & 14 \\
\hline $\mathrm{H}$ & 3.14 & & \\
\hline $\mathrm{C}$ & 4.792194 & 850 & \\
\hline $\mathrm{H}$ & -0.11 & 632 & -3.0 \\
\hline G & & & \\
\hline a & -0.5 & & \\
\hline 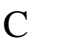 & 0.90 & 4.8 & 0.9 \\
\hline $\mathrm{C}$ & & 4.322261 & 1.269089 \\
\hline 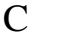 & 4.3 & 3.755993 & 0.445098 \\
\hline G & 4.750477 & 2.259463 & -1.41767 \\
\hline
\end{tabular}




$\begin{array}{lrrr}\mathrm{H} & 2.969271 & 1.356263 & -2.239362 \\ \mathrm{H} & -0.848978 & -3.976731 & -2.897270 \\ \mathrm{C} & 0.739287 & -4.191908 & -1.438718 \\ \mathrm{C} & 2.495169 & -4.277771 & 0.260308 \\ \mathrm{C} & 5.218085 & -1.016844 & 0.736976 \\ \mathrm{C} & 4.725650 & -2.541763 & -1.111825 \\ \mathrm{H} & 5.525928 & 0.410784 & 2.338958 \\ \mathrm{H} & -1.480704 & 4.523808 & -3.823563 \\ \mathrm{C} & -1.199105 & 5.683428 & -2.021798 \\ \mathrm{H} & -0.712142 & 6.617489 & -0.146991 \\ \mathrm{H} & 0.443083 & 5.477005 & 1.706597 \\ \mathrm{H} & 2.580298 & 4.632260 & 2.211111 \\ \mathrm{H} & 4.792419 & 4.440893 & 1.190698 \\ \mathrm{C} & 5.260326 & 3.119189 & -0.440263 \\ \mathrm{H} & 5.419230 & 1.762535 & -2.113662 \\ \mathrm{C} & 0.662338 & -5.609767 & -1.385197 \\ \mathrm{C} & 2.384509 & -5.649890 & 0.302005 \\ \mathrm{H} & 3.188082 & -3.779982 & 0.926708 \\ \mathrm{C} & 6.591136 & -1.359225 & 0.609873 \\ \mathrm{C} & 6.067604 & -2.834212 & -1.225623 \\ \mathrm{H} & 4.021195 & -2.993373 & -1.799852 \\ \mathrm{H} & -1.790503 & 6.524837 & -2.370117 \\ \mathrm{H} & 6.329494 & 3.298161 & -0.375715 \\ \mathrm{H} & -0.061084 & -6.113863 & -2.020930 \\ \mathrm{C} & 1.472306 & -6.329376 & -0.538615 \\ \mathrm{H} & 3.000799 & -6.216152 & 0.994474 \\ \mathrm{H} & 7.307208 & -0.886806 & 1.277583 \\ \mathrm{C} & 7.011798 & -2.252744 & -0.347933 \\ \mathrm{H} & 6.403448 & -3.516664 & -2.001115 \\ \mathrm{H} & 1.403800 & -7.412620 & -0.501537 \\ \mathrm{H} & 8.064659 & -2.501957 & -0.441675 \\ \mathrm{C} & -2.617901 & 0.530140 & -2.588799 \\ \mathrm{H} & -1.587577 & 0.540059 & -2.960059 \\ \mathrm{H} & -3.204997 & -0.150381 & -3.214718 \\ \mathrm{H} & -3.006526 & 1.547787 & -2.726543 \\ \mathrm{C} & -6.400683 & -2.803502 & -1.500195 \\ \mathrm{O} & -4.413968 & -2.178561 & -2.329172 \\ \mathrm{C} & -5.552086 & -2.893526 & -2.560413 \\ \mathrm{H} & -5.599470 & -3.403871 & -3.510279 \\ \mathrm{H} & -7.376351 & -3.262674 & -1.417139 \\ \mathrm{C} & -0.017047 & -2.055719 & 4.235747 \\ \mathrm{H} & -0.884073 & -2.178405 & 4.893188 \\ \mathrm{H} & 0.716606 & -2.835295 & 4.441437 \\ \mathrm{H} & 0.417480 & -1.071964 & 4.443193\end{array}$

\section{S34a}

M06-2X/6-31+G(d) SCF energy: $\quad-1256.10234869$ a.u.

M06-2X/6-31+G(d) enthalpy: $\quad-1255.773871$ a.u.

M06-2X/6-31+G(d) free energy: -1255.863737 a.u. 
M06-2X/6-311++G(d,p) SCF energy in solution: -1256.81880611 a.u.

M06-2X/6-311++G(d,p) enthalpy: $\quad-1256.490328$ a.u.

M06-2X/6-311++G(d,p) free energy: $\quad-1256.580194$ a.u.

Cartesian coordinates

$\begin{array}{lrcc}\text { ATOM } & \text { X } & \text { Y } & \text { Z } \\ \text { C } & -1.677106 & -0.567420 & 1.235472 \\ \text { C } & -0.518532 & -0.938212 & 1.574460 \\ \text { C } & -2.938932 & -0.288200 & 0.778441 \\ \text { C } & -3.582484 & 0.878968 & 0.334752 \\ \text { C } & -1.836126 & 2.363995 & -0.825188 \\ \text { H } & -1.990039 & 3.268822 & -1.423957 \\ \text { C } & -0.484135 & 2.452973 & -0.152818 \\ \text { C } & 0.369311 & 1.444155 & -0.033000 \\ \text { C } & 1.536752 & 1.010644 & 0.596151 \\ \text { H } & 1.569483 & 0.818143 & 1.664076 \\ \text { O } & 2.780828 & 1.350782 & 0.064090 \\ \text { C } & 3.870110 & 0.714003 & 0.634001 \\ \text { O } & 3.737414 & -0.153810 & 1.463255 \\ \text { H } & -1.870144 & 1.515752 & -1.514935 \\ \text { C } & -3.011500 & 2.261953 & 0.209250 \\ \text { H } & -2.670900 & 2.608922 & 1.192042 \\ \text { H } & -3.821390 & 2.933320 & -0.089156 \\ \text { Rh } & 0.517639 & -0.671898 & -0.385008 \\ \text { C } & 1.438852 & -0.181018 & -2.030754 \\ \text { O } & 1.967191 & 0.117443 & -2.995212 \\ \text { C } & -0.130444 & 3.803487 & 0.439854 \\ \text { H } & 0.810474 & 3.777908 & 0.994028 \\ \text { H } & -0.920832 & 4.156802 & 1.114686 \\ \text { H } & -0.036960 & 4.549652 & -0.359762 \\ \text { C } & -0.946608 & -1.688563 & -1.424894 \\ \text { O } & -1.770041 & -2.234578 & -1.992217 \\ \text { C } & 0.204550 & -1.367782 & 2.811207 \\ \text { H } & -0.332474 & -1.050118 & 3.710179 \\ \text { H } & 0.266315 & -2.462804 & 2.819510 \\ \text { H } & 1.227999 & -0.986604 & 2.843270 \\ \text { C } & -4.873005 & 0.474848 & -0.071750 \\ \text { O } & -3.806295 & -1.371456 & 0.622875 \\ \text { C } & -4.949668 & -0.884241 & 0.136554 \\ \text { H } & -5.653240 & 1.106623 & -0.473137 \\ \text { H } & -5.745578 & -1.600834 & -0.012815 \\ \text { C } & 5.156716 & 1.255007 & 0.079361 \\ \text { H } & 5.993016 & 0.684144 & 0.483153 \\ \text { H } & 5.154510 & 1.201115 & -1.014075 \\ \text { H } & 5.264320 & 2.310442 & 0.352473 \\ \text { C } & 1.763292 & -2.288861 & 0.124114 \\ \text { O } & 2.480333 & -3.124668 & 0.405772\end{array}$

S34b 
M06-2X/6-31+G(d) SCF energy: $\quad-1256.15073215$ a.u.

M06-2X/6-31+G(d) enthalpy: $\quad-1255.820822$ a.u.

M06-2X/6-31+G(d) free energy: -1255.907560 a.u.

M06-2X/6-311++G(d,p) SCF energy in solution: -1256.86758309 a.u.

M06-2X/6-311++G(d,p) enthalpy: $\quad-1256.537673$ a.u.

M06-2X/6-311++G(d,p) free energy: $\quad-1256.624411$ a.u.

Cartesian coordinates

$\begin{array}{lccc}\text { ATOM } & \text { X } & \text { Y } & \text { Z } \\ \text { C } & 1.456631 & -0.231975 & -0.445665 \\ \text { C } & 0.776619 & -1.372990 & -0.675174 \\ \text { C } & 2.851110 & 0.104858 & -0.636040 \\ \text { C } & 3.676050 & 0.909652 & 0.118478 \\ \text { C } & 2.075503 & 0.997242 & 2.116469 \\ \text { H } & 2.043428 & 1.363477 & 3.146488 \\ \text { C } & 0.786657 & 1.356277 & 1.418522 \\ \text { C } & 0.532779 & 0.798384 & 0.168106 \\ \text { C } & -0.609702 & 1.006759 & -0.706054 \\ \text { H } & -0.481901 & 0.768150 & -1.755971 \\ \text { O } & -1.493451 & 2.030990 & -0.483091 \\ \text { C } & -2.502977 & 2.194448 & -1.451913 \\ \text { O } & -2.645164 & 1.396985 & -2.337897 \\ \text { H } & 2.201005 & -0.090944 & 2.152069 \\ \text { C } & 3.323113 & 1.605983 & 1.404761 \\ \text { H } & 3.166110 & 2.681907 & 1.247464 \\ \text { H } & 4.170023 & 1.525784 & 2.096358 \\ \text { Rh } & -1.013955 & -0.929231 & 0.296667 \\ \text { C } & -2.606960 & -0.178633 & 1.399983 \\ \text { O } & -3.480512 & 0.263671 & 1.978297 \\ \text { C } & -0.013214 & 2.441887 & 2.070674 \\ \text { H } & -0.925768 & 2.707119 & 1.544336 \\ \text { H } & 0.618379 & 3.339659 & 2.151071 \\ \text { H } & -0.249817 & 2.157264 & 3.103940 \\ \text { C } & -0.695776 & -2.551553 & 1.523639 \\ \text { O } & -0.516154 & -3.499924 & 2.125579 \\ \text { C } & 1.174177 & -2.642832 & -1.344028 \\ \text { H } & 0.613211 & -2.793252 & -2.275691 \\ \text { H } & 2.239184 & -2.619825 & -1.598792 \\ \text { H } & 0.981032 & -3.516760 & -0.710803 \\ \text { C } & 4.963300 & 0.840271 & -0.507226 \\ \text { O } & 3.553776 & -0.461818 & -1.666081 \\ \text { C } & 4.830566 & 0.003500 & -1.573812 \\ \text { H } & 5.511529 & -0.344277 & -2.335627 \\ \text { H } & 5.868026 & 1.342723 & -0.193652 \\ \text { C } & -3.298351 & 3.434155 & -1.175604 \\ \text { H } & -2.646980 & 4.313859 & -1.214785 \\ \text { H } & -4.089800 & 3.526105 & -1.919494 \\ \text { H } & -3.733126 & 3.391114 & -0.171281 \\ \text { C } & -2.050548 & -1.608866 & -1.197331 \\ \text { O } & -2.627298 & -2.051916 & -2.074599\end{array}$




\section{TS1}

M06-2X/6-31+G(d) SCF energy: $\quad-3457.81472612$ a.u.

M06-2X/6-31+G(d) enthalpy: $\quad-3457.008709$ a.u.

M06-2X/6-31+G(d) free energy: -3457.157842 a.u.

M06-2X/6-311++G(d,p) SCF energy in solution: -3457.88127795 a.u.

M06-2X/6-311++G(d,p) enthalpy: $\quad-3457.075261$ a.u.

M06-2X/6-311++G(d,p) free energy: $\quad-3457.224394$ a.u.

Imaginary frequency: $\quad-208.5525 \mathrm{~cm}-1$

Cartesian coordinates

$\begin{array}{lccc}\text { ATOM X } & \text { Y } & \text { Z } \\ \text { C } & -1.436619 & 1.836690 & 1.401503 \\ \text { C } & -1.763623 & 0.847657 & 2.134203 \\ \text { C } & -0.853644 & 3.147952 & 1.422047 \\ \text { C } & -0.406384 & 4.114579 & 0.546342 \\ \text { C } & -0.958765 & 2.960568 & -1.696404 \\ \text { H } & -0.890350 & 3.148049 & -2.775144 \\ \text { C } & -2.405280 & 2.787830 & -1.304924 \\ \text { C } & -2.780614 & 1.868317 & -0.417793 \\ \text { C } & -3.869795 & 1.144615 & 0.081094 \\ \text { H } & -4.247810 & 1.262956 & 1.091170 \\ \text { O } & -4.835962 & 0.753539 & -0.848383 \\ \text { C } & -5.906636 & 0.053114 & -0.363864 \\ \text { O } & -5.983873 & -0.347357 & 0.770379 \\ \text { H } & -0.402699 & 2.042841 & -1.488883 \\ \text { C } & -0.302901 & 4.140966 & -0.955276 \\ \text { H } & -0.728554 & 5.087848 & -1.312345 \\ \text { H } & 0.758555 & 4.170475 & -1.236209 \\ \text { Rh } & -2.039066 & -0.086425 & 0.223943 \\ \text { C } & -2.990288 & -1.621056 & 1.112134 \\ \text { O } & -3.648101 & -2.353427 & 1.687840 \\ \text { C } & -3.419976 & 3.760904 & -1.860854 \\ \text { H } & -4.435189 & 3.539010 & -1.525711 \\ \text { H } & -3.172550 & 4.787758 & -1.563299 \\ \text { H } & -3.402241 & 3.729576 & -2.957559 \\ \text { C } & -2.167215 & -0.617207 & -1.683792 \\ \text { O } & -2.282745 & -0.887909 & -2.786791 \\ \text { P } & 0.331967 & -0.958275 & 0.162507 \\ \text { O } & 1.499522 & -0.494810 & 1.212692 \\ \text { O } & 0.850513 & -0.298819 & -1.271243 \\ \text { N } & 0.640614 & -2.618073 & 0.235614 \\ \text { C } & 2.154688 & 0.738501 & 1.163451 \\ \text { C } & 2.169872 & -0.463160 & -1.714427 \\ \text { C } & 1.797012 & -3.205501 & 0.884852 \\ \text { C } & -0.380210 & -3.548423 & -0.170154 \\ \text { C } & 2.040946 & 1.529605 & 2.327305 \\ \text { C } & 2.941624 & 1.099801 & 0.079475 \\ \text { C } & 3.210470 & 0.156322 & -1.043911 \\ \text { C } & 2.351535 & -1.248687 & -2.872537\end{array}$




\begin{tabular}{|c|c|c|c|}
\hline & 5807 & 455 & \\
\hline & 1.648415 & -3.740648 & 1662 \\
\hline & 271 & 416 & \\
\hline & 647314 & -3.735753 & \\
\hline & 1.459350 & & \\
\hline & .680683 & & \\
\hline & .501923 & 2.431283 & 0 . \\
\hline & 4.553453 & -0.1 & \\
\hline & .477929 & & \\
\hline & 965 & -1.4 & \\
\hline & 3.11 & -2.8 & \\
\hline & 4.15 & -3.72 & \\
\hline & 2.801575 & -4.2 & 47 \\
\hline & 0.35 & & \\
\hline & 05 & -4 & \\
\hline & 2 & & \\
\hline & -1.6 & & \\
\hline & -0.0 & & 076 \\
\hline & 2.62 & & \\
\hline & 3.35 & & \\
\hline & 4.12 & & 50 \\
\hline & 2 & -0.9 & \\
\hline & & & \\
\hline & 3.7 & & \\
\hline & 5.12 & -3.7 & \\
\hline & 4.04 & & \\
\hline & 2.70 & -4.6 & \\
\hline & & & \\
\hline & 37 & & 09 \\
\hline I & -2.7 & & 703 \\
\hline & -2.4 & & -0. \\
\hline & -1.86 & -4.7 & 661 \\
\hline & & & \\
\hline & 4.66 & & \\
\hline & 4.20 & & \\
\hline & $6.0^{\circ}$ & -1.2 & -3 . \\
\hline & $6.9^{\prime}$ & -0.0 & -1 . \\
\hline & & & \\
\hline & 4.91 & & \\
\hline & -3.2 & & 256 \\
\hline & & & \\
\hline & 4.60 & & \\
\hline 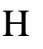 & 5.1 & & 69 \\
\hline $\mathrm{H}$ & 6.200147 & 905 & 188 \\
\hline$C$ & 7.163454 & -0.8 & 1670 \\
\hline & & & \\
\hline & & & \\
\hline 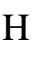 & & -1.086396 & 2641 \\
\hline $\mathrm{C}$ & 1.900139 & & 5001 \\
\hline $\mathrm{H}$ & -1.279497 & -0.592537 & 3.635402 \\
\hline $\mathrm{H}$ & -1.590883 & 1.058734 & 4.233624 \\
\hline
\end{tabular}




$\begin{array}{lrrl}\mathrm{H} & -2.936789 & 0.017060 & 3.720938 \\ \mathrm{C} & 0.024151 & 5.207917 & 1.371544 \\ \mathrm{O} & -0.710159 & 3.593054 & 2.723734 \\ \mathrm{C} & -0.182317 & 4.841373 & 2.662957 \\ \mathrm{H} & -0.029841 & 5.332572 & 3.612337 \\ \mathrm{H} & 0.437246 & 6.144258 & 1.022260 \\ \mathrm{C} & -6.950518 & -0.172103 & -1.439264 \\ \mathrm{H} & -6.520724 & -0.725491 & -2.277687 \\ \mathrm{H} & -7.779826 & -0.726772 & -1.003482 \\ \mathrm{Cl} & -7.586214 & 1.385323 & -2.087924\end{array}$

\section{TS1-rot}

M06-2X/6-31+G(d) SCF energy: $\quad-3457.81623555$ a.u.

M06-2X/6-31+G(d) enthalpy: $\quad-3457.010178$ a.u.

M06-2X/6-31+G(d) free energy: -3457.158095 a.u.

M06-2X/6-311++G(d,p) SCF energy in solution: -3457.87985013 a.u.

M06-2X/6-311++G(d,p) enthalpy: $\quad-3457.073793$ a.u.

M06-2X/6-311++G(d,p) free energy: $\quad-3457.221710$ a.u.

Imaginary frequency: $\quad-201.3951 \mathrm{~cm}-1$

Cartesian coordinates

\begin{tabular}{lccc} 
ATOM & X & Y & \multicolumn{2}{l}{ Z } \\
C & -0.844205 & -1.193628 & 2.104503 \\
C & -0.738122 & -2.062882 & 1.171283 \\
C & -0.155044 & -0.536035 & 3.155259 \\
C & -0.368472 & 0.467684 & 4.089729 \\
C & -2.449924 & 1.647309 & 3.125900 \\
H & -3.109079 & 2.488769 & 3.371996 \\
C & -3.292700 & 0.443736 & 2.795771 \\
C & -2.938561 & -0.464909 & 1.884405 \\
C & -3.478973 & -1.556953 & 1.173786 \\
H & -3.202122 & -2.584251 & 1.383888 \\
O & -4.788738 & -1.425863 & 0.699113 \\
C & -5.269419 & -2.479100 & -0.031327 \\
O & -4.627715 & -3.470181 & -0.276135 \\
H & -1.838198 & 1.948904 & 2.270095 \\
C & -1.542382 & 1.374298 & 4.343759 \\
H & -2.146301 & 0.973579 & 5.169900 \\
H & -1.148948 & 2.330896 & 4.708128 \\
Rh & -1.878987 & -0.678488 & 0.000727 \\
C & -1.972962 & -1.902827 & -1.588910 \\
O & -2.152429 & -2.624497 & -2.454158 \\
C & -4.537661 & 0.233700 & 3.628654 \\
H & -5.031498 & -0.715245 & 3.410514 \\
H & -4.300007 & 0.262734 & 4.700023 \\
H & -5.252032 & 1.045826 & 3.439379 \\
C & -3.151432 & 0.690032 & -0.701131 \\
O & -4.033248 & 1.348898 & -0.997119 \\
P & 0.239035 & 0.592527 & -0.679876
\end{tabular}




\begin{tabular}{|c|c|c|c|}
\hline D & 1.184441 & 0.001236 & \\
\hline & 1.259247 & 0.462030 & 0500021 \\
\hline & 0.162702 & 222353 & -1.098713 \\
\hline & 1.733556 & -1.277491 & -1.802641 \\
\hline & 2.658638 & 0.526700 & \\
\hline & -0.996171 & 2.760440 & -1.757369 \\
\hline & 1.279363 & 3.133963 & -0.986190 \\
\hline & 1.226020 & -2.273362 & -2.666291 \\
\hline & 2.768978 & -1.509758 & -0.9 \\
\hline & 3.408096 & -0.3 & \\
\hline & 3.233130 & 1.5 & \\
\hline & -1.370106 & 2.287172 & -3.021087 \\
\hline & -1.731574 & 3.785030 & 0479 \\
\hline & 1.170803 & 4.21 & -0.0 \\
\hline & 2.43 & 2.9 & -1 . \\
\hline & 0.47 & -1.9 & \\
\hline & 1.7 & -3.5 & \\
\hline & 3.204937 & -2.8 & -0. \\
\hline & 4.837695 & -0.1 & -0.2 \\
\hline & 2.57 & 2.1 & \\
\hline & $4.5 \mathrm{C}$ & 1.6 & \\
\hline & -0.7 & 1.5 & \\
\hline & -2.5 & 2.7 & \\
\hline & -2.8 & 4.2 & \\
\hline & -1.3 & 4.3 & \\
\hline & -0.06 & 4.5 & \\
\hline & 2.29 & 5.0 & \\
\hline & 3.52 & 3.8 & \\
\hline & 2.4 & 2.1 & \\
\hline & 1.3 & -4.3 & \\
\hline & 2.682918 & -3.8 & -1. \\
\hline & 4.111737 & -3.27 & \\
\hline & 5.430199 & 0.842495 & \\
\hline & 5.6 & -0.8 & \\
\hline & 5.0 & 2.4 & \\
\hline & -2.7 & 2.4 & -4 \\
\hline & -3.259116 & 3.790359 & -3.0 \\
\hline & -3.461445 & 5.053793 & -1.2 \\
\hline & -2.139450 & 4.805088 & \\
\hline & 0.050933 & 5.10 & \\
\hline & 2.22 & 5.88 & 0.7 \\
\hline & 3.45 & 4.85 & -0.6 \\
\hline & 4.422275 & 3.652801 & 2988 \\
\hline & 3.125714 & -5.239359 & -1.451078 \\
\hline & 4.504178 & -4.584470 & 8275 \\
\hline & 4.489425 & -2.524644 & \\
\hline & & 1.041 & \\
\hline & 7.053942 & -0.661633 & -1.115415 \\
\hline & 5.271424 & -1.628937 & -1.766060 \\
\hline & -4.142459 & 4.192799 & -3.512544 \\
\hline & 4.301238 & 5.517791 & -0.524382 \\
\hline
\end{tabular}




$\begin{array}{lrrc}\mathrm{H} & 2.731701 & -5.995958 & -2.124822 \\ \mathrm{C} & 4.021107 & -5.579588 & -0.463881 \\ \mathrm{H} & 5.191553 & -4.859652 & 1.213125 \\ \mathrm{H} & 7.266506 & 1.803196 & 1.223039 \\ \mathrm{C} & 7.637819 & 0.302842 & -0.262272 \\ \mathrm{H} & 7.681837 & -1.223484 & -1.800999 \\ \mathrm{H} & 4.349181 & -6.608719 & -0.351844 \\ \mathrm{H} & 8.711003 & 0.467677 & -0.283583 \\ \mathrm{C} & -0.299532 & -3.444582 & 0.859988 \\ \mathrm{H} & 0.314241 & -3.466096 & -0.044940 \\ \mathrm{H} & 0.284270 & -3.850687 & 1.692884 \\ \mathrm{H} & -1.163066 & -4.099115 & 0.686329 \\ \mathrm{C} & 0.851734 & 0.572150 & 4.827135 \\ \mathrm{O} & 1.140126 & -1.015602 & 3.289543 \\ \mathrm{C} & 1.712815 & -0.349301 & 4.311047 \\ \mathrm{H} & 2.723270 & -0.640190 & 4.557102 \\ \mathrm{H} & 1.048409 & 1.258146 & 5.639709 \\ \mathrm{C} & -6.692961 & -2.225383 & -0.482390 \\ \mathrm{H} & -7.113267 & -3.155314 & -0.861537 \\ \mathrm{H} & -7.300529 & -1.822999 & 0.329433 \\ \mathrm{Cl} & -6.712184 & -1.015676 & -1.825410\end{array}$

\section{TS1b}

M06-2X/6-31+G(d) SCF energy: $\quad-3457.81469912$ a.u.

M06-2X/6-31+G(d) enthalpy: $\quad-3457.008687$ a.u.

M06-2X/6-31+G(d) free energy: -3457.154607 a.u.

M06-2X/6-311++G(d,p) SCF energy in solution: -3457.87793246 a.u.

M06-2X/6-311++G(d,p) enthalpy: $\quad-3457.071920$ a.u.

M06-2X/6-311++G(d,p) free energy: $\quad-3457.217840$ a.u.

Imaginary frequency: $\quad-199.5581 \mathrm{~cm}-1$

Cartesian coordinates

\begin{tabular}{lrrr} 
ATOM & X & Y & \multicolumn{2}{l}{ Z } \\
C & 3.653533 & 1.773625 & 0.525125 \\
C & 4.271362 & 0.686771 & 0.236313 \\
C & 3.655118 & 3.169789 & 0.321107 \\
C & 2.928767 & 4.284333 & 0.722906 \\
C & 0.684659 & 3.225679 & 1.326692 \\
H & -0.300642 & 3.548877 & 1.678852 \\
C & 1.088142 & 1.985064 & 2.073556 \\
C & 1.821522 & 0.995942 & 1.554766 \\
C & 2.269162 & -0.292809 & 1.915047 \\
H & 3.284350 & -0.464818 & 2.261011 \\
O & 1.341555 & -1.145266 & 2.531199 \\
C & 1.789653 & -2.384346 & 2.843535 \\
O & 2.876819 & -2.812262 & 2.531786 \\
H & 0.601014 & 3.034121 & 0.254538 \\
C & 1.689068 & 4.380323 & 1.564375 \\
H & 1.952942 & 4.432862 & 2.629331
\end{tabular}




\begin{tabular}{|c|c|c|c|}
\hline & 197996 & 1123 & \\
\hline & 353753 & -0.154589 & 7709 \\
\hline & 3.216055 & 769 & \\
\hline & 3900125 & -2.857961 & 140 \\
\hline & 004 & & \\
\hline & 1.121880 & & \\
\hline & 0.99 & 2.8 & 861 \\
\hline & -0.400981 & 1.8 & 3.6 \\
\hline & 68 & & \\
\hline & & & \\
\hline & 037 & & \\
\hline & 5.671592 & -0.7 & \\
\hline & 3.5 & & \\
\hline 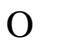 & & & \\
\hline & & & \\
\hline & & & \\
\hline & & & \\
\hline & & -3.2 & \\
\hline & & & \\
\hline & & & \\
\hline & & -0.7 & \\
\hline & & & \\
\hline & & & \\
\hline & & & \\
\hline & & & \\
\hline & & & \\
\hline & 1 & & \\
\hline & 1 & & \\
\hline & -0. & & \\
\hline & -2.1 & & \\
\hline & & & \\
\hline & & & \\
\hline & & & \\
\hline & & & \\
\hline & & & 69 \\
\hline C & -2.7 & & \\
\hline & & & \\
\hline 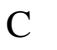 & -0.71 & & 714 \\
\hline $\mathrm{C}$ & -2.3 & & \\
\hline & & & \\
\hline $\mathrm{H}$ & -2.4 & & \\
\hline 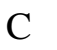 & -4.3 & -0.9 & \\
\hline $\mathrm{H}$ & & & 226 \\
\hline $\mathrm{C}$ & 369 & -3.4 & \\
\hline C & & & \\
\hline 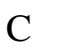 & & & \\
\hline G & & & \\
\hline $\mathrm{C}$ & -3.268872 & -4.5 & -0.1 \\
\hline $\mathrm{C}$ & -4.058000 & 174 & 5840 \\
\hline $\mathrm{H}$ & -2.5 & 006 & 100 \\
\hline 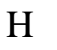 & -0.218427 & 4.419670 & -3.083848 \\
\hline
\end{tabular}




$\begin{array}{lrrr}\mathrm{C} & -1.621133 & 4.203370 & -1.444725 \\ \mathrm{C} & -3.143112 & 3.806360 & 0.430322 \\ \mathrm{C} & -5.087394 & -0.004893 & 0.830069 \\ \mathrm{C} & -5.104328 & 1.719193 & -0.905560 \\ \mathrm{H} & -4.935909 & -1.569576 & 2.322547 \\ \mathrm{H} & 2.437992 & -3.147743 & -4.625583 \\ \mathrm{C} & 2.485167 & -4.675928 & -3.097856 \\ \mathrm{H} & 2.290390 & -6.041797 & -1.449568 \\ \mathrm{H} & 1.030542 & -5.581469 & 0.578117 \\ \mathrm{H} & -1.188495 & -5.325349 & 1.318197 \\ \mathrm{H} & -3.456721 & -5.376119 & 0.502936 \\ \mathrm{C} & -4.310357 & -3.946639 & -0.857982 \\ \mathrm{H} & -4.862167 & -2.424273 & -2.286858 \\ \mathrm{C} & -1.833596 & 5.599115 & -1.277776 \\ \mathrm{C} & -3.308564 & 5.166370 & 0.581526 \\ \mathrm{H} & -3.646457 & 3.129136 & 1.109531 \\ \mathrm{C} & -6.507550 & 0.033867 & 0.835022 \\ \mathrm{C} & -6.482651 & 1.716177 & -0.892862 \\ \mathrm{H} & -4.580386 & 2.361629 & -1.603213 \\ \mathrm{H} & 3.233691 & -5.278717 & -3.603120 \\ \mathrm{H} & -5.313226 & -4.354929 & -0.775811 \\ \mathrm{H} & -1.317483 & 6.285100 & -1.944881 \\ \mathrm{C} & -2.662423 & 6.075802 & -0.288782 \\ \mathrm{H} & -3.943919 & 5.543670 & 1.377742 \\ \mathrm{H} & -7.041435 & -0.631870 & 1.508435 \\ \mathrm{C} & -7.196160 & 0.879013 & -0.004355 \\ \mathrm{H} & -7.026195 & 2.361346 & -1.577207 \\ \mathrm{H} & -2.815508 & 7.144137 & -0.167635 \\ \mathrm{H} & -8.281993 & 0.895363 & 0.002508 \\ \mathrm{H} & 1.310389 & -3.907033 & 4.242976 \\ \mathrm{H} & 0.237106 & -3.829810 & 2.827662 \\ \mathrm{Cl} & -0.455503 & -2.340132 & 4.538170\end{array}$

\section{TS1b-rot}

M06-2X/6-31+G(d) SCF energy: $\quad-3457.81433560$ a.u.

M06-2X/6-31+G(d) enthalpy: $\quad-3457.008262$ a.u.

M06-2X/6-31+G(d) free energy: -3457.155343 a.u.

M06-2X/6-311++G(d,p) SCF energy in solution: -3457.87758378 a.u.

M06-2X/6-311++G(d,p) enthalpy: $\quad-3457.071510$ a.u.

M06-2X/6-311++G(d,p) free energy: $\quad-3457.218591$ a.u.

Imaginary frequency: $\quad-205.5083 \mathrm{~cm}-1$

Cartesian coordinates

$\begin{array}{lrrrr}\text { ATOM } & \text { X } & \text { Y } & \text { Z } \\ \text { C } & 2.995288 & 2.753496 & -0.084662 \\ \text { C } & 3.876795 & 1.879140 & -0.409342 \\ \text { C } & 2.521833 & 4.050618 & -0.383994 \\ \text { C } & 1.553683 & 4.939733 & 0.065922 \\ \text { C } & -0.112156 & 3.352583 & 1.178919\end{array}$




\begin{tabular}{|c|c|c|c|}
\hline 1 & -1.074238 & 409543 & \\
\hline & 0.788497 & 2.404301 & \\
\hline & 705682 & 624163 & 40333 \\
\hline & .597426 & & \\
\hline & .656773 & & \\
\hline & 2.130822 & -0.404594 & 2.572720 \\
\hline & 052 & 767 & \\
\hline J & 4.159657 & 516 & 2.4 \\
\hline & 30 & & \\
\hline & & & \\
\hline $\mathrm{H}$ & & & \\
\hline $\mathrm{H}$ & 700 & & \\
\hline NII & 2.271132 & & \\
\hline f & & & \\
\hline 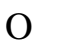 & & -1. & \\
\hline 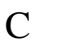 & & & \\
\hline $\mathrm{H}$ & & & \\
\hline $\mathrm{H}$ & 10 & & \\
\hline $\mathrm{H}$ & 97 & & \\
\hline & & & \\
\hline $\mathrm{H}$ & & & \\
\hline & & & \\
\hline $\mathrm{H}$ & & & \\
\hline $\mathrm{C}$ & & & \\
\hline $\mathrm{O}$ & & & \\
\hline$C$ & & & \\
\hline $\mathrm{H}$ & & & \\
\hline 11 & & & \\
\hline 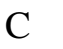 & & 88 & \\
\hline $\mathrm{C}$ & & & \\
\hline $\mathrm{O}$ & & & \\
\hline 1 & 2 & & \\
\hline $\mathrm{O}$ & & & \\
\hline $\mathrm{O}$ & & & \\
\hline $\mathrm{N}$ & & & \\
\hline $\mathrm{C}$ & 6 & & \\
\hline $\mathrm{C}$ & & & \\
\hline $\mathrm{C}$ & -0.9 & -2.8 & -1 . \\
\hline $\mathrm{C}$ & & -3.3 & \\
\hline $\mathrm{C}$ & & & \\
\hline $\mathrm{C}$ & & & \\
\hline $\mathrm{C}$ & -2. & -0. & \\
\hline $\mathrm{C}$ & & & \\
\hline $\mathrm{C}$ & -2.2 & 78 & -1 . \\
\hline $\mathrm{C}$ & -0.6 & -3.0 & -3 . \\
\hline $\mathrm{C}$ & & & \\
\hline $\mathrm{C}$ & & & \\
\hline $\mathrm{H}$ & -0.8 & 701 & -3.0 \\
\hline $\mathrm{C}$ & & & \\
\hline $\mathrm{C}$ & -3.3 & 250 & -0.1 \\
\hline $\mathrm{C}$ & -4.341671 & -0.648922 & 0.69795 \\
\hline
\end{tabular}




$\begin{array}{lrrr}\mathrm{H} & -1.386585 & -2.277056 & 2.681171 \\ \mathrm{C} & -3.518184 & -2.267243 & 2.369298 \\ \mathrm{H} & -2.523726 & -3.046930 & -0.298529 \\ \mathrm{C} & -3.251796 & -3.607415 & -2.249107 \\ \mathrm{C} & -1.633233 & -3.506855 & -4.035559 \\ \mathrm{C} & 0.745485 & -2.977528 & -3.622167 \\ \mathrm{C} & 1.874510 & -3.262590 & -2.932185 \\ \mathrm{C} & 2.992161 & -4.608417 & -1.176500 \\ \mathrm{C} & 2.175786 & -4.701467 & 1.094021 \\ \mathrm{H} & 0.432443 & -3.492315 & 1.503863 \\ \mathrm{H} & -2.086980 & 3.971095 & -2.856105 \\ \mathrm{C} & -3.133015 & 3.367602 & -1.055546 \\ \mathrm{C} & -4.201532 & 2.562198 & 0.994269 \\ \mathrm{C} & -4.597130 & -1.707847 & 1.634160 \\ \mathrm{C} & -5.433791 & -0.171954 & -0.079436 \\ \mathrm{H} & -3.723134 & -3.050228 & 3.094368 \\ \mathrm{H} & -4.259056 & -3.809782 & -1.897823 \\ \mathrm{C} & -2.930636 & -3.756855 & -3.600770 \\ \mathrm{H} & -1.373935 & -3.650850 & -5.081174 \\ \mathrm{H} & 0.851309 & -2.768428 & -4.685183 \\ \mathrm{H} & 2.810956 & -3.270764 & -3.487540 \\ \mathrm{H} & 3.703444 & -4.926880 & -1.933834 \\ \mathrm{C} & 3.096541 & -5.100566 & 0.119887 \\ \mathrm{H} & 2.235853 & -5.104928 & 2.100966 \\ \mathrm{C} & -3.803086 & 4.608499 & -0.877039 \\ \mathrm{C} & -4.817923 & 3.784943 & 1.150926 \\ \mathrm{H} & -4.355672 & 1.787931 & 1.736217 \\ \mathrm{C} & -5.921898 & -2.196920 & 1.794921 \\ \mathrm{C} & -6.702818 & -0.682490 & 0.086593 \\ \mathrm{H} & -5.260216 & 0.593991 & -0.825681 \\ \mathrm{H} & -3.687084 & -4.077875 & -4.310716 \\ \mathrm{H} & 3.887384 & -5.801191 & 0.370254 \\ \mathrm{H} & -3.638502 & 5.396163 & -1.608135 \\ \mathrm{C} & -4.632663 & 4.816480 & 0.200462 \\ \mathrm{H} & -5.453813 & 3.957898 & 2.014459 \\ \mathrm{H} & -6.097915 & -2.988792 & 2.518459 \\ \mathrm{C} & -6.957499 & -1.694168 & 1.042037 \\ \mathrm{H} & -7.516215 & -0.306657 & -0.527455 \\ \mathrm{H} & -5.137268 & 5.769441 & 0.329441 \\ \mathrm{H} & -7.965006 & -2.079244 & 1.168231 \\ & 2.816923 & -3.294074 & 3.708031 \\ & 0.885625 & -2.187333 & 4.543146\end{array}$

\section{TS1c}

M06-2X/6-31+G(d) SCF energy: $\quad-3457.81218125$ a.u.

M06-2X/6-31+G(d) enthalpy: $\quad-3457.006353$ a.u.

M06-2X/6-31+G(d) free energy: -3457.153085 a.u.

M06-2X/6-311++G(d,p) SCF energy in solution: -3457.87453784 a.u. 


\begin{tabular}{|c|c|c|c|c|}
\hline \multicolumn{4}{|c|}{ M06-2X/6-311++G(d,p) enthalpy: } & \multirow{2}{*}{$\begin{array}{l}-3457.068710 \text { a.u. } \\
-3457.215442 \text { a.u. } \\
1\end{array}$} \\
\hline & inary freque & ncy: & $90.7432 \mathrm{~cm}-1$ & \\
\hline \multicolumn{5}{|c|}{ Cartesian coordinates } \\
\hline & $\mathrm{X}$ & $\mathrm{Y}$ & $\mathrm{Z}$ & \\
\hline $\mathrm{C}$ & 2.591206 & -2.496786 & -0.820348 & \\
\hline $\mathrm{C}$ & 1.385729 & -2.288336 & -0.430075 & \\
\hline $\mathrm{C}$ & 3.364425 & -3.210711 & -1.765263 & \\
\hline $\mathrm{C}$ & 4.695864 & -3.370365 & -2.128265 & \\
\hline $\mathrm{C}$ & 5.725030 & -1.221874 & -1.203006 & \\
\hline $\mathrm{H}$ & 6.704866 & -0.732201 & -1.159028 & \\
\hline $\mathrm{C}$ & 5.066301 & -1.106058 & 0.146260 & \\
\hline $\mathrm{C}$ & 3.748114 & -0.997933 & 0.336004 & \\
\hline $\mathrm{C}$ & 2.850915 & -0.592326 & 1.347860 & \\
\hline $\mathrm{H}$ & 2.223714 & -1.275113 & 1.912993 & \\
\hline $\mathrm{O}$ & 3.326695 & 0.501981 & 2.103818 & \\
\hline $\mathrm{C}$ & 2.553699 & 0.977413 & 3.118501 & \\
\hline $\mathrm{O}$ & 1.454061 & 0.570019 & 3.384928 & \\
\hline $\mathrm{H}$ & 5.139719 & -0.713314 & -1.975434 & \\
\hline $\mathrm{C}$ & 5.935198 & -2.700645 & -1.607005 & \\
\hline $\mathrm{H}$ & 6.343540 & -3.261597 & -0.755428 & \\
\hline $\mathrm{H}$ & 6.699154 & -2.751495 & -2.390784 & \\
\hline $\mathrm{Rh}$ & 1.929740 & -0.204659 & -0.560994 & \\
\hline $\mathrm{C}$ & 5.981985 & -1.228355 & 1.345339 & \\
\hline $\mathrm{H}$ & 5.435364 & -1.266424 & 2.289284 & \\
\hline $\mathrm{H}$ & 6.601432 & -2.131595 & 1.268080 & \\
\hline $\mathrm{H}$ & 6.670116 & -0.373131 & 1.376530 & \\
\hline $\mathrm{C}$ & 2.923652 & 1.507793 & -0.696199 & \\
\hline $\mathrm{O}$ & 3.667101 & 2.369845 & -0.775176 & \\
\hline $\mathrm{C}$ & 0.184495 & -3.062956 & -0.035698 & \\
\hline $\mathrm{H}$ & -0.693339 & -2.714419 & -0.584288 & \\
\hline $\mathrm{H}$ & 0.346266 & -4.125468 & -0.248724 & \\
\hline $\mathrm{H}$ & -0.031629 & -2.947782 & 1.031312 & \\
\hline $\mathrm{C}$ & 4.693500 & -4.280314 & -3.226688 & \\
\hline $\mathrm{O}$ & 2.569053 & -3.966206 & -2.621035 & \\
\hline $\mathrm{C}$ & 3.392349 & -4.613046 & -3.467944 & \\
\hline $\mathrm{H}$ & 2.916227 & -5.272874 & -4.178429 & \\
\hline $\mathrm{H}$ & 5.561508 & -4.630899 & -3.767861 & \\
\hline $\mathrm{C}$ & 3.337575 & 2.064790 & 3.838911 & \\
\hline $\mathrm{C}$ & 1.414883 & -0.210387 & -2.533980 & \\
\hline $\mathrm{O}$ & 1.151781 & -0.278893 & -3.640410 & \\
\hline $\mathrm{P}$ & -0.427539 & 0.667830 & -0.086130 & \\
\hline $\mathrm{O}$ & -1.335937 & 0.503057 & 1.291374 & \\
\hline $\mathrm{O}$ & -1.310032 & -0.234757 & -1.147224 & \\
\hline $\mathrm{N}$ & -0.686618 & 2.297753 & -0.429510 & \\
\hline $\mathrm{C}$ & -1.764686 & -0.753084 & 1.735940 & \\
\hline $\mathrm{C}$ & -2.715123 & -0.294377 & -1.185428 & \\
\hline $\mathrm{C}$ & 0.331933 & 3.283638 & -0.178156 & \\
\hline $\mathrm{C}$ & -1.967297 & 2.858275 & -0.809499 & \\
\hline $\mathrm{C}$ & -1.182554 & -1.231525 & 2.930215 & \\
\hline
\end{tabular}




\begin{tabular}{|c|c|c|c|}
\hline & 36 & 88 & \\
\hline & -3.439322 & -0.819627 & \\
\hline & .308494 & 0.136136 & \\
\hline & .778609 & 3.518516 & \\
\hline & & 4.038205 & 000 \\
\hline & -2.08 & & 655 \\
\hline & -3.053688 & 2.837198 & 876 \\
\hline & -0.450874 & -0.611674 & 3.432754 \\
\hline & 328 & -2.4 & 3.422516 \\
\hline & & & \\
\hline & -4.8 & & \\
\hline & -2.6 & & \\
\hline & -4.673739 & & -2.5 \\
\hline & 0.34 & & \\
\hline & & & \\
\hline & 1.8 & & -0.9 \\
\hline & & & \\
\hline & -0.9 & & \\
\hline & -3.3 & & \\
\hline & -4.2 & & \\
\hline & -2.9 & & \\
\hline & -1. & & \\
\hline & -2.4 & & \\
\hline & -3.9 & & \\
\hline & & & \\
\hline & -5.7 & & \\
\hline & & & \\
\hline & & & \\
\hline & & & \\
\hline & & & \\
\hline & & & 69 \\
\hline & & & \\
\hline & & & \\
\hline & & & -1. \\
\hline & & & \\
\hline & & & \\
\hline & -4.2 & -4 & \\
\hline & & & -0.1 \\
\hline & & & \\
\hline & & & \\
\hline & & & \\
\hline & & & \\
\hline & -5.4 & & -1. \\
\hline & & & 644 \\
\hline & -3.68 & 680 & 550 \\
\hline & & & 361 \\
\hline & & & \\
\hline & & & -0.4 \\
\hline & & -1.168473 & \\
\hline$\Pi$ & -3.9 & -6.36 & 2.81 \\
\hline & -8.783552 & -0.485625 & -0.51432 \\
\hline
\end{tabular}




$\begin{array}{llll}\mathrm{H} & 4.157327 & 1.603548 & 4.396916 \\ \mathrm{H} & 3.765915 & 2.750984 & 3.105933 \\ \mathrm{Cl} & 2.326995 & 2.991216 & 4.981400\end{array}$

\footnotetext{
TS1c-rot

M06-2X/6-31+G(d) SCF energy: $\quad-3457.80849319$ a.u.

M06-2X/6-31+G(d) enthalpy: $\quad-3457.002726$ a.u.

M06-2X/6-31+G(d) free energy: -3457.150236 a.u.

M06-2X/6-311++G(d,p) SCF energy in solution: -3457.87426357 a.u.

M06-2X/6-311++G(d,p) enthalpy: $\quad-3457.068496$ a.u.

M06-2X/6-311++G(d,p) free energy: $\quad-3457.216006$ a.u.

Imaginary frequency: $\quad-234.1104 \mathrm{~cm}-1$

Cartesian coordinates

$\begin{array}{llll}\text { C } & -2.981392 & 2.045119 & 1.095119\end{array}$

$\begin{array}{llll}\text { C } & -2.107910 & 1.339459 & 1.720695\end{array}$

$\begin{array}{llll}\text { C } & -3.494176 & 3.361714 & 0.989052\end{array}$

$\begin{array}{llll}\text { C } & -4.400939 & 4.080434 & 0.220768\end{array}$

$\begin{array}{llll}\text { C } & -4.588697 & 2.574326 & -1.840569\end{array}$

$\begin{array}{llll}\mathrm{H} & -5.119745 & 2.545187 & -2.799476\end{array}$

$\begin{array}{lllll}\text { C } & -4.686218 & 1.220664 & -1.188684\end{array}$

$\begin{array}{llll}\text { C } & -3.716174 & 0.689875 & -0.434154\end{array}$

$\begin{array}{llll}\text { C } & -3.321465 & -0.572169 & 0.086850\end{array}$

$\begin{array}{llll}\mathrm{H} & -3.461340 & -0.847157 & 1.126601\end{array}$

$\begin{array}{lllll}\text { O } & -3.453763 & -1.674291 & -0.799139\end{array}$

$\begin{array}{llll}\text { C } & -4.431476 & -2.562390 & -0.512997\end{array}$

$\begin{array}{llll}\text { O } & -5.200000 & -2.464892 & 0.414980\end{array}$

$\begin{array}{llll}\mathrm{H} & -3.547901 & 2.839447 & -2.050984\end{array}$

$\begin{array}{llll}\text { C } & -5.233008 & 3.669084 & -0.960633\end{array}$

$\begin{array}{llll}\mathrm{H} & -6.225024 & 3.335977 & -0.626400\end{array}$

$\begin{array}{lllll}\mathrm{H} & -5.410279 & 4.560895 & -1.572633\end{array}$

$\begin{array}{lllll}\mathrm{Rh} & -1.575188 & 0.607237 & -0.221885\end{array}$

$\begin{array}{llll}\text { C } & -6.010818 & 0.504831 & -1.335674\end{array}$

$\begin{array}{llll}\mathrm{H} & -6.113683 & -0.336696 & -0.648423\end{array}$

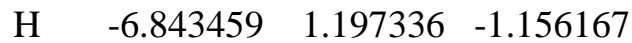

$\begin{array}{llll}\mathrm{H} & -6.124202 & 0.140071 & -2.366174\end{array}$

C $\quad-1.373516 \quad-0.120216 \quad-2.066139$

$\begin{array}{llll}\text { O } & -1.286715 & -0.540084 & -3.120887\end{array}$

$\begin{array}{llll}\text { C } & -1.690837 & 1.011067 & 3.107627\end{array}$

$\begin{array}{llll}\mathrm{H} & -0.629389 & 0.759371 & 3.164542\end{array}$

$\begin{array}{llll}\mathrm{H} & -1.897750 & 1.860368 & 3.769491\end{array}$

$\begin{array}{llll}\mathrm{H} & -2.257322 & 0.149797 & 3.482047\end{array}$

$\begin{array}{llll}\text { C } & -4.354997 & 5.418274 & 0.717448\end{array}$

$\begin{array}{llll}\text { O } & -2.889963 & 4.221473 & 1.900564\end{array}$

$\begin{array}{llll}\text { C } & -3.443543 & 5.438823 & 1.730970\end{array}$

$\begin{array}{llll}\mathrm{H} & -3.097698 & 6.212749 & 2.400424\end{array}$

$\begin{array}{llll}\mathrm{H} & -4.931769 & 6.255522 & 0.349262\end{array}$

C $\quad-4.539056 \quad-3.715807 \quad-1.498204$
} 


\begin{tabular}{|c|c|c|c|}
\hline & 0.497752 & 2.292859 & \\
\hline & 0.102850 & 7541 & \\
\hline & 0.515237 & -0.576879 & \\
\hline & 1.542327 & & 174 \\
\hline & 821 & -0.475887 & \\
\hline & 0.622493 & -2.112587 & \\
\hline & 2.048487 & 1.479640 & \\
\hline & 2.747994 & -0.574922 & \\
\hline & -0.551359 & 5297 & \\
\hline & 379 & 377 & \\
\hline & 42 & & \\
\hline & 2.9 & & \\
\hline & 3.571740 & 571 & \\
\hline & 3.228407 & -1.6 & \\
\hline & 1.3 & -2. & \\
\hline & 0.8 & -3.5 & \\
\hline & & -3.8 & \\
\hline & & -2.4 & \\
\hline & 0.9 & & \\
\hline & & & \\
\hline & & 2.9 & \\
\hline & & 0.1 & \\
\hline & & -2. & \\
\hline$y$ & & -1.7 & \\
\hline I & 55 & & \\
\hline & -2.4 & -3.3 & 05 \\
\hline & -2.0 & -4. & \\
\hline & -0.0 & -4 & \\
\hline & & -4 & \\
\hline & & -4. & \\
\hline & & $-3 .($ & \\
\hline & 2.4 & -1.5 & \\
\hline & & & \\
\hline & & & \\
\hline & & & \\
\hline & & -0.5 & \\
\hline $\mathrm{C}$ & 5.9 & 0.9 & \\
\hline 1 & & -2.6 & \\
\hline 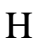 & -3.07 & -3.1 & \\
\hline $\mathrm{C}$ & -2.8 & & \\
\hline & 20 & -5. & \\
\hline & -0. & -4.9 & -1 \\
\hline $\mathrm{H}$ & & -4.8 & \\
\hline $\mathrm{H}$ & & -5.3 & \\
\hline $\mathrm{C}$ & 4.325038 & -4.0 & \\
\hline & & & \\
\hline & & & \\
\hline $\mathrm{C}$ & & 4.478507 & -1.753 \\
\hline & & 2.364313 & -2.024 \\
\hline $\mathrm{C}$ & 6.8 & -1.1 & -1.50 \\
\hline$c$ & 7.293872 & 0.745152 & -0.03160 \\
\hline
\end{tabular}




$\begin{array}{lrrr}\mathrm{H} & 5.587687 & 1.789110 & 0.704893 \\ \mathrm{H} & -3.701561 & -5.027900 & 2.195247 \\ \mathrm{H} & 5.279407 & -4.583891 & 1.888308 \\ \mathrm{H} & 2.976556 & 6.207355 & 0.724752 \\ \mathrm{C} & 4.125537 & 5.579855 & -0.972463 \\ \mathrm{H} & 5.156659 & 4.648493 & -2.636835 \\ \mathrm{H} & 7.258594 & -1.947666 & -2.115595 \\ \mathrm{C} & 7.783392 & -0.307420 & -0.839233 \\ \mathrm{H} & 7.991976 & 1.370745 & 0.517313 \\ \mathrm{H} & 4.422991 & 6.586713 & -1.250149 \\ \mathrm{H} & 8.853087 & -0.476993 & -0.919161 \\ \mathrm{H} & -4.519731 & -4.643770 & -0.925157 \\ \mathrm{H} & -5.511180 & -3.642389 & -1.991443 \\ \mathrm{Cl} & -3.280822 & -3.815295 & -2.775320\end{array}$

\section{TS2}

M06-2X/6-31+G(d) SCF energy: $\quad-3457.80926993$ a.u.

M06-2X/6-31+G(d) enthalpy: $\quad-3457.003368$ a.u.

M06-2X/6-31+G(d) free energy: -3457.152407 a.u.

M06-2X/6-311++G(d,p) SCF energy in solution: -3457.87568552 a.u.

M06-2X/6-311++G(d,p) enthalpy: $\quad-3457.069784$ a.u.

M06-2X/6-311++G(d,p) free energy: $\quad-3457.218823$ a.u.

Imaginary frequency: $\quad-197.9857 \mathrm{~cm}-1$

Cartesian coordinates

\begin{tabular}{lccc} 
ATOM & X & Y & \multicolumn{2}{l}{ Z } \\
C & -1.271402 & 1.395026 & -2.096135 \\
C & -1.649504 & 0.237194 & -2.489083 \\
C & -0.271993 & 2.386717 & -2.284457 \\
C & 0.065177 & 3.645956 & -1.812940 \\
C & -1.345153 & 3.754805 & 0.336810 \\
H & -1.526626 & 4.440811 & 1.172893 \\
C & -2.668324 & 3.264722 & -0.187242 \\
C & -2.863590 & 2.032838 & -0.657919 \\
C & -3.893348 & 1.147150 & -1.005189 \\
H & -4.128328 & 0.886114 & -2.031605 \\
O & -4.996867 & 1.100093 & -0.144615 \\
C & -5.944211 & 0.157577 & -0.427275 \\
O & -5.872231 & -0.604672 & -1.359645 \\
H & -0.747610 & 2.925531 & 0.721181 \\
C & -0.543841 & 4.509533 & -0.742386 \\
H & -1.174577 & 5.281781 & -1.204960 \\
H & 0.272456 & 5.050223 & -0.249083 \\
Rh & -2.150255 & -0.048117 & -0.432891 \\
C & -2.998302 & -1.828614 & -0.801121 \\
O & -3.495941 & -2.822221 & -1.060965 \\
C & -3.793086 & 4.274793 & -0.257103 \\
H & -4.682639 & 3.878444 & -0.750816 \\
H & -3.470925 & 5.176529 & -0.793575
\end{tabular}




\begin{tabular}{|c|c|c|c|}
\hline & -4.071685 & 4.589239 & 0.1 \\
\hline & -2.518780 & 0.094827 & \\
\hline & -2.760735 & 185973 & 623843 \\
\hline & 0.120232 & -0.992043 & \\
\hline & 1178 & -0.617580 & 7998 \\
\hline & 1.118080 & -0.373424 & -1.051320 \\
\hline & 0.336501 & -2.679725 & 0.139377 \\
\hline & 1.219329 & 0.700010 & 1.765577 \\
\hline & 2.515633 & -0.306809 & -0.907206 \\
\hline & 0.865031 & -3.45 & \\
\hline & & -3.3 & 601 \\
\hline & 3774 & 1.371948 & 4273 \\
\hline & 2.270754 & 1.257886 & \\
\hline & 3.090659 & 0.43 & \\
\hline & 3.26 & -0.9 & \\
\hline & & -3.7 & \\
\hline & & & \\
\hline & & -3.7 & \\
\hline & -1.0 & -3.5 & \\
\hline & -0.1 & & \\
\hline & 0.8 & 2.6 & \\
\hline & 17 & 2.6 & \\
\hline & & & \\
\hline & & & \\
\hline & 4.6 & -0.9 & \\
\hline & -0.9 & & \\
\hline & 0.5 & -4.4 & -3 \\
\hline & 2.6 & -4. & \\
\hline & 3.0 & -3. & \\
\hline & & -3.6 & \\
\hline & 152 & -4.3 & \\
\hline & -1.260620 & -4.1 & 8245 \\
\hline & -1.9 & -3.2 & \\
\hline & 28 & & \\
\hline & 47 & 3.3 & \\
\hline & 15 & 32 & \\
\hline & 043 & -0.2 & 555 \\
\hline & 5.227211 & 0.904287 & 8860 \\
\hline & 5.222426 & -1.4 & 1213 \\
\hline & 944 & -4.6 & 0200 \\
\hline & 55 & -4.8 & \\
\hline $\mathrm{H}$ & 3.7 & -4 & -1 . \\
\hline 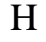 & 4.119550 & -3.7 & 6627 \\
\hline 1 & 3.474486 & -3.637980 & 2.322145 \\
\hline 11 & 2.010446 & -4.618133 & \\
\hline & & & \\
\hline $\mathrm{H}$ & & -4.27 & 3.6 \\
\hline $2+$ & 2.161265 & 4.723648 & 2.572363 \\
\hline $\mathrm{C}$ & 3.713848 & 4.727907 & 0.724495 \\
\hline 11 & 3.967930 & 2.899332 & -0.343349 \\
\hline & 6.714643 & -0.348601 & -0.655844 \\
\hline
\end{tabular}




$\begin{array}{lrrr}\mathrm{C} & 6.599744 & 0.820433 & 1.450340 \\ \mathrm{H} & 4.668883 & 1.370345 & 2.161646 \\ \mathrm{H} & 2.324396 & -5.423577 & -3.964134 \\ \mathrm{H} & -0.250788 & -4.924460 & 4.928910 \\ \mathrm{H} & 1.648132 & 5.229595 & 3.386367 \\ \mathrm{C} & 3.066855 & 5.400870 & 1.788276 \\ \mathrm{H} & 4.415268 & 5.268330 & 0.094993 \\ \mathrm{H} & 7.280525 & -0.846569 & -1.439151 \\ \mathrm{C} & 7.357170 & 0.203227 & 0.428065 \\ \mathrm{H} & 7.104404 & 1.228366 & 2.321440 \\ \mathrm{H} & 3.282734 & 6.448066 & 1.978770 \\ \mathrm{H} & 8.438960 & 0.153617 & 0.509074 \\ \mathrm{C} & -1.815307 & -0.623468 & -3.685251 \\ \mathrm{H} & -1.211521 & -1.533013 & -3.589578 \\ \mathrm{H} & -2.858895 & -0.930881 & -3.821550 \\ \mathrm{H} & -1.490014 & -0.084710 & -4.581645 \\ \mathrm{C} & 1.243819 & 4.023101 & -2.529357 \\ \mathrm{O} & 0.662717 & 1.987427 & -3.230220 \\ \mathrm{C} & 1.547318 & 2.996049 & -3.370774 \\ \mathrm{H} & 2.326862 & 2.840805 & -4.101972 \\ \mathrm{H} & 1.792668 & 4.946703 & -2.409418 \\ \mathrm{C} & -7.072129 & 0.198245 & 0.582945 \\ \mathrm{H} & -7.415759 & 1.219758 & 0.752707 \\ \mathrm{H} & -7.885278 & -0.433825 & 0.230381 \\ \mathrm{Cl} & -6.504869 & -0.453398 & 2.171580\end{array}$

\section{TS2-rot}

M06-2X/6-31+G(d) SCF energy: $\quad-3457.80598597$ a.u.

M06-2X/6-31+G(d) enthalpy: $\quad-3457.000070$ a.u.

M06-2X/6-31+G(d) free energy: -3457.148979 a.u.

M06-2X/6-311++G(d,p) SCF energy in solution: -3457.86686161 a.u.

M06-2X/6-311++G(d,p) enthalpy: $\quad-3457.060946$ a.u.

M06-2X/6-311++G(d,p) free energy: $\quad-3457.209855$ a.u.

Imaginary frequency: $\quad-216.6743 \mathrm{~cm}-1$

Cartesian coordinates

$\begin{array}{lrrr}\text { ATOM } & \text { X } & \text { Y } & \text { Z } \\ \text { C } & 2.461283 & -1.030063 & 1.812967 \\ \text { C } & 1.707276 & -1.909981 & 1.261383 \\ \text { C } & 2.708144 & -0.315830 & 3.014876 \\ \text { C } & 3.620515 & 0.597856 & 3.523695 \\ \text { C } & 4.647948 & 1.549165 & 1.369472 \\ \text { H } & 5.392579 & 2.297320 & 1.071396 \\ \text { C } & 4.883074 & 0.286610 & 0.587223 \\ \text { C } & 3.907183 & -0.542750 & 0.209857 \\ \text { C } & 3.732557 & -1.657294 & -0.637524 \\ \text { H } & 3.622567 & -2.669161 & -0.261810 \\ \text { O } & 4.360636 & -1.597360 & -1.887514 \\ \text { C } & 4.133037 & -2.651514 & -2.728221\end{array}$




\begin{tabular}{|c|c|c|c|}
\hline & 5154 & -3.599475 & \\
\hline & 659459 & 1.963659 & \\
\hline & & 87 & \\
\hline & 13477 & & \\
\hline & & & \\
\hline & 26866 & -0.672653 & \\
\hline & 0.759603 & 795 & -1 \\
\hline 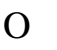 & 0.217208 & 507 & -2.17 \\
\hline & & -0.0 & \\
\hline & & & \\
\hline I & & & \\
\hline & .784712 & & \\
\hline & .227248 & & \\
\hline 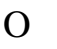 & & & \\
\hline & 7 & 0.6 & \\
\hline & & & \\
\hline ) & & & \\
\hline & 74 & & -0. \\
\hline & & -0.7 & \\
\hline & & & \\
\hline & & & \\
\hline & & & \\
\hline & & & \\
\hline & & & \\
\hline & & & \\
\hline & & & \\
\hline & & & \\
\hline & -2.5 & & \\
\hline & -0.4 & & \\
\hline & & & \\
\hline$T$ & -0.6 & & \\
\hline & -1.8 & & \\
\hline & & & \\
\hline & & & \\
\hline & -2.5 & & \\
\hline & -4.5 & & \\
\hline & -0.1 & & \\
\hline & -2.01 & & -3.3 \\
\hline & $-3.4^{\prime}$ & & \\
\hline & & & \\
\hline $\mathrm{C}$ & -1.8 & & \\
\hline S & 0.2 & 4.5 & \\
\hline $\mathrm{C}$ & & & \\
\hline $\mathrm{H}$ & 2.12 & 3.2 & -0.5 \\
\hline 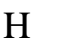 & 37 & & \\
\hline 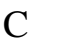 & & & \\
\hline & & -3.4 & \\
\hline $\mathrm{C}$ & -5.4 & 0.071999 & -1.6 \\
\hline $\mathrm{C}$ & & -0.841935 & \\
\hline 11 & -5.03 & 0.91 & -3.6 \\
\hline 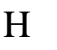 & -1.804309 & 3.991649 & -4.44799 \\
\hline
\end{tabular}




$\begin{array}{lrrr}\mathrm{C} & -3.219968 & 4.309420 & -2.845573 \\ \mathrm{H} & -4.401014 & 4.521577 & -1.061764 \\ \mathrm{H} & -3.852374 & 3.508371 & 1.086594 \\ \mathrm{H} & -2.265257 & 3.648414 & 2.816224 \\ \mathrm{H} & -0.293264 & 4.913472 & 3.443654 \\ \mathrm{C} & 1.593490 & 4.842649 & 2.416579 \\ \mathrm{H} & 3.319061 & 4.659458 & 1.129057 \\ \mathrm{C} & -3.134007 & -4.556870 & 2.734342 \\ \mathrm{C} & -4.366857 & -4.757558 & 0.669343 \\ \mathrm{H} & -4.345521 & -3.082462 & -0.650151 \\ \mathrm{C} & -6.859301 & 0.177772 & -1.758067 \\ \mathrm{C} & -7.113279 & -0.706273 & 0.470877 \\ \mathrm{H} & -5.337489 & -1.215945 & 1.530682 \\ \mathrm{H} & -3.952136 & 4.804799 & -3.476500 \\ \mathrm{H} & 2.096253 & 5.472820 & 3.144483 \\ \mathrm{H} & -2.782608 & -4.968516 & 3.677206 \\ \mathrm{C} & -3.939264 & -5.301009 & 1.903421 \\ \mathrm{H} & -4.979911 & -5.356504 & 0.002120 \\ \mathrm{H} & -7.278051 & 0.583725 & -2.675431 \\ \mathrm{C} & -7.680921 & -0.207018 & -0.724037 \\ \mathrm{H} & -7.759931 & -0.982013 & 1.299028 \\ \mathrm{H} & -4.237312 & -6.306682 & 2.184761 \\ \mathrm{H} & -8.759187 & -0.116081 & -0.816039 \\ \mathrm{C} & 1.140011 & -3.271130 & 1.429738 \\ \mathrm{H} & 0.083585 & -3.299959 & 1.150051 \\ \mathrm{H} & 1.670642 & -3.989838 & 0.792038 \\ \mathrm{H} & 1.235696 & -3.595630 & 2.471131 \\ \mathrm{C} & 3.220357 & 0.836787 & 4.874627 \\ \mathrm{O} & 1.769926 & -0.612405 & 3.998143 \\ \mathrm{C} & 2.114284 & 0.077388 & 5.106143 \\ \mathrm{H} & 1.486888 & -0.075411 & 5.971912 \\ \mathrm{H} & 3.706615 & 1.501788 & 5.575086 \\ \mathrm{C} & 4.852195 & -2.462579 & -4.048063 \\ \mathrm{H} & 5.897418 & -2.190298 & -3.892315 \\ \mathrm{H} & 4.771539 & -3.380828 & -4.627133 \\ \mathrm{Cl} & 4.081614 & -1.131200 & -4.997200\end{array}$

\section{TS2b}

M06-2X/6-31+G(d) SCF energy: $\quad-3457.81095305$ a.u.

M06-2X/6-31+G(d) enthalpy: $\quad-3457.004922$ a.u.

M06-2X/6-31+G(d) free energy: -3457.153112 a.u.

M06-2X/6-311++G(d,p) SCF energy in solution: -3457.87526715 a.u.

M06-2X/6-311++G(d,p) enthalpy: $\quad-3457.069236$ a.u.

M06-2X/6-311++G(d,p) free energy: $\quad-3457.217426$ a.u.

Imaginary frequency: $\quad-223.0012 \mathrm{~cm}-1$

Cartesian coordinates

\begin{tabular}{lrcr} 
ATOM & X & Y & \multicolumn{2}{l}{ Z } \\
C & 3.803438 & -0.289430 & 1.130352
\end{tabular}




\begin{tabular}{|c|c|c|c|}
\hline C & 2.746158 & 0.219455 & \\
\hline & 5.212507 & -0.150001 & 060100 \\
\hline & 299930 & -0.701881 & \\
\hline & 146127 & & \\
\hline & & 3106 & \\
\hline & 3.965860 & -2.446896 & -0.929121 \\
\hline & 10 & 435 & -0 \\
\hline & 693053 & 170 & \\
\hline & & -2.0 & \\
\hline 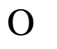 & & -2.6 & \\
\hline $\mathrm{C}$ & & -3.9 & \\
\hline $\mathrm{O}$ & & -4.4 & \\
\hline 1 & 028 & -0.7 & 673 \\
\hline & 17 & -1.7 & -0.6 \\
\hline & & -2.7 & \\
\hline$I$ & & -1.5 & \\
\hline $\mathrm{Rh}$ & & & \\
\hline & 002 & -0.2 & \\
\hline 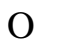 & & & \\
\hline & & -3.9 & \\
\hline & & -4.3 & \\
\hline & & -4 & \\
\hline H & & & \\
\hline $\mathrm{C}$ & & & \\
\hline O & & & \\
\hline & & & \\
\hline D & & & \\
\hline $\mathrm{O}$ & & & \\
\hline $\mathrm{N}$ & & & \\
\hline $\mathrm{C}$ & & -0.7 & \\
\hline $\mathrm{C}$ & & & \\
\hline 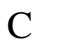 & & & \\
\hline $\mathrm{C}_{2}$ & & & \\
\hline$a_{1}$ & & & \\
\hline 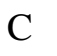 & & -1.2 & \\
\hline$C$ & -3 . & -0.3 & -0 . \\
\hline $\mathrm{C}$ & & & $-2 .($ \\
\hline $\mathrm{C}$ & & & \\
\hline $\mathrm{C}$ & & & \\
\hline 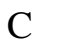 & & & \\
\hline 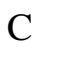 & -2.5 & & \\
\hline $\mathrm{H}$ & -0.2 & -1.1 & 21 \\
\hline $\mathrm{C}$ & -1.3 & -2.8 & 507 \\
\hline $\mathrm{C}$ & -2.99 & -2.6 & .751 \\
\hline $\mathrm{C}$ & -4.779655 & 703 & 225 \\
\hline-1 & & & \\
\hline$a^{2}$ & & & \\
\hline $\mathrm{H}$ & & 3.27 & 75 \\
\hline $\mathrm{C}$ & & & \\
\hline $\mathrm{C}$ & 1.70 & & -1.10 \\
\hline $\mathrm{G}$ & -0.403653 & 4.393997 & -2.01357 \\
\hline
\end{tabular}




$\begin{array}{lrrr}\mathrm{C} & -1.731855 & 4.233212 & -1.814397 \\ \mathrm{C} & -3.703183 & 4.561839 & -0.357819 \\ \mathrm{C} & -3.766201 & 3.783141 & 1.925489 \\ \mathrm{H} & -2.027376 & 2.686979 & 2.582938 \\ \mathrm{H} & -0.931966 & -3.466417 & 3.616054 \\ \mathrm{C} & -2.371025 & -3.432446 & 1.995834 \\ \mathrm{C} & -3.912976 & -3.262637 & 0.102393 \\ \mathrm{C} & -5.415433 & 0.538947 & -1.150440 \\ \mathrm{C} & -5.603445 & -0.716532 & 0.942476 \\ \mathrm{H} & -5.107118 & 1.667147 & -2.974660 \\ \mathrm{H} & 3.209442 & 4.891692 & 1.922571 \\ \mathrm{C} & 2.636513 & 5.478028 & -0.077170 \\ \mathrm{H} & 1.797620 & 5.901856 & -2.010806 \\ \mathrm{H} & -0.074035 & 4.670256 & -3.013338 \\ \mathrm{H} & -2.384930 & 4.388968 & -2.671230 \\ \mathrm{H} & -4.166629 & 5.094404 & -1.184129 \\ \mathrm{C} & -4.371306 & 4.449774 & 0.856372 \\ \mathrm{H} & -4.279996 & 3.694837 & 2.877965 \\ \mathrm{C} & -2.725493 & -4.803350 & 2.118828 \\ \mathrm{C} & -4.223435 & -4.599472 & 0.238599 \\ \mathrm{H} & -4.369899 & -2.688612 & -0.694191 \\ \mathrm{C} & -6.830788 & 0.660217 & -1.165487 \\ \mathrm{C} & -6.972613 & -0.562481 & 0.909771 \\ \mathrm{H} & -5.143903 & -1.233423 & 1.776882 \\ \mathrm{H} & 3.453257 & 6.183654 & -0.196283 \\ \mathrm{H} & -5.359435 & 4.884708 & 0.972716 \\ \mathrm{H} & -2.248545 & -5.393396 & 2.896906 \\ \mathrm{C} & -3.637662 & -5.378416 & 1.263891 \\ \mathrm{H} & -4.924533 & -5.059109 & -0.452183 \\ \mathrm{H} & -7.296082 & 1.203531 & -1.984049 \\ \mathrm{C} & -7.598282 & 0.118919 & -0.159966 \\ \mathrm{H} & -7.577230 & -0.965366 & 1.717317 \\ \mathrm{H} & -1.477359 & -4.055529 & -1.124158 \\ \mathrm{H} & -3.899625 & -6.427418 & 1.365237 \\ \mathrm{H} & -8.679081 & 0.223455 & -0.177729 \\ \mathrm{C} & 2.221146 & 0.648887 & 2.977896 \\ \mathrm{H} & 2.727331 & 1.572412 & 3.287398 \\ \mathrm{H} & 1.145782 & 0.833105 & 2.964955 \\ \mathrm{H} & 2.439534 & -0.110872 & 3.737596 \\ \mathrm{C} & 7.443809 & 0.012689 & 0.873867 \\ \mathrm{O} & 5.657668 & 0.880918 & 1.890312 \\ \mathrm{H} & 6.999257 & 0.938077 & 1.770647 \\ \mathrm{H} & -0.467508 & -0.149503 & 0.565698 \\ \mathrm{H} & -0.516046 & -4.572521 & -1.157966 \\ \mathrm{H} & & -53831 & -2.871883\end{array}$

\section{TS2b-rot}


M06-2X/6-31+G(d) SCF energy: $\quad-3457.81115702$ a.u.

M06-2X/6-31+G(d) enthalpy: $\quad-3457.005200$ a.u.

M06-2X/6-31+G(d) free energy: -3457.152897 a.u.

M06-2X/6-311++G(d,p) SCF energy in solution: -3457.87343620 a.u.

M06-2X/6-311++G(d,p) enthalpy: $\quad-3457.067479$ a.u.

M06-2X/6-311++G(d,p) free energy: $\quad-3457.215176$ a.u.

Imaginary frequency: $\quad-229.3098 \mathrm{~cm}-1$

Cartesian coordinates

$\begin{array}{lccc}\text { ATOM } & \text { X } & \text { Y } & \text { Z } \\ \text { C } & -2.238563 & -1.617128 & 2.091956 \\ \text { C } & -1.388320 & -0.654373 & 2.139291 \\ \text { C } & -2.511600 & -2.903305 & 2.610091 \\ \text { C } & -3.435192 & -3.929873 & 2.445003 \\ \text { C } & -4.353423 & -3.396130 & 0.119626 \\ \text { H } & -5.096868 & -3.786525 & -0.585466 \\ \text { C } & -4.504480 & -1.901433 & 0.205648 \\ \text { C } & -3.482724 & -1.056938 & 0.392997 \\ \text { C } & -3.202060 & 0.330379 & 0.264414 \\ \text { H } & -3.154478 & 0.998528 & 1.118084 \\ \text { O } & -3.728741 & 0.946778 & -0.899410 \\ \text { C } & -4.742273 & 1.835519 & -0.715858 \\ \text { O } & -5.156050 & 2.184177 & 0.363268 \\ \text { H } & -3.364763 & -3.676012 & -0.257418 \\ \text { C } & -4.590992 & -4.062674 & 1.495453 \\ \text { H } & -5.504418 & -3.653879 & 1.947988 \\ \text { H } & -4.783841 & -5.131309 & 1.348312 \\ \text { Rh } & -1.386560 & -0.749929 & -0.015677 \\ \text { C } & -1.732847 & -0.853575 & -1.960707 \\ \text { O } & -1.987647 & -0.893214 & -3.072118 \\ \text { C } & -5.922815 & -1.374810 & 0.187699 \\ \text { H } & -5.993468 & -0.342237 & 0.534309 \\ \text { H } & -6.569873 & -1.994113 & 0.822279 \\ \text { H } & -6.328455 & -1.427396 & -0.831154 \\ \text { C } & -0.101947 & -2.356778 & 0.016470 \\ \text { O } & 0.496711 & -3.319095 & 0.131630 \\ \text { P } & 0.568743 & 0.806751 & -0.173930 \\ \text { O } & 1.522099 & 0.720913 & -1.542099 \\ \text { O } & 1.597596 & 0.229234 & 0.968090 \\ \text { N } & 0.453605 & 2.467665 & 0.052550 \\ \text { C } & 2.043423 & -0.531270 & -1.887111 \\ \text { C } & 3.000084 & 0.304986 & 0.949558 \\ \text { C } & -0.671882 & 3.204751 & -0.462090 \\ \text { C } & 1.525267 & 3.297976 & 0.555384 \\ \text { C } & 1.498502 & -1.161180 & -3.028350 \\ \text { C } & 3.076554 & -1.080106 & -1.140973 \\ \text { C } & 3.735897 & -0.290393 & -0.061940 \\ \text { C } & 3.588437 & 0.933860 & 2.066834 \\ \text { C } & -0.910511 & 3.255524 & -1.838929 \\ \text { C } & -1.488064 & 3.916359 & 0.443724 \\ \text { C } & 1.317144 & 3.982311 & 1.772823\end{array}$




\begin{tabular}{|c|c|c|c|}
\hline & .725663 & 8804 & 146911 \\
\hline & .752806 & .631894 & -3.611582 \\
\hline & 1.949078 & -2.406377 & 181 \\
\hline & 3.478427 & -2.429699 & -1.456594 \\
\hline & 5.167173 & -0.091858 & -0 \\
\hline & .943358 & 1.343107 & 36184 \\
\hline & 954057 & 1.028786 & 5064 \\
\hline & .238232 & 2.731340 & -2.511557 \\
\hline & 801 & 362 & -2.3 \\
\hline & 8511 & 367 & \\
\hline & 154 & & 410 \\
\hline & 0.023289 & 4.014382 & 5670 \\
\hline & 389969 & 4.726901 & 8974 \\
\hline & .762061 & 4.195864 & \\
\hline & 841 & 81 & -1. \\
\hline & 89 & -2. & \\
\hline & 2.915621 & 021 & -2 \\
\hline & 4.386197 & -3 & -0. \\
\hline & 5.774537 & 0.5 & 377 \\
\hline & 203 & -0.4 & 737 \\
\hline & 89 & 06 & \\
\hline & 327 & & \\
\hline & -2.8 & & \\
\hline & -3.2 & & \\
\hline & -2.0 & & \\
\hline & 0.063879 & 4.2 & 3.5 \\
\hline & 2.25 & 462 & 3.2 \\
\hline C & 3.59 & 4.8 & \\
\hline H & 4.6 & 23 & -0 . \\
\hline & 3.32 & -4.4 & -2 \\
\hline & 4.742992 & -4.4 & -0.9 \\
\hline & 4.793704 & 334 & \\
\hline & 563 & 90 & \\
\hline & 793 & -0.2 & 64 \\
\hline & 27 & -0. & \\
\hline & -3.6 & & -1 . \\
\hline & 4.405015 & 841 & 2.0 \\
\hline & 2.898331 & -4.882245 & -3.8 \\
\hline & 220524 & -5.0 & -2.1 \\
\hline & 432174 & -5.0 & -0 \\
\hline & 7.62 & 15 & \\
\hline & 7.97 & 0.3 & 0.067802 \\
\hline & 7.993063 & -0.559203 & -1.910202 \\
\hline & 4.520864 & -6.096302 & -2.382271 \\
\hline & 047711 & & \\
\hline & -0.737690 & 0.2 & 7220 \\
\hline & 0.345977 & & 3.102930 \\
\hline & -0.921887 & 1.296214 & 2.897054 \\
\hline & -1.110309 & 0.022719 & 4.133788 \\
\hline & -3.022385 & -4.969142 & 3.330105 \\
\hline & -1.550848 & -3.304034 & 3.534126 \\
\hline
\end{tabular}




$\begin{array}{llll}\mathrm{C} & -1.897057 & -4.531195 & 3.965585 \\ \mathrm{H} & -3.509105 & -5.925551 & 3.462606 \\ \mathrm{H} & -1.259221 & -4.963366 & 4.722778 \\ \mathrm{C} & -5.288411 & 2.318755 & -2.047353 \\ \mathrm{H} & -4.482181 & 2.670659 & -2.689890 \\ \mathrm{H} & -6.013629 & 3.108951 & -1.861432 \\ \mathrm{Cl} & -6.134249 & 0.985783 & -2.930230\end{array}$

\section{TS2c}

M06-2X/6-31+G(d) SCF energy: $\quad-3457.81015707$ a.u.

M06-2X/6-31+G(d) enthalpy: $\quad-3457.004333$ a.u.

M06-2X/6-31+G(d) free energy: -3457.151512 a.u.

M06-2X/6-311++G(d,p) SCF energy in solution: -3457.87509143 a.u.

M06-2X/6-311++G(d,p) enthalpy: $\quad-3457.069267$ a.u.

M06-2X/6-311++G(d,p) free energy: $\quad-3457.216446$ a.u.

Imaginary frequency: $\quad-227.9760 \mathrm{~cm}-1$

Cartesian coordinates

\begin{tabular}{lrcr} 
ATOM & X & Y & \multicolumn{2}{l}{ Z } \\
C & -3.024764 & -1.805238 & 1.919139 \\
C & -3.894436 & -0.927669 & 1.561097 \\
C & -2.577726 & -2.533255 & 3.047911 \\
C & -1.617024 & -3.483190 & 3.368720 \\
C & 0.025881 & -3.157527 & 1.439216 \\
H & 0.977598 & -3.568666 & 1.084562 \\
C & -0.910749 & -3.027431 & 0.267739 \\
C & -1.792140 & -2.030314 & 0.127297 \\
C & -2.673348 & -1.508718 & -0.849621 \\
H & -3.738290 & -1.719114 & -0.823811 \\
O & -2.185448 & -1.389627 & -2.162152 \\
C & -3.057951 & -0.876515 & -3.070324 \\
O & -4.145499 & -0.434443 & -2.787709 \\
H & 0.237072 & -2.178395 & 1.877771 \\
C & -0.542266 & -4.102551 & 2.522627 \\
H & -0.910817 & -5.026114 & 2.055960 \\
H & 0.272009 & -4.411827 & 3.187694 \\
Rh & -2.283267 & 0.044426 & 0.561940 \\
C & -0.911683 & -4.167353 & -0.725898 \\
H & -1.606009 & -4.002767 & -1.551752 \\
H & -1.188130 & -5.104969 & -0.223771 \\
H & 0.093351 & -4.307373 & -1.137951 \\
C & -1.808480 & 1.020820 & 2.295742 \\
O & -1.641406 & 1.497176 & 3.317885 \\
C & -5.338158 & -0.597274 & 1.632344 \\
H & -5.485485 & 0.409335 & 2.040702 \\
H & -5.801487 & -0.614900 & 0.638410 \\
H & -5.860422 & -1.315291 & 2.273644 \\
C & -1.768581 & -3.731999 & 4.765628 \\
O & -3.275104 & -2.185563 & 4.200101
\end{tabular}




\begin{tabular}{|c|c|c|c|}
\hline C & -2.784745 & -2.935765 & \\
\hline & -1.180068 & -4.417320 & \\
\hline & -3.254871 & -2.799567 & 160 \\
\hline & -2.492614 & -0.873953 & -4.47 \\
\hline & -0.057869 & & -0.2 \\
\hline & 0.880035 & 0.443878 & -1.501849 \\
\hline & 9336 & 549 & 53332 \\
\hline & 0.003067 & 2.581323 & -0.369432 \\
\hline & 1.538200 & -0.7 & -1.575 \\
\hline & 2.286089 & & \\
\hline & -1.105697 & & \\
\hline & 1.182359 & & -0.148109 \\
\hline & 1.147405 & 206 & -2.635322 \\
\hline & 2.582322 & -1.0 & -0.713 \\
\hline & 60 & $-0 .($ & \\
\hline & 2.7 & & \\
\hline & -1.4 & & -2 \\
\hline & -1.7 & & -0. \\
\hline & 1.146076 & & \\
\hline & 2.3 & 3.2 & $-0.94 \varsigma$ \\
\hline & 0.3 & -1. & -3 . \\
\hline C & 1.7 & -2 & -2 \\
\hline & & -2 & -0.8 \\
\hline & 4.5 & & \\
\hline & 2.0 & & \\
\hline & 4.11 & & \\
\hline & -0.9 & & -2.8 \\
\hline & -2.5 & & -2.8 \\
\hline & -2.8 & 4.9 & -0 \\
\hline & -1.3 & & 1.2 \\
\hline & -0.06 & & \\
\hline & 2.31 & 5.0 & 1.15 \\
\hline & 3.457179 & 4.0 & -0.6 \\
\hline & 56 & & \\
\hline & 1.4 & -3.4 & -3 . \\
\hline & 03 & -3.2 & -1.8 \\
\hline & 369 & -2.9 & \\
\hline & 5.022854 & 1.05 & 1.367 \\
\hline & 5.462132 & -0.2 & -0.65 \\
\hline & 4.49 & & \\
\hline & -2.8 & 3.5 & -3.8 \\
\hline & -3.2 & 4.7 & -2.02 \\
\hline & -3.40 & 5.6 & -0.113608 \\
\hline & -2.113542 & 4.994035 & 1.870703 \\
\hline$\pi$ & 0.100544 & 5.033929 & 2.664380 \\
\hline & & 5.8 & \\
\hline & & 4.91 & 0.380114 \\
\hline & 4.342385 & 3.884440 & -1.298564 \\
\hline & 3.328056 & -4.582701 & -1.985537 \\
\hline & 4.569513 & -4.211445 & 0.049276 \\
\hline & 4.330483 & -2.323759 & 1.010914 \\
\hline
\end{tabular}




$\begin{array}{lrrc}\mathrm{C} & 6.412347 & 1.343655 & 1.444759 \\ \mathrm{C} & 6.800996 & 0.067380 & -0.563754 \\ \mathrm{H} & 5.111868 & -0.845089 & -1.486330 \\ \mathrm{H} & -4.105552 & 5.244739 & -2.445017 \\ \mathrm{H} & 4.345512 & 5.506214 & 0.593142 \\ \mathrm{H} & 3.035453 & -5.213564 & -2.821150 \\ \mathrm{C} & 4.221561 & -5.038825 & -1.043813 \\ \mathrm{H} & 5.254065 & -4.581630 & 0.807154 \\ \mathrm{H} & 6.767448 & 1.971632 & 2.257960 \\ \mathrm{C} & 7.289141 & 0.856136 & 0.503304 \\ \mathrm{H} & 7.487579 & -0.291045 & -1.325407 \\ \mathrm{H} & 4.650223 & -6.032909 & -1.129885 \\ \mathrm{H} & 8.348434 & 1.086648 & 0.567503 \\ \mathrm{C} & -3.543907 & 1.377526 & -0.246617 \\ \mathrm{O} & -4.427560 & 1.989623 & -0.628509 \\ \mathrm{H} & -1.665338 & -0.162296 & -4.547987 \\ \mathrm{H} & -3.288376 & -0.582574 & -5.162434 \\ \mathrm{Cl} & -1.866965 & -2.483716 & -4.994886\end{array}$

\section{TS2c-rot}

M06-2X/6-31+G(d) SCF energy: $\quad-3457.81213130$ a.u.

M06-2X/6-31+G(d) enthalpy: $\quad-3457.006180$ a.u.

M06-2X/6-31+G(d) free energy: -3457.151565 a.u.

M06-2X/6-311++G(d,p) SCF energy in solution: -3457.87743195 a.u.

M06-2X/6-311++G(d,p) enthalpy: $\quad-3457.071481$ a.u.

M06-2X/6-311++G(d,p) free energy: $\quad-3457.216866$ a.u.

Imaginary frequency: $\quad-217.2212 \mathrm{~cm}-1$

Cartesian coordinates

\begin{tabular}{lccc}
\multicolumn{2}{l}{ ATOM } & X & \multicolumn{2}{l}{ Y } \\
C & -3.935648 & -1.844960 & 0.650201 \\
C & -3.011712 & -2.727024 & 0.796956 \\
C & -5.195869 & -1.623112 & 0.067092 \\
C & -6.141163 & -0.605650 & 0.000170 \\
C & -4.673404 & 1.445154 & 0.317225 \\
C & -3.703519 & 1.034884 & 1.390356 \\
C & -2.859901 & -0.003184 & 1.305588 \\
C & -1.855540 & -0.578223 & 2.124977 \\
H & -2.067202 & -1.431669 & 2.763162 \\
O & -0.931259 & 0.303435 & 2.699419 \\
C & 0.039835 & -0.269646 & 3.450819 \\
O & 0.180900 & -1.462924 & 3.580440 \\
H & -4.302822 & 1.193964 & -0.678774 \\
C & -6.066101 & 0.792631 & 0.533965 \\
H & -6.326635 & 0.816125 & 1.600185 \\
Rh & -1.557435 & -1.312923 & 0.140176 \\
C & -3.805276 & 1.810964 & 2.686032 \\
H & -3.199835 & 1.384364 & 3.486653 \\
H & -4.849016 & 1.865994 & 3.023994
\end{tabular}




\begin{tabular}{|c|c|c|c|}
\hline & -3.467993 & 12622 & \\
\hline & 1.956273 & -1.753839 & \\
\hline & 247213 & -2.061171 & 67781 \\
\hline & -2.834923 & -4.120261 & \\
\hline & -2.329064 & -4.730514 & \\
\hline & -2.216668 & -4.148931 & 2.187987 \\
\hline & -3.805790 & -4.573483 & 8665 \\
\hline & -7.199293 & 023 & -0.80904 \\
\hline & -5.6 & -2.7 & \\
\hline & -6.8 & -2.3 & \\
\hline & -8.1 & -0.5 & \\
\hline & -7.340940 & -3.133601 & -1.773163 \\
\hline & 0.978950 & & \\
\hline & 1 & & \\
\hline & 97 & -0.2 & \\
\hline & 1.2 & & \\
\hline & -0.1 & & \\
\hline & & & \\
\hline & 2.5 & & \\
\hline & -1.3 & & \\
\hline & 6 & & \\
\hline & & -2.5 & \\
\hline & 29 & & \\
\hline & 57 & -0.1 & \\
\hline & 3.0 & & \\
\hline & -1.7 & & \\
\hline & -2.10 & & \\
\hline & $0.6^{\prime}$ & & \\
\hline & 8 & & \\
\hline & 0.6 & -2.4 & -3 \\
\hline & 547 & -3.7 & \\
\hline & 3.58 & -2.6 & -0. \\
\hline & 4.82 & & \\
\hline & 9 & & \\
\hline & 4.37 & & \\
\hline & -1.0 & 0.7 & -3 . \\
\hline & -2.8 & & -3. \\
\hline & -3.341249 & & -2.0 \\
\hline & -1.824129 & & \\
\hline & -0.5 & & \\
\hline & 1.70 & & \\
\hline & 3.00 & 3.7 & \\
\hline & 2.12 & 652 & -2.5 \\
\hline$\Pi$ & 1.867968 & -4.5 & -2.790991 \\
\hline & 3.18 & -3.8 & -1.2 \\
\hline & 4.5 & & \\
\hline & & & \\
\hline & 5.749539 & -0.4 & -1.10570 \\
\hline $\mathrm{H}$ & 4.739145 & & \\
\hline 11 & -3.140295 & 1.3 & -4.559530 \\
\hline & -3.697176 & 2.895008 & -3.185641 \\
\hline
\end{tabular}




$\begin{array}{lrrr}\mathrm{H} & -3.970443 & 4.407278 & -1.682374 \\ \mathrm{H} & -2.668468 & 4.516777 & 0.393766 \\ \mathrm{H} & -0.513253 & 5.098389 & 1.132696 \\ \mathrm{H} & 1.569196 & 5.977045 & 0.212373 \\ \mathrm{C} & 2.855377 & 4.907719 & -1.140703 \\ \mathrm{H} & 3.892849 & 3.639832 & -2.548248 \\ \mathrm{C} & 3.815821 & -5.078388 & -0.998229 \\ \mathrm{C} & 5.149419 & -4.080950 & 0.748203 \\ \mathrm{H} & 4.859024 & -2.004721 & 1.137481 \\ \mathrm{C} & 6.653242 & 1.818244 & 0.343594 \\ \mathrm{C} & 7.058534 & 0.018055 & -1.208748 \\ \mathrm{H} & 5.415074 & -1.247554 & -1.692538 \\ \mathrm{H} & 3.630053 & 5.668654 & -1.123988 \\ \mathrm{H} & 3.510382 & -5.937588 & -1.589876 \\ \mathrm{C} & 4.783946 & -5.204714 & -0.029273 \\ \mathrm{H} & 5.893761 & -4.189177 & 1.531776 \\ \mathrm{H} & 6.989407 & 2.687536 & 0.903068 \\ \mathrm{C} & 7.524485 & 1.127896 & -0.466715 \\ \mathrm{H} & 7.738522 & -0.508675 & -1.872149 \\ \mathrm{H} & 5.258526 & -6.164848 & 0.150044 \\ \mathrm{H} & 8.561421 & 1.439538 & -0.549998 \\ \mathrm{C} & -0.107684 & -2.617555 & 0.596969 \\ \mathrm{O} & 0.577470 & -3.443103 & 0.984094 \\ \mathrm{H} & -4.602877 & 3.161382 & -3.722587 \\ \mathrm{H} & -6.828374 & 1.391329 & 0.023950 \\ \mathrm{H} & -4.799257 & 2.532406 & 0.352651 \\ \mathrm{H} & 1.153140 & 0.369454 & 5.141169 \\ \mathrm{H} & 1.929127 & 0.697715 & 3.581985 \\ \mathrm{Cl} & 0.424097 & 2.423480 & 4.194625\end{array}$

\section{TS3}

M06-2X/6-31+G(d) SCF energy: $\quad-1715.66711034$ a.u.

M06-2X/6-31+G(d) enthalpy: $\quad-1715.347647$ a.u.

M06-2X/6-31+G(d) free energy: -1715.438363 a.u.

M06-2X/6-311++G(d,p) SCF energy in solution: -1716.38653619 a.u.

M06-2X/6-311++G(d,p) enthalpy: $\quad-1716.067073$ a.u.

M06-2X/6-311++G(d,p) free energy: $\quad-1716.157789$ a.u.

Imaginary frequency: $\quad-223.9618 \mathrm{~cm}-1$

Cartesian coordinates

\begin{tabular}{lrrr} 
ATOM & X & Y & \multicolumn{2}{l}{ Z } \\
C & 1.833173 & -0.278775 & -0.889360 \\
C & 1.149244 & -1.324190 & -1.210944 \\
C & 3.128696 & 0.172066 & -0.581073 \\
C & 3.755982 & 1.341101 & -0.153393 \\
C & 1.828871 & 2.562325 & 0.996123 \\
H & 1.662970 & 3.504028 & 1.530934 \\
C & 0.682583 & 2.331247 & 0.050119 \\
C & 0.225614 & 1.122727 & -0.303119
\end{tabular}




$\begin{array}{lccc}\mathrm{C} & -0.877594 & 0.575099 & -0.997974 \\ \mathrm{H} & -0.782022 & 0.150531 & -1.992967 \\ \mathrm{O} & -2.125703 & 1.149342 & -0.754313 \\ \mathrm{C} & -3.199951 & 0.519241 & -1.332970 \\ \mathrm{O} & -3.096515 & -0.474777 & -2.006362 \\ \mathrm{H} & 1.900252 & 1.767312 & 1.744346 \\ \mathrm{C} & 3.171029 & 2.671209 & 0.223104 \\ \mathrm{H} & 3.033915 & 3.298412 & -0.667454 \\ \mathrm{H} & 3.901271 & 3.194877 & 0.848766 \\ \mathrm{Rh} & -0.180028 & -0.907149 & 0.395568 \\ \mathrm{C} & -1.270966 & -0.142142 & 1.920263 \\ \mathrm{O} & -1.853134 & 0.314333 & 2.783224 \\ \mathrm{C} & 0.119637 & 3.564858 & -0.620177 \\ \mathrm{H} & -0.609405 & 3.330075 & -1.397326 \\ \mathrm{H} & 0.924988 & 4.163156 & -1.066196 \\ \mathrm{H} & -0.367444 & 4.200181 & 0.131202 \\ \mathrm{C} & 1.061708 & -1.963674 & 1.694583 \\ \mathrm{O} & 1.793243 & -2.549317 & 2.338514 \\ \mathrm{C} & 1.088301 & -2.378170 & -2.256702 \\ \mathrm{H} & 1.953220 & -2.306323 & -2.924098 \\ \mathrm{H} & 1.078913 & -3.373395 & -1.797601 \\ \mathrm{H} & 0.174670 & -2.288966 & -2.856857 \\ \mathrm{C} & 5.131636 & 1.010342 & -0.005083 \\ \mathrm{O} & 4.075342 & -0.846244 & -0.657797 \\ \mathrm{C} & 5.265055 & -0.309209 & -0.337154 \\ \mathrm{H} & 5.919069 & 1.676858 & 0.318560 \\ \mathrm{H} & 6.115060 & -0.973958 & -0.393170 \\ \mathrm{C} & -4.494867 & 1.227265 & -0.997485 \\ \mathrm{C} & -1.450699 & -2.416873 & -0.110544 \\ \mathrm{O} & -2.154253 & -3.251346 & -0.428834 \\ \mathrm{H} & -5.298516 & 0.789679 & -1.587263 \\ \mathrm{H} & -4.417775 & 2.300515 & -1.179463 \\ \mathrm{Cl} & -4.887323 & 0.996828 & 0.750782\end{array}$

\section{TS4}

M06-2X/6-31+G(d) SCF energy: $\quad$-2998.23541804 a.u.

M06-2X/6-31+G(d) enthalpy: $\quad-2997.421869$ a.u.

M06-2X/6-31+G(d) free energy: -2997.567485 a.u.

M06-2X/6-311++G(d,p) SCF energy in solution: -2998.29616000 a.u.

M06-2X/6-311++G(d,p) enthalpy: $\quad$-2997.482611 a.u.

M06-2X/6-311++G(d,p) free energy: $\quad-2997.628227$ a.u.

Imaginary frequency: $\quad-213.5709 \mathrm{~cm}-1$

Cartesian coordinates

\begin{tabular}{lrrr} 
ATOM & X & \multicolumn{2}{l}{ Y Z } \\
C & -1.541329 & 2.139495 & 1.136323 \\
C & -2.041644 & 1.229602 & 1.875199 \\
C & -0.794571 & 3.365087 & 1.165371 \\
C & -0.163841 & 4.231882 & 0.298291
\end{tabular}




\begin{tabular}{|c|c|c|c|}
\hline & .702069 & 064747 & \\
\hline & 0.533162 & 3.193491 & \\
\hline & 3074 & 860 & \\
\hline & -2.737733 & & \\
\hline & 722 & & \\
\hline & 4.368925 & & \\
\hline & 865 & 386 & -1 \\
\hline & 6.058140 & & \\
\hline & 49 & & \\
\hline & 575 & & \\
\hline & & & \\
\hline & -0.228408 & & \\
\hline & 1.12 & & \\
\hline & 4005 & 745 & -0 \\
\hline & -3.4 & -1.1 & \\
\hline & -4.2 & & \\
\hline & -3.0 & & \\
\hline & -4.0 & & \\
\hline & -2.6 & & \\
\hline & -2.9 & & -3 . \\
\hline & -2.3 & -0.3 & \\
\hline & -2.4 & & \\
\hline & -0.0 & & \\
\hline ) & & & \\
\hline ) & & & \\
\hline & & & \\
\hline & & & \\
\hline & 1.9 & & \\
\hline C & 1.0 & & \\
\hline & -1.0 & & \\
\hline & & & \\
\hline & & & \\
\hline & 3.02 & & \\
\hline & & & \\
\hline $\mathrm{C}$ & 2.3 & 4 & \\
\hline $\mathrm{C}$ & 0.7 & & \\
\hline C & & & \\
\hline 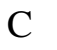 & 072 & -3.5 & 970 \\
\hline & & & \\
\hline & 9 & 2.5 & \\
\hline $\mathrm{C}$ & 2 & & \\
\hline $\mathrm{C}$ & & & \\
\hline $\mathrm{H}$ & 1.242552 & & 6776 \\
\hline $\mathrm{C}$ & 3.383668 & -1.9 & 3310 \\
\hline $\mathrm{H}$ & & & \\
\hline & & & \\
\hline$\sigma$ & & & \\
\hline $\mathrm{C}$ & $-0.5 \varepsilon$ & & \\
\hline $\mathrm{C}$ & -1.7 & -3.834455 & 2.200627 \\
\hline $\mathrm{C}$ & -3.038712 & -4.706686 & 0.26881 \\
\hline 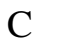 & -2.348281 & -4.340093 & -2.01730 \\
\hline
\end{tabular}




$\begin{array}{lrcc}\mathrm{H} & -0.544370 & -3.177334 & -2.260849 \\ \mathrm{H} & 2.535380 & 3.191812 & 3.289382 \\ \mathrm{C} & 3.428486 & 2.917430 & 1.333898 \\ \mathrm{C} & 4.305018 & 2.500177 & -0.912575 \\ \mathrm{C} & 4.514214 & -1.571431 & -2.343069 \\ \mathrm{C} & 5.483160 & -0.412791 & -0.416174 \\ \mathrm{H} & 3.526843 & -2.590759 & -3.981884 \\ \mathrm{H} & 4.322808 & -4.232506 & 0.777663 \\ \mathrm{C} & 3.072056 & -4.513812 & 2.516764 \\ \mathrm{H} & 1.584006 & -4.696259 & 4.058603 \\ \mathrm{H} & -0.631933 & -3.744500 & 3.958150 \\ \mathrm{H} & -2.660271 & -3.939831 & 2.794707 \\ \mathrm{H} & -3.731235 & -5.144648 & 0.982659 \\ \mathrm{C} & -3.243409 & -4.891141 & -1.095150 \\ \mathrm{H} & -2.495370 & -4.488424 & -3.082910 \\ \mathrm{C} & 4.163427 & 4.133062 & 1.358085 \\ \mathrm{C} & 4.986323 & 3.697636 & -0.867613 \\ \mathrm{H} & 4.359248 & 1.888545 & -1.805368 \\ \mathrm{C} & 5.811607 & -2.056270 & -2.662079 \\ \mathrm{C} & 6.723836 & -0.916662 & -0.741059 \\ \mathrm{H} & 5.371812 & 0.201589 & 0.468931 \\ \mathrm{H} & 3.853297 & -4.977287 & 3.111835 \\ \mathrm{H} & -4.094022 & -5.471611 & -1.439889 \\ \mathrm{H} & 4.095540 & 4.761770 & 2.242252 \\ \mathrm{C} & 4.931779 & 4.517876 & 0.283502 \\ \mathrm{H} & 5.571920 & 4.013973 & -1.726122 \\ \mathrm{H} & 5.925523 & -2.700487 & -3.530142 \\ \mathrm{C} & 6.898012 & -1.733985 & -1.882432 \\ \mathrm{H} & 7.577441 & -0.686802 & -0.109865 \\ \mathrm{H} & 5.486389 & 5.451241 & 0.310825 \\ \mathrm{H} & 7.884124 & -2.114775 & -2.131124 \\ \mathrm{C} & -2.343335 & 0.768199 & 3.250768 \\ \mathrm{H} & -1.850802 & -0.188016 & 3.461487 \\ \mathrm{H} & -1.995162 & 1.514591 & 3.973162 \\ \mathrm{H} & -3.420274 & 0.622289 & 3.395980 \\ \mathrm{C} & 0.347261 & 5.291815 & 1.121228 \\ \mathrm{O} & -0.685546 & 3.837519 & 2.460667 \\ \mathrm{C} & 0.004233 & 5.004522 & 2.403601 \\ \mathrm{H} & 0.152742 & 5.507893 & 3.347212 \\ \mathrm{H} & 0.903566 & 6.152912 & 0.777066 \\ \mathrm{C} & -7.015461 & 0.628578 & -2.026426 \\ \mathrm{H} & -6.549652 & 0.040837 & -2.824304 \\ \mathrm{H} & -7.916276 & 0.128530 & -1.670274 \\ & -7.275359 & 1.604743 & -2.449526\end{array}$

TS5

M06-2X/6-31+G(d) SCF energy: $\quad-2998.22885656$ a.u.

M06-2X/6-31+G(d) enthalpy: $\quad-2997.415508$ a.u.

M06-2X/6-31+G(d) free energy: -2997.561581 a.u. 
M06-2X/6-311++G(d,p) SCF energy in solution: -2998.28933249 a.u.

M06-2X/6-311++G(d,p) enthalpy: $\quad-2997.475984$ a.u.

M06-2X/6-311++G(d,p) free energy: $\quad-2997.622057$ a.u.

Imaginary frequency: $\quad-201.5608 \mathrm{~cm}-1$

Cartesian coordinates

$\begin{array}{lccc}\text { ATOM } & \text { X } & \text { Y } & \text { Z } \\ \text { C } & -1.725990 & 1.246895 & -1.905900 \\ \text { C } & -2.074027 & 0.065360 & -2.252742 \\ \text { C } & -0.804555 & 2.287878 & -2.203734 \\ \text { C } & -0.489890 & 3.569605 & -1.781377 \\ \text { C } & -1.714332 & 3.643422 & 0.480545 \\ \text { H } & -1.857979 & 4.331299 & 1.322542 \\ \text { C } & -3.052077 & 3.088110 & 0.070807 \\ \text { C } & -3.229049 & 1.839392 & -0.361713 \\ \text { C } & -4.242548 & 0.901943 & -0.599292 \\ \text { H } & -4.532096 & 0.585407 & -1.595914 \\ \text { O } & -5.267863 & 0.832118 & 0.340482 \\ \text { C } & -6.194925 & -0.177959 & 0.161976 \\ \text { O } & -6.078027 & -1.009459 & -0.704420 \\ \text { H } & -1.051631 & 2.845671 & 0.822470 \\ \text { C } & -1.035960 & 4.418148 & -0.665757 \\ \text { H } & -1.731190 & 5.164059 & -1.076197 \\ \text { H } & -0.200930 & 4.991445 & -0.245775 \\ \text { Rh } & -2.380744 & -0.194821 & -0.154021 \\ \text { C } & -3.171630 & -2.021649 & -0.420282 \\ \text { O } & -3.620922 & -3.051750 & -0.619526 \\ \text { C } & -4.222509 & 4.047435 & 0.070853 \\ \text { H } & -5.134028 & 3.595601 & -0.325281 \\ \text { H } & -3.991201 & 4.941243 & -0.522816 \\ \text { H } & -4.422452 & 4.387917 & 1.095306 \\ \text { C } & -2.604549 & -0.022442 & 1.803001 \\ \text { O } & -2.792709 & 0.078185 & 2.925910 \\ \text { P } & -0.029168 & -1.014083 & 0.175437 \\ \text { O } & 0.774732 & -0.582614 & 1.551980 \\ \text { O } & 0.842016 & -0.360668 & -1.035011 \\ \text { N } & 0.288354 & -2.686709 & 0.238692 \\ \text { C } & 1.104047 & 0.758117 & 1.754658 \\ \text { C } & 2.239815 & -0.212655 & -1.003163 \\ \text { C } & 0.756624 & -3.448005 & -0.890221 \\ \text { C } & 0.280025 & -3.350772 & 1.521171 \\ \text { C } & 0.486762 & 1.406598 & 2.846831 \\ \text { C } & 2.059973 & 1.365166 & 0.952247 \\ \text { C } & 2.849651 & 0.576081 & -0.040296 \\ \text { C } & 2.941437 & -0.834479 & -2.056292 \\ \text { C } & -0.122023 & -3.782485 & -1.924791 \\ \text { C } & 2.108442 & -3.854886 & -0.937289 \\ \text { C } & 1.504368 & -3.687796 & 2.137435 \\ \text { C } & -0.934421 & -3.615953 & 2.158805 \\ \text { H } & -0.185385 & 0.837263 & 3.479270 \\ \text { C } & 0.778196 & 2.723627 & 3.103229\end{array}$




\begin{tabular}{|c|c|c|c|}
\hline C & 276454 & 779407 & 1.143159 \\
\hline & 4.294935 & .594248 & -0.026484 \\
\hline & 380689 & -1.386586 & -2.801630 \\
\hline & 307878 & -0.723703 & -2.110970 \\
\hline & -1.168601 & -3.512720 & -1 \\
\hline & 0.334094 & -4.470617 & -3.049181 \\
\hline & .545819 & -4.543790 & 5191 \\
\hline & .057173 & -3.610587 & 362 \\
\hline & .796848 & -3.533163 & 636 \\
\hline & 458085 & -4.23 & \\
\hline & -0.955086 & -4 & 607 \\
\hline & 1.863097 & -3.391095 & 1754 \\
\hline & 0.330664 & 3.228493 & 100 \\
\hline & 640780 & 3.452216 & \\
\hline & 3.07 & 3.5 & \\
\hline & 30 & -0.0 & \\
\hline & 5.04 & & \\
\hline & 4.861909 & -1. & 771 \\
\hline & 0.35 & -4.71 & 938 \\
\hline & 1.678059 & -4.84 & 536 \\
\hline & 31 & -4. & -2 \\
\hline & 3 & -3 & \\
\hline & 3.6 & -3. & \\
\hline & 2.39 & -4 & \\
\hline & 0.24 & -4.4 & 27 \\
\hline & 1.903892 & -4.3 & 970 \\
\hline & 1.87 & 4.8 & 28 \\
\hline & 03 & & \\
\hline $\mathrm{H}$ & 3.53 & 3.0 & -0 . \\
\hline E & 6.44 & -0.0 & -1. \\
\hline & 6.424766 & & \\
\hline & 4.52 & 1.6 & 83 \\
\hline & 2.04 & -5.3 & 267 \\
\hline & 0.24 & -4. & \\
\hline & 1.39 & 5.3 & \\
\hline C & 2.66 & & \\
\hline & 3.87 & & 085 \\
\hline & 6.971498 & -0.4 & 7154 \\
\hline & 7.13 & 0.5 & -0.0 \\
\hline & 6.97 & & 03 \\
\hline $\mathrm{H}$ & 2.83 & 6.6 & 71 \\
\hline 11 & 8.21 & 0.6 & -0.0 \\
\hline $\mathrm{C}$ & -2.292186 & -0.827901 & -3.415702 \\
\hline $\mathrm{H}$ & -1.628977 & -1.698588 & -3.360691 \\
\hline & -3.323460 & -1.198092 & $\$ 751$ \\
\hline & -2.079005 & -0.2 & -4.3 \\
\hline $\mathrm{C}$ & & & \\
\hline $\mathrm{O}$ & 0.050864 & 1.923104 & -3.23 \\
\hline 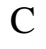 & 0.863046 & 2.973937 & -3.472918 \\
\hline 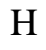 & 1.573617 & 2.847496 & -4.276349 \\
\hline 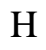 & 1.097974 & 4.949561 & -2.561775 \\
\hline
\end{tabular}




$\begin{array}{rrrr}\mathrm{C} & -7.303185 & -0.066215 & 1.171373 \\ \mathrm{H} & -7.834657 & 0.882378 & 1.039699 \\ \mathrm{H} & -7.995701 & -0.897863 & 1.040284 \\ \mathrm{H} & -6.895030 & -0.072913 & 2.187355\end{array}$

\section{TS6}

M06-2X/6-31+G(d) SCF energy: $\quad-1256.08762791$ a.u.

M06-2X/6-31+G(d) enthalpy: $\quad-1255.760675$ a.u.

M06-2X/6-31+G(d) free energy: -1255.848277 a.u.

M06-2X/6-311++G(d,p) SCF energy in solution: -1256.80075791 a.u.

M06-2X/6-311++G(d,p) enthalpy: $\quad-1256.473805$ a.u.

M06-2X/6-311++G(d,p) free energy: $\quad-1256.561407$ a.u.

Imaginary frequency: $\quad-222.9708 \mathrm{~cm}-1$

Cartesian coordinates

$\begin{array}{lccc}\text { ATOM } & \text { X } & \text { Y } & \text { Z } \\ \text { C } & 1.374742 & -0.314128 & -0.942004 \\ \text { C } & 0.555040 & -1.249124 & -1.281648 \\ \text { C } & 2.733282 & -0.032594 & -0.709152 \\ \text { C } & 3.521017 & 1.023457 & -0.255499 \\ \text { C } & 1.832297 & 2.363930 & 1.115954 \\ \text { H } & 1.814115 & 3.275574 & 1.723499 \\ \text { C } & 0.606752 & 2.335435 & 0.244261 \\ \text { C } & -0.015724 & 1.216671 & -0.148974 \\ \text { C } & -1.222283 & 0.854610 & -0.790150 \\ \text { H } & -1.244033 & 0.477351 & -1.808499 \\ \text { O } & -2.367669 & 1.549038 & -0.423840 \\ \text { C } & -3.560686 & 1.082266 & -0.964484 \\ \text { O } & -3.602879 & 0.074752 & -1.624516 \\ \text { H } & 1.857745 & 1.511855 & 1.801810 \\ \text { C } & 3.124560 & 2.377751 & 0.256467 \\ \text { H } & 3.006764 & 3.084570 & -0.575328 \\ \text { H } & 3.951370 & 2.763458 & 0.862045 \\ \text { Rh } & -0.599059 & -0.803646 & 0.451238 \\ \text { C } & -1.500185 & -0.029757 & 2.077863 \\ \text { O } & -2.005188 & 0.441693 & 2.982276 \\ \text { C } & 0.153928 & 3.670159 & -0.305331 \\ \text { H } & -0.659968 & 3.577176 & -1.025961 \\ \text { H } & 0.990207 & 4.193007 & -0.787955 \\ \text { H } & -0.185329 & 4.310584 & 0.519405 \\ \text { C } & 0.598941 & -2.085577 & 1.581467 \\ \text { O } & 1.299023 & -2.794053 & 2.130261 \\ \text { C } & 0.296284 & -2.209560 & -2.385323 \\ \text { H } & -0.641656 & -1.975494 & -2.903236 \\ \text { H } & 1.113441 & -2.184624 & -3.113568 \\ \text { H } & 0.204746 & -3.228903 & -1.993047 \\ \text { C } & 4.856997 & 0.533466 & -0.245324 \\ \text { O } & 3.547623 & -1.139024 & -0.935236 \\ \text { C } & 4.812241 & -0.762490 & -0.677681\end{array}$




$\begin{array}{lrrc}\mathrm{H} & 5.737910 & 1.082698 & 0.056975 \\ \mathrm{H} & 5.573038 & -1.510250 & -0.849464 \\ \mathrm{C} & -4.699922 & 1.990668 & -0.602899 \\ \mathrm{H} & -5.632703 & 1.562719 & -0.970432 \\ \mathrm{H} & -4.749302 & 2.129349 & 0.481911 \\ \mathrm{H} & -4.545604 & 2.978149 & -1.051451 \\ \mathrm{C} & -2.067390 & -2.118988 & -0.067424 \\ \mathrm{O} & -2.874962 & -2.852448 & -0.388083\end{array}$

MonoPhos-alkene (8)

M06-2X/6-31+G(d) SCF energy: $\quad-1855.43782154$ a.u.

M06-2X/6-31+G(d) enthalpy: $\quad-1854.943673$ a.u.

M06-2X/6-31+G(d) free energy: -1855.030957 a.u.

M06-2X/6-311++G(d,p) SCF energy in solution: -1854.75396705 a.u.

M06-2X/6-311++G(d,p) enthalpy: $\quad-1854.259819$ a.u.

M06-2X/6-311++G(d,p) free energy: $\quad-1854.347103$ a.u.

Cartesian coordinates

\begin{tabular}{lrrr} 
ATOM & X & Y & \multicolumn{2}{l}{ Z } \\
P & 1.154191 & -1.359782 & -0.460104 \\
O & 0.142305 & -1.247467 & 0.904887 \\
O & 0.279449 & -0.350952 & -1.475846 \\
N & 2.465248 & -0.422086 & 0.114430 \\
C & -1.136291 & -1.741421 & 0.695001 \\
C & -0.568288 & 0.675042 & -1.096526 \\
C & 3.730460 & -1.101267 & 0.162965 \\
C & 2.433806 & 0.953465 & 0.522269 \\
C & -1.428169 & -3.040745 & 1.170000 \\
C & -2.076558 & -0.956752 & 0.041415 \\
C & -1.716171 & 0.434628 & -0.351153 \\
C & -0.244831 & 1.961706 & -1.588277 \\
C & 3.883829 & -2.198666 & 1.015984 \\
C & 4.784264 & -0.713016 & -0.694297 \\
C & 3.134810 & 1.923140 & -0.231649 \\
C & 1.678779 & 1.336944 & 1.636077 \\
H & -0.650965 & -3.583200 & 1.698886 \\
C & -2.674002 & -3.578265 & 0.963948 \\
C & -3.356786 & -1.545987 & -0.256372 \\
C & -2.503863 & 1.571841 & 0.055998 \\
H & 0.641865 & 2.064607 & -2.203802 \\
C & -1.039711 & 3.034821 & -1.279569 \\
H & 3.063688 & -2.460484 & 1.678058 \\
C & 5.060059 & -2.949083 & 1.006969 \\
C & 5.958513 & -1.488146 & -0.689467 \\
C & 4.706281 & 0.457129 & -1.566397 \\
C & 3.996590 & 1.589493 & -1.365504 \\
C & 3.005829 & 3.273672 & 0.143651 \\
C & 1.574601 & 2.681543 & 1.990285 \\
H & 1.153969 & 0.573482 & 2.197295
\end{tabular}




$\begin{array}{lrrr}\mathrm{H} & -2.912736 & -4.571902 & 1.334783 \\ \mathrm{C} & -3.657662 & -2.862957 & 0.230266 \\ \mathrm{C} & -4.334484 & -0.896478 & -1.061426 \\ \mathrm{C} & -2.168715 & 2.878844 & -0.432090 \\ \mathrm{C} & -3.591410 & 1.466213 & 0.968386 \\ \mathrm{H} & -0.797545 & 4.024477 & -1.658669 \\ \mathrm{H} & 5.158482 & -3.809108 & 1.663254 \\ \mathrm{C} & 6.100052 & -2.592576 & 0.146449 \\ \mathrm{H} & 6.770424 & -1.207212 & -1.355996 \\ \mathrm{H} & 5.377218 & 0.438316 & -2.423724 \\ \mathrm{H} & 4.151651 & 2.399813 & -2.076527 \\ \mathrm{H} & 3.532264 & 4.027499 & -0.437221 \\ \mathrm{C} & 2.236924 & 3.654612 & 1.238642 \\ \mathrm{H} & 0.969298 & 2.966760 & 2.846020 \\ \mathrm{C} & -4.924139 & -3.441068 & -0.054853 \\ \mathrm{C} & -5.546350 & -1.492471 & -1.333959 \\ \mathrm{H} & -4.111372 & 0.079669 & -1.475545 \\ \mathrm{C} & -2.953467 & 3.996168 & -0.040247 \\ \mathrm{C} & -4.323263 & 2.572572 & 1.342222 \\ \mathrm{H} & -3.837950 & 0.496832 & 1.385236 \\ \mathrm{H} & 7.018556 & -3.172461 & 0.126879 \\ \mathrm{H} & 2.156019 & 4.704445 & 1.506465 \\ \mathrm{H} & -5.137922 & -4.434781 & 0.332031 \\ \mathrm{C} & -5.854665 & -2.772488 & -0.816343 \\ \mathrm{H} & -6.270349 & -0.975720 & -1.958243 \\ \mathrm{H} & -2.688774 & 4.976018 & -0.431266 \\ \mathrm{C} & -4.013714 & 3.851947 & 0.825110 \\ \mathrm{H} & -5.143293 & 2.460261 & 2.046539 \\ \mathrm{H} & -6.816832 & -3.228376 & -1.033038 \\ \mathrm{H} & -4.602864 & 4.715395 & 1.121754\end{array}$

\section{CO}

M06-2X/6-31+G(d) SCF energy: $\quad-113.3069125$ a.u.

M06-2X/6-31+G(d) enthalpy: $\quad-113.298571$ a.u.

M06-2X/6-31+G(d) free energy: -113.321014 a.u.

M06-2X/6-311++G(d,p) SCF energy in solution: -113.2801719 a.u.

M06-2X/6-311++G(d,p) enthalpy: $\quad-113.2718304$ a.u.

M06-2X/6-311++G(d,p) free energy: $\quad-113.2942734$ a.u.

Cartesian coordinates

\begin{tabular}{lrrr} 
ATOM & X & Y & \multicolumn{2}{l}{ Z } \\
C & 0.000000 & 0.000000 & -0.650136 \\
O & 0.000000 & 0.000000 & 0.487602
\end{tabular}


1. Kelly, A. R.; Kerrigan, M. H.; Walsh, P. J., Addition/Oxidative Rearrangement of 3-Furfurals and 3-Furyl Imines: New Approaches to Substituted Furans and Pyrroles. J. Am. Chem. Soc. 2008, 130, 40974104.

2. Mironov, M. E.; Bagryanskaya, I. Y.; Shults, E. E., Synthesis of 3-trimethylsiloxy-1-(furan-3yl)butadiene and its Reactions with Dienophiles. Chem. Heterocycl Compd. 2016, 52, 364-373.

3. Fujii, M.; Aida, T.; Yoshihara, M.; Ohno, A., NAD(P)+-NAD(P)H Models. 71. A Convenient Route to the Synthesis of Juvabione. Bull. Chem. Soc. Jpn. 1990, 63, 1255-1257.

4. $\quad$ Nakamura, K.; Fujii, M.; Ohno, A.; Oka, S., NAD(P)+-NAD(P)H model. 52. Reduction of Olefins by Hantzsch Ester on Silica Gel. Tetrahedron Lett. 1984, 25, 3983-3986.

5. $\quad$ Burrows, L. C.; Jesikiewicz, L. T.; Lu, G.; Geib, S. J.; Liu, P.; Brummond, K. M., Computationally Guided Catalyst Design in the Type I Dynamic Kinetic Asymmetric Pauson-Khand Reaction of Allenyl Acetates. J. Am. Chem. Soc. 2017, 139, 15022-15032.

6. Kajiro, H.; Mitamura, S.; Mori, A.; Hiyama, T., Scandium Trifluoromethanesulfonate-Catalyzed Cleavage of Esters Bearing a Coordinative Group at a Vicinal Position. Bull. Chem. Soc. Jpn. 1999, 72, 1553-1560.

7. Dai, H.; Yu, S.; Cheng, W.; Xu, Z.-F.; Li, C.-Y., Rhodium-Catalyzed Synthesis of 1,2dihydropyridine by a Tandem Reaction of 4-(1-acetoxyallyl)-1-sulfonyl-1,2,3-triazole. Chem. Commun. 2017, 53, 6417-6420.

8. Lalonde, M. P.; McGowan, M. A.; Rajapaksa, N. S.; Jacobsen, E. N., Enantioselective Formal Aza-Diels-Alder Reactions of Enones with Cyclic Imines Catalyzed by Primary Aminothioureas. J. Am. Chem. Soc. 2013, 135, 1891-1894.

9. $\quad$ Frisch, M. J.; Trucks, G. W.; Schlegel, H. B.; Scuseria, G. E.; Robb, M. A.; Cheeseman, J. R.; Scalmani, G.; Barone, V.; Mennucci, B.; Petersson, G. A.; Nakatsuji, H.; Caricato, M.; Li, X.; Hratchian, H. P.; Izmaylov, A. F.; Bloino, J.; Zheng, G.; Sonnenberg, J. L.; Hada, M.; Ehara, M.; Toyota, K.; Fukuda, R.; Hasegawa, J.; Ishida, M.; Nakajima, T.; Honda, Y.; Kitao, O.; Nakai, H.; Vreven, T.; Montgomery, J. A., Jr.; Peralta, J. E.; Ogliaro, F.; Bearpark, M.; Heyd, J. J.; Brothers, E.; Kudin, K. N.; Staroverov, V. N.; Kobayashi, R.; Normand, J.; Raghavachari, K.; Rendell, A.; Burant, J. C.; Iyengar, S. S.; Tomasi, J.; Cossi, M.; Rega, N.; Millam, J. M.; Klene, M.; Knox, J. E.; Cross, J. B.; Bakken, V.; Adamo, C.; Jaramillo, J.; Gomperts, R.; Stratmann, R. E.; Yazyev, O.; Austin, A. J.; Cammi, R.; Pomelli, C.; Ochterski, J. W.; Martin, R. L.; Morokuma, K.; Zakrzewski, V. G.; Voth, G. A.; Salvador, P.; Dannenberg, J. J.; Dapprich, S.; Daniels, A. D.; Farkas, O.; Foresman, J. B.; Ortiz, J. V.; Cioslowski, J.; Fox, D. J. Gaussian 09, Revision D.01, Gaussian, Inc., Wallingford CT, 2013.

10. Towns, J.; Cockerill, T.; Dahan, M.; Foster, I.; Gaither, K.; Grimshaw, A.; Hazlewood, V.; Lathrop, S.; Lifka, D.; Peterson, G.D.; Roskies, R.; Scott, J.R.; Wilkins-Diehr, N. XSEDE: Accelerating Scientific Discovery. Comput. Sci. Eng. 2014, 16, 62-74.

11. Becke, A.D., A new mixing of Hartree-Fock and local density-functional theories. J. Chem. Phys. 1993, 98, 1372-1377.

12. Zhao, Y.; Truhlar, D. G., The M06 suite of density functionals for main group thermochemistry, thermochemical kinetics, noncovalent interactions, excited states, and transition elements: two new functionals and systematic testing of four M06-class functionals and 12 other functionals. Theor. Chem. Acc. 2008, 120, 215-241.

13. Zhao, Y.; Truhlar, D. G., Density Functionals with Broad Applicability in Chemistry. Acc. Chem. Res. 2008, 41, 157-167.

14. Marenich, A. V.; Cramer, C. J.; Truhlar, D. G., Universal Solvation Model Based on Solute Electron Density and on a Continuum Model of the Solvent Defined by the Bulk Dielectric Constant and Atomic Surface Tensions. J. Phys. Chem. B 2009, 113, 6378-6396. 